\title{
GEOS SEISMOGRAMS RECORDED FOR AFTERSHOCKS OF THE EARTHQUAKES OF DECEMBER 7, 1988, NEAR SPITAK, ARMENIA SSR, DURING THE TIME PERIOD \\ 3 JANUARY 1989 THROUGH 2 FEBRUARY 1989 (UTC)
}

\author{
compiled by \\ Gary Glassmoyer and Edward Cranswick
}

\author{
Volume V \\ of
}

Results and Data from Seismologic and Geologic Studies Following Earthquakes of December 7, 1988, near Spitak, Armenia SSR, R. D. Borcherdt, editor.

\section{OPEN-FILE REPORT 89-163E}

This report is preliminary and has not been reviewed for conformity with U.S. Geological Survey editorial standards and stratigraphic nomenclature. Any use of trade names is for descriptive purposes only and does not imply endorsement by the USGS. 


\section{Preface}

The earthquakes of December 7, 1988, near Spitak, Armenia SSR, serve as another grim reminder of the serious hazard that earthquakes pose throughout the world. We extend our heartfelt sympathies to the families of the earthquake victims and intend that our cooperative scientific endeavours will help reduce losses in future earthquakes. Only through a better understanding of earthquake hazards can earthquake losses be reduced for all peoples in seismically active regions of the world.

The tragic consequences of these earthquakes remind scientists and public officials alike of their urgent responsibilities to understand and mitigate the effects of earthquakes. On behalf of the U.S. Geological Survey, I would like to express appreciation to our Soviet colleagues for their kind invitation to participate in joint scientific and engineering studies. Without their cooperation and generous assistance, the conduct of these studies would not have been possible.

This report provides seismologic and geologic data collected during the time period December 21, 1988, through February 2, 1989. These data are presented in their entirety to expedite analysis of the data set for inferences regarding hazard mitigation actions, applicable not only in Armenia but other regions of the world exposed to high seismic risk.

Dallas L. Peck

Director, United States Geological Survey 


\section{FORWARD}

This volume represents one of a five-part set, which present preliminary results and data for the seismologic and geologic investigations conducted following the earthquakes of December 7, 1988, near Spitak, Armenia SSR. The first volume of this set describes the field experiments, instrumentation, the data set, formats for data dissemination, and preliminary conclusions permitted by the data analyzed as of this writing. Volumes II, III, IV and V present the seismograms for the digital recordings of aftershocks obtained during the time periods indicated. Because of limited computer facilities and data format incompatibilities, the digital-event recordings are presented as analog seismograms. The seismograms are included to expedite analysis of the data for developing appropriate hazard mitigation measures for future potentially damaging earthquakes.

This data set is a testament to the generous cooperation and assistance provided by our colleagues in the Union of Soviet Socialist Republics. Without their contributions this data set would not have been possible. For their contributions, we express our most sincere appreciation.

Roger D. Borcherdt

March 10, 1989 


\section{INTRODUCTION}

This report provides seismograms corresponding to the digital recordings of the aftershock sequence of the earthquakes of December 7, 1988, near Spitak, Armenia SSR. This volume includes those seismograms recorded during the time period 3 January 1989 through 2 February 1989 (UTC). Seismograms obtained during other time periods are included in companion volumes.

Three types of seismograms are included: those corresponding to the recordings of the three-component velocity transducers, those corresponding to some recordings of the three-component force-balance accelerometers, and those resulting from the integration of the accelerometer recordings. These three types of seismograms are presented successively in each volume. The seismograms in each set are arranged chronologically.

The seismograms corresponding to the accelerometer recordings have been included to provide on-scale recordings for those events for which the velocity transducer recordings exceeded full-scale amplitude (32,767 digital counts) of the analog-to-digital converter at the gain selected for the field recorders. Integrated versions of these recordings are provided to expedite comparison with other velocity recordings provided for the events. Any recognized uncertainties in station locations or event timing are summarized in the data description provided in Volume I.

\section{SEISMOGRAM LISTING}

During data playback and processing, seismograms are identified in the computer by a 13-character filename (constructed from the start-of-record time, uncorrected for clock drift, component, and station name): characters 1-3 = Julian day (001-366), characters 4$5=$ hour $(00-23)$, characters $6-7=$ minute $(00-59)$, character $8=$ second code $(A-T$, where $\mathrm{A}=0.000-2.999, \mathrm{~B}=3.000-5.999, \ldots, \mathrm{T}=57.000-59.999)$, character $9=$ component code (1-3 for acceleration, 4-6 for velocity), character $10=$ ".", and characters $11-13=$ station name. The seismogram data set listings in Table 1 (velocity), Table 2 (acceleration), and Table 3 (integrated acceleration) are based on this filenaming scheme. In the tables, each line summarizes one event, which is generally identified by a computer algorithm that counts triggers in a sliding time window (22 seconds long, this report) and/or by direct 
inspection of data plots. Events are specified in the left column by the filename time of the earliest recording (rather than the actual event origin time, which is generally unknown at early stages of data processing), and recordings are specified in the station columns by the filename second-code character. For example, an aftershock at Julian day 359, hour 14, minute 14 was recorded at stations ART, KIR, MOO, NAB (with start-of-record time in second-bin $\mathrm{Q}=48.000-50.999$ ), and $\mathrm{KET}$ (with start-of-record time in second-bin $\mathrm{R}=$ 51.000-53.999). This aftershock is identified in Table 1 as 3591414Q. Station locations are mapped in Figure 1.

\section{SEISMOGRAM LABELS}

Each three-component seismogram is identified by a representation of the filename (e.g., 3561633PV.GSS) which corresponds to the earthquake identification as described above. The seismograms as arranged, proceeding from the top of the plot, represent recordings of the vertical, and the two horizontal seismometer channels. Orientations of the horizontal seismometers were obtained by operators in the field at time of sensor installation with a Brunton compass. Such installations are usually accurate to within 4-5 degrees. (Orientations have not been checked for consistency using polarization analyses.) Orientations for each seismogram are indicated by a two-number code (e.g., 090/164) with the numbers indicating direction of positive motion (up on plots) from up (e.g., 090) and clockwise from North (e.g., 164) measured in degrees.

Labels on the top of each plot identify the parameters used to plot and record the data. The parameters include: 1) start time of the record (year, Julian day, hour, minute, and second of the internal clock, and the clock correction in seconds), 2) duration of plot (seconds unless otherwise stated), 3) sampling rate (samples per second), 4) number of the event on the tape, 5) station latitude and longitude (degrees and minutes), 6) elevation (not used here), 7) GEOS unit number and tape number, 8) number of channels recorded and total number of samples recorded per channel, 9) orientation of positive direction for each sensor (degrees), 10) sample lag (seconds), 11) sensor serial number (not used here), 12) sensor natural frequency $(\mathrm{Hz}), 13)$ sensor damping (fraction of critical), 14) sensor sensitivity (volts/ground motion unit), 15) gain (decibels), 16) low-cut filter parameters 
(corner frequency $(\mathrm{Hz})$ and number of poles, e.g., $\mathrm{L} 1, .10$ indicates single-pole low-cut filter with corner at $0.10 \mathrm{~Hz}$ ), and 17) high-cut anti-aliasing filter parameters (e.g., H7,50 indicates seven-pole Butterworth with corner at $50 \mathrm{~Hz}$ ).

Labels for the ordinates of each trace include: 1) maximum ground motion value for time interval plotted, 2) direction of positive ground motion, 3) maximum digital counts for time histories. Tick marks on the ordinate scale are provided in powers of two; however, the time histories themselves are plotted on a linear scale defined by the indicated maximum ground-motion value. Absolute time (clock-drift corrected; in seconds) of the start of the plotted record is to the left of the lower time axis.

\section{ACKNOWLEDGMENTS}

The authors would like to acknowledge those that expended tremendous effort on the coordination, experiment execution, and acquisition of the data sets reported herein. In addition to our colleagues who participated on the initial U.S. Investigation Team and on Soviet teams, we would like to acknowledge the efforts of Chuck Mueller, Tom Noce, and Mary Andrews, who upon subsequent visits helped maintain the instrumentation after the departure of the original team on January 6, 1989. Gary Maxwell provided several computer programs necessary for processing the digital data. Howard Bundock contributed the digitized map data used in Figure 1. Chuck Mueller also provided the computer program used to create the seismogram listing, its description, and a review of this report. 


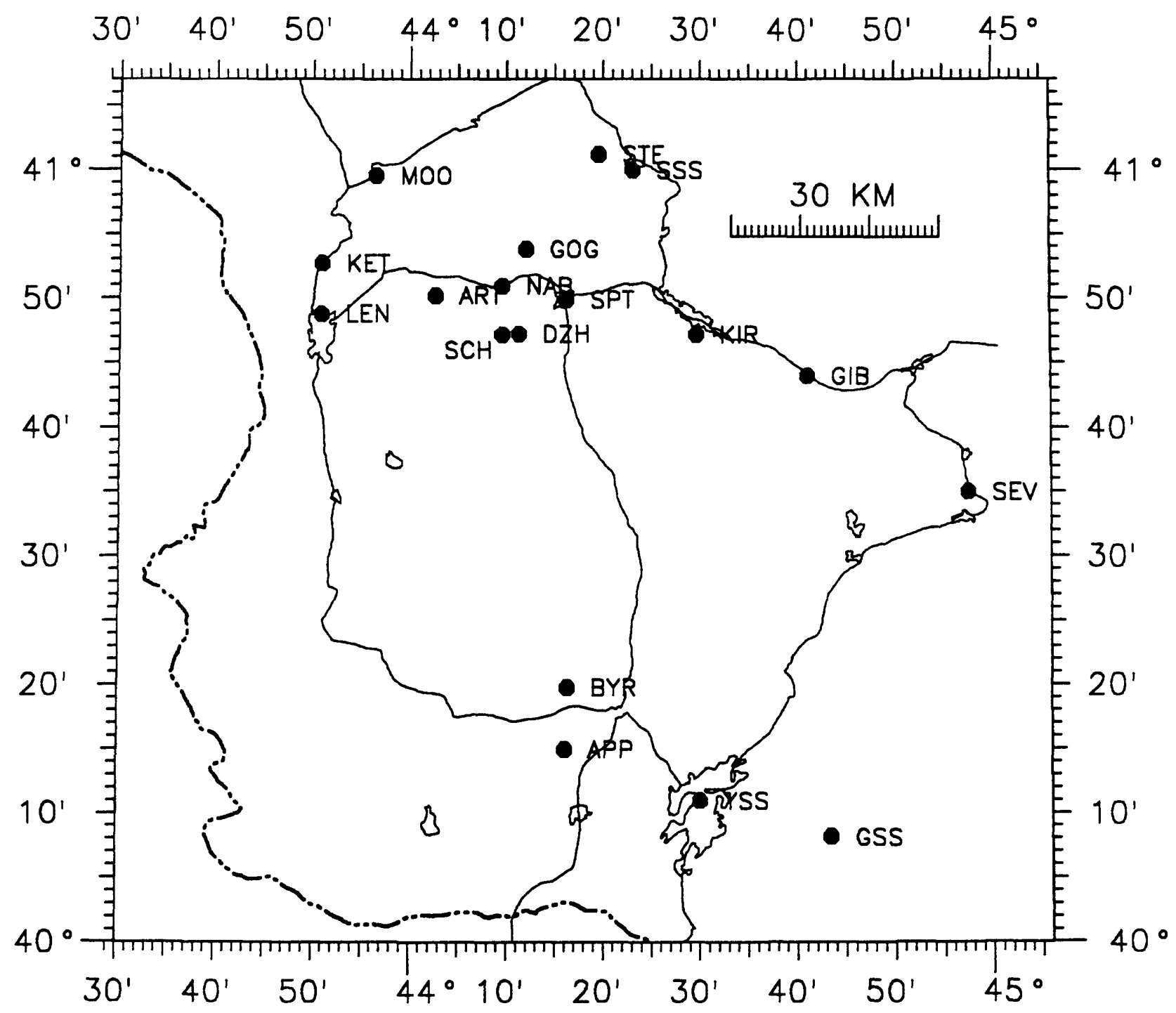

Figure 1. Location map for deployment sites of digital recorders (GEOS) used during the time period of December 21, 1988 through about March 1, 1989. Time periods for deployment at each site are given in the first volume of this report. Cities, roads, ArmeniaTurkey international boundary, and three-letter station identification codes are indicated on the map. 
Table 1 - Listing of seismograms derived from the digital recordings of the velocity transducers.

$0030021 \mathrm{~S}$ $0030041 \mathrm{~K}$ $0030117 \mathrm{~L}$ 00301208 00301260 $0030201 \mathrm{P}$ $0030238 R$ 00303220 $0030334 \mathrm{~F}$ 00303460 $0030445 \mathrm{~J}$ $0030452 \mathrm{~L}$ $0030512 \mathrm{~F}$ $0030525 \mathrm{~A}$ $0030531 \mathrm{M}$ $0030623 \mathrm{~A}$ $0030720 I$ $0030747 \mathrm{E}$ $0030819 \mathrm{~S}$ 00308210 $0030827 I$ $0030836 \mathrm{G}$ $0030927 \mathrm{~A}$ $0031328 \mathrm{~F}$ $0031335 \mathrm{C}$ $0031348 \mathrm{P}$ $0031445 \mathrm{C}$ 0031600R $0031653 L$ $0031705 \mathrm{M}$ $0031810 \mathrm{~F}$ $0031941 \mathrm{~J}$ 0032000E O032050F $0032133 \mathrm{G}$ $0032138 \mathrm{~J}$ $0032229 \mathrm{~T}$ $0032303 \mathrm{~J}$ 00323271 $0040127 \mathrm{C}$ $0040154 \mathrm{~A}$ $0040209 B$ $0040247 \mathrm{~A}$ $0040250 I$ $0040258 \mathrm{P}$ 00404562 $0040601 \mathrm{~S}$ $0040729 \mathrm{~N}$ $0040735 \mathrm{M}$ $0040738 \mathrm{~J}$ 0040740P 0040809E $0040901 \mathrm{~T}$ $0041016 \mathrm{H}$ $0041024 \mathrm{P}$ $0041029 \mathrm{G}$ $0041030 \mathrm{~J}$ $0041055 \mathrm{D}$ 00411220 $0041724 \mathrm{H}$ $0041726 \mathrm{D}$ $0042356 \mathrm{E}$ 0050815E $0051354 \mathrm{~S}$ 0051530I 0051851B $0051917 \mathrm{~A}$ $0060322 \mathrm{~K}$ $0060735 \mathrm{~T}$ $0060827 \mathrm{E}$ $0061214 \mathrm{P}$ $0061601 \mathrm{~s}$
APP ART BYR DZH GIB GOG GSS KET KIR KI2 LEN MOO MO2 NAB SCH SEV SPT SSS STE YSS

$$
\mathrm{T}
$$

Q

$\mathbf{R}$

F

\section{$\mathrm{S}$}

o

0
L

$\mathbf{N}$

A

T

S

$T$

I

B

D

Q

P

c

Q

E

F

E
E

E

$J$

J

I

B

C

A

B
B

P

A

P

$\begin{array}{ll} & S \\ \text { M } & N \\ & M \\ & J \\ & Q \\ & E \\ & T\end{array}$

N

0

N

Q

0

Q

$$
\text { N }
$$

$\begin{array}{ll}K & \text { J } \\ R & \text { P }\end{array}$

H
P
G
J
D

H

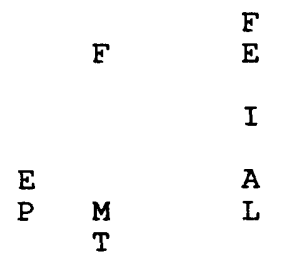

G

$\begin{array}{cc} & \\ & \\ & H \\ & D \\ & G \\ & G \\ & E \\ & S\end{array}$

B

$\mathrm{B}$
$\mathrm{K}$

E

P 
Table 1 - (continued)

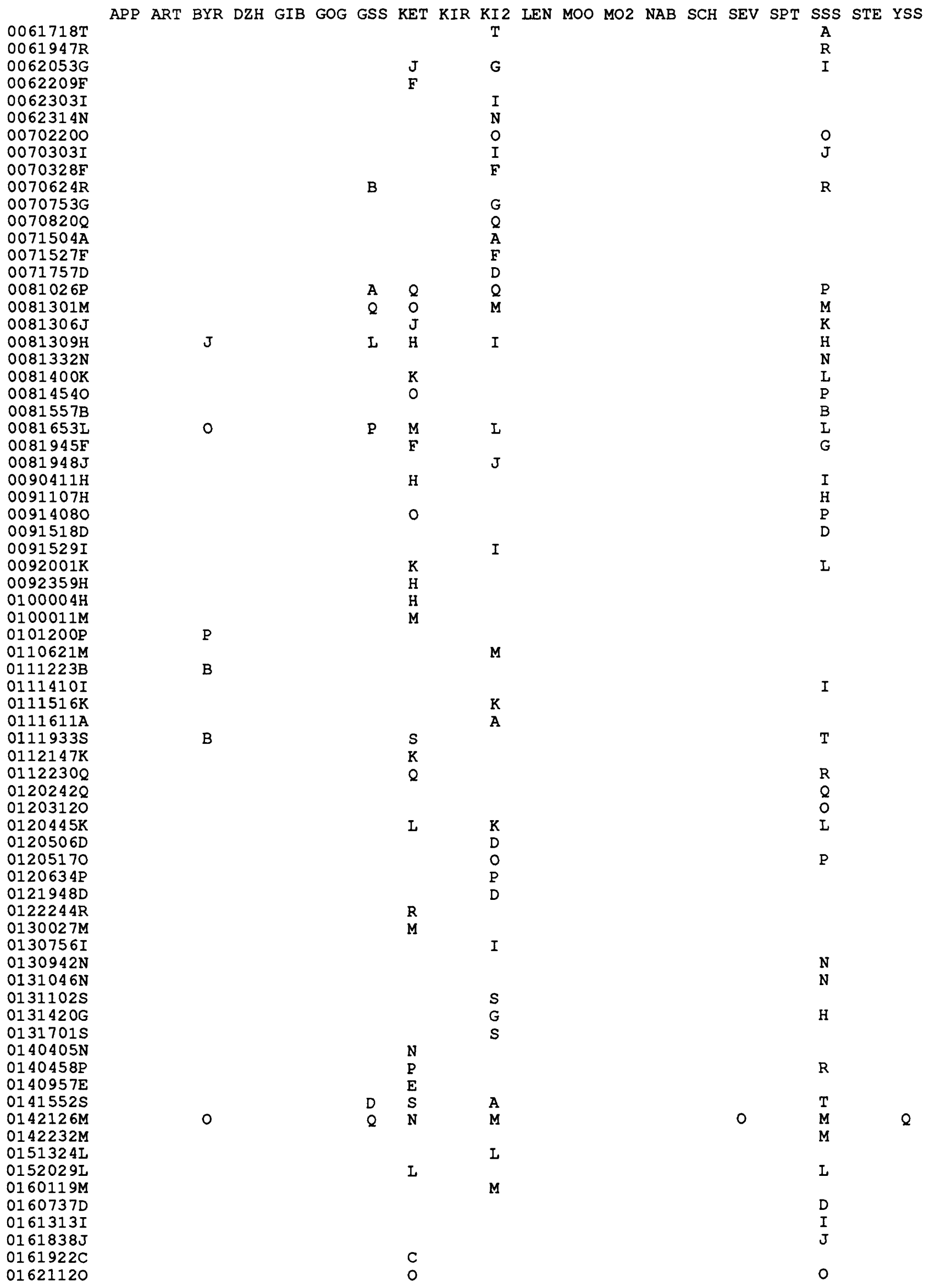


Table 1 - (continued)

APP ART BYR DZH GIB GOG GSS KET KIR KI2 LEN MOO MO2 NAB SCH SEV SPT SSS STE YSS

$0170049 \mathrm{H}$

01712068

$0171926 \mathrm{~S}$

01811560

$0190441 \mathrm{~N}$

01904480

$0190727 R$

$0190813 \mathrm{~N}$

$0191404 \mathrm{E}$

$0191645 \mathrm{P}$

$0191902 E$

0200240G

$0200324 \mathrm{C}$

$0200450 \mathrm{~B}$

$0200626 \mathrm{~J}$

020112 OE

0201833R

$0202026 \mathrm{H}$

$0202345 \mathrm{~J}$

$0210100 \mathrm{H}$

0210151D

$0210154 \mathrm{M}$

$0210809 \mathrm{~K}$

0210920P

$0211518 \mathrm{C}$

02202160

$0220222 \mathrm{E}$

$0220813 \mathrm{E}$

$0221357 \mathrm{E}$

$0221526 \mathrm{E}$

0221705E

02223358

$0230141 \mathrm{C}$

$0230505 \mathrm{M}$

$0230744 \mathrm{P}$

$0230914 \mathrm{~L}$

$0231614 \mathrm{~F}$

02317290

02320431

$0232347 \mathrm{~L}$

$0240208 \mathrm{C}$

$0240231 \mathrm{C}$

$0241207 \mathrm{H}$

$0241249 \mathrm{~S}$

02414330

$0251556 \mathrm{~T}$

$0252206 \mathrm{R}$

$0260808 \mathrm{I}$

$0261515 I$

$0261942 \mathrm{~J}$

$0270243 I$

$0270343 \mathrm{D}$

$0270635 \mathrm{~K}$

$0271034 \mathrm{M}$

$0272028 \mathrm{~F}$

02722350

$0272356 \mathrm{D}$

$0280149 \mathrm{~F}$

$0291711 \mathrm{G}$

$0300033 \mathrm{M}$

$0300204 \mathrm{E}$

03015570

$0310432 \mathrm{~J}$

03119560

$0312008 \mathrm{~J}$

$0312118 \mathrm{~L}$

$0320346 \mathrm{H}$

03204070

$0321005 \mathrm{~N}$

03214208

$0332128 \mathrm{~B}$ $\begin{array}{ll} & H \\ S & Q \\ T & S\end{array}$

N

T R

N

$\mathrm{Q}$
$\mathrm{S}$
$\mathrm{N}$
$\mathrm{R}$

R

S

Q

0

S

E

$\begin{array}{lll}\text { K } & \mathrm{H} & \mathrm{G}\end{array}$

P

E

C

B

$\mathrm{R}$
$\mathrm{H}$

$\mathrm{H}$

D

P

K

C

E

E E E

G

C

M

M

L

L

H D $\quad$ D

G

$S$

Q

I

S R

$\begin{array}{lll} & \mathrm{J} & \mathrm{J}\end{array}$

$\mathbf{J}$

$\mathrm{J}$

$\mathrm{K}$
$\mathrm{M}$
$\mathrm{E}$

Q

D

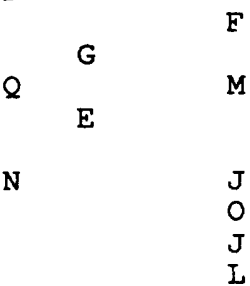

0 
Table 2 - Listing of seismograms derived from the digital recordings of the accelerometers.

APP ART BYR DZH GIB GOG GSS KET KIR KI2 IEN MOO MO2 NAB SCH SEV SPT SSS STE YSS

$0030819 \mathrm{~s}$

$0031348 \mathrm{P}$

$0040729 \mathrm{~N}$

$0040738 \mathrm{~J}$

$0040740 \mathrm{P}$

$0050815 \mathrm{H}$

Q

$\begin{array}{cccc} & \text { S } & \text { S } & \text { T } \\ & \text { P } & \text { P } & \text { Q } \\ \text { Q } & \text { N } & \text { N } & 0 \\ \text { M } & \text { J } & \text { J } & \text { K } \\ & \text { Q } & \text { P } & \text { R } \\ \text { H } & & & \end{array}$

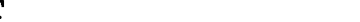

T

Table 3 - Listing of seismograms derived from integration and hi-pass filtering of the digital recordings of the accelerometers.

APP ART BYR DZH GIB GOG GSS KET KIR KI2 LEN MOO MO2 NAB SCH SEV SPT SSS STE YSS

$0030819 \mathrm{~s}$

$0031348 \mathrm{P}$

$0040729 \mathrm{~N}$

$0040738 \mathrm{~J}$

$0040740 \mathrm{P}$

$\begin{array}{llll} & & & \\ & N & P & \\ & N & N & \\ & & \text { J } & \\ & & P & \end{array}$




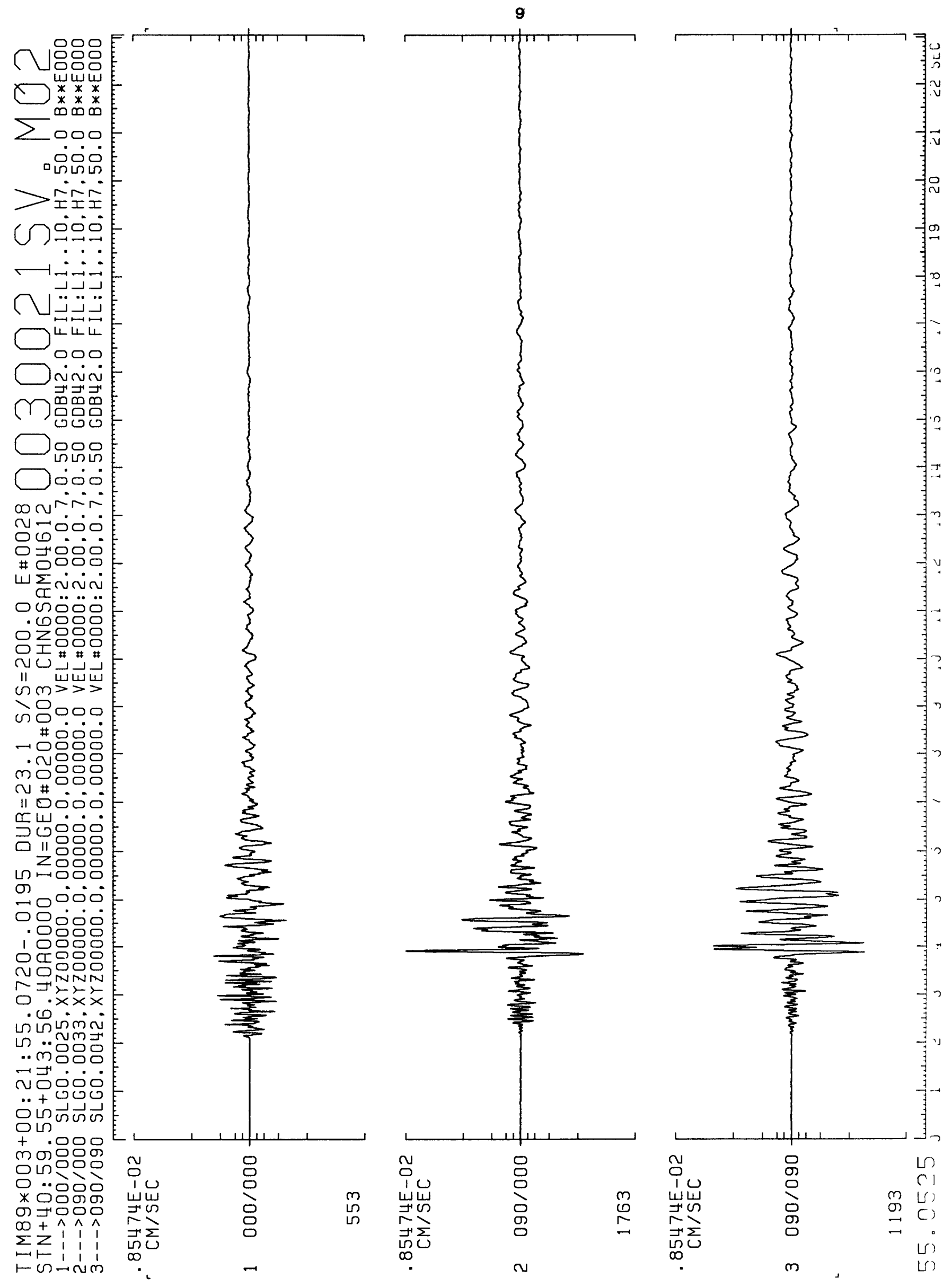


10

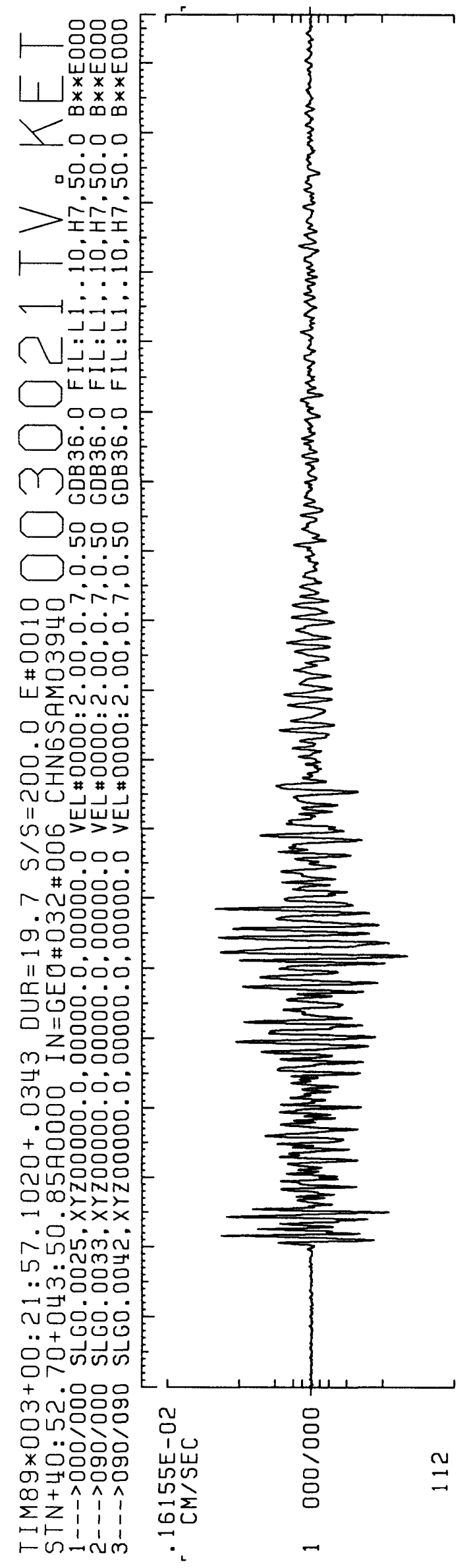

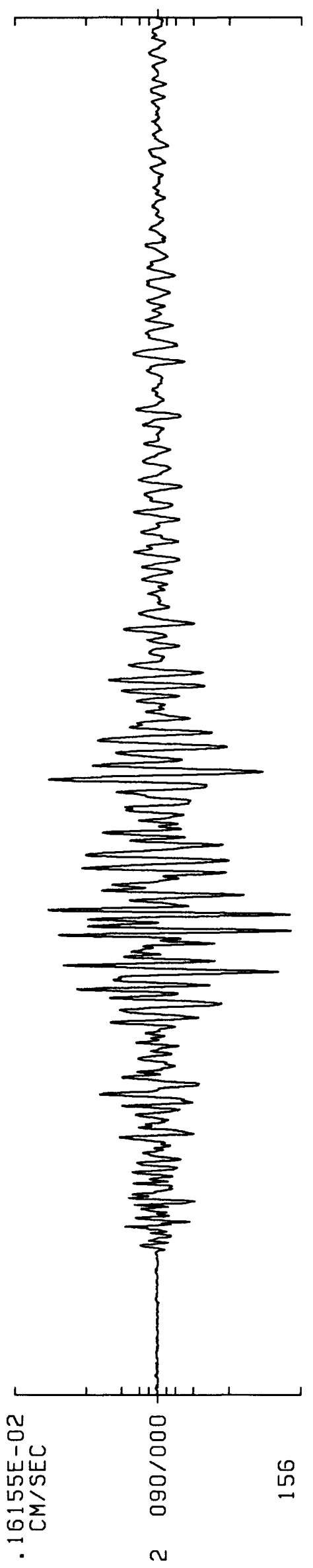

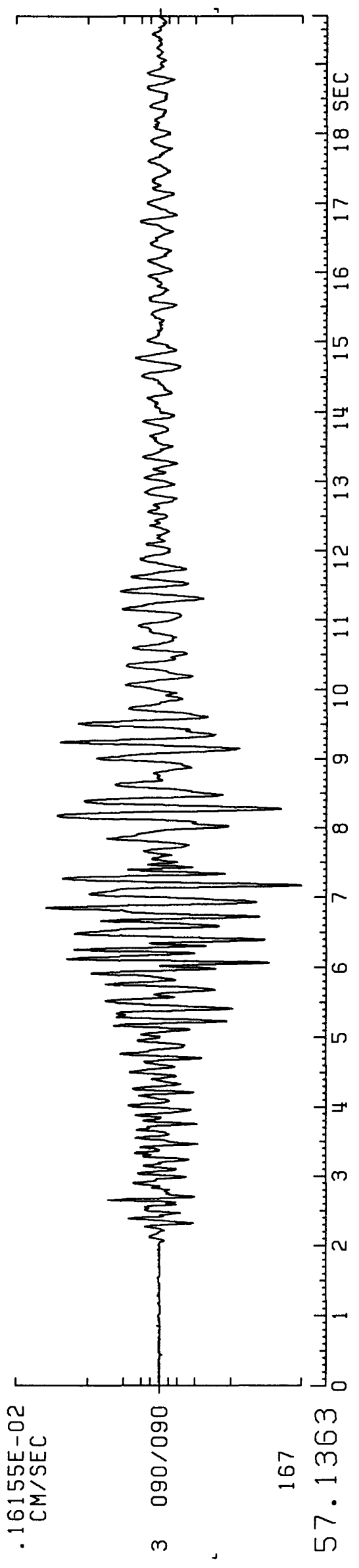


11

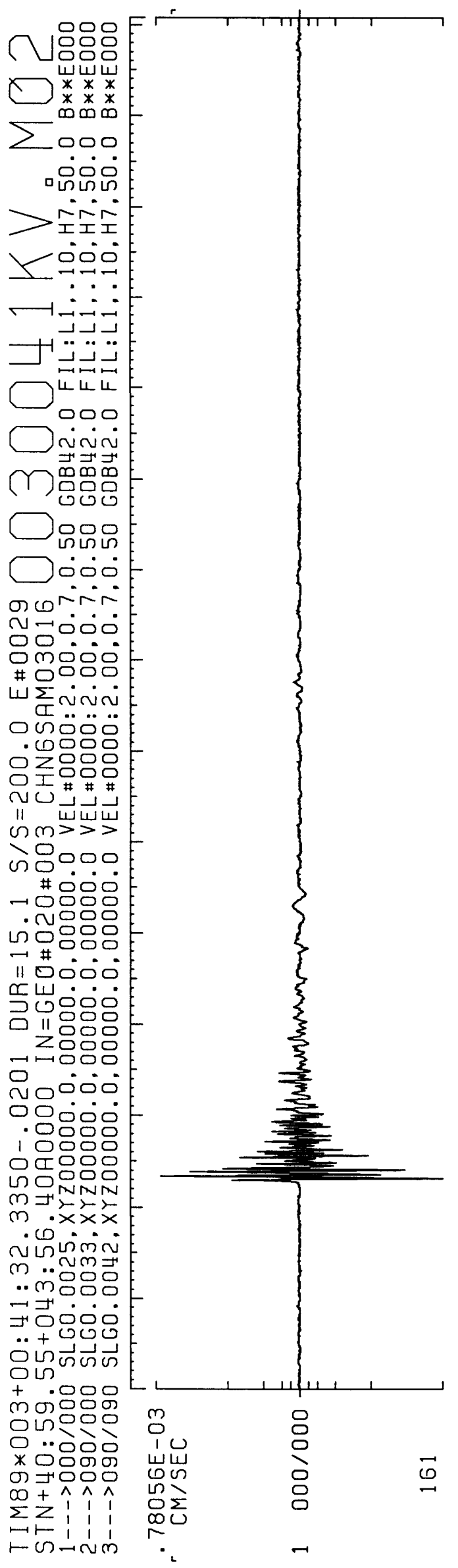

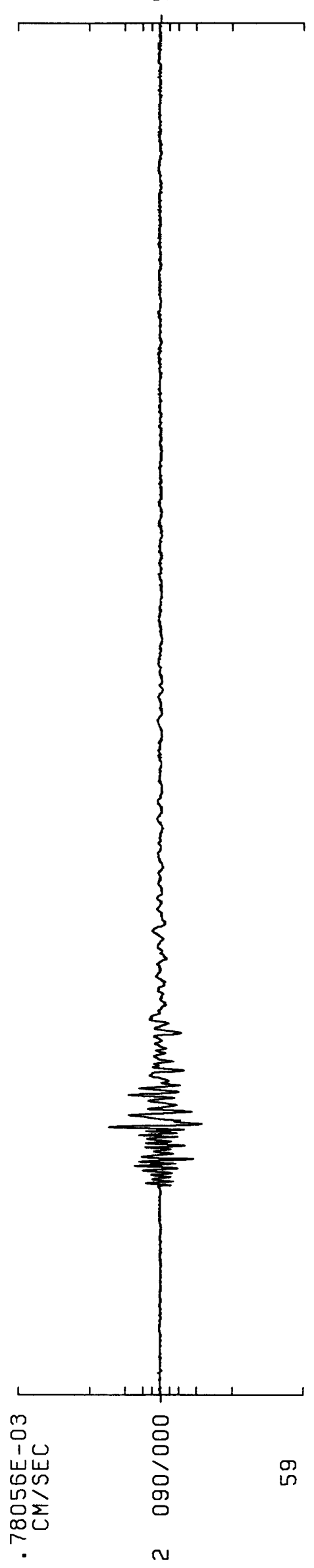

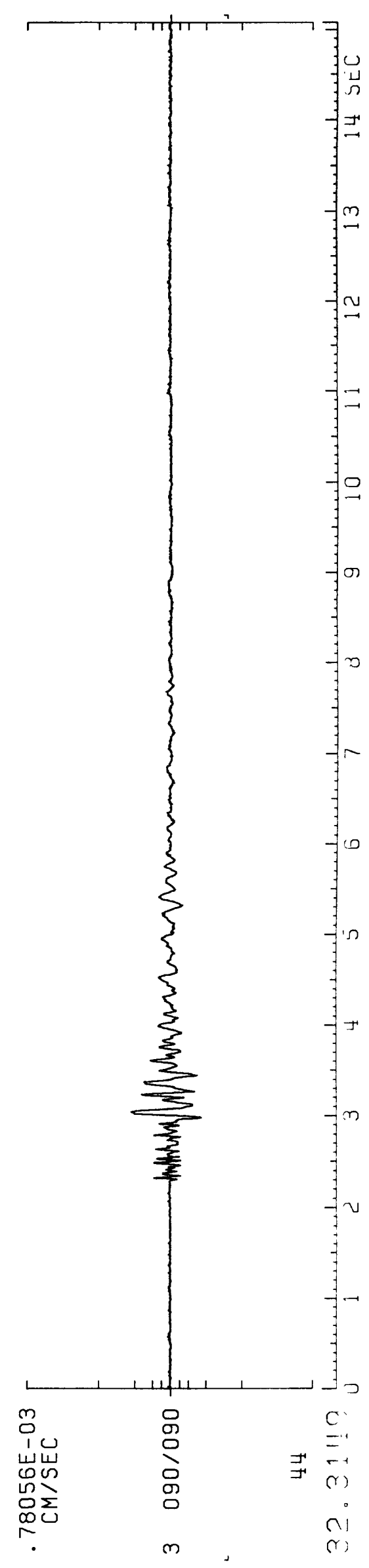


12

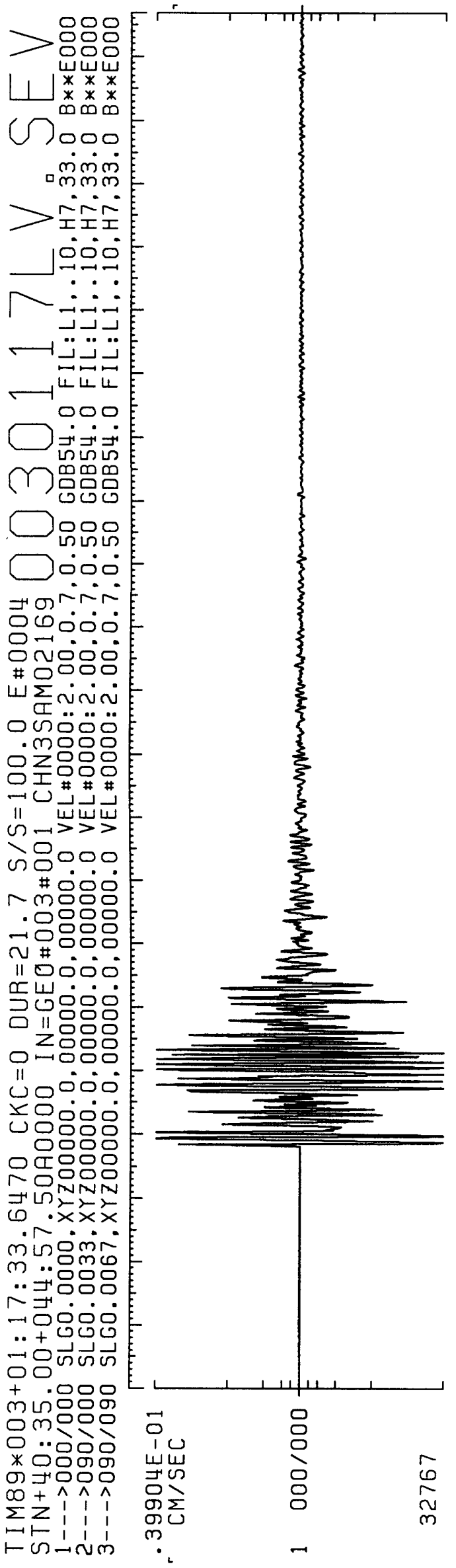

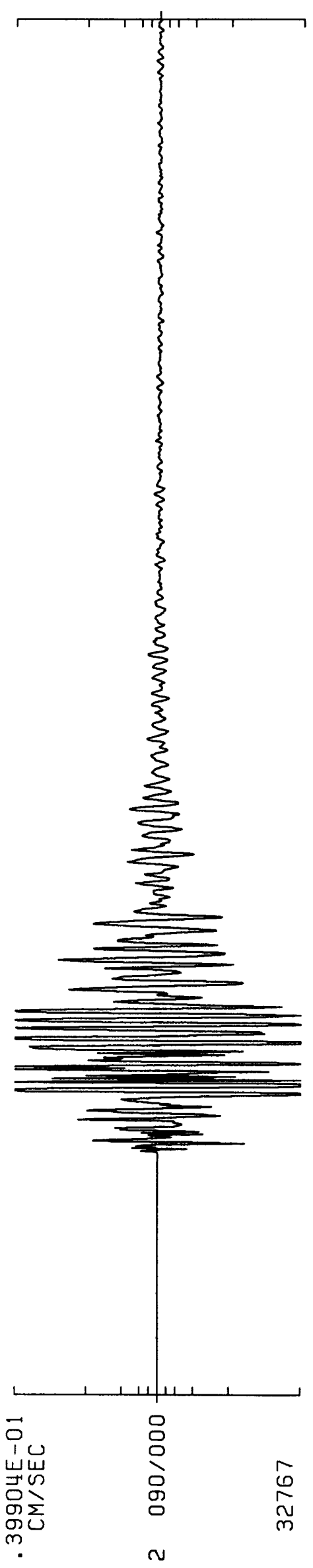

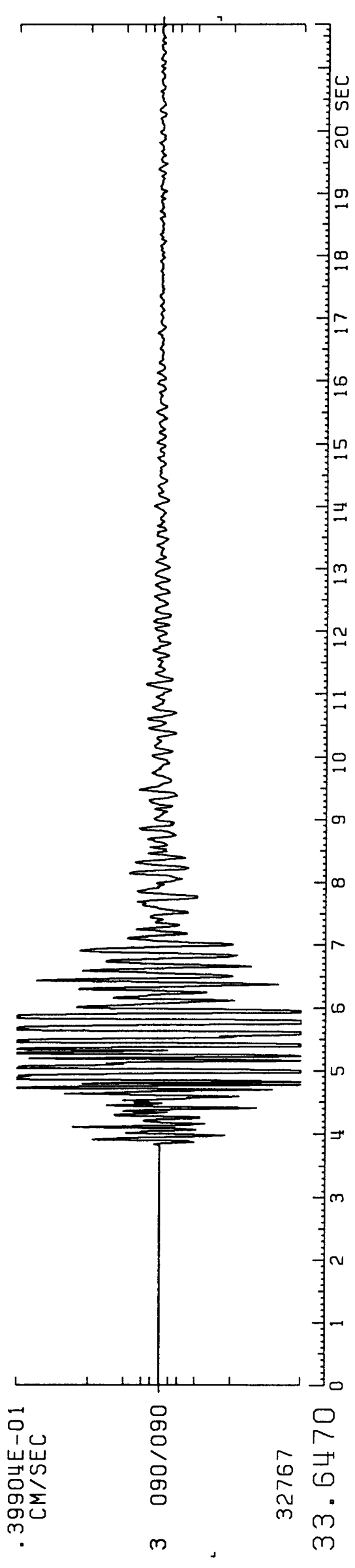


13

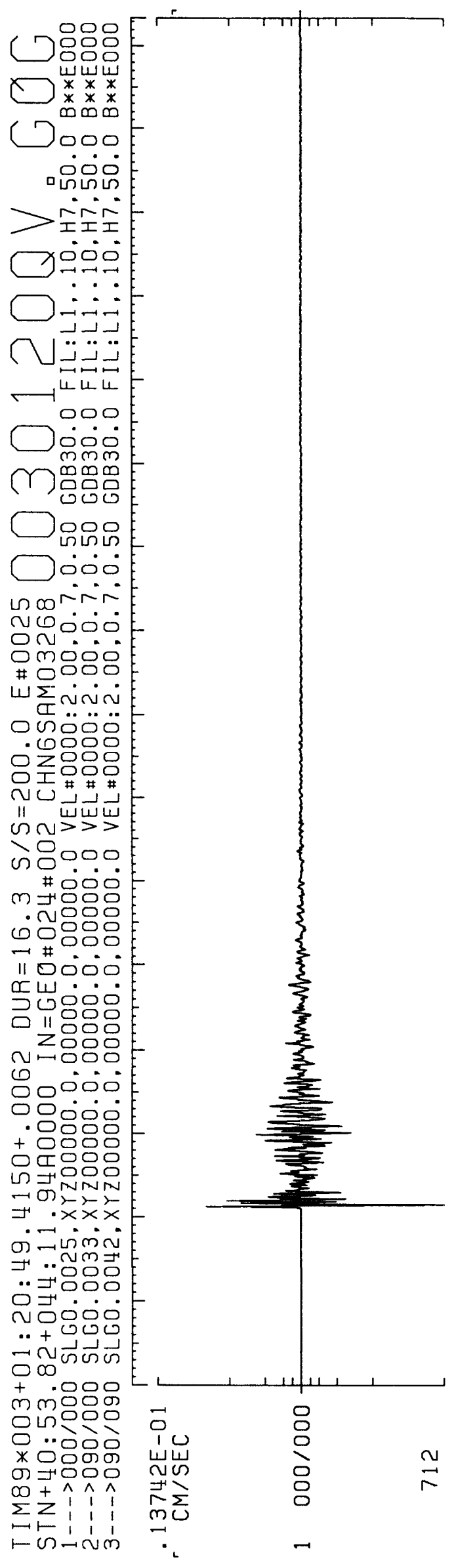

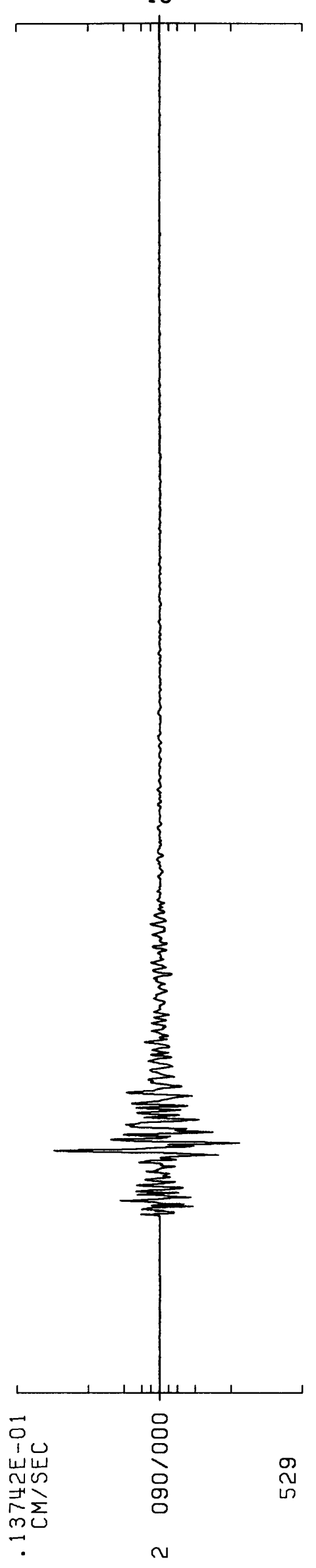

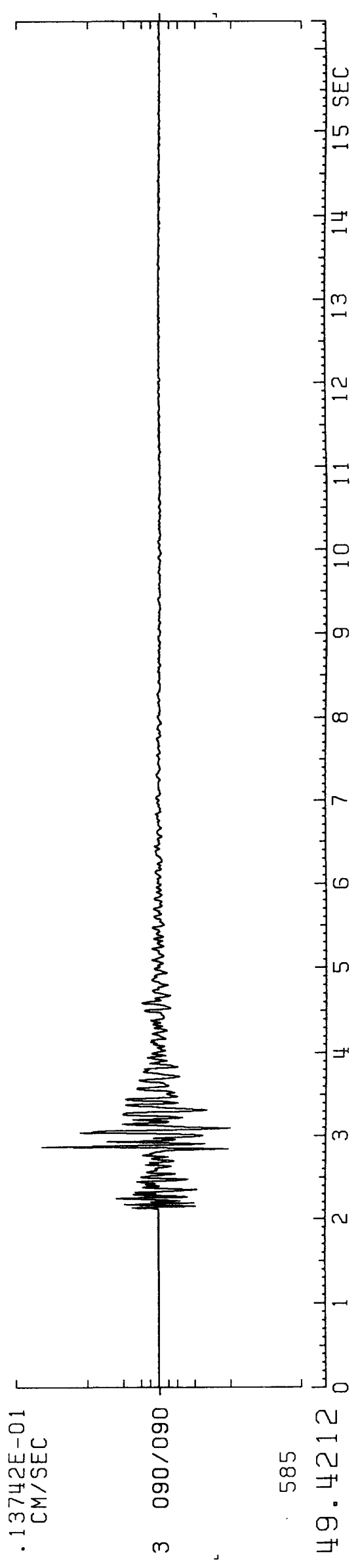



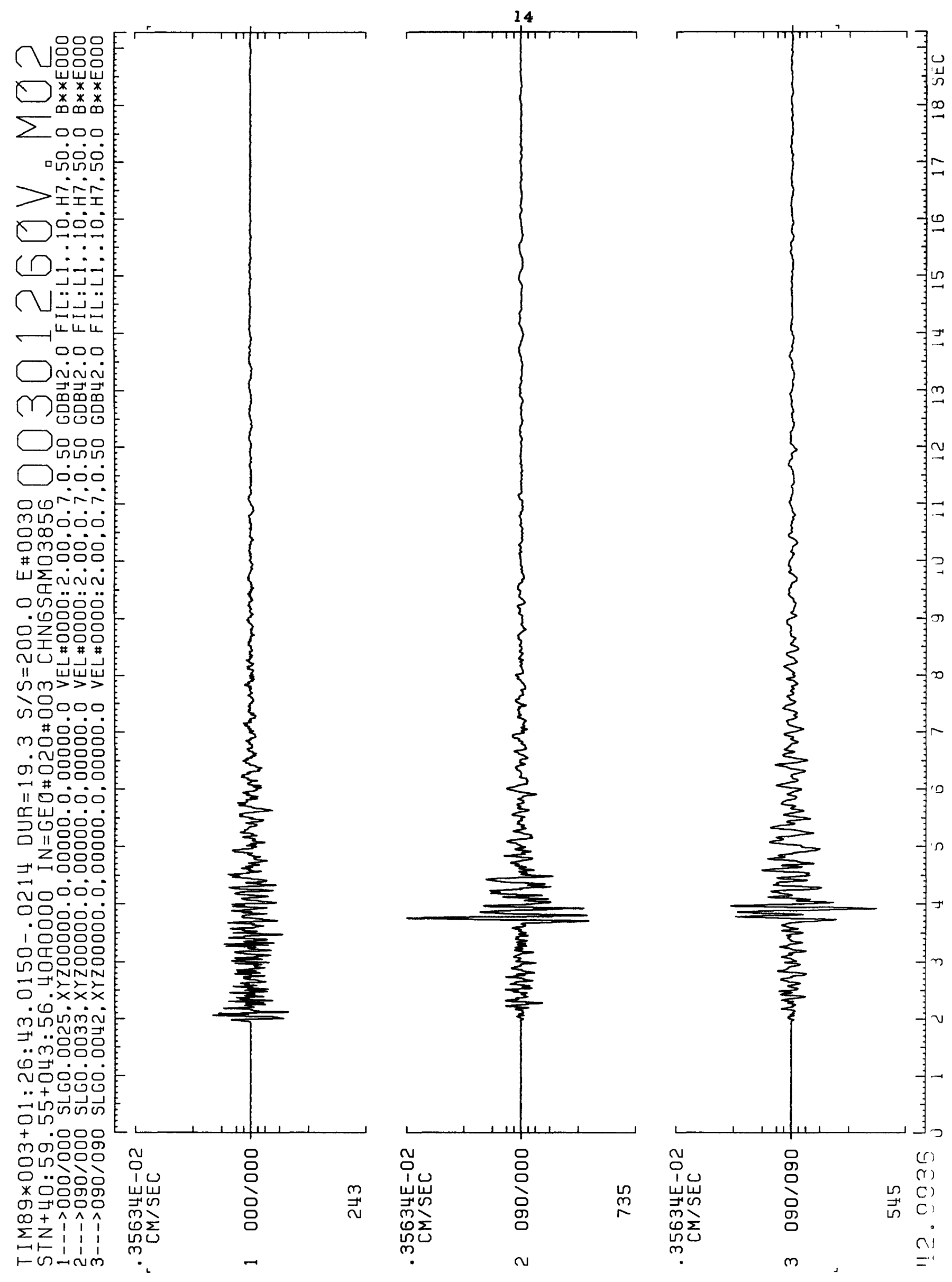
บำ:ำ

$\left\ulcorner\underset{*}{*} * \frac{w}{*}\right.$ $* * *$
$m \infty$

$\sum 000$ i.ं. ดับย์

$\rightarrow$ ririr i०० ๑ー

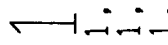
1 d ....... こロロ

๑レル $\square \square$ บก

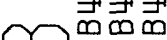
뭉요 $\square$ 으음 $\square$ Qio. moris mनिं0ं móo \# แ ه正苑 - 1000 ○Z匀口 $\bigcirc$ 工員 NU $-\frac{1}{-1}$ o $m>>>$ 认े \# 一잉ㅇㅇ - 능음 잉ㅇㅁ응 11 \# 0 エ山ய.

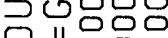
ㄴ11응음

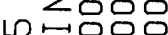
บั00ं ○ - 0 000 1 응응 똥으음 OONNN m.x又文 0.ंin

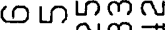

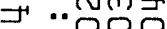
.. m음요

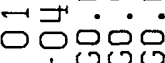
$\cdots+600$ तथजिज 는 + 응음 moro임 으일 * の $\infty+\wedge \wedge \wedge$ $\infty \pm \hat{\imath} \hat{\imath} \hat{i}$ $\sum \sum 1$

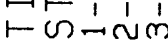

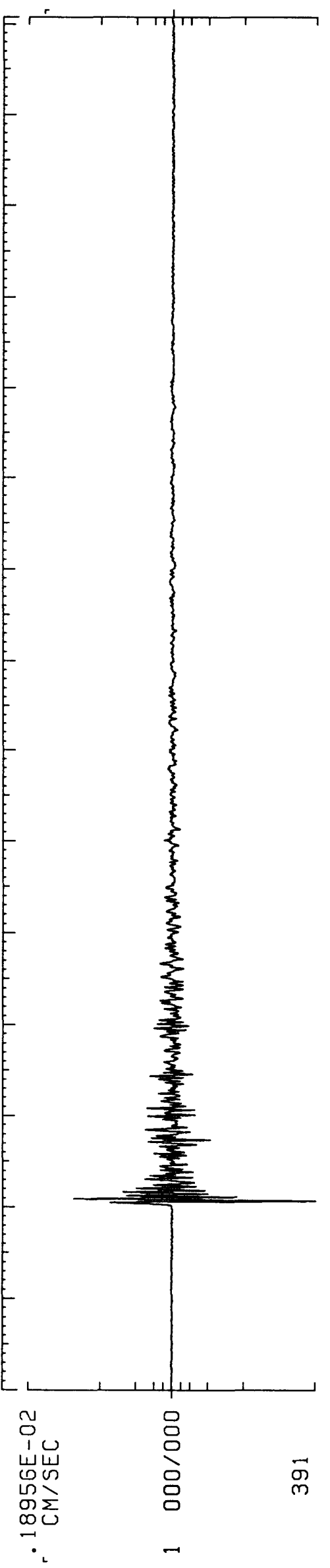

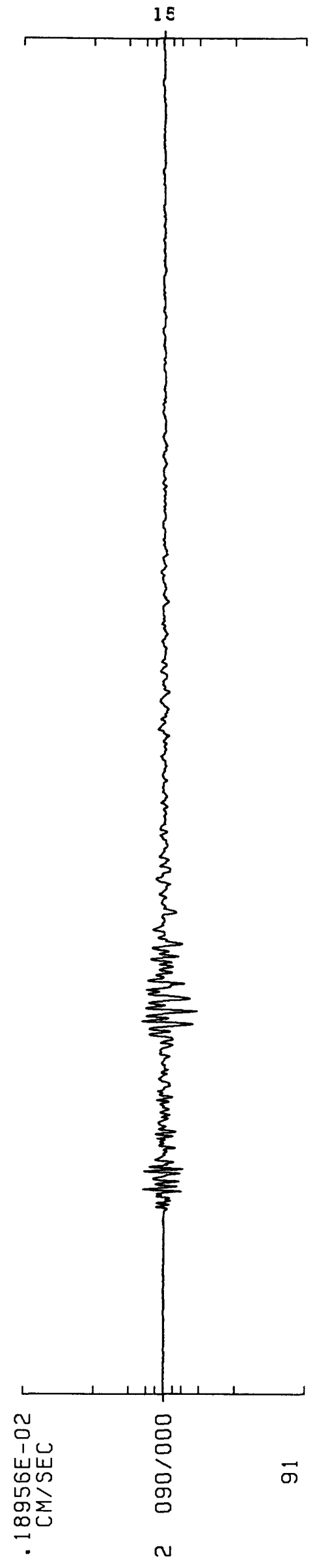

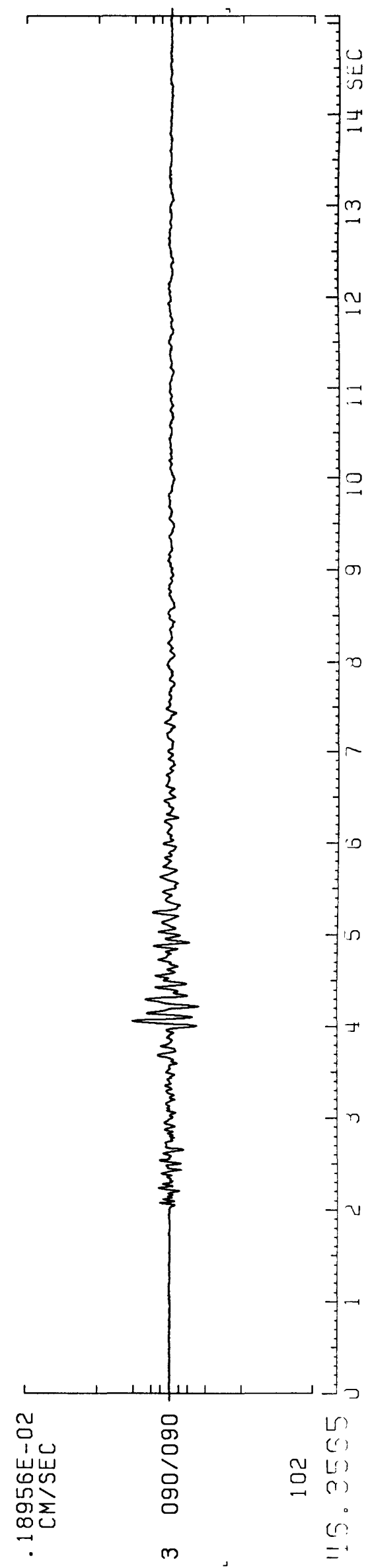


16
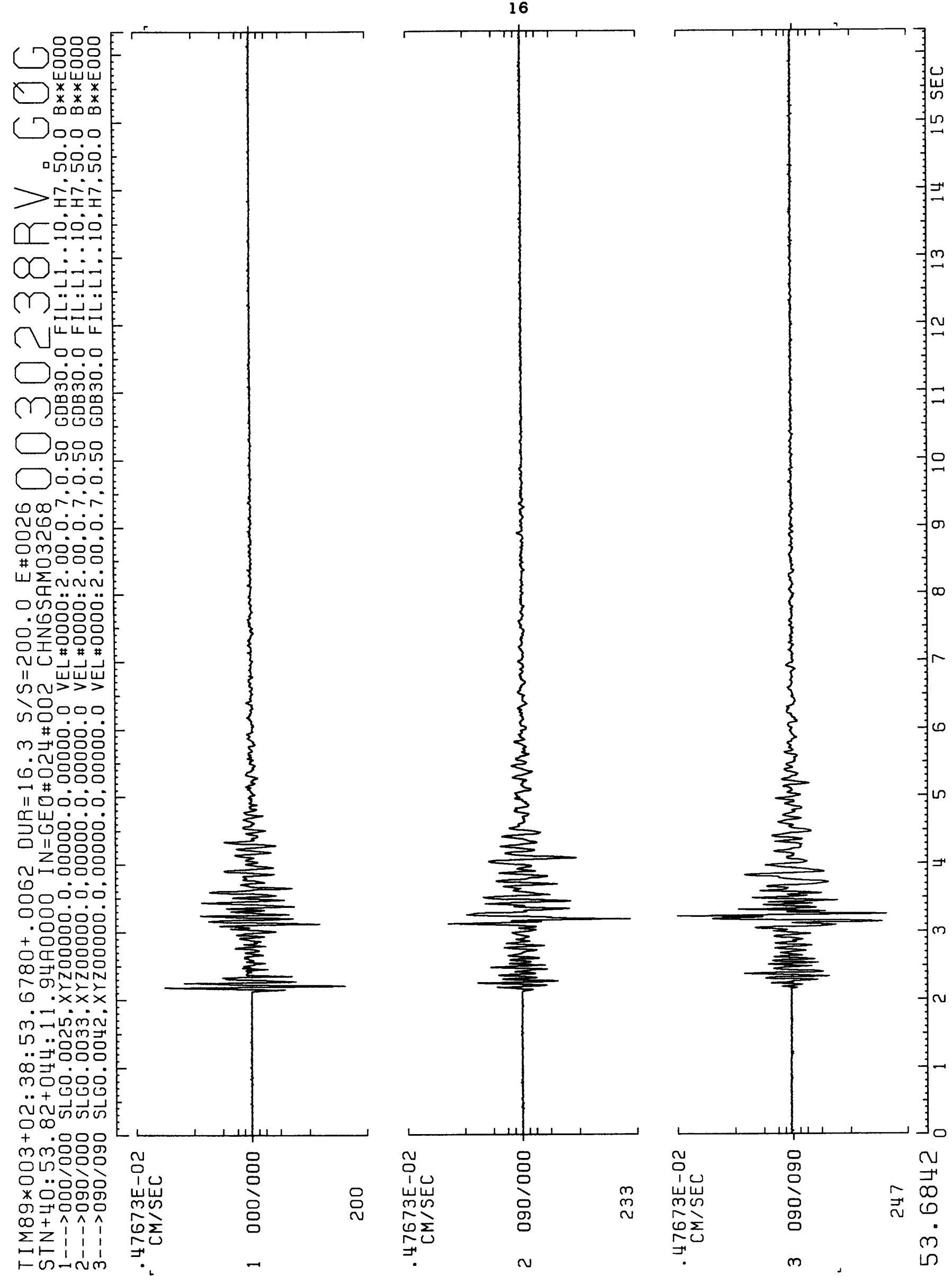
17
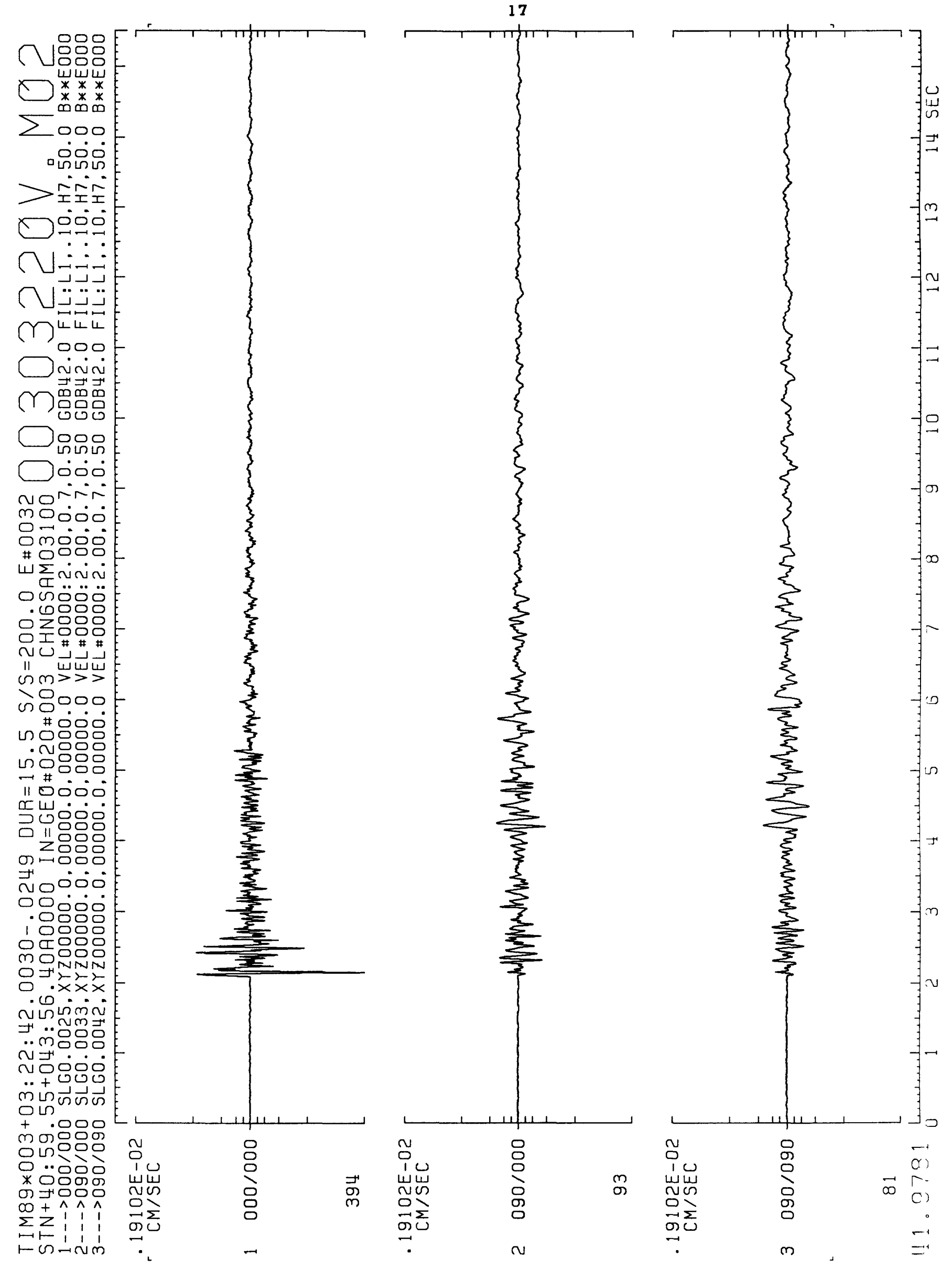


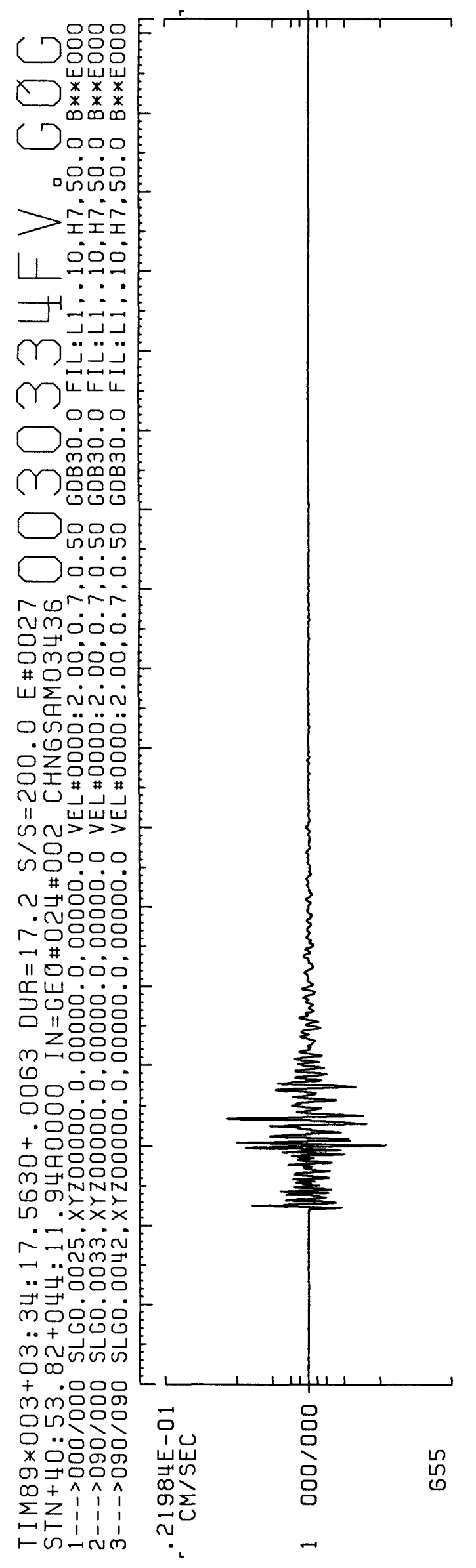

18

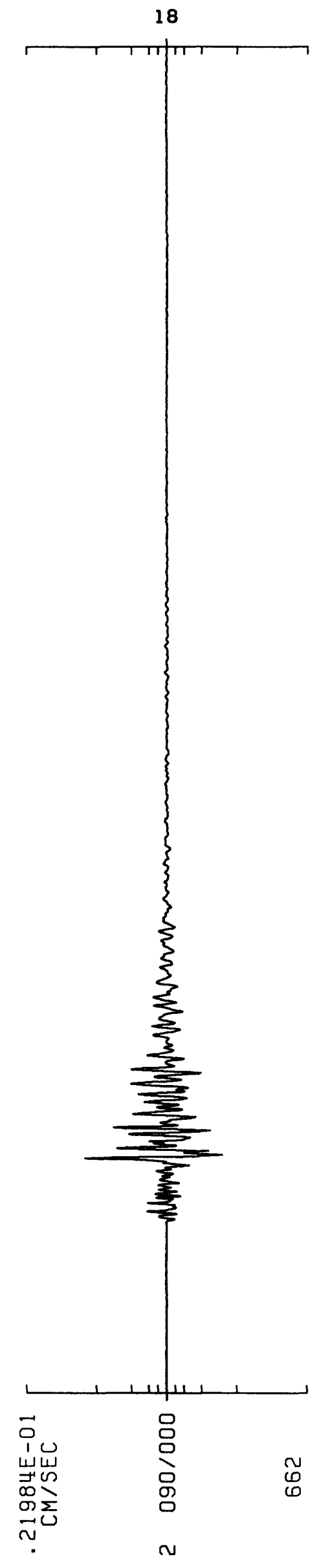

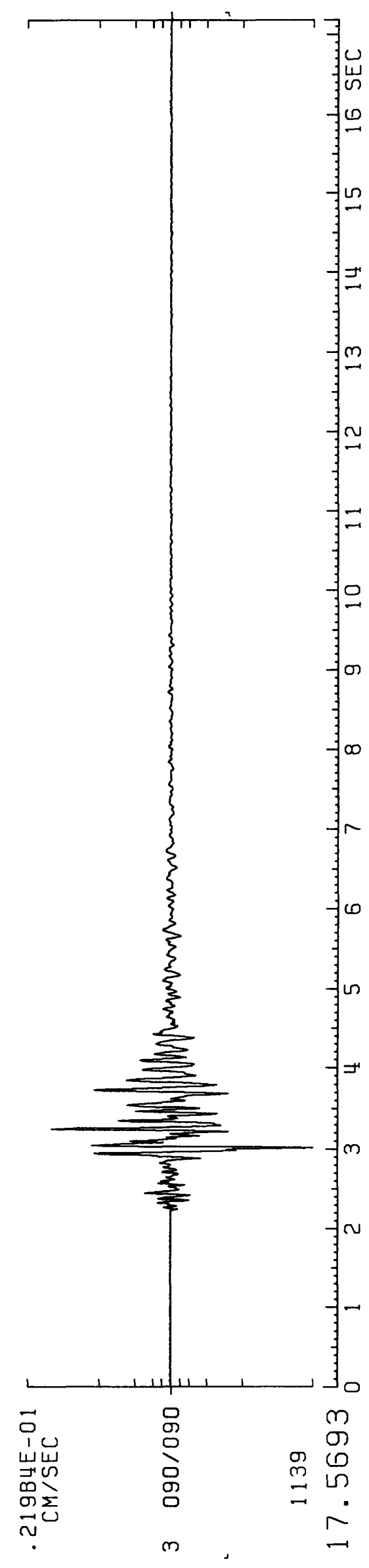


19
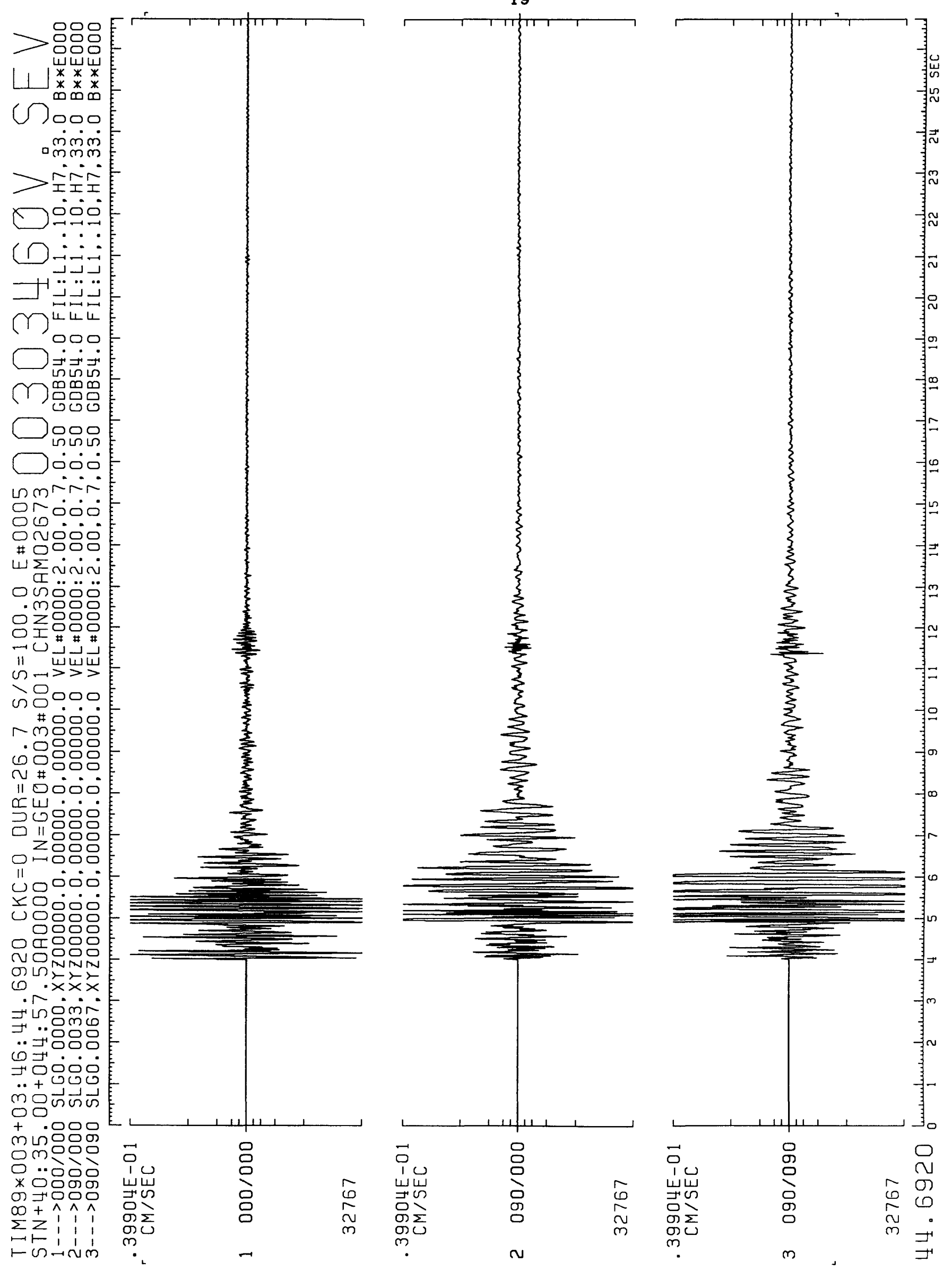

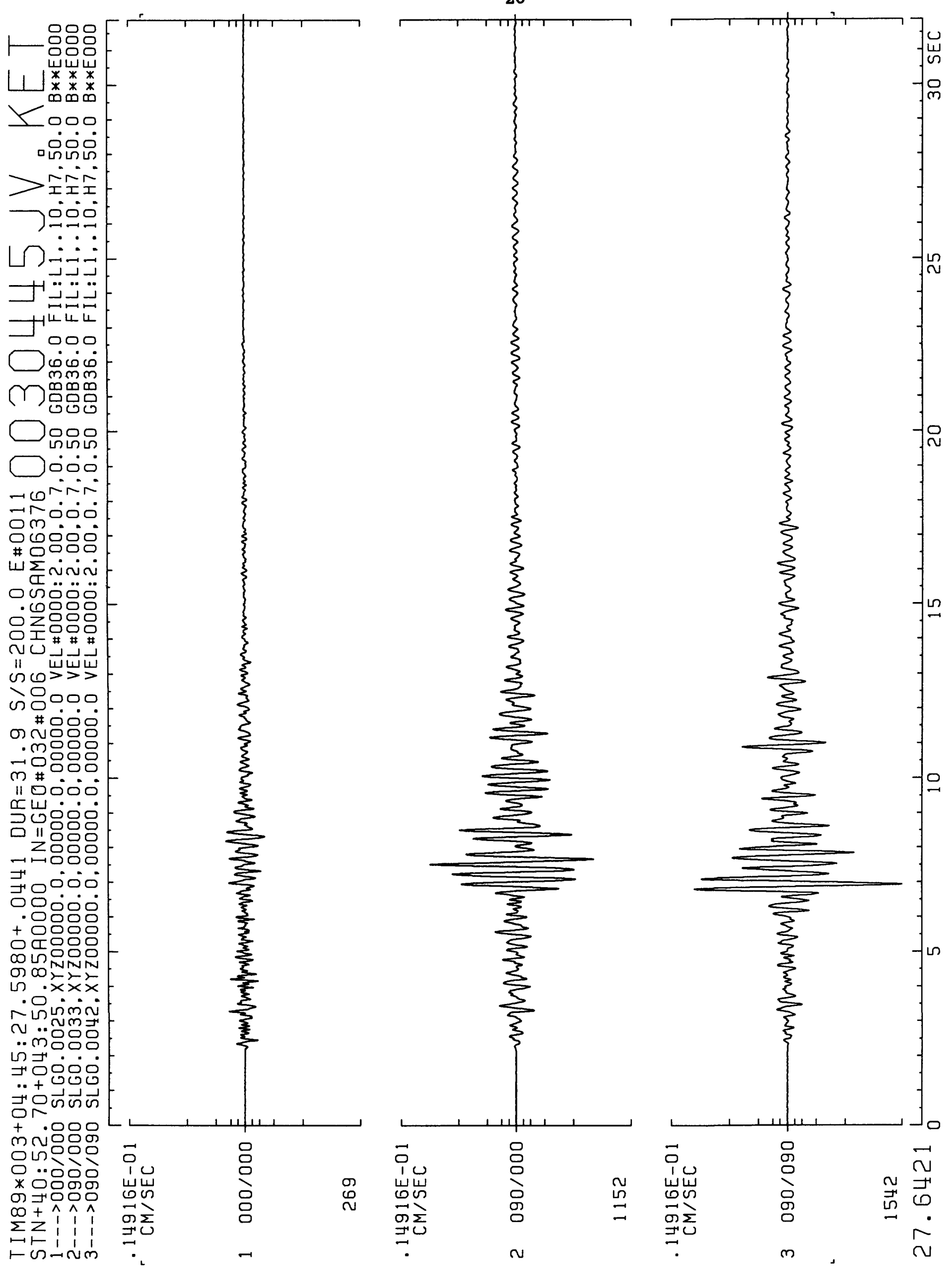
21
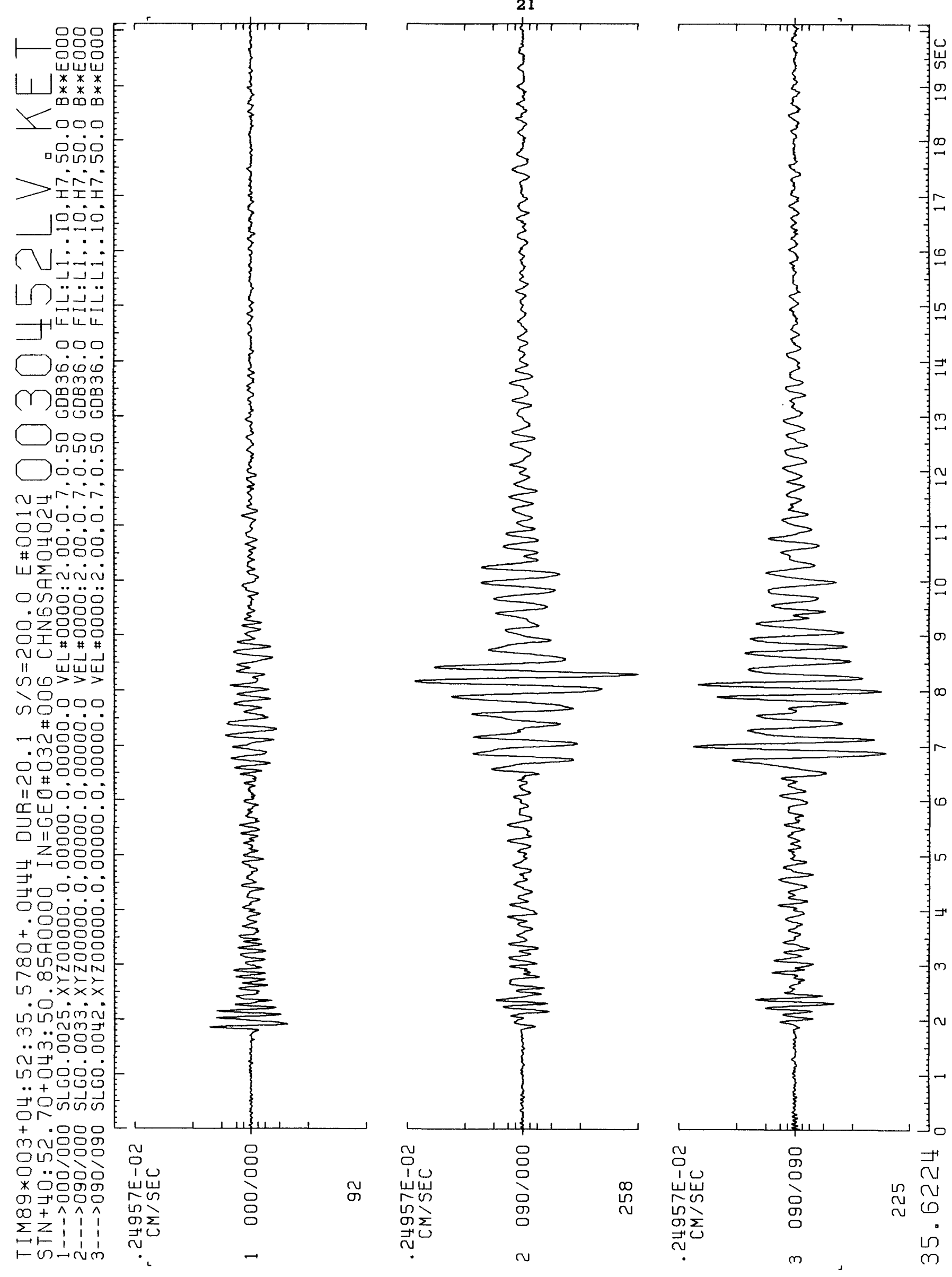
22
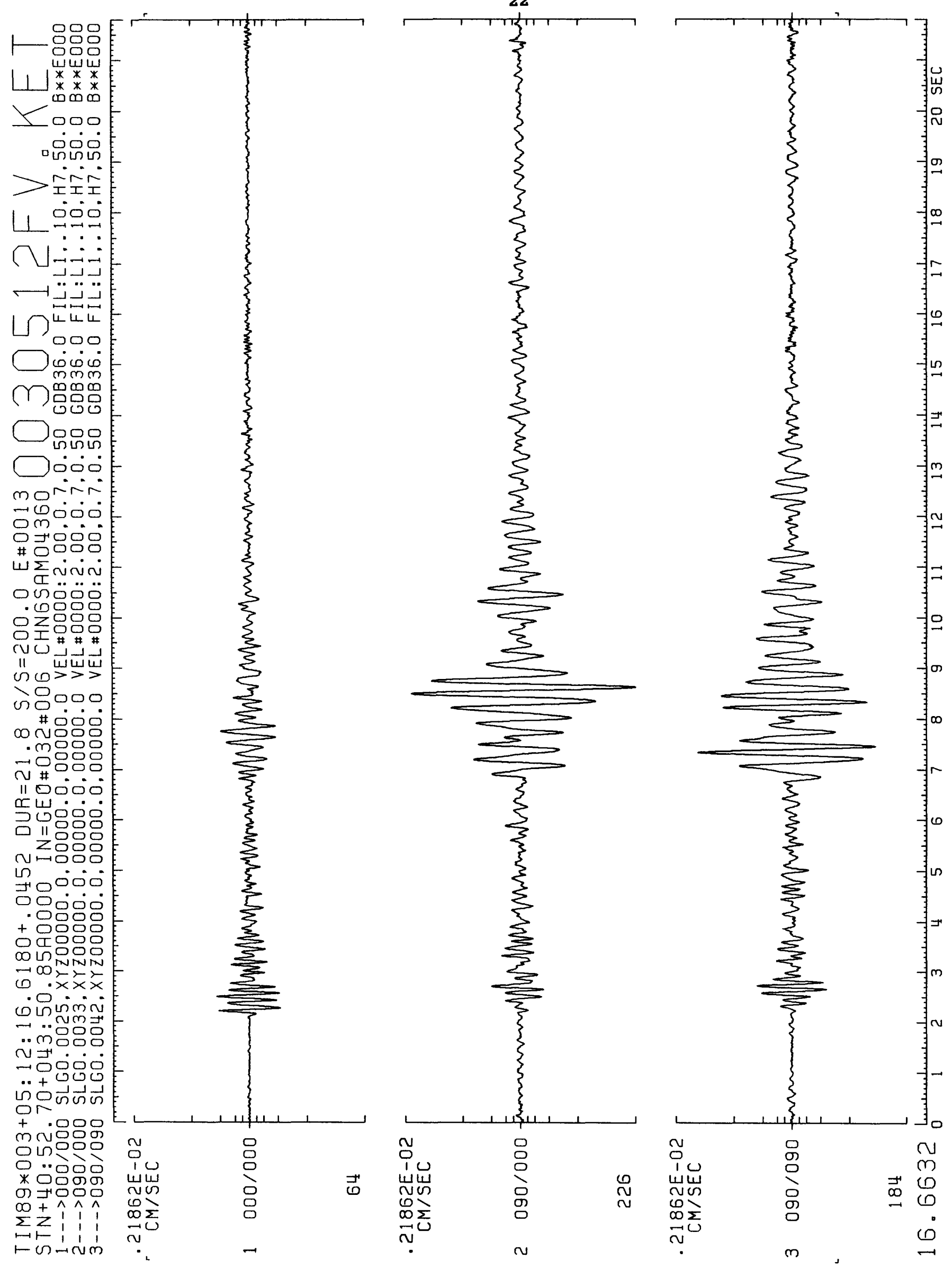

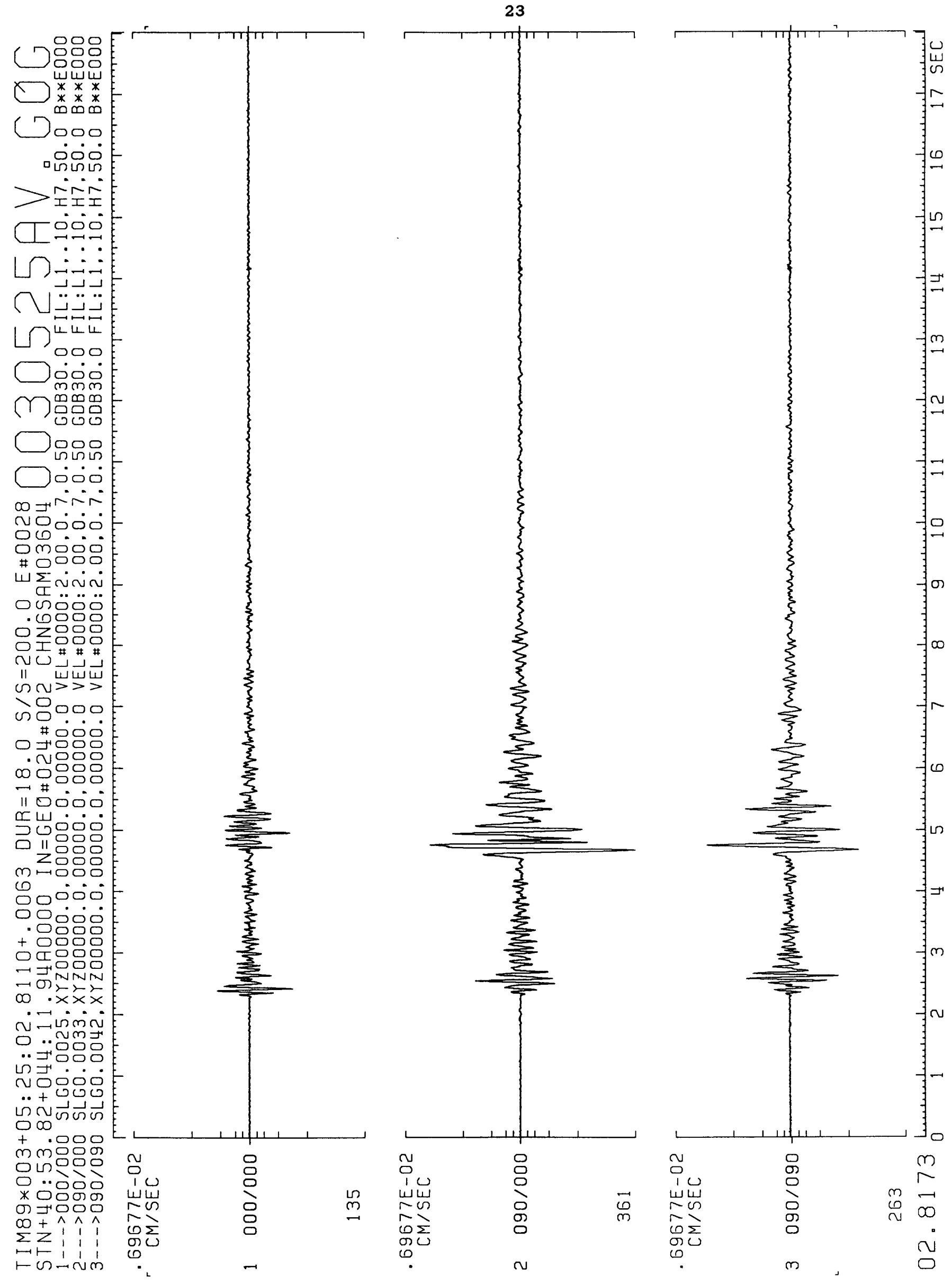


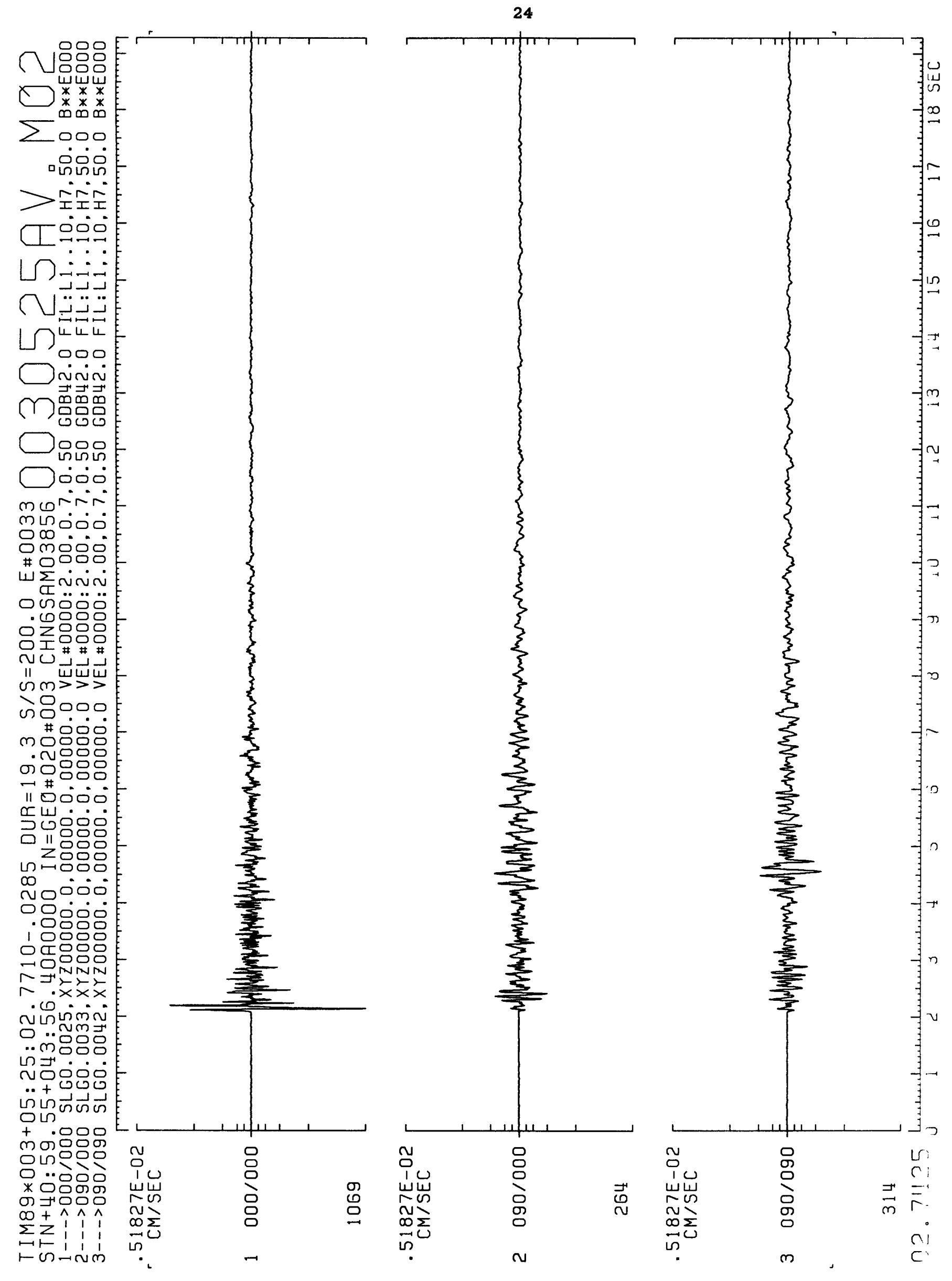



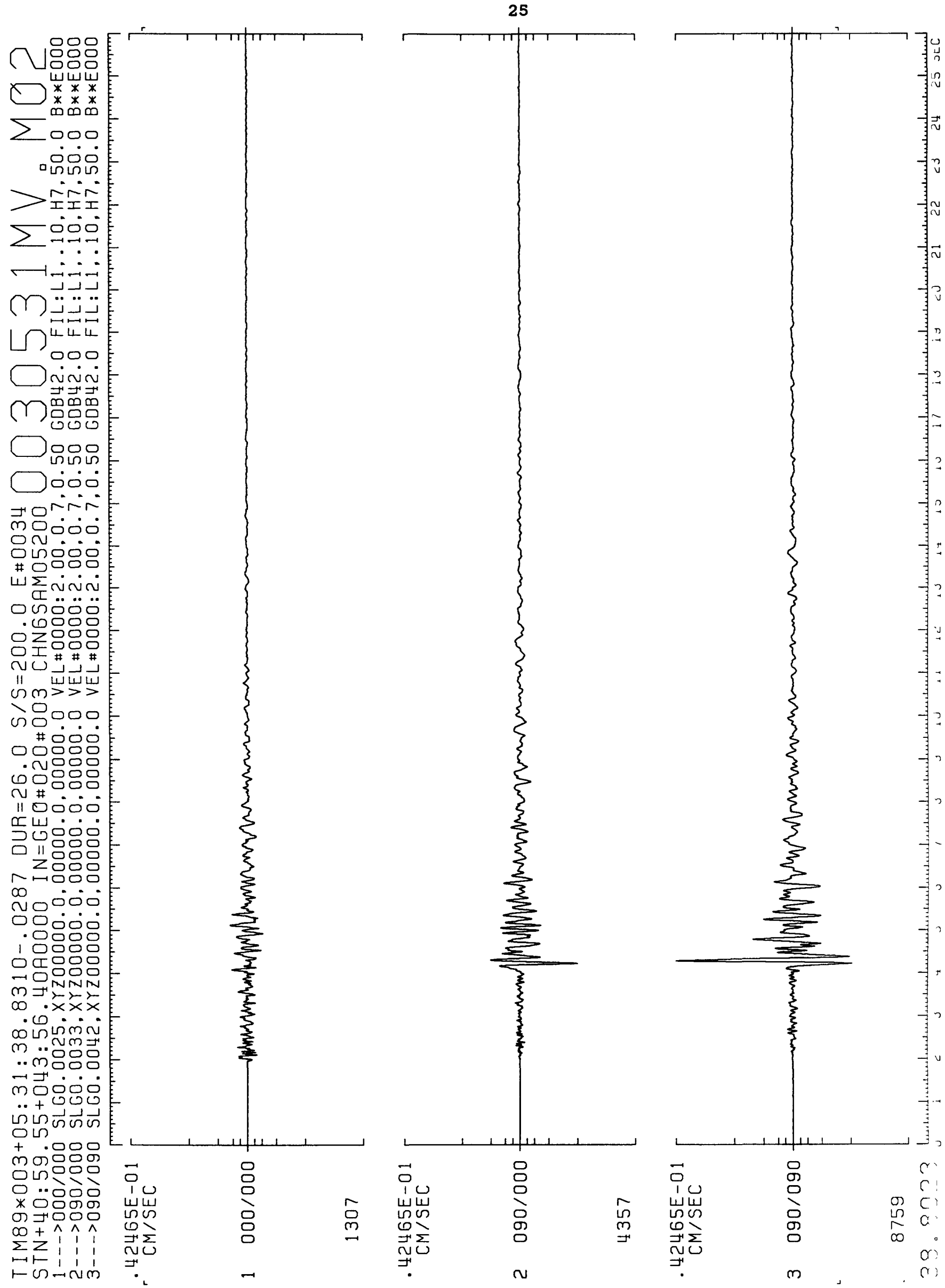
26
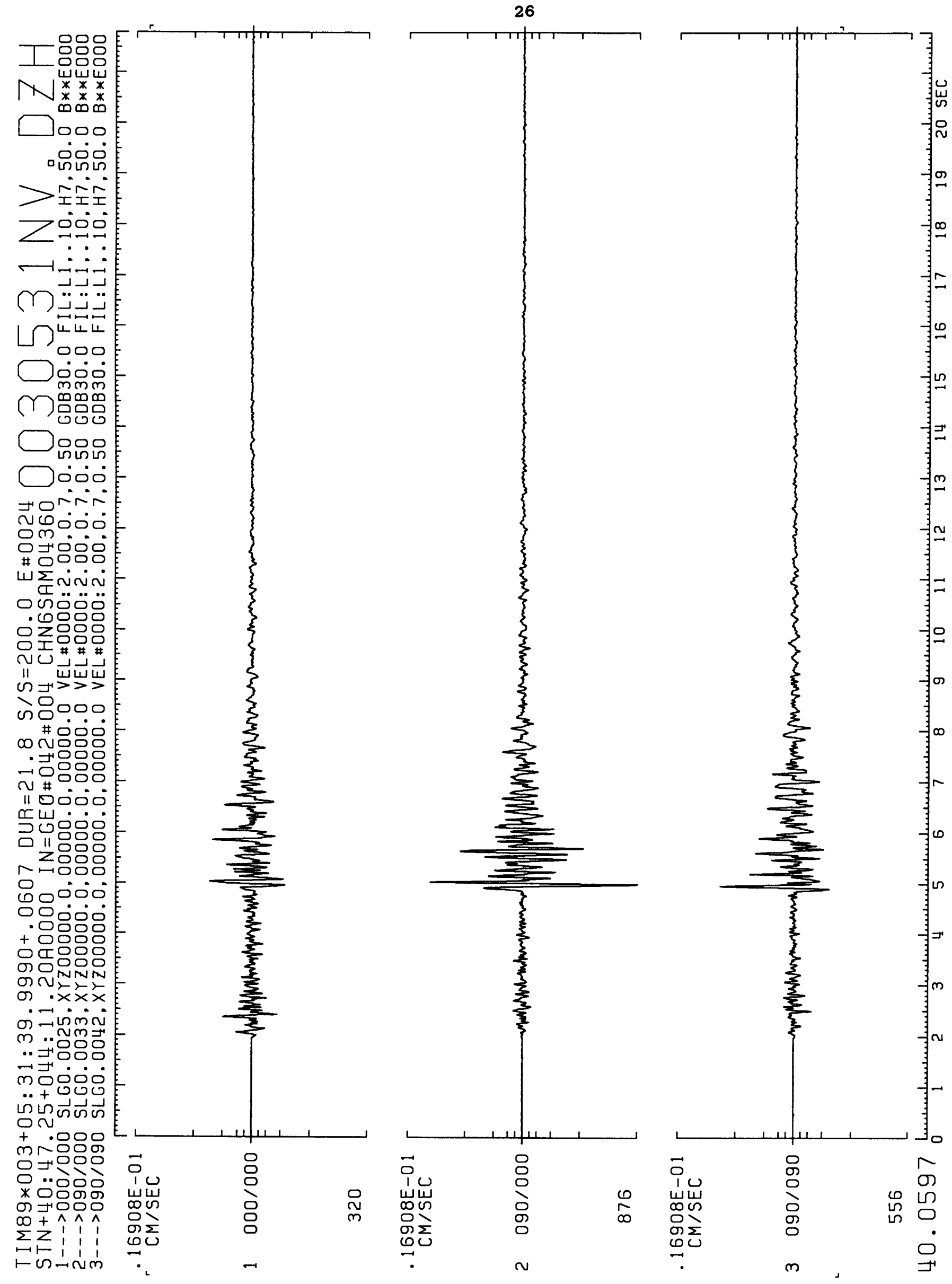


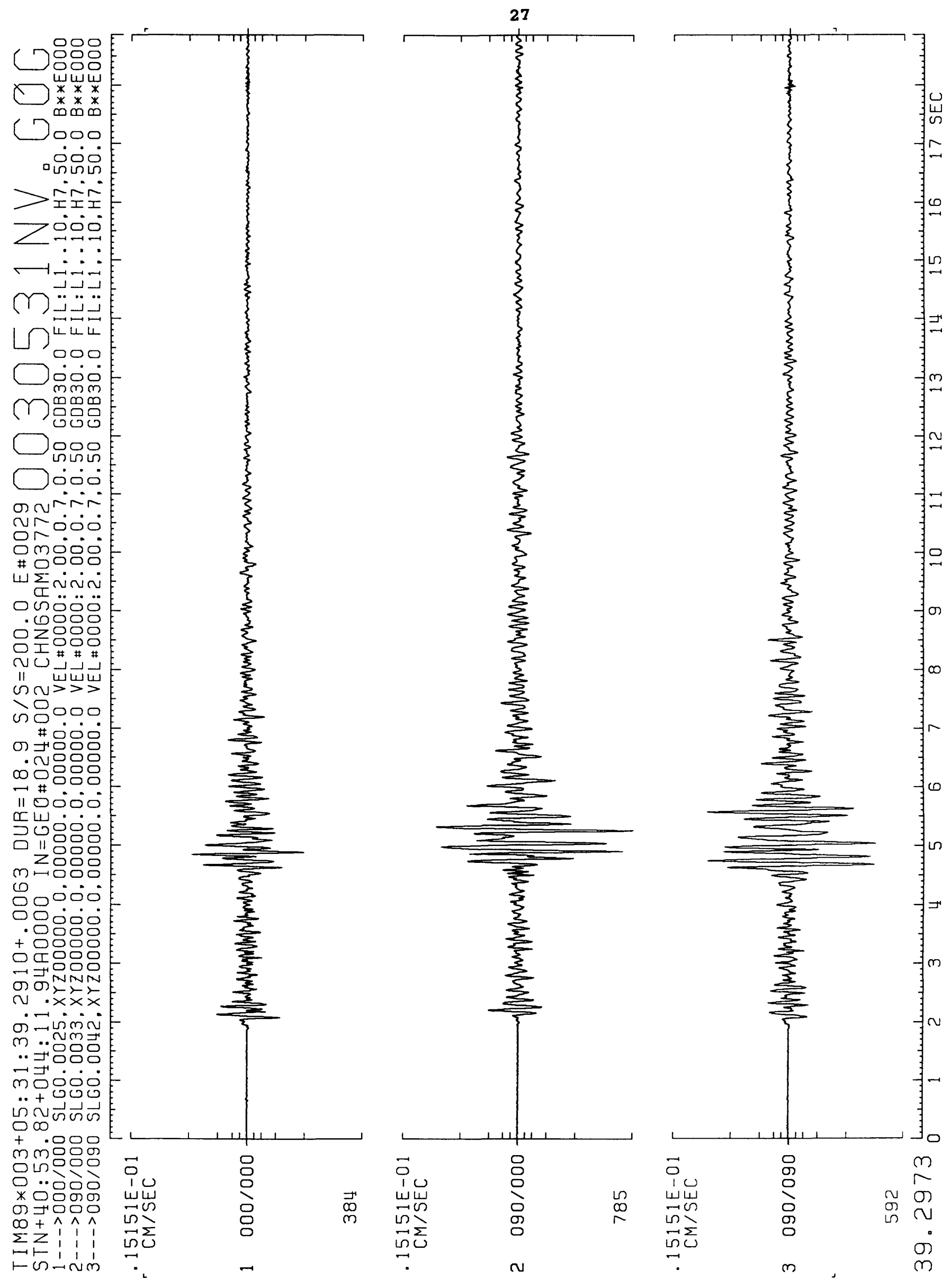



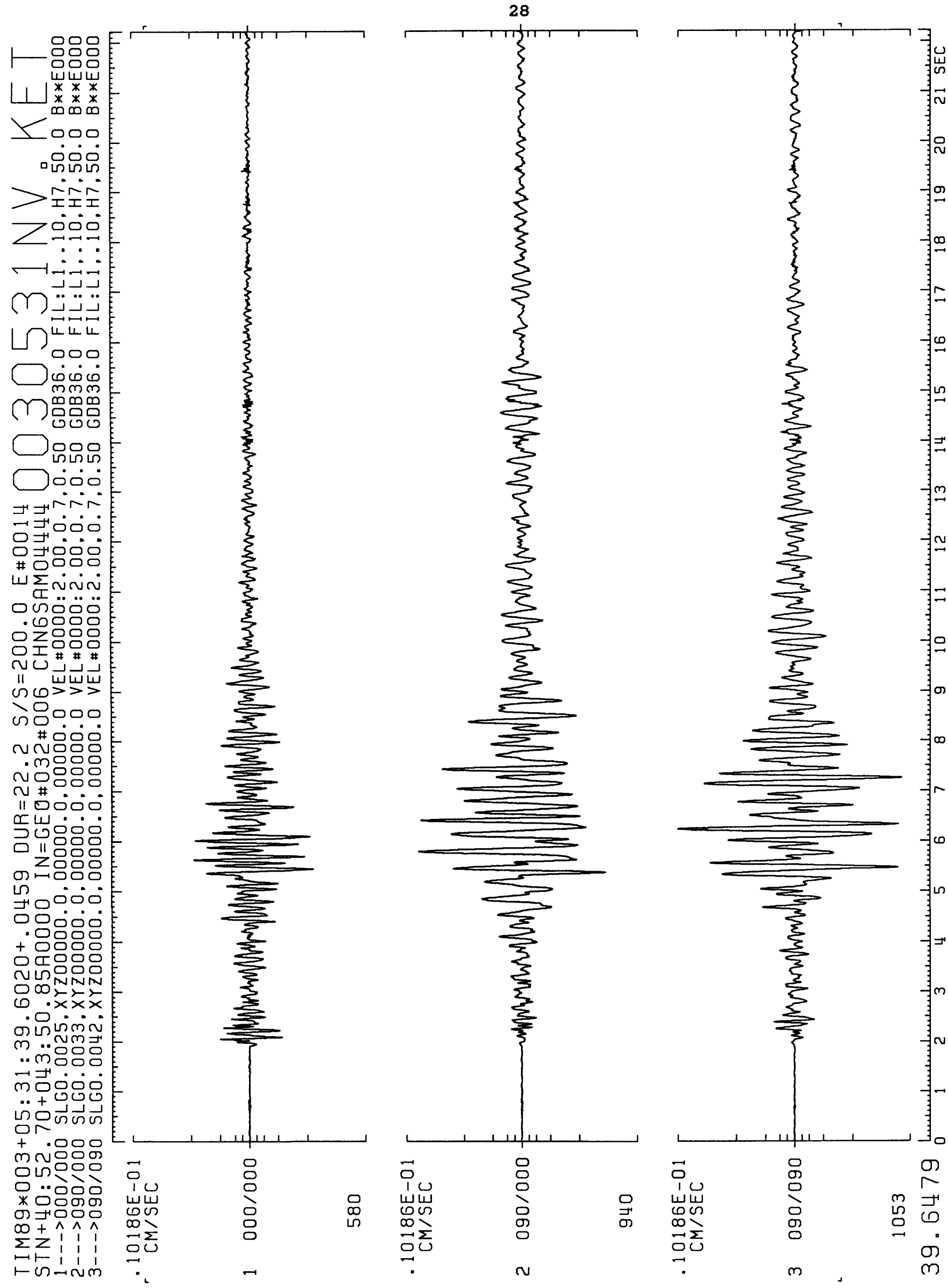
29

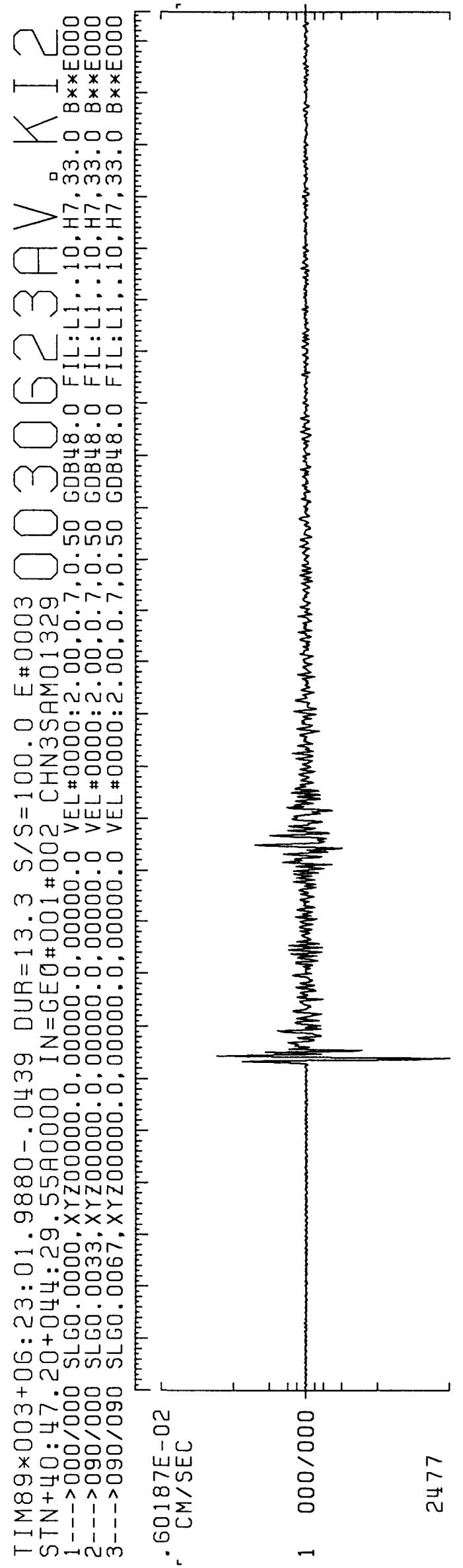

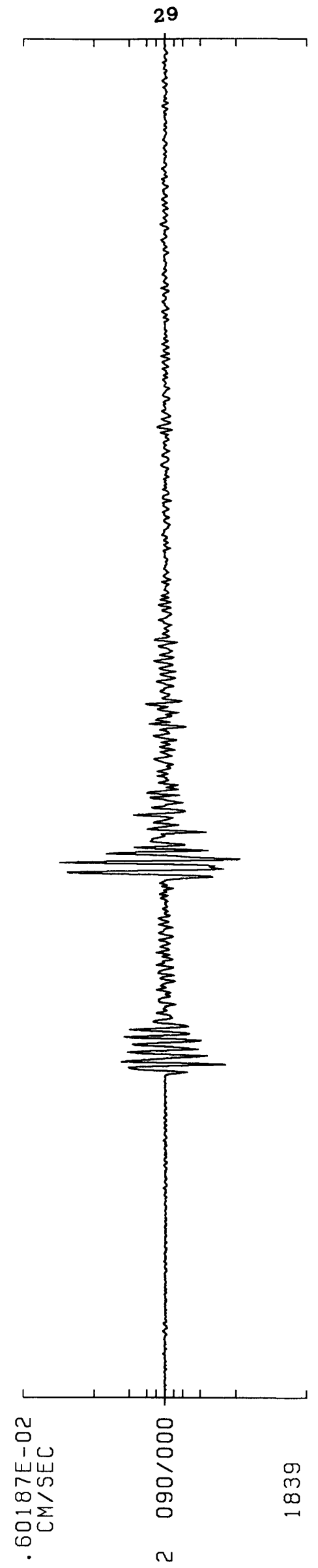

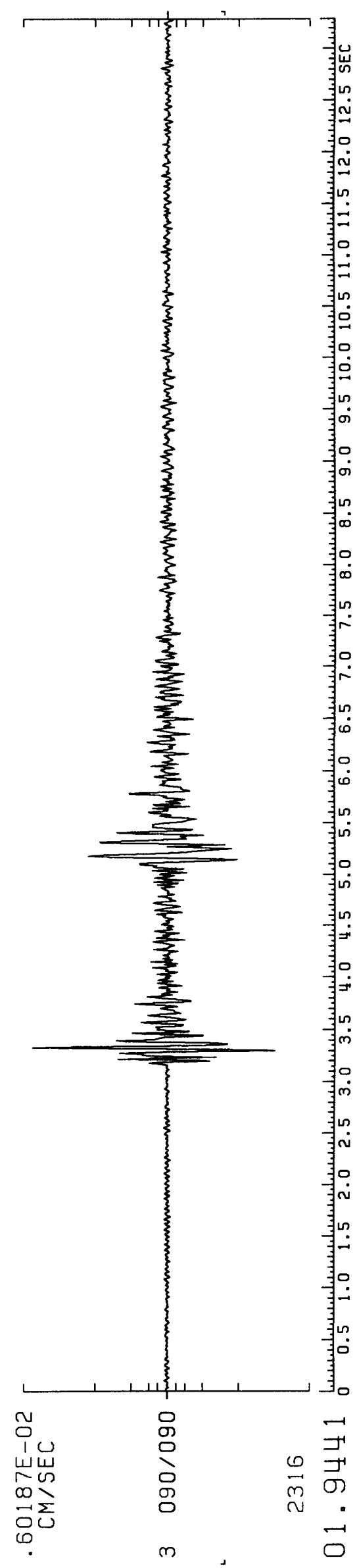


30
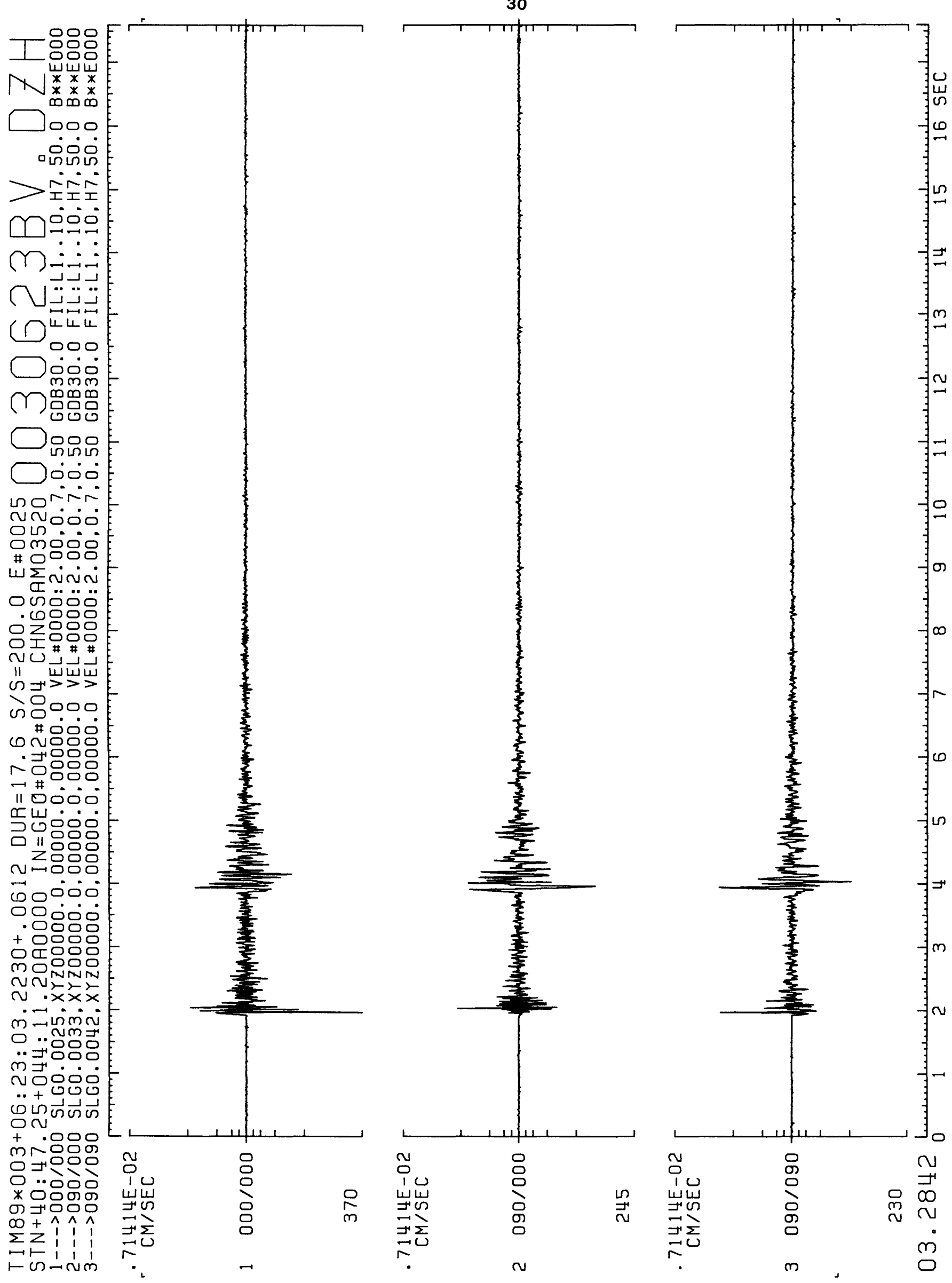
31

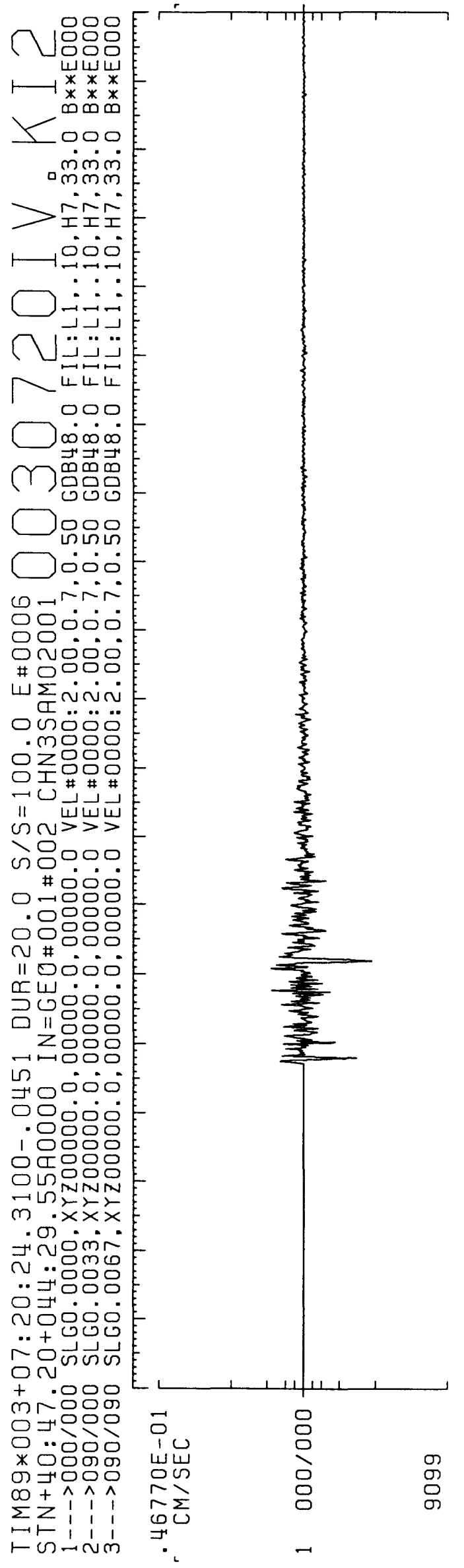

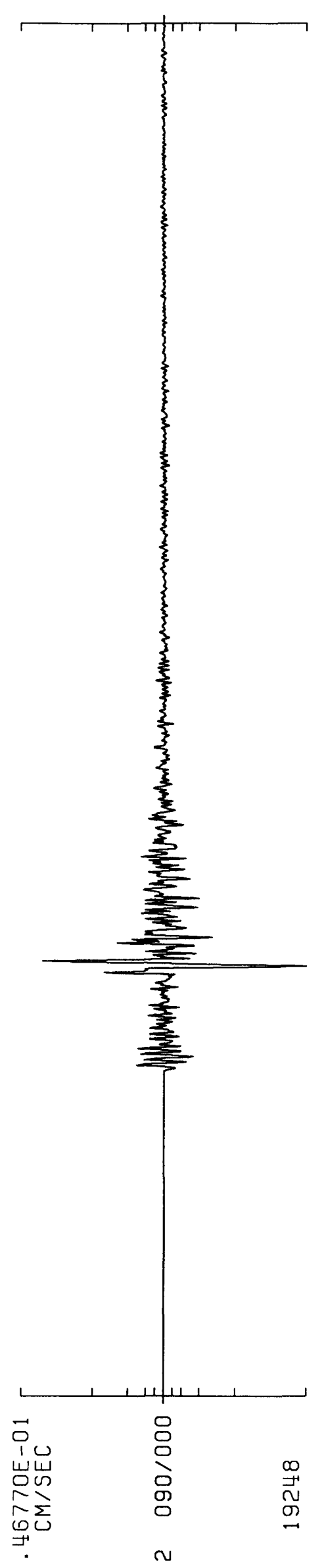

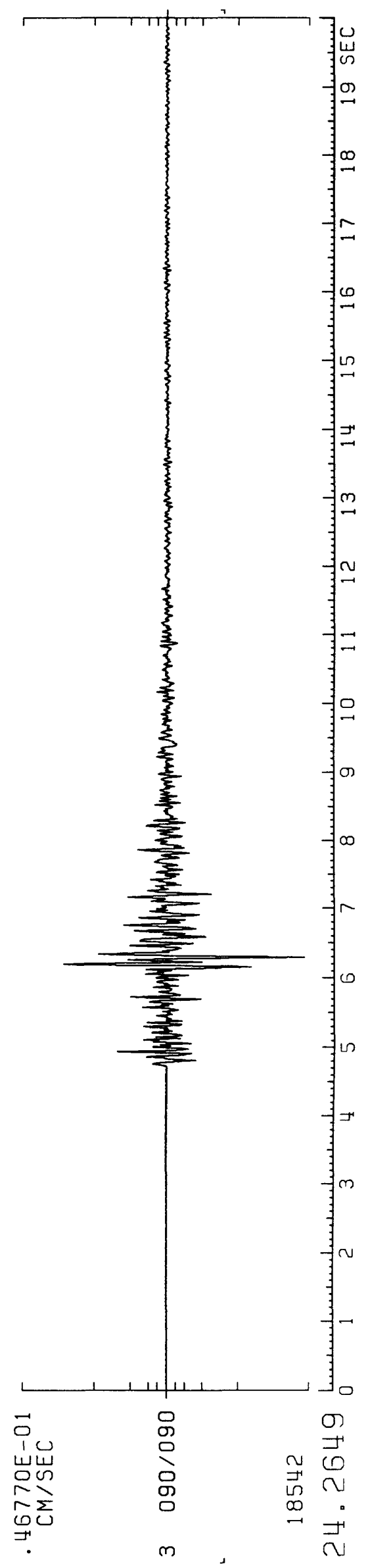


32
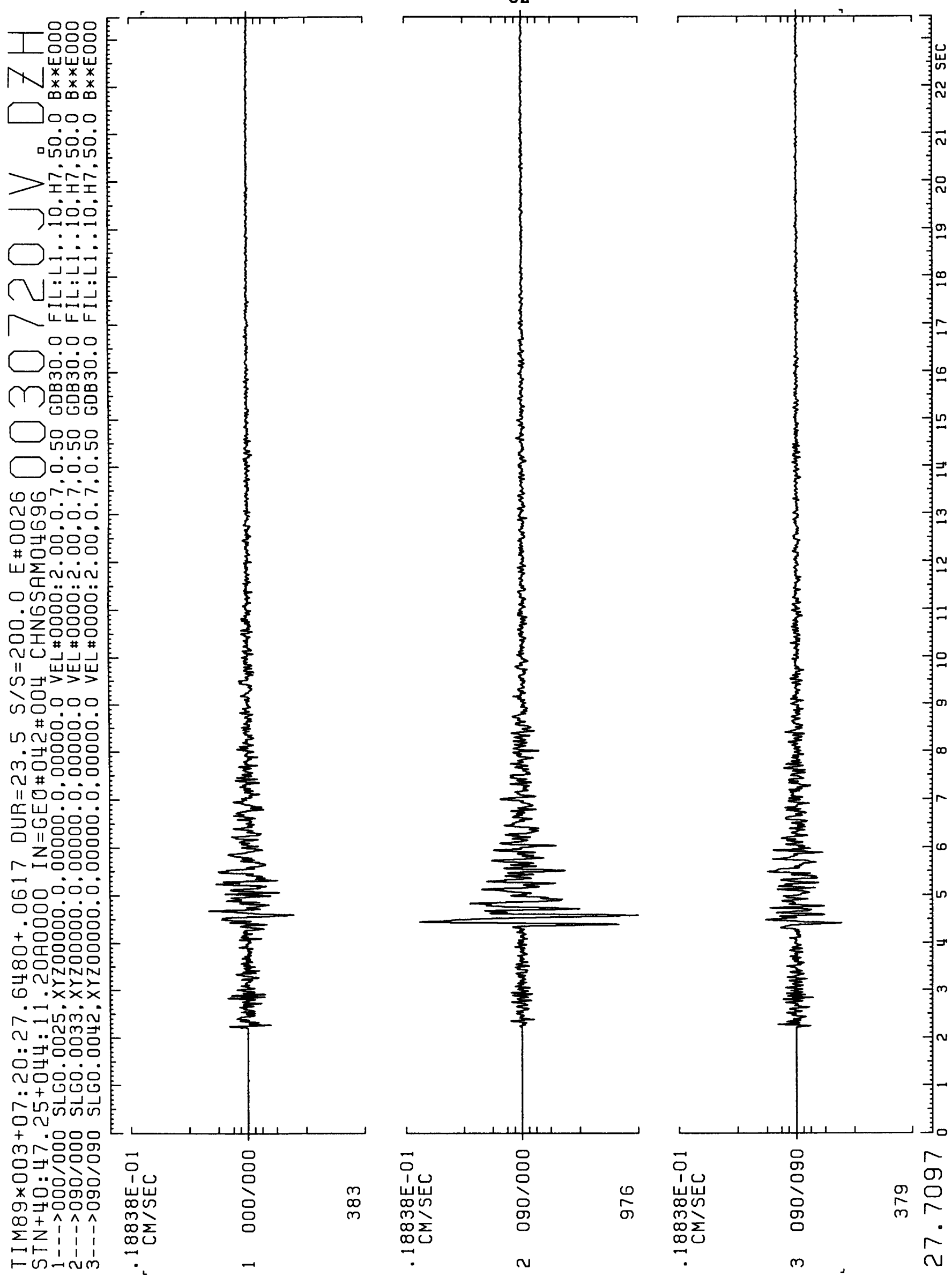
33
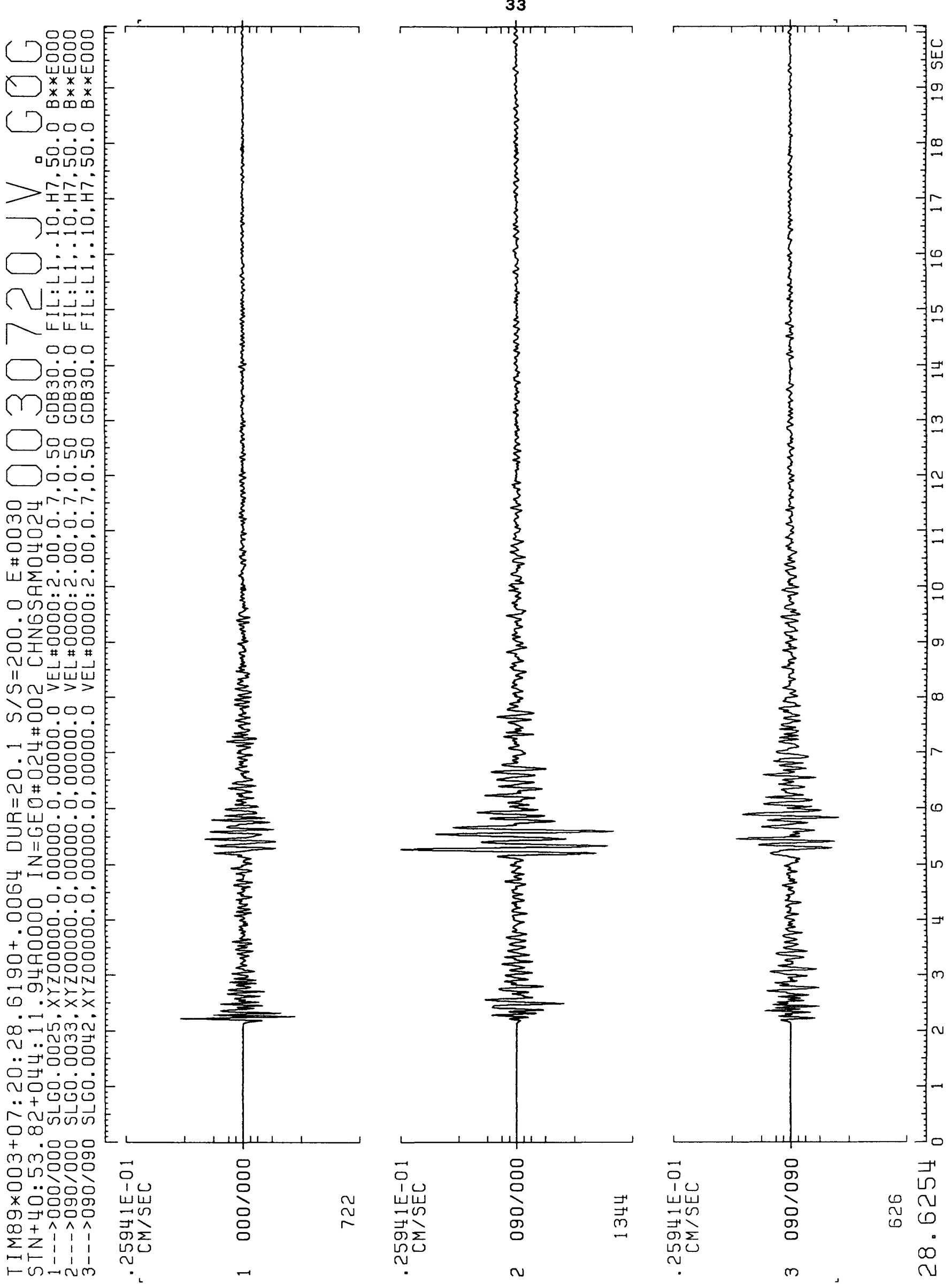


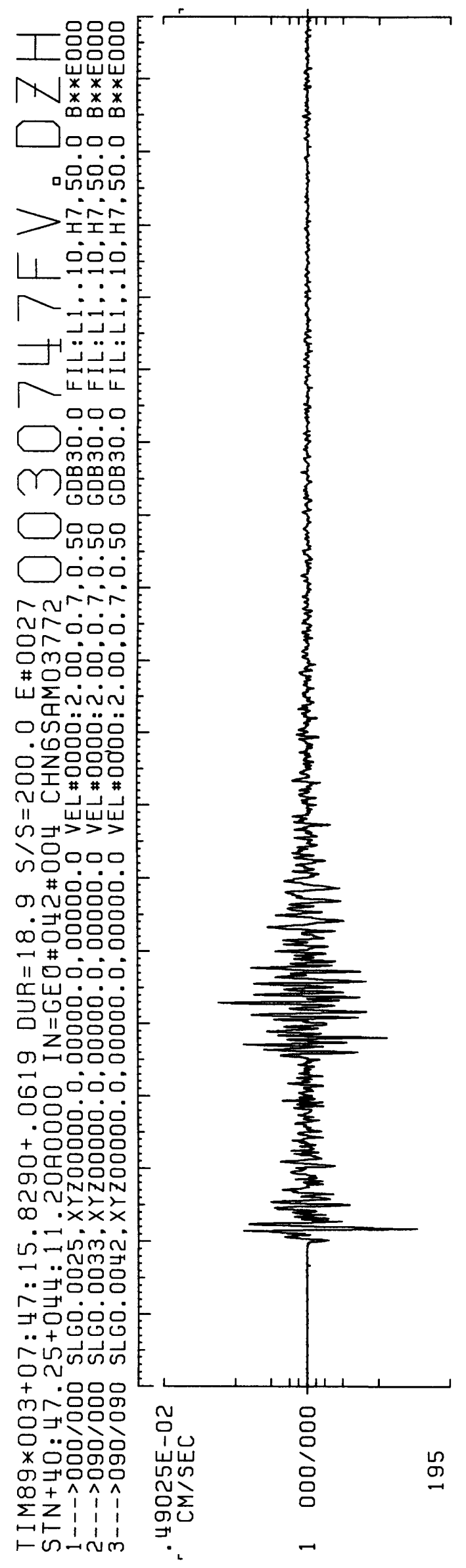

34

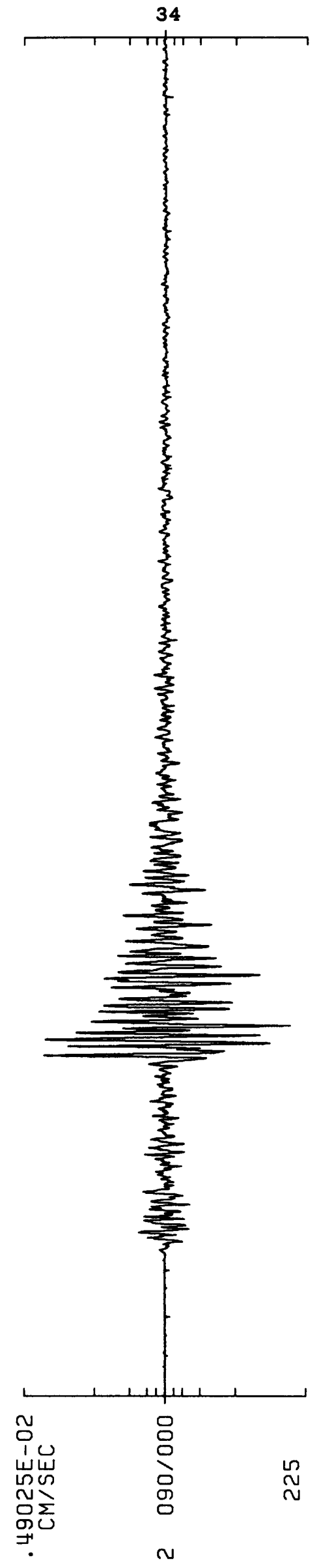

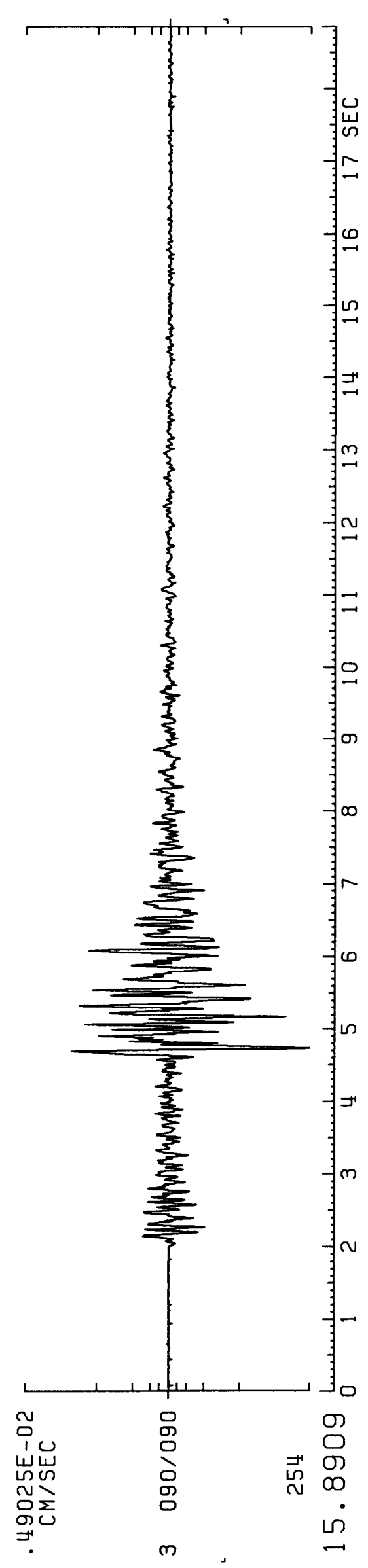


35
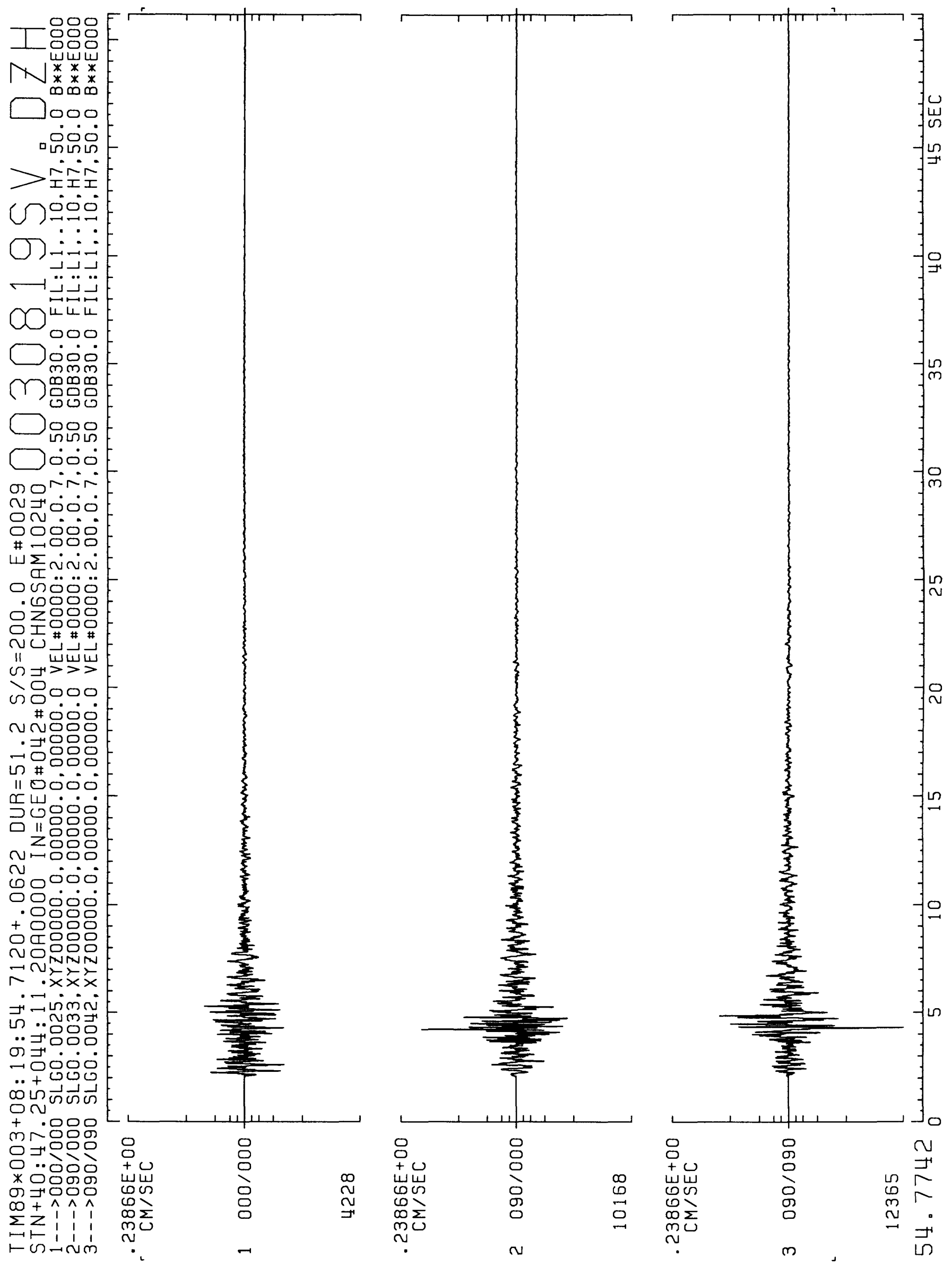
36
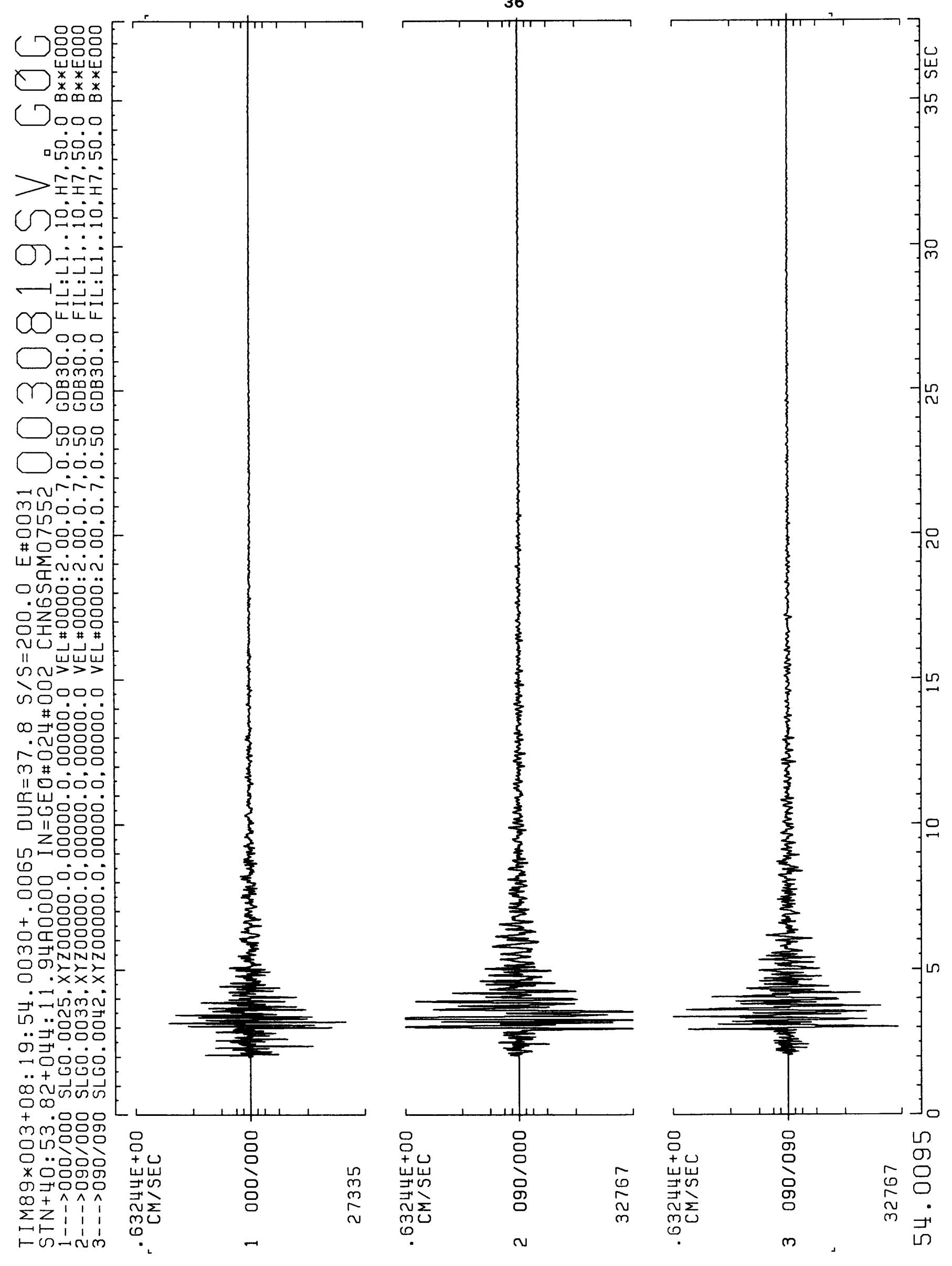

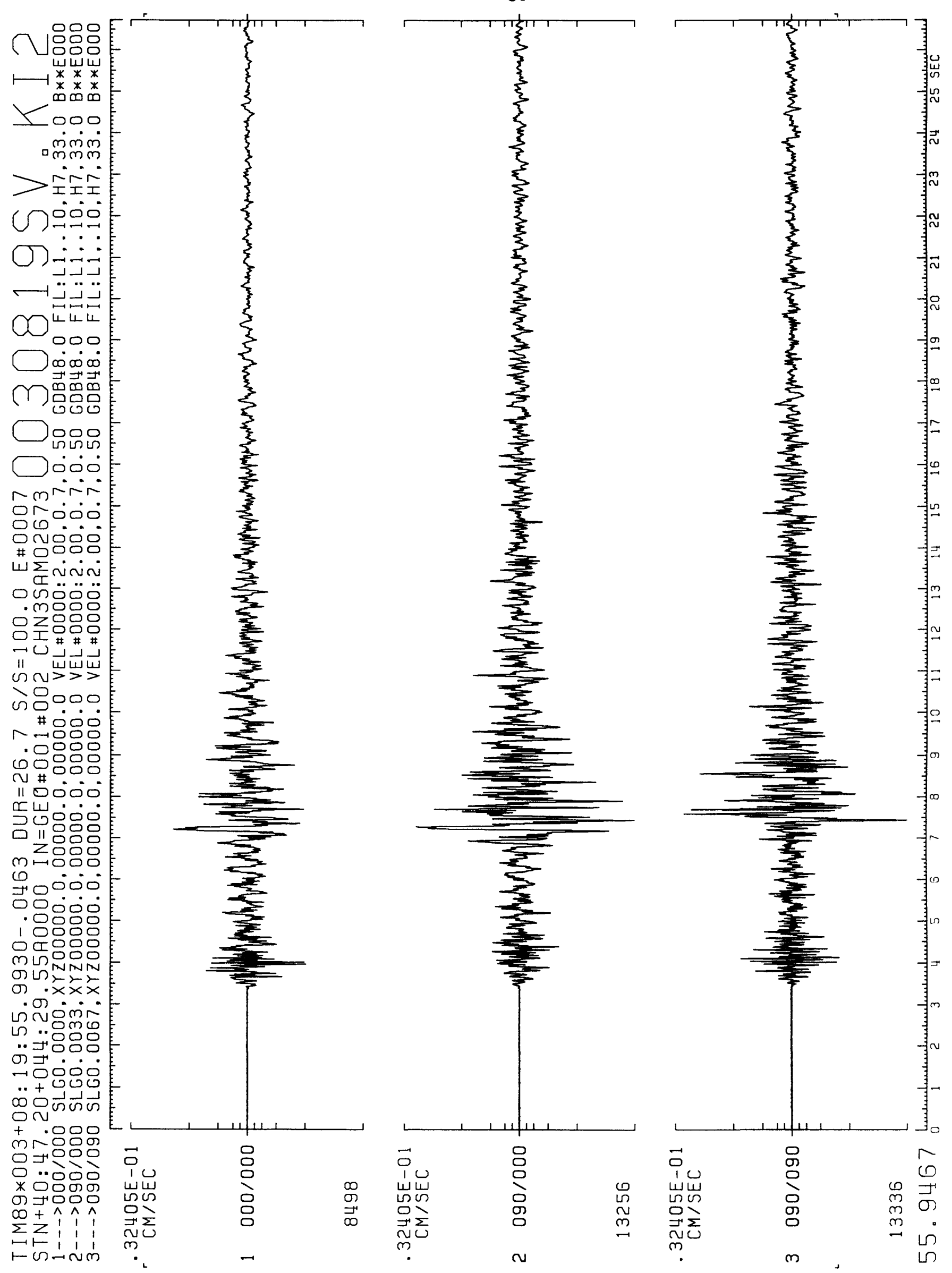
38

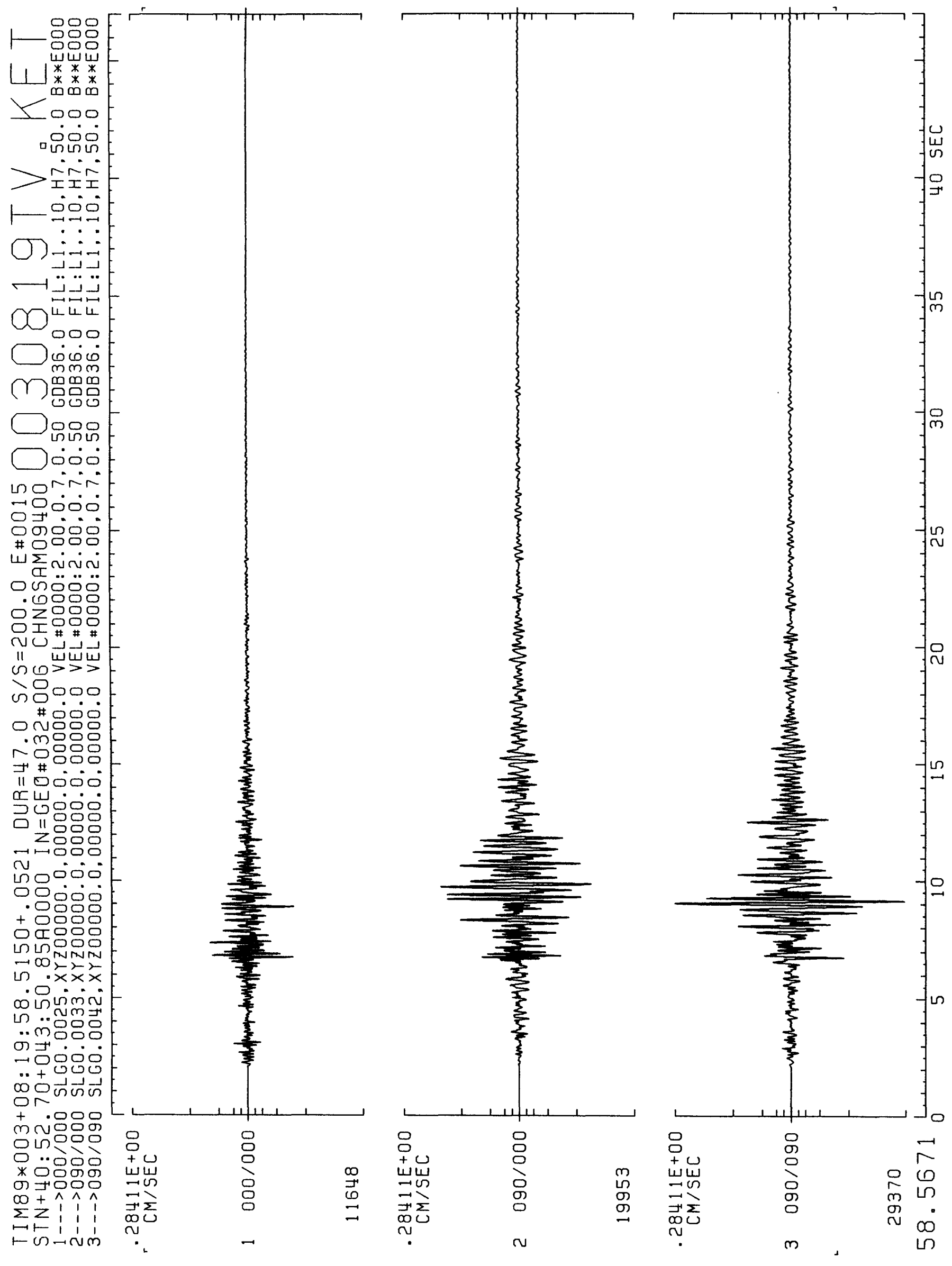



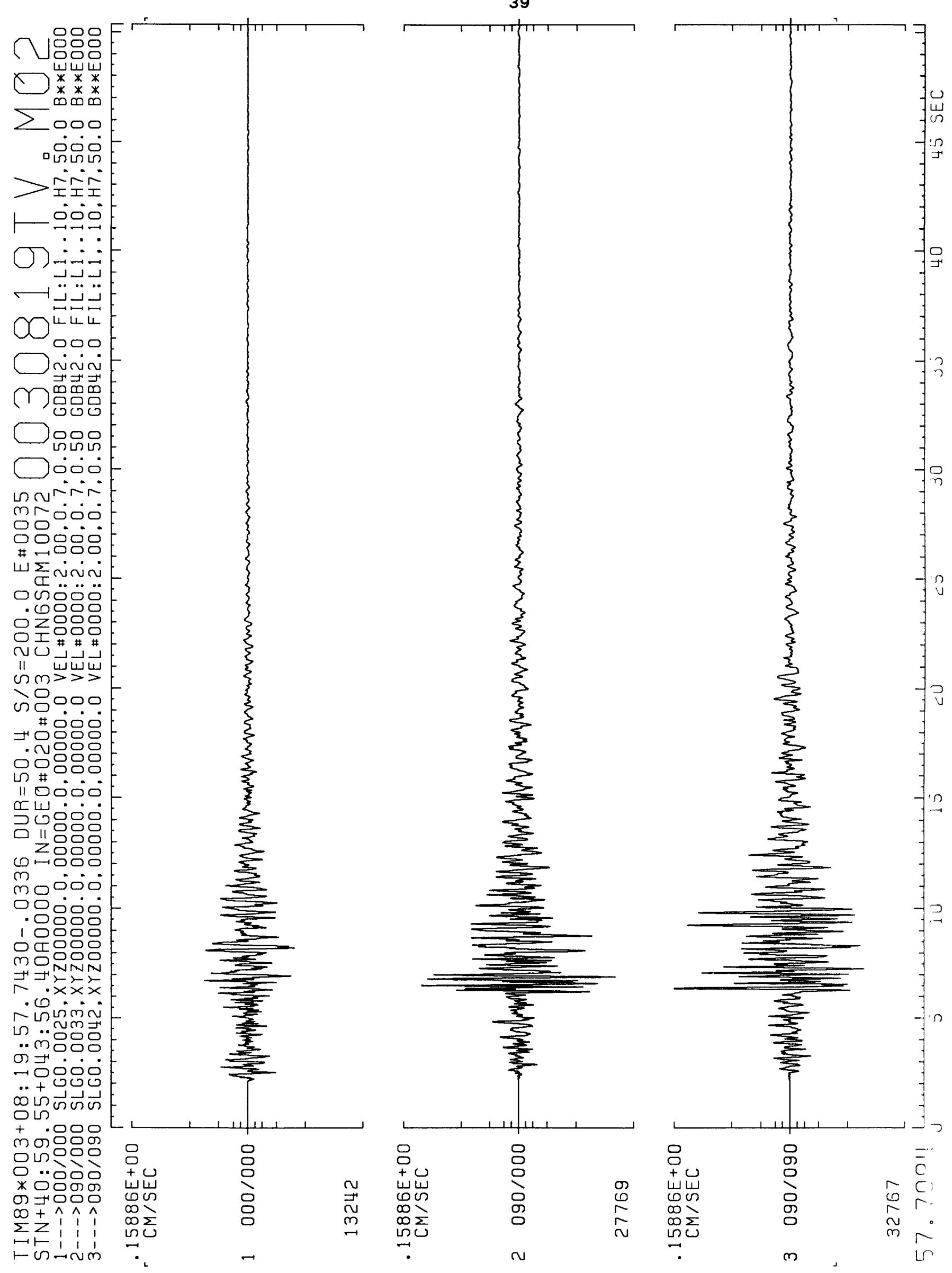


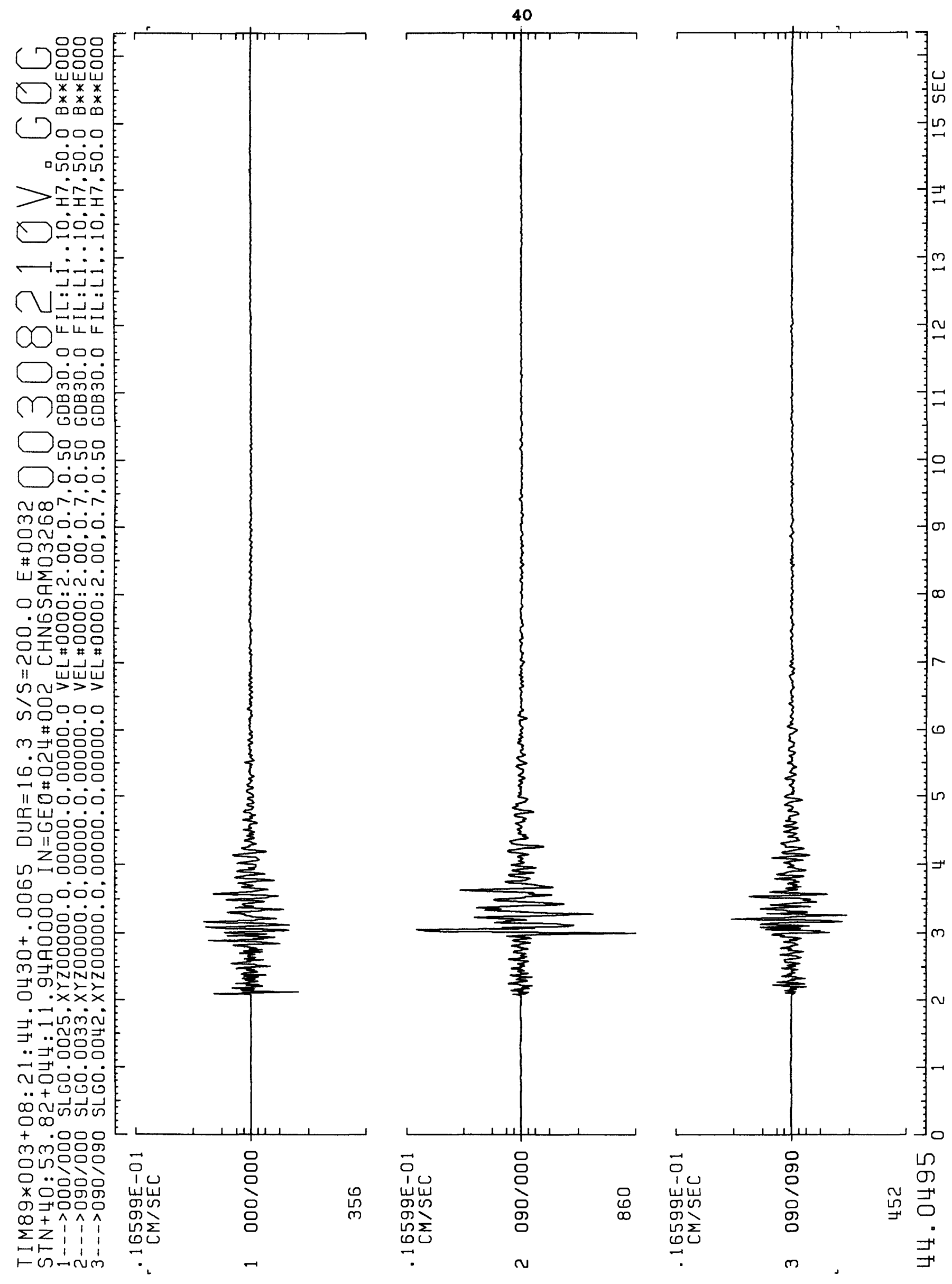


41
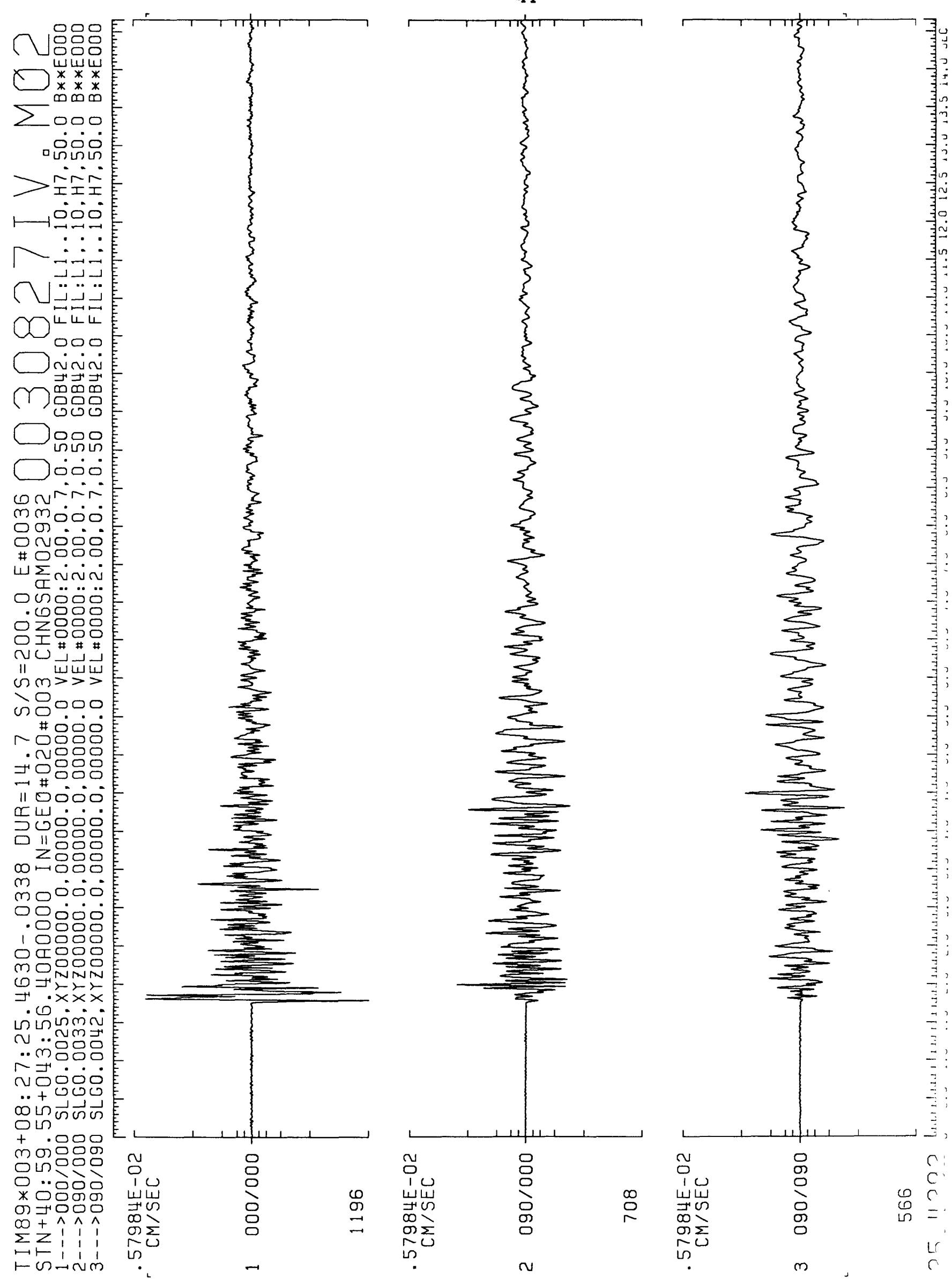
42

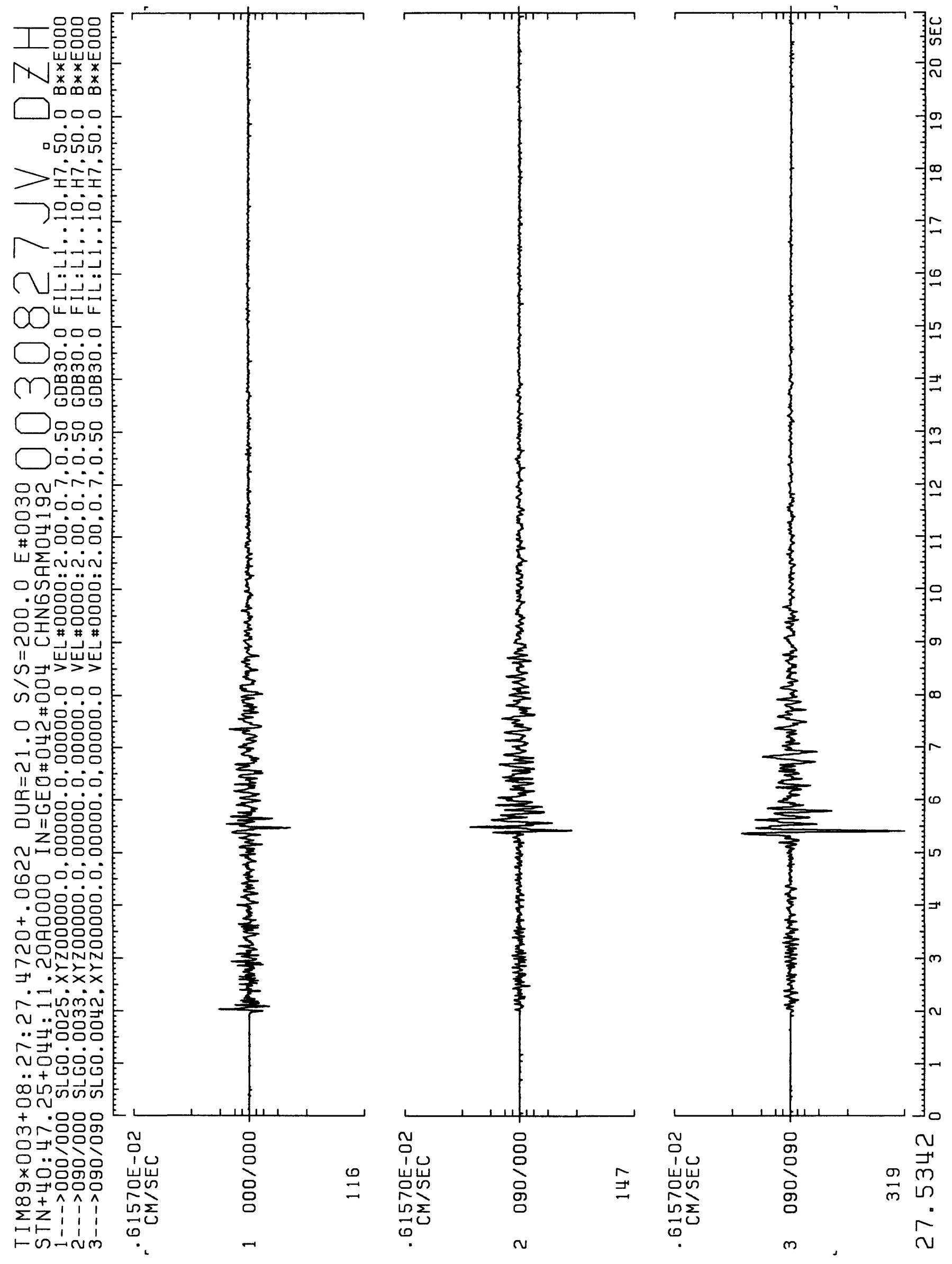




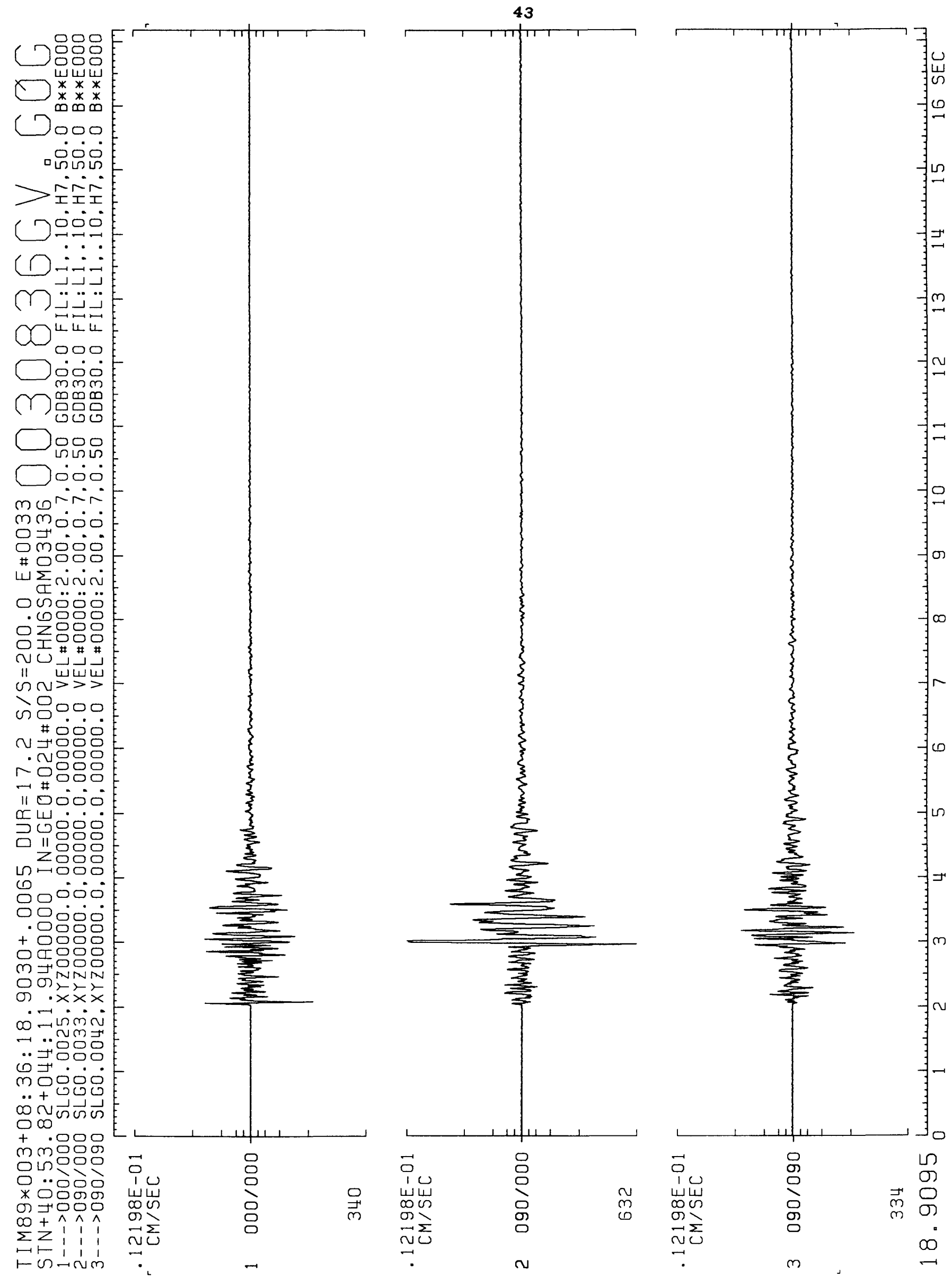



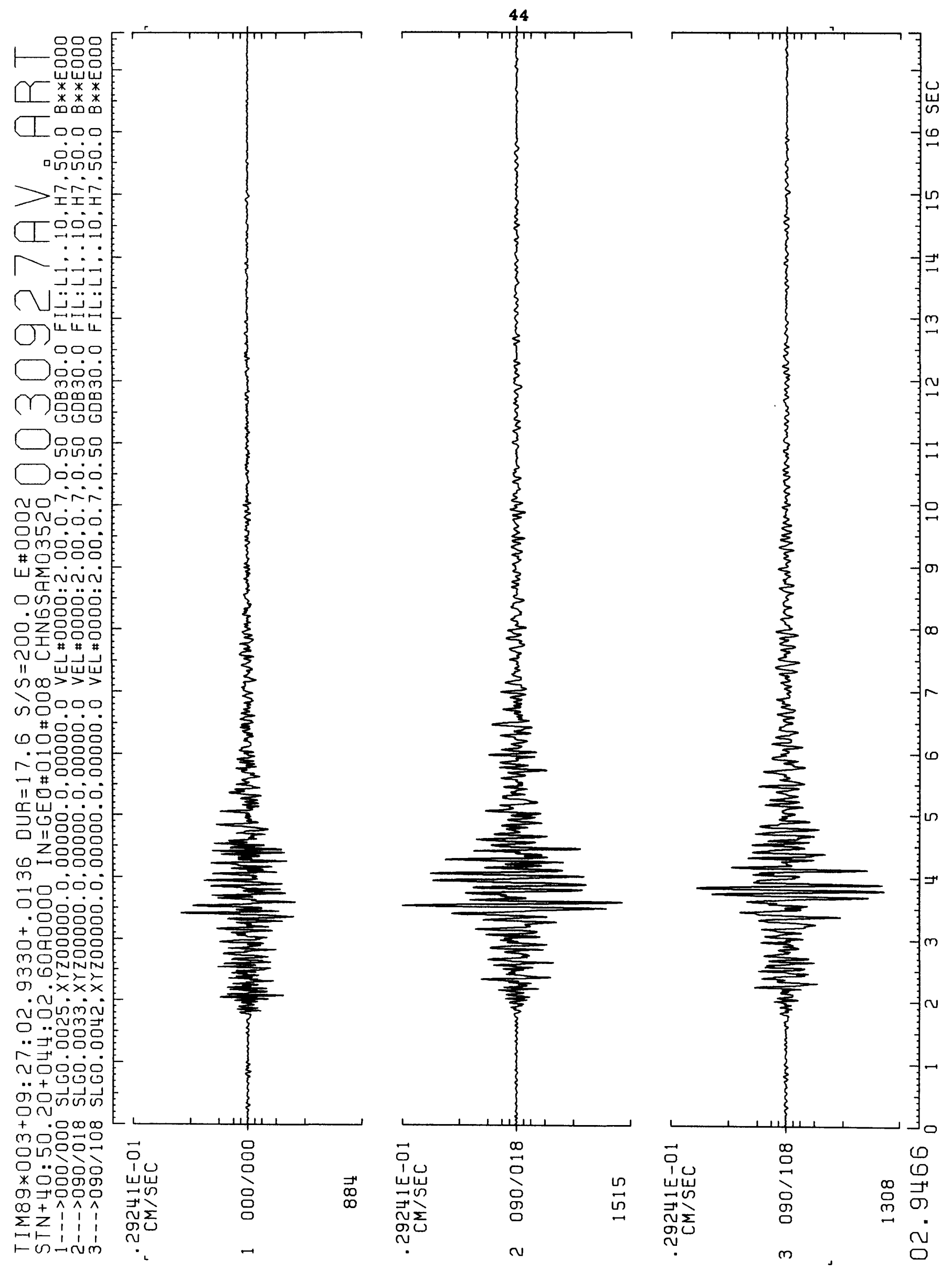


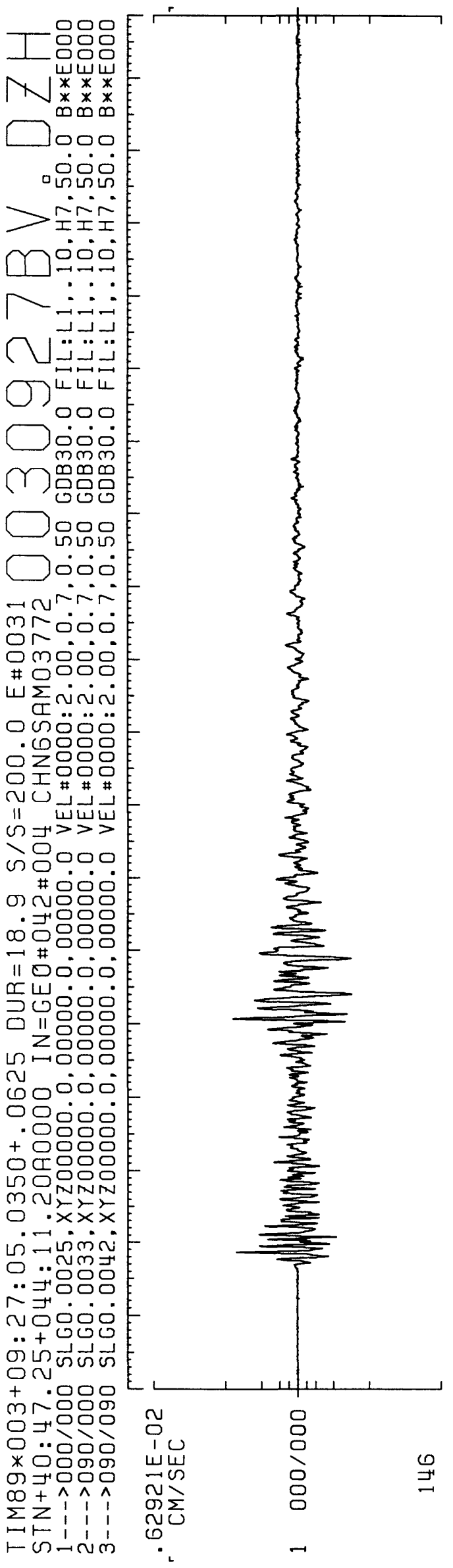

45

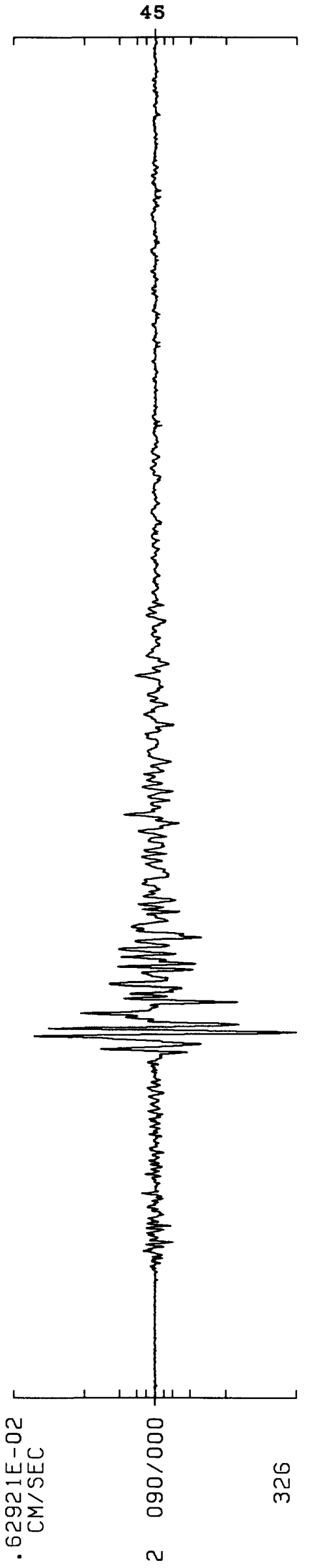

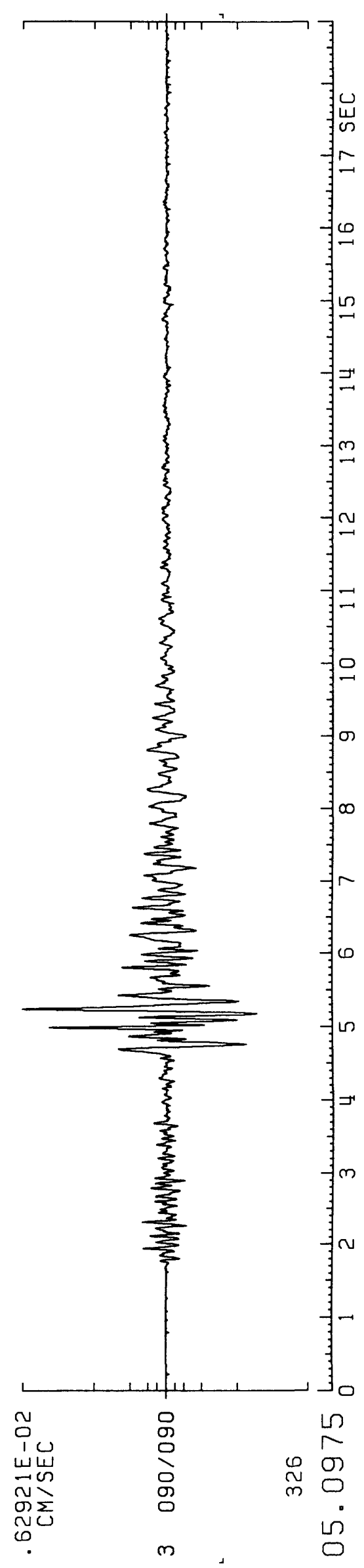


46
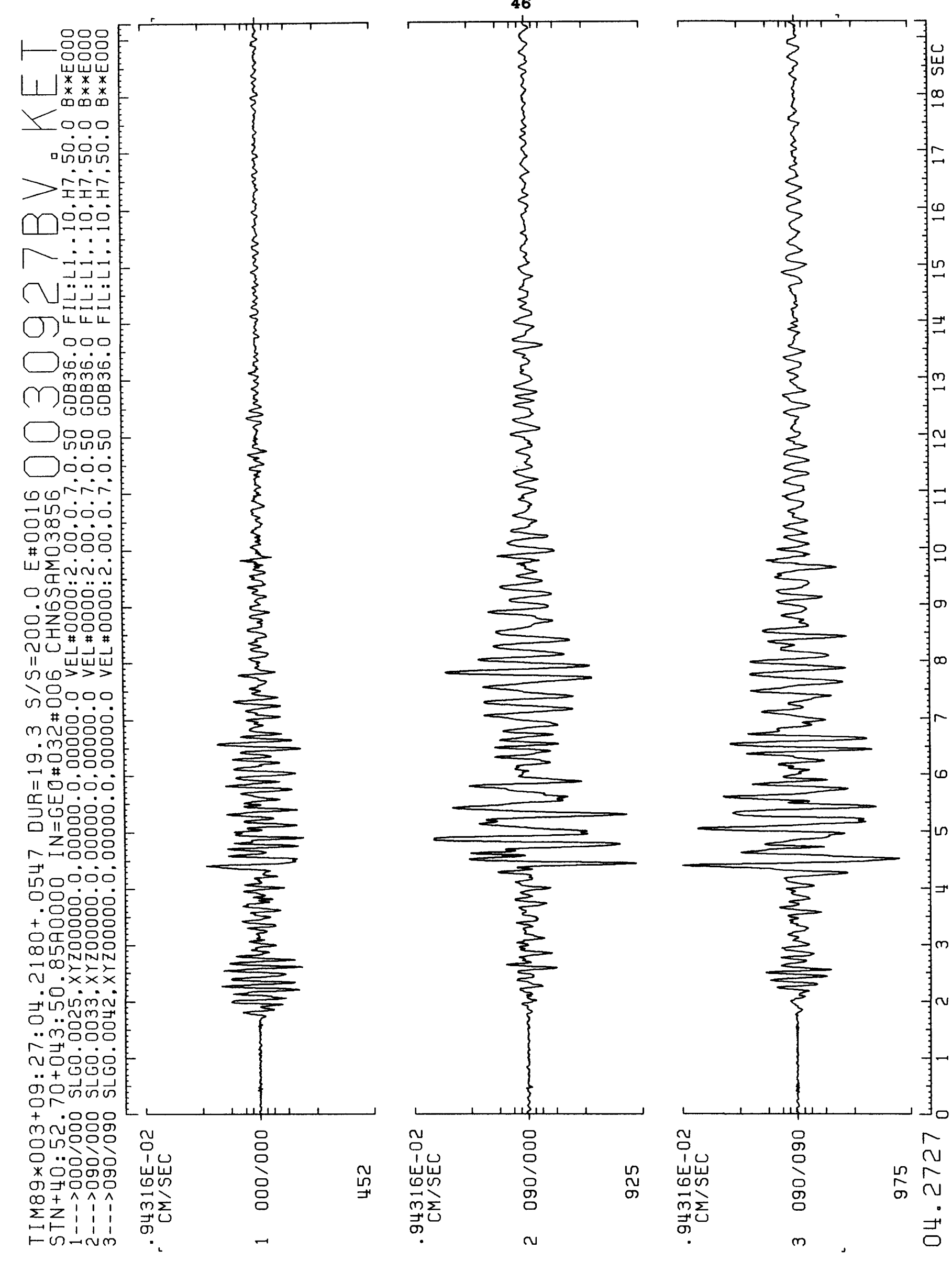
47

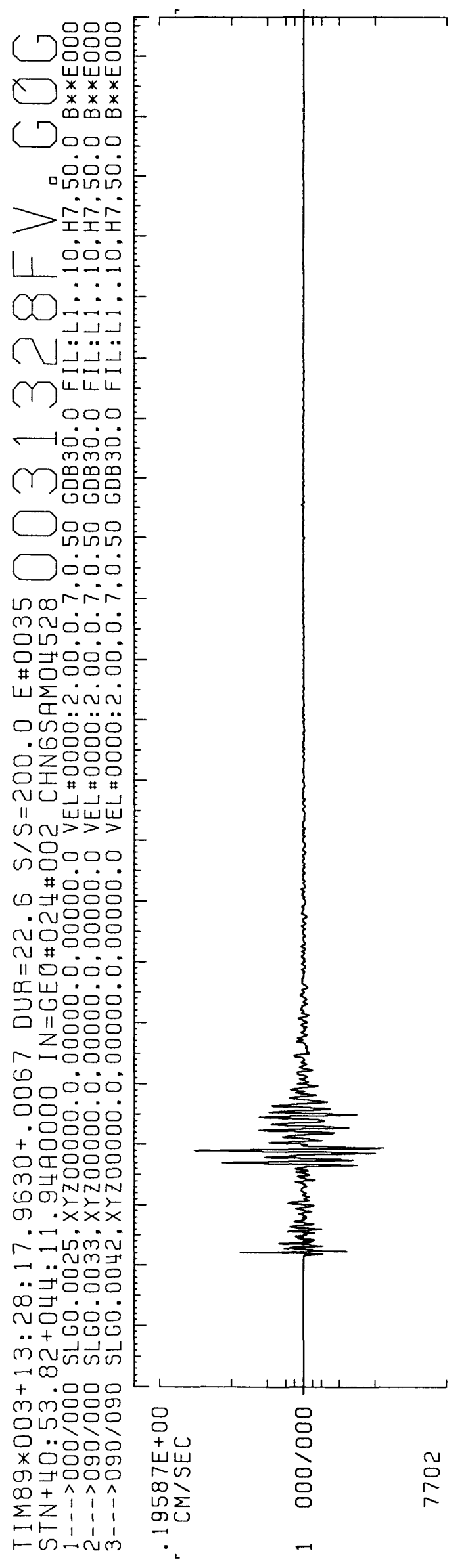

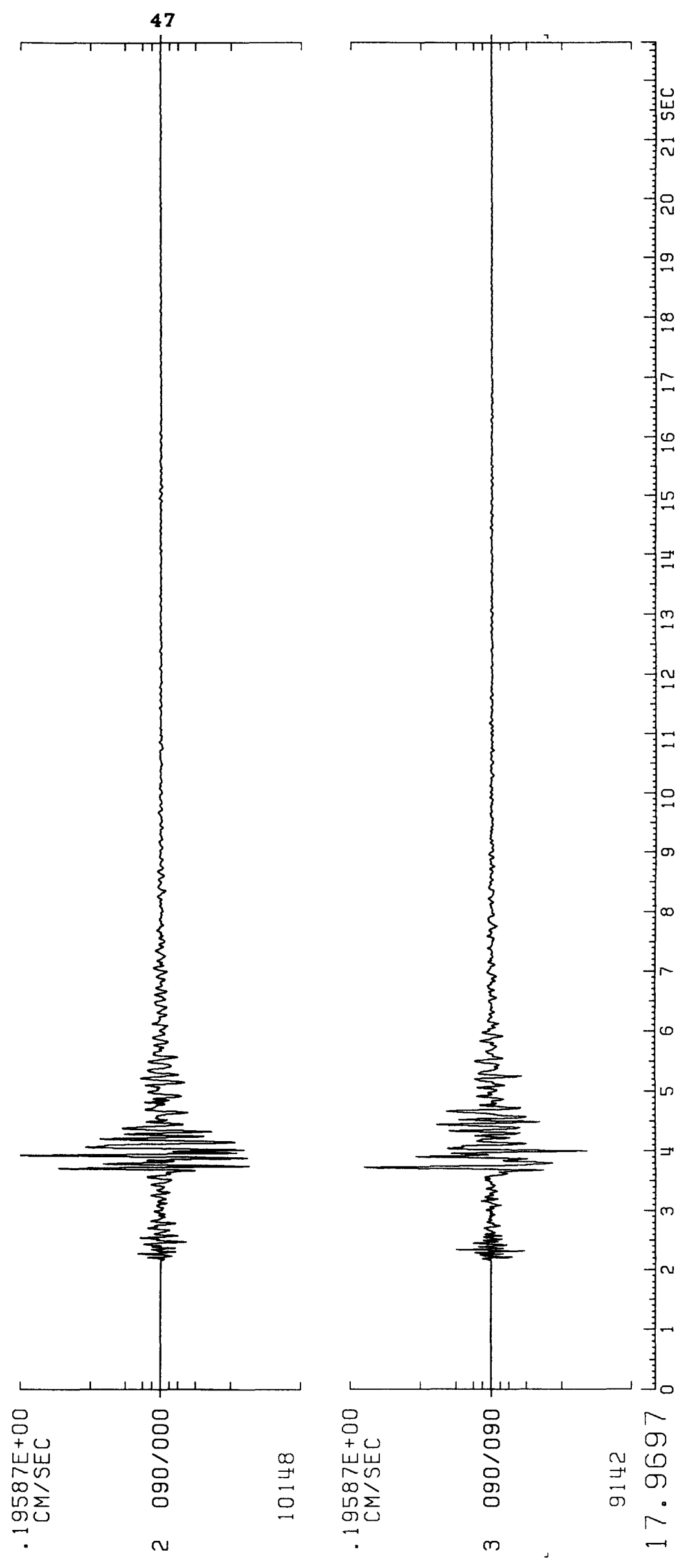


48

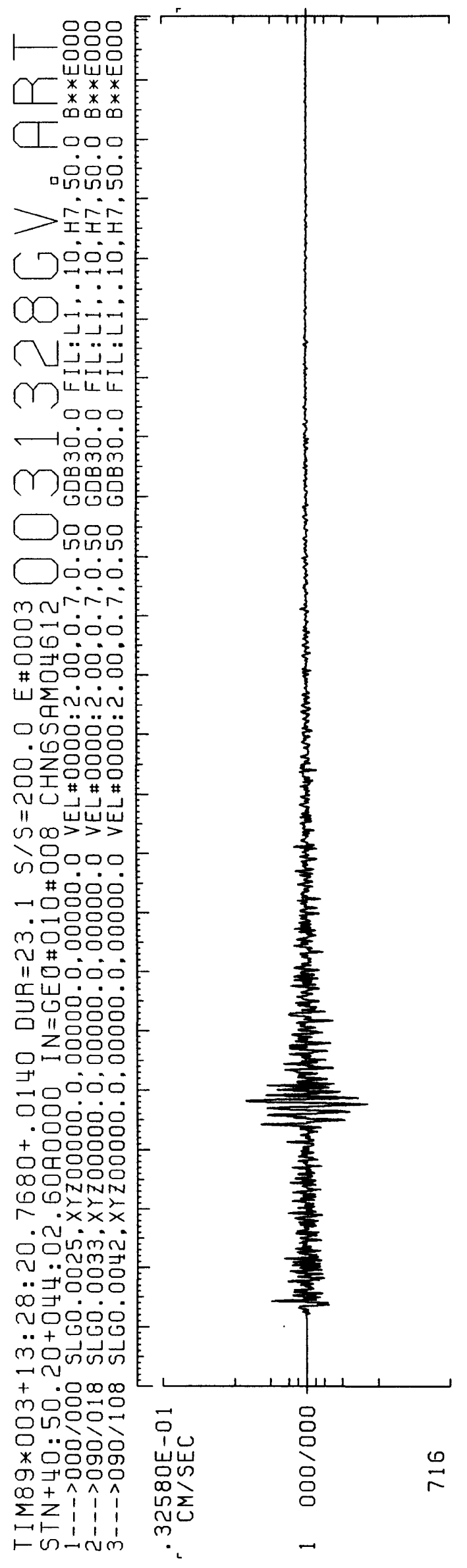

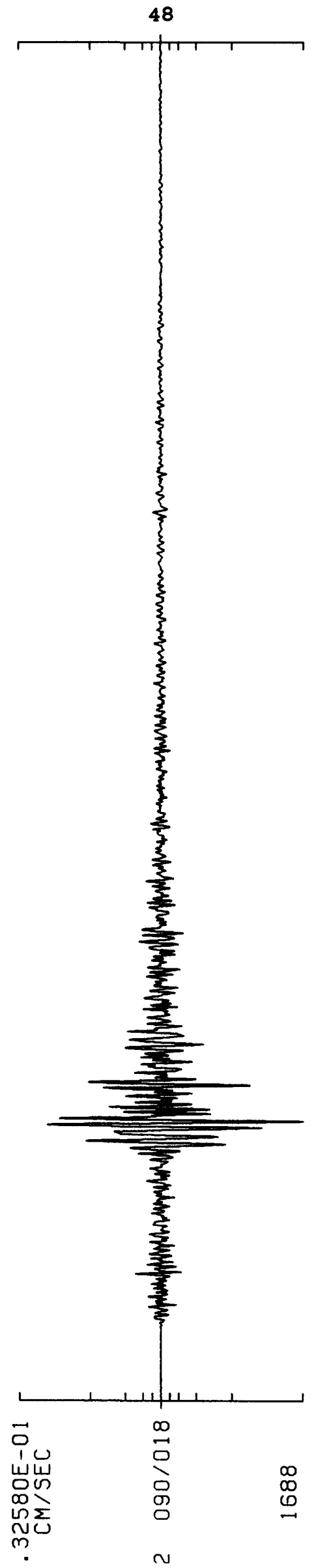

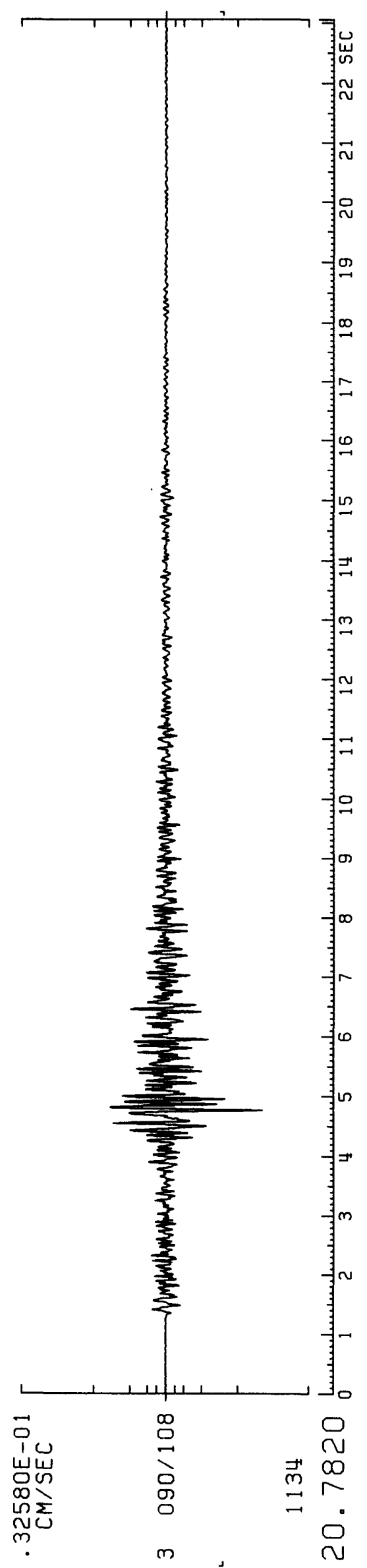


49

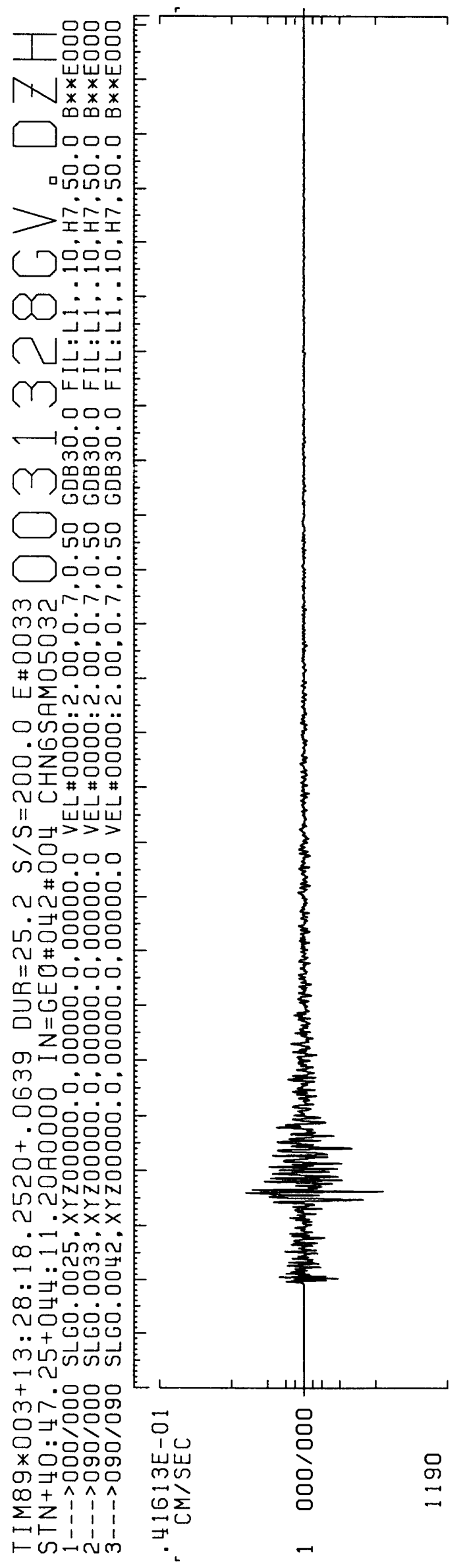

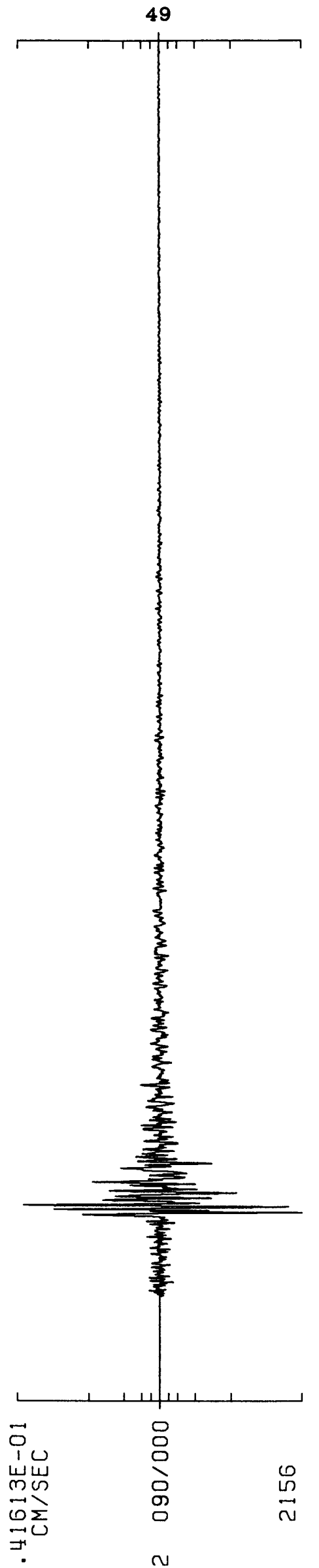

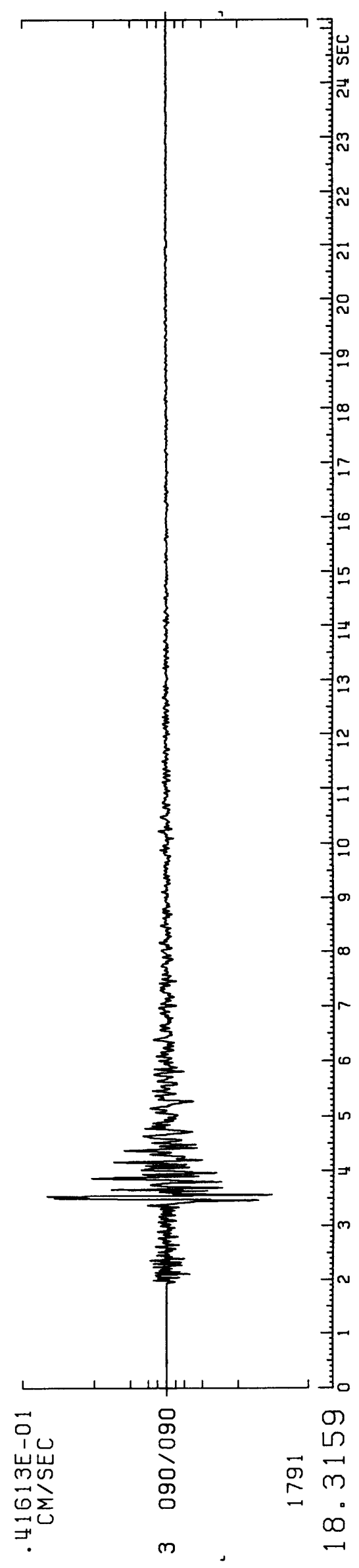




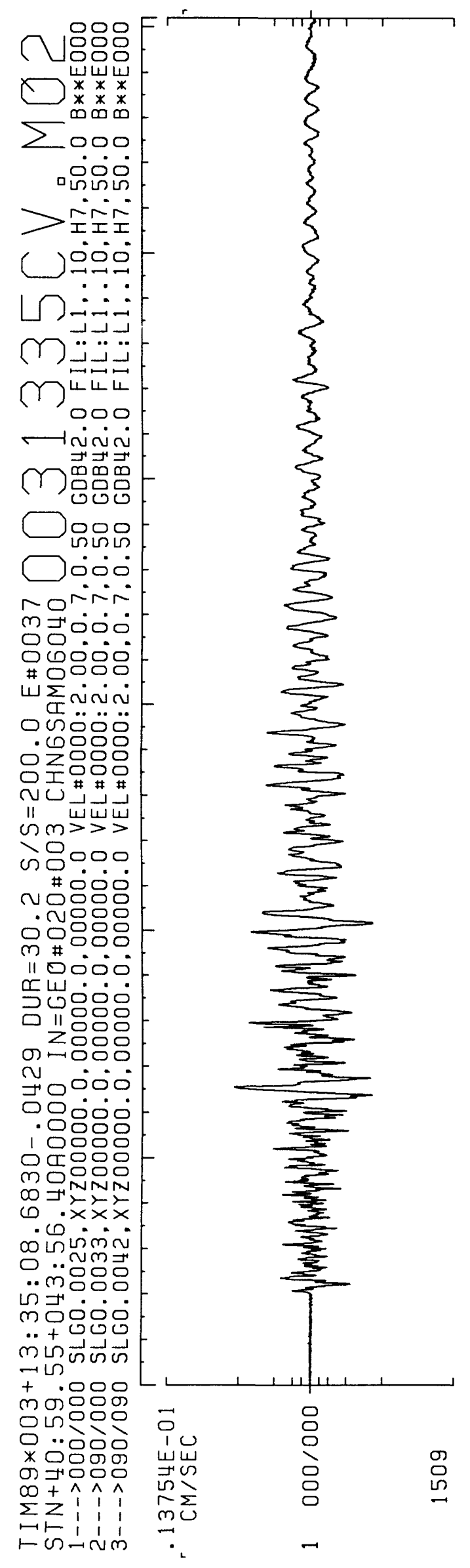

50

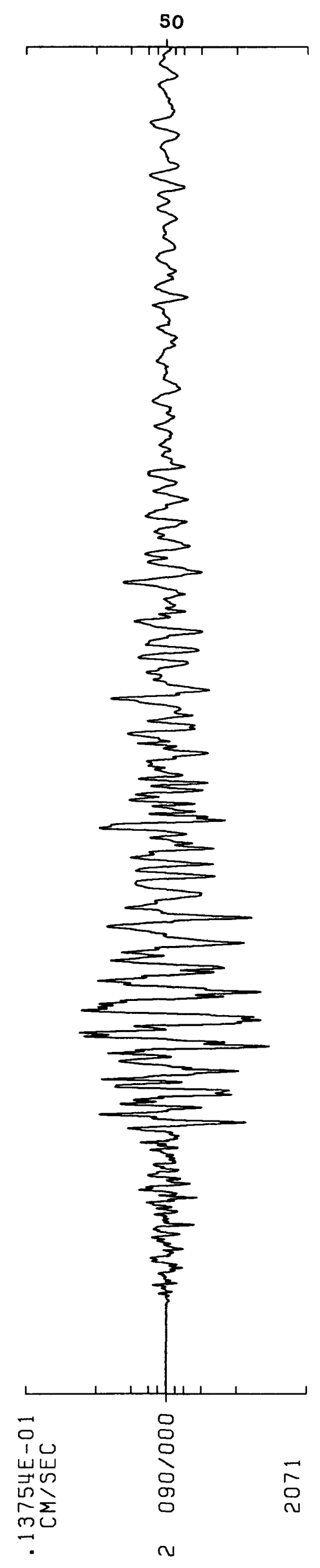

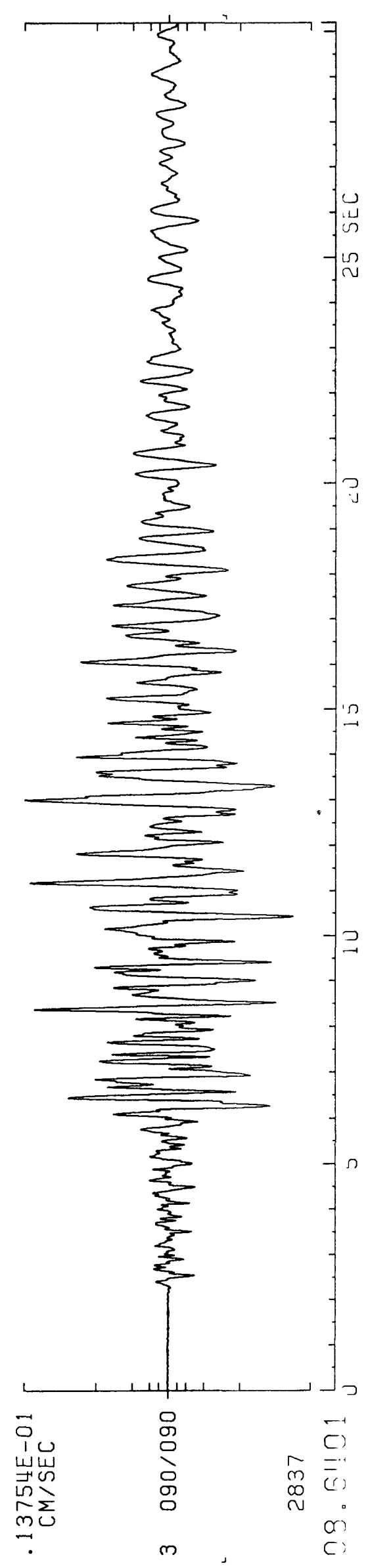




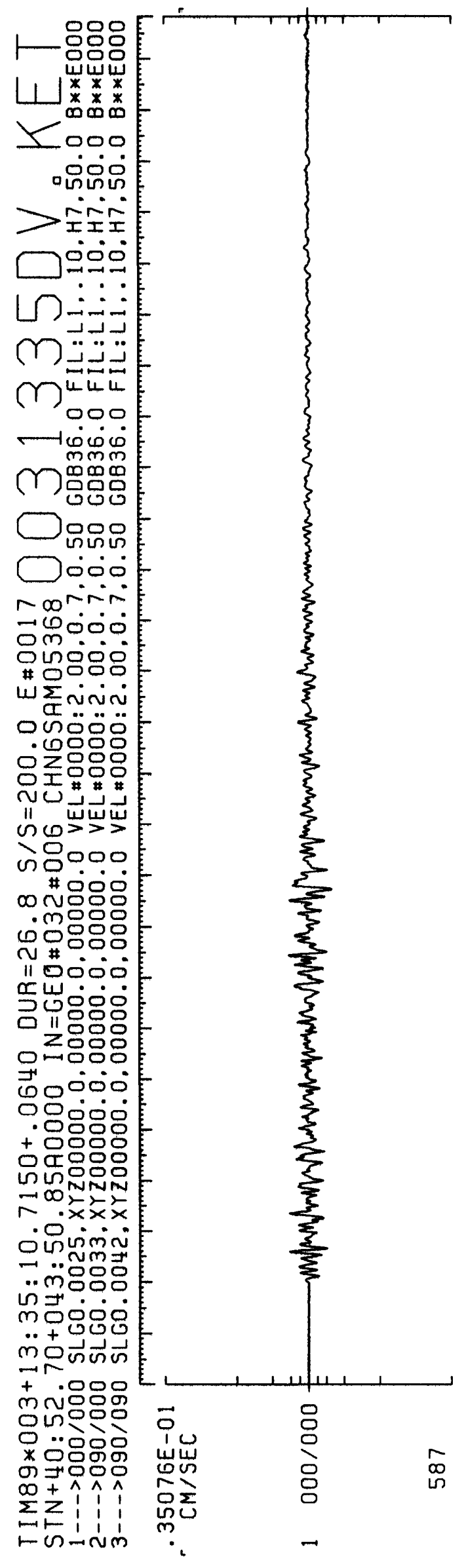

51

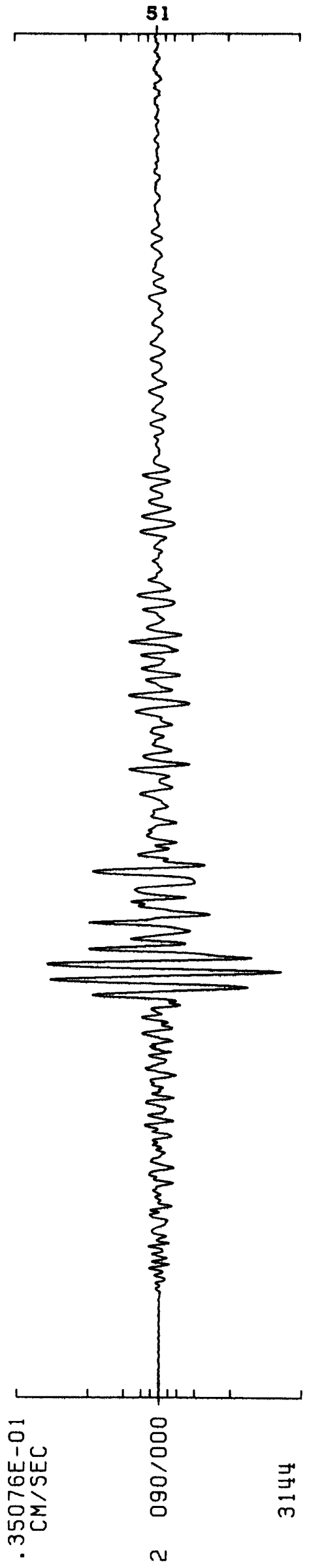

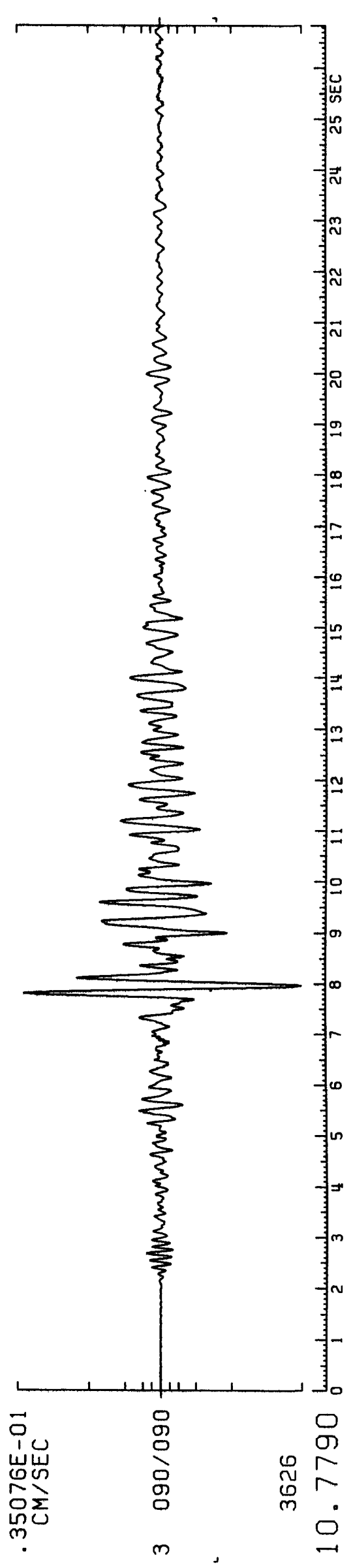




\section{2}
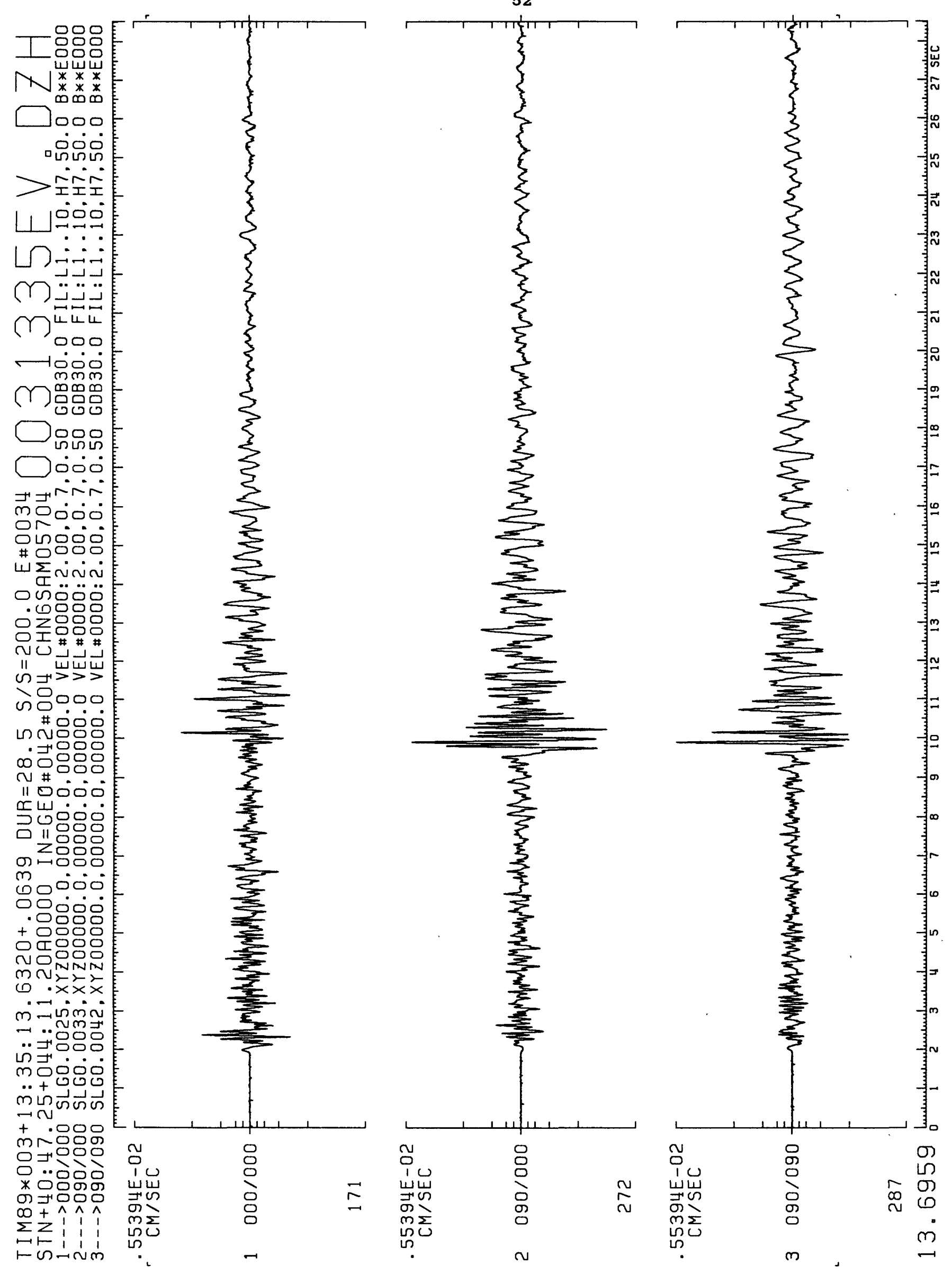
53
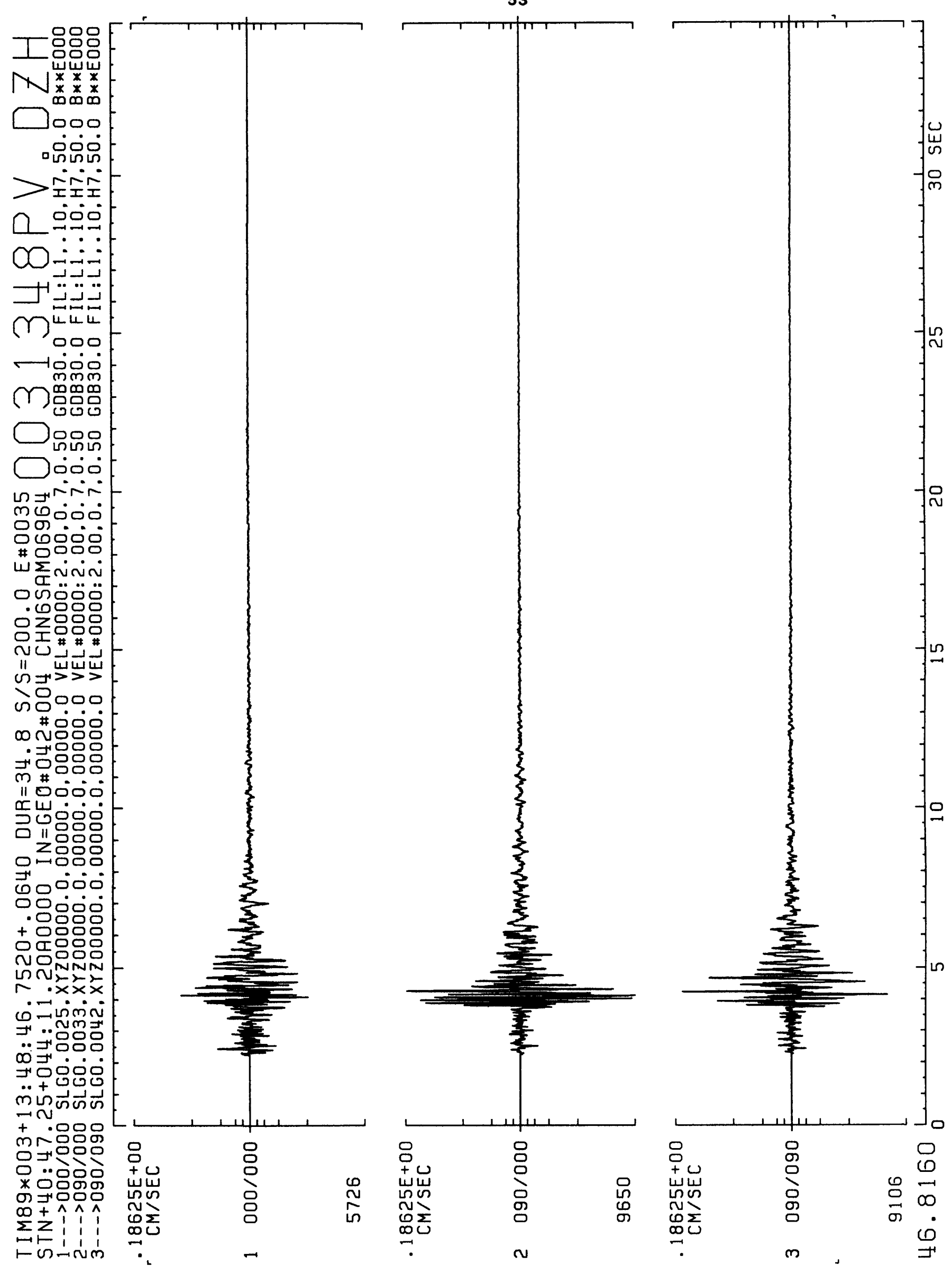
54
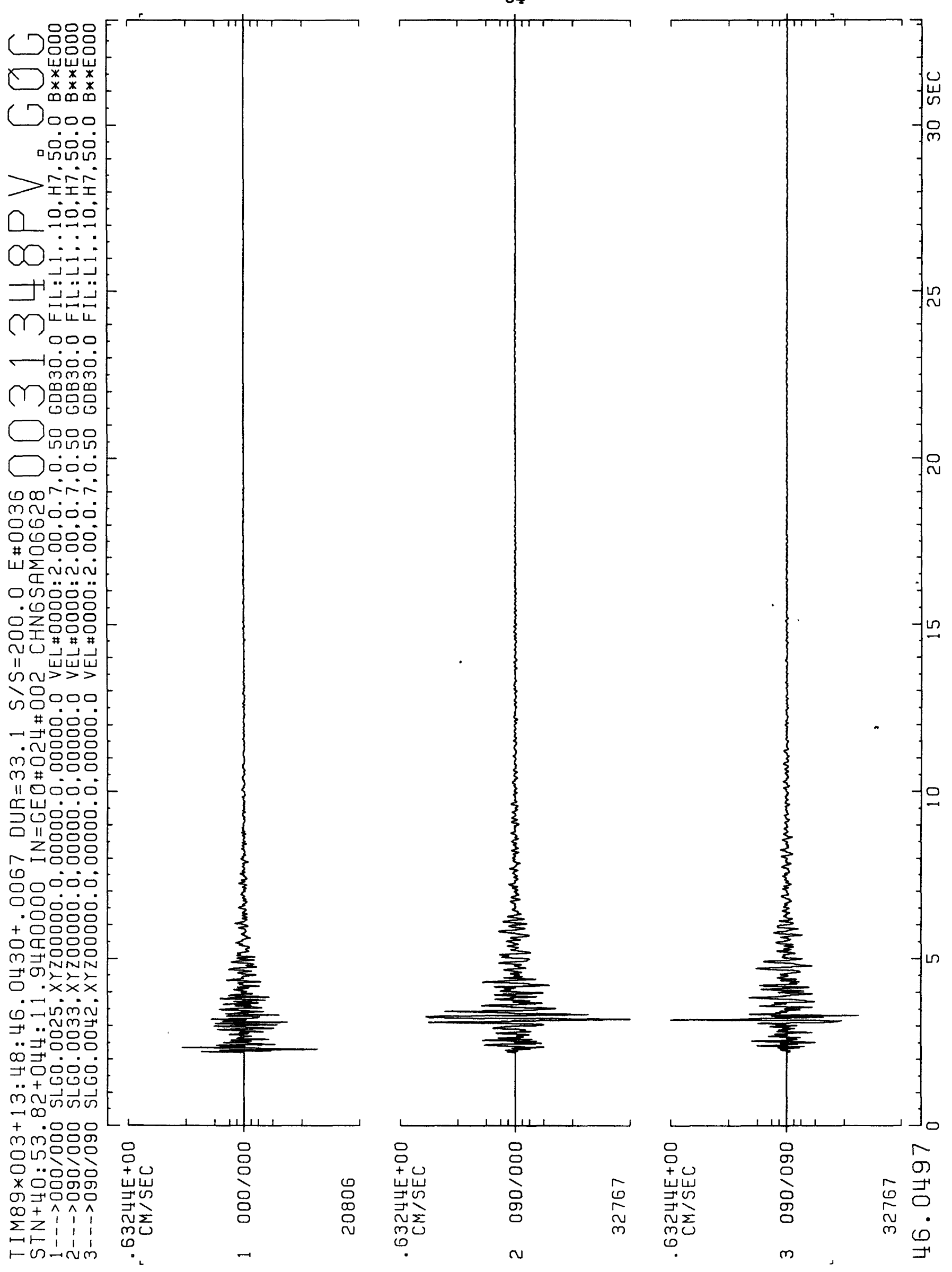


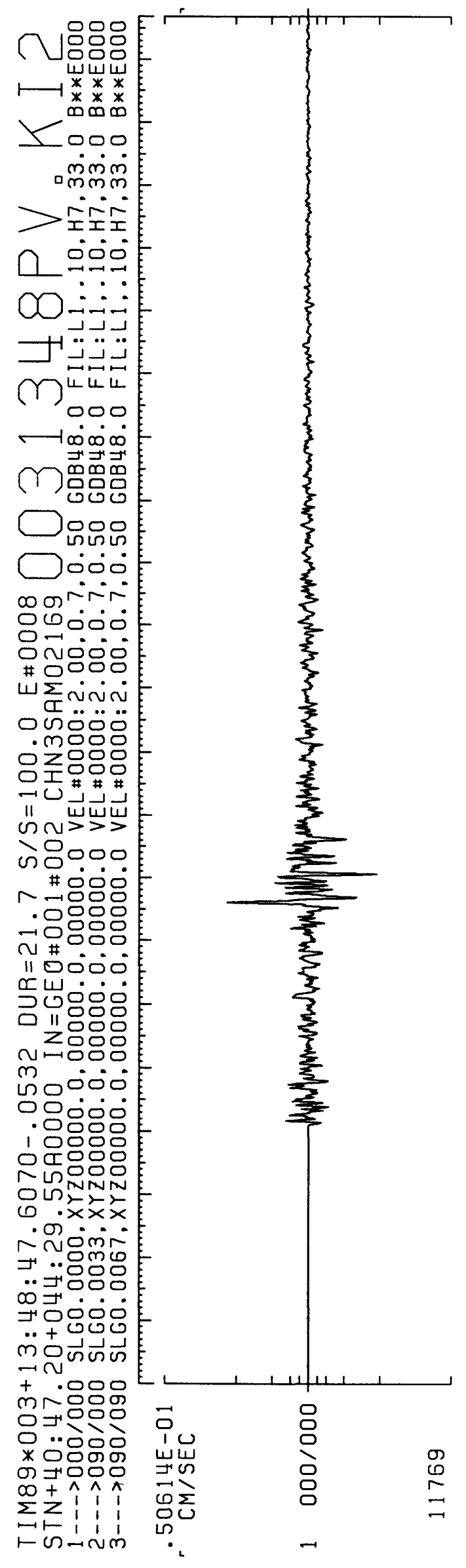

55

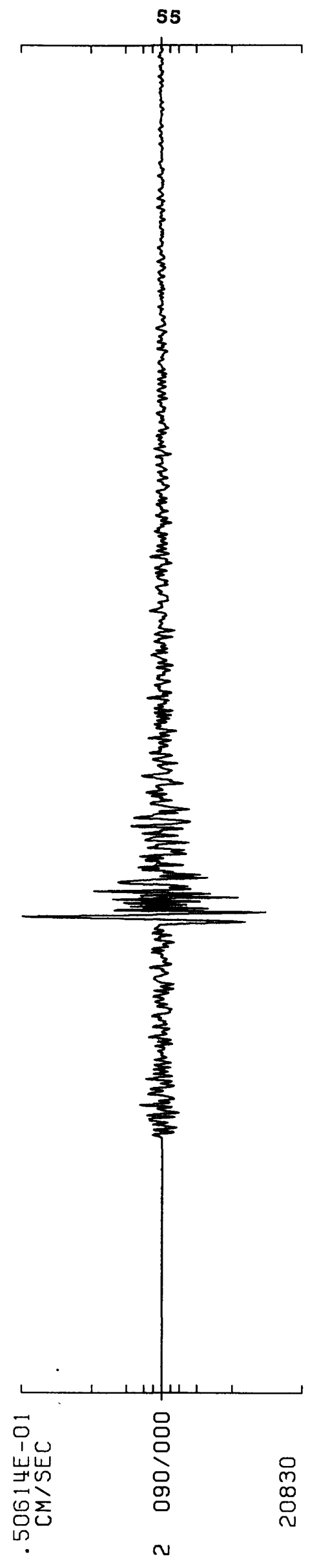




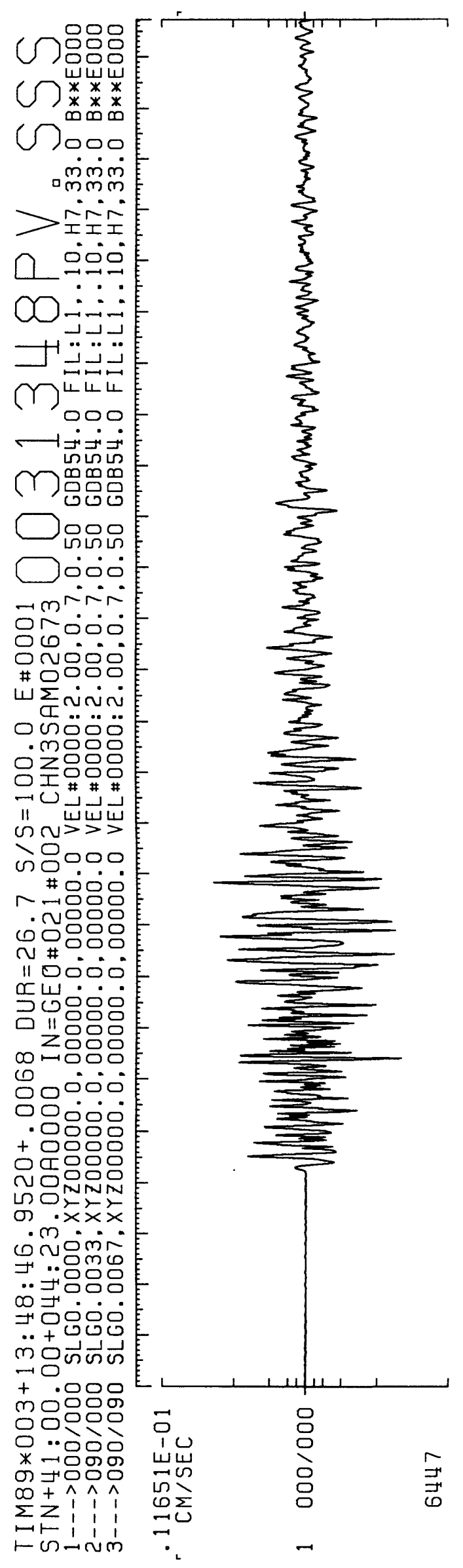

56

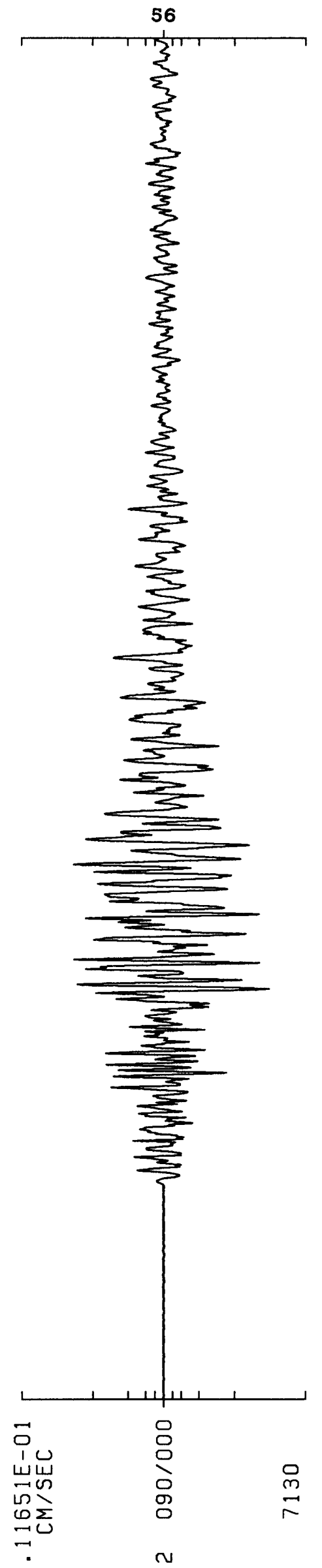

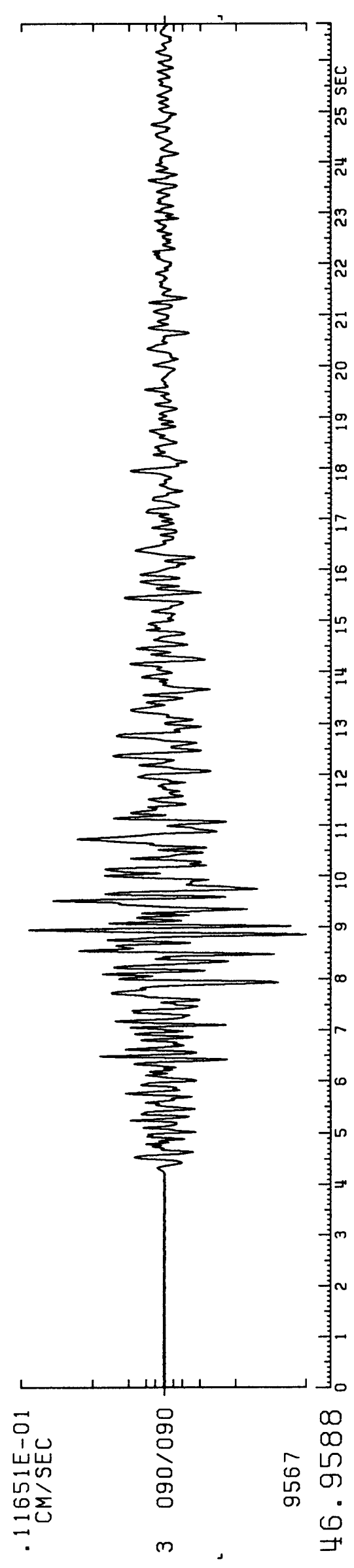


57
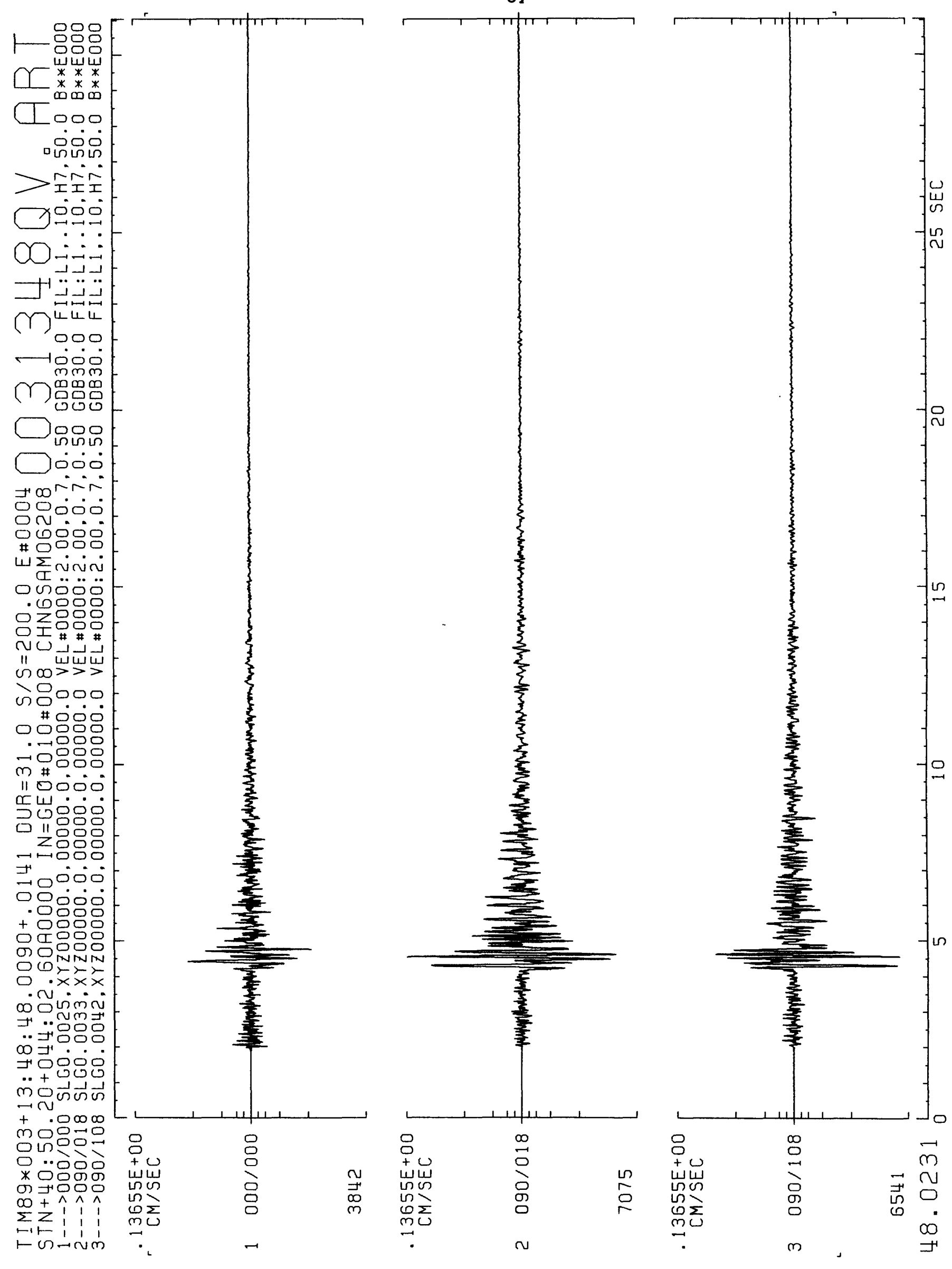


\section{8}
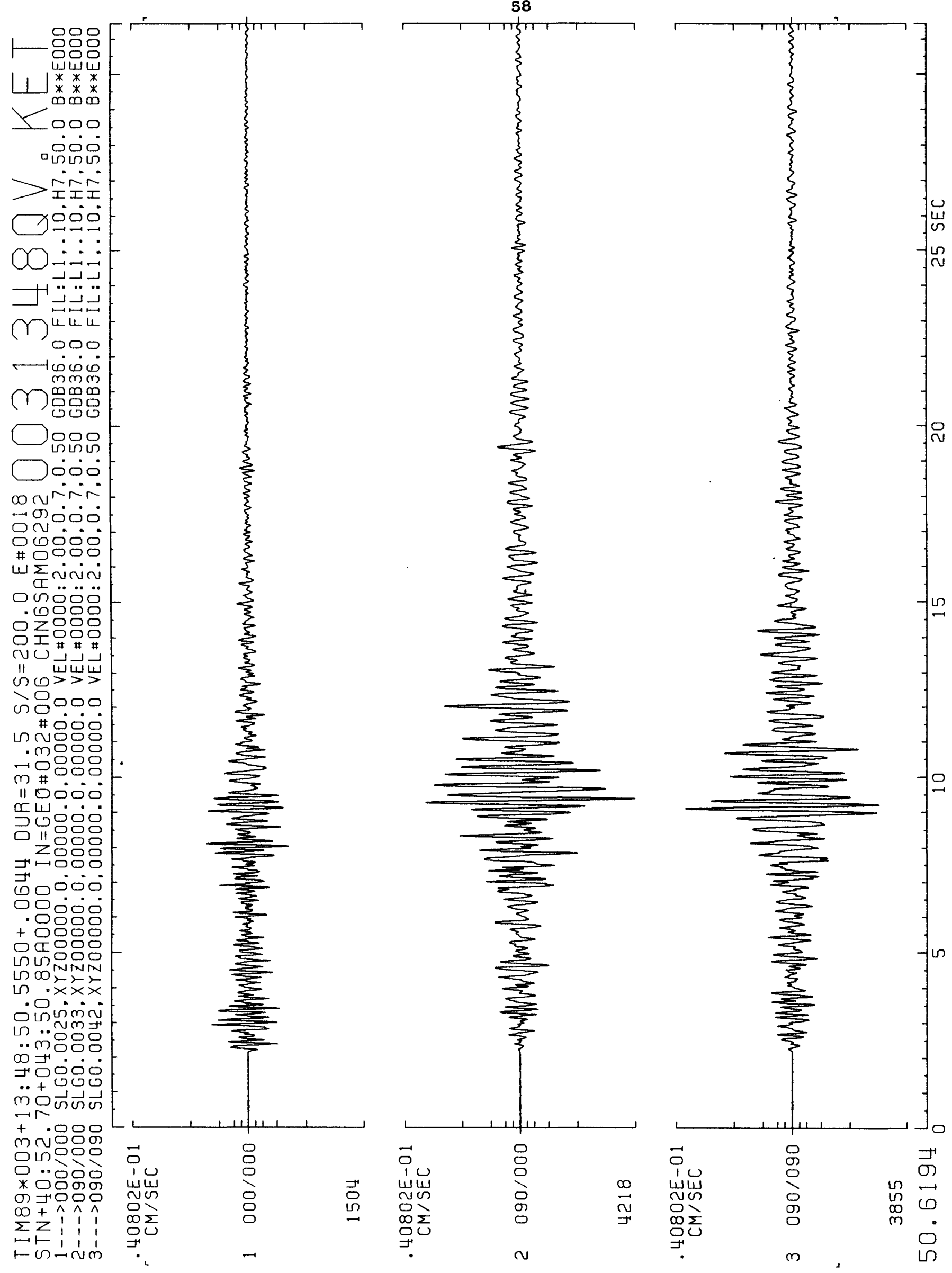


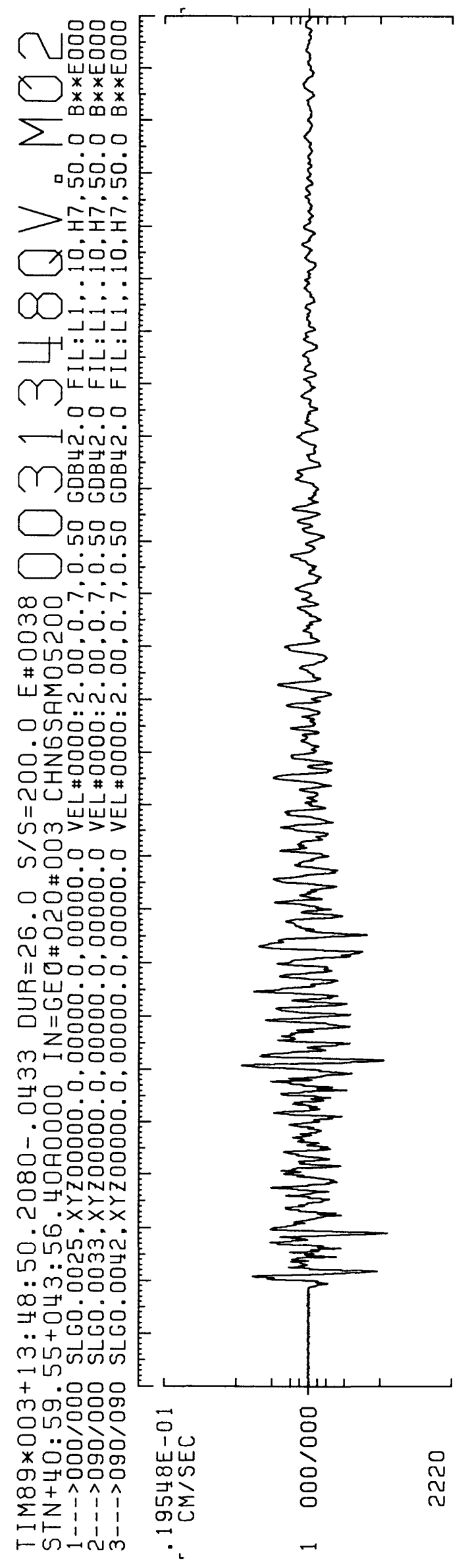

59

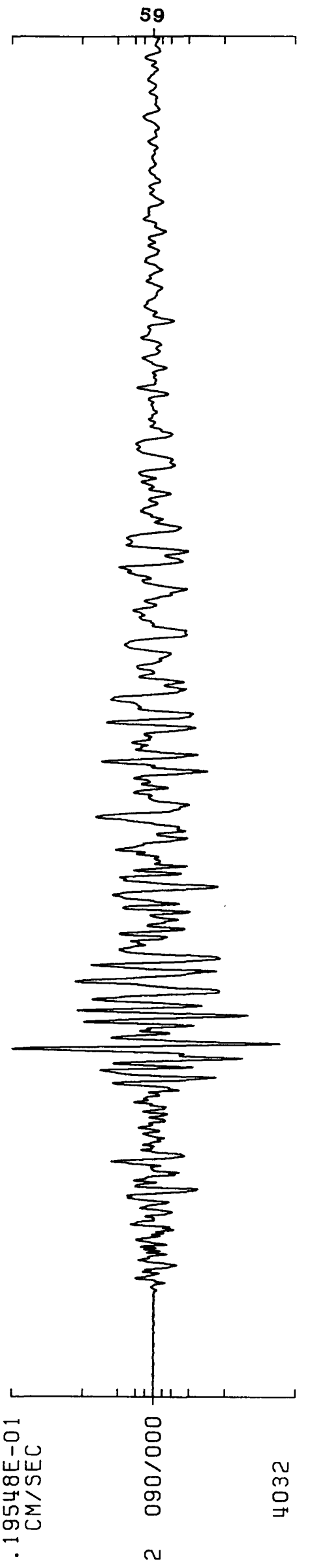

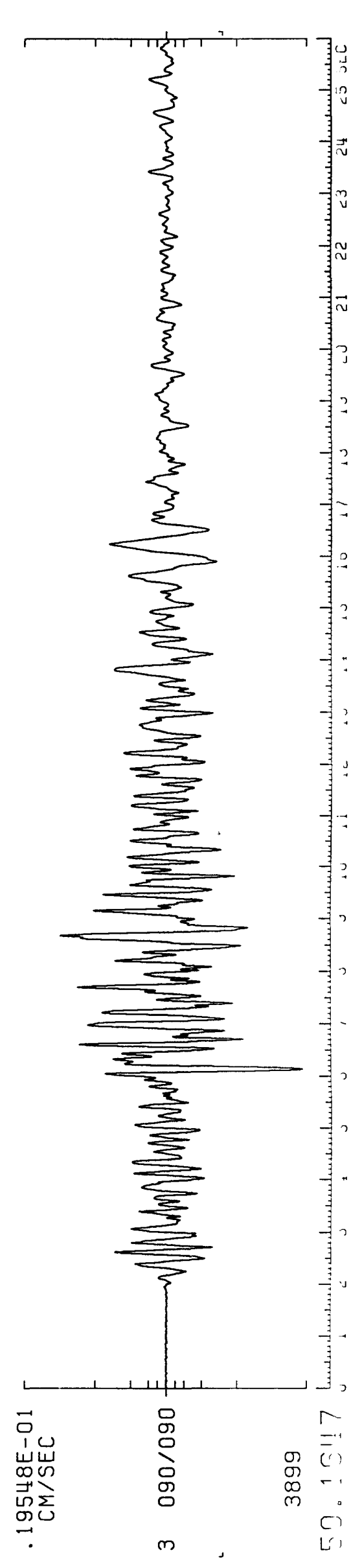


60

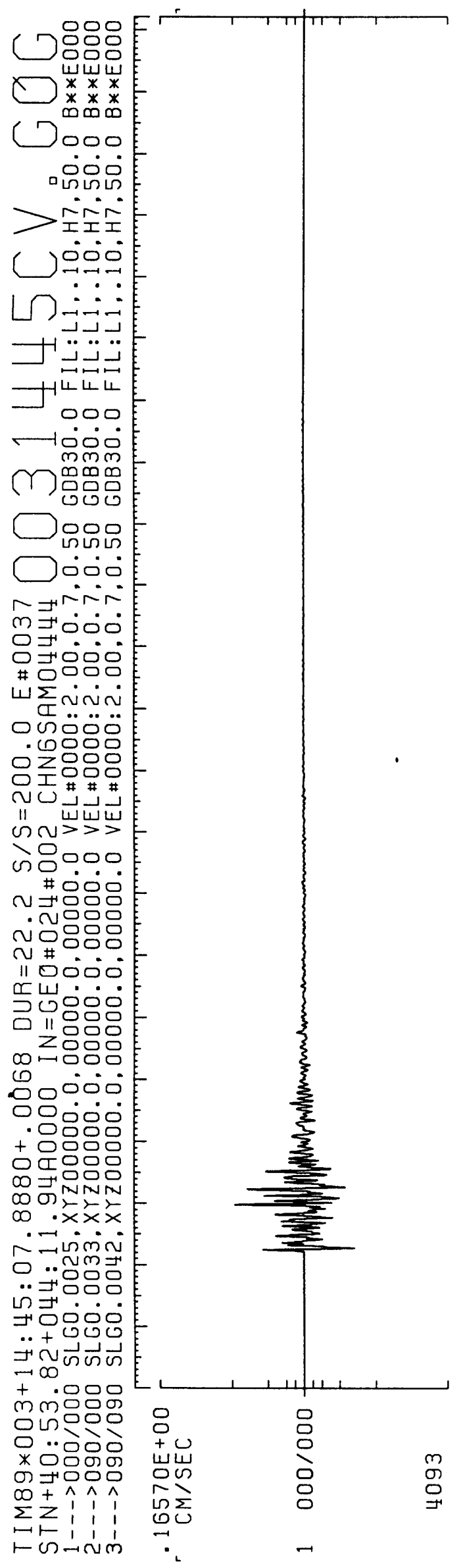

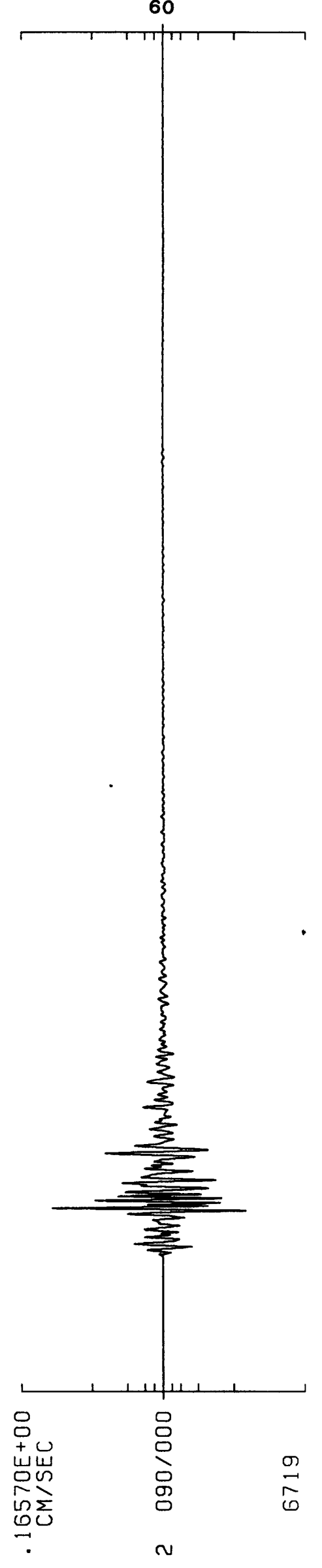

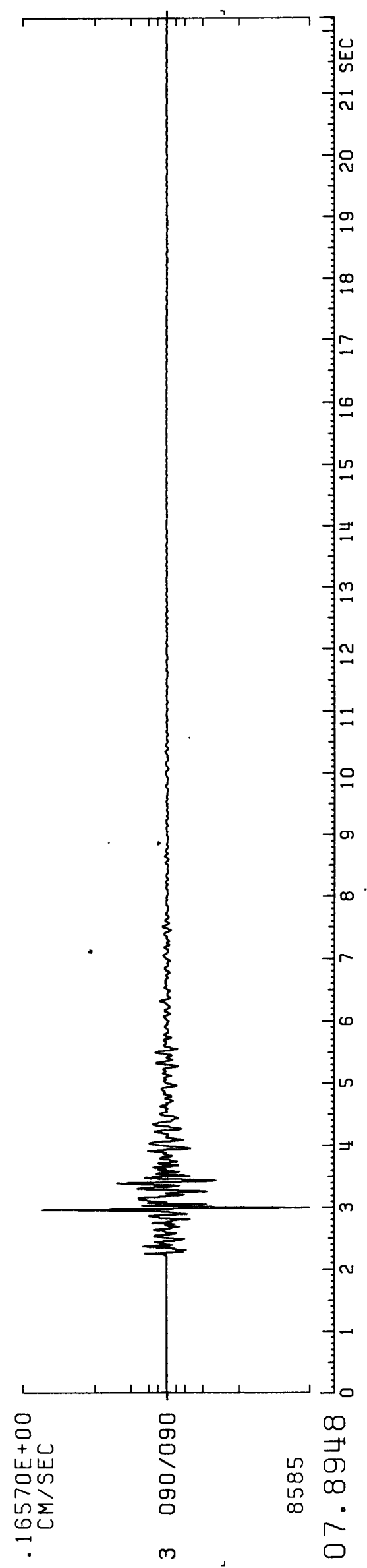


61
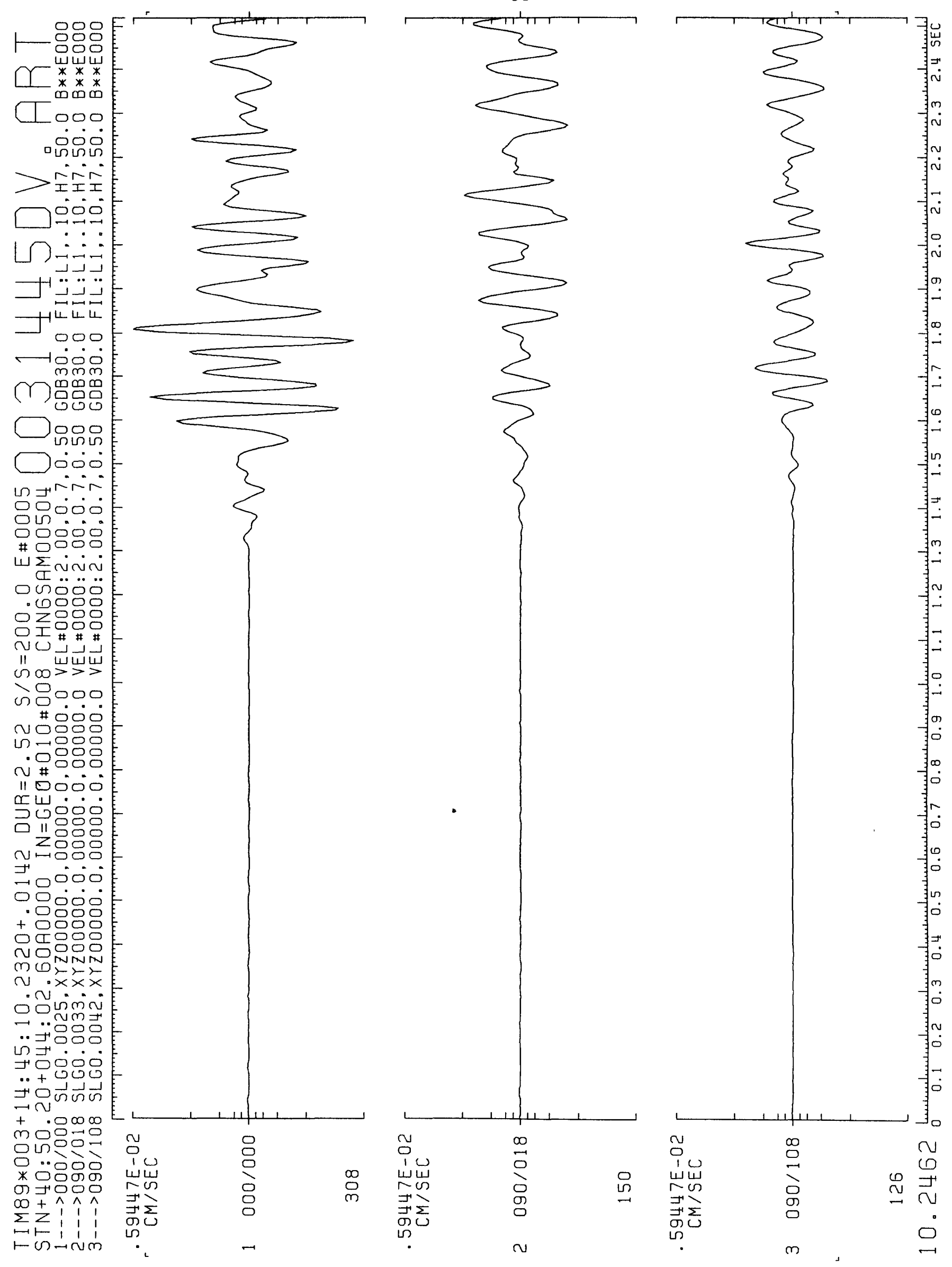
62
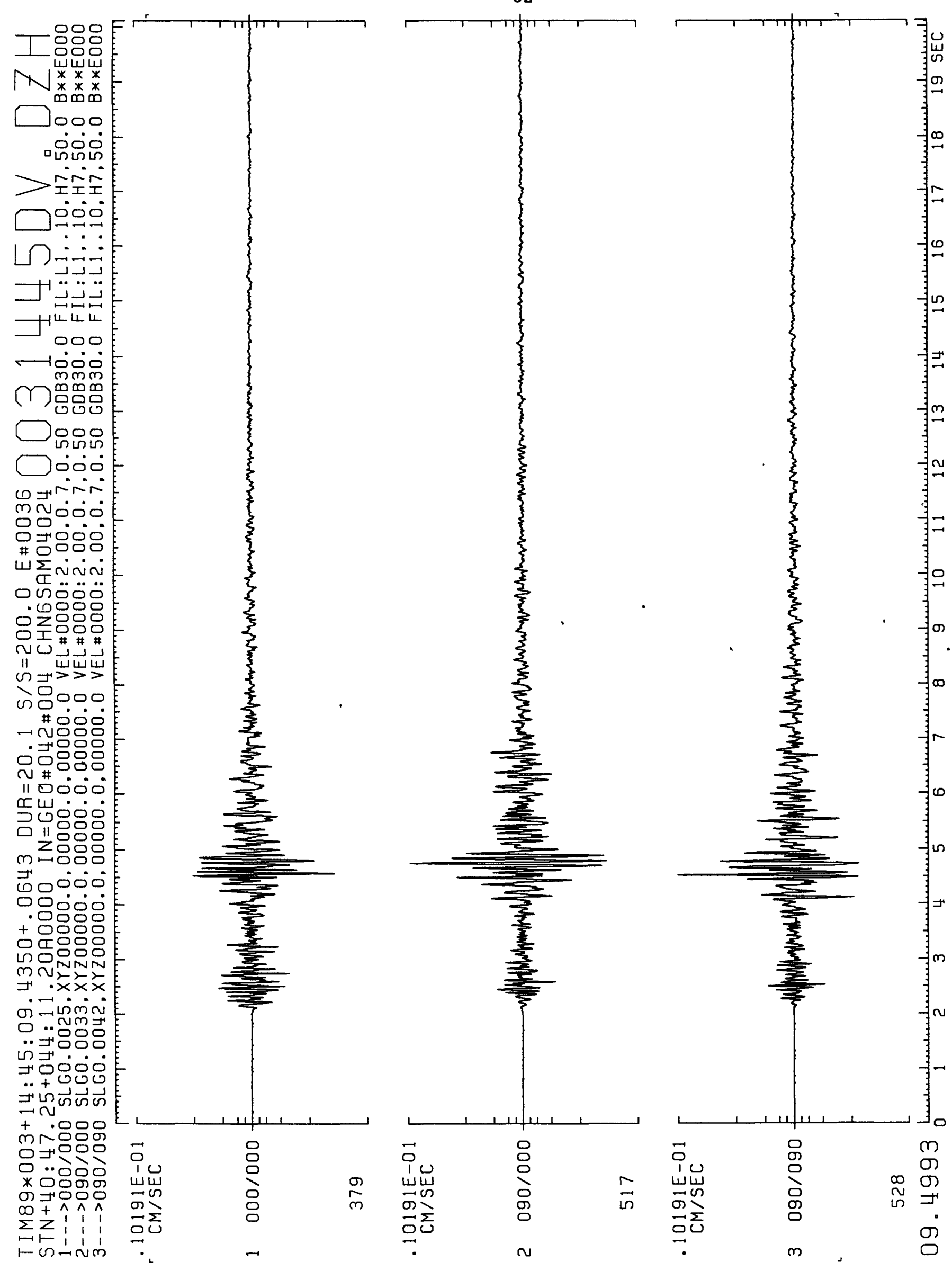
63

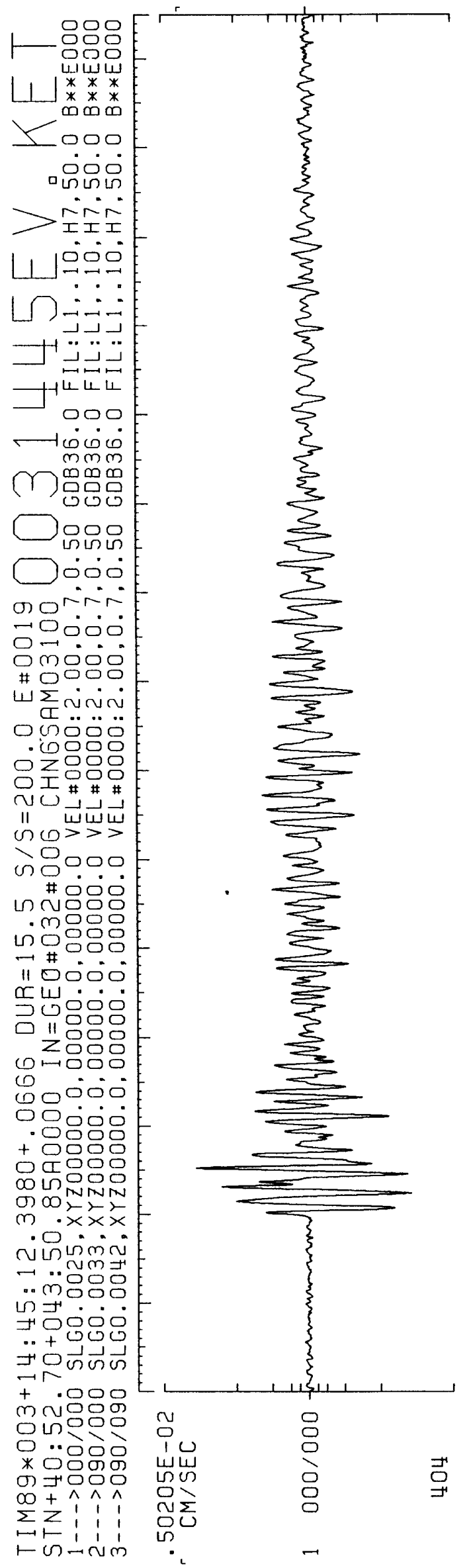

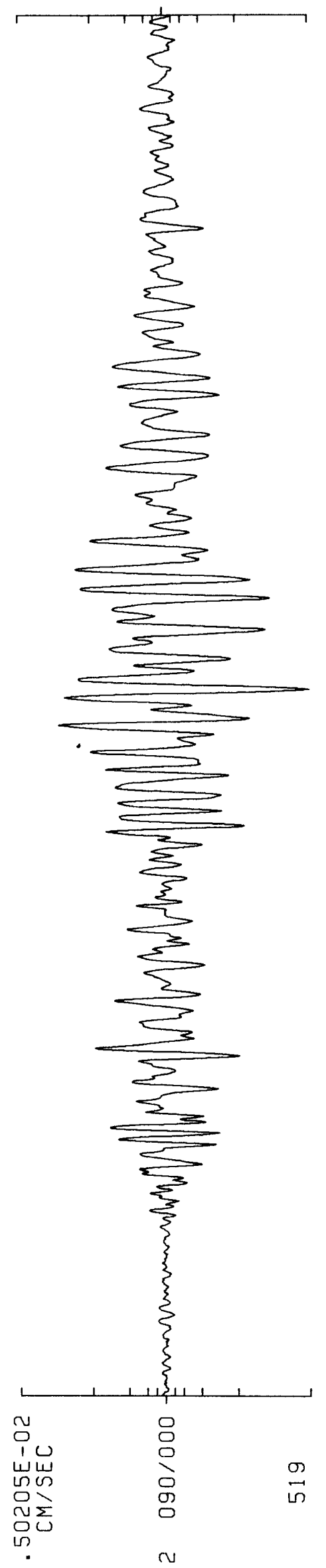

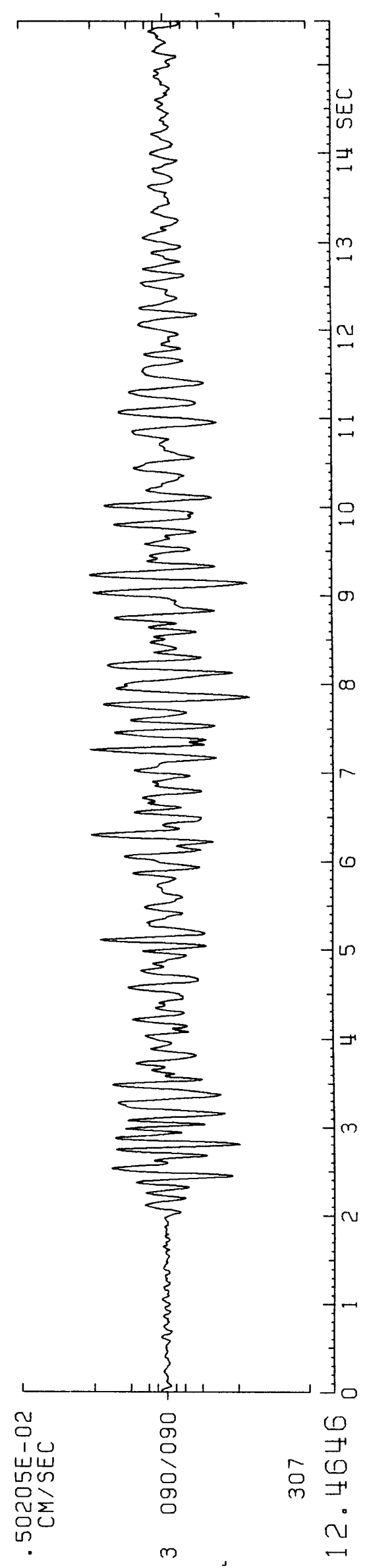


64

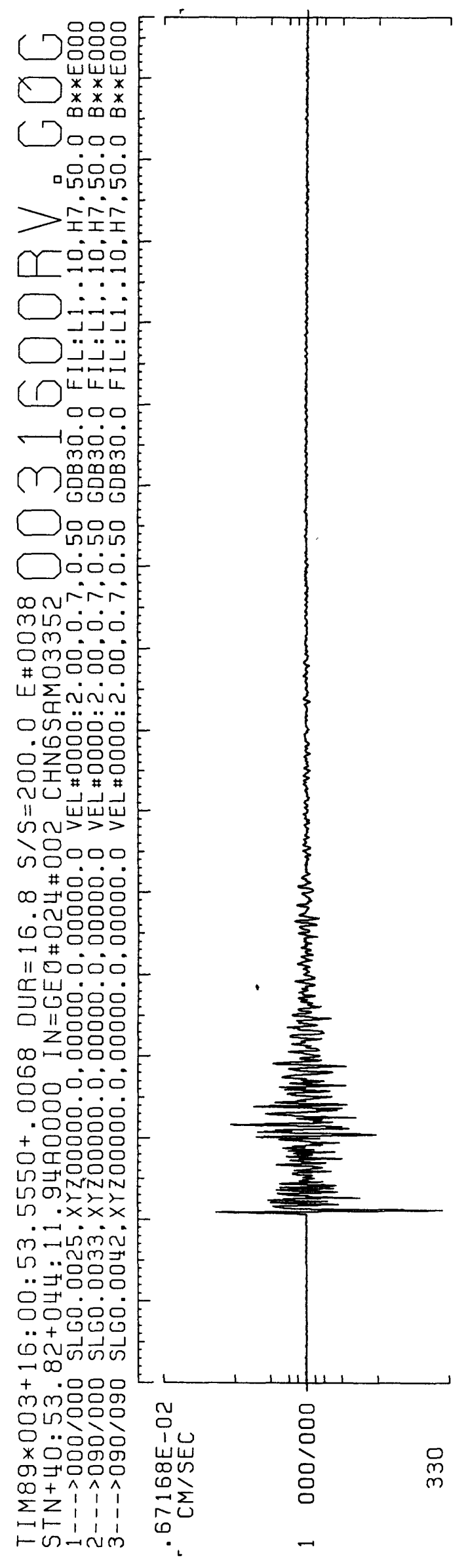

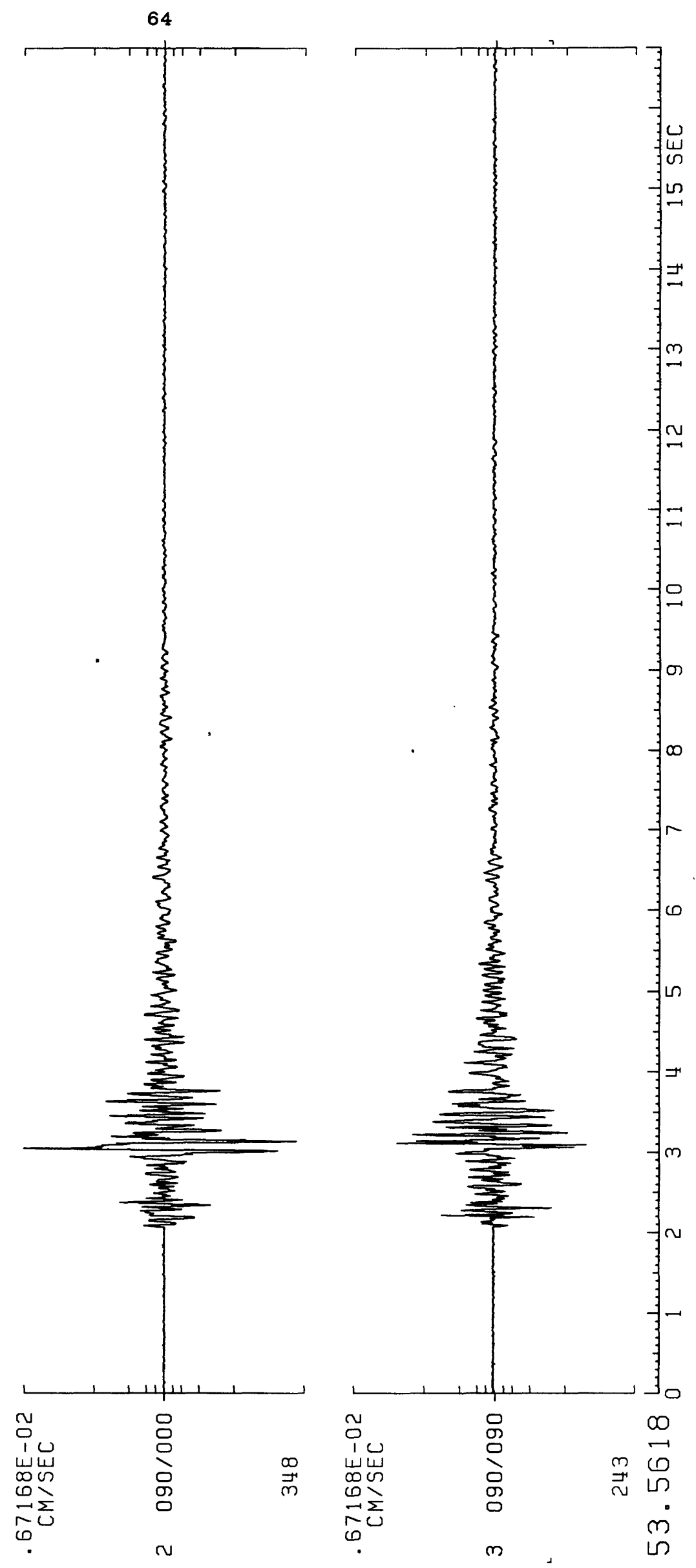


65

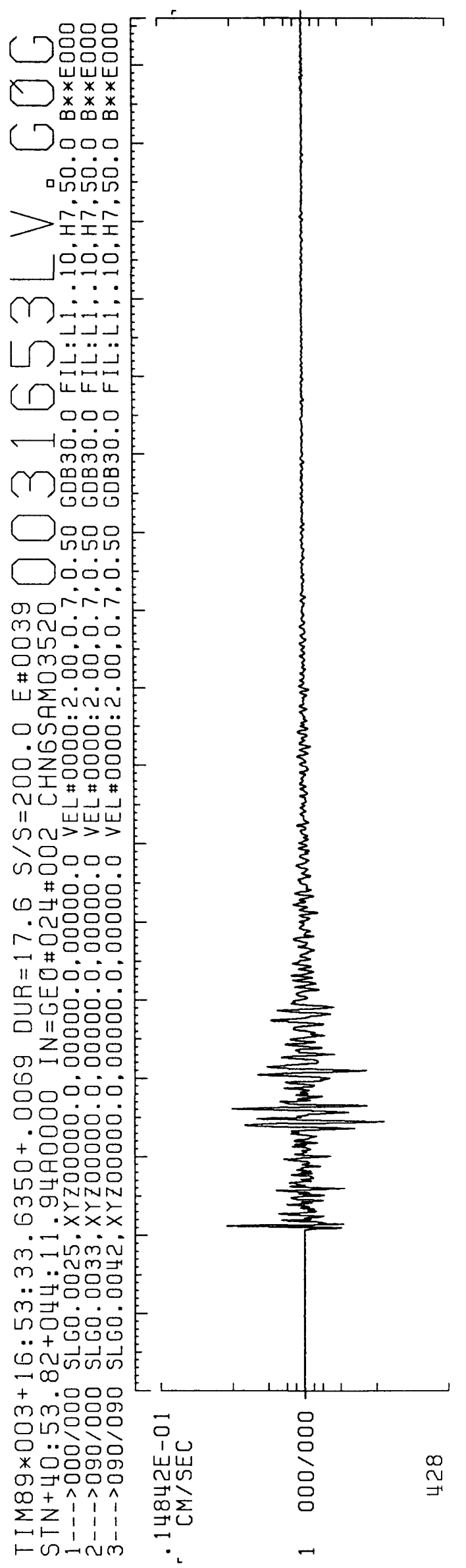

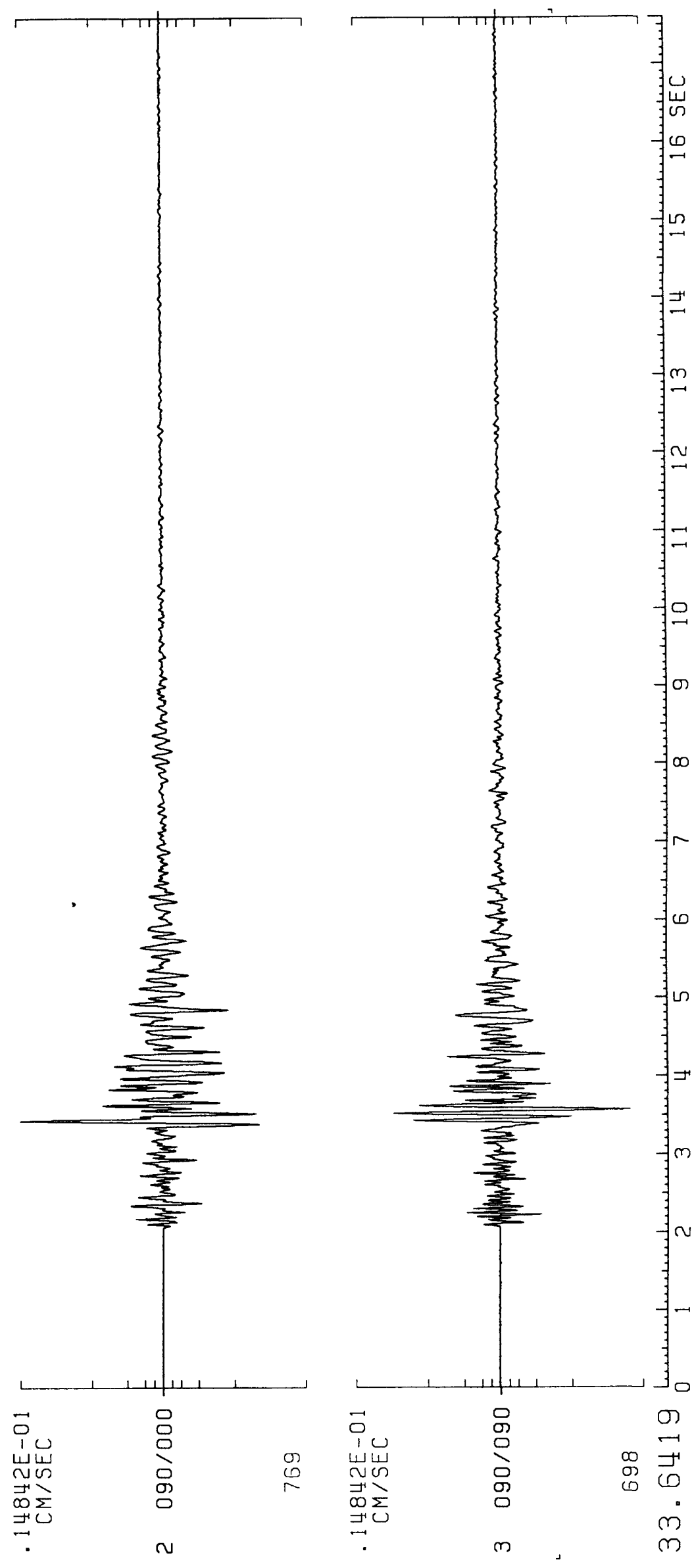


66
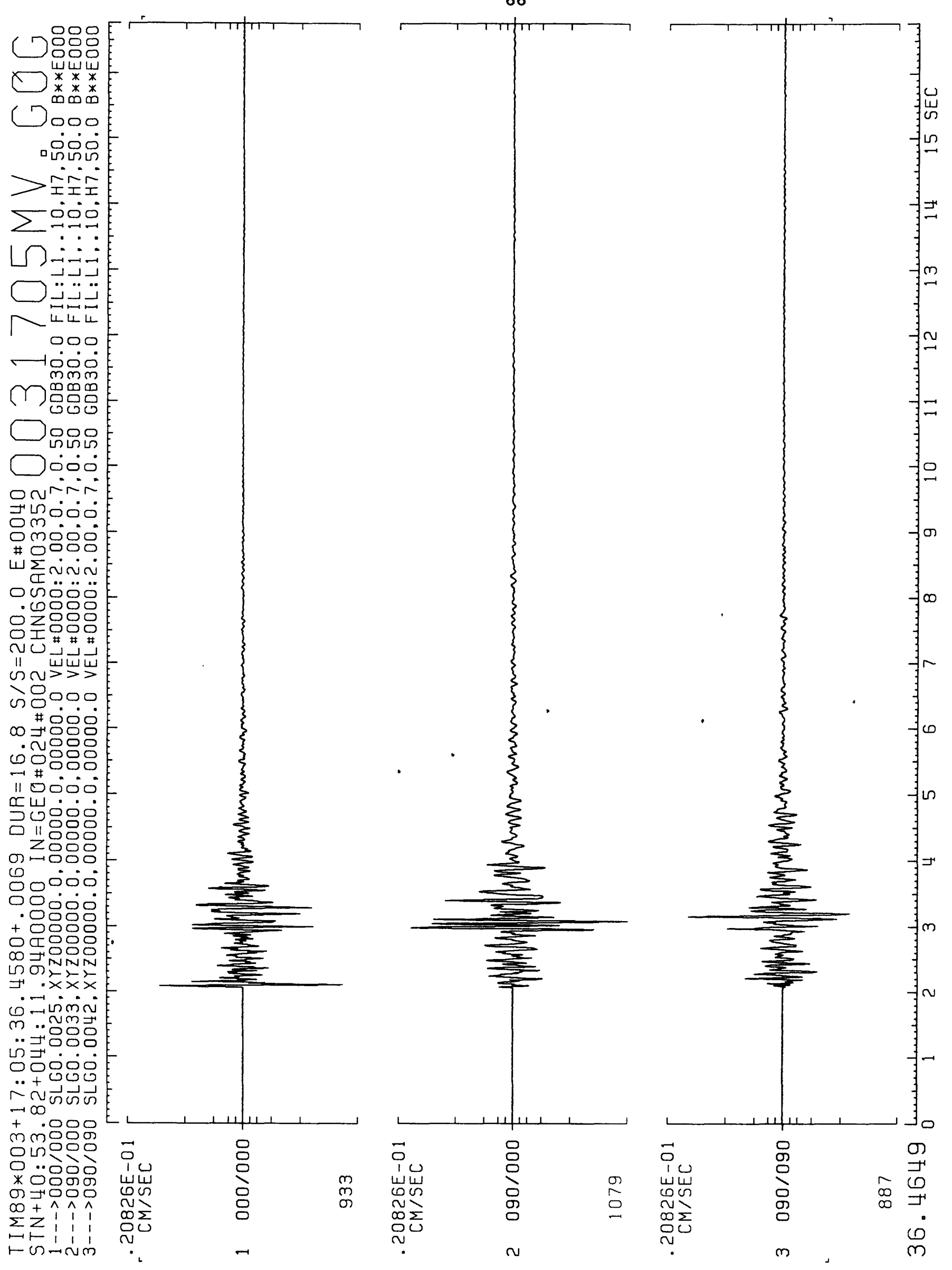
67

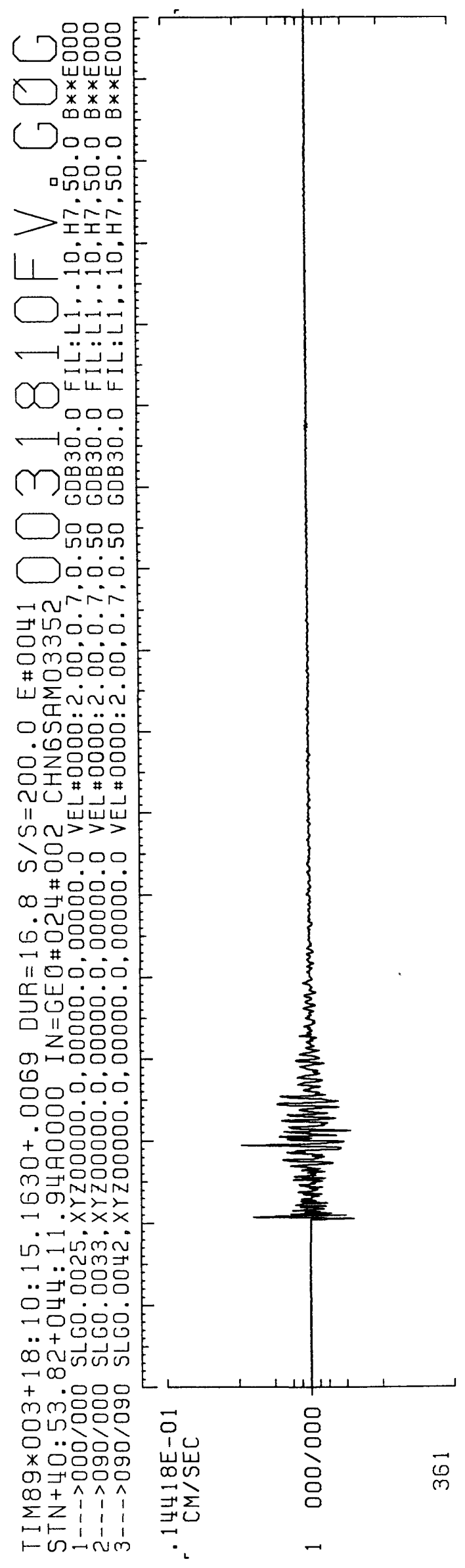

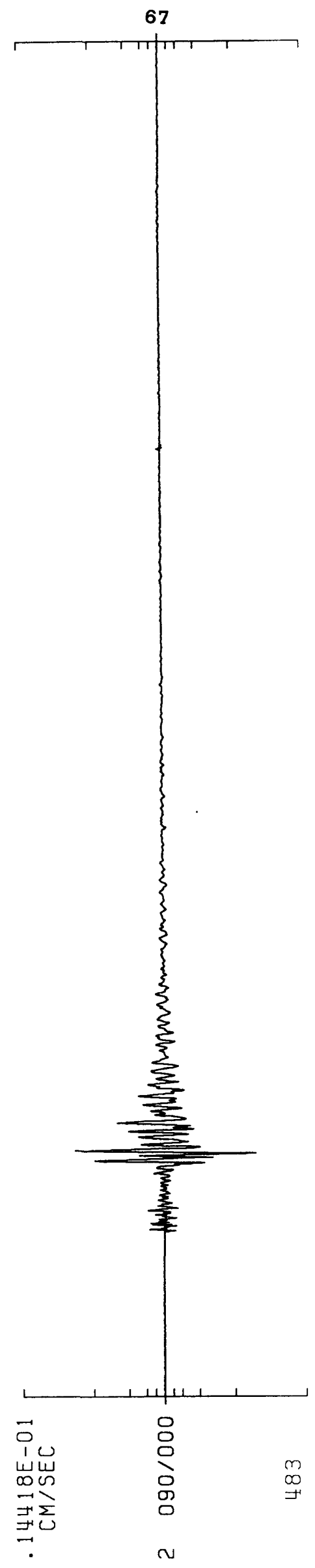

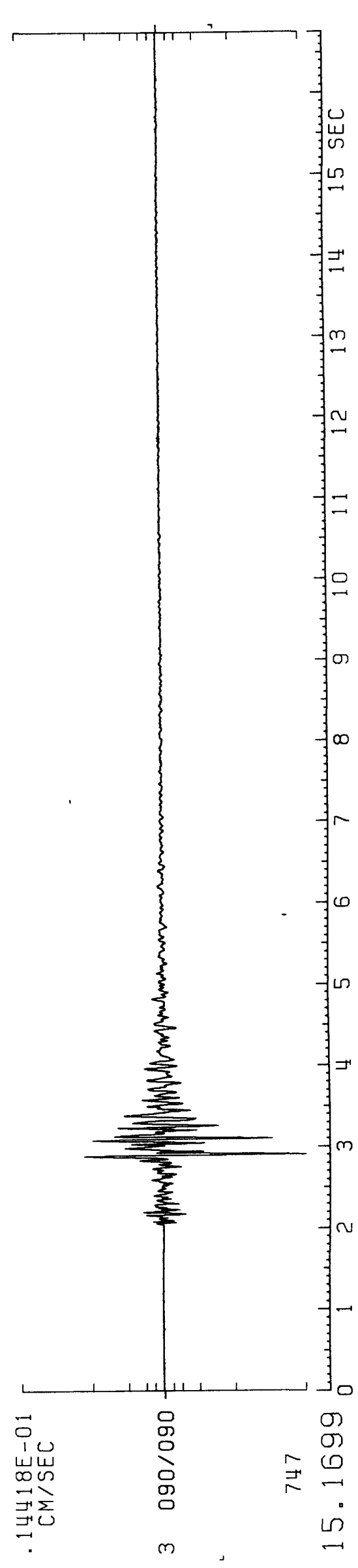




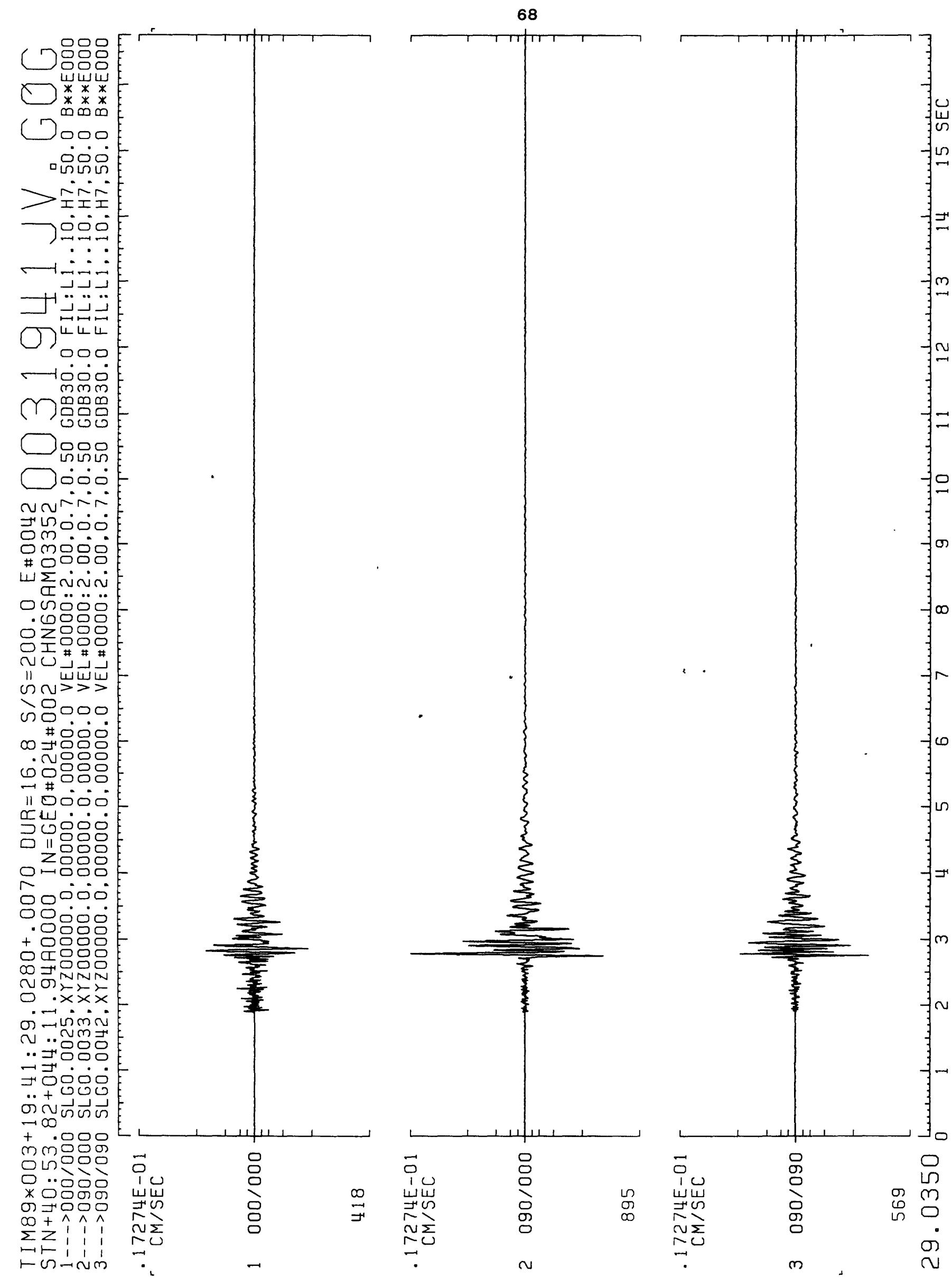


69

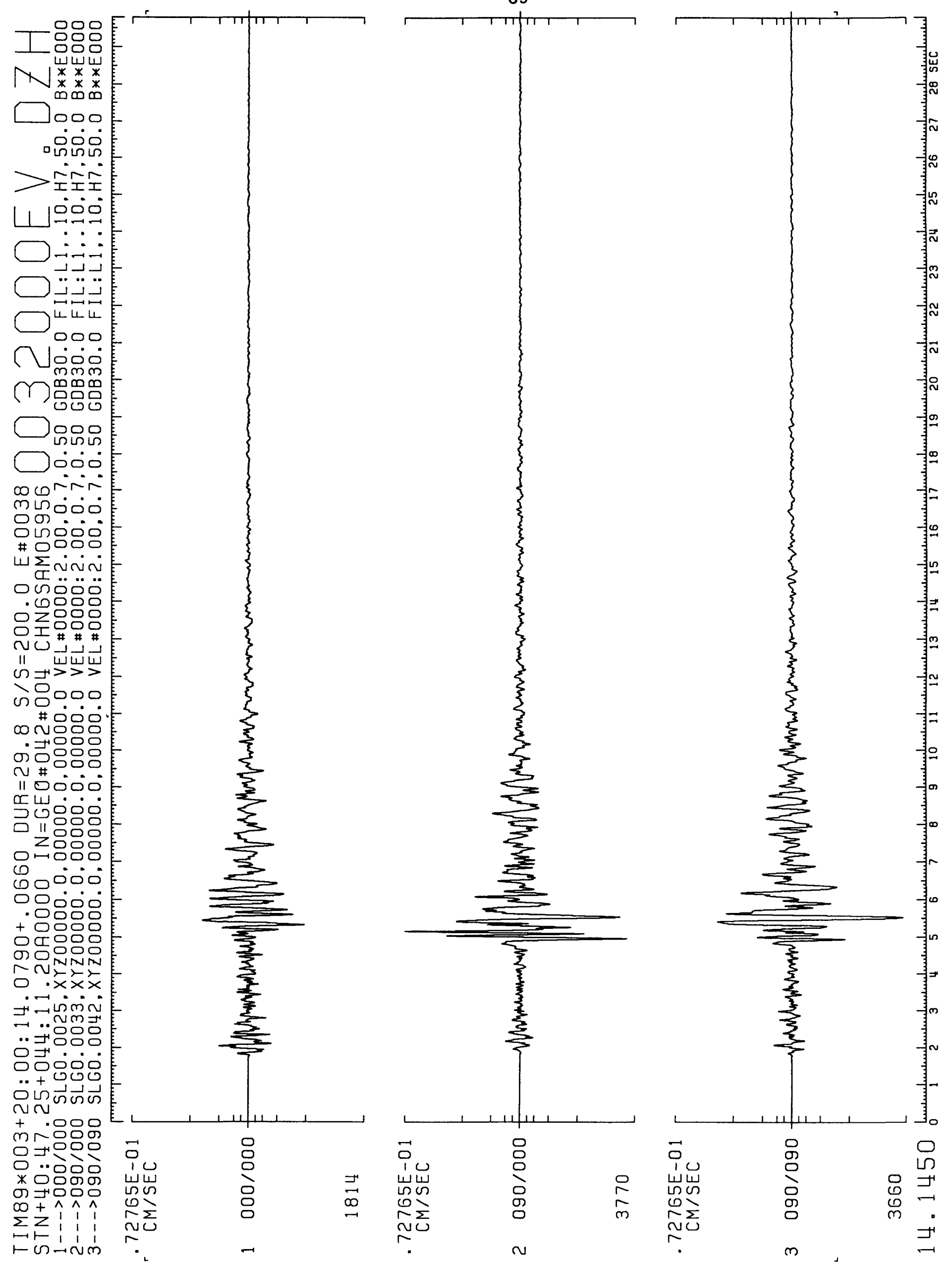


70

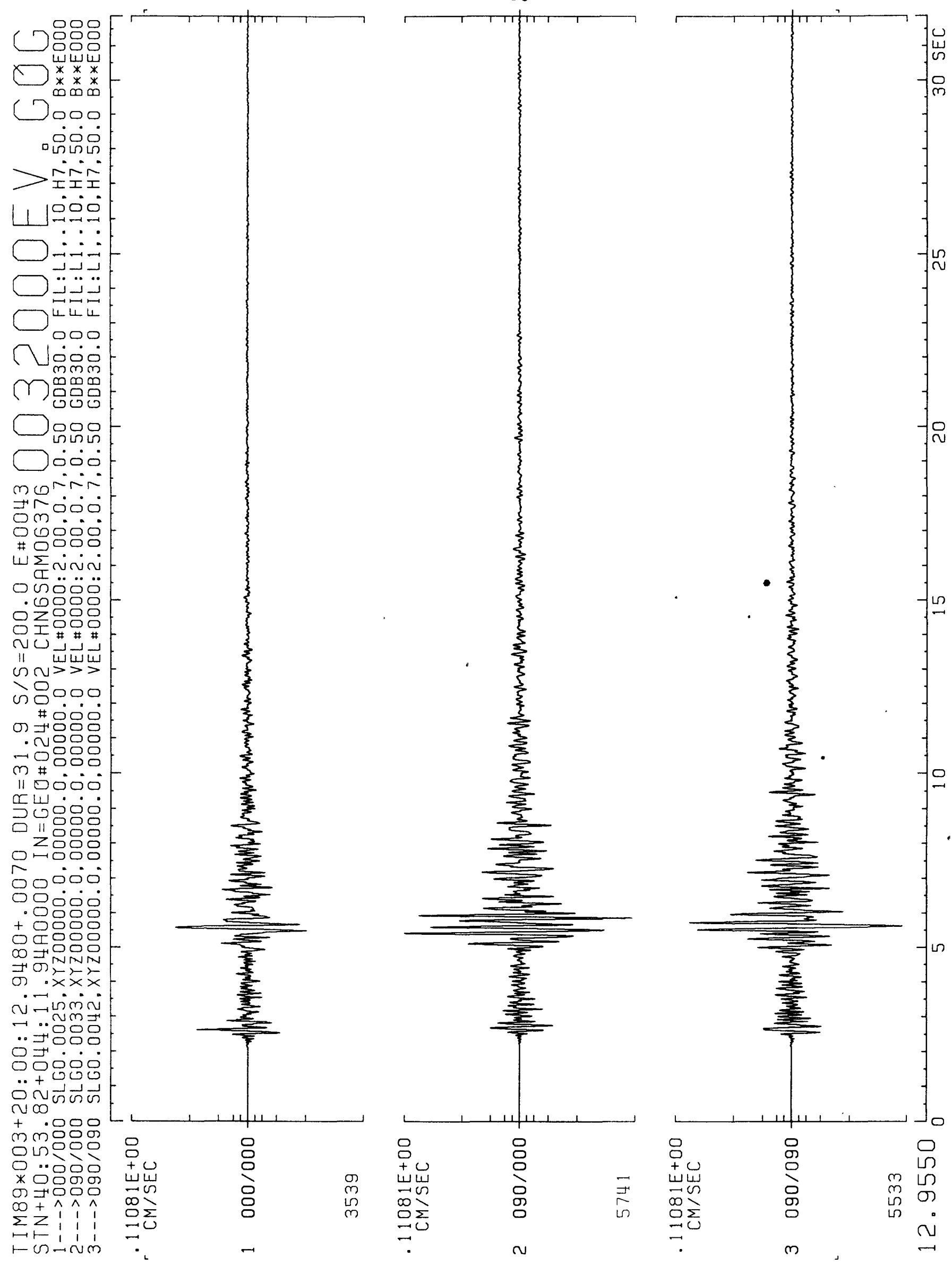


71

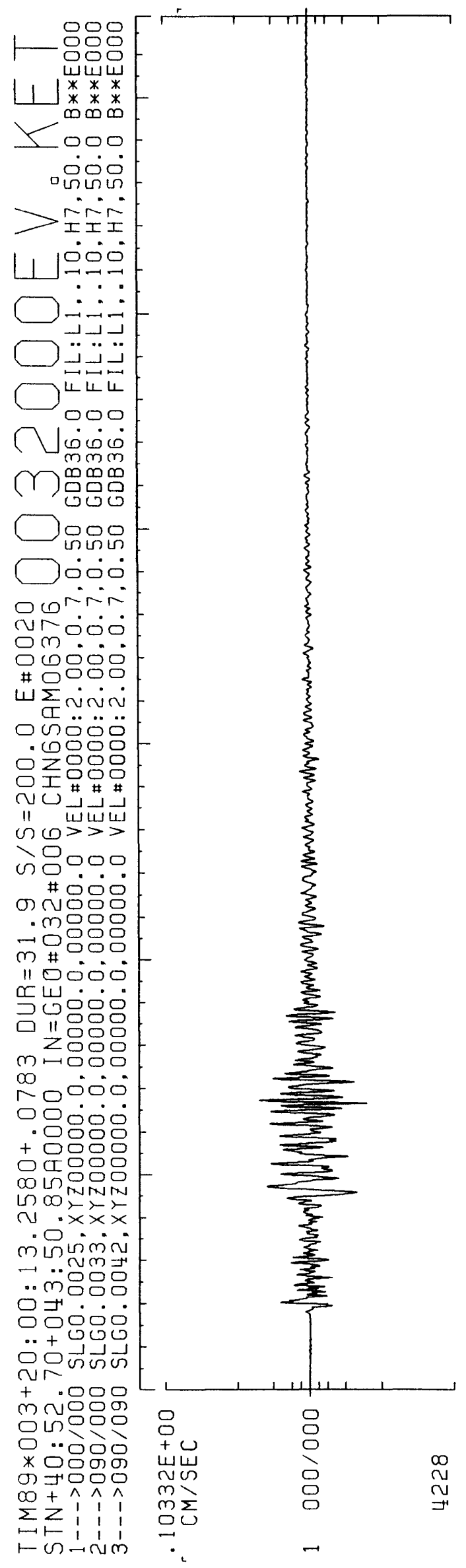

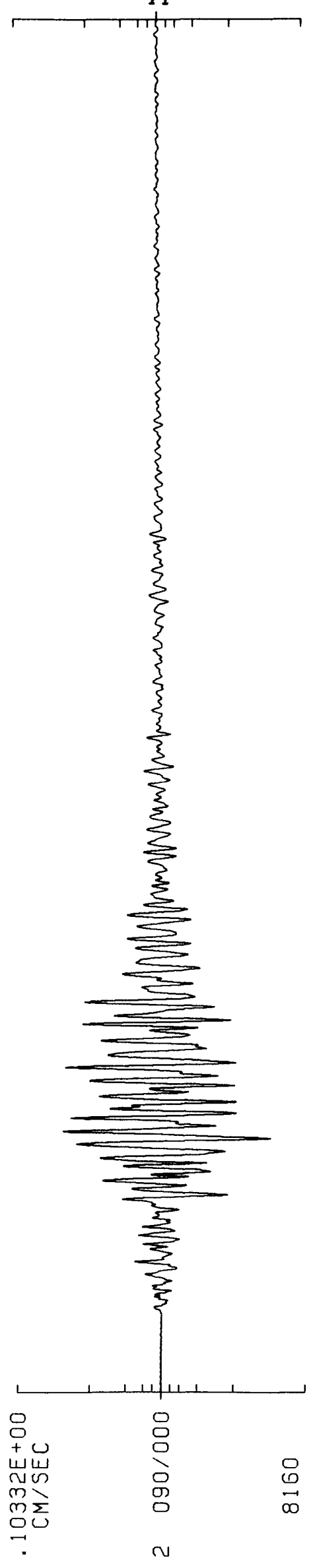

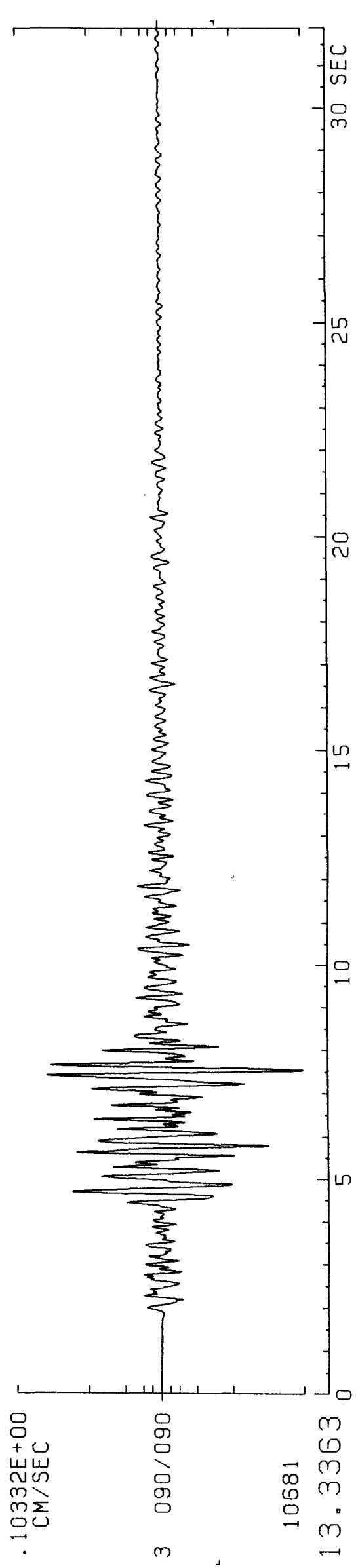




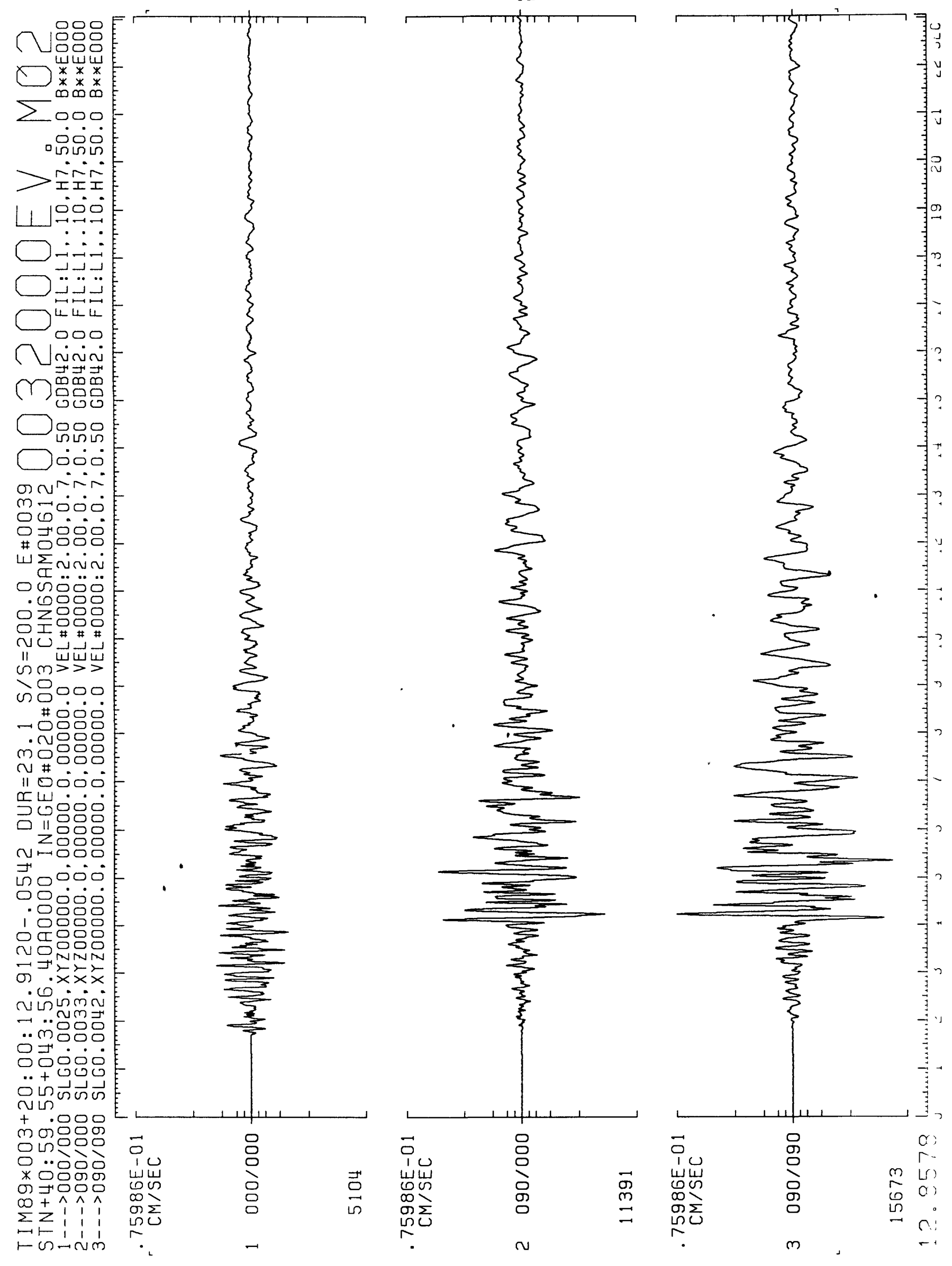


73

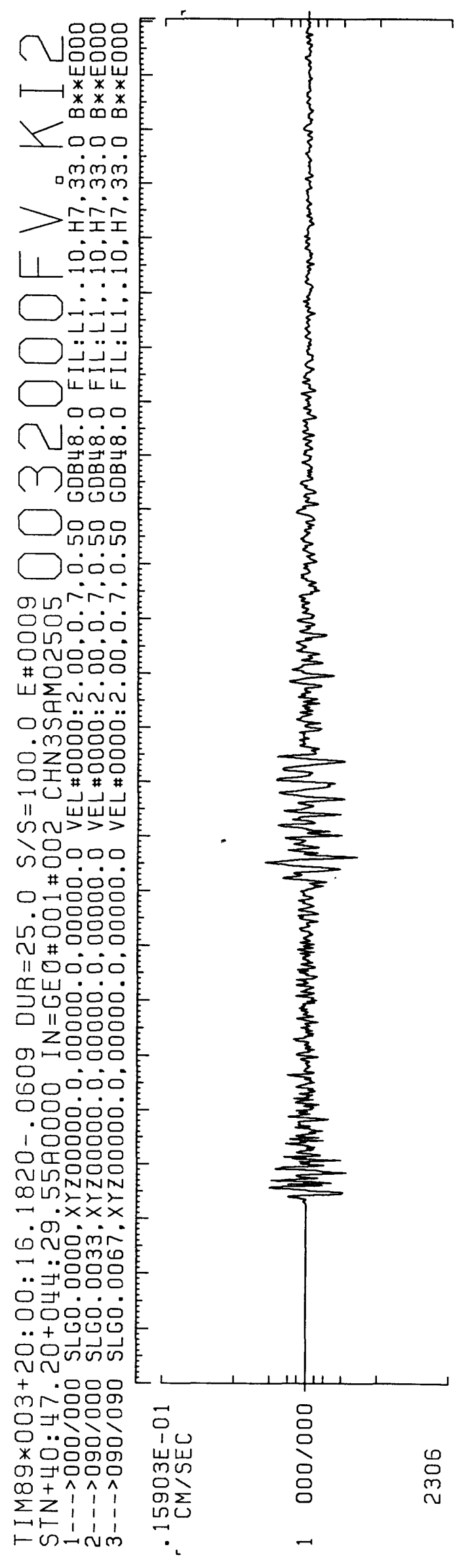

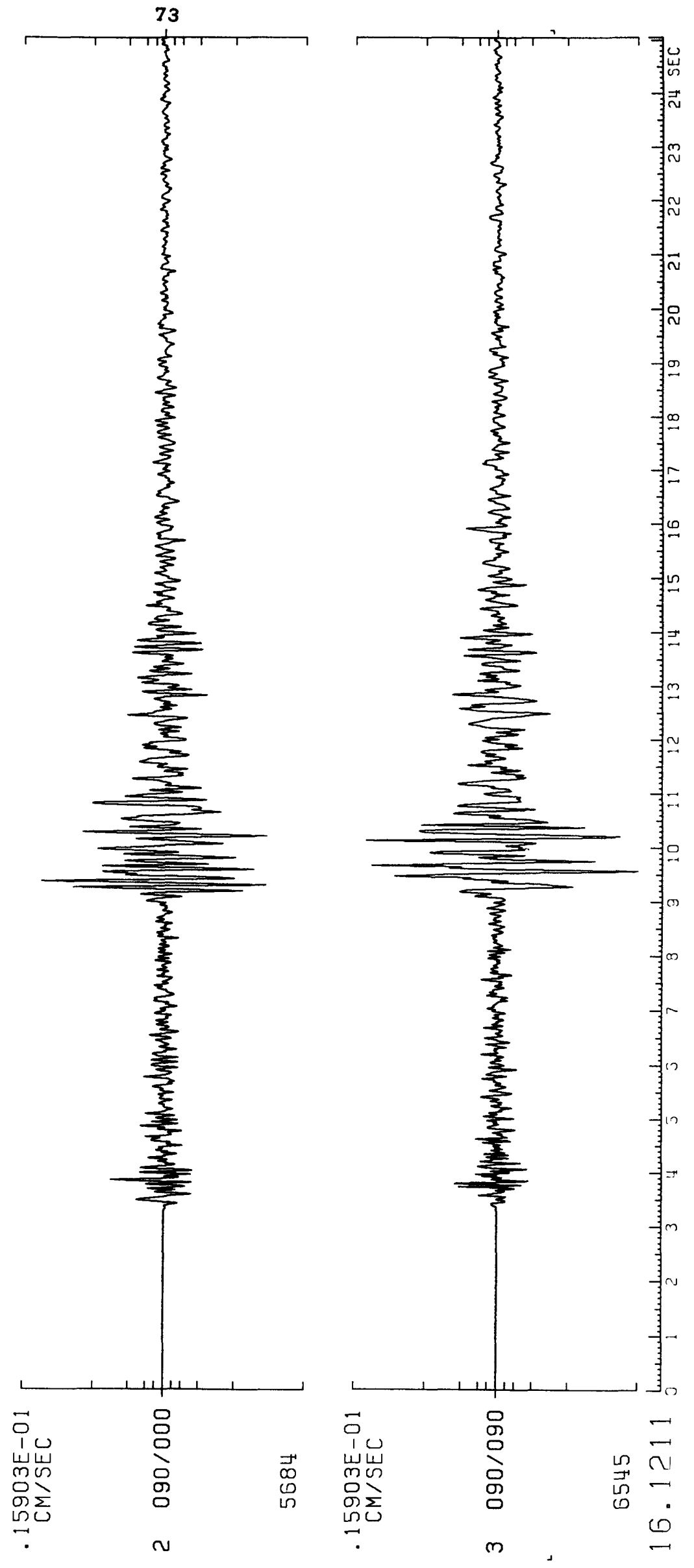




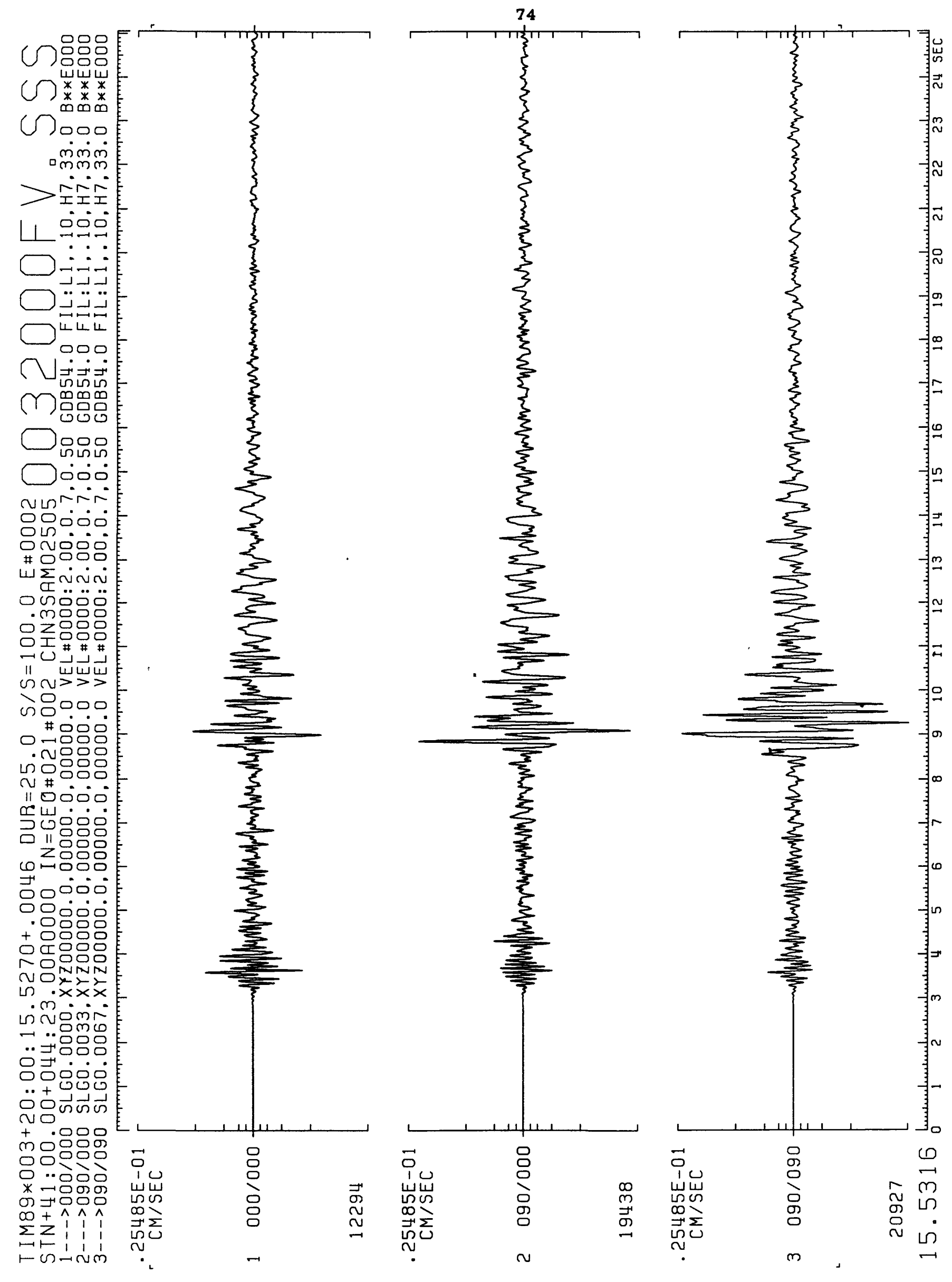




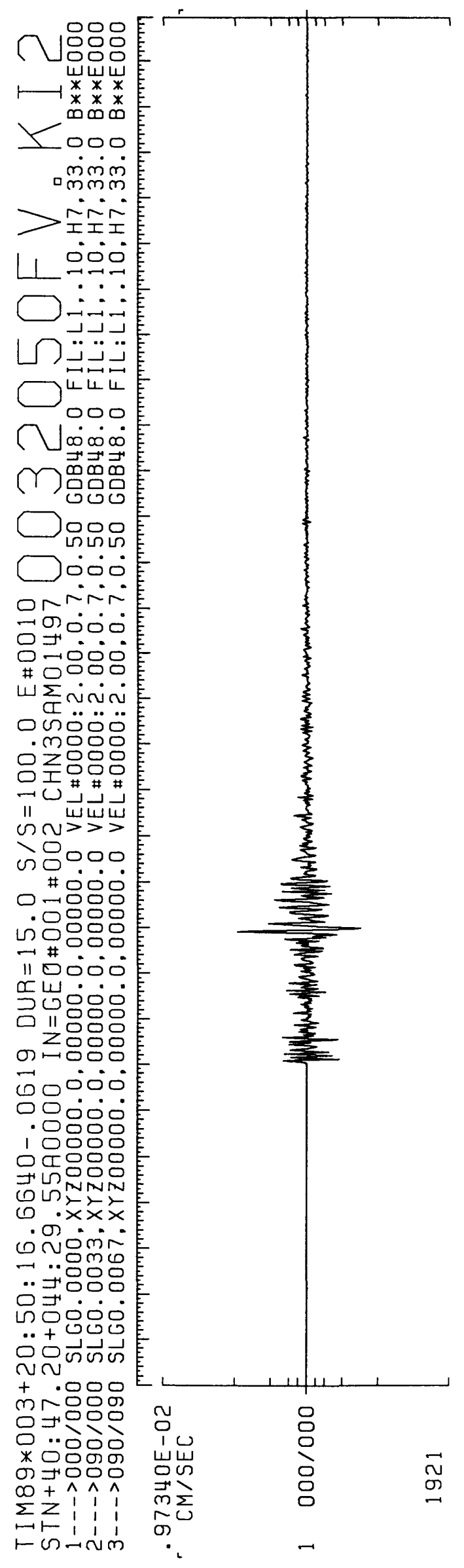

75

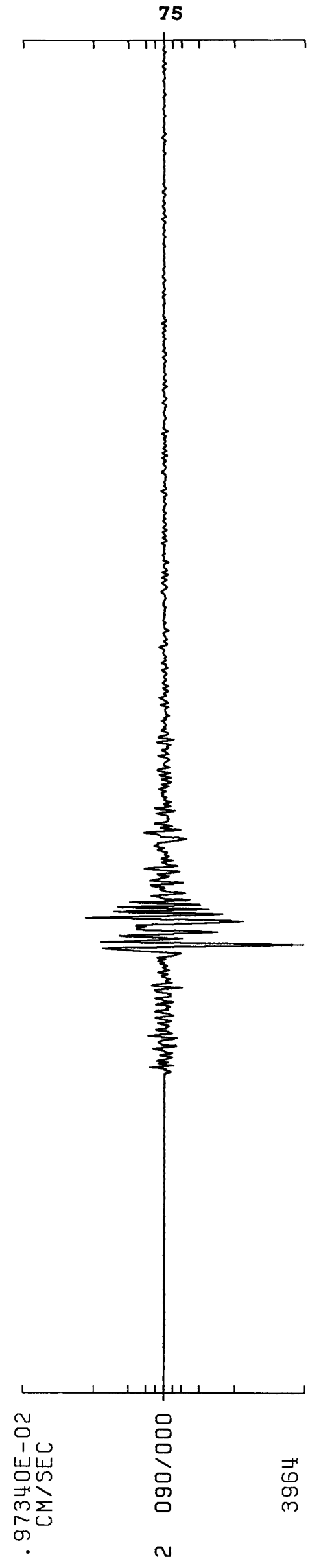

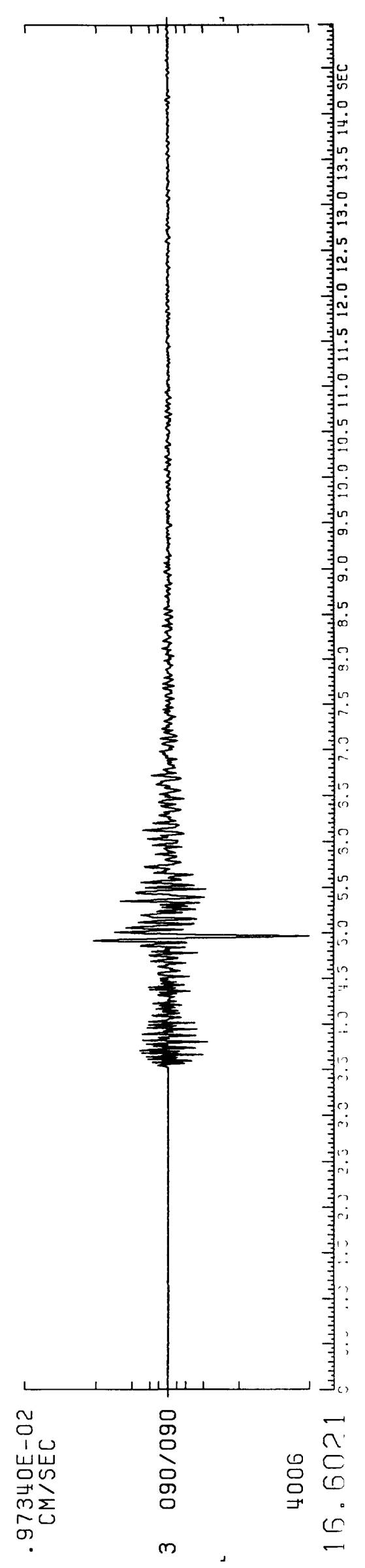



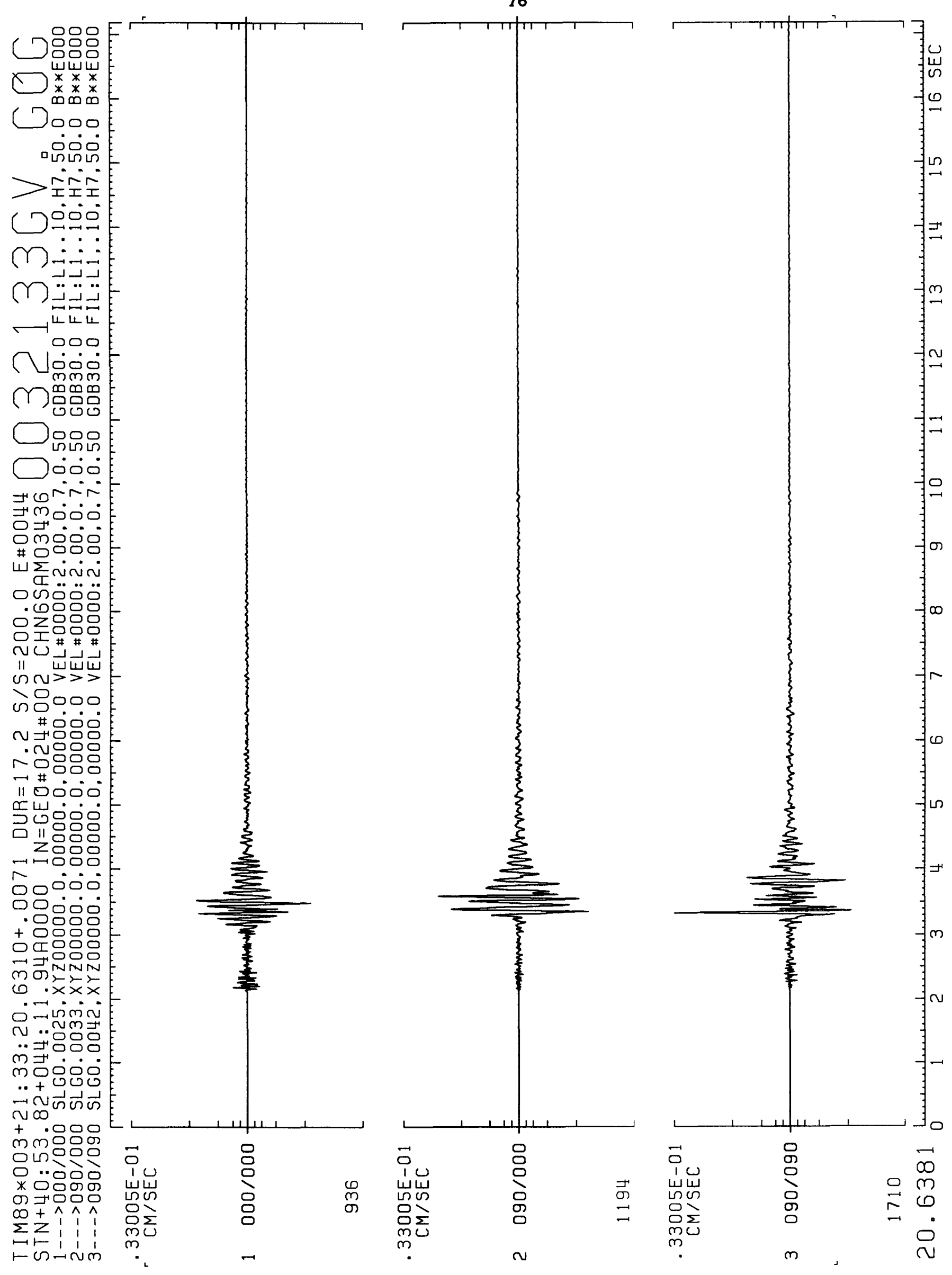
77

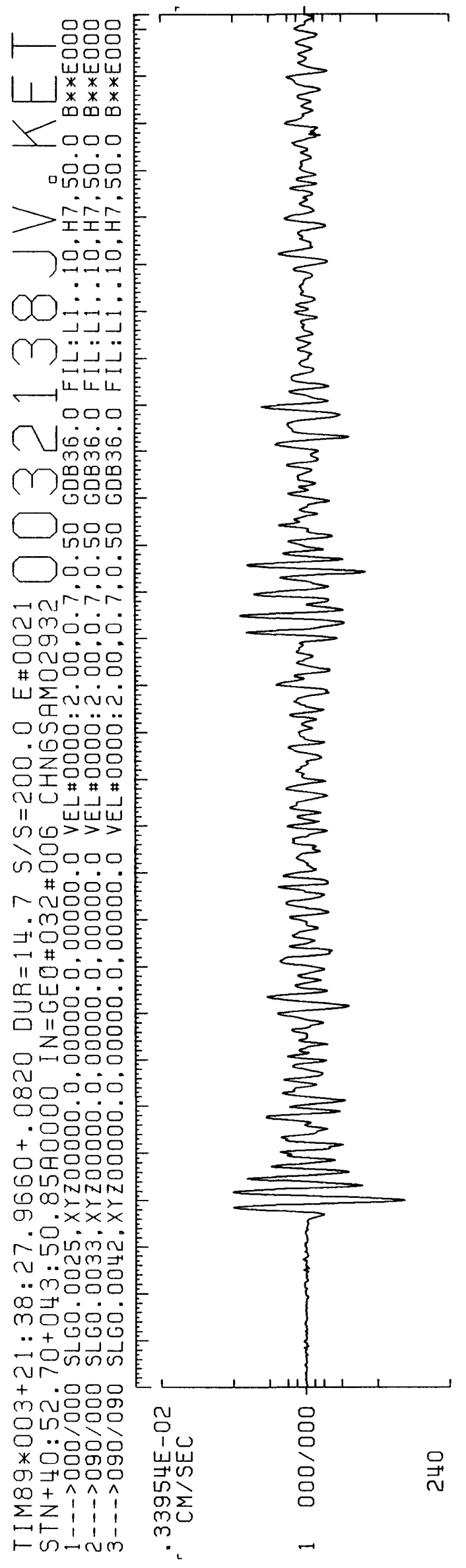

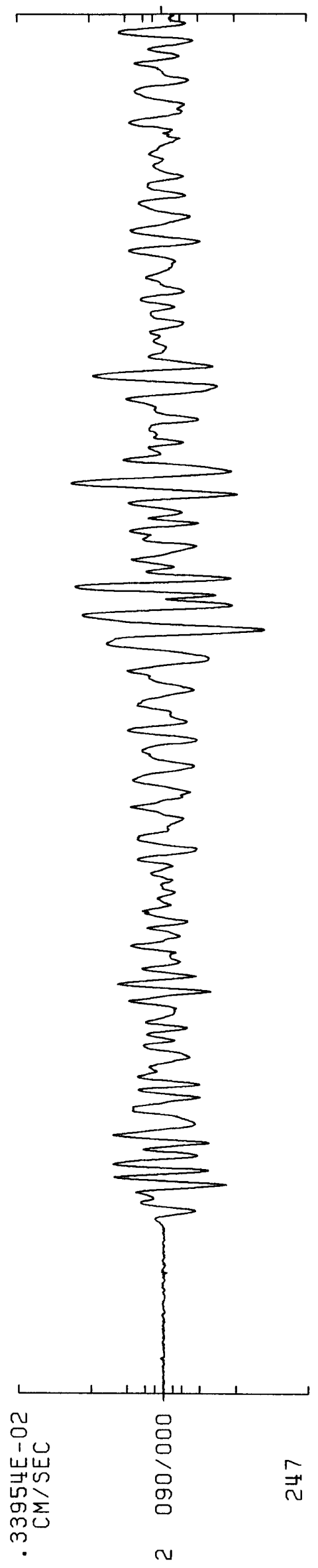

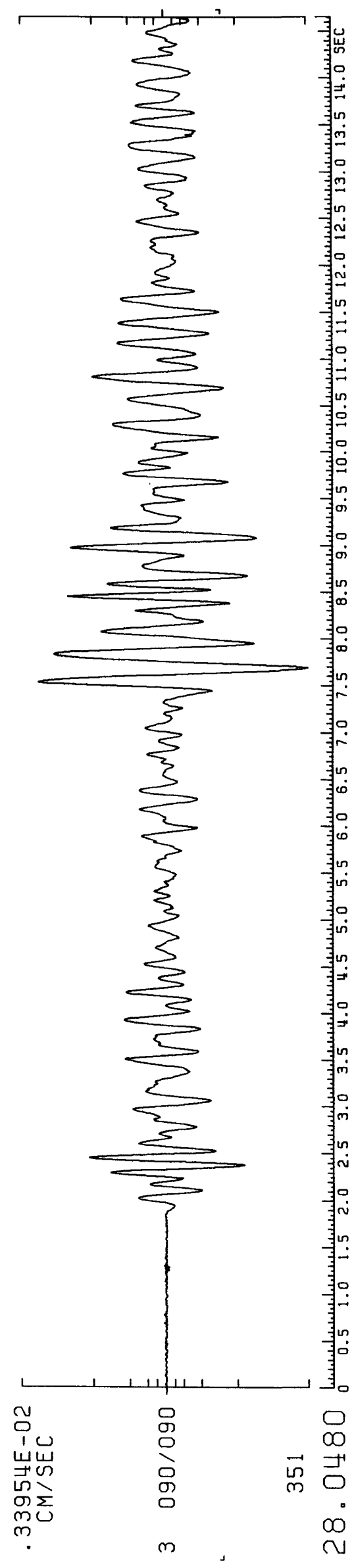


78

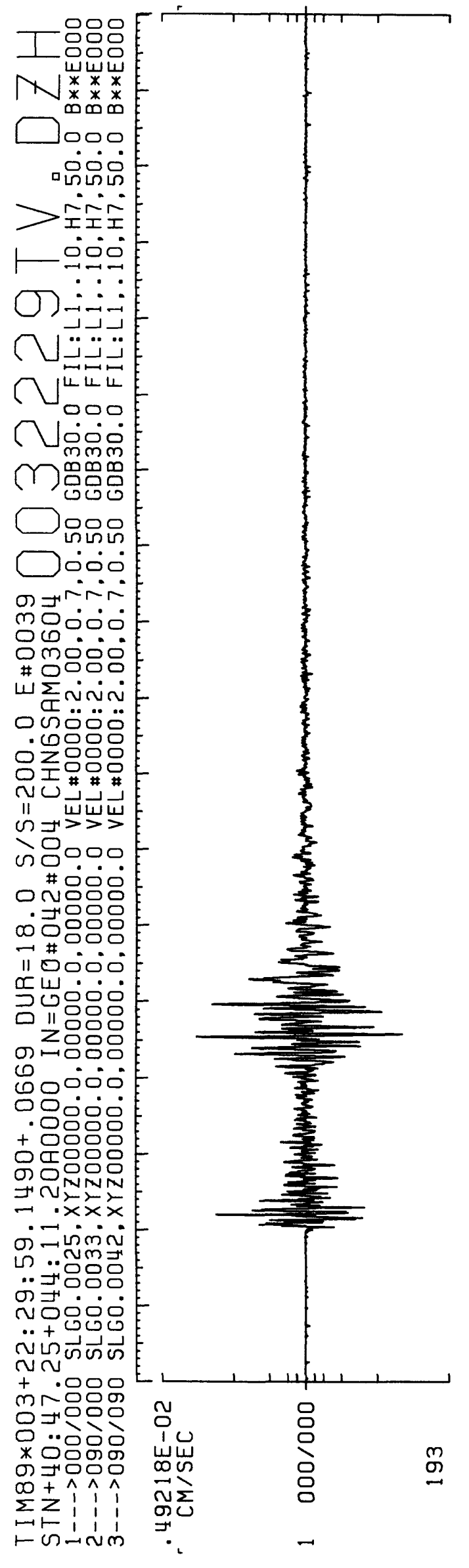

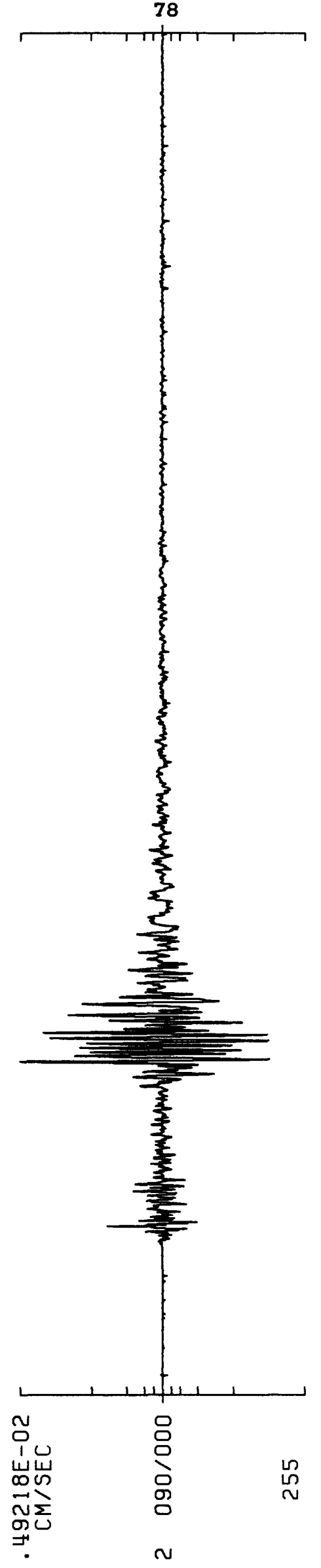

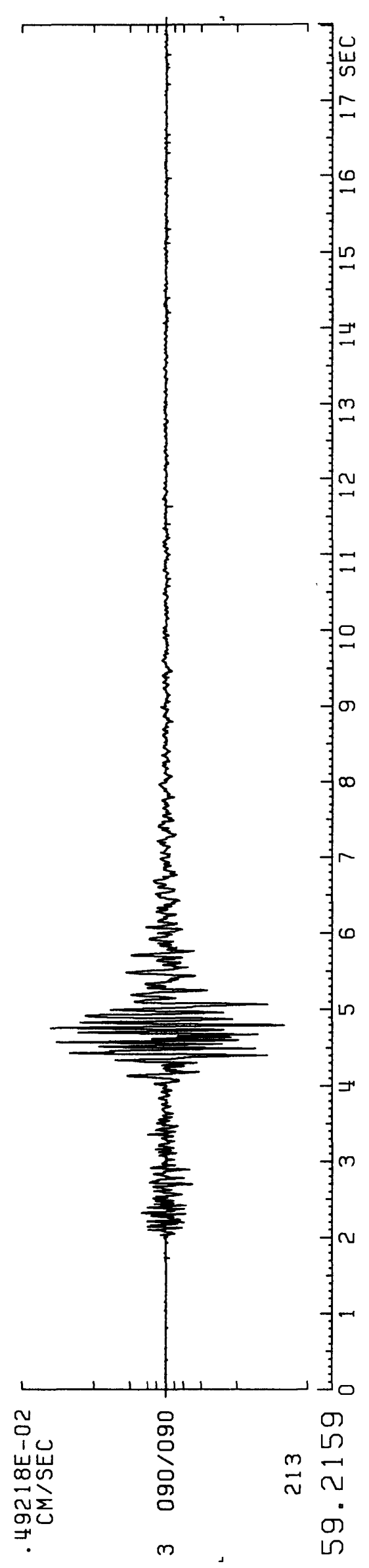




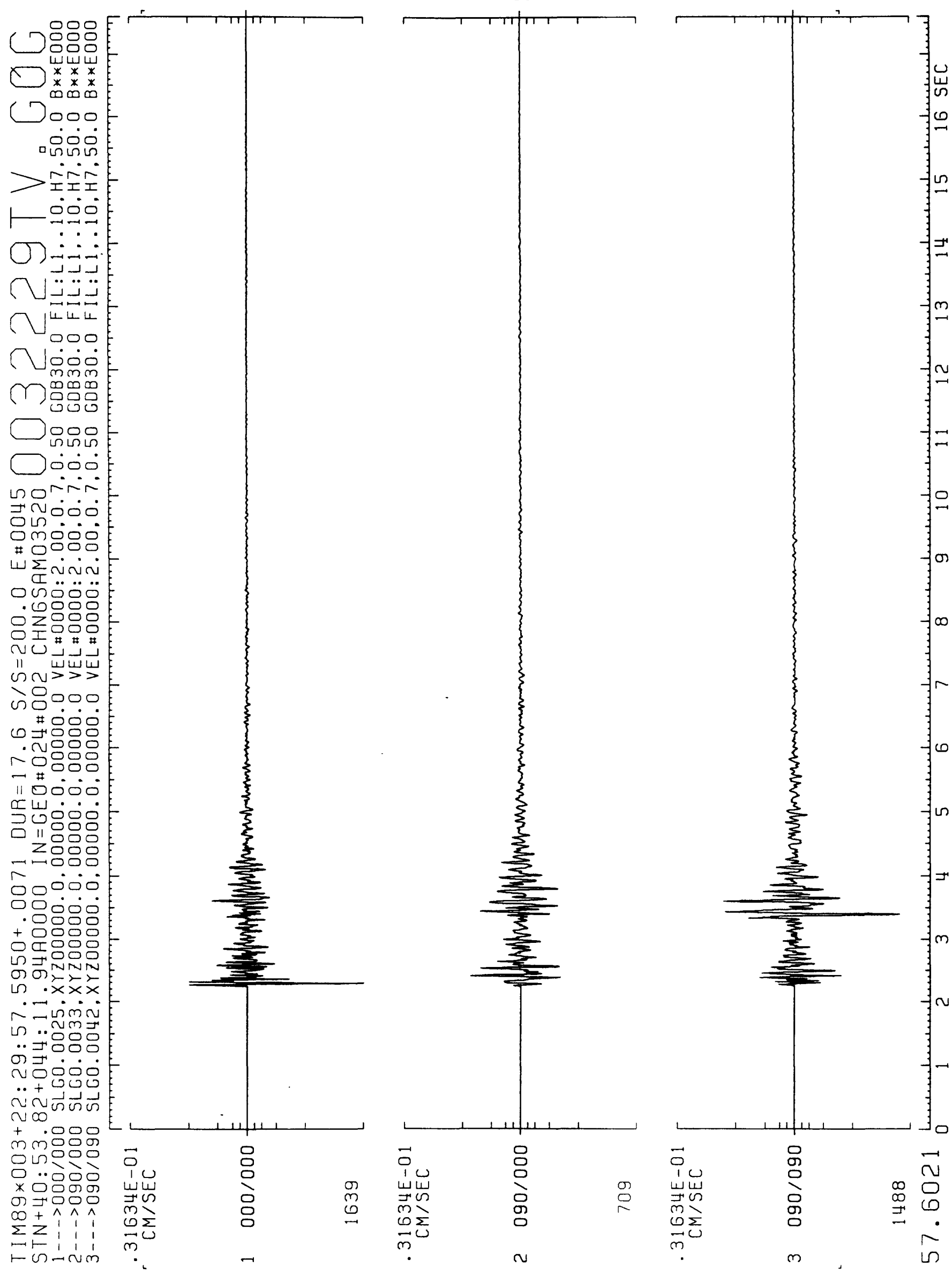




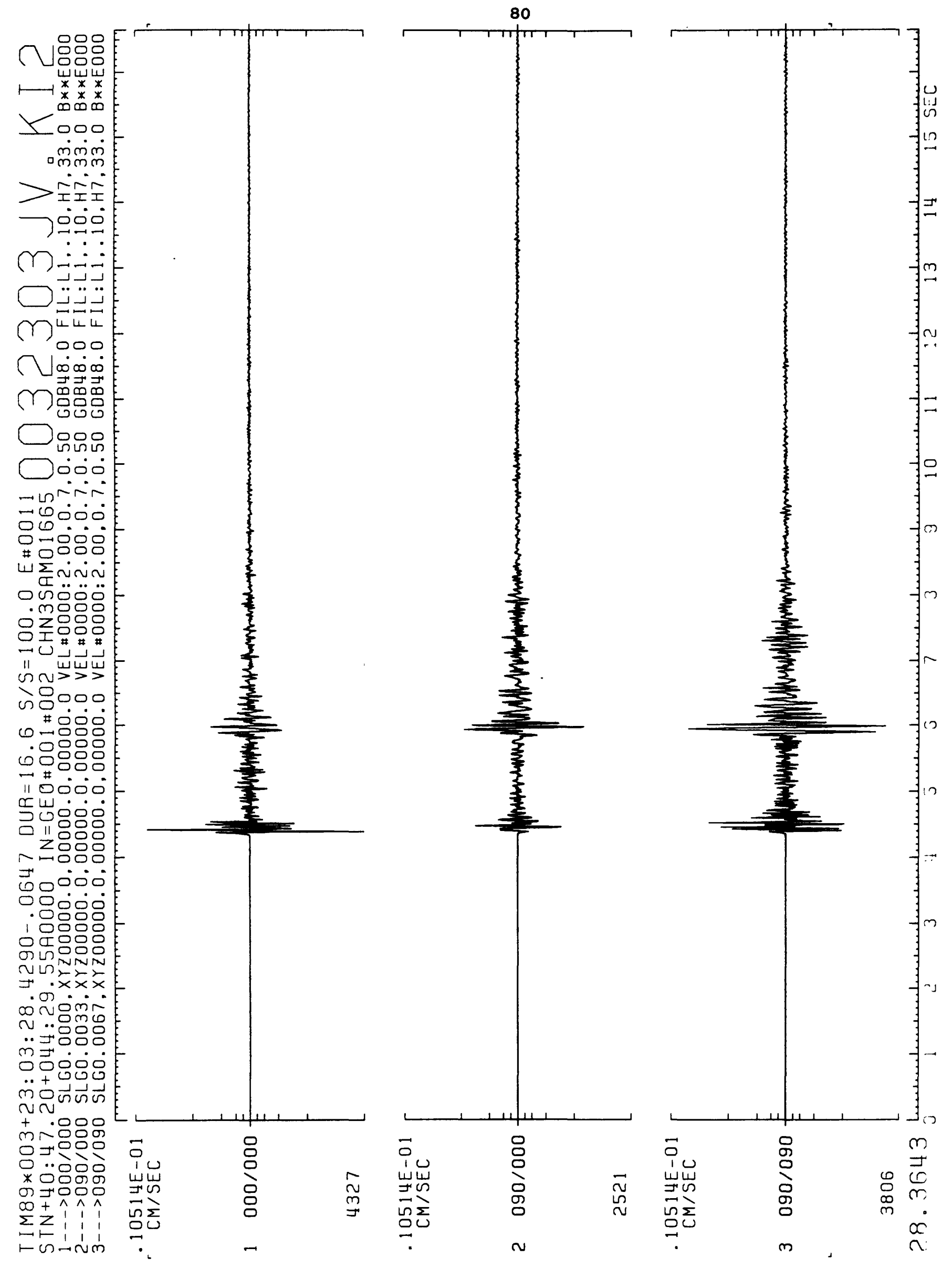



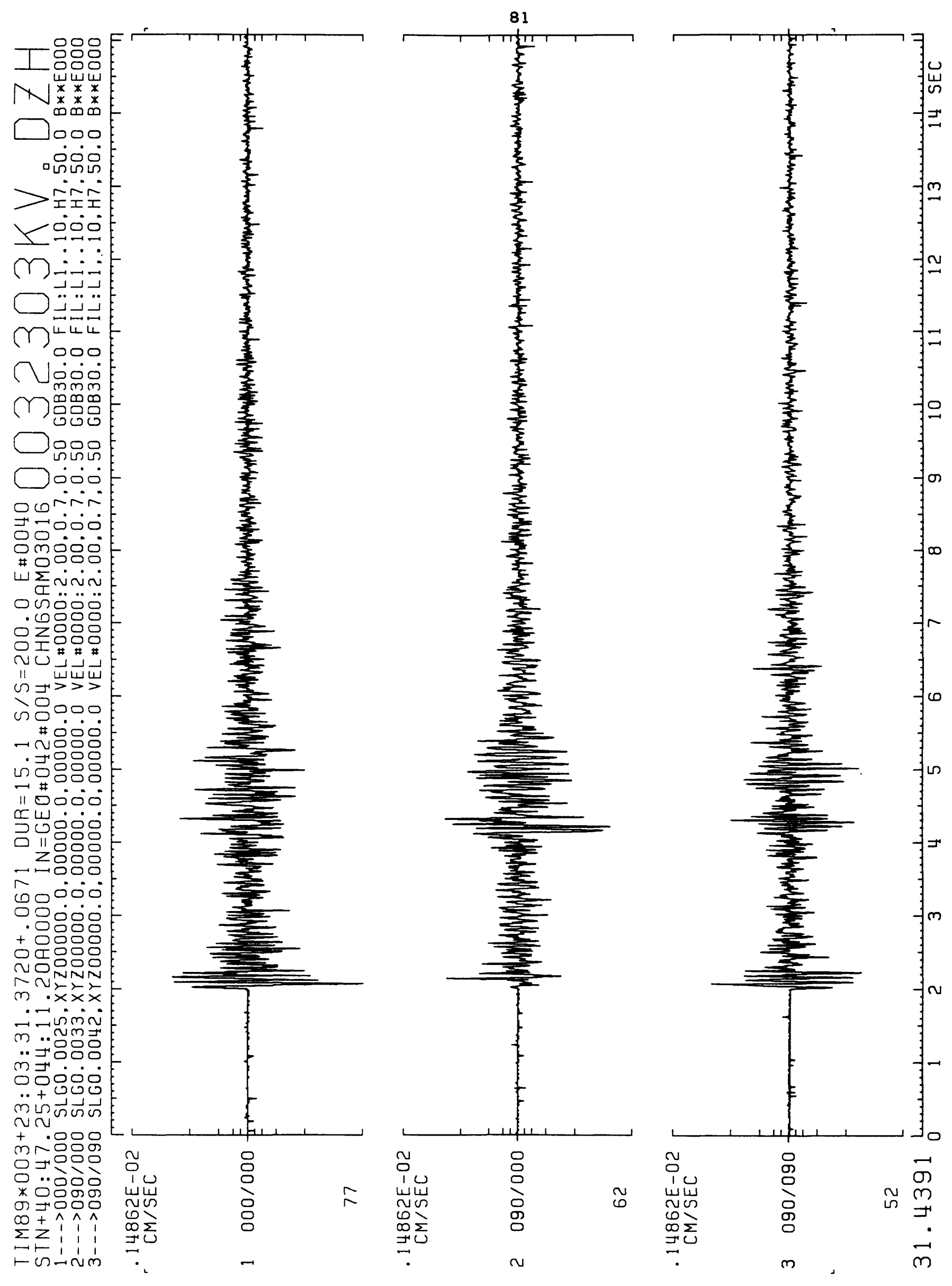
82
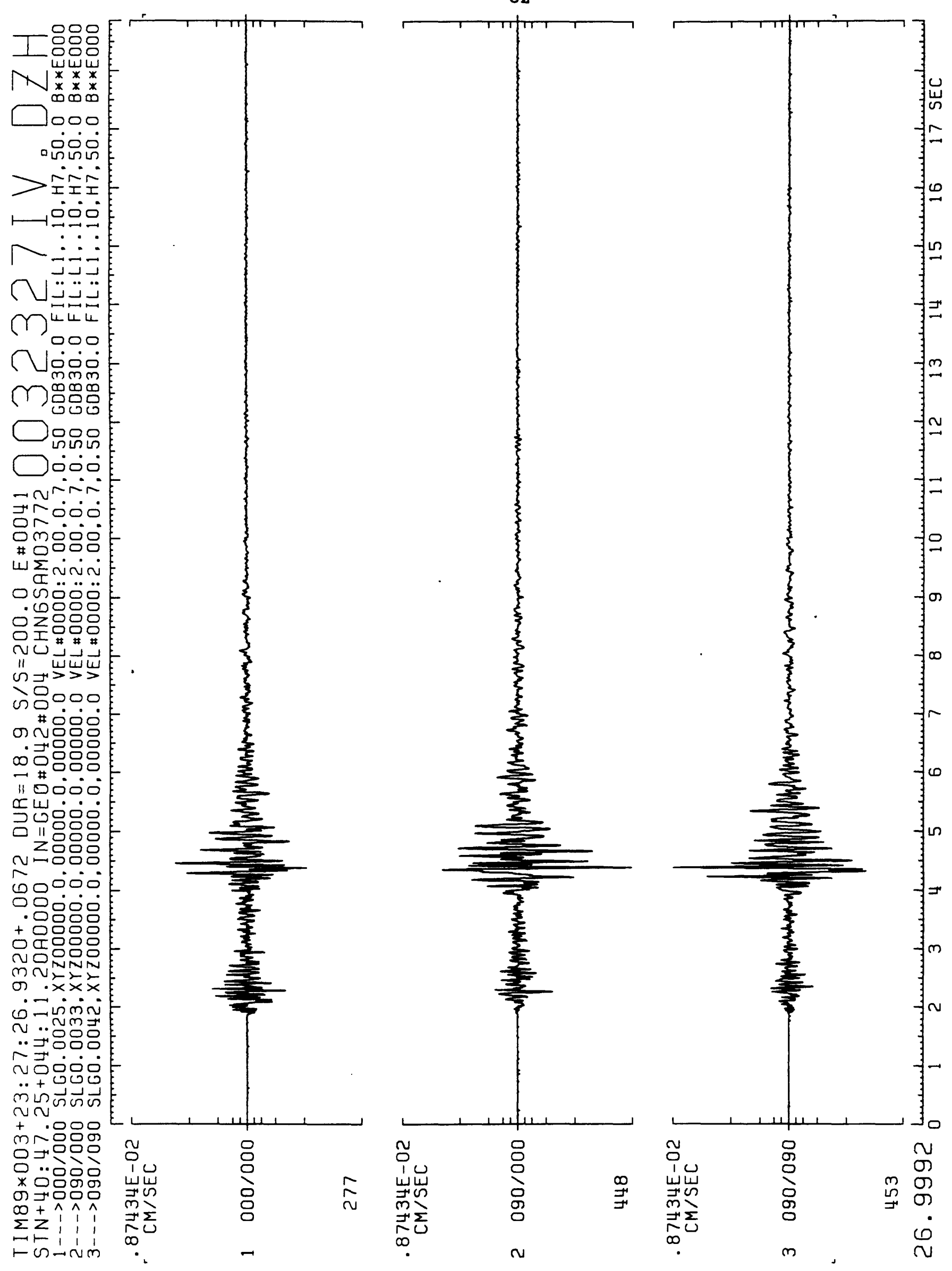


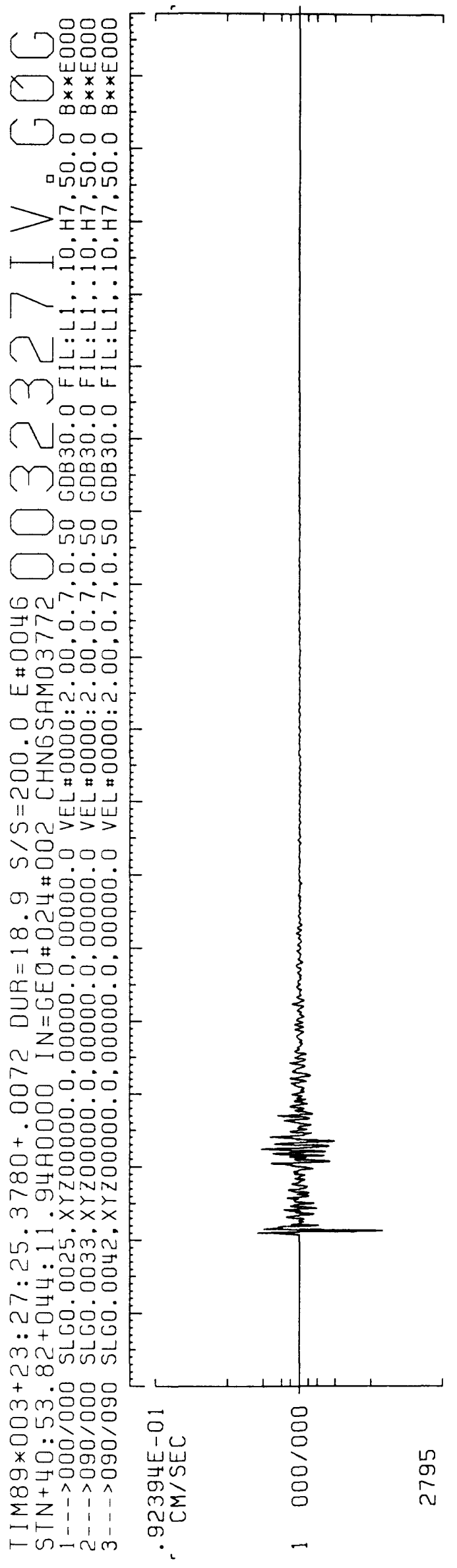

83

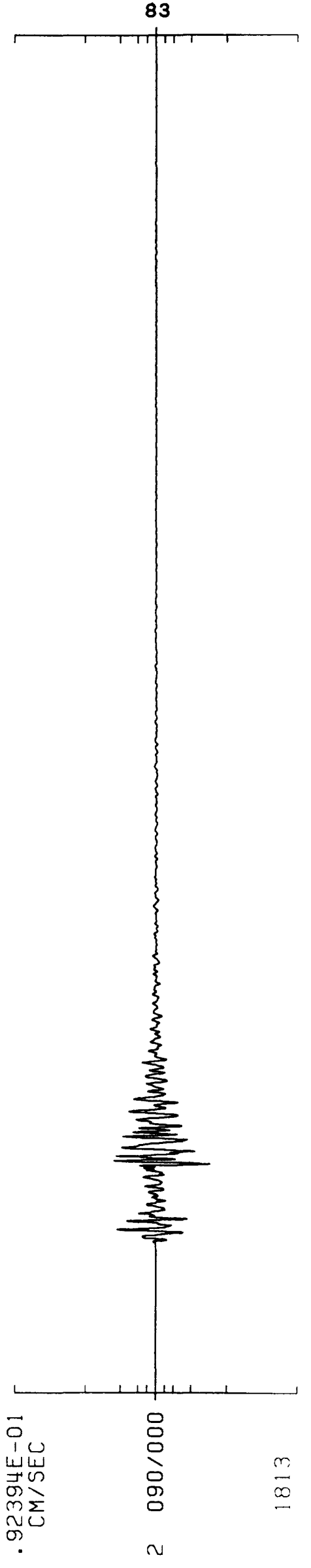




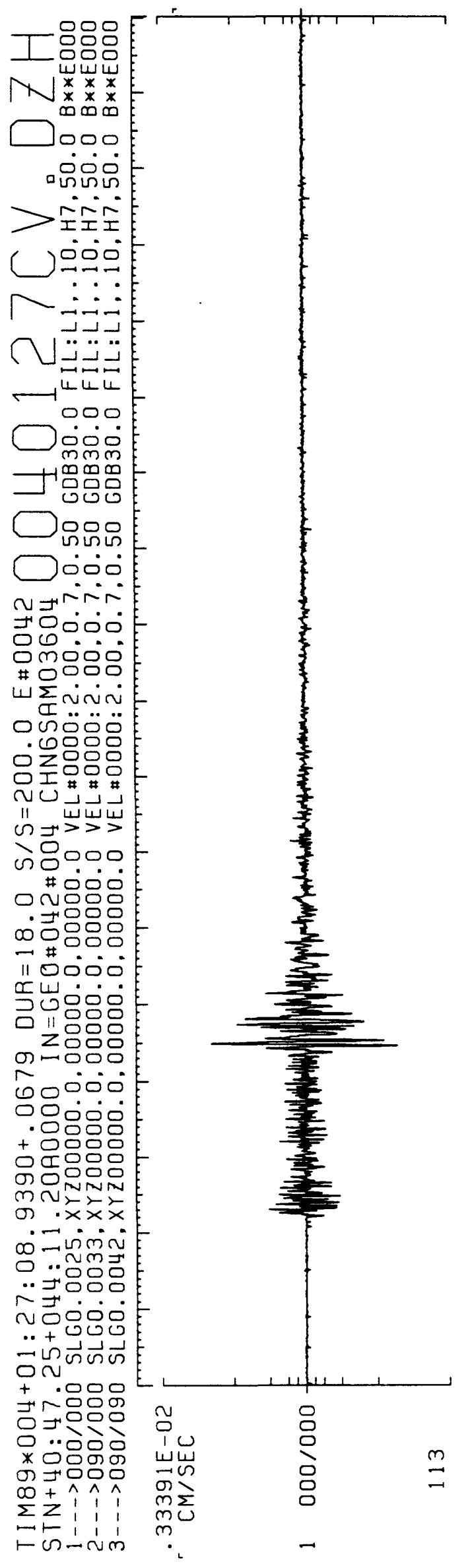

84

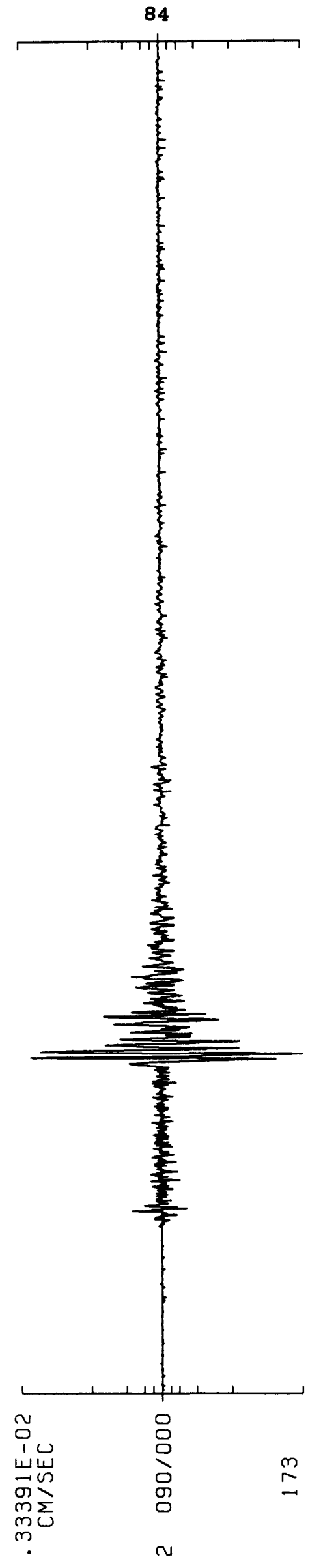

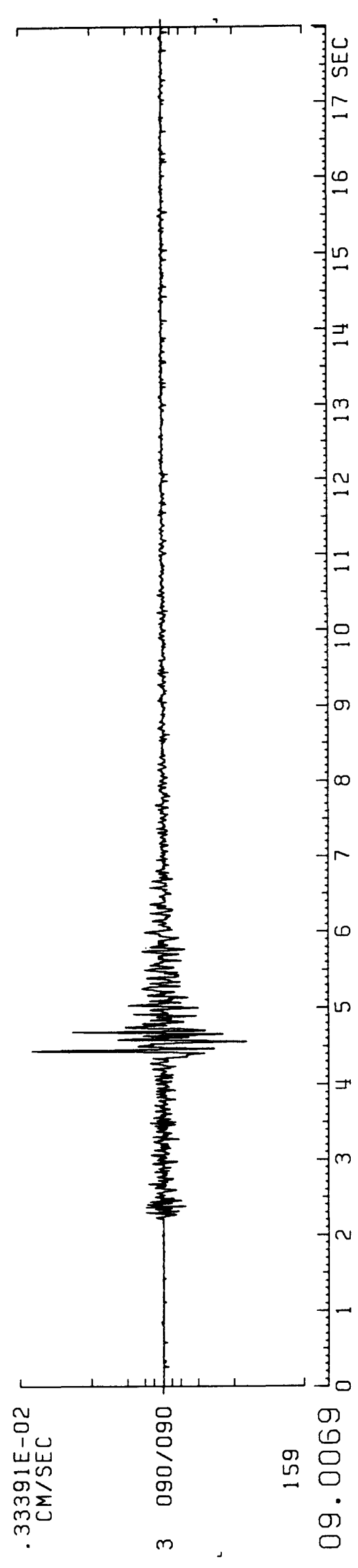




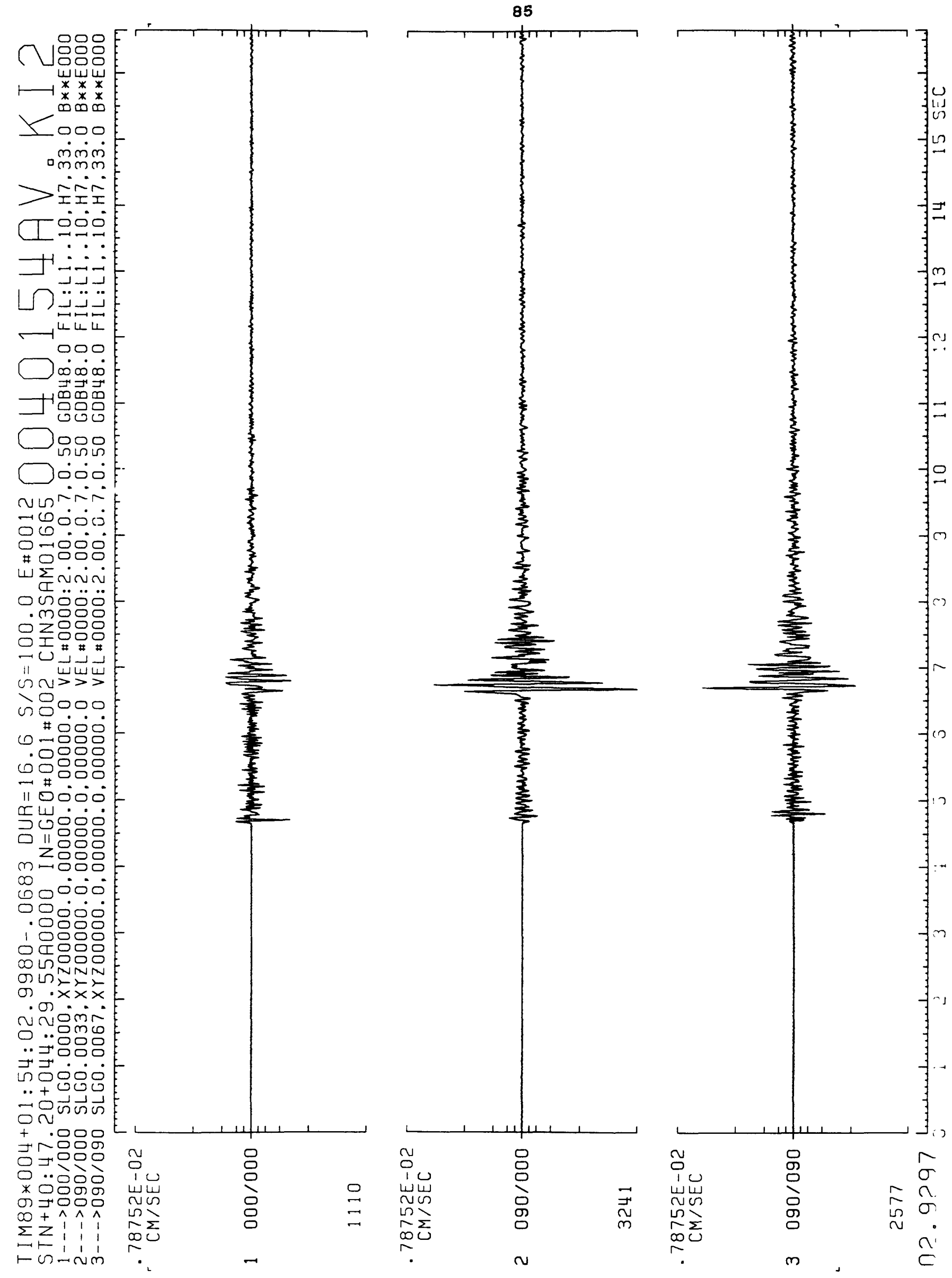




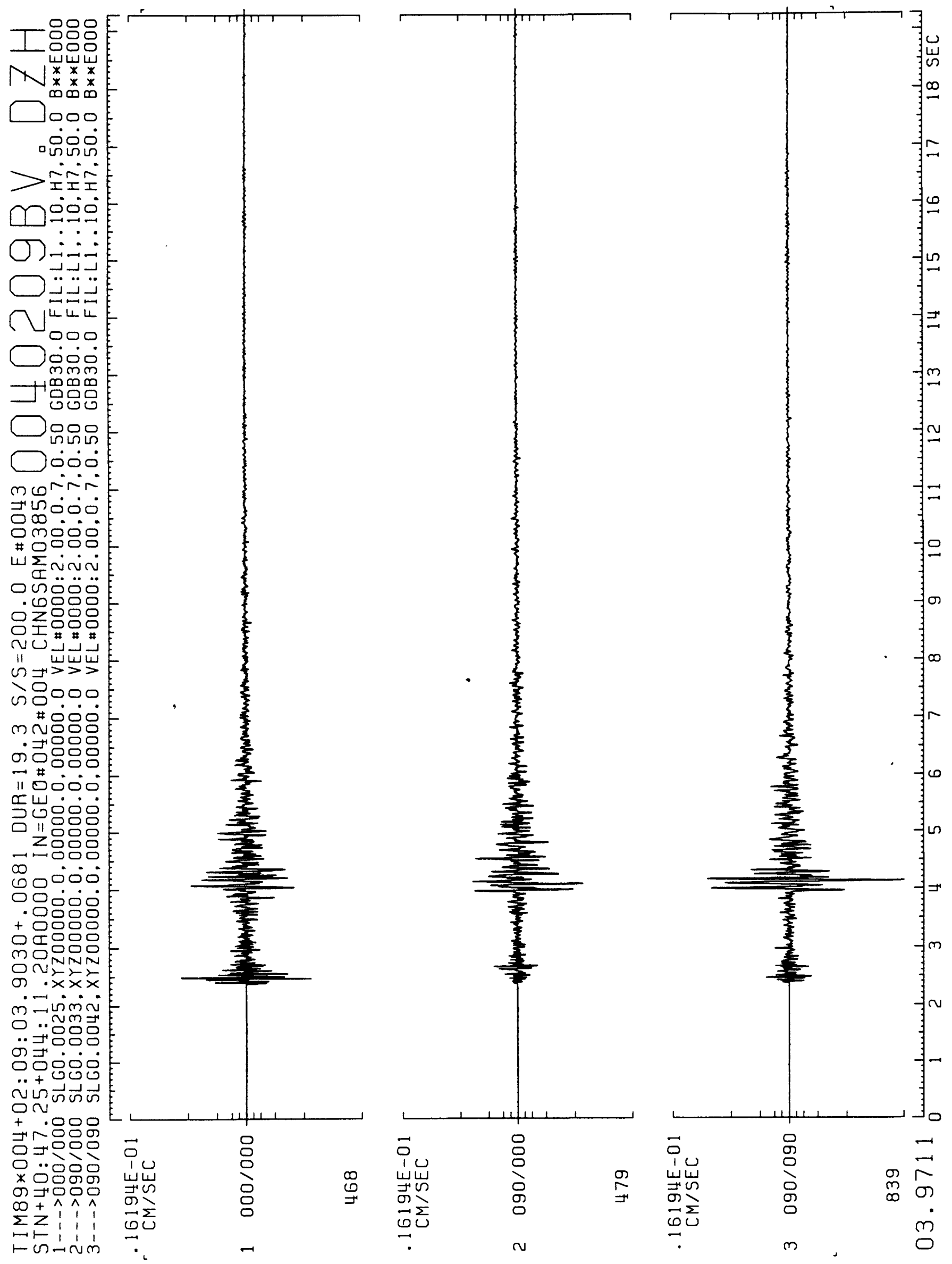



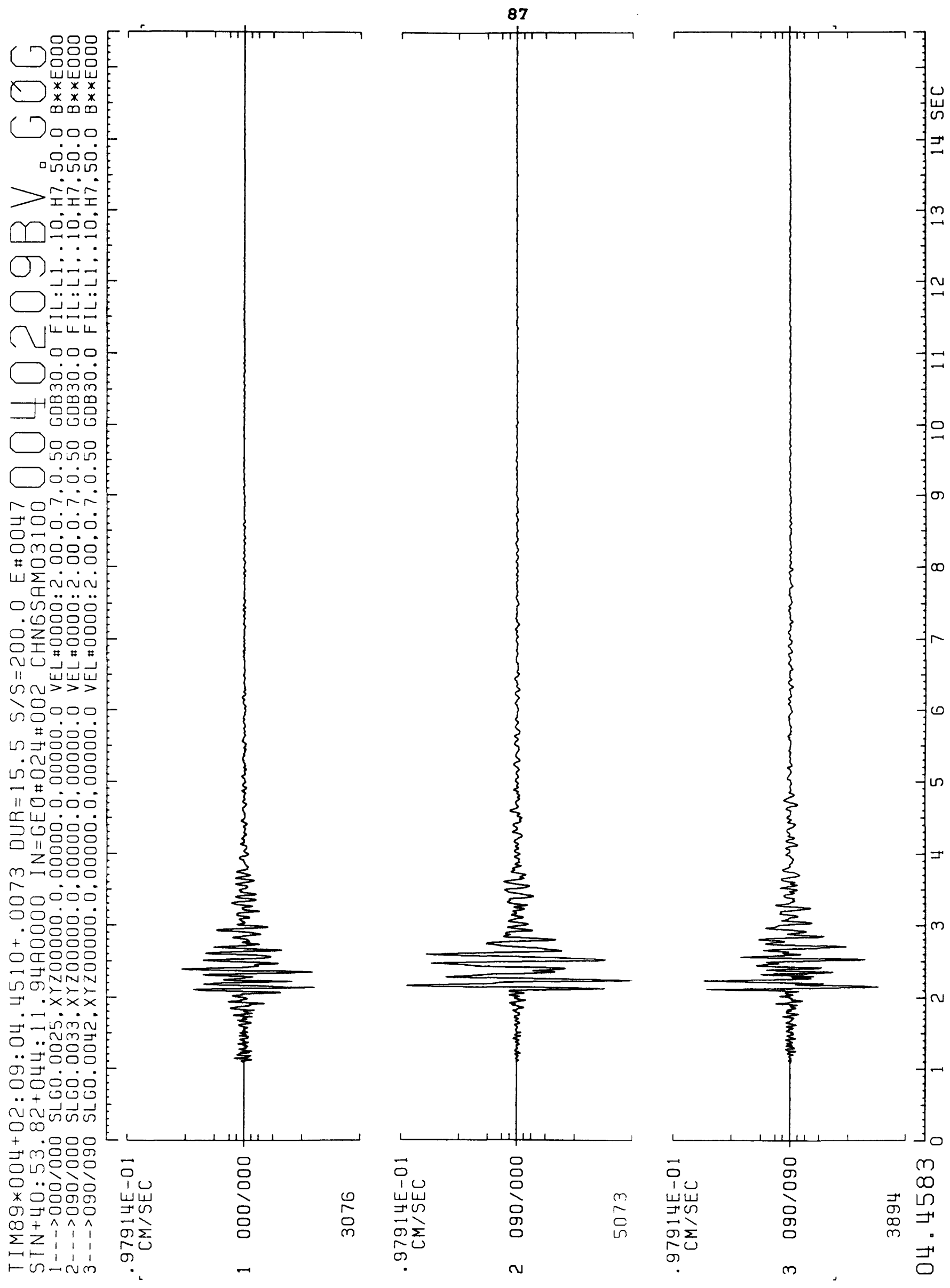


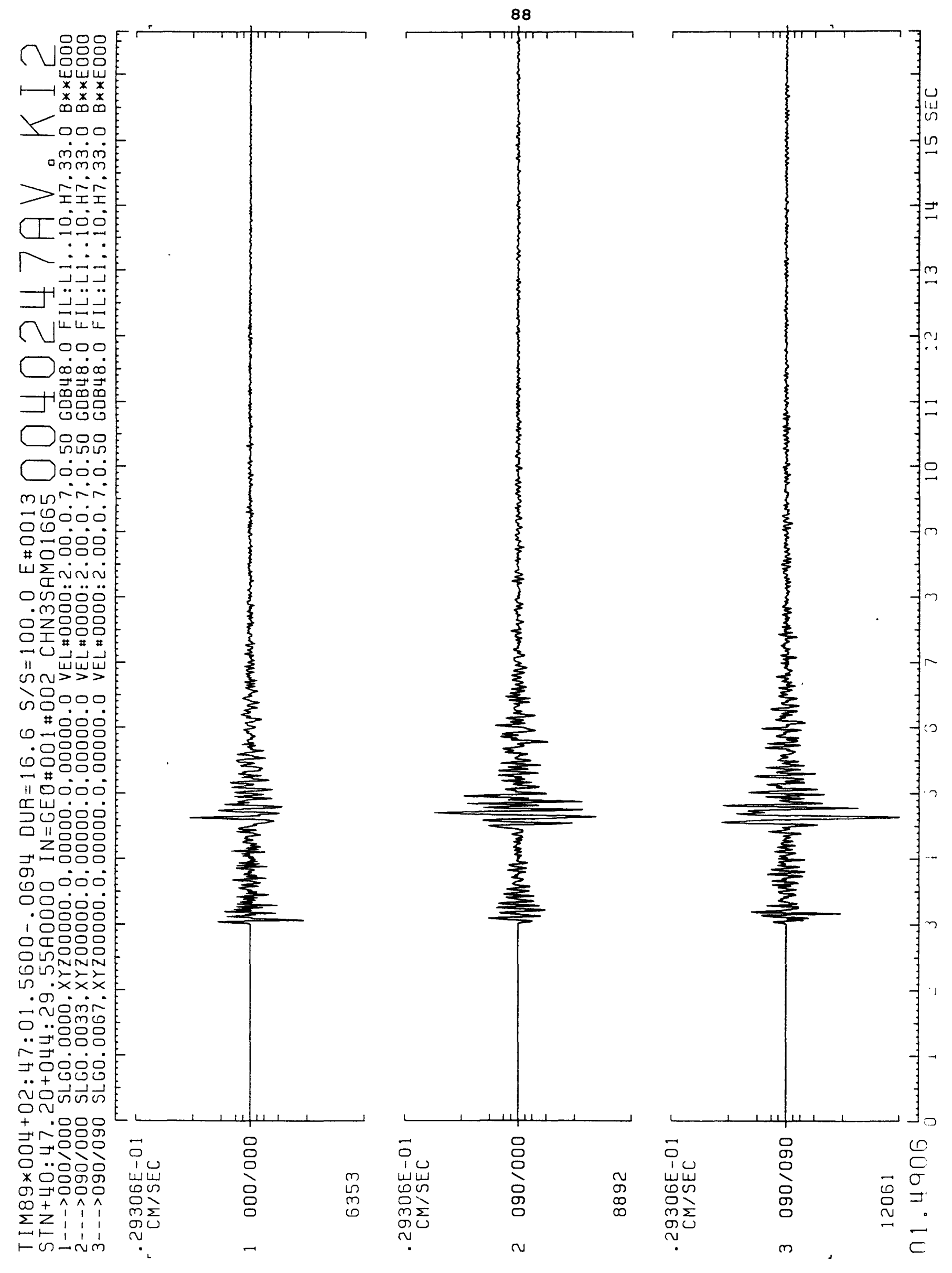




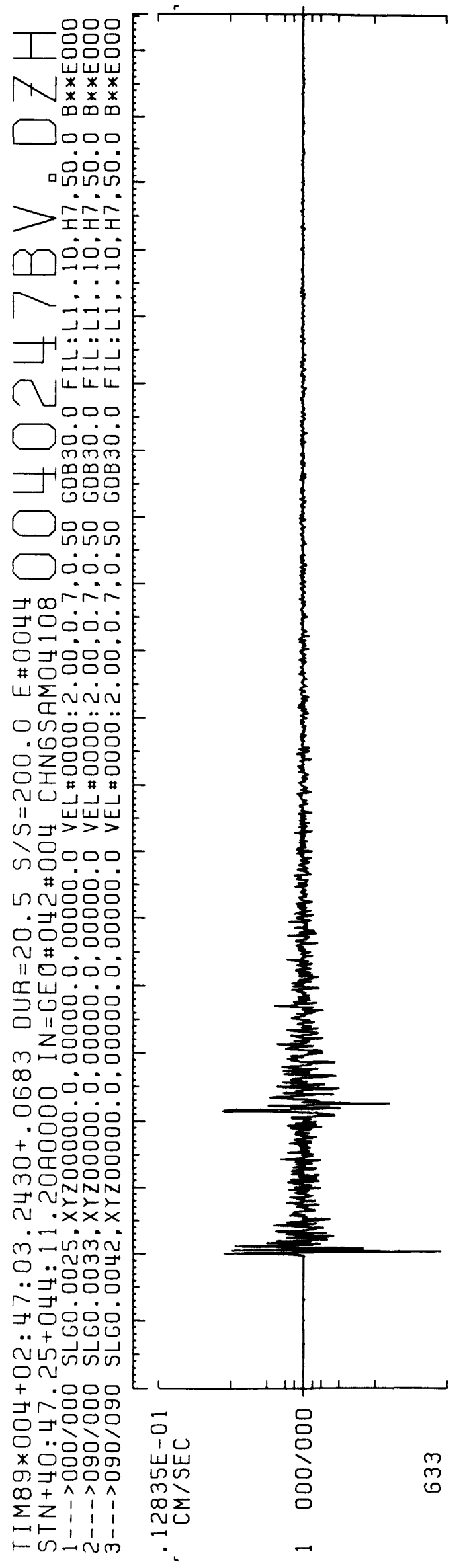

89

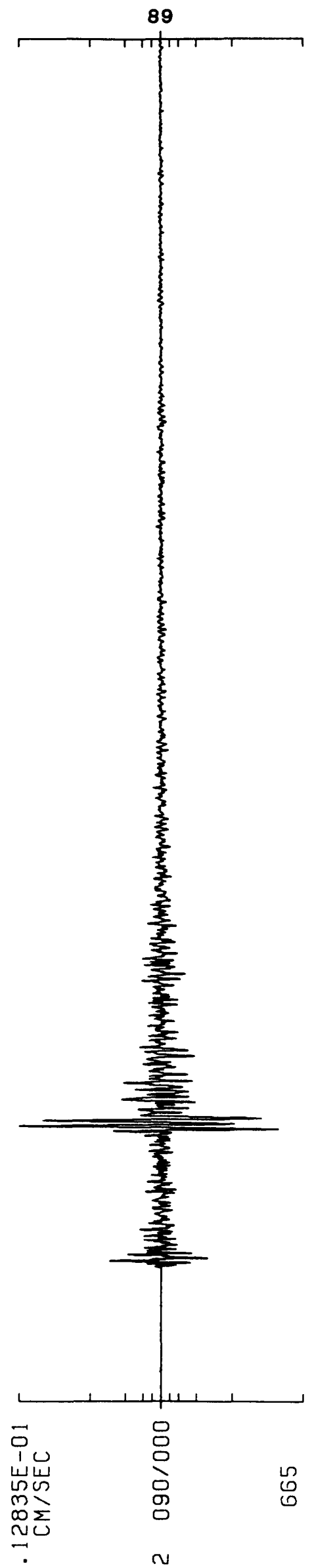



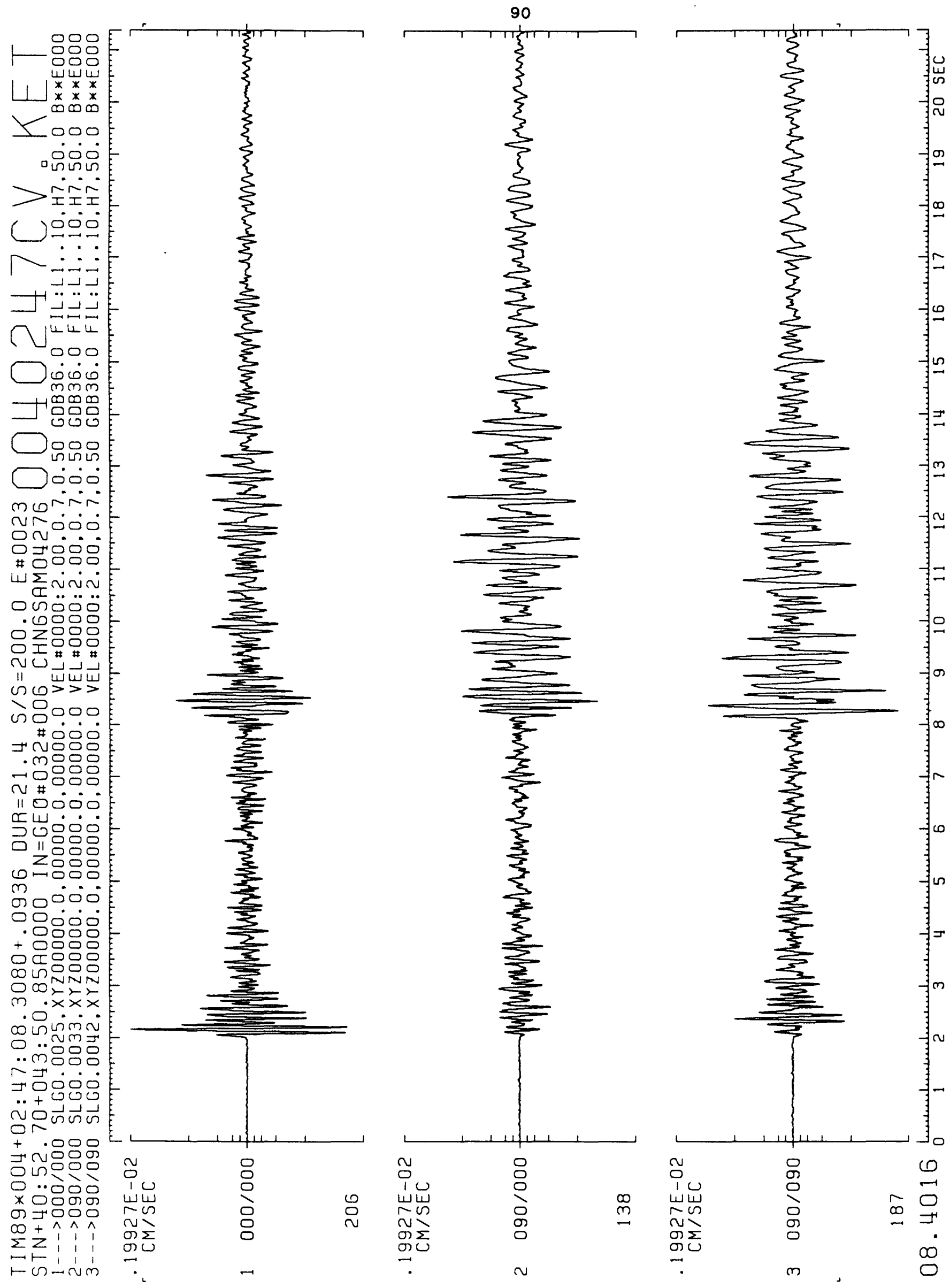
91
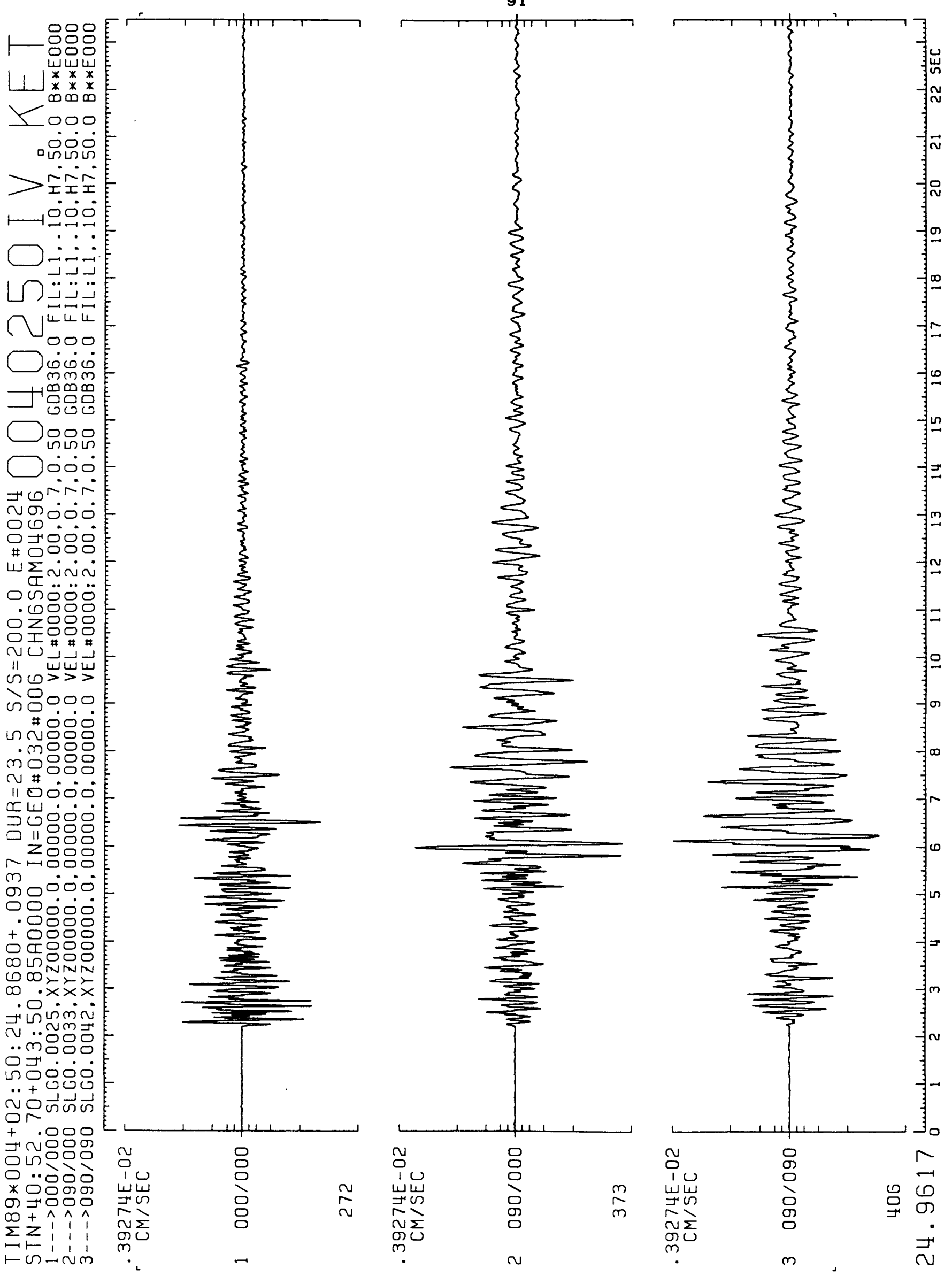


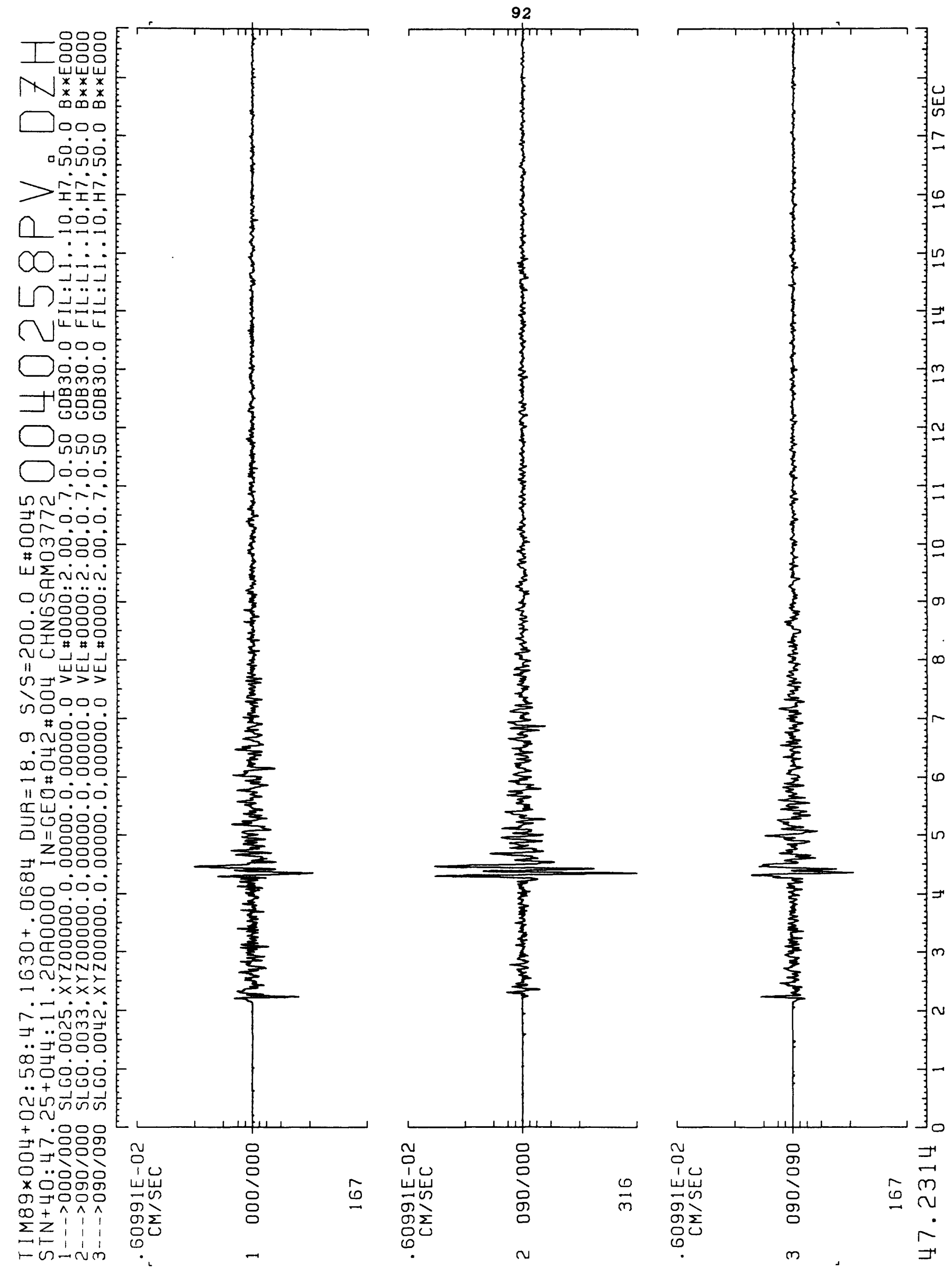




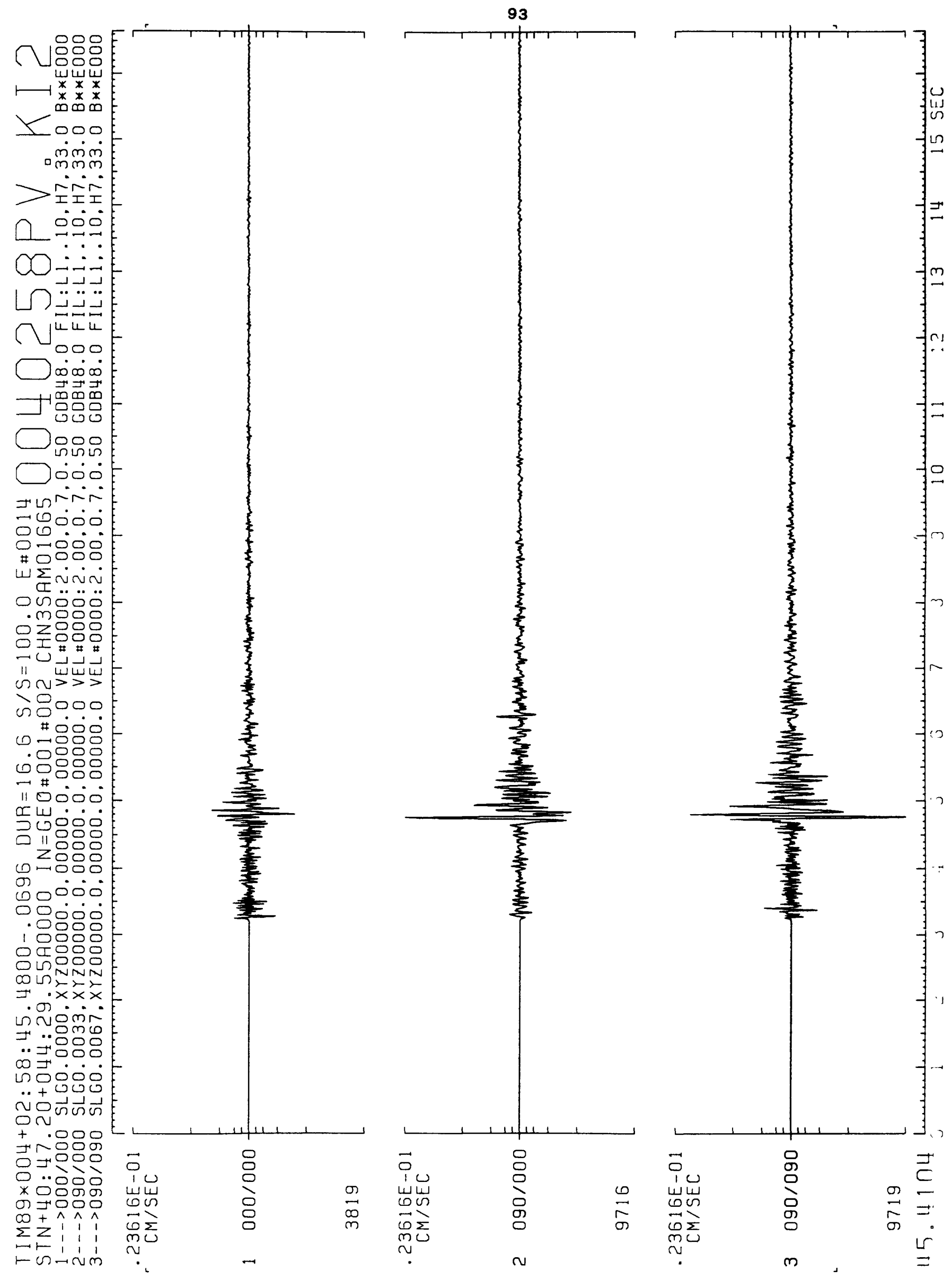



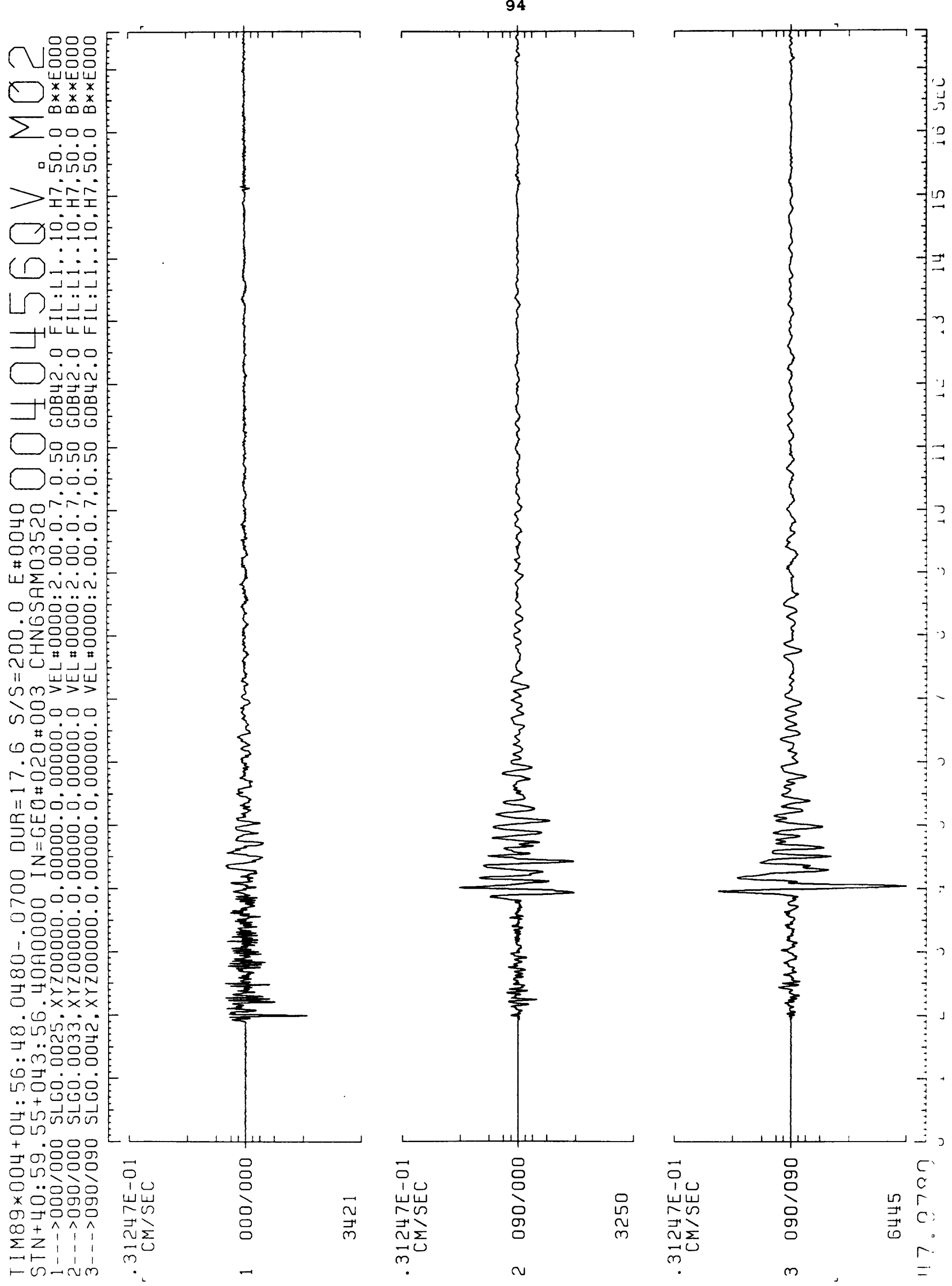
95
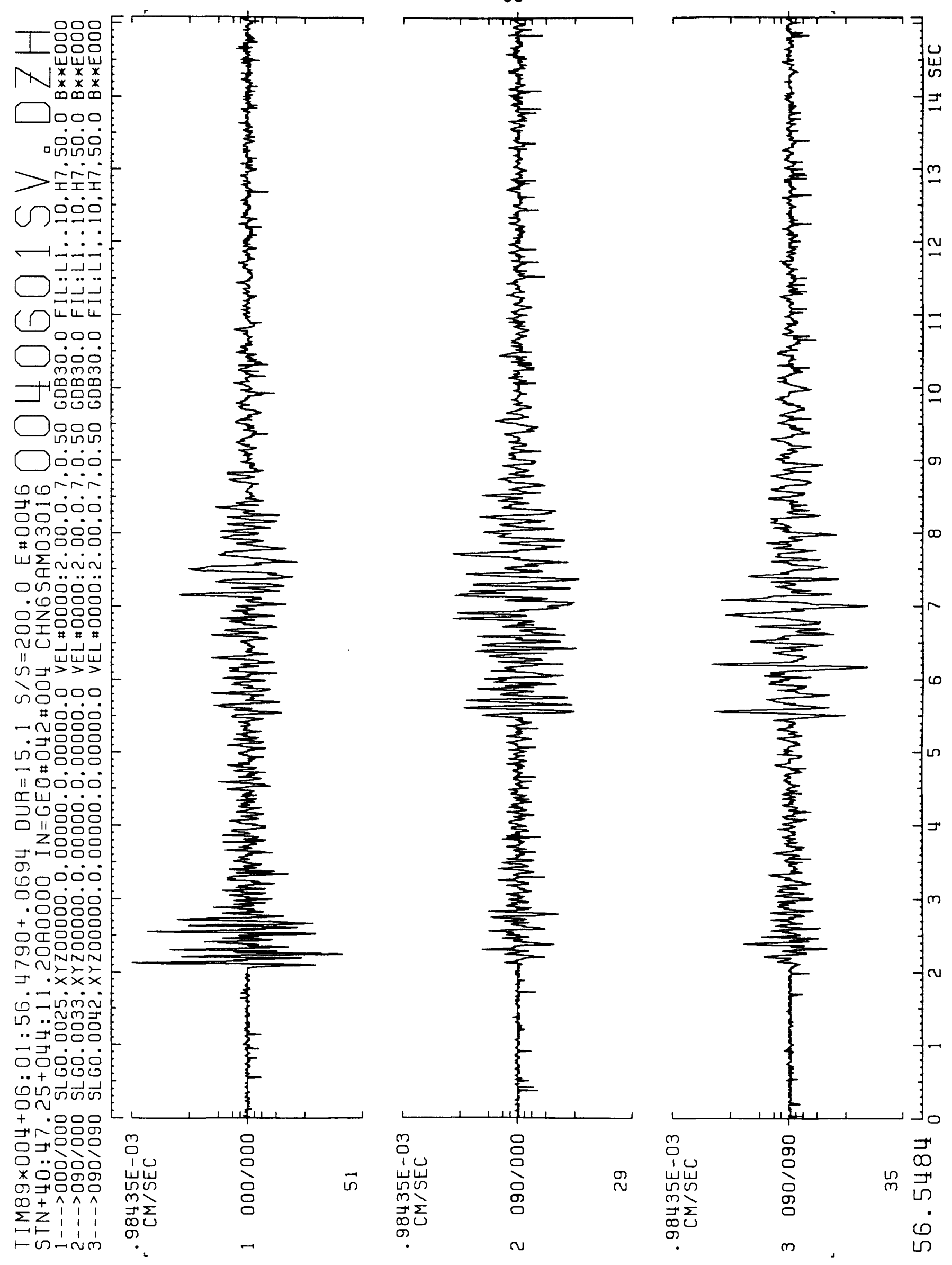
96

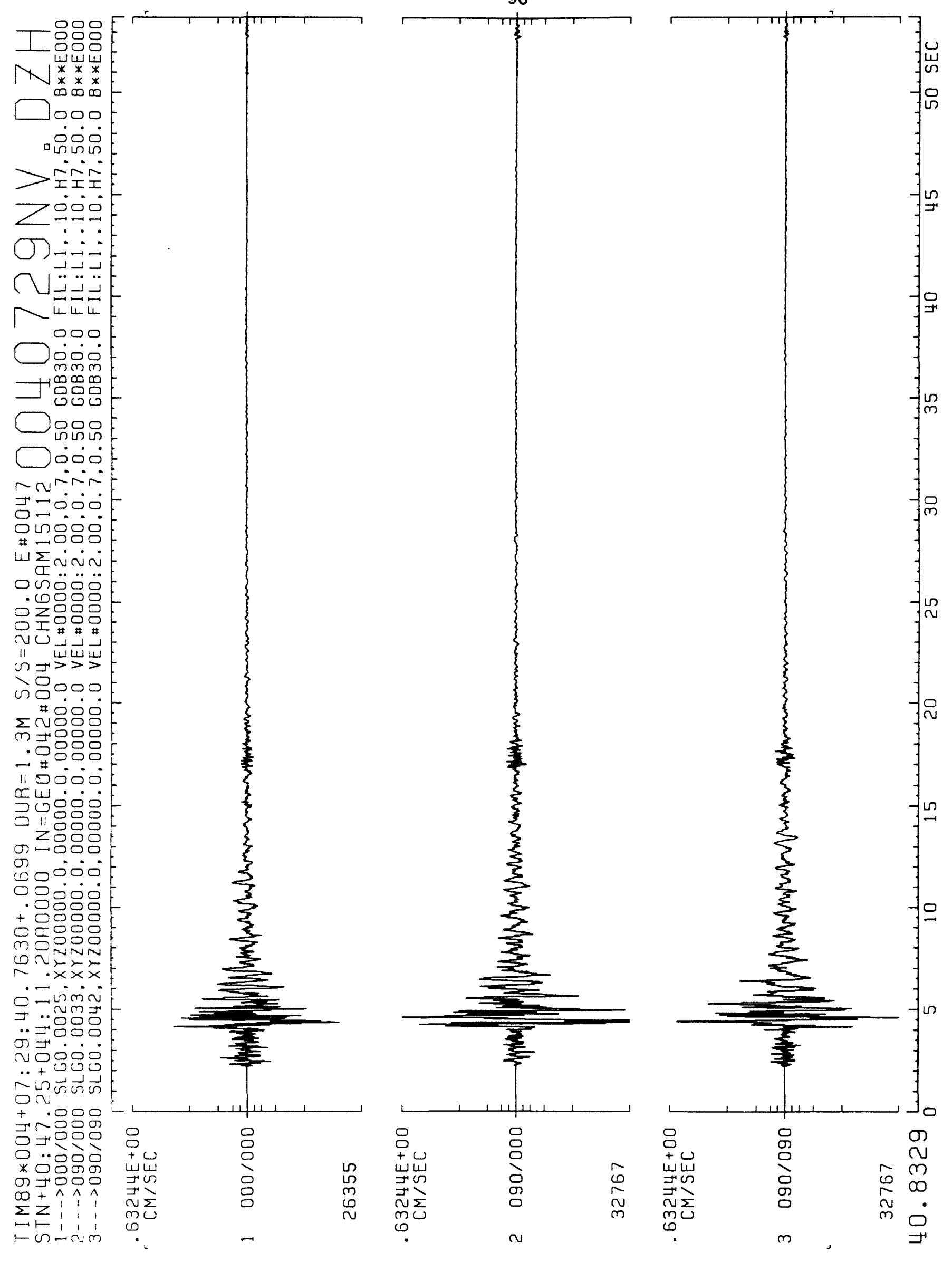




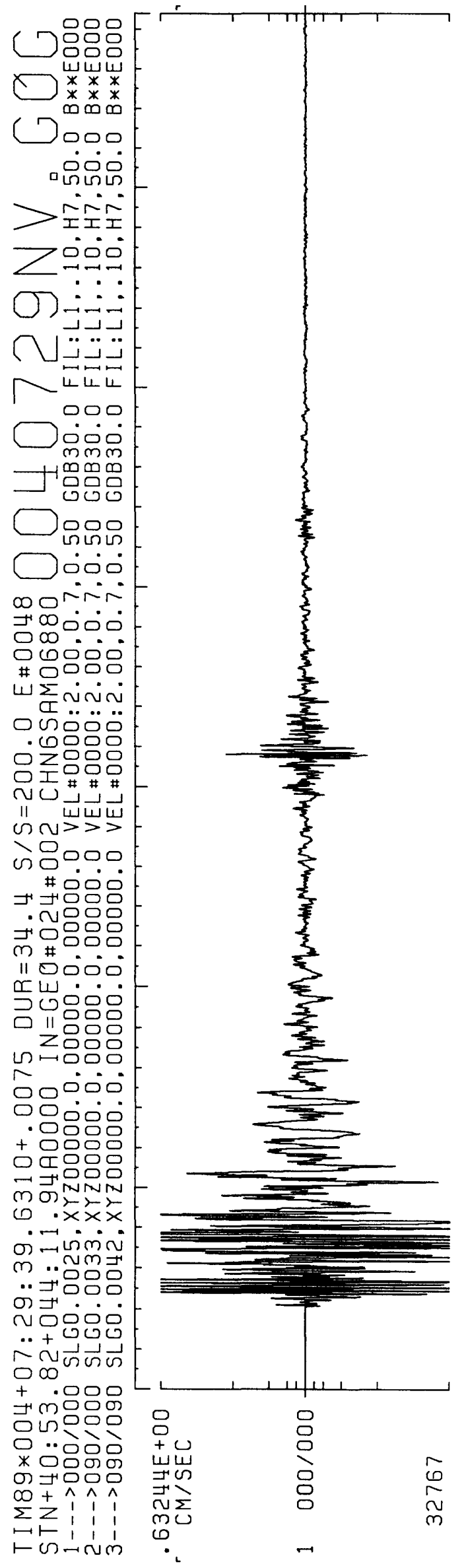

97

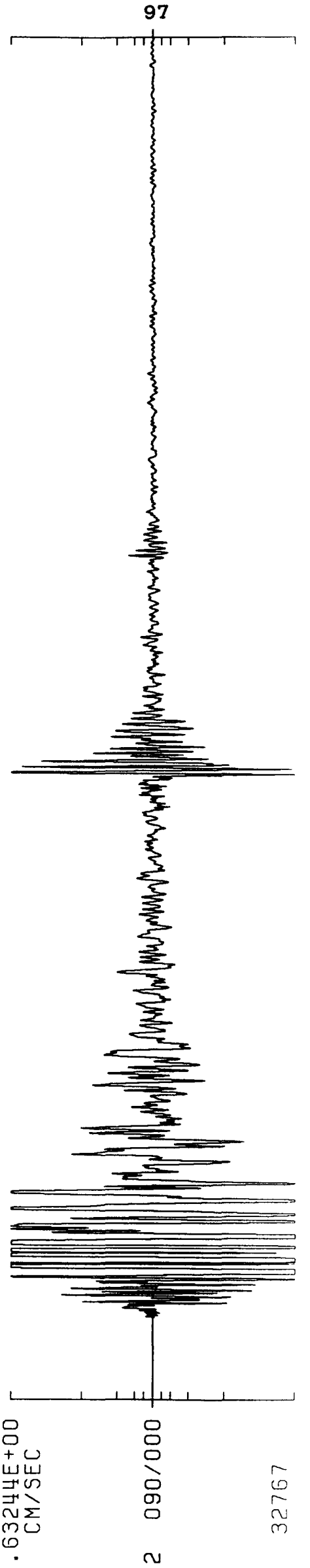

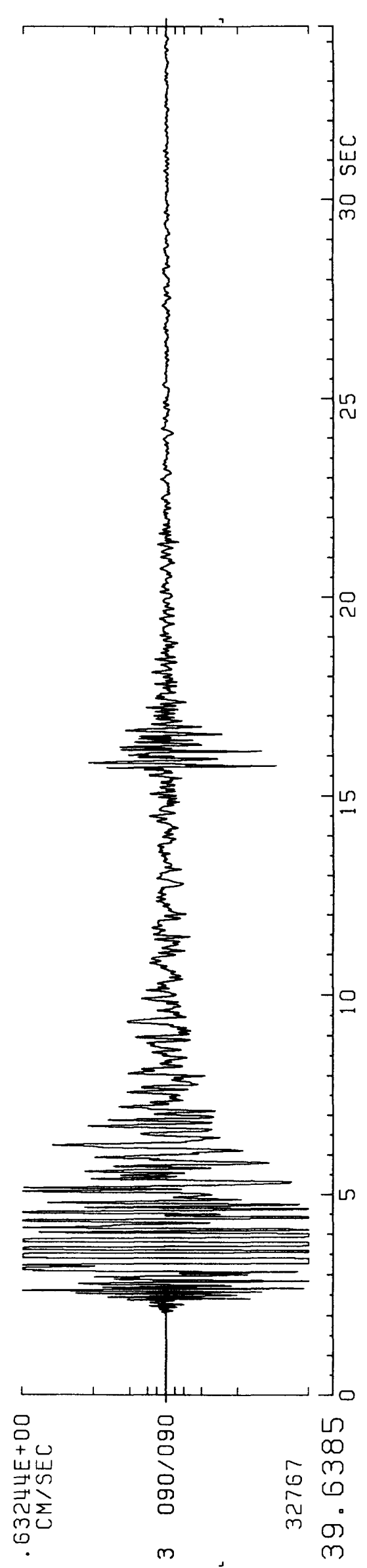



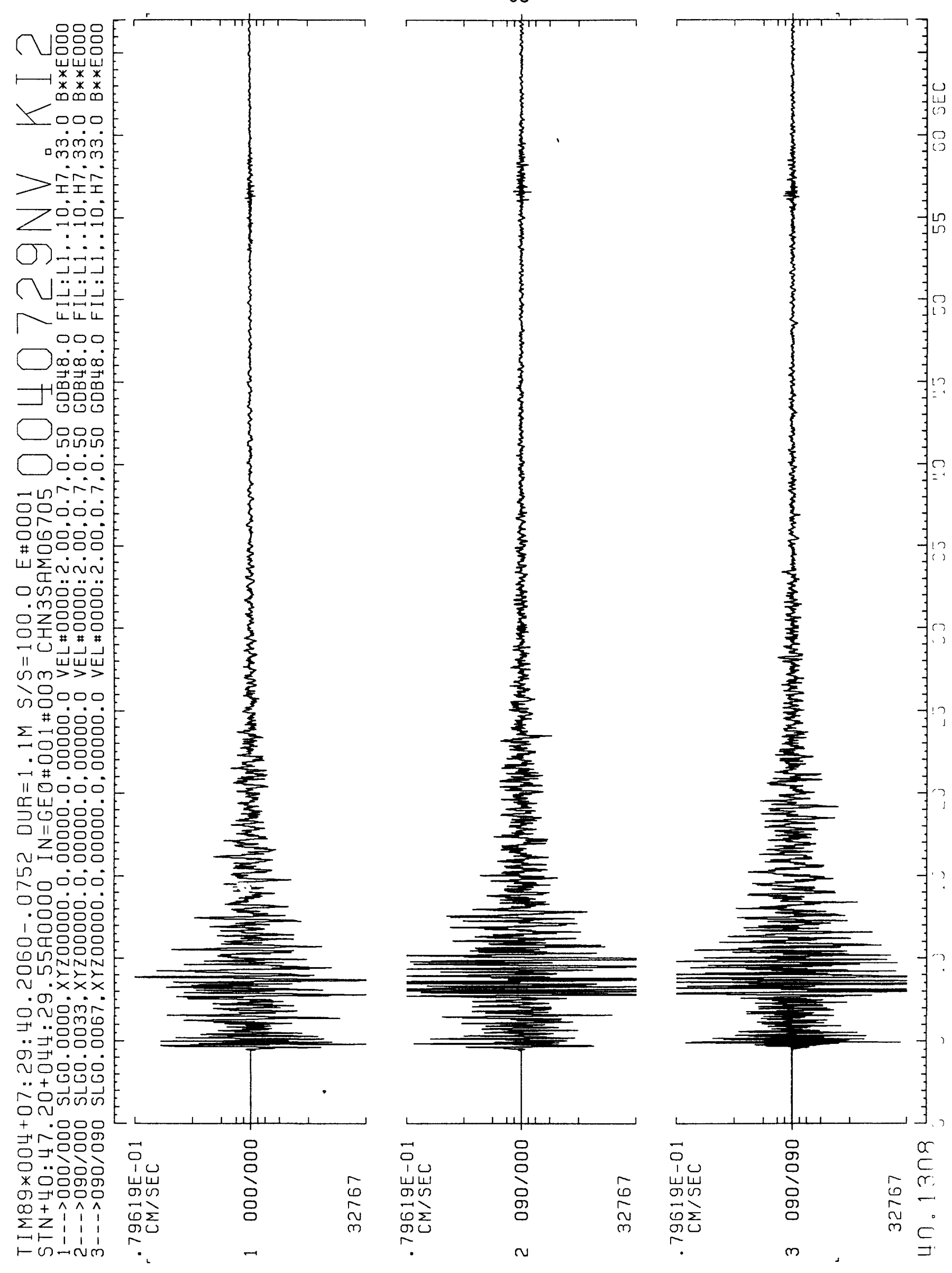
99

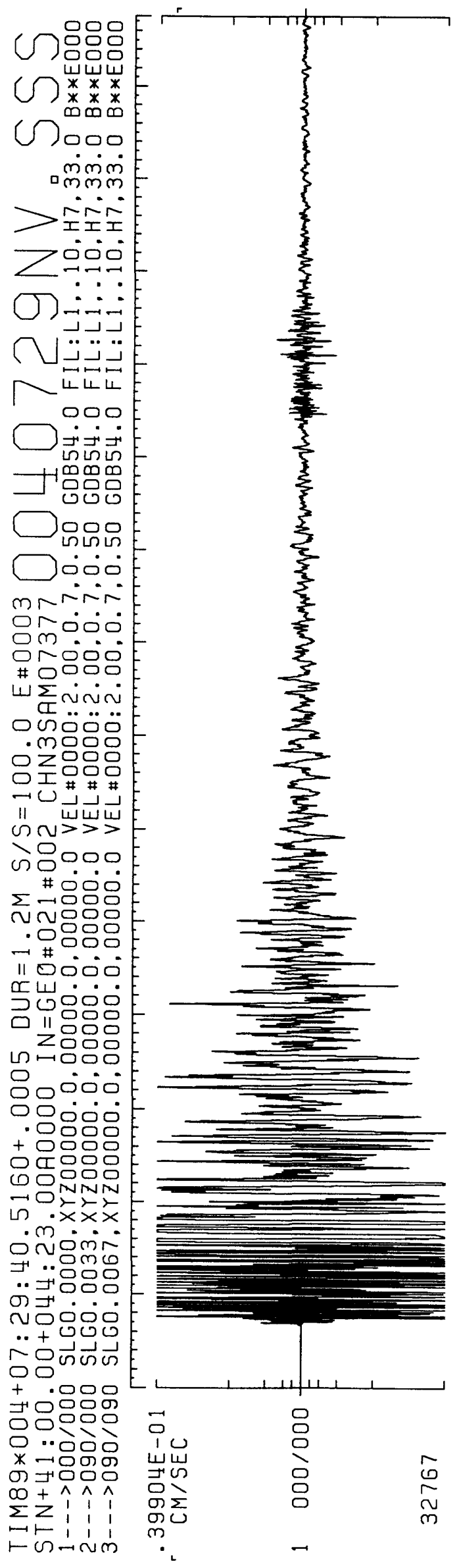

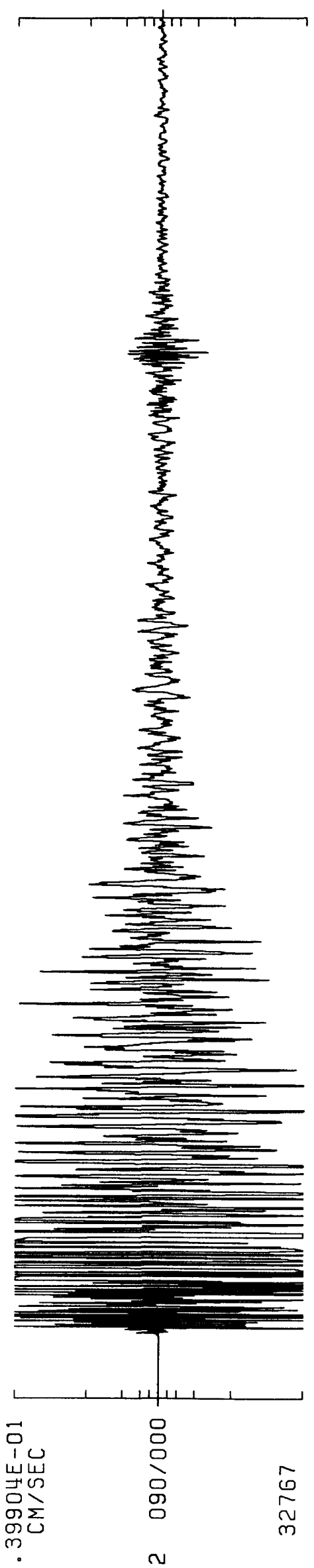

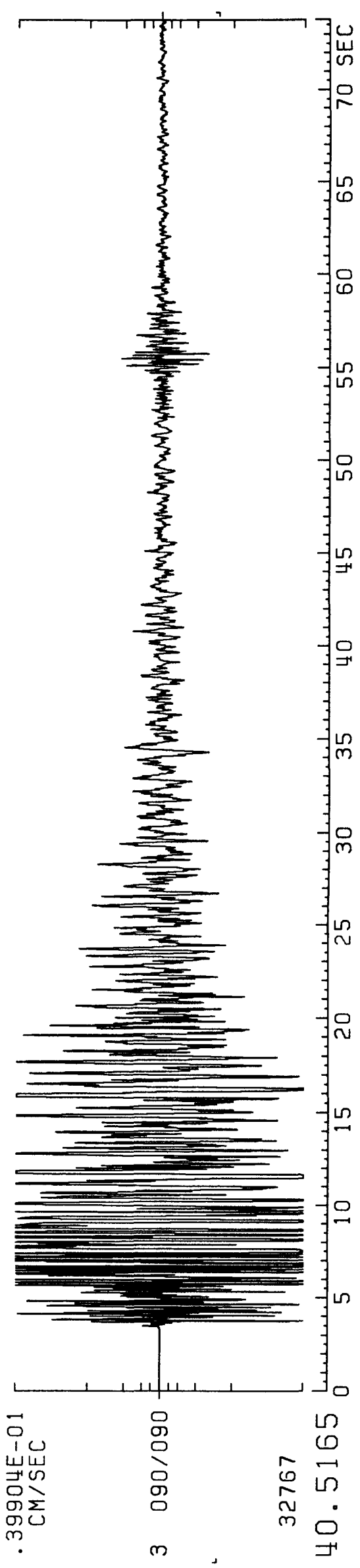




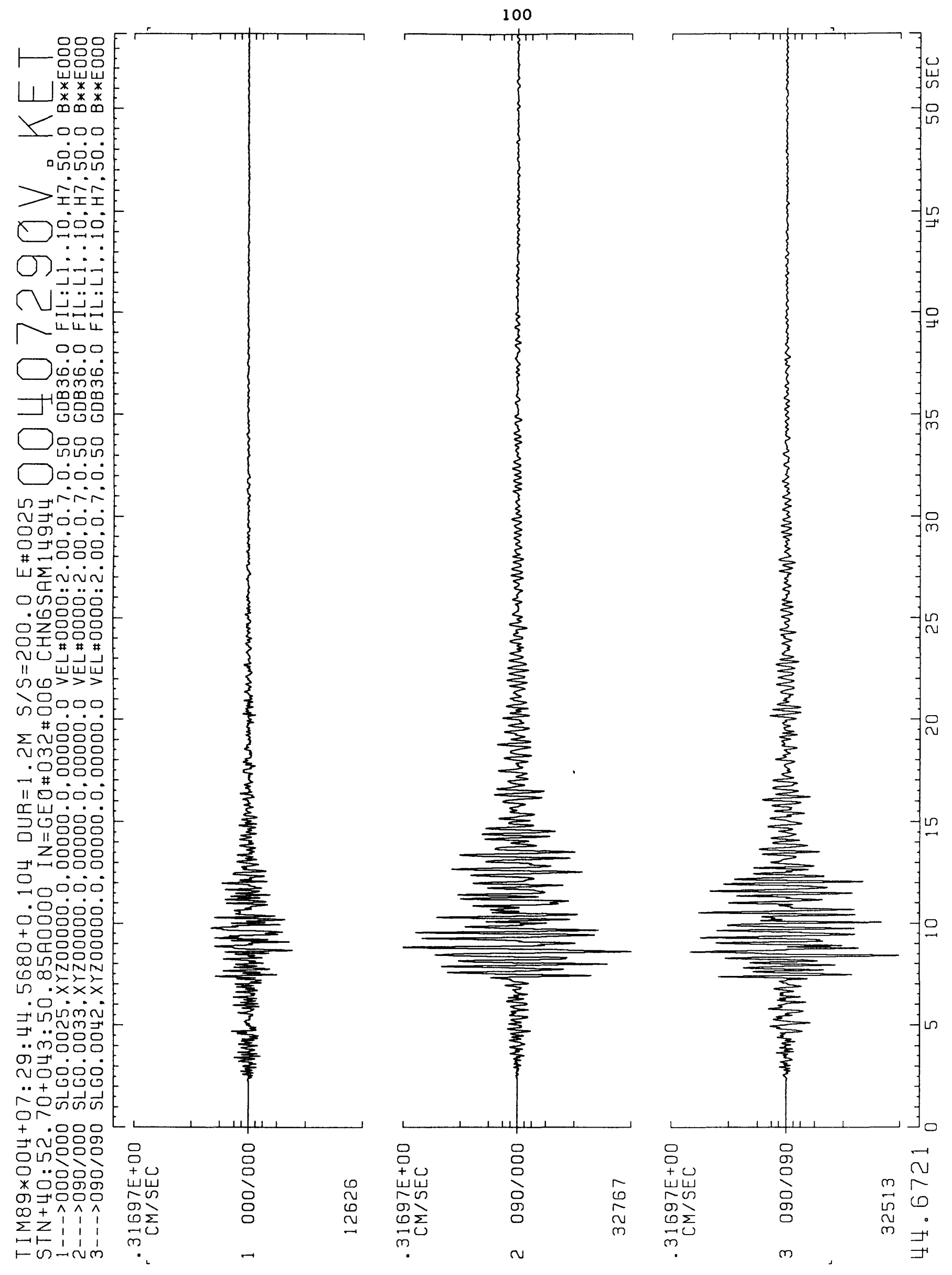


101

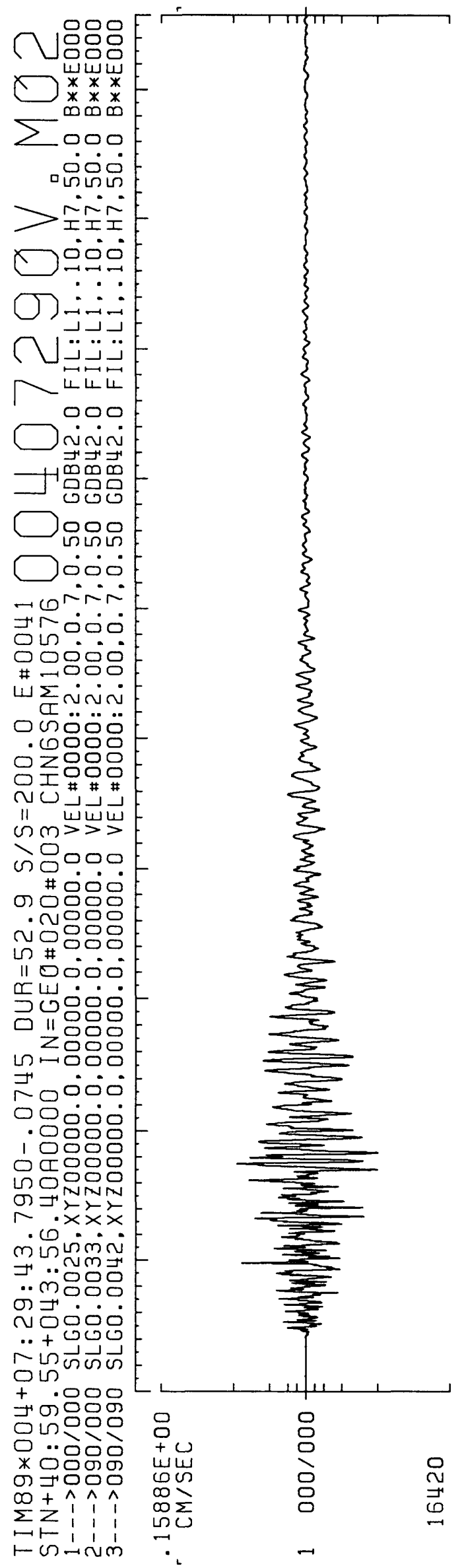

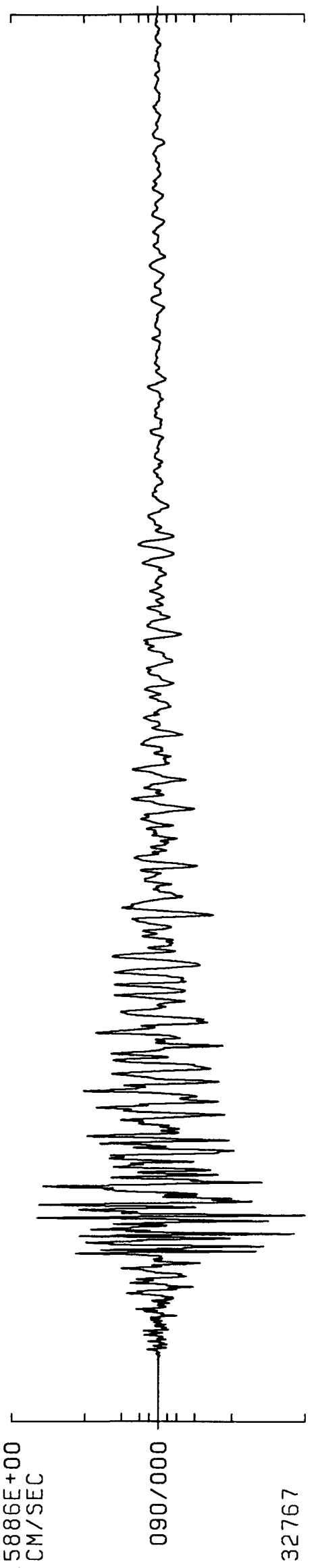

N

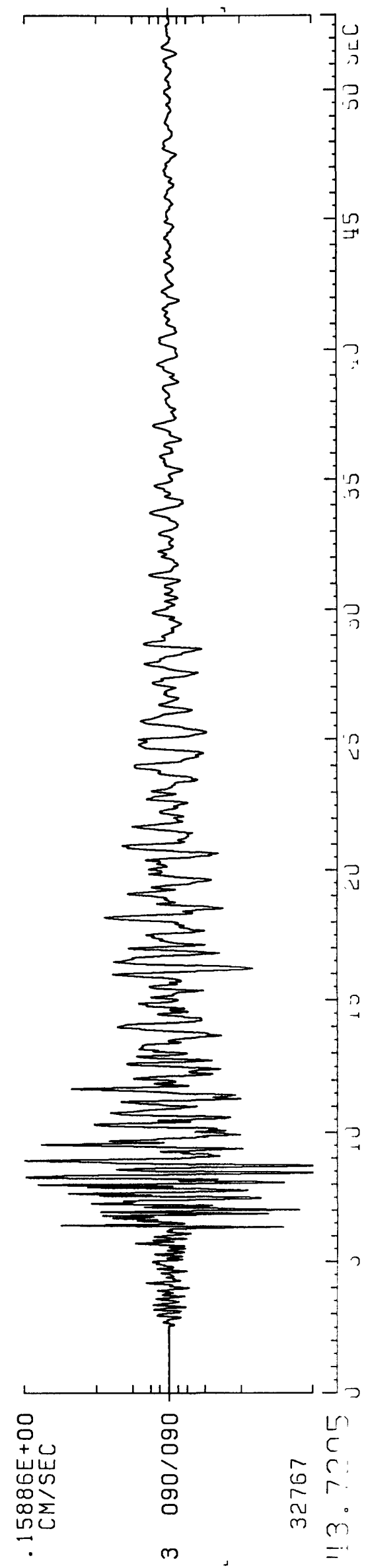



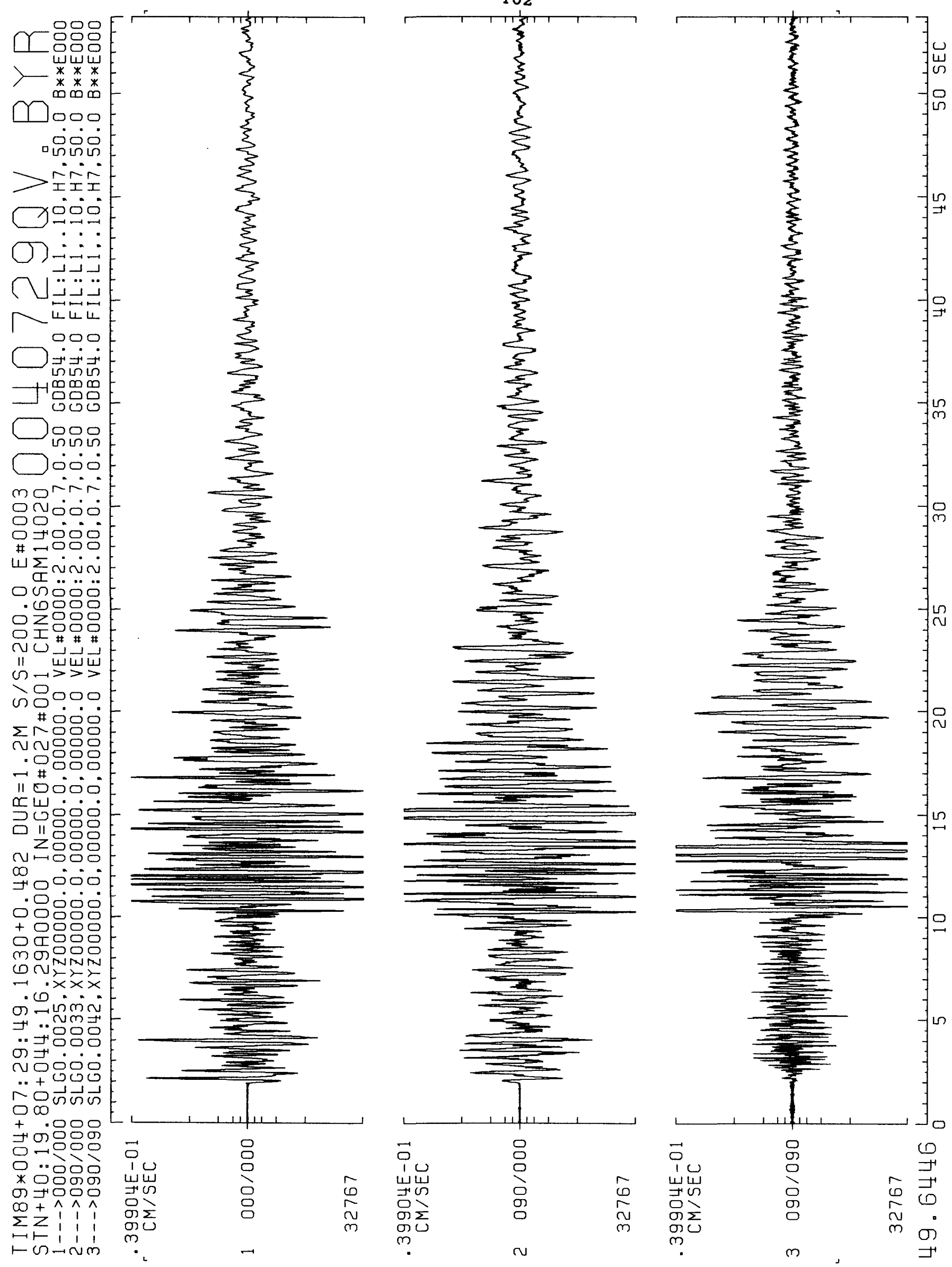
103

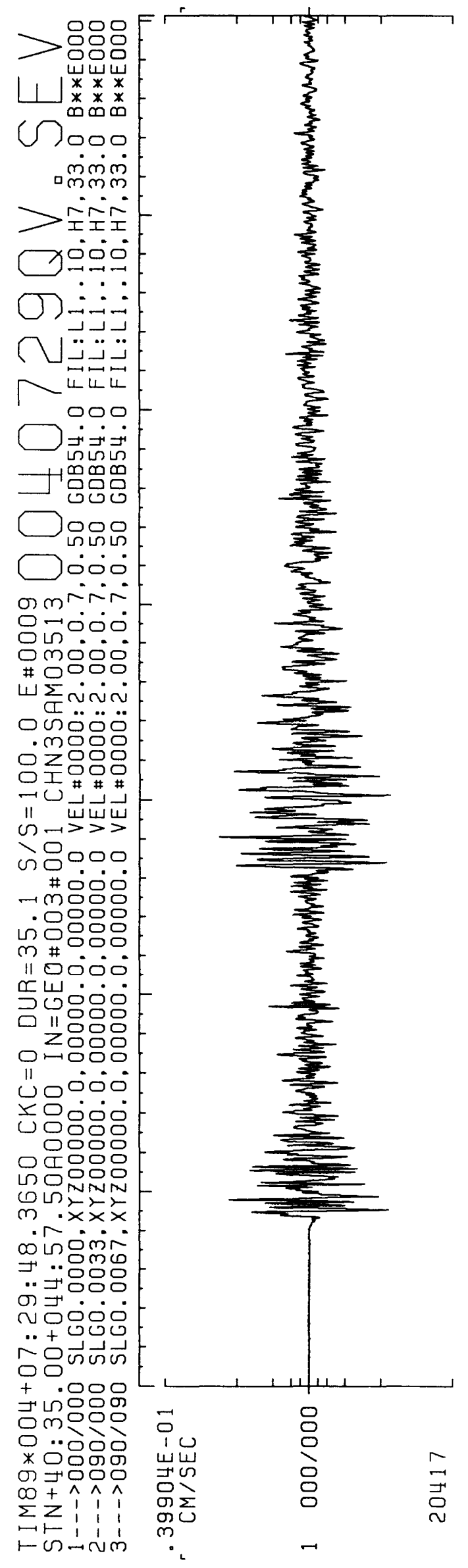

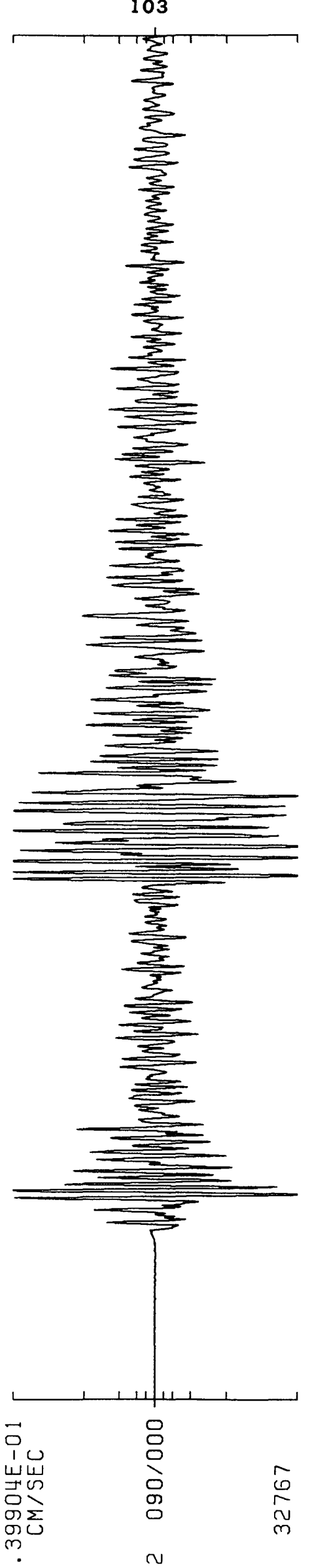

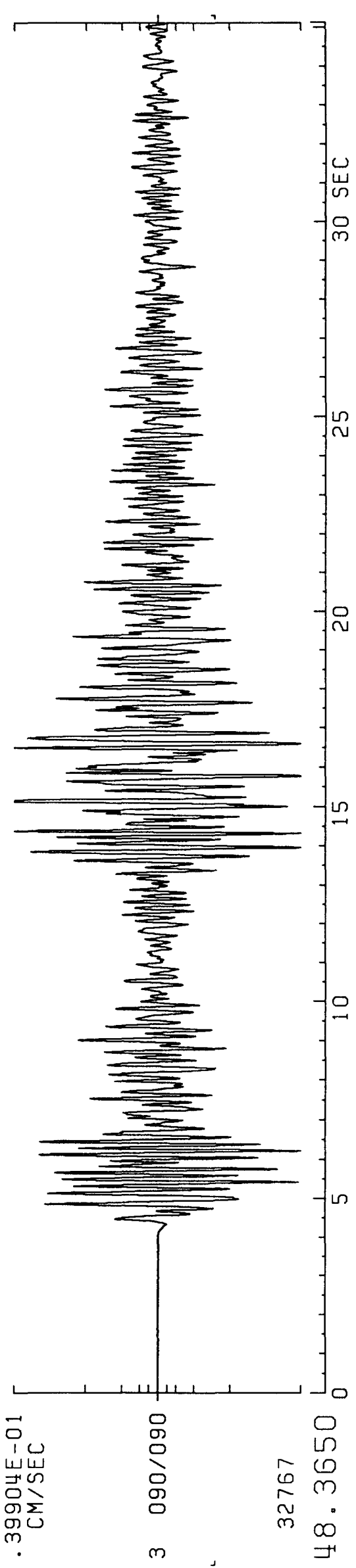


104
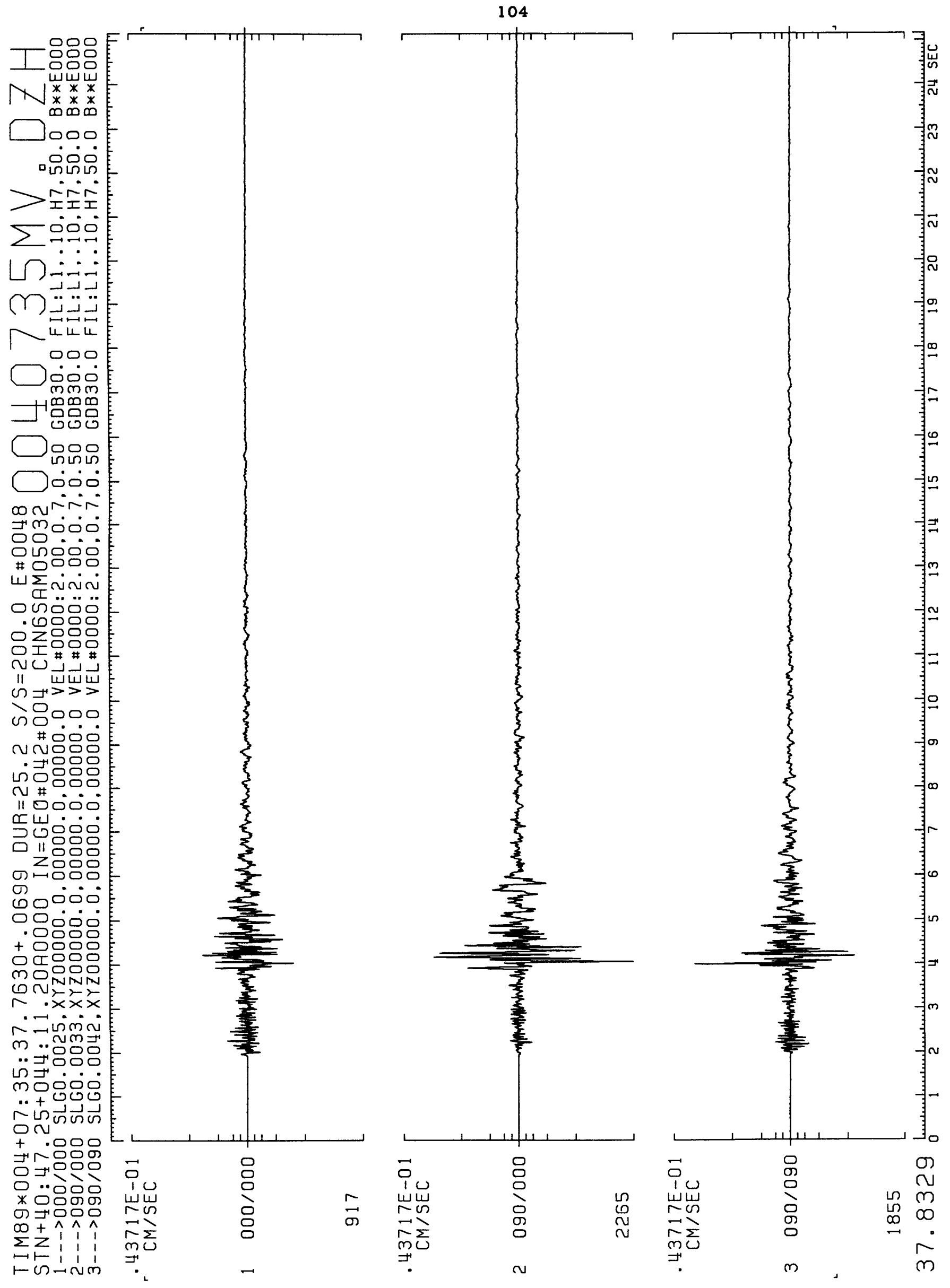

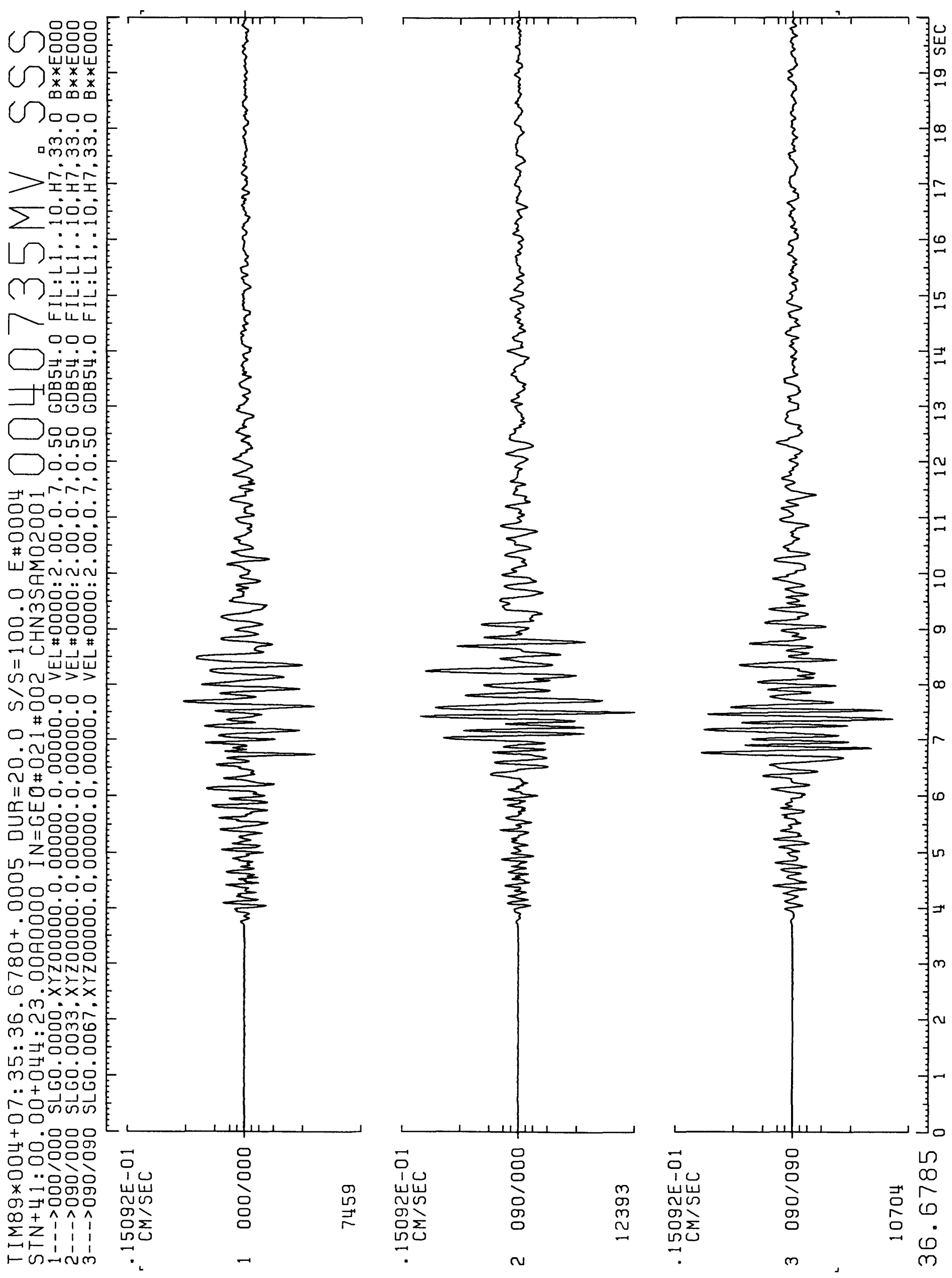

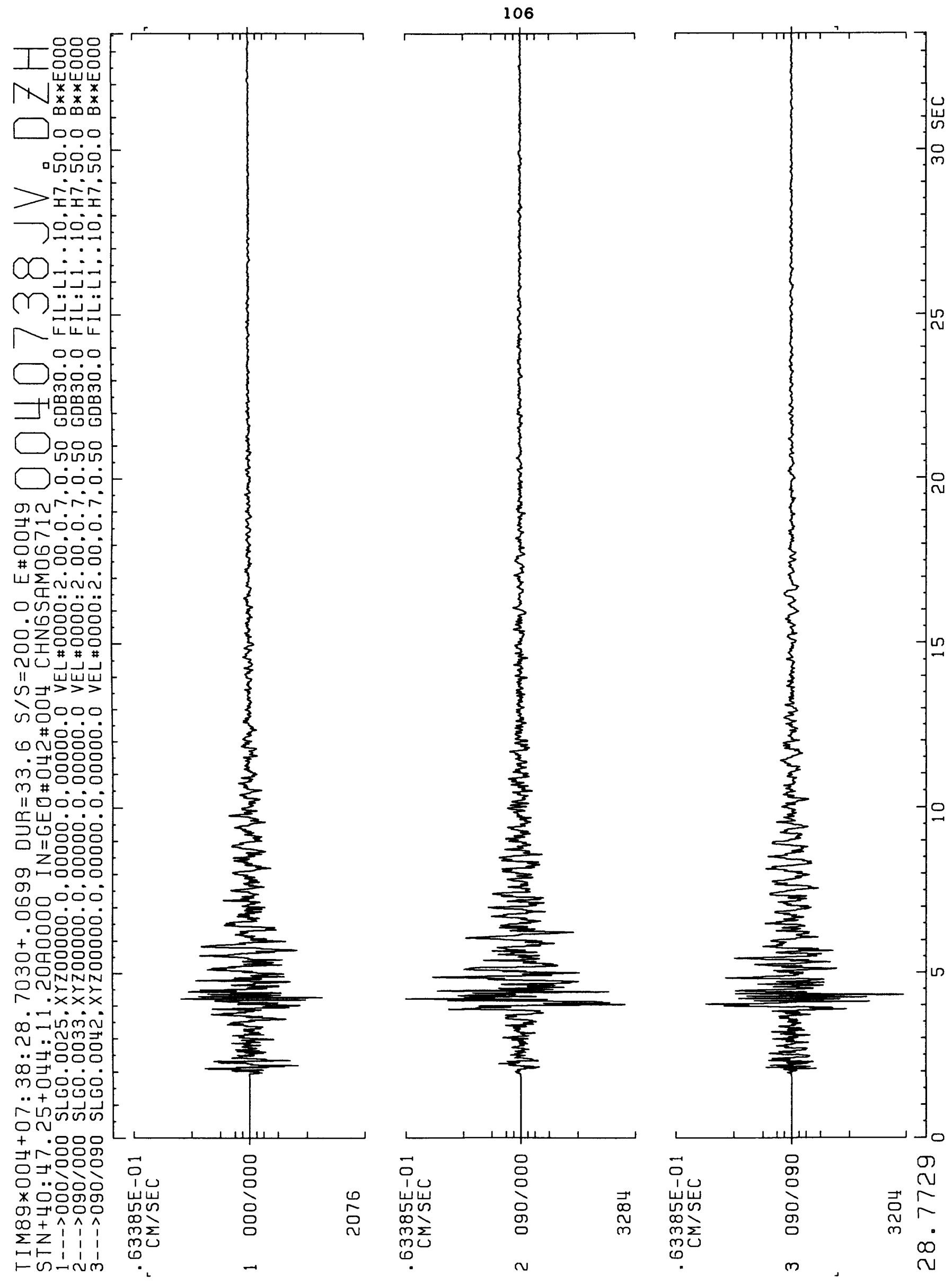


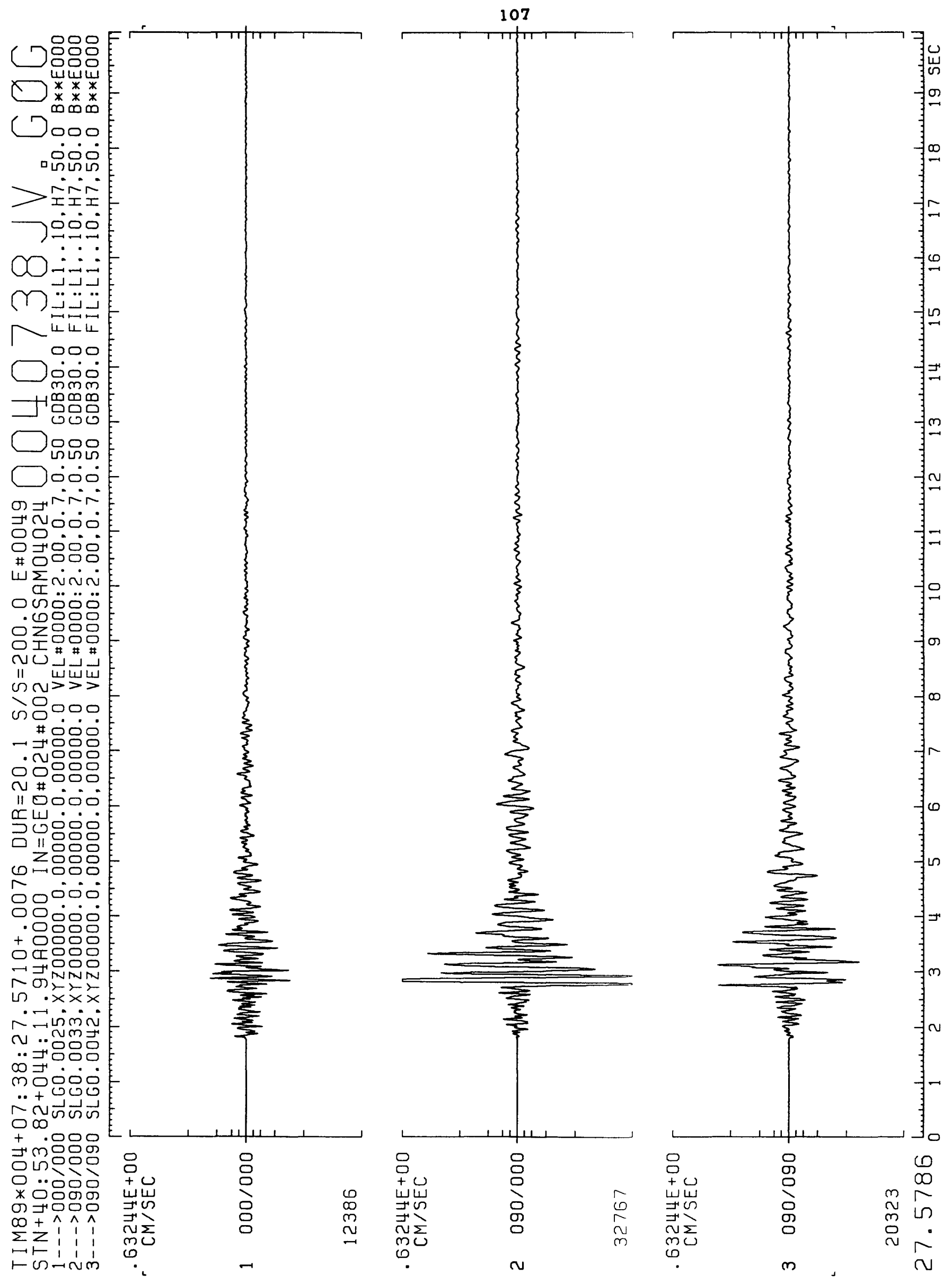


108
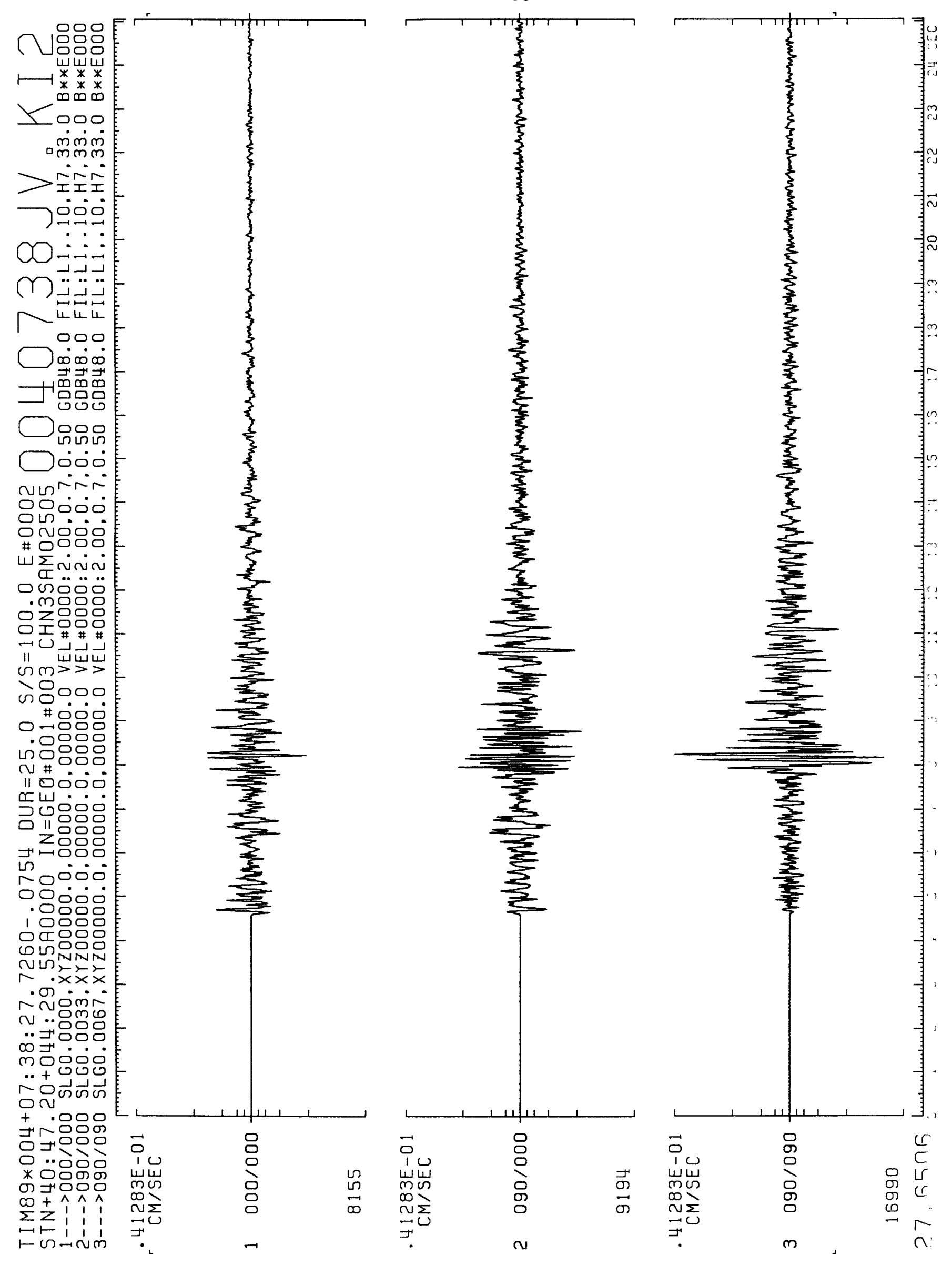

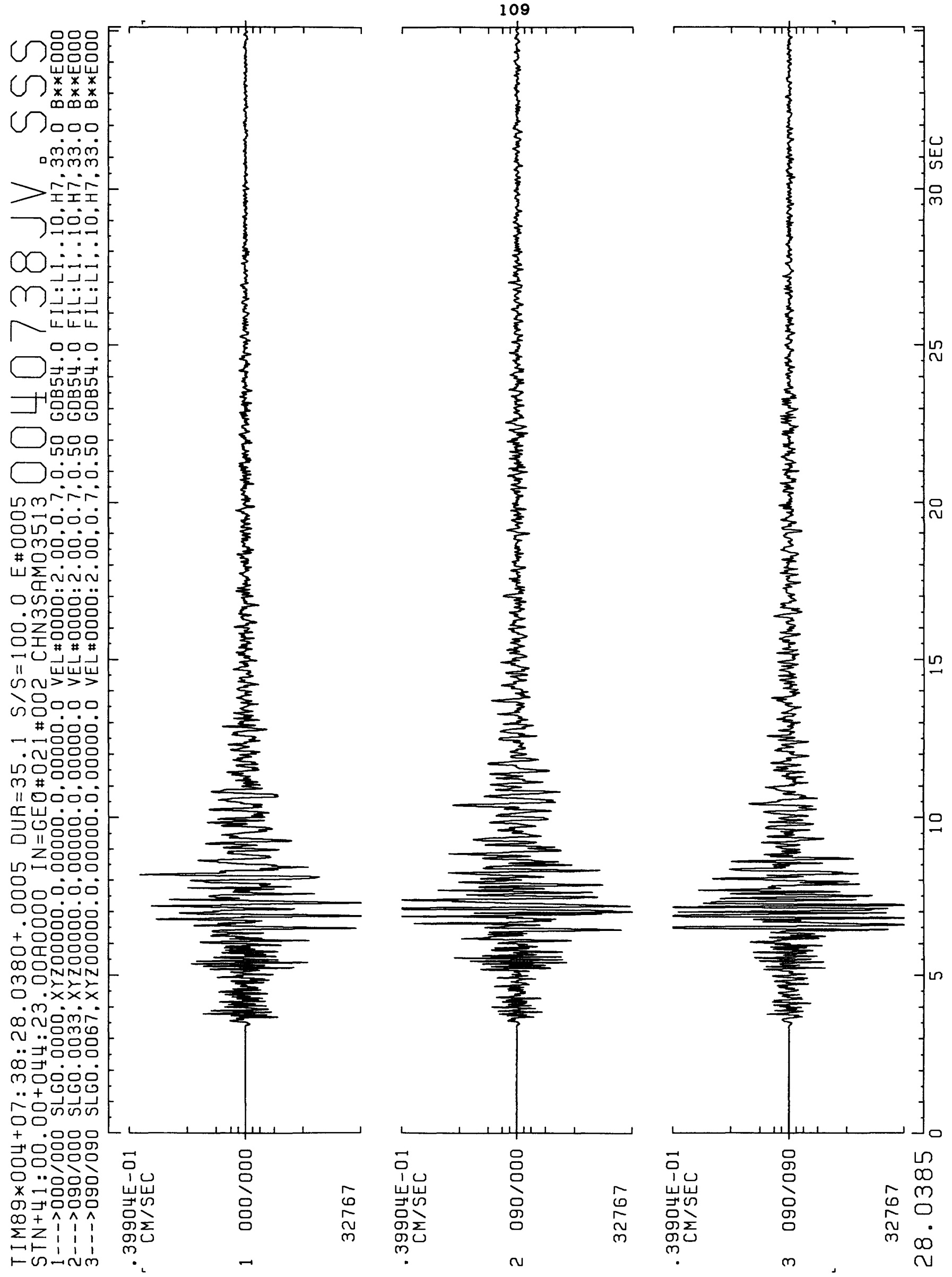
110

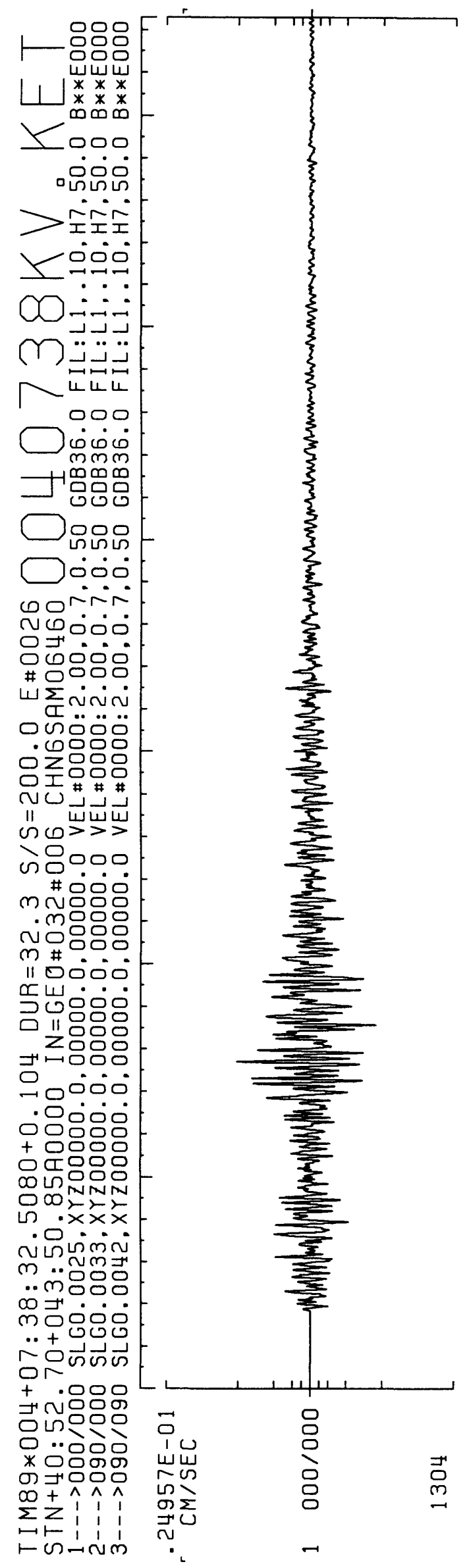

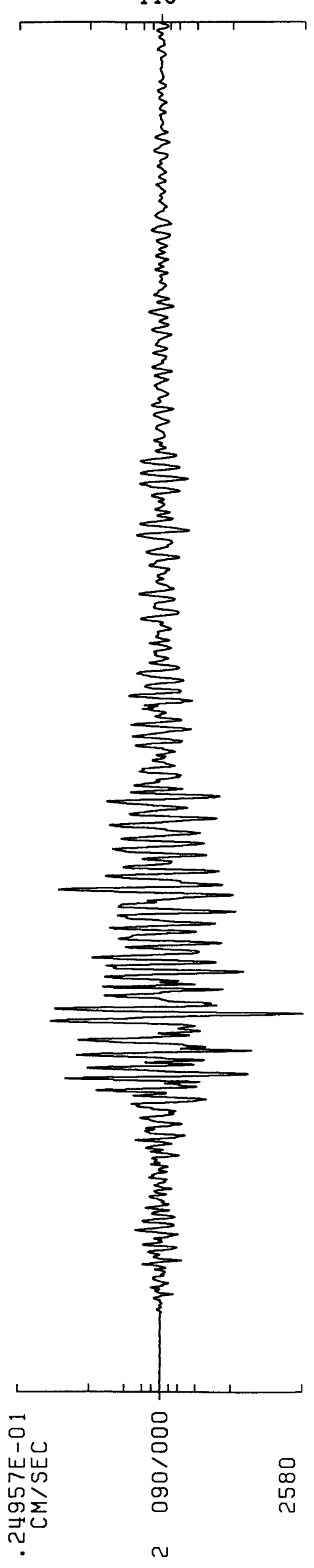

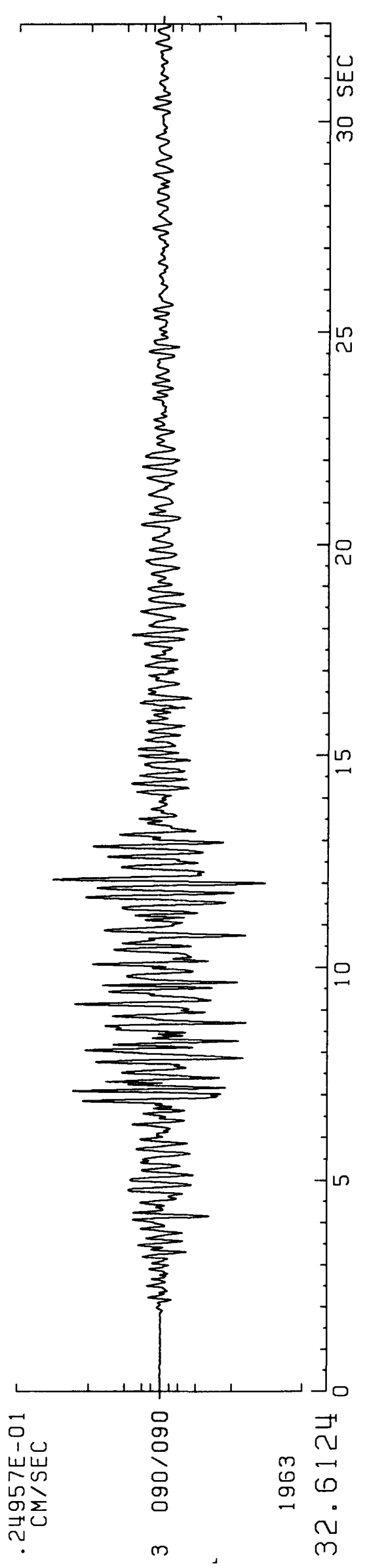


111
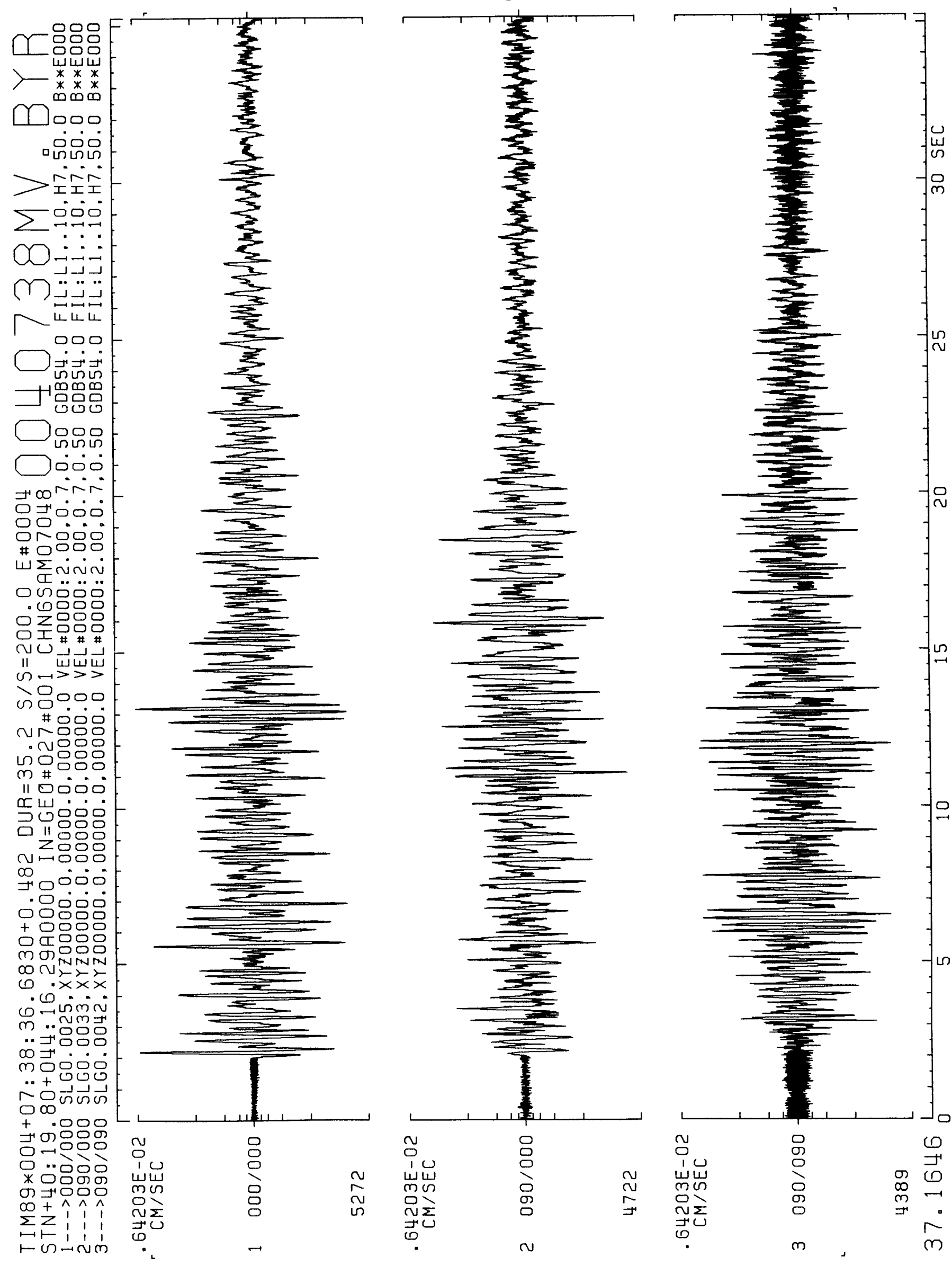
112

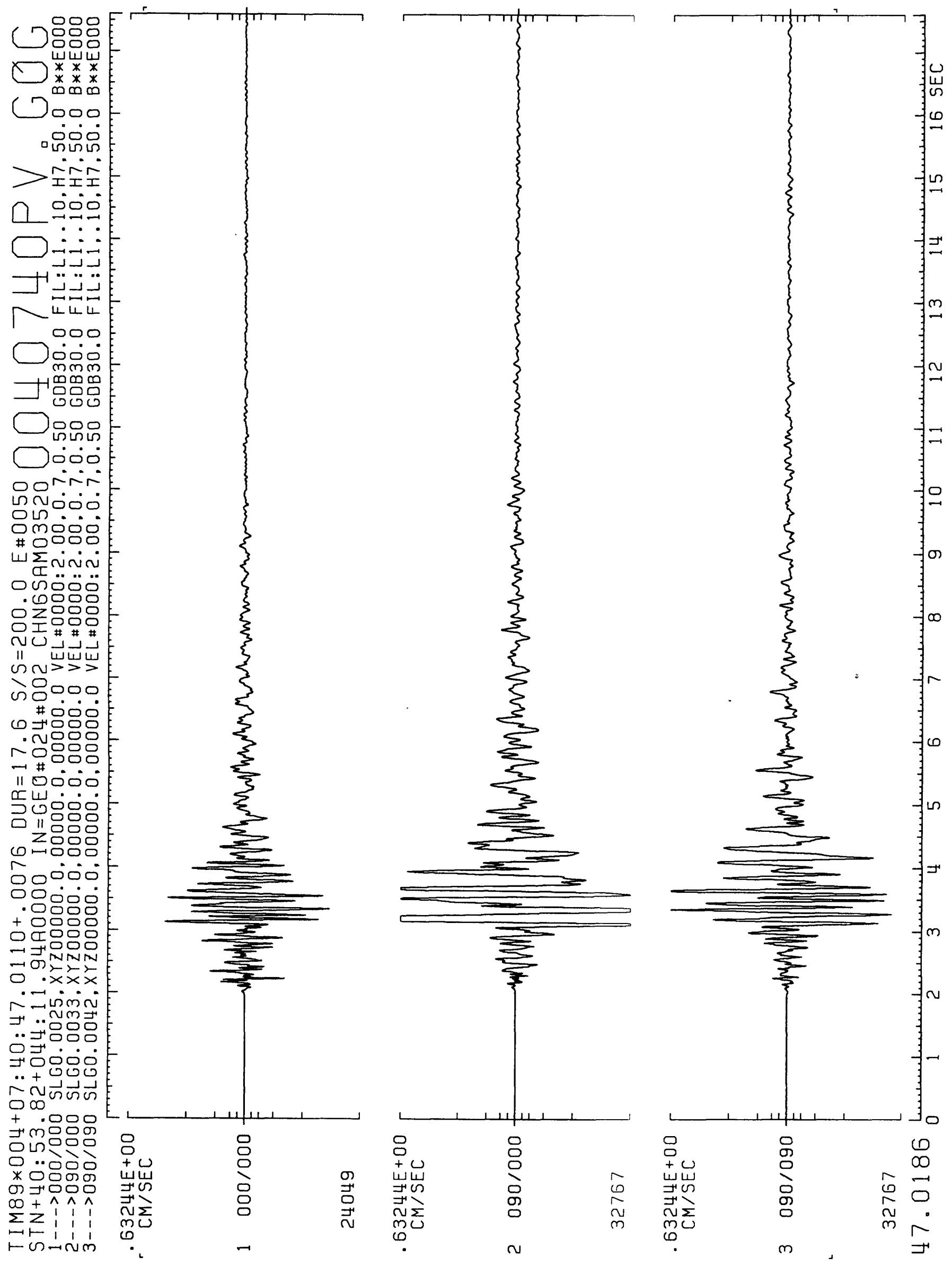



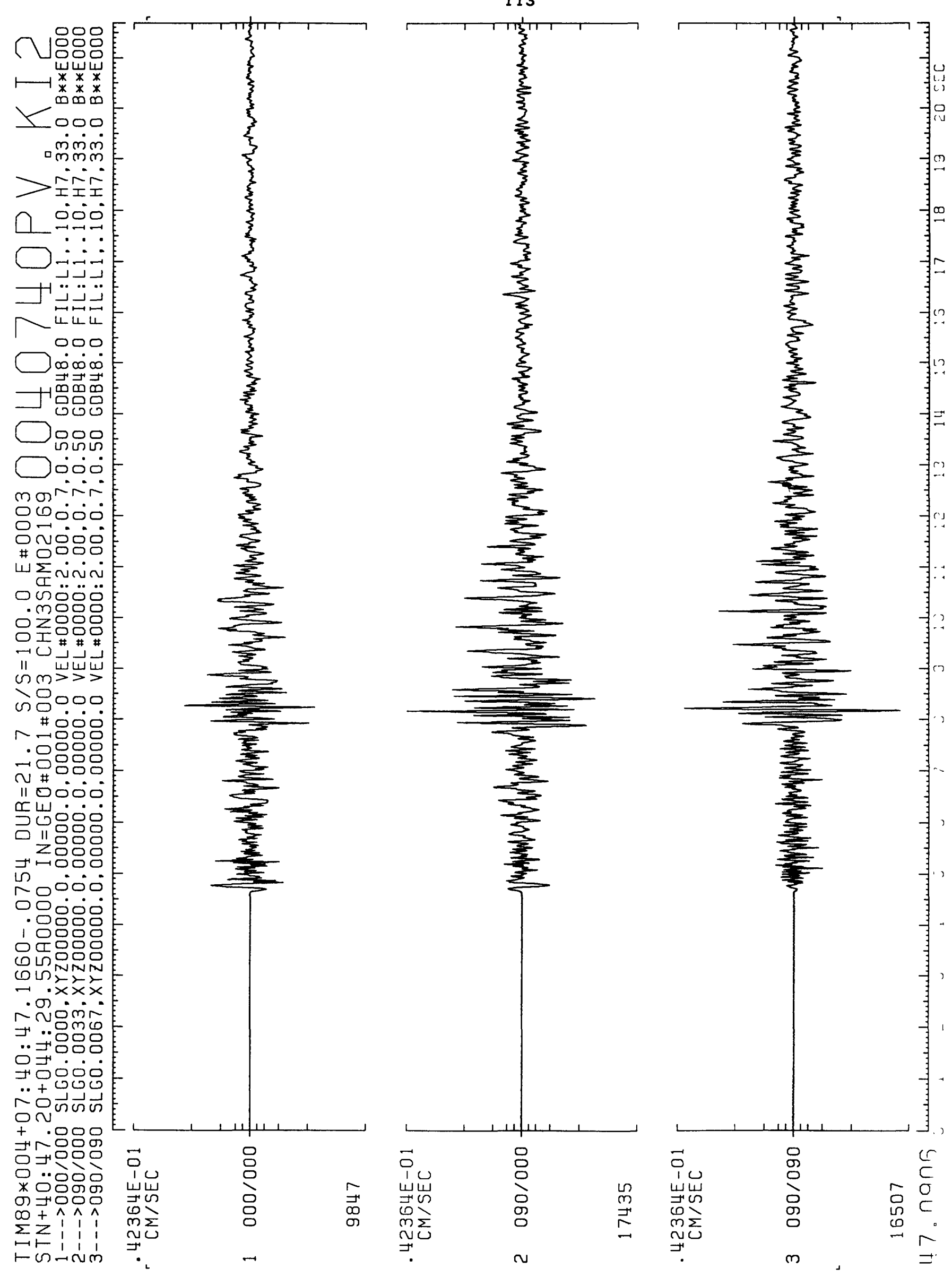
114
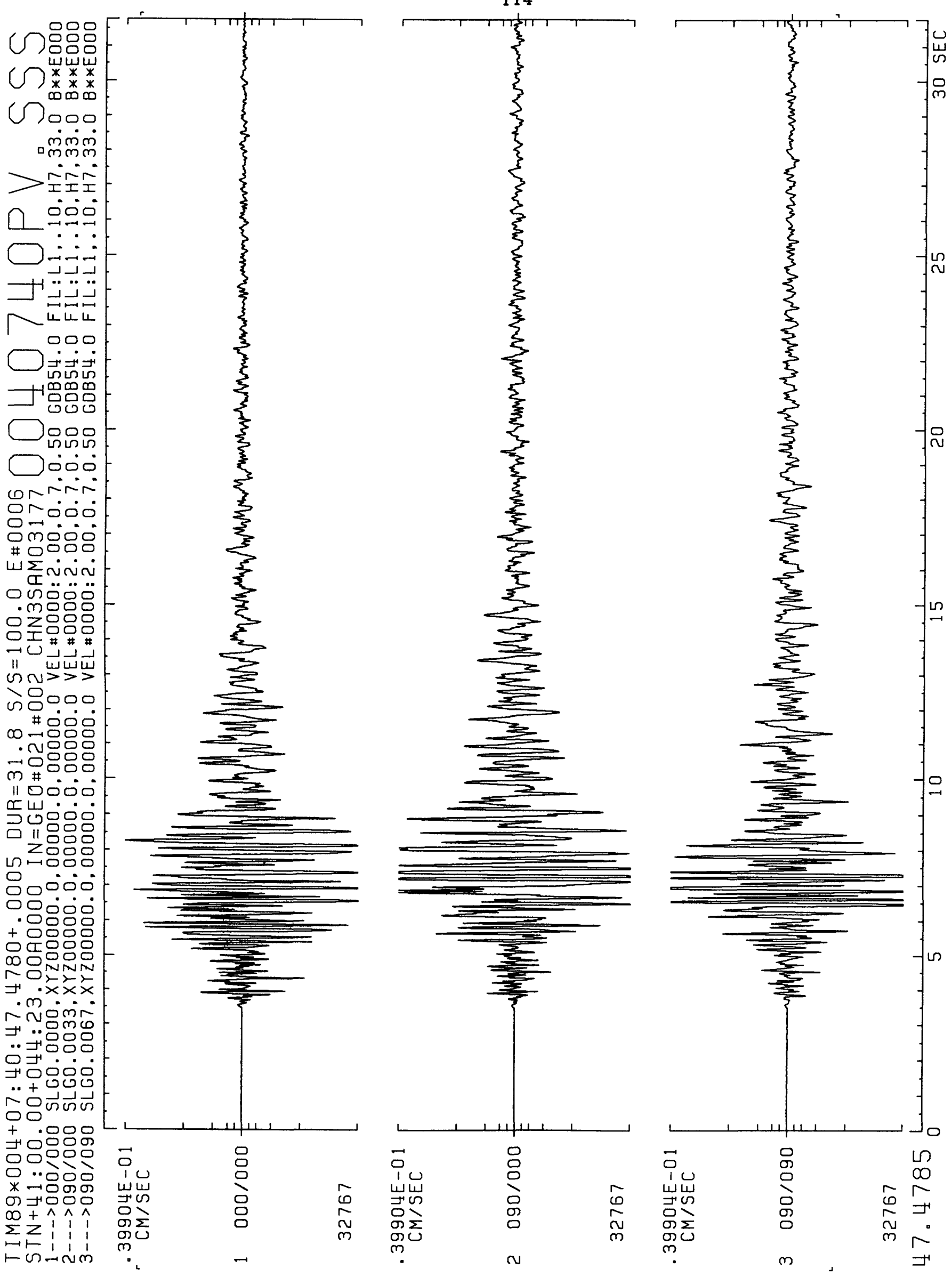

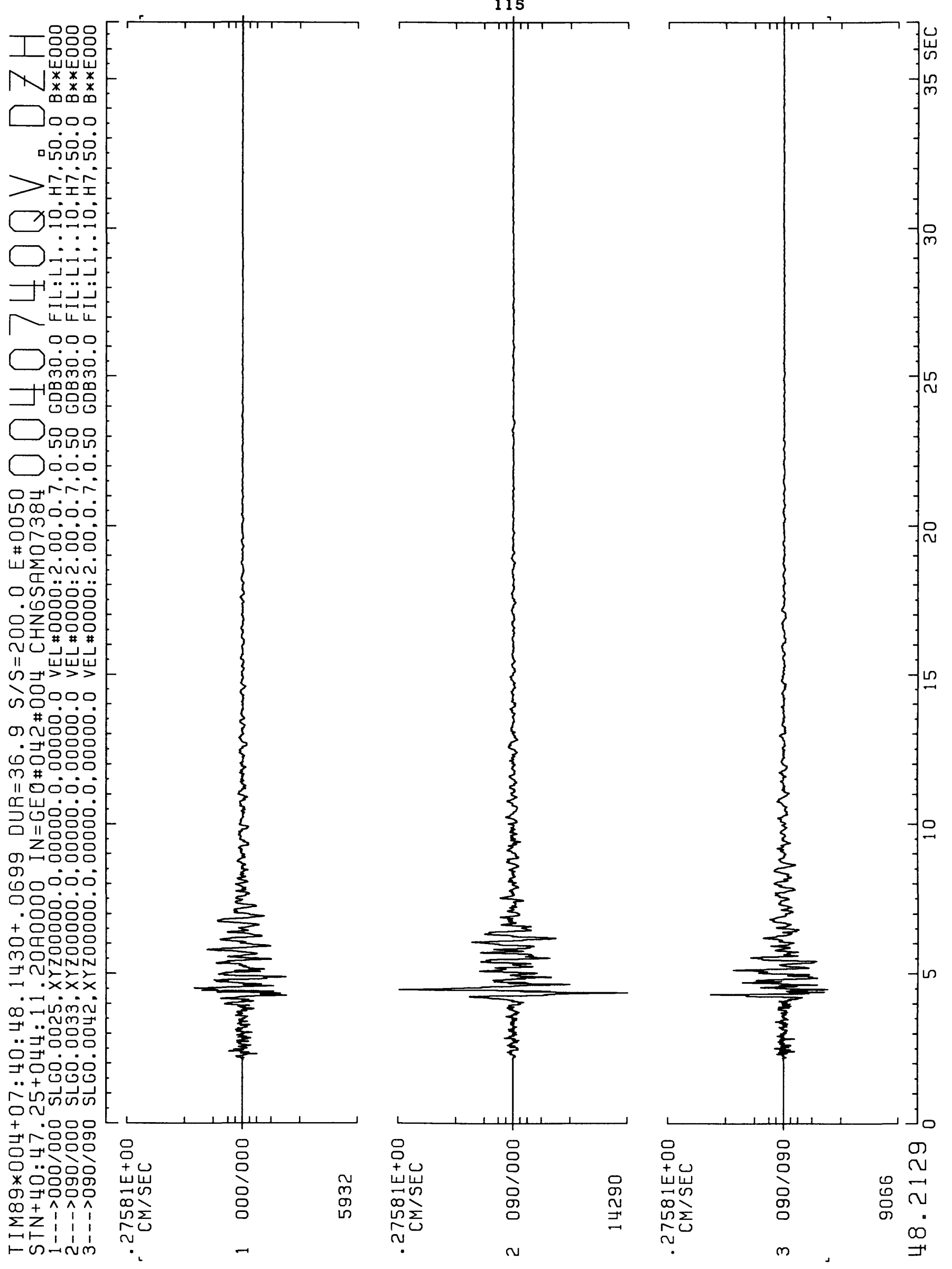
116

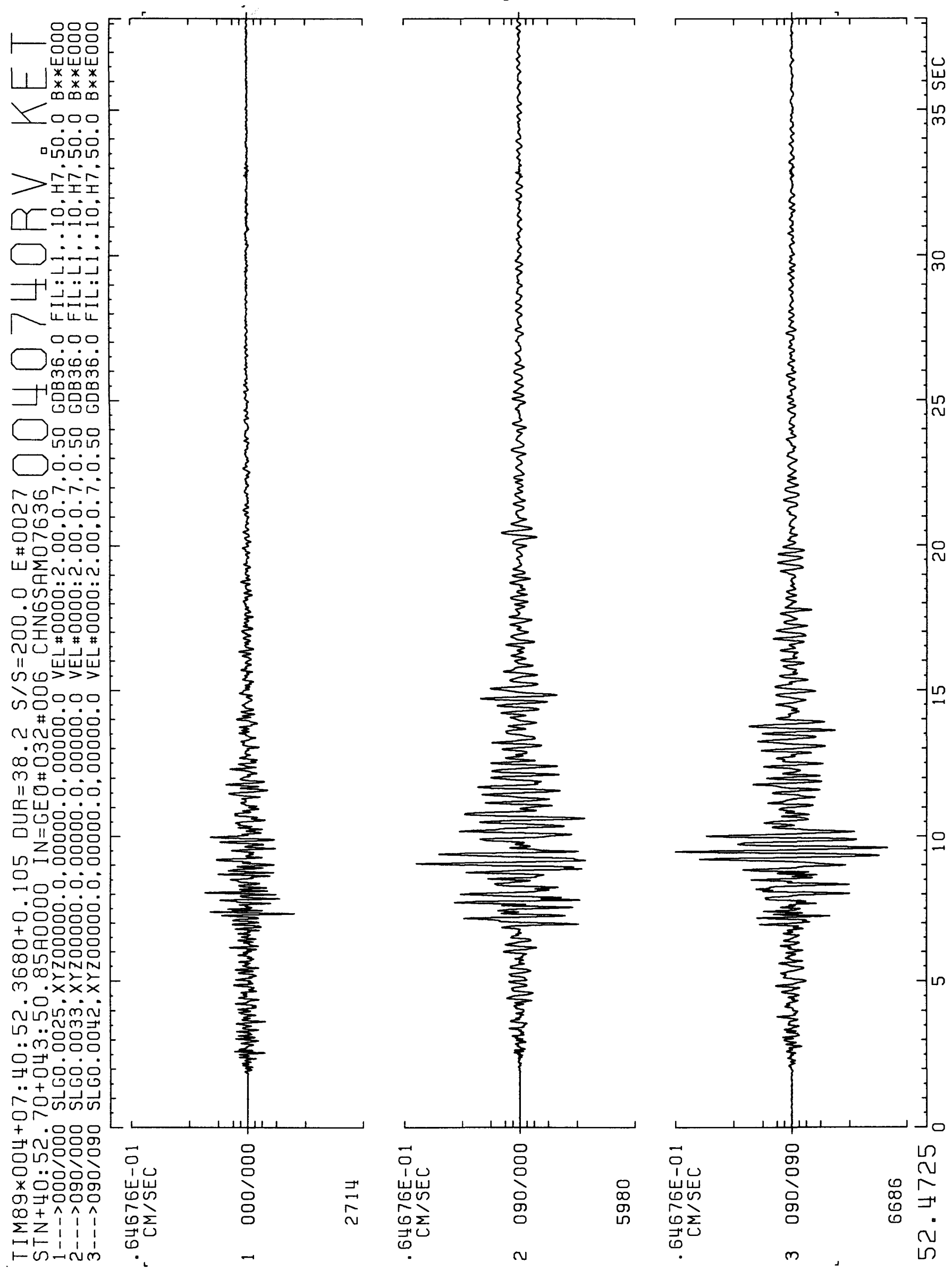


117
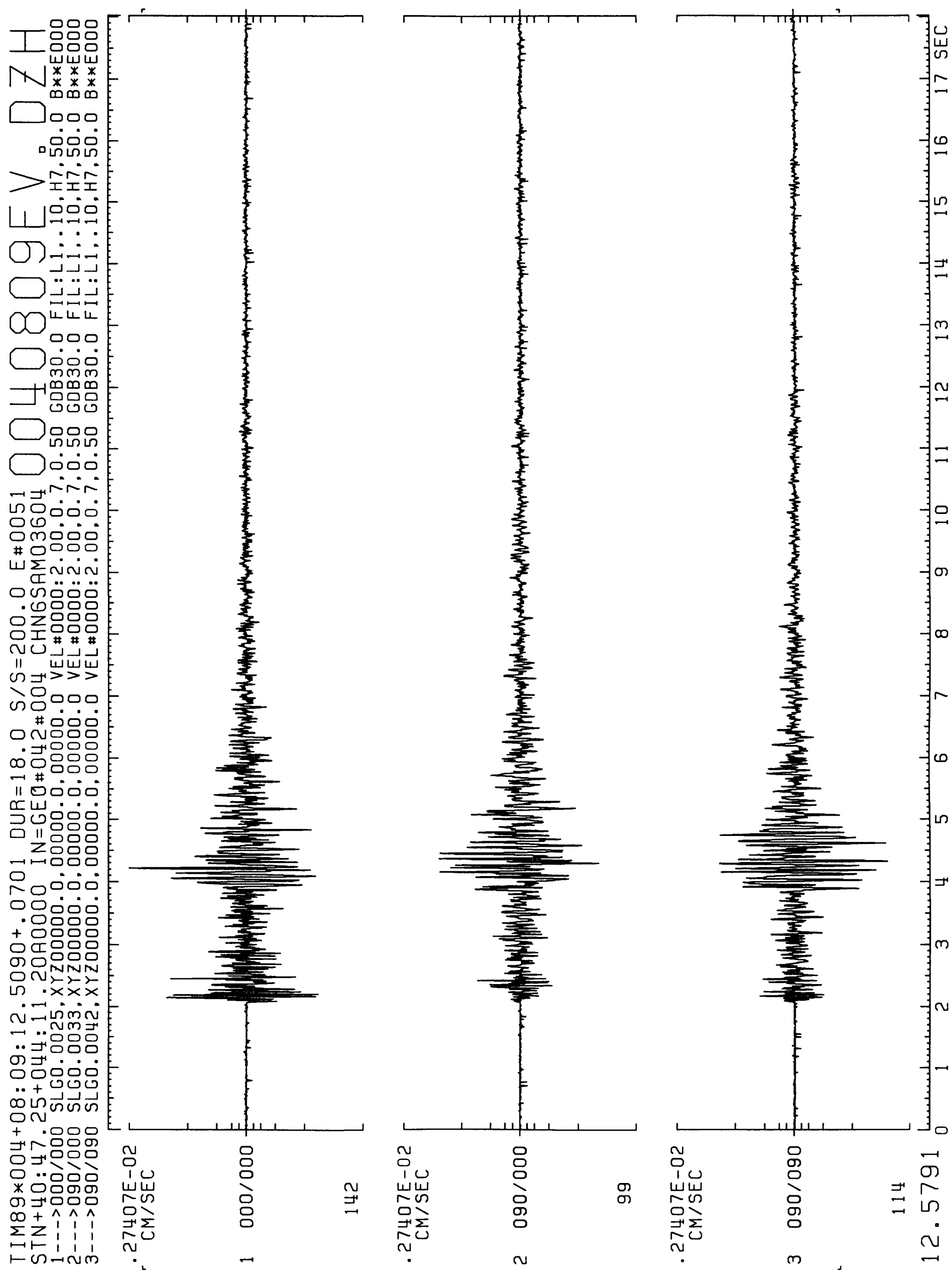
118

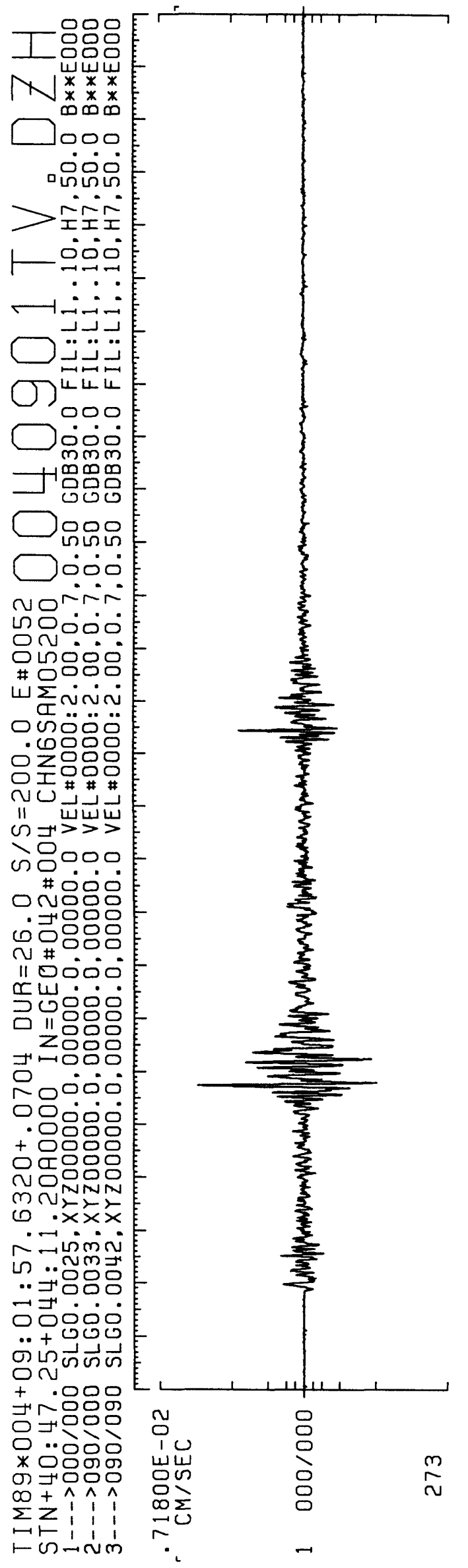

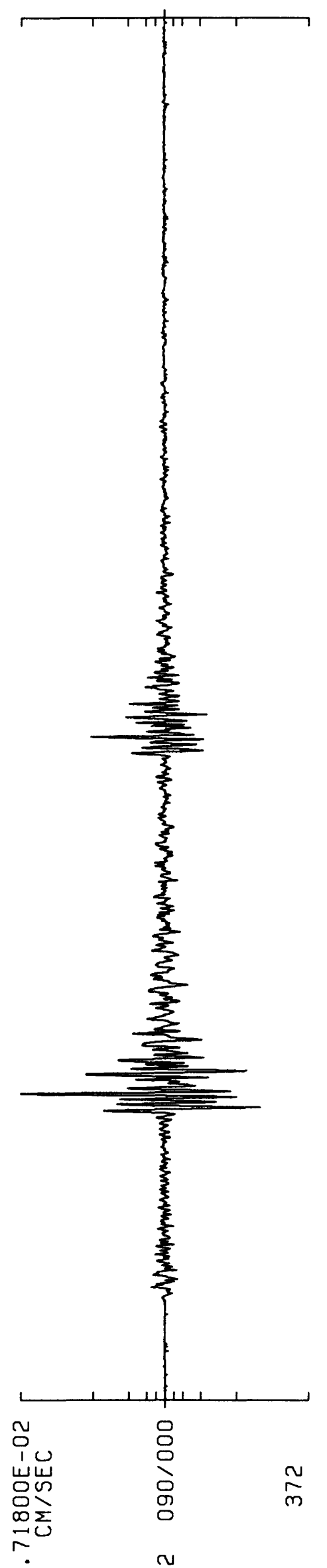

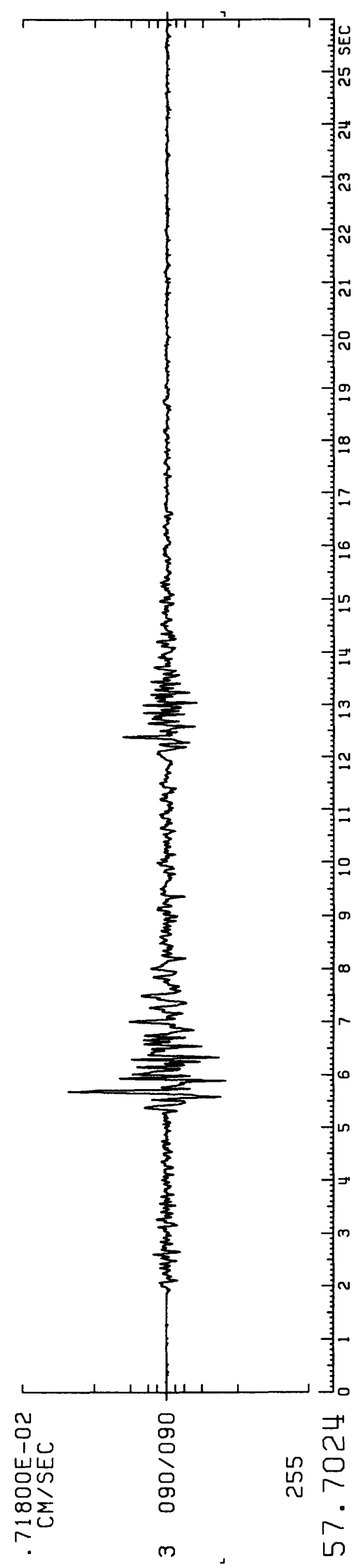


119

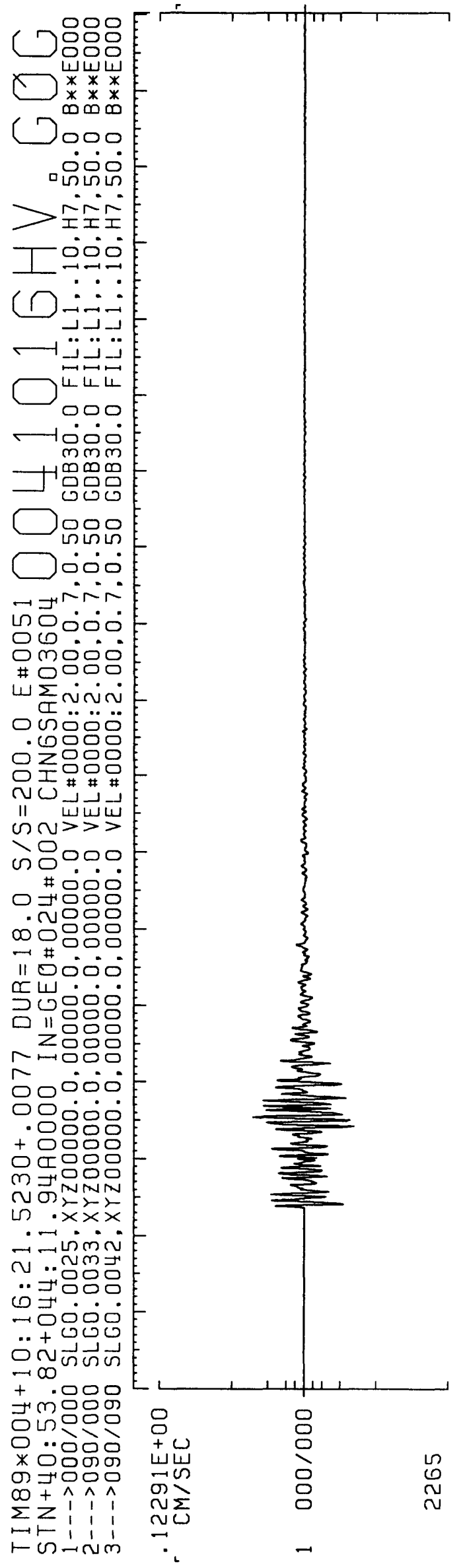

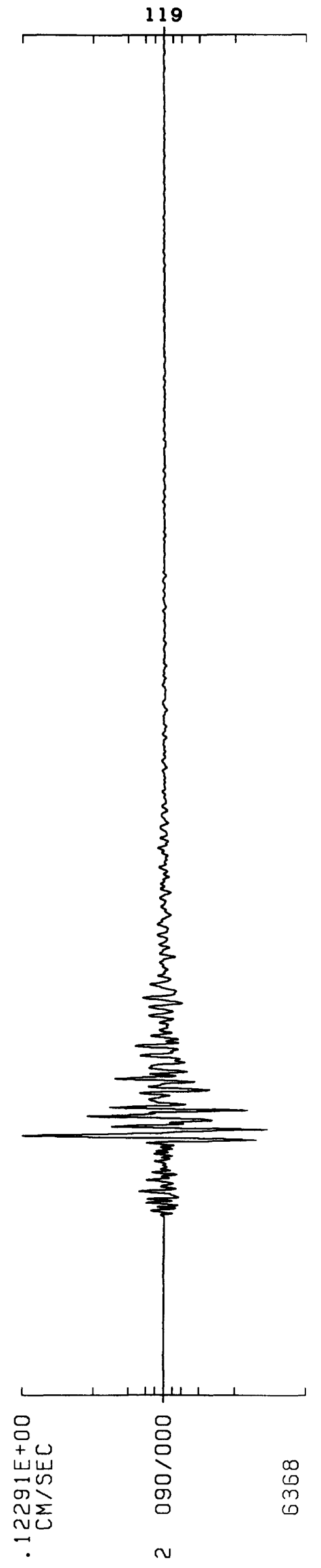

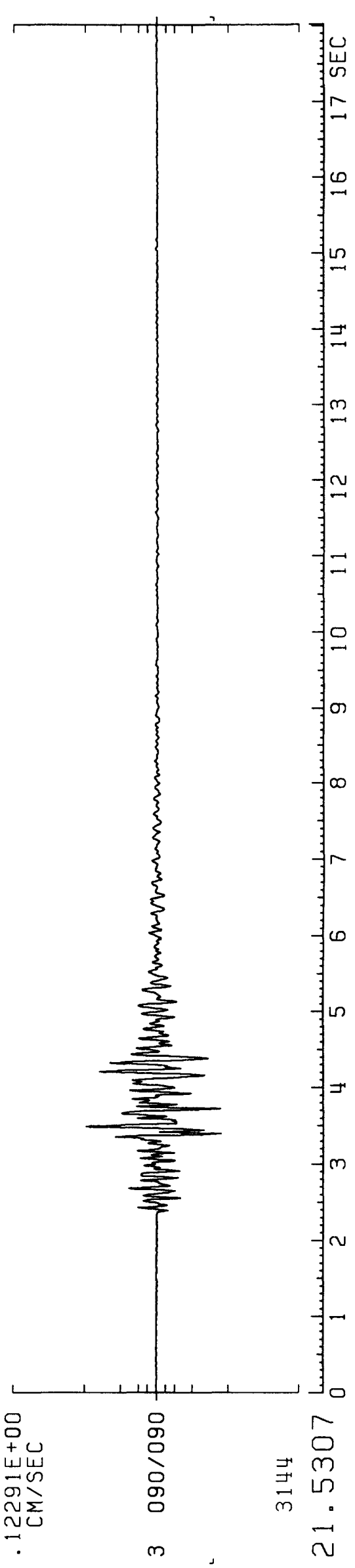




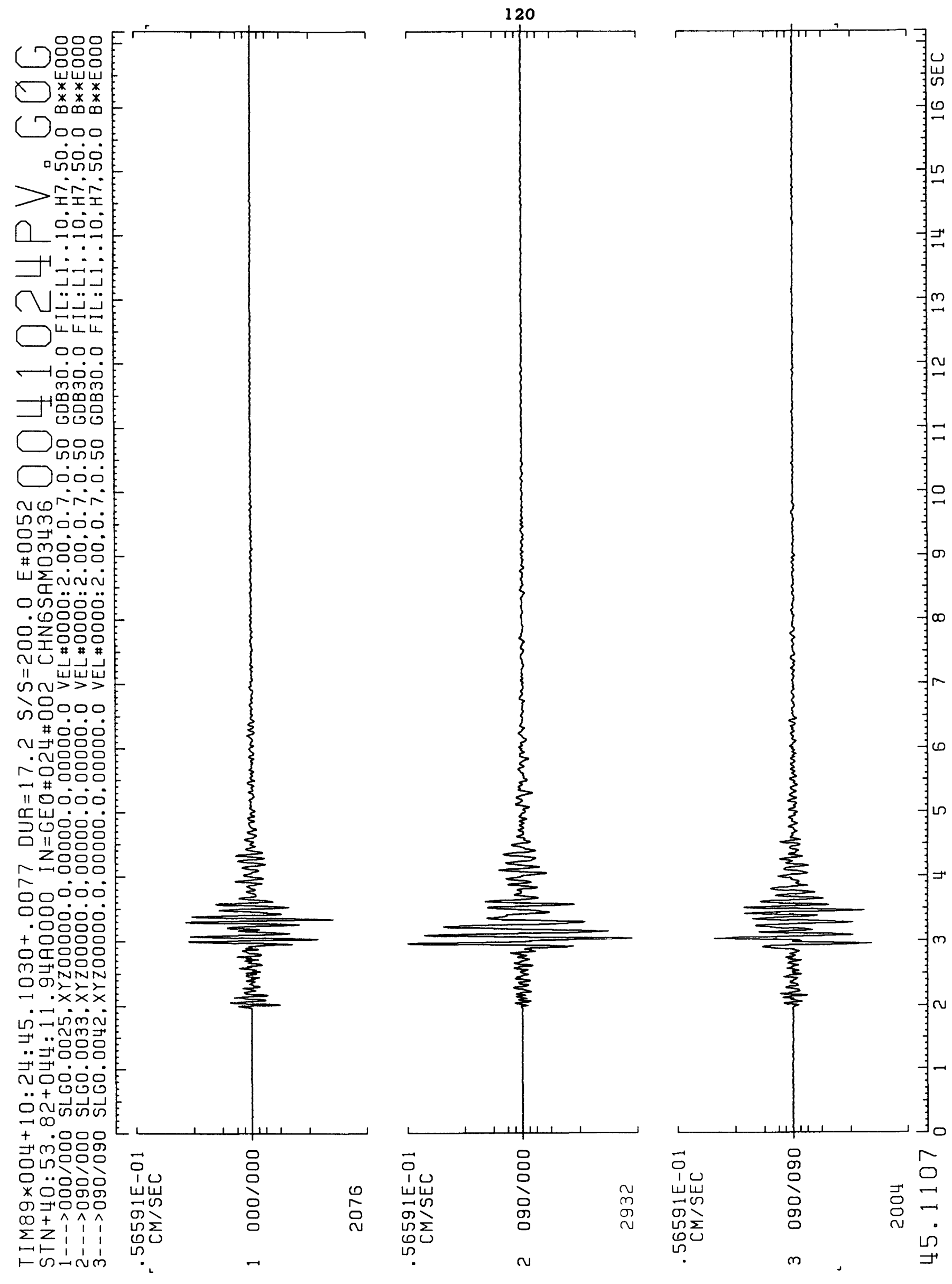


121
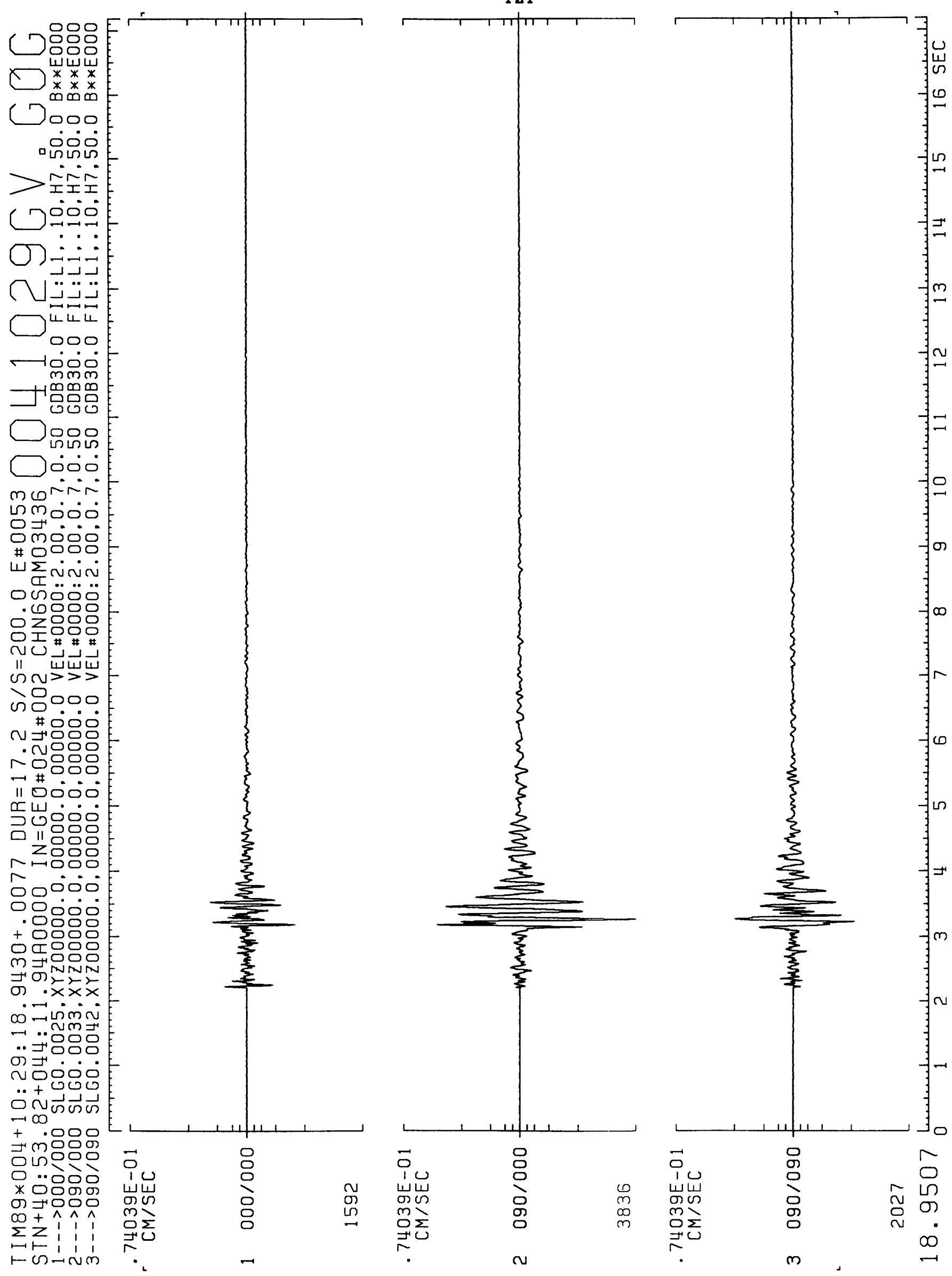


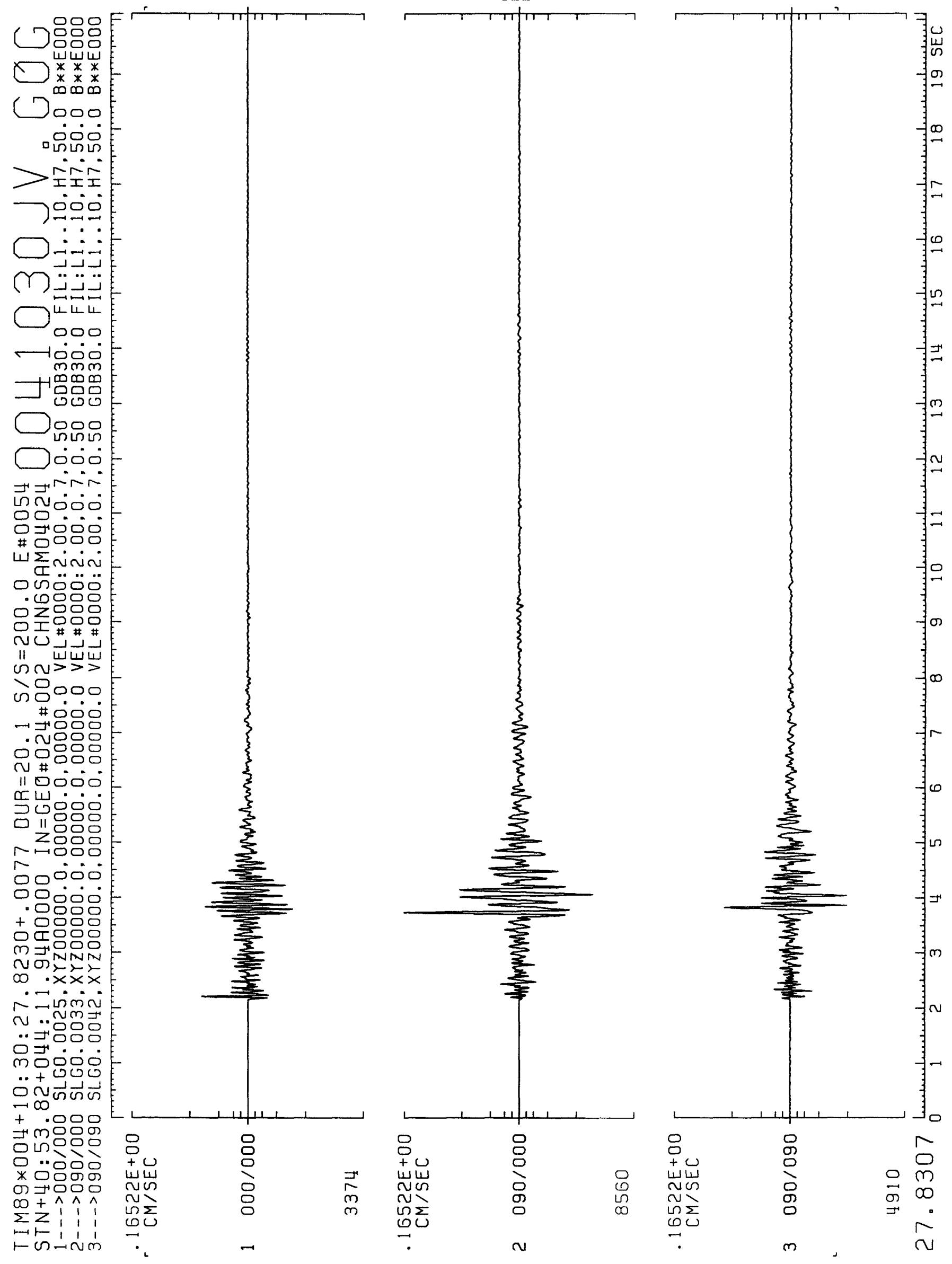



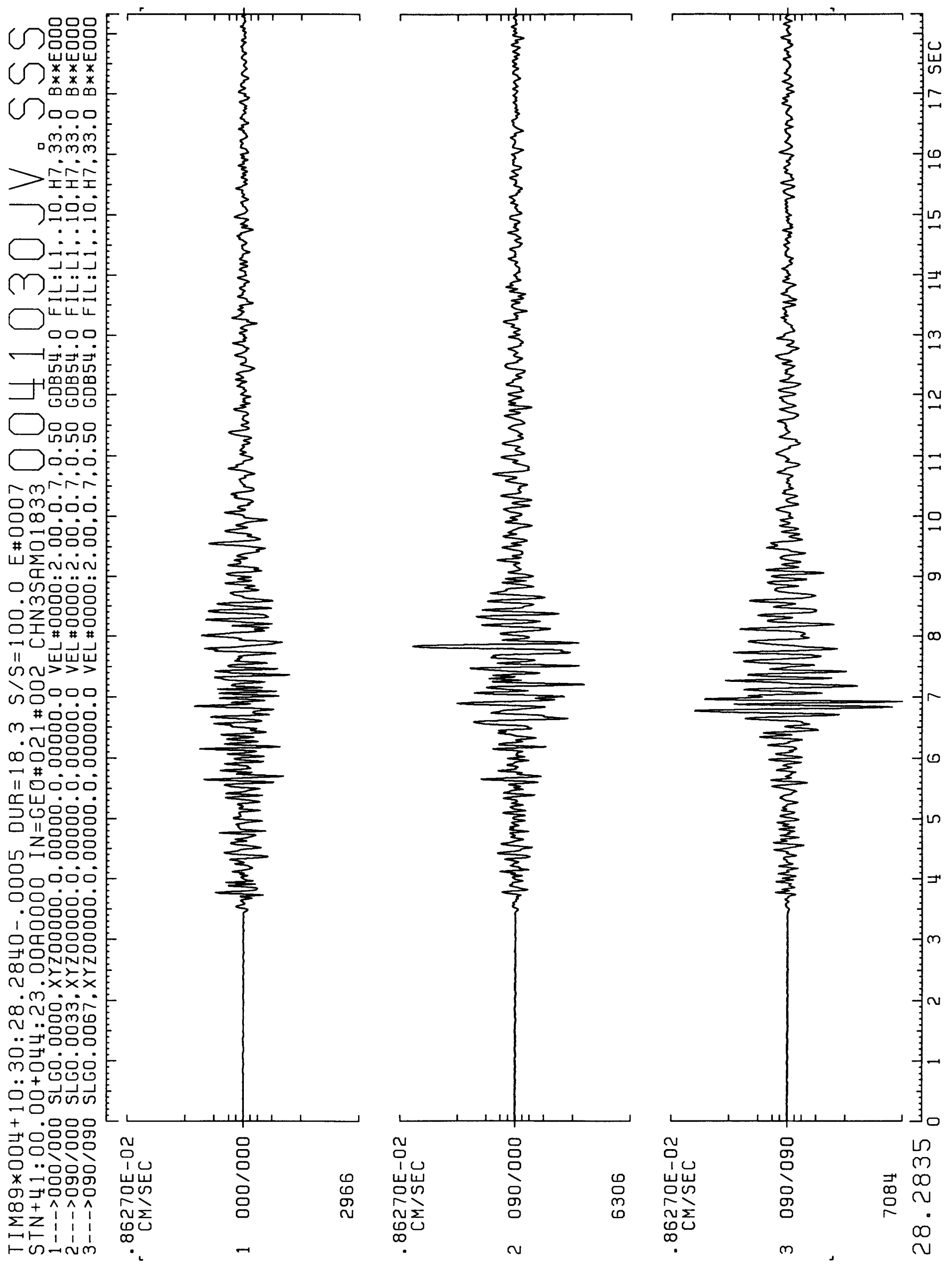


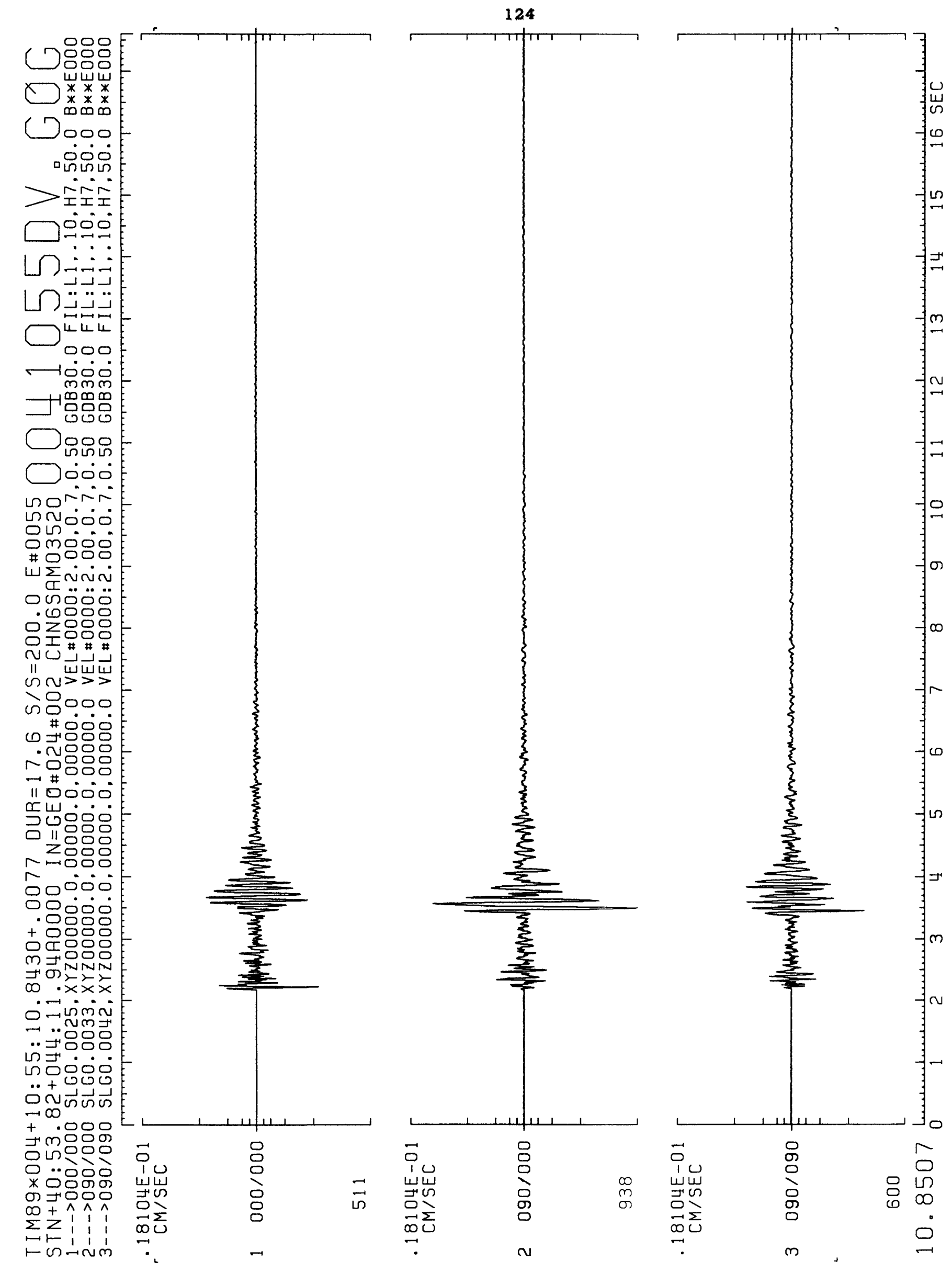




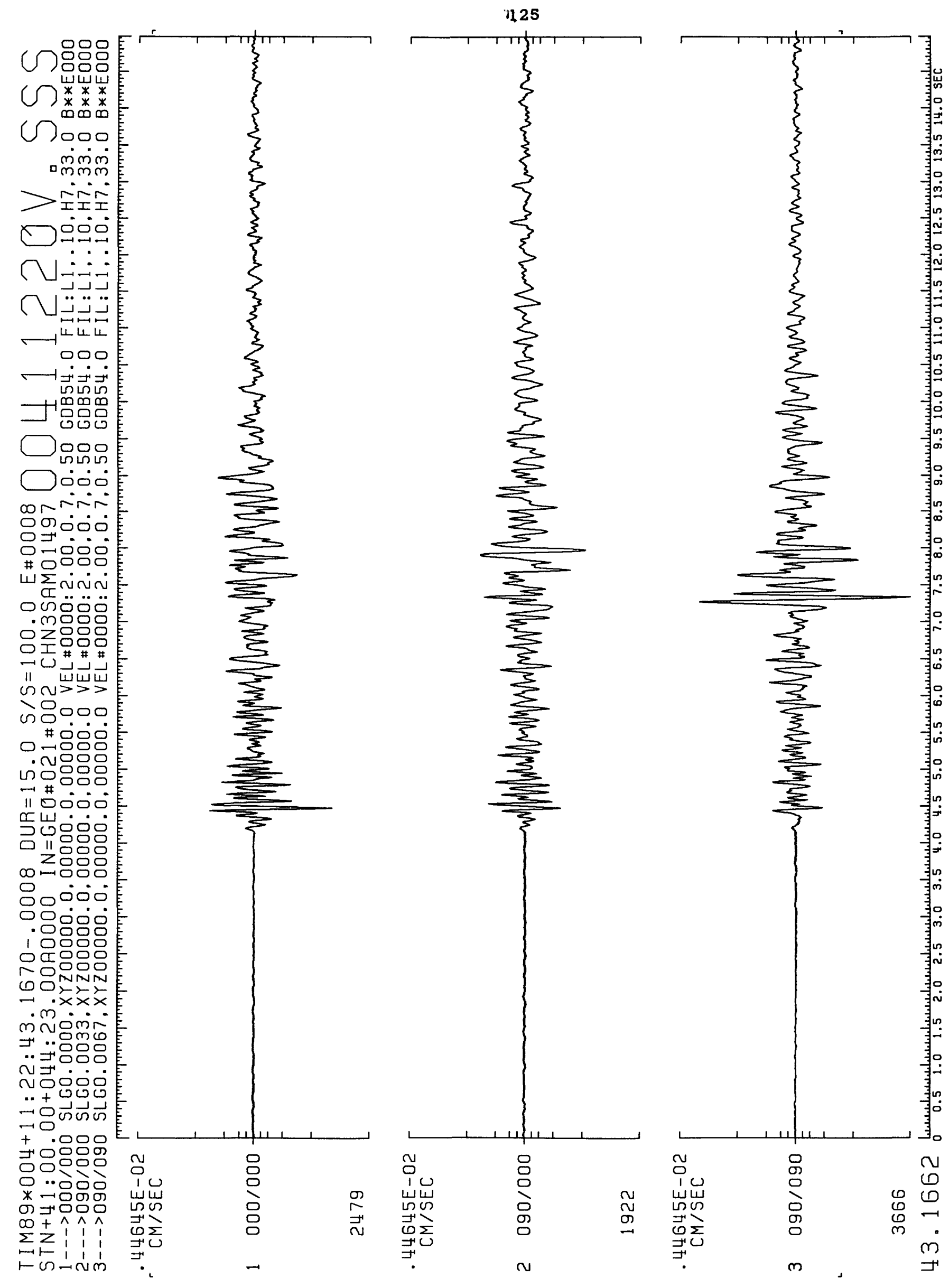




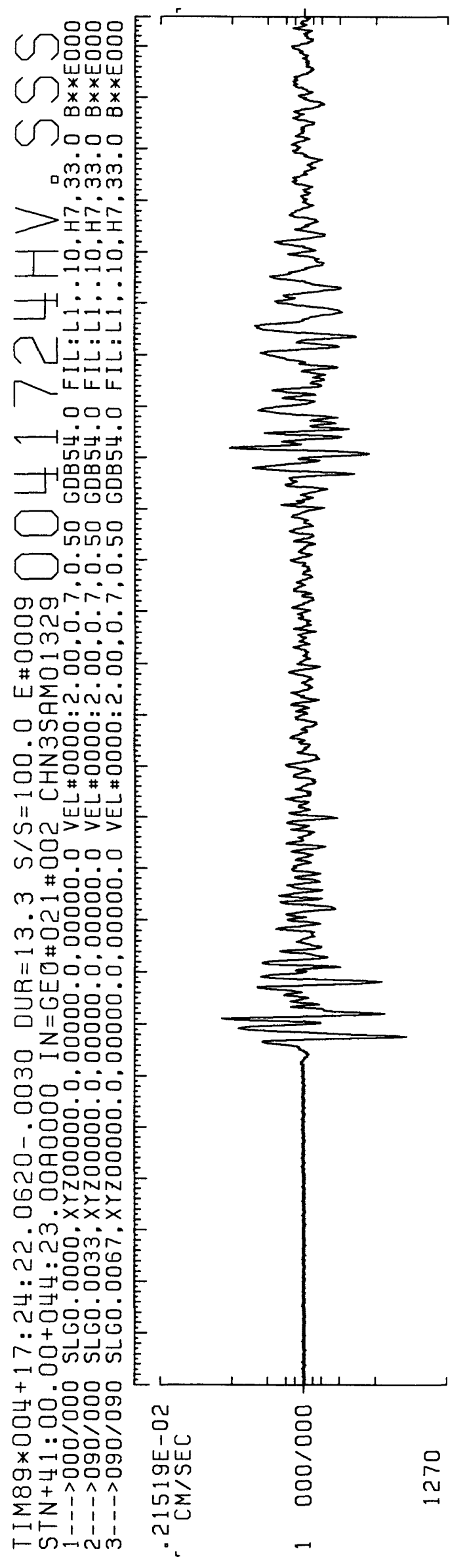

126

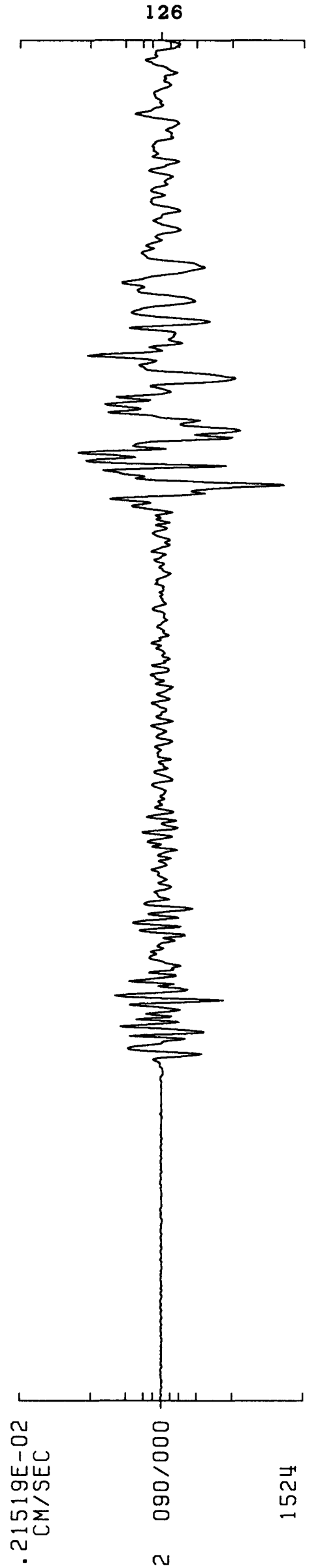

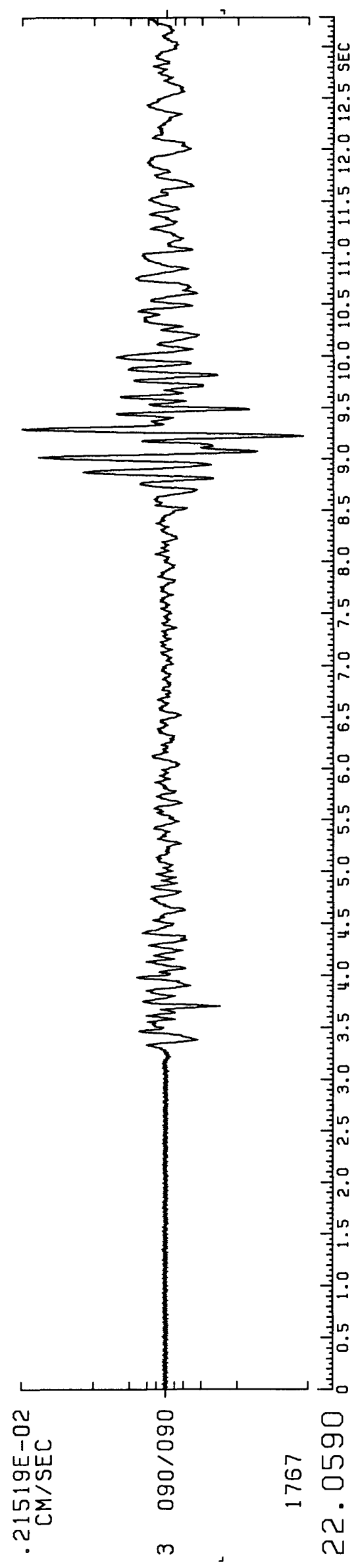


127
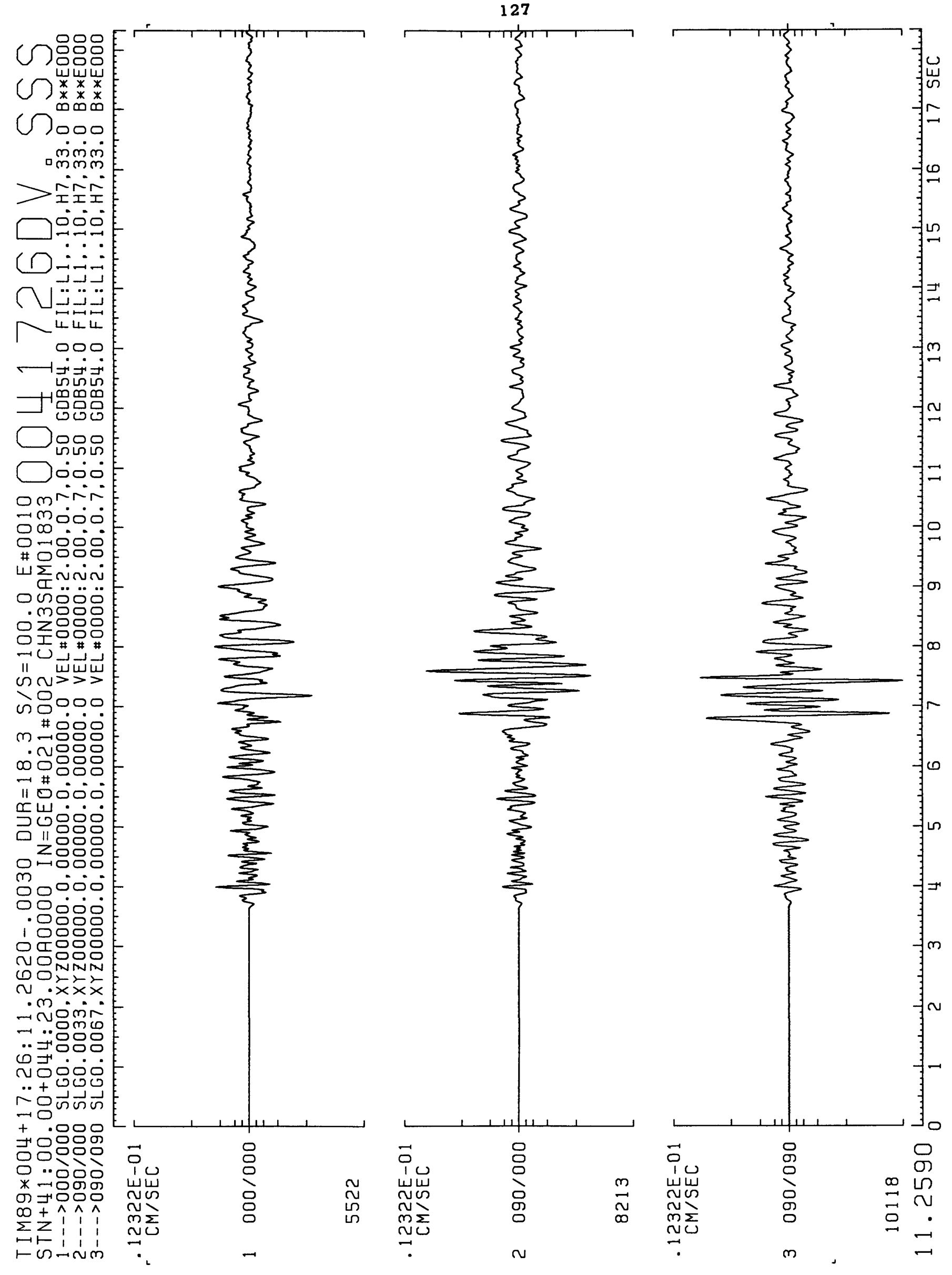
128

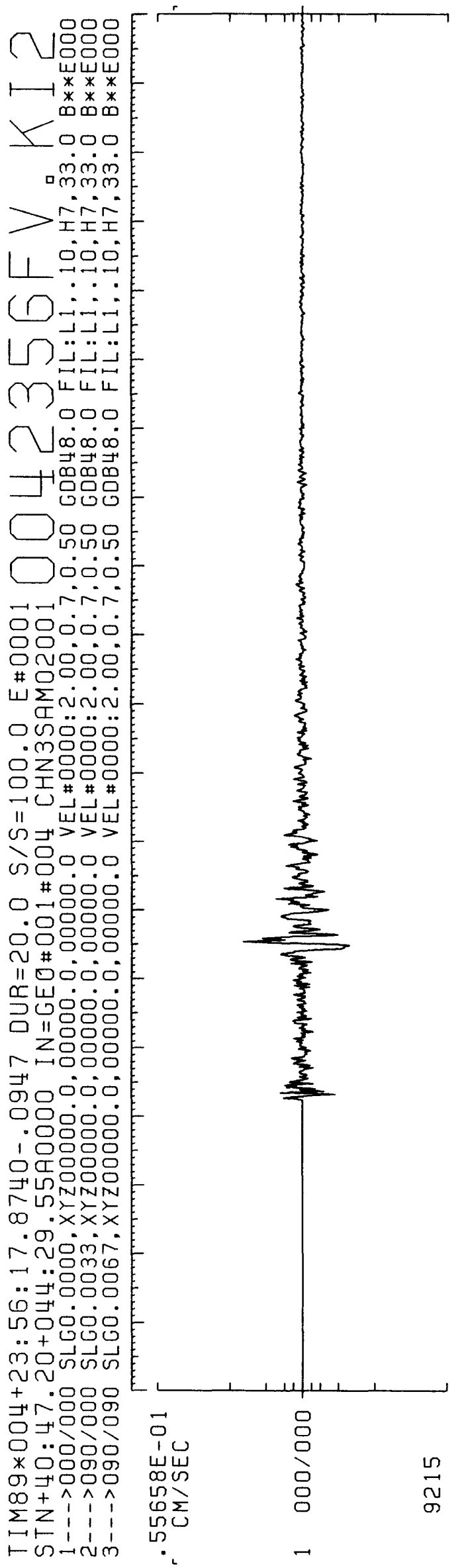

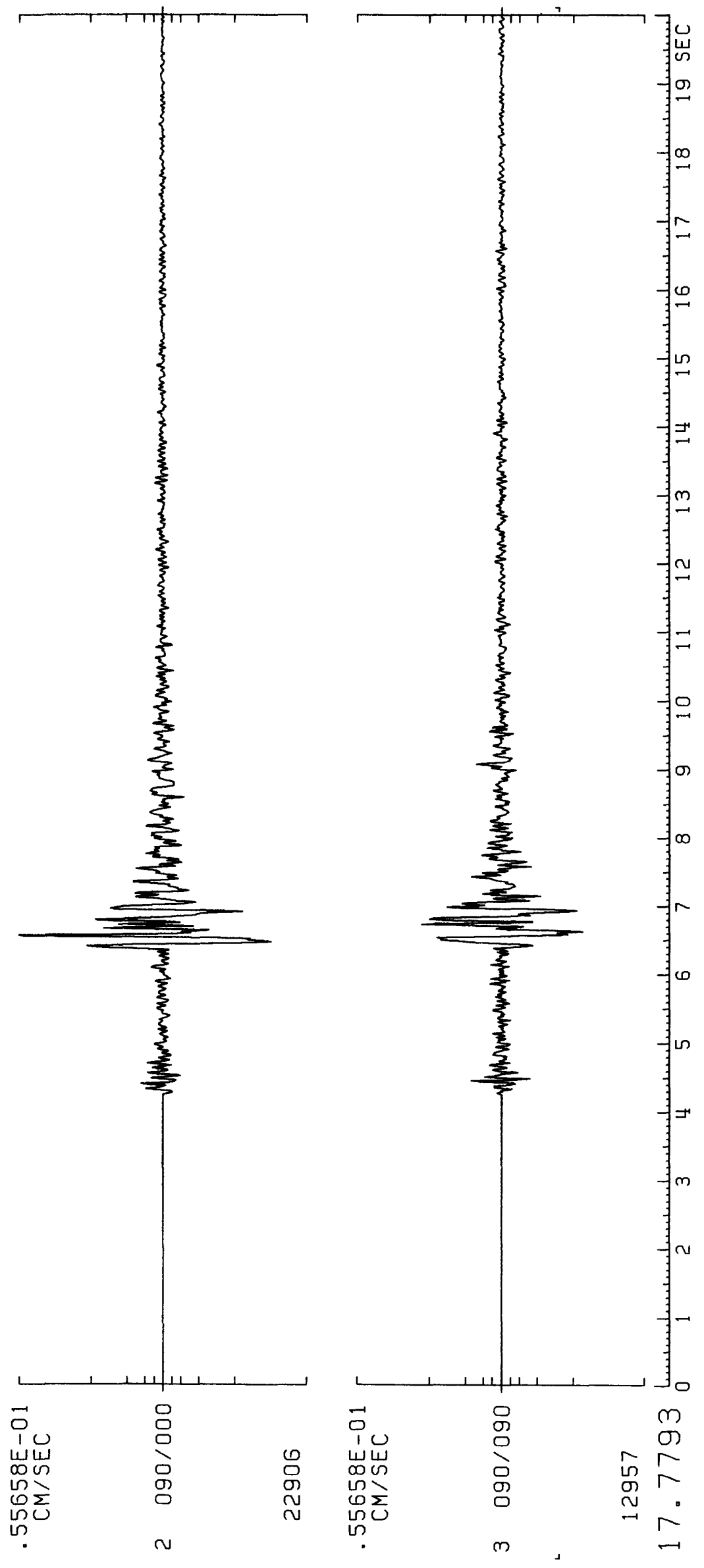




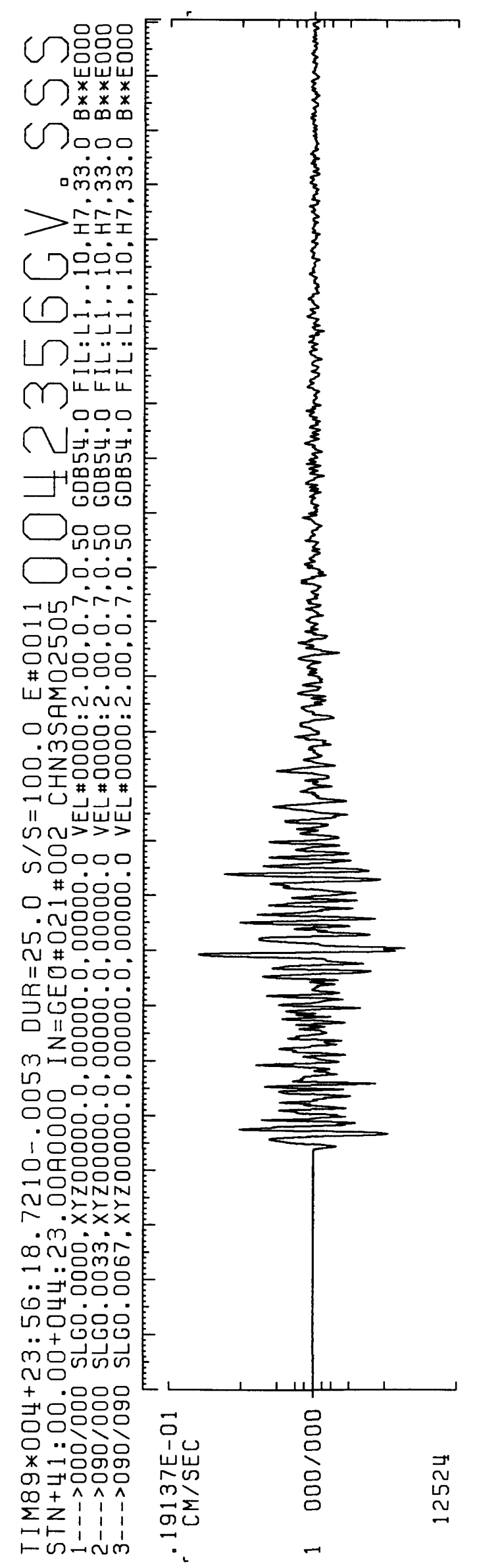

129

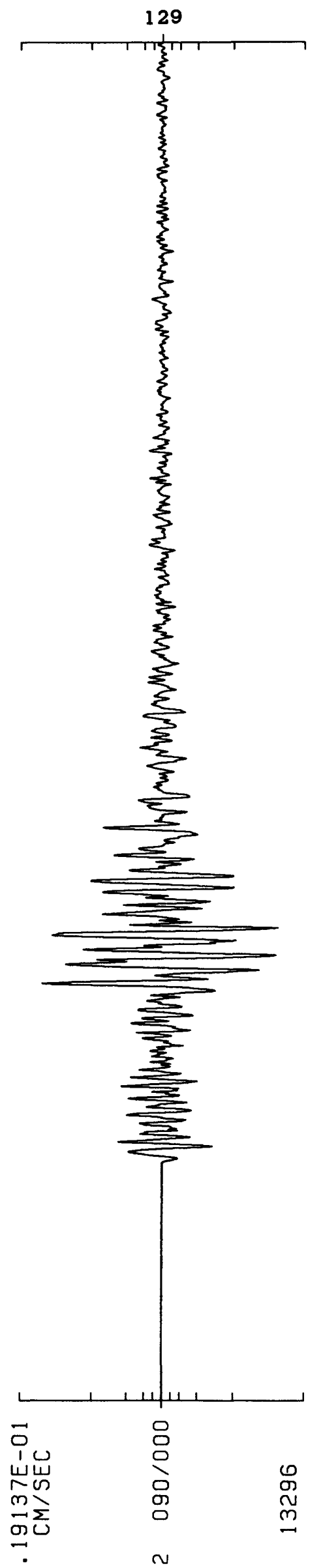

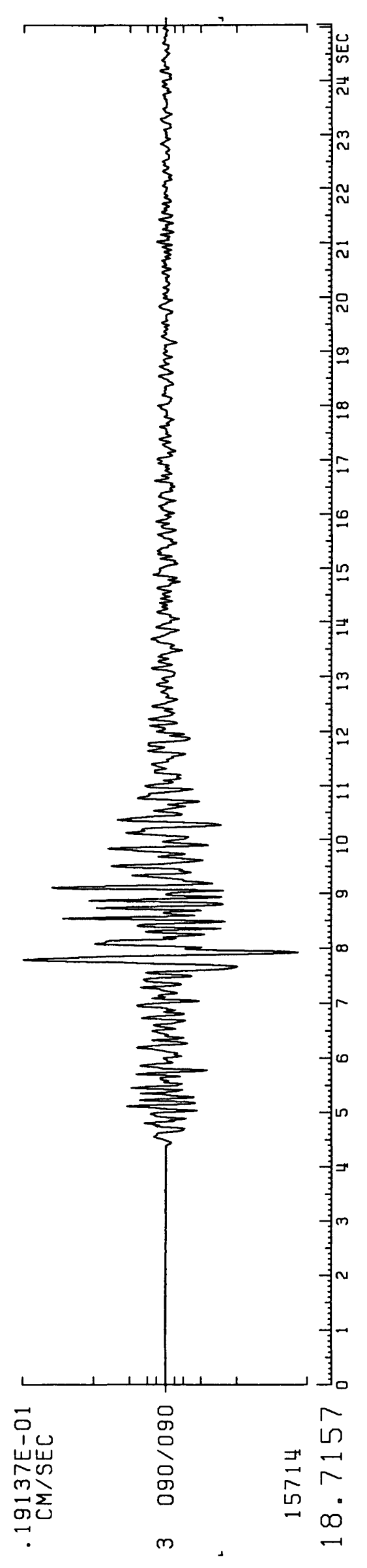


130
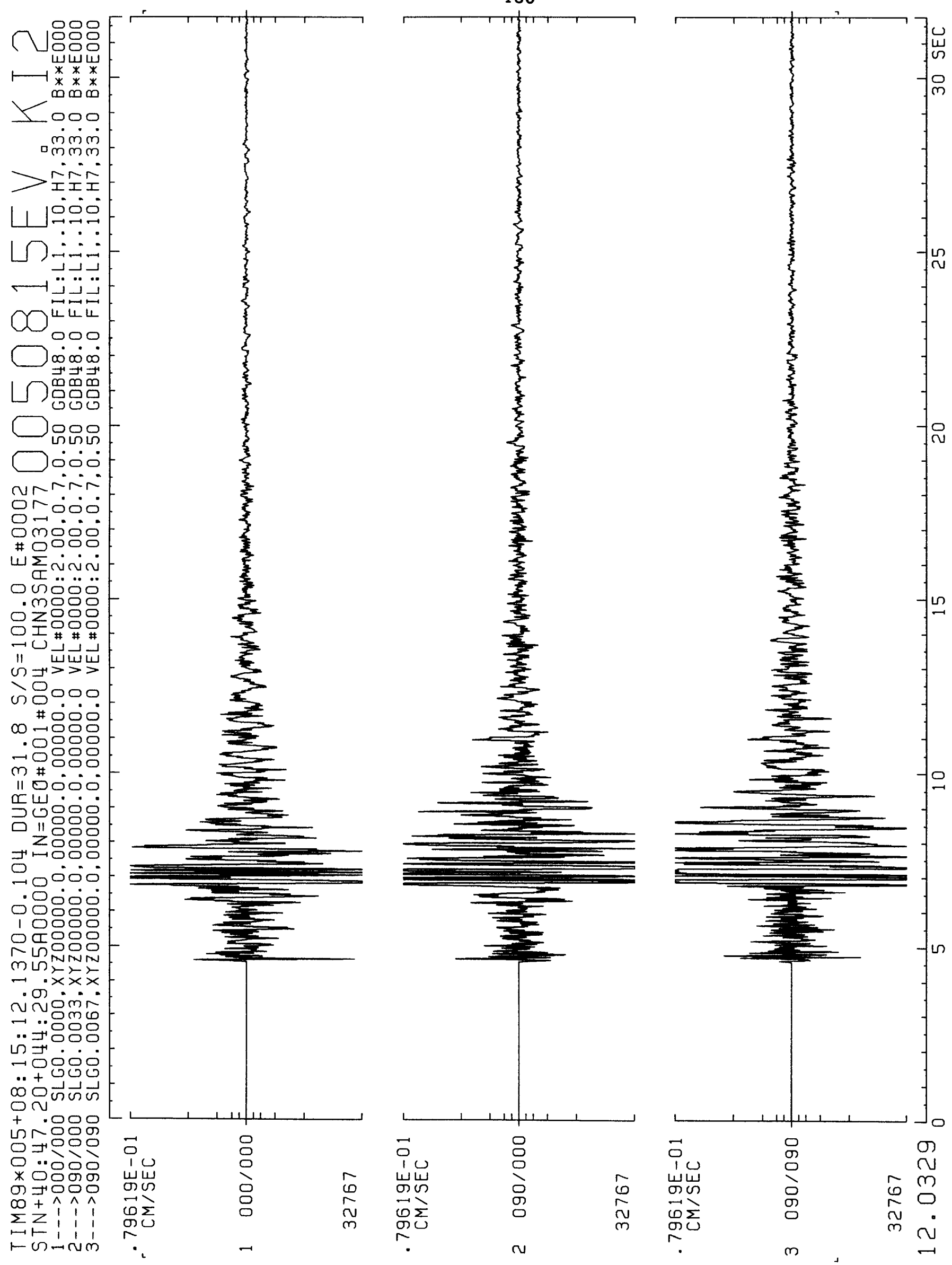

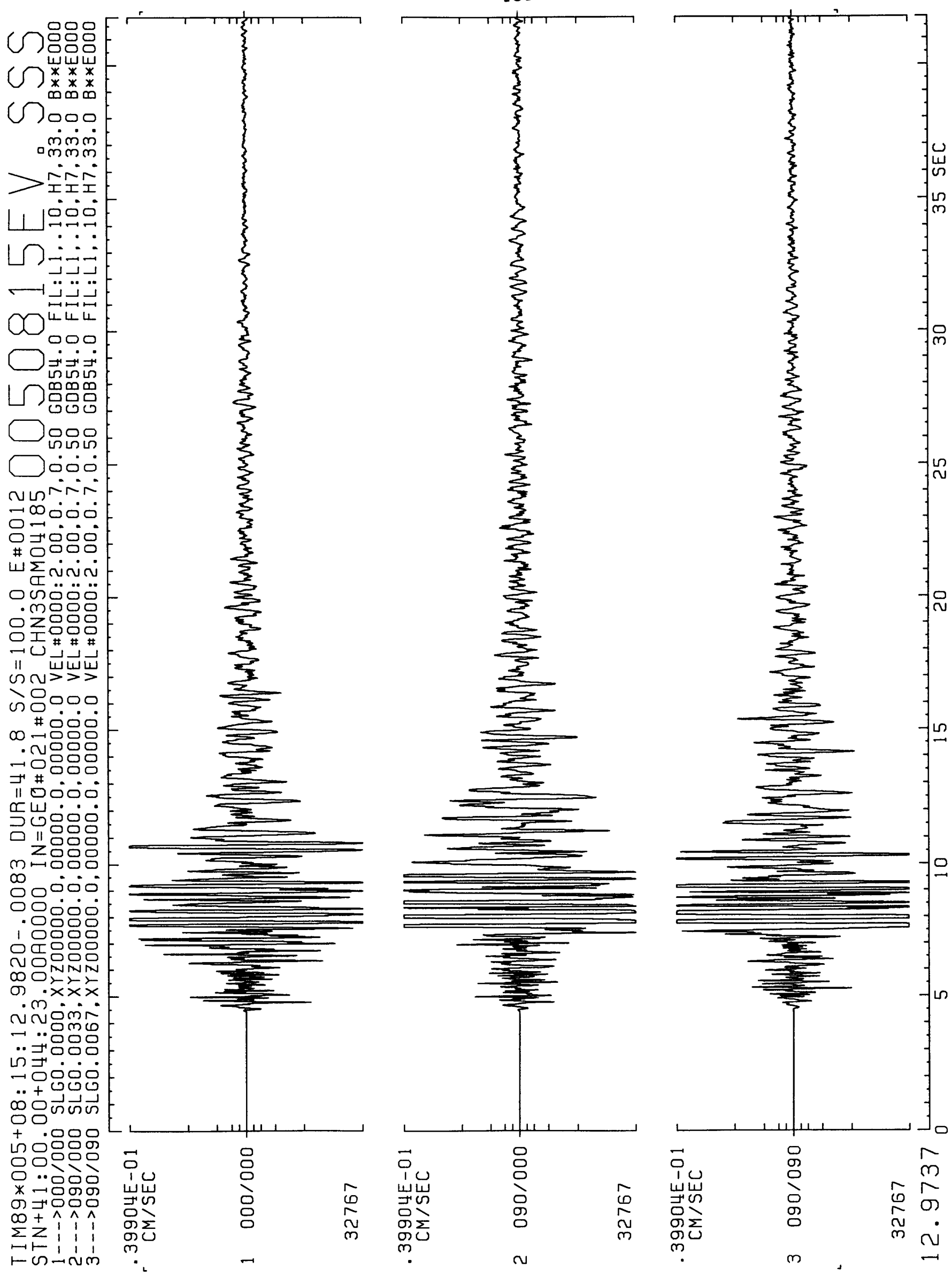
132

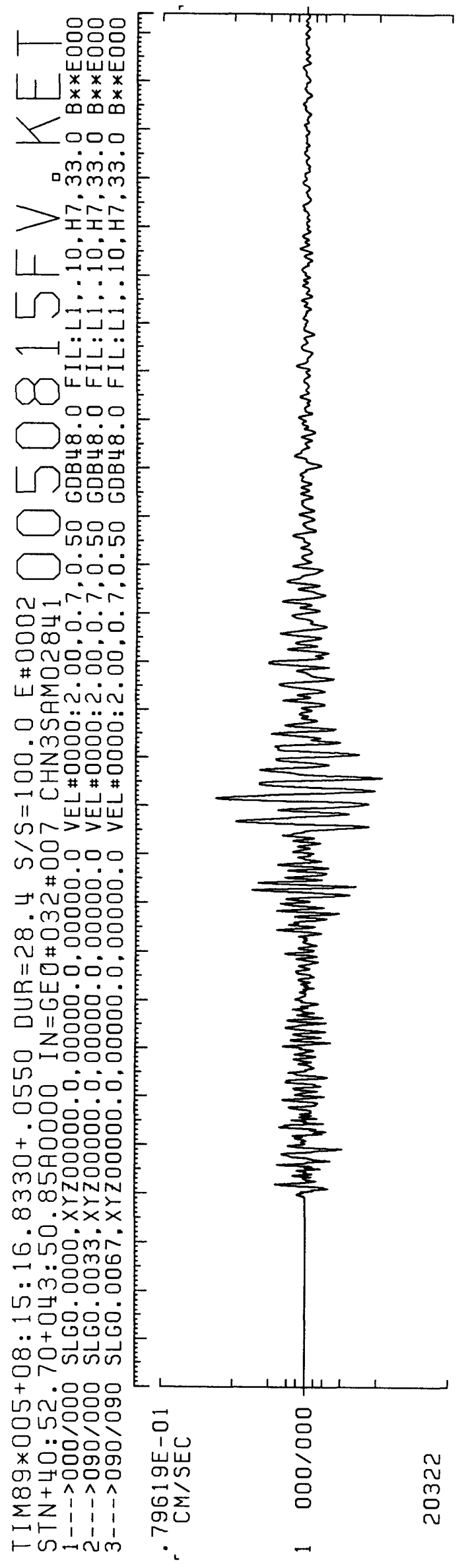

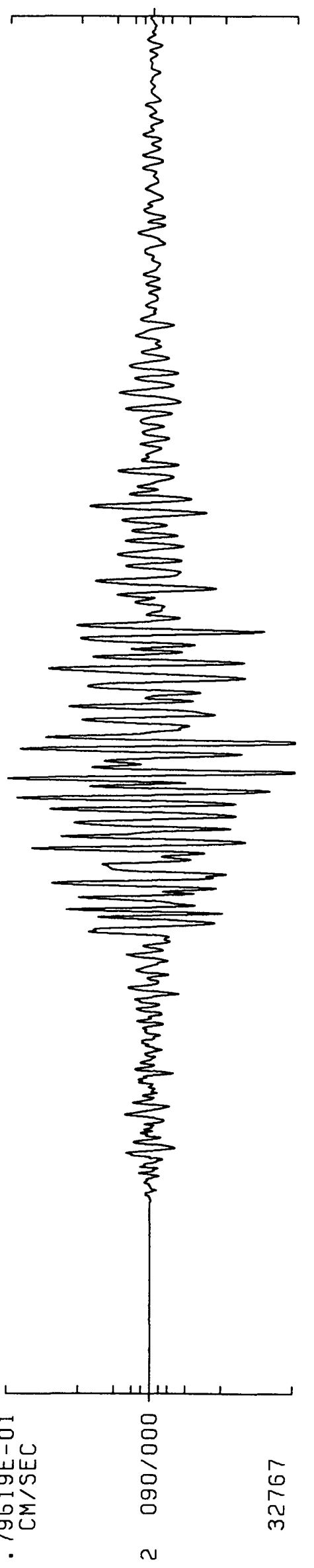

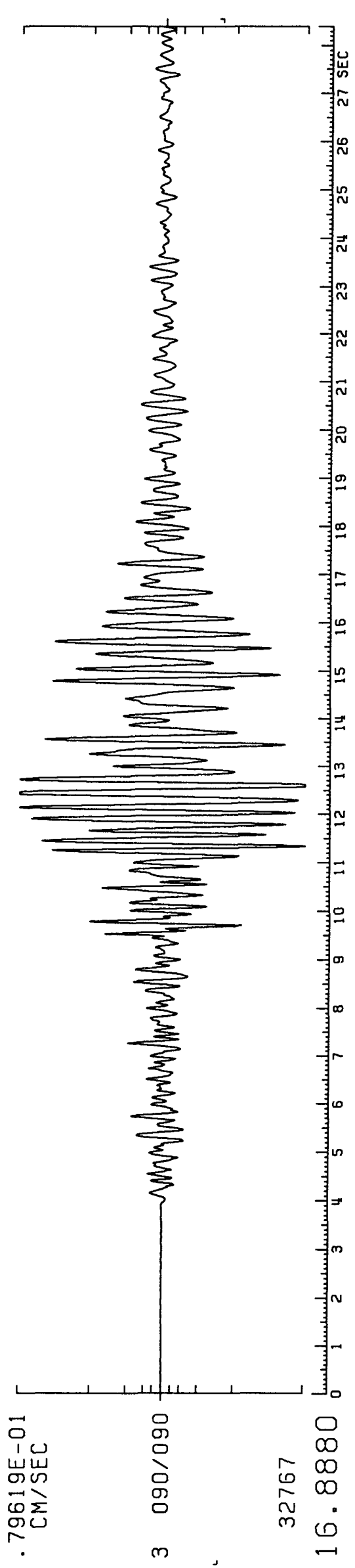


133

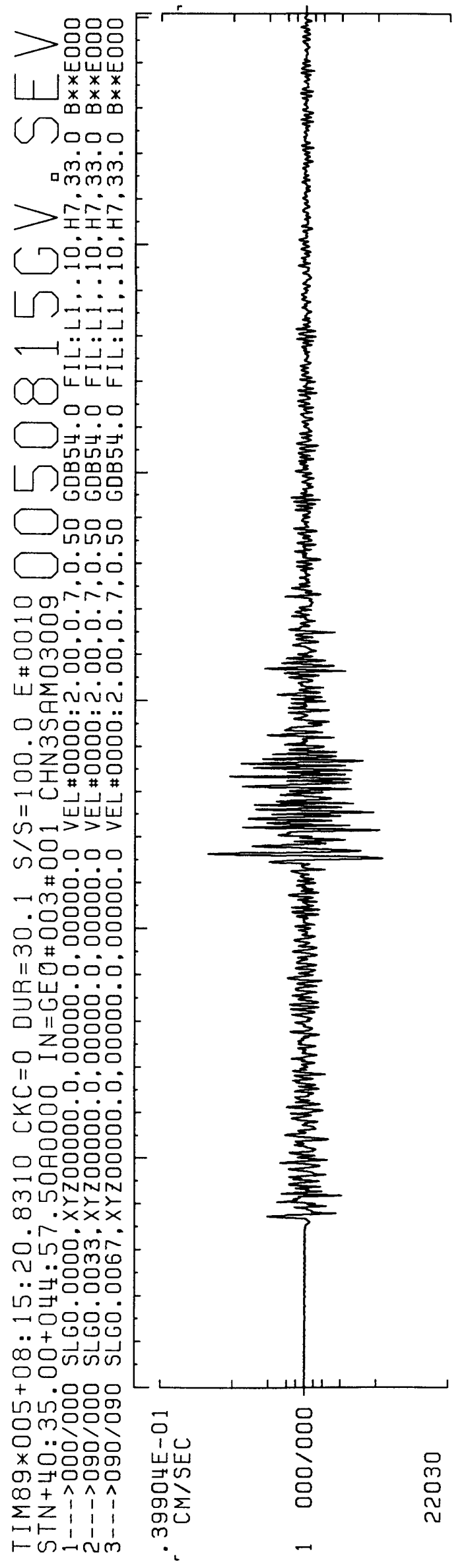

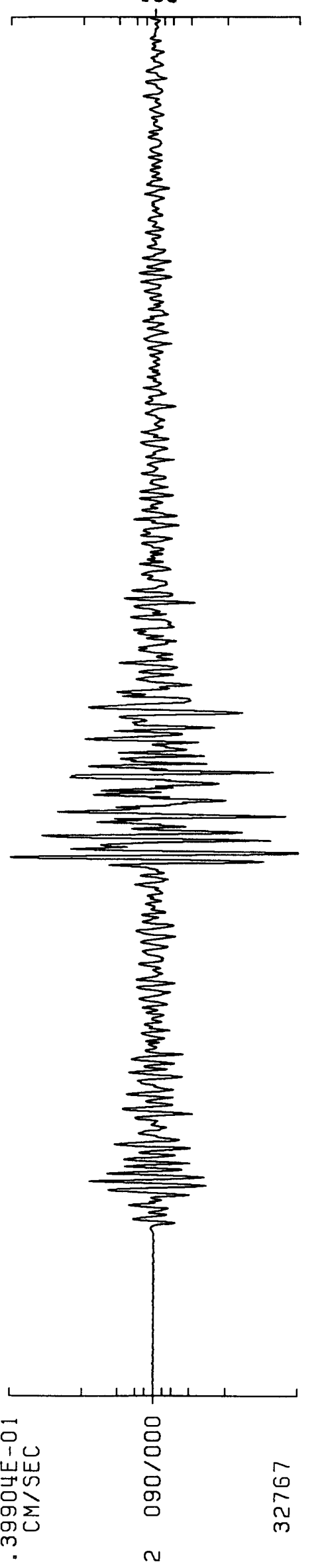

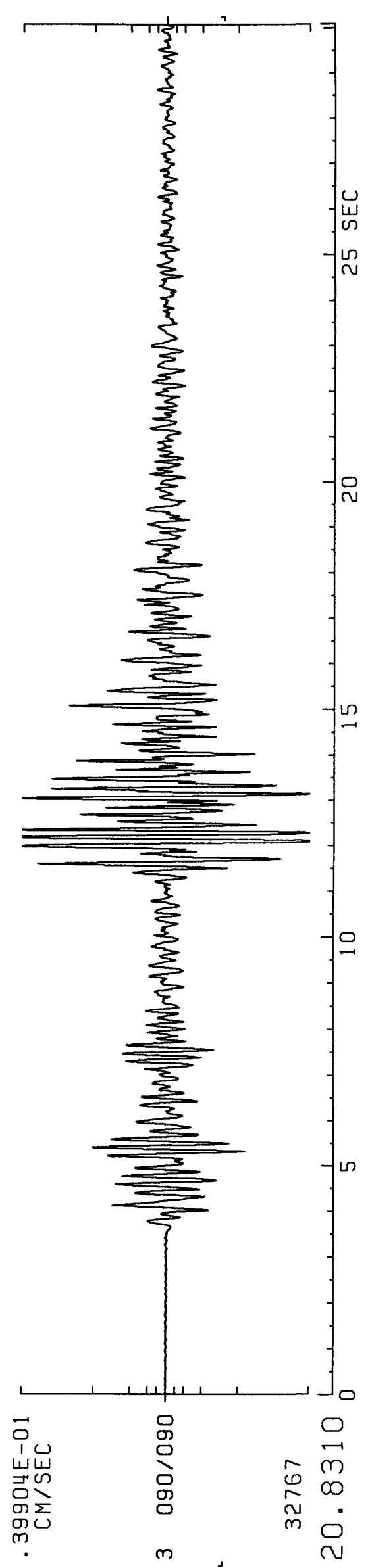


134

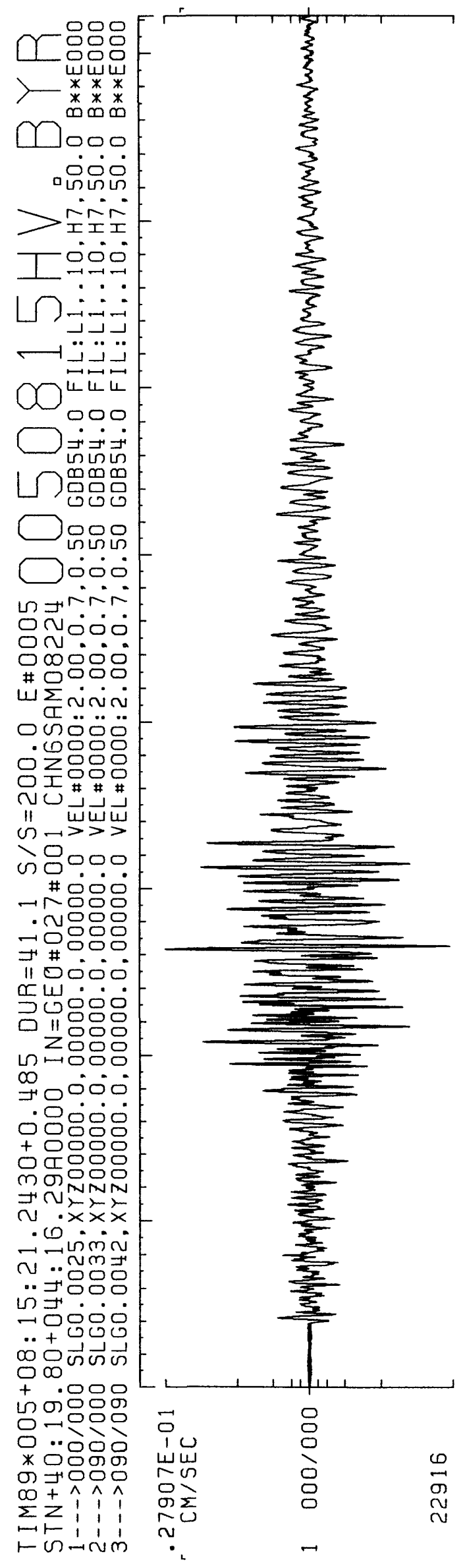

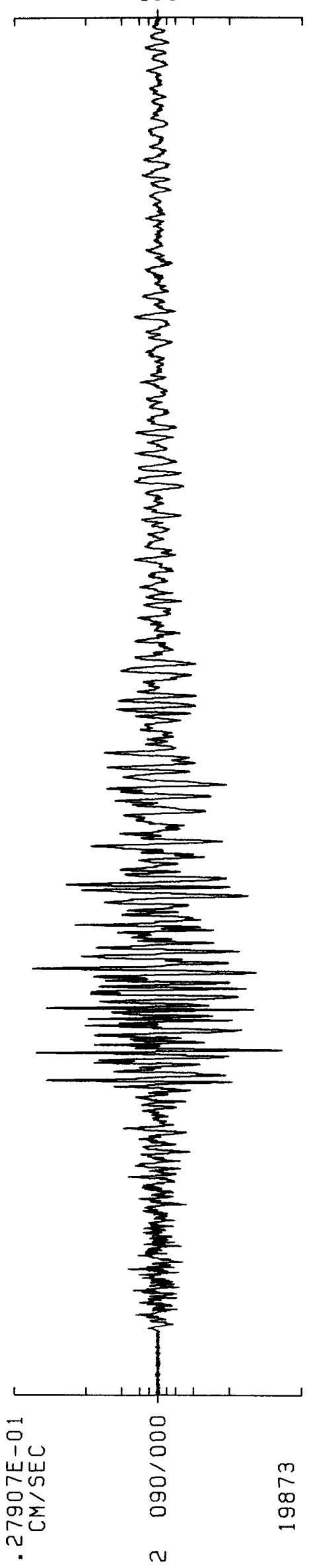

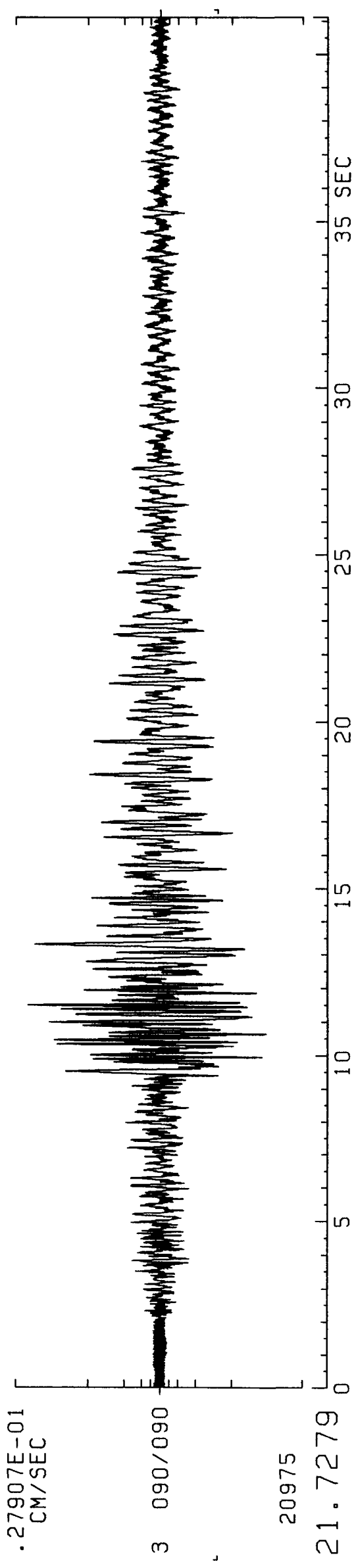


135

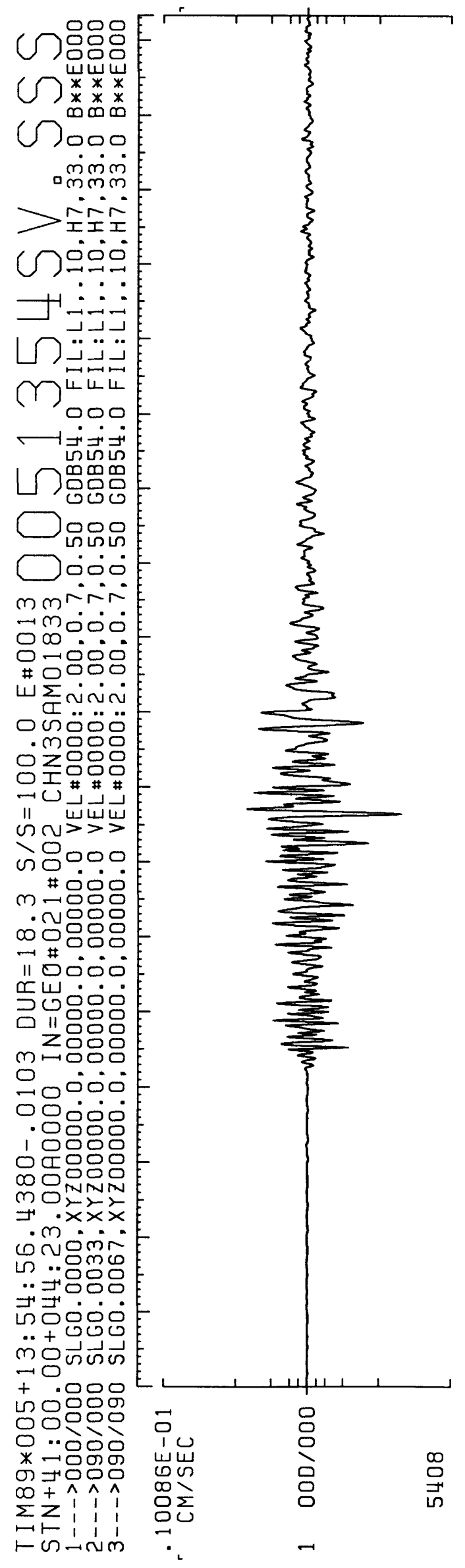

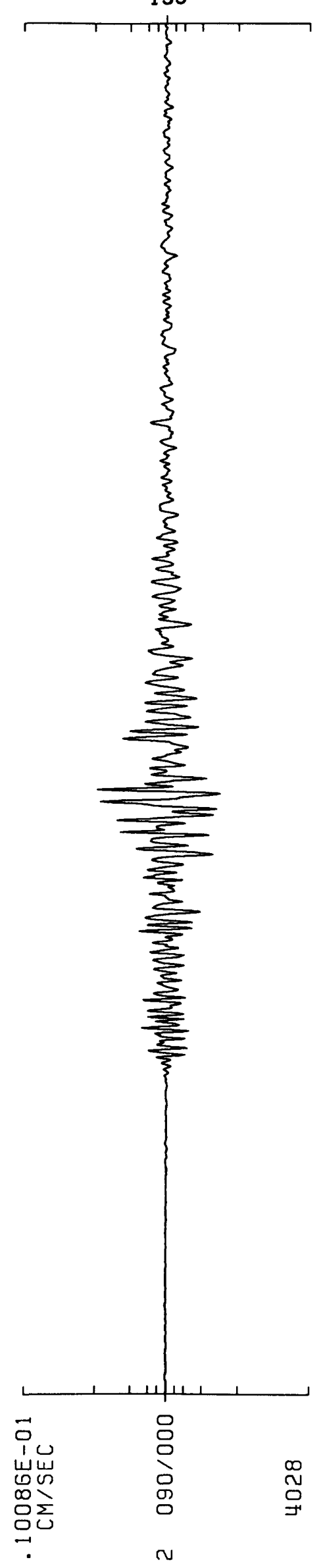

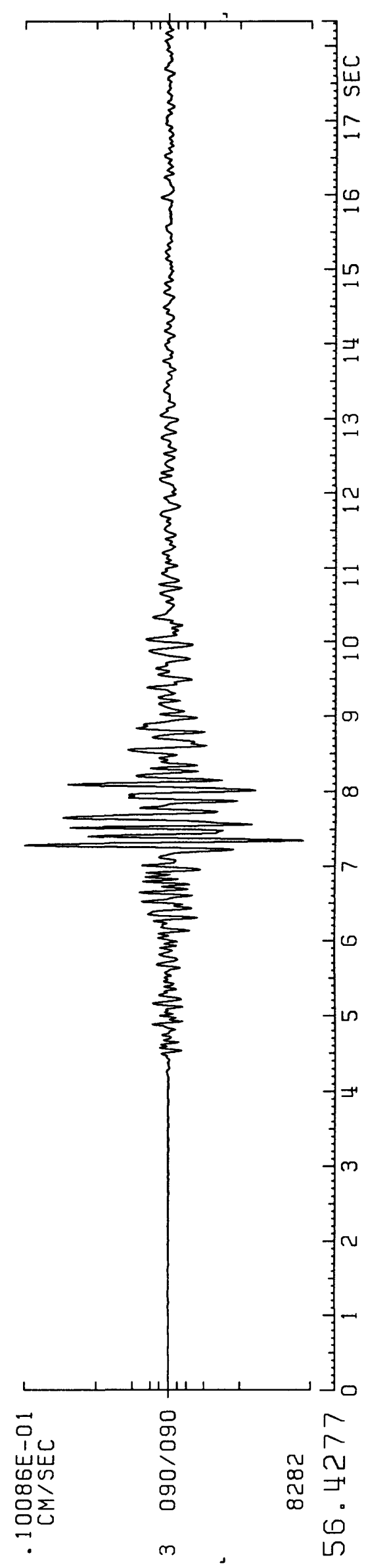




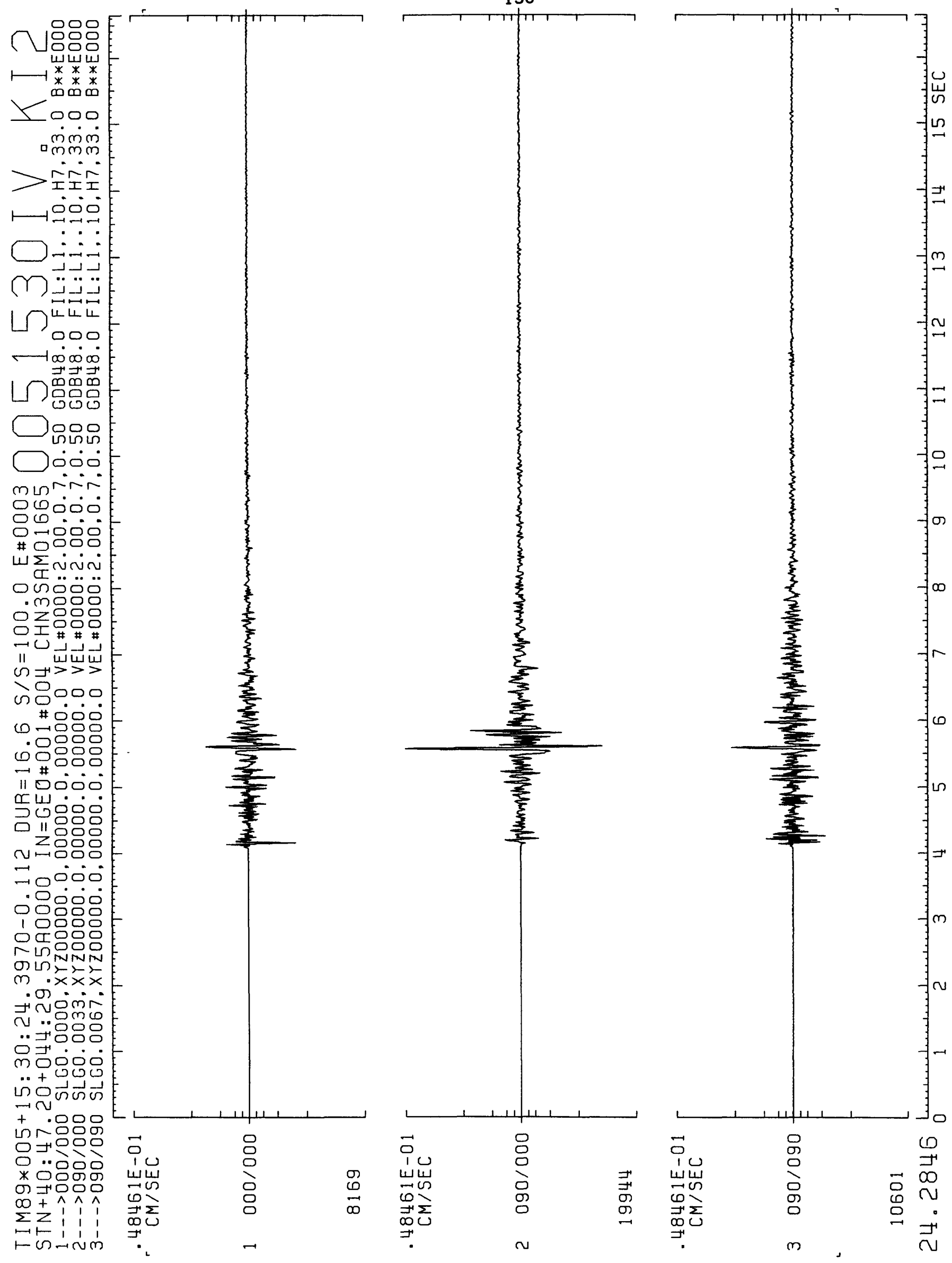




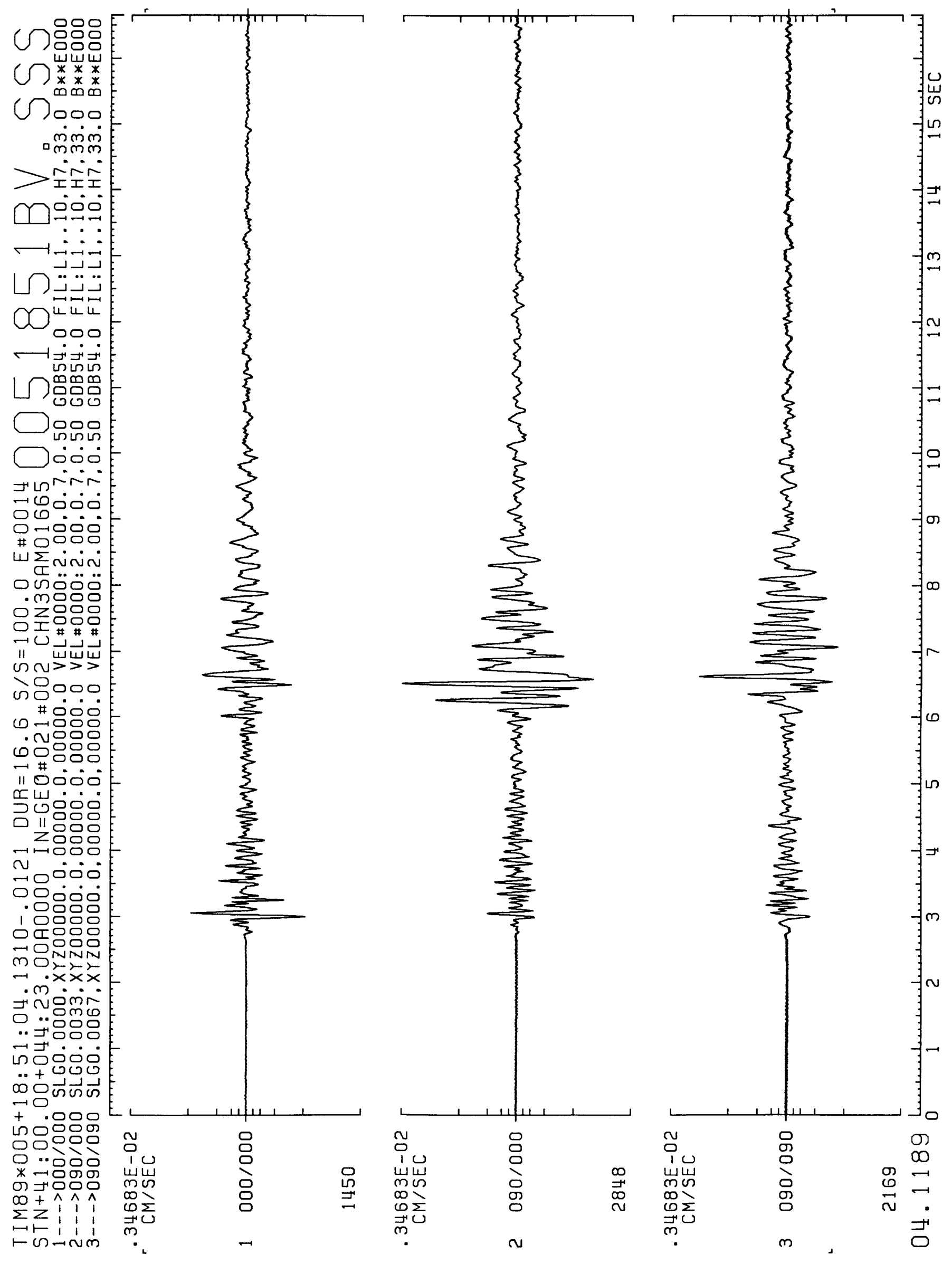


139

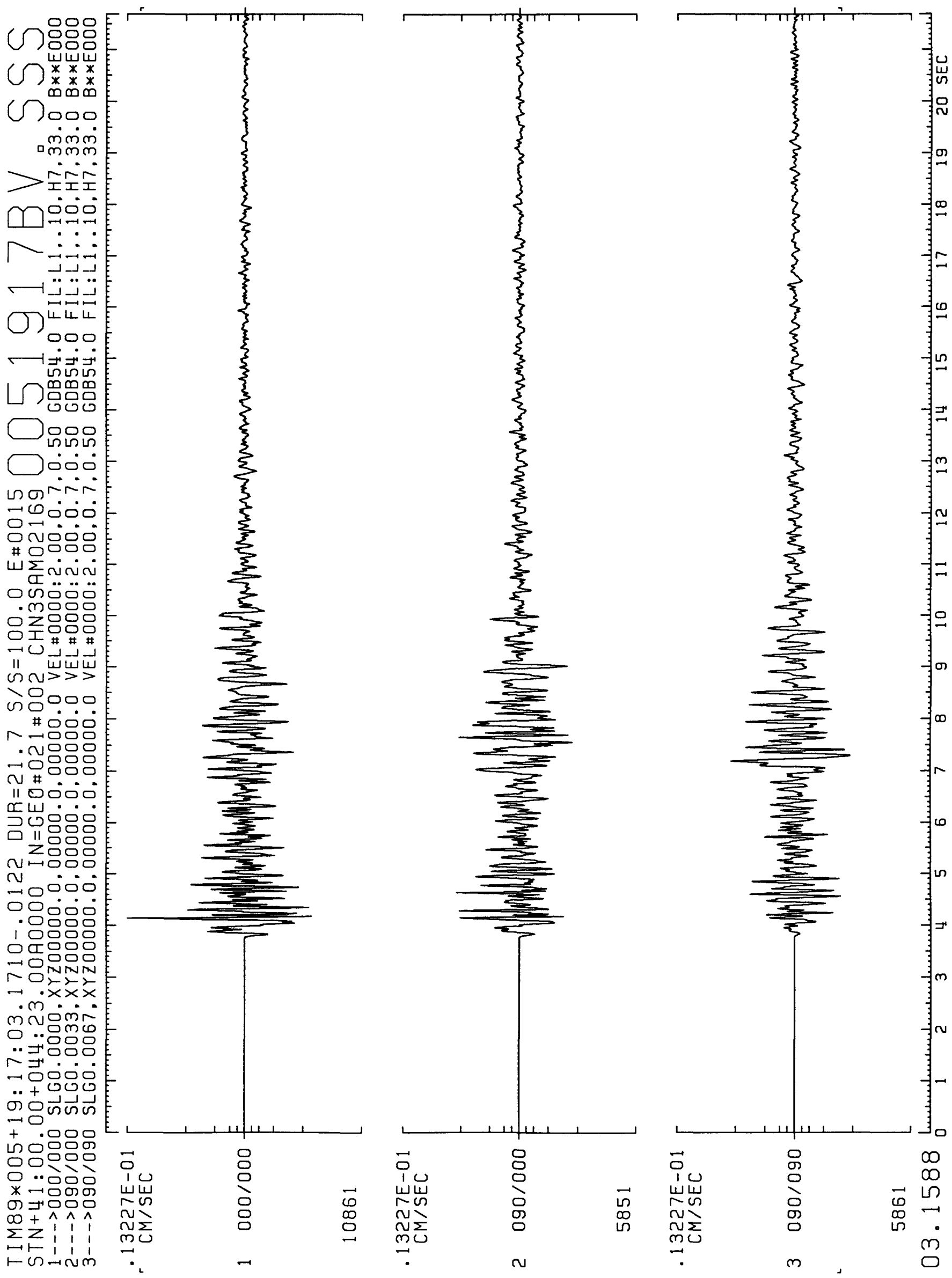


140

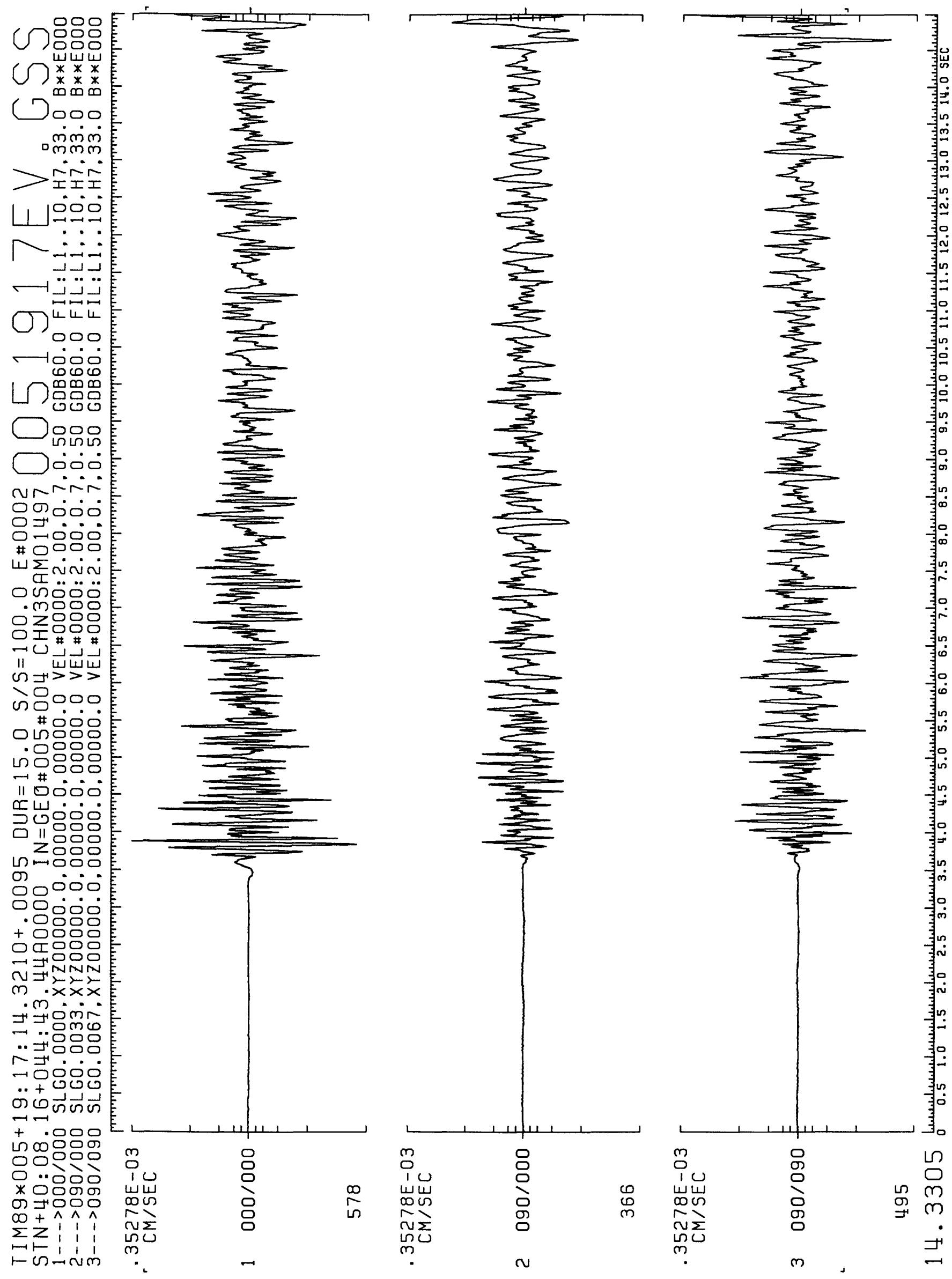



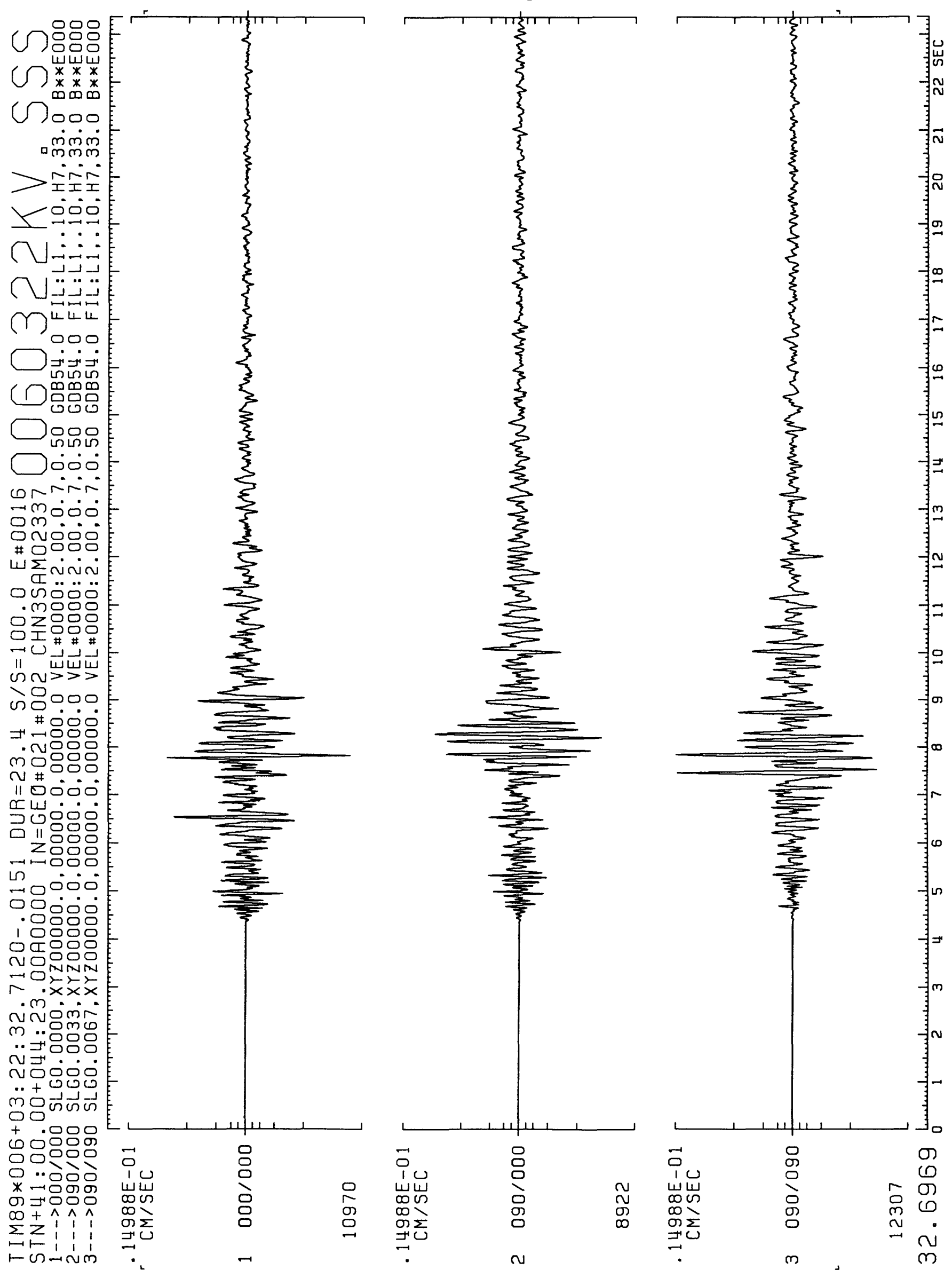
142
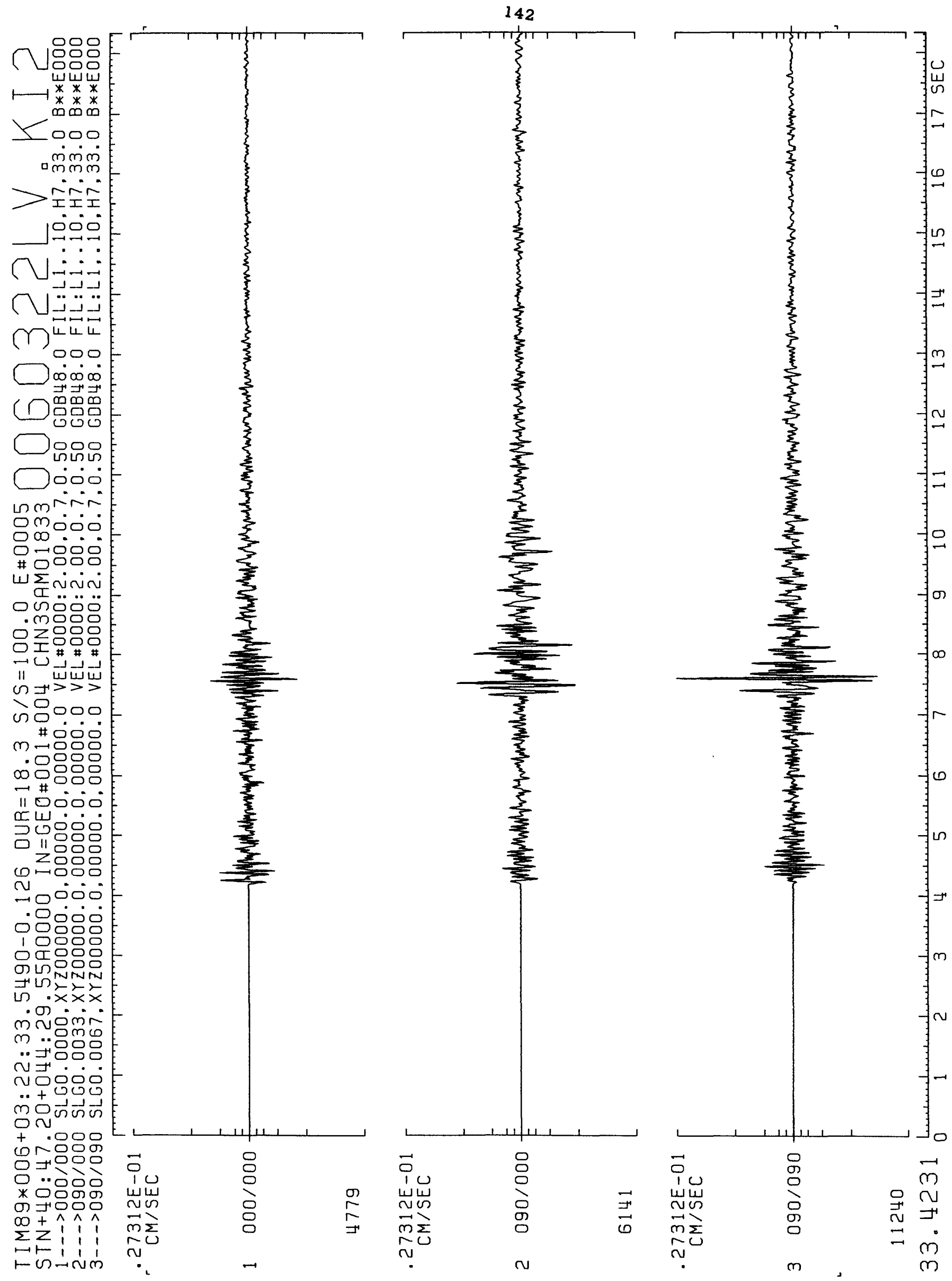
143

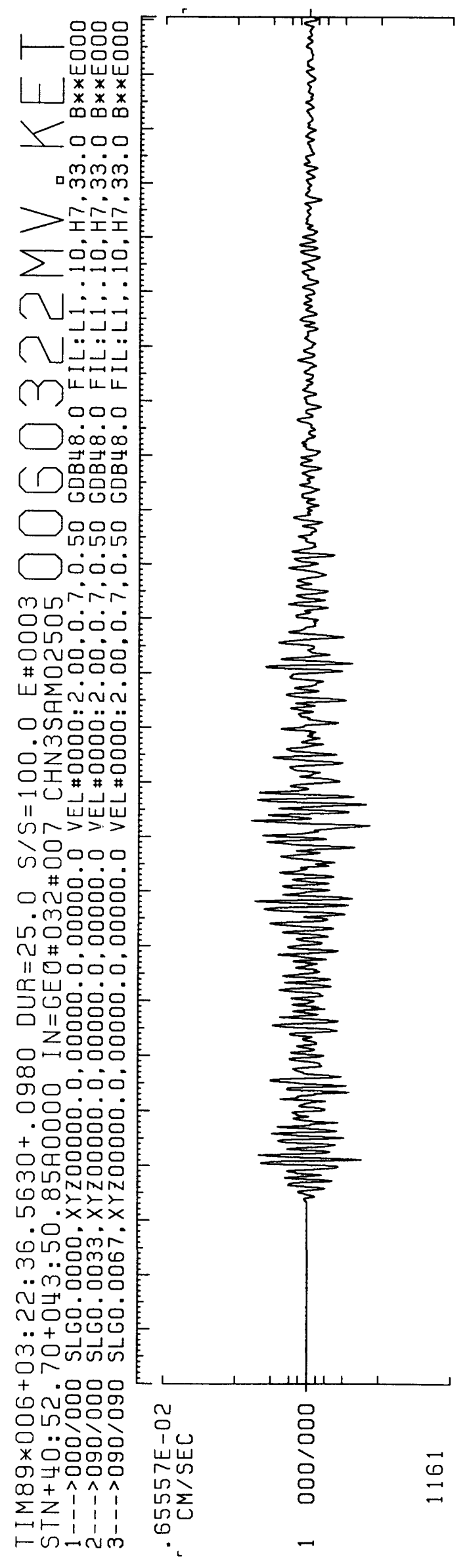

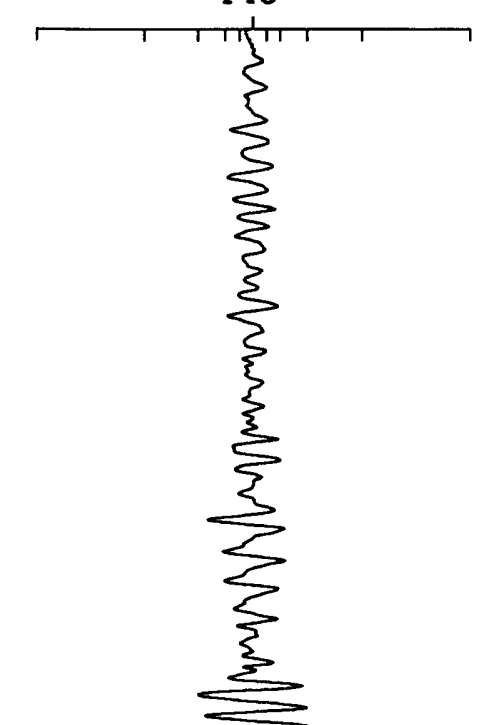


144
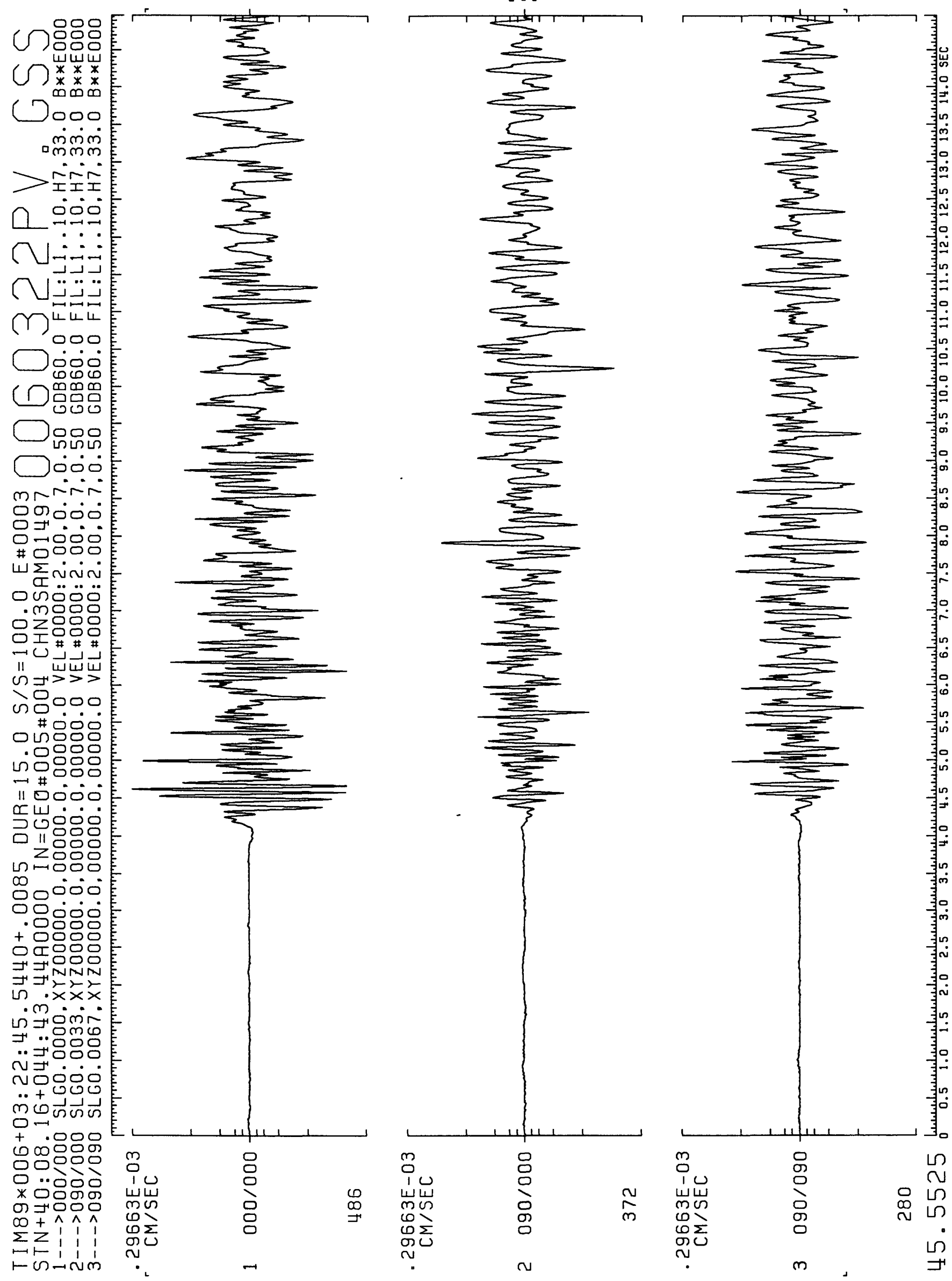


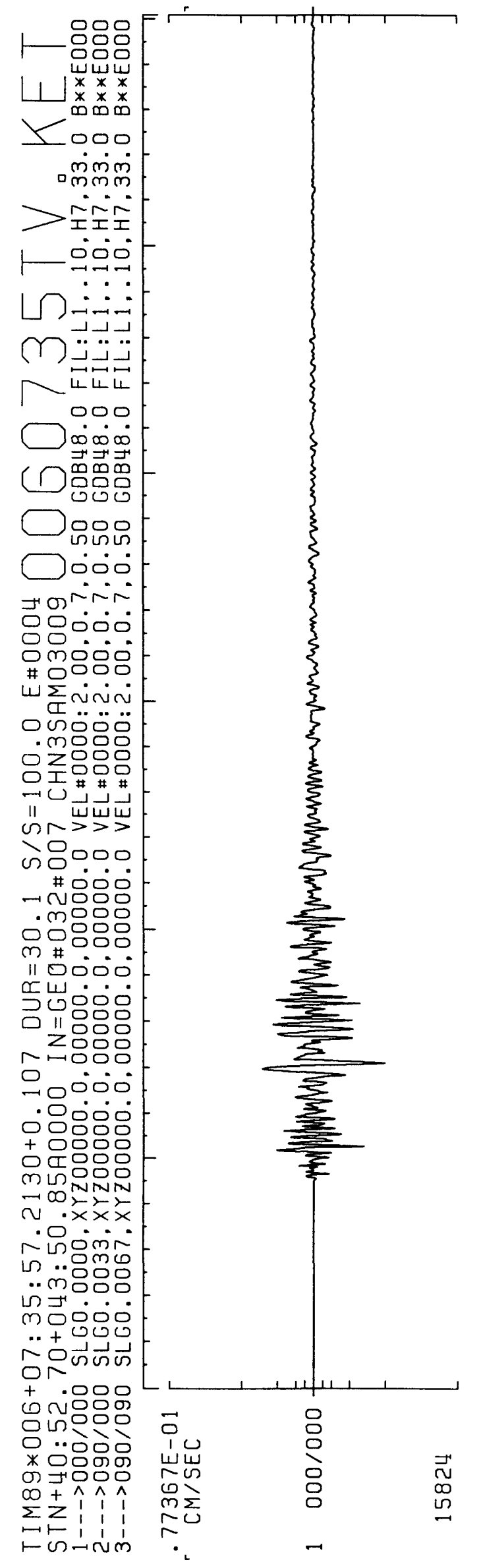

145

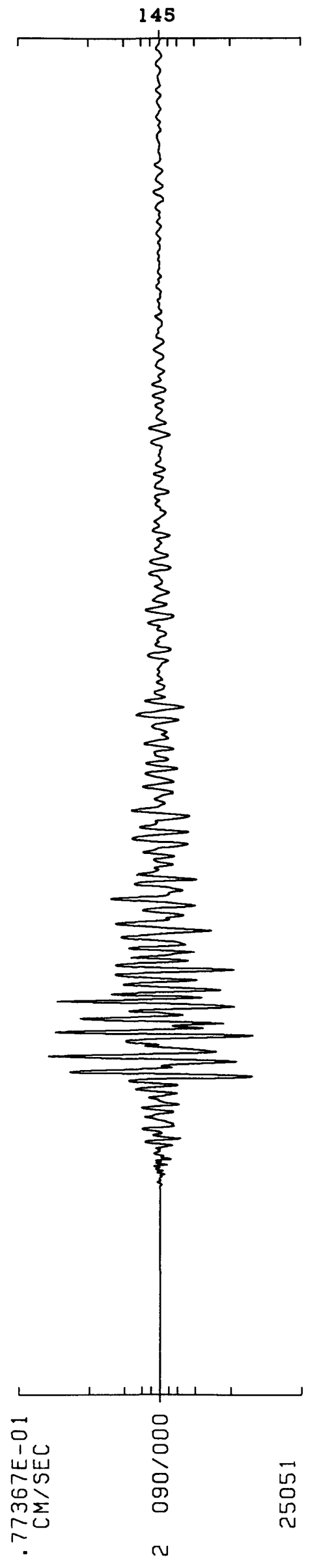

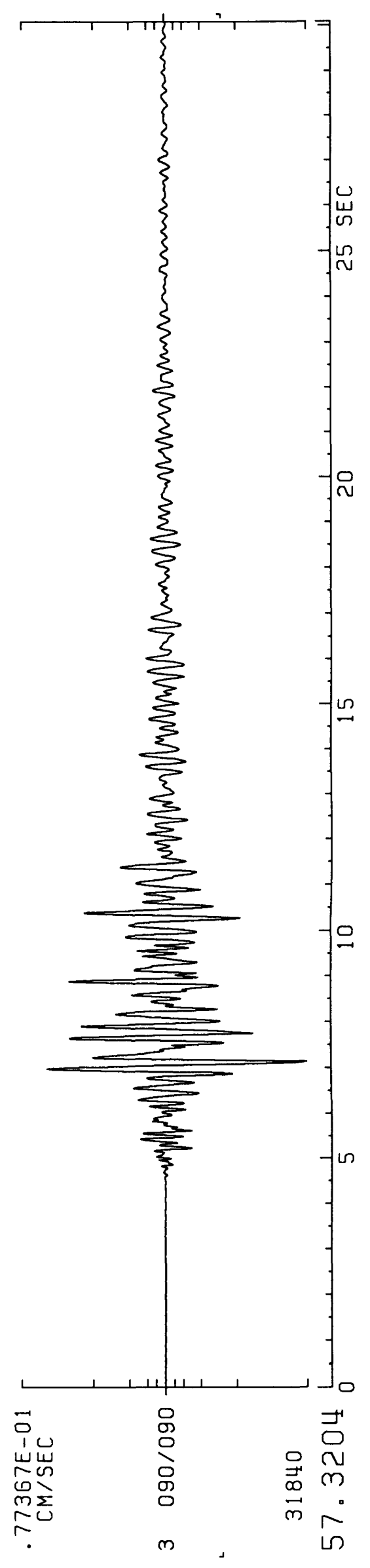




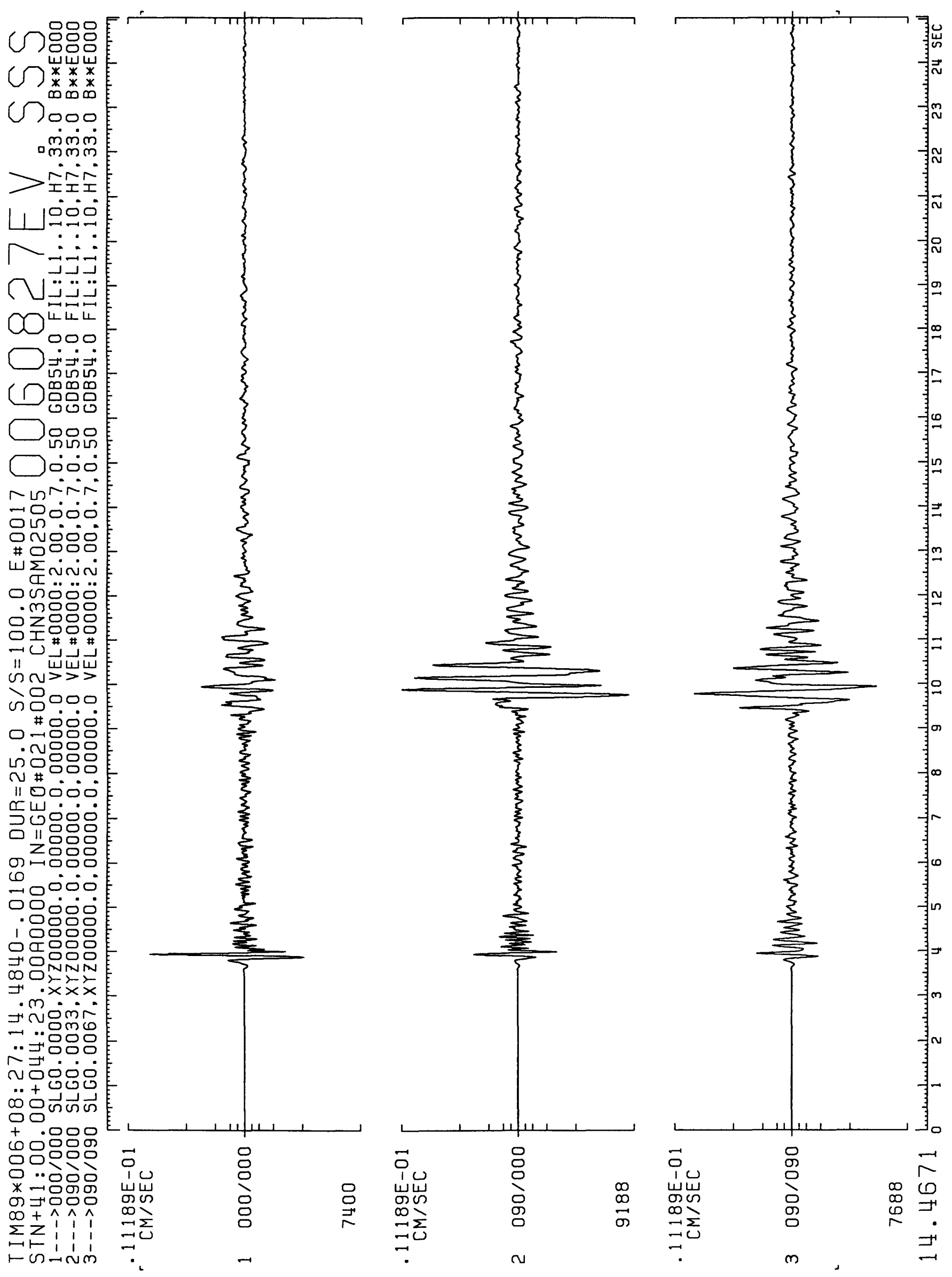


147

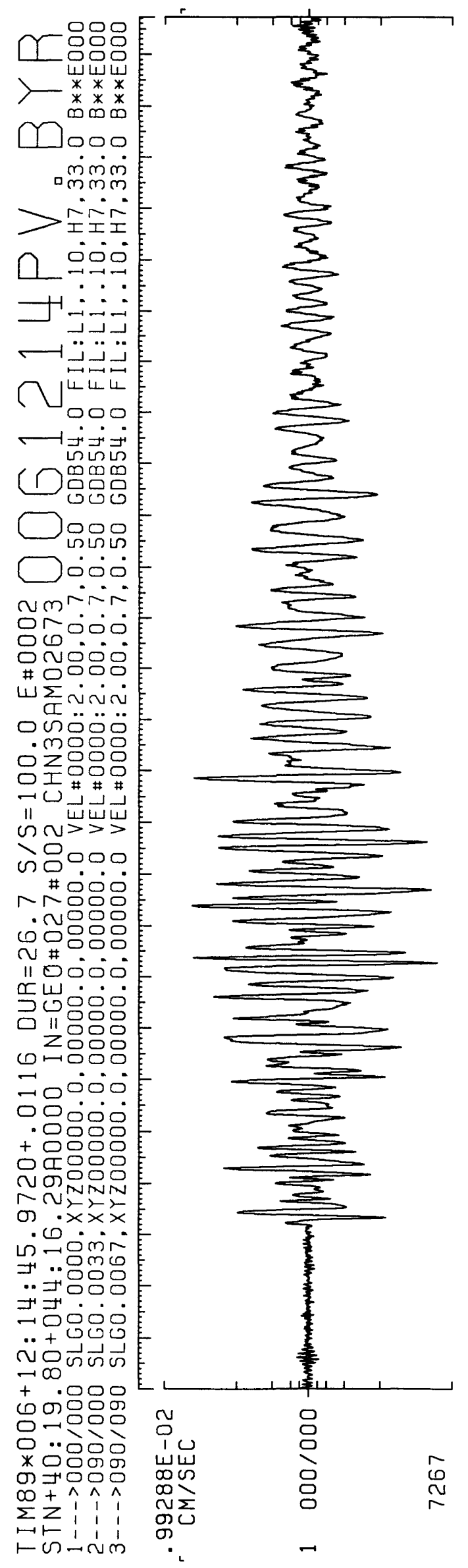

1
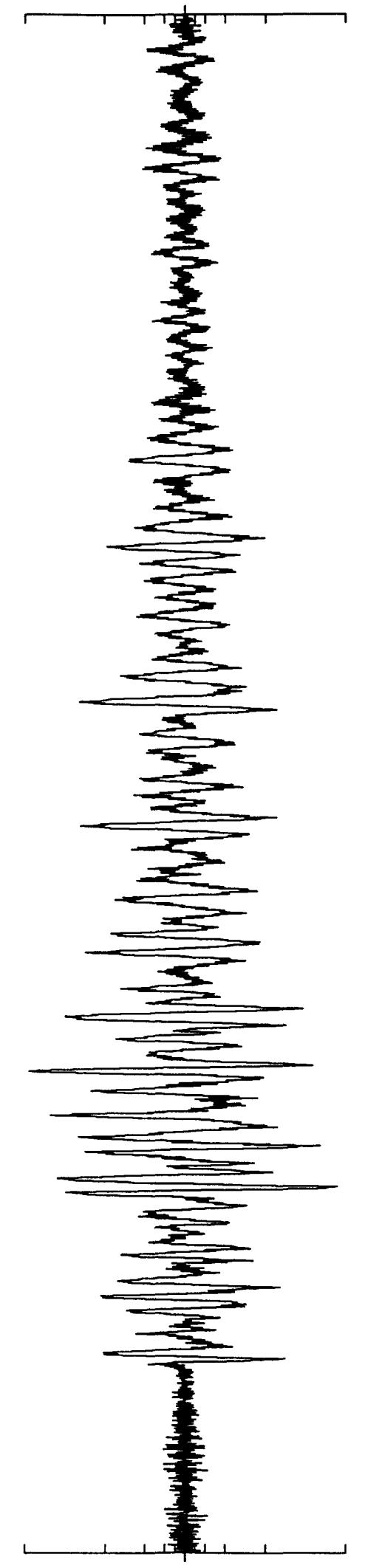

ऽ

10

山以

禹

证

の

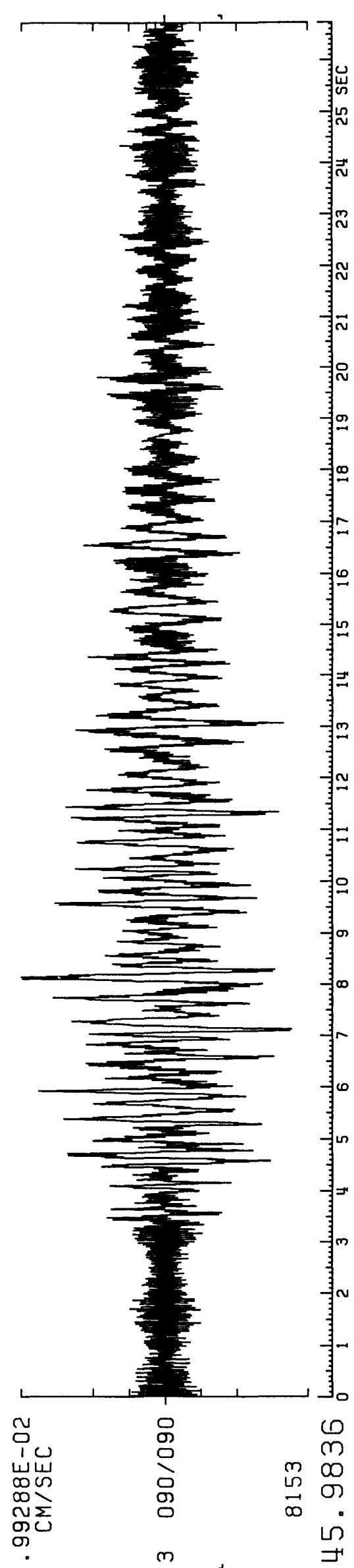


148
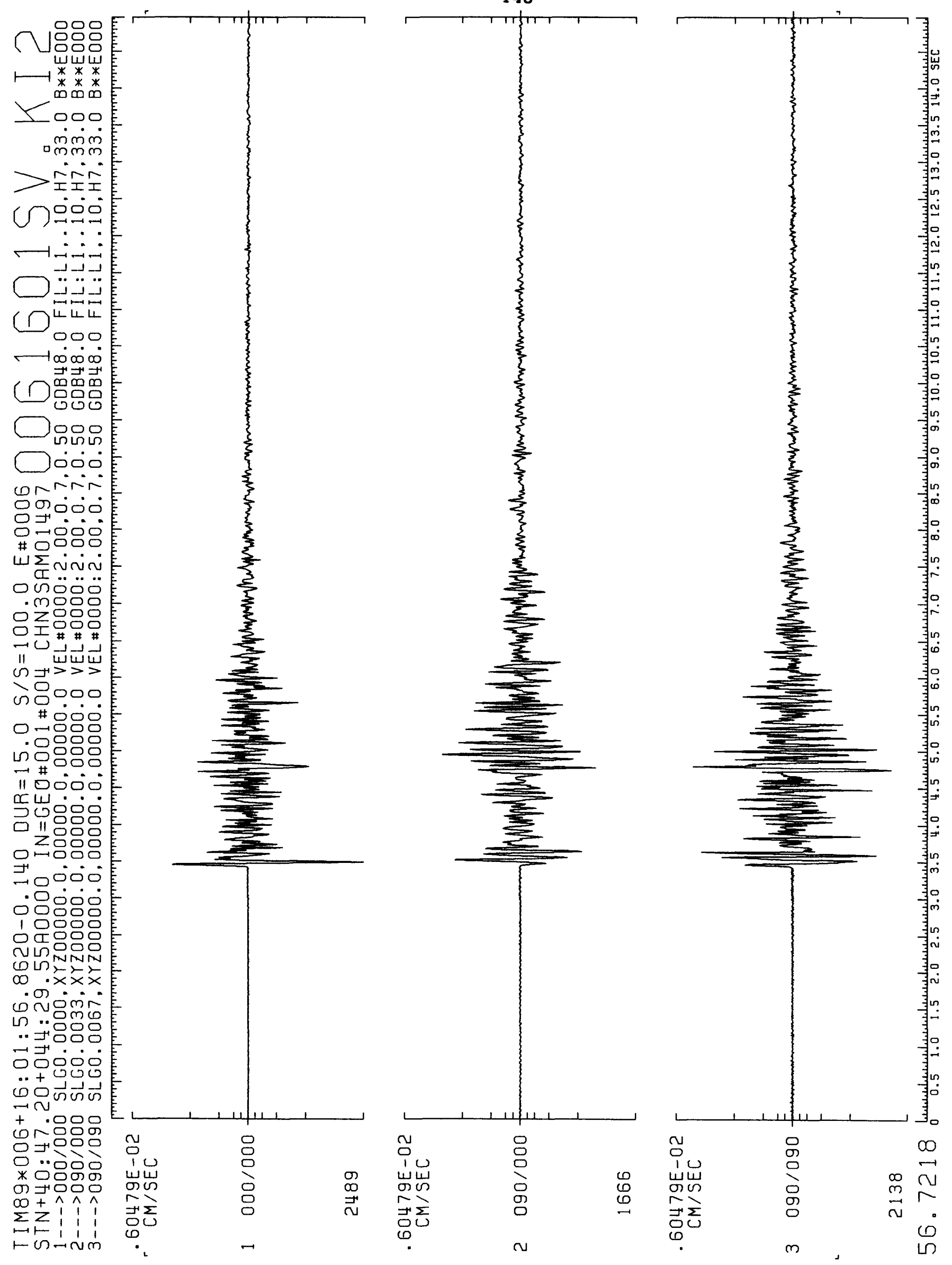

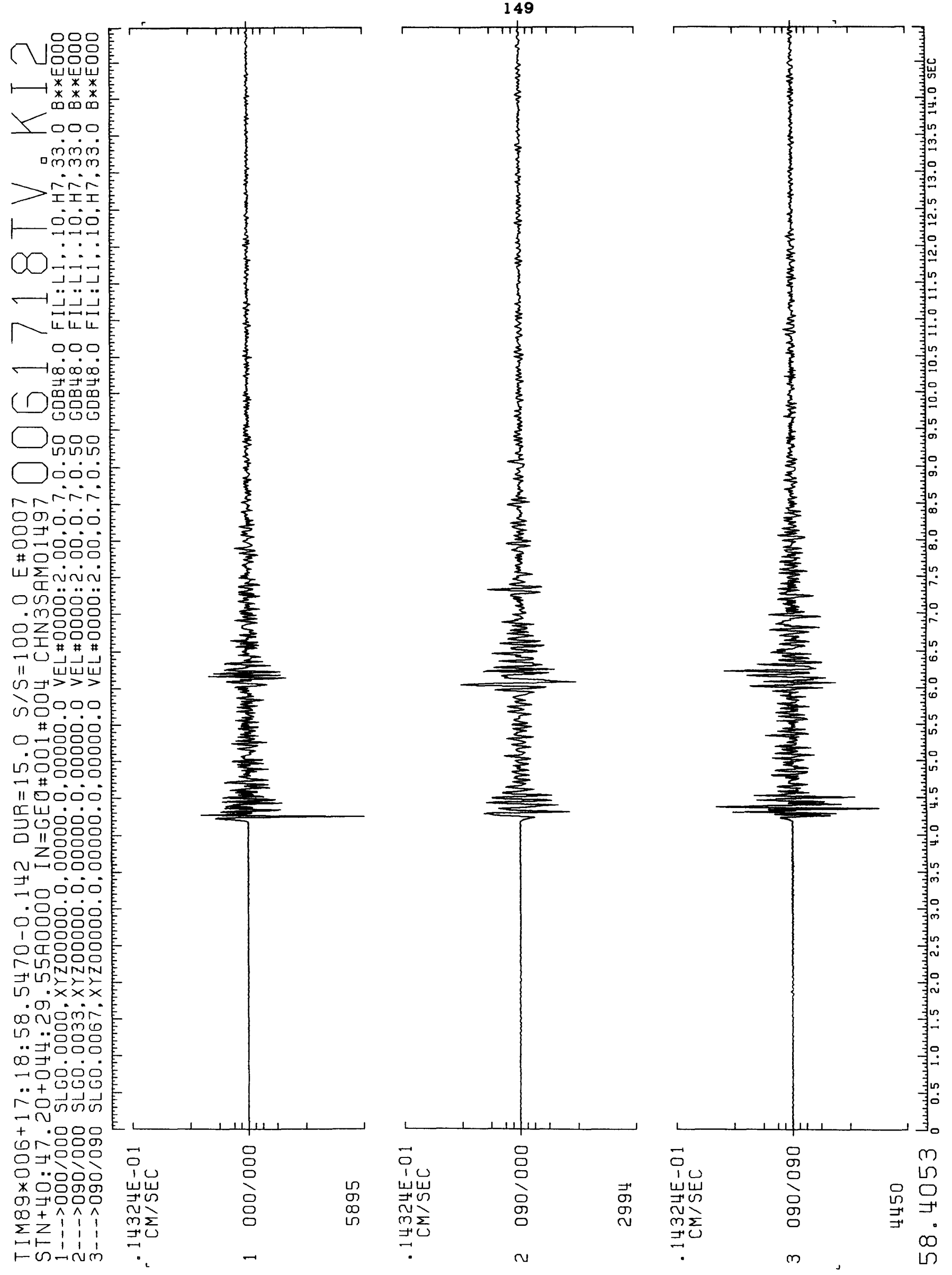
150

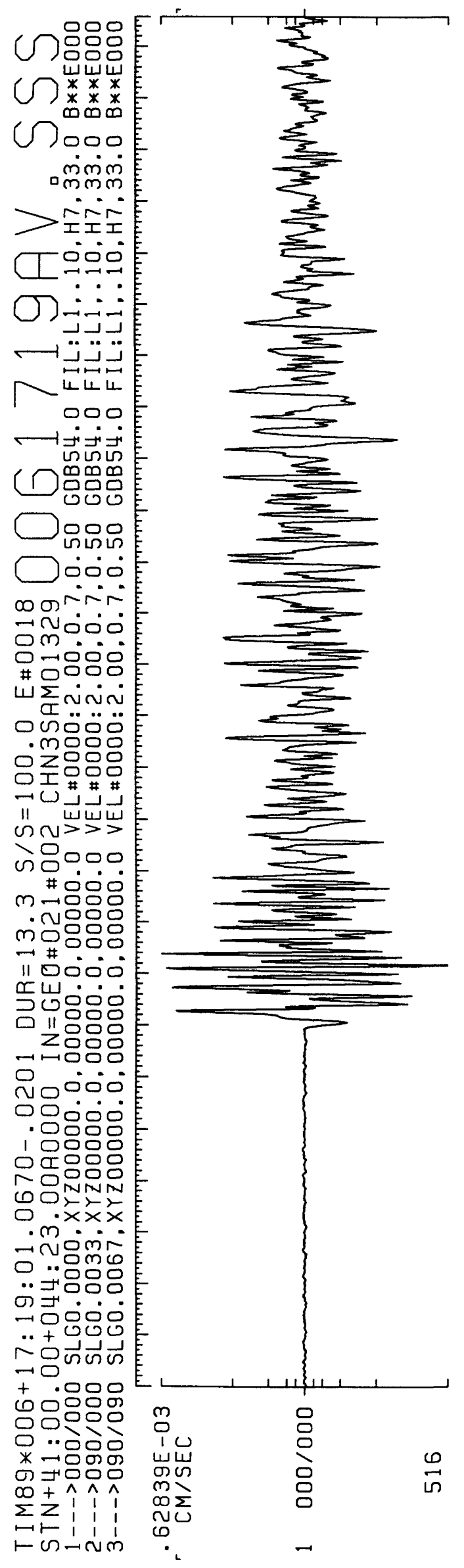

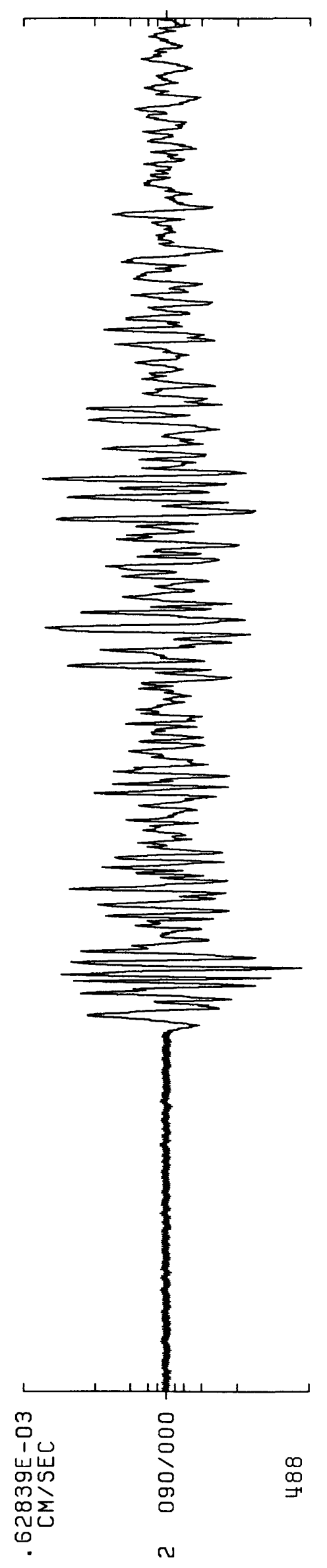

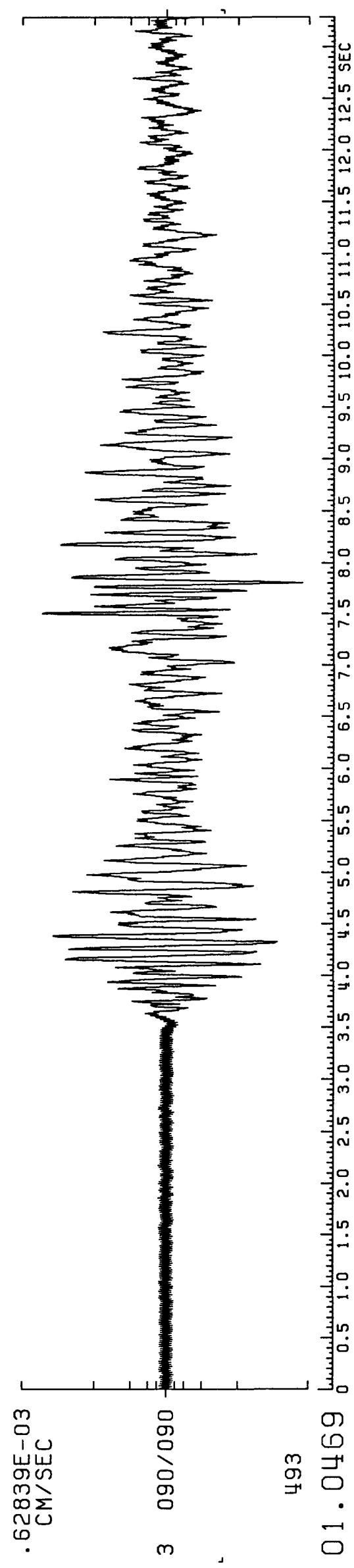




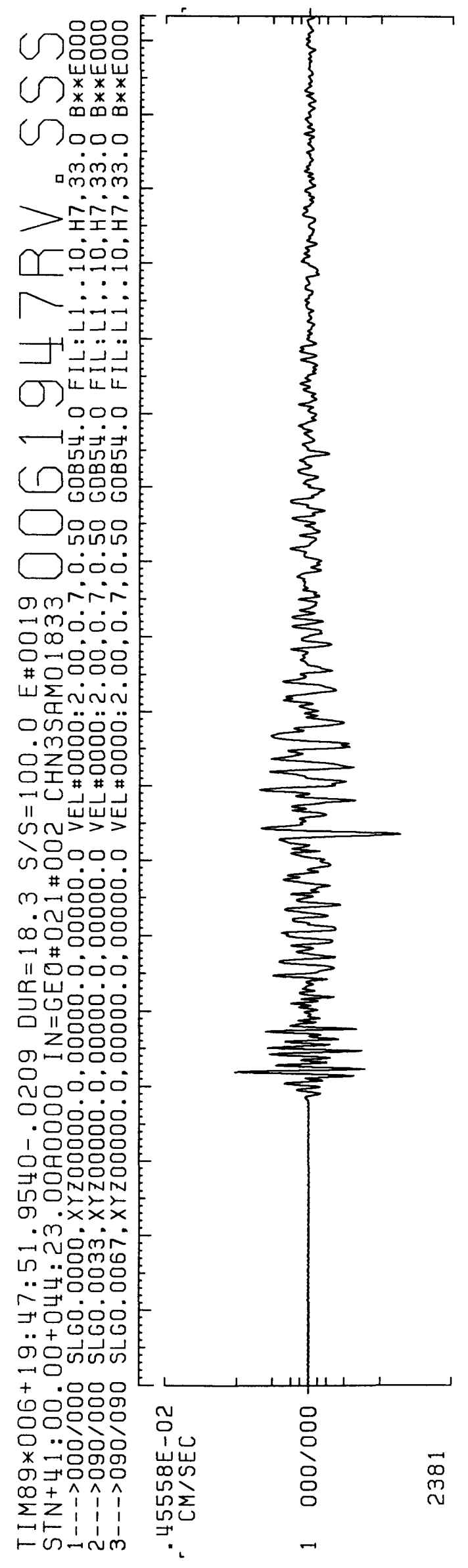

151

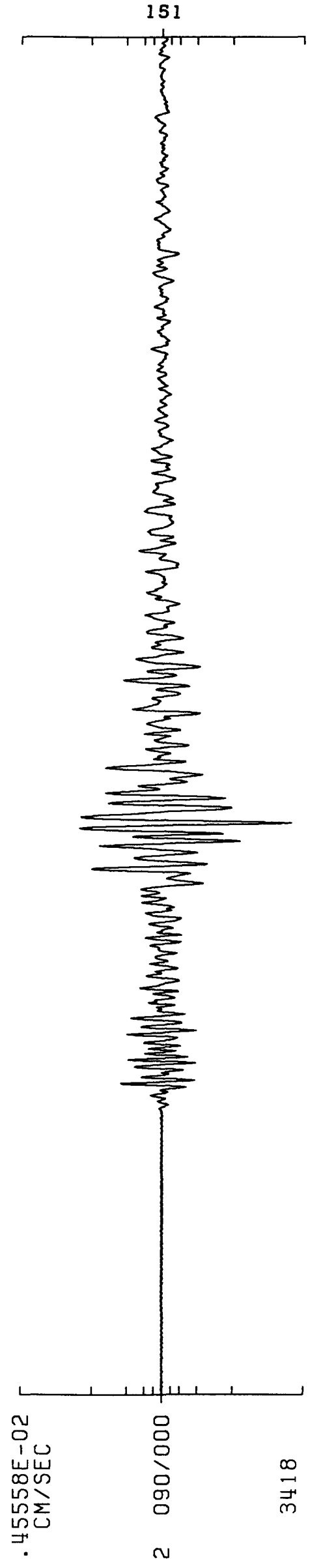

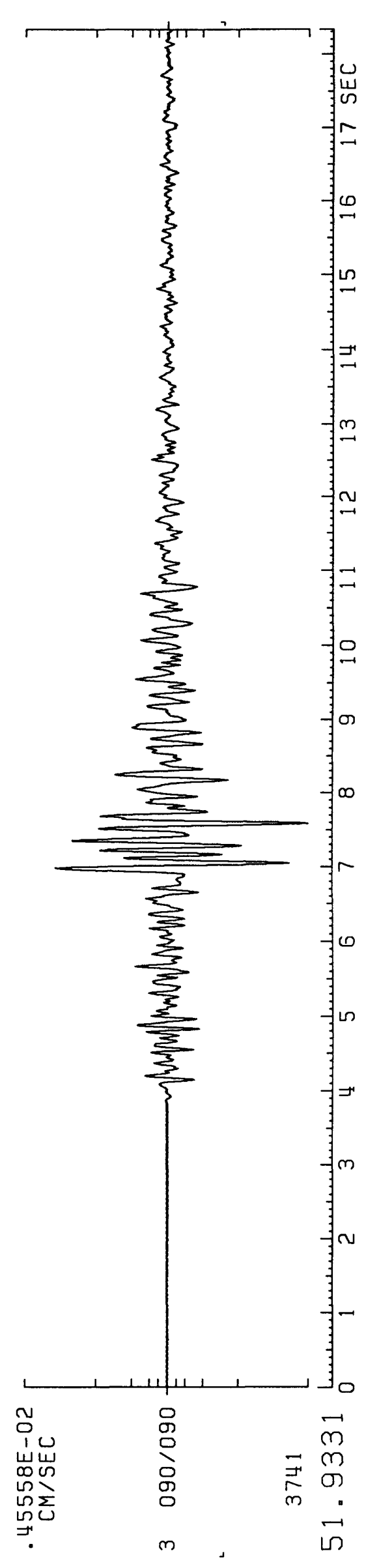


152

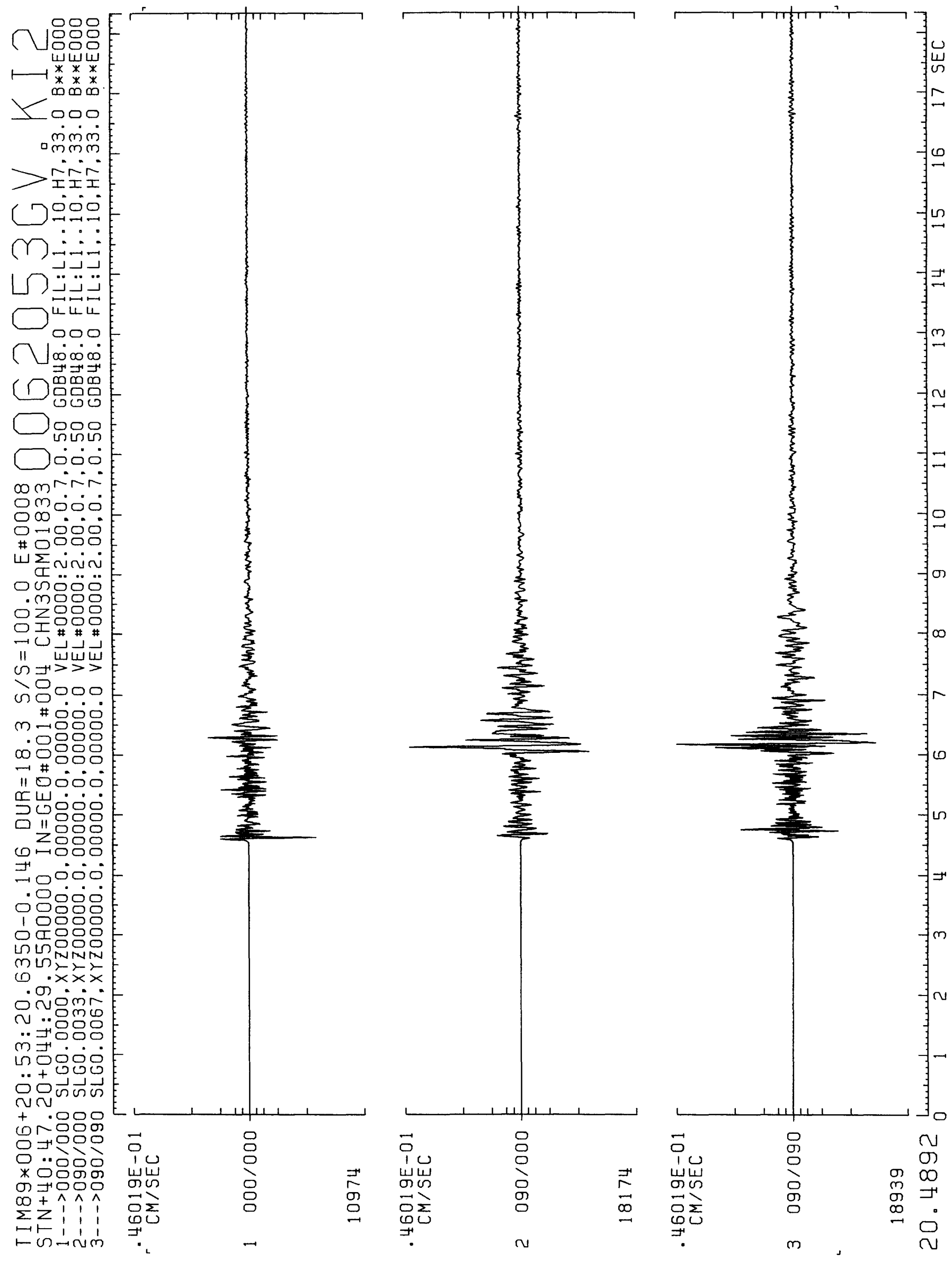


153
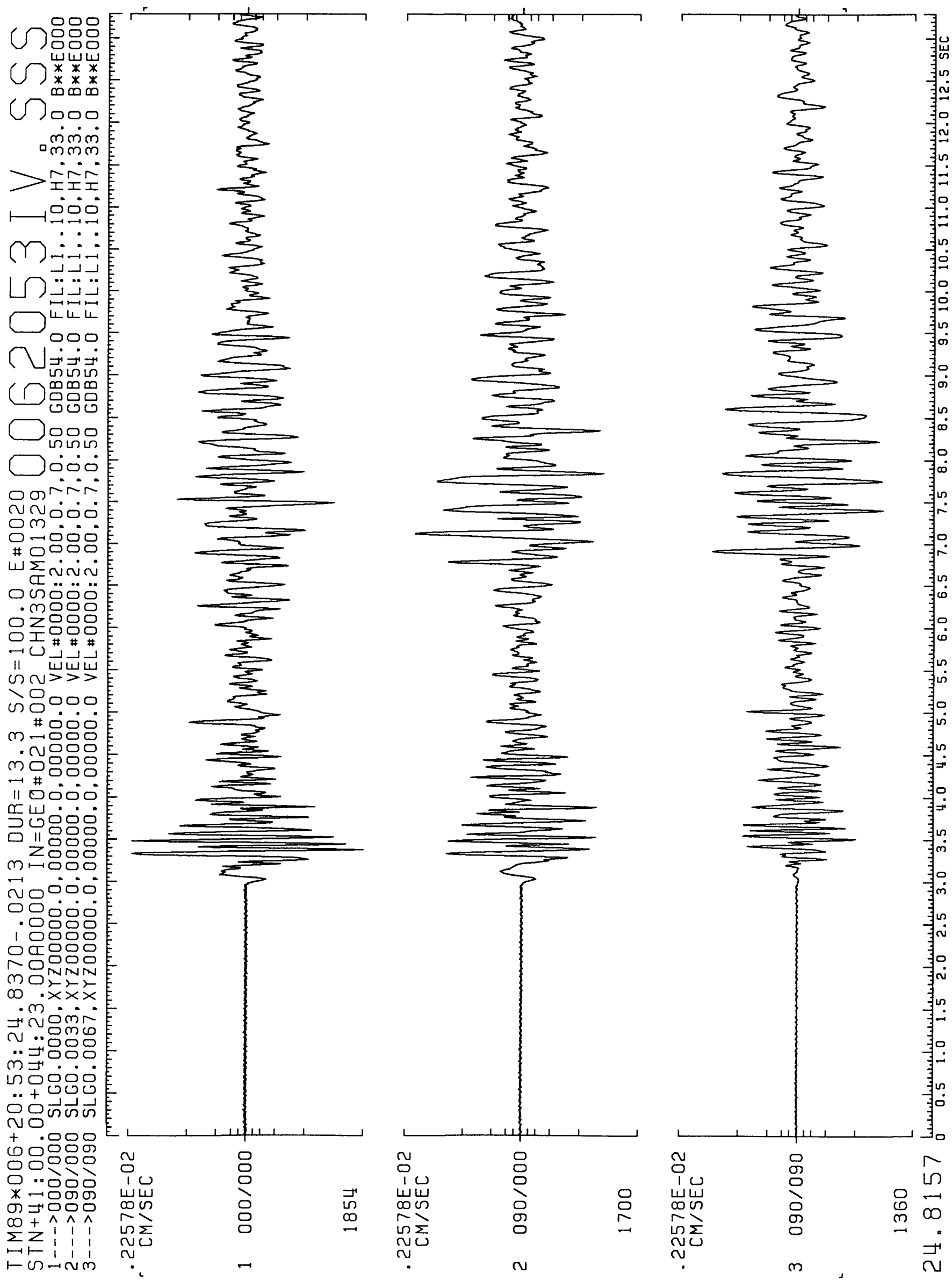
154

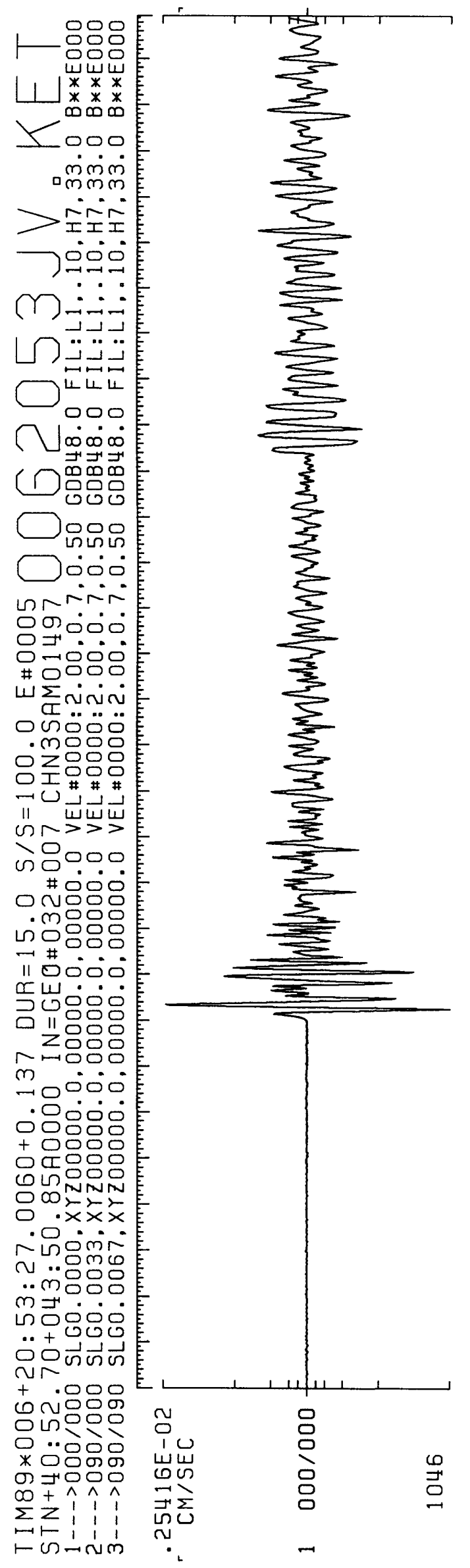

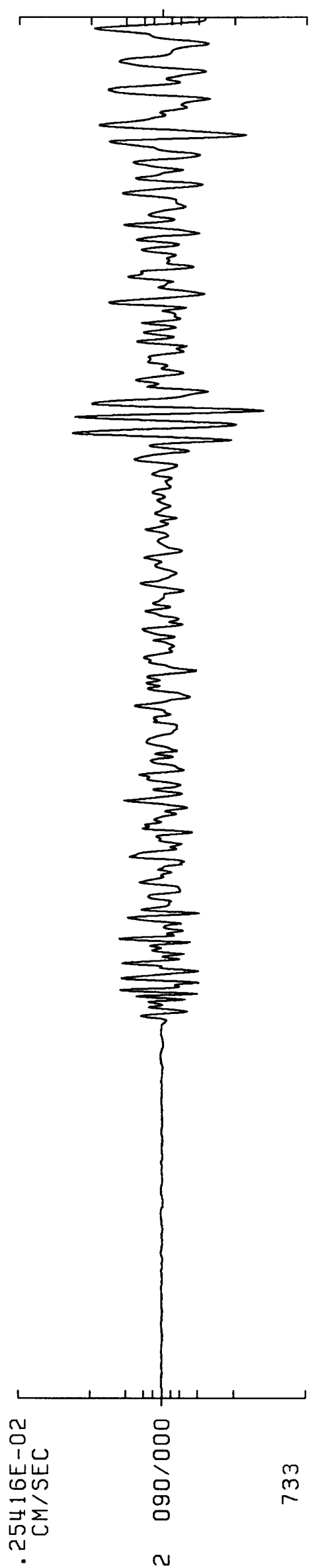

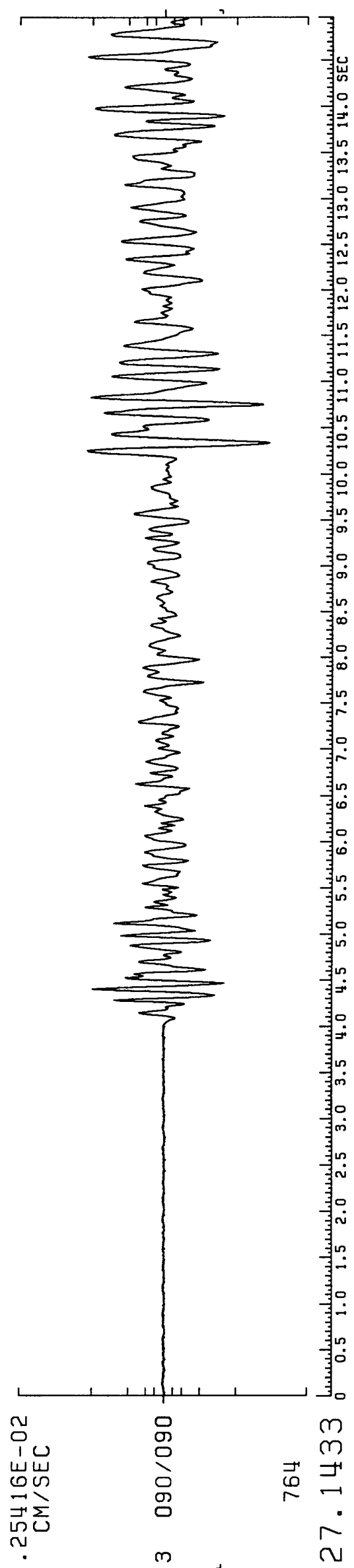




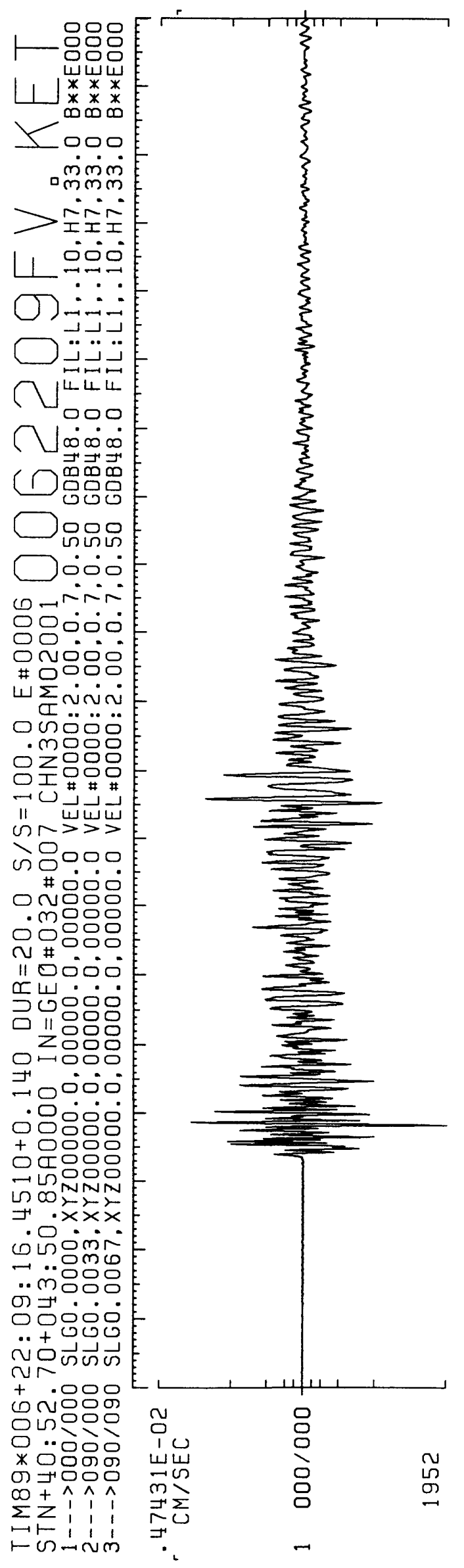

155

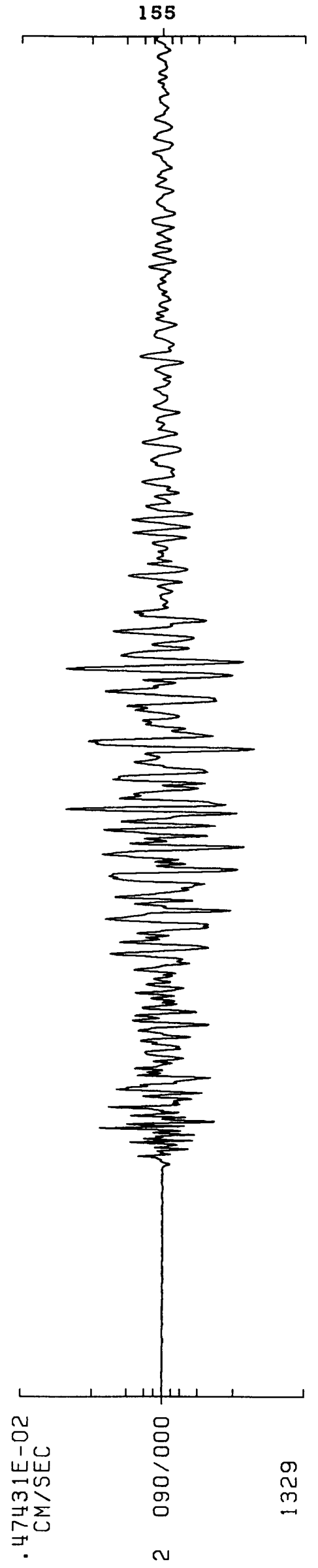

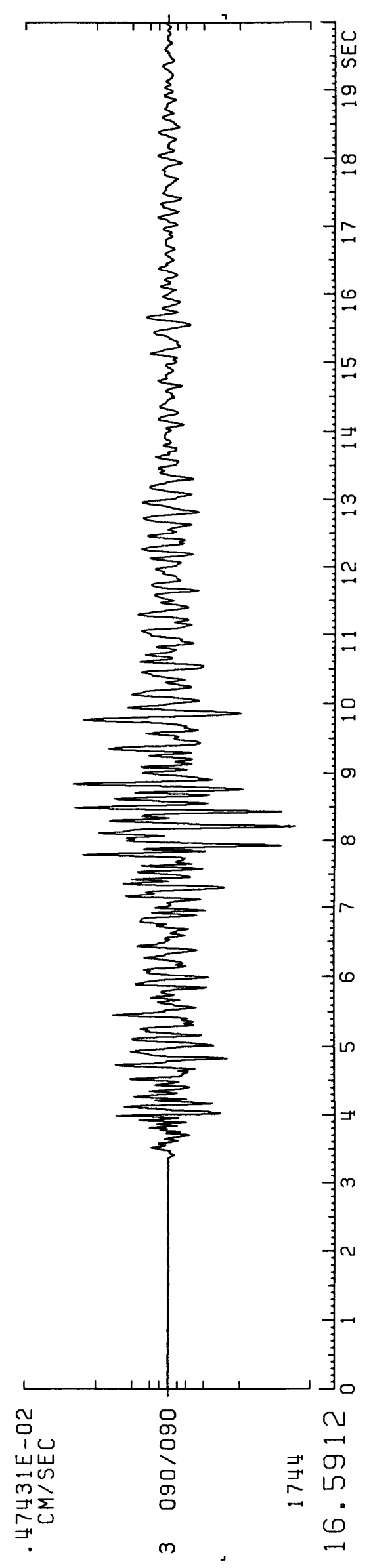


156
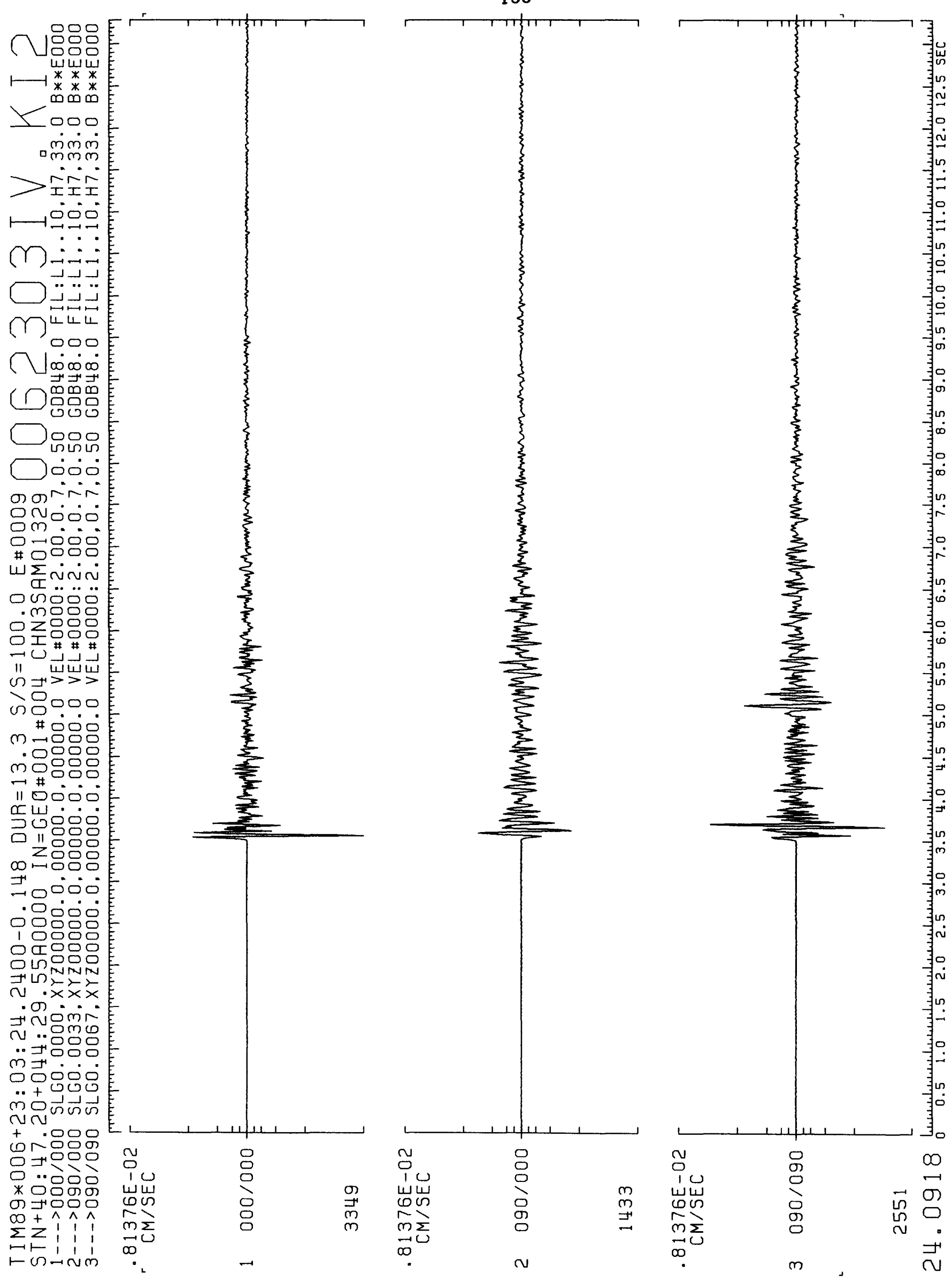


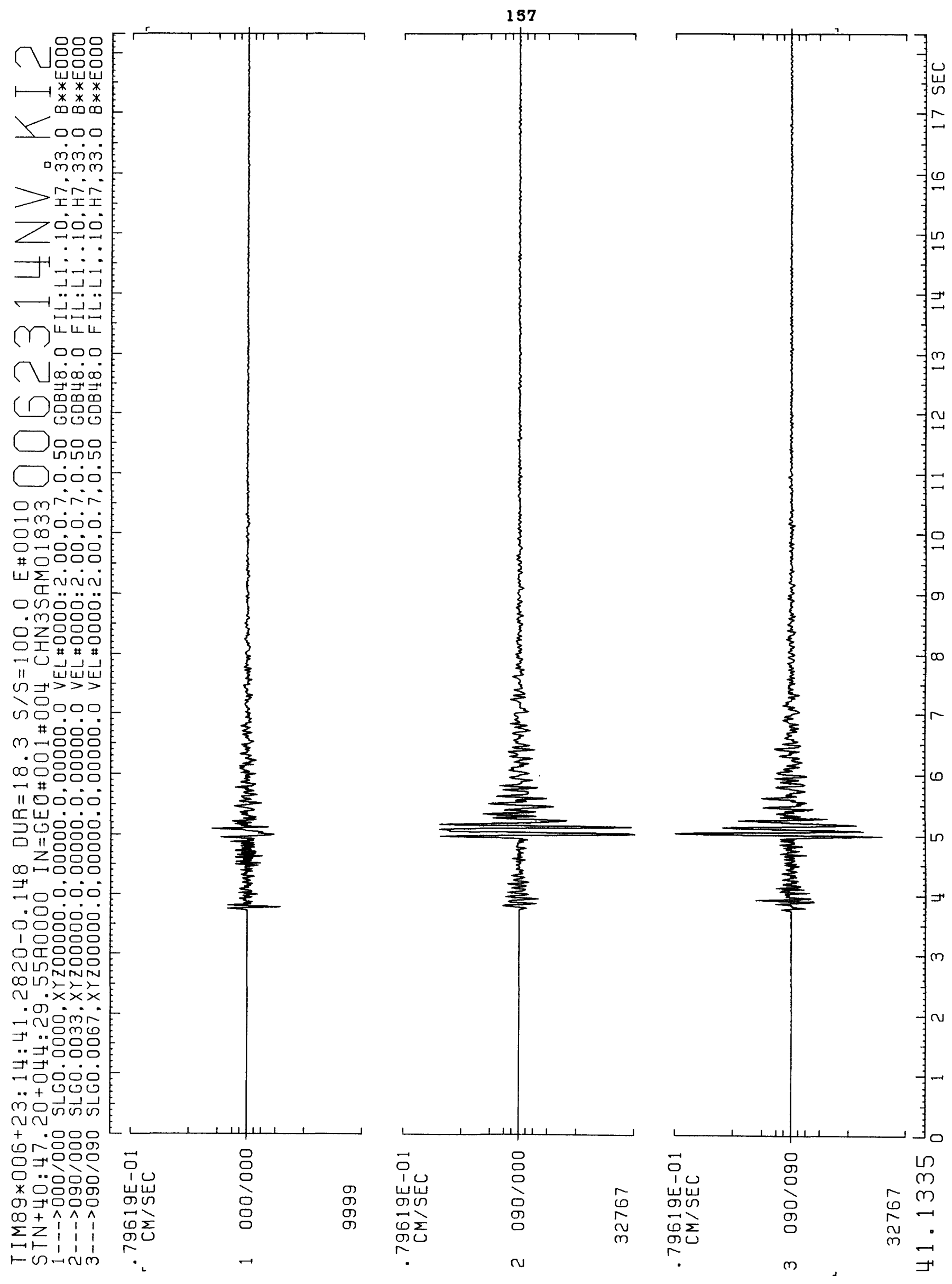


158

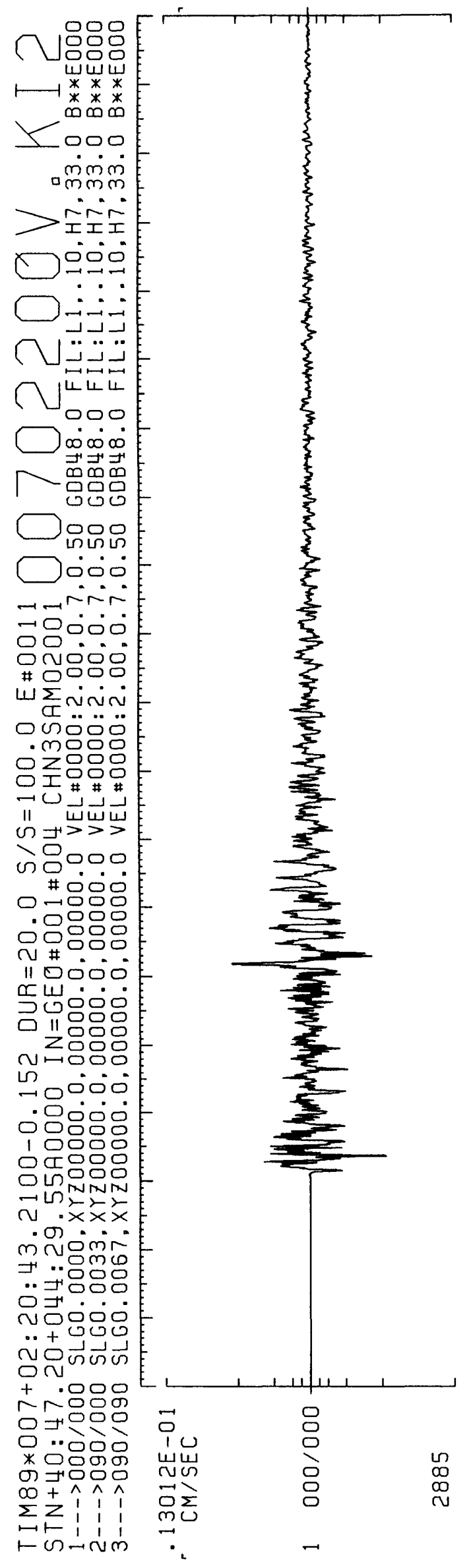

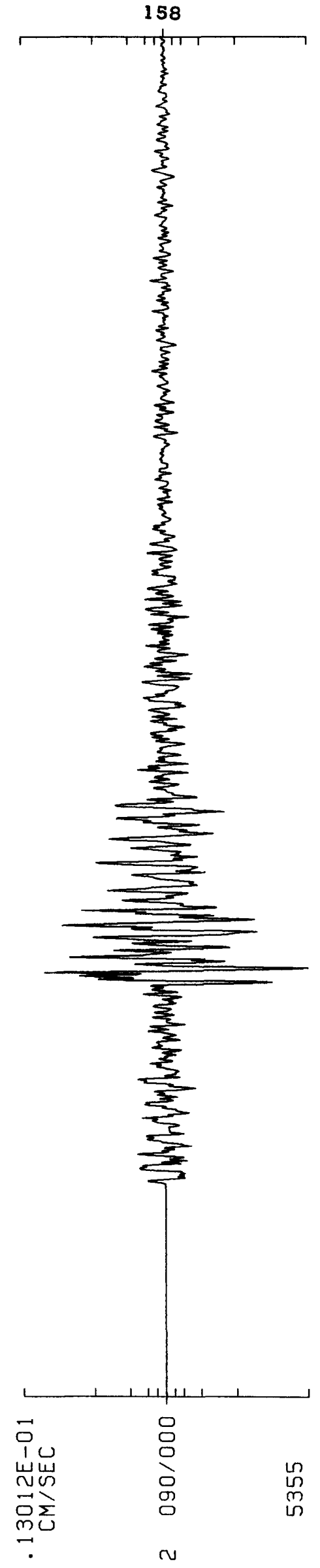

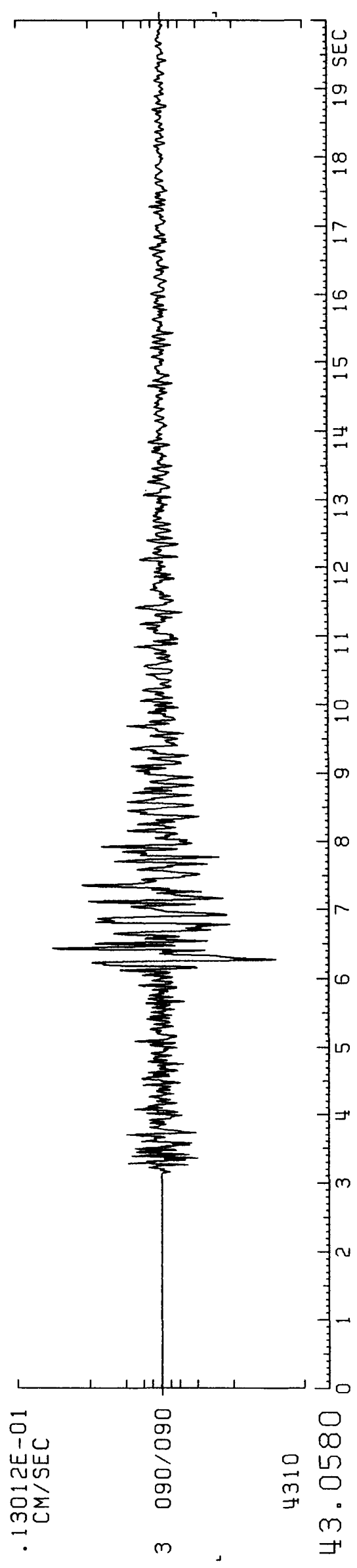




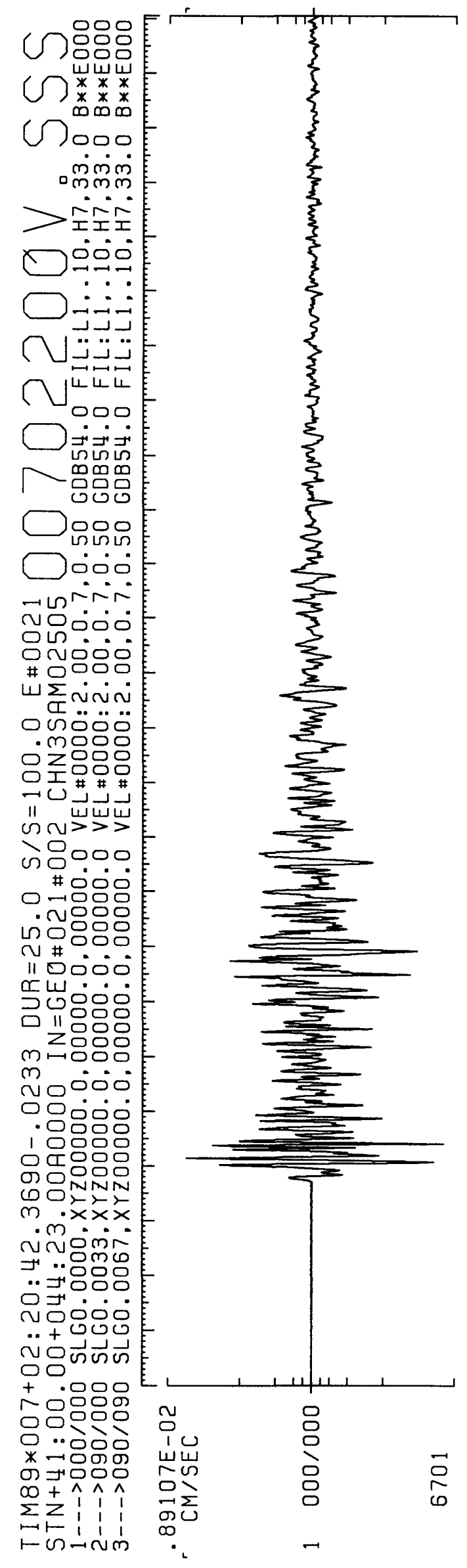

159

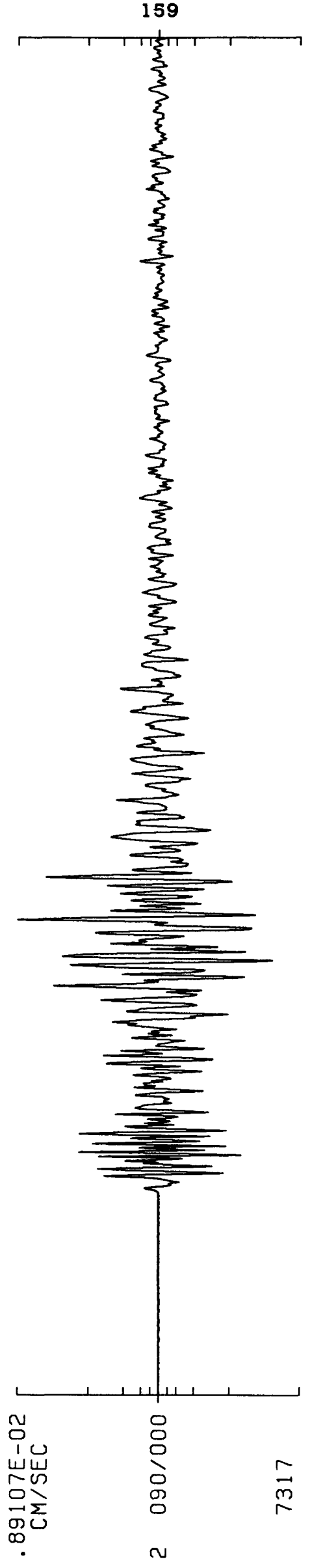

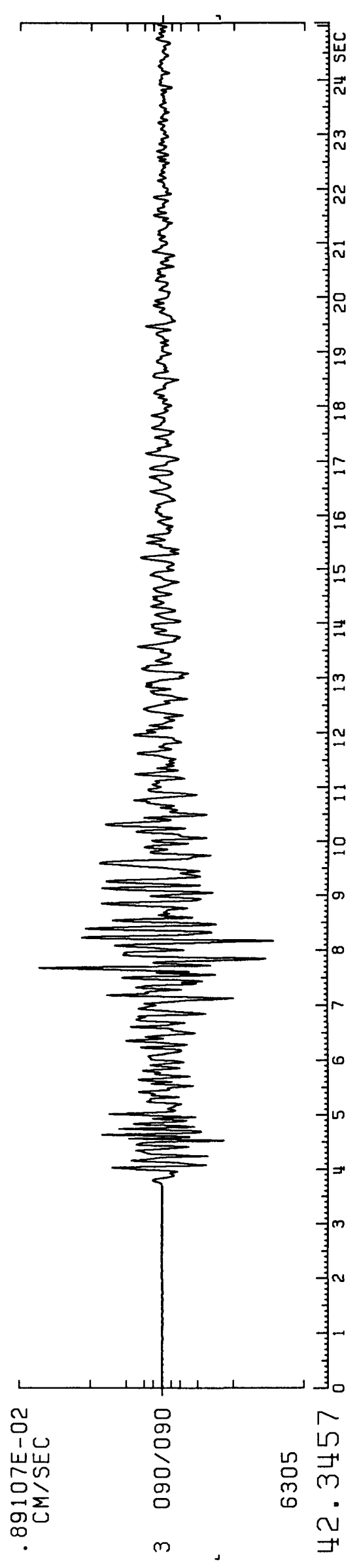


160

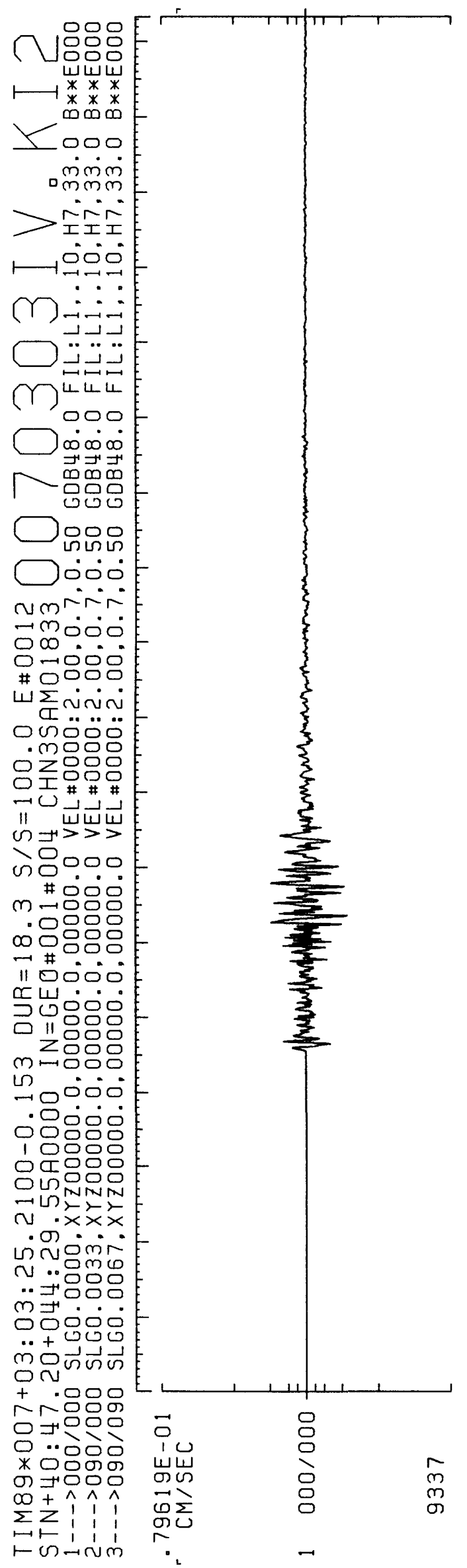

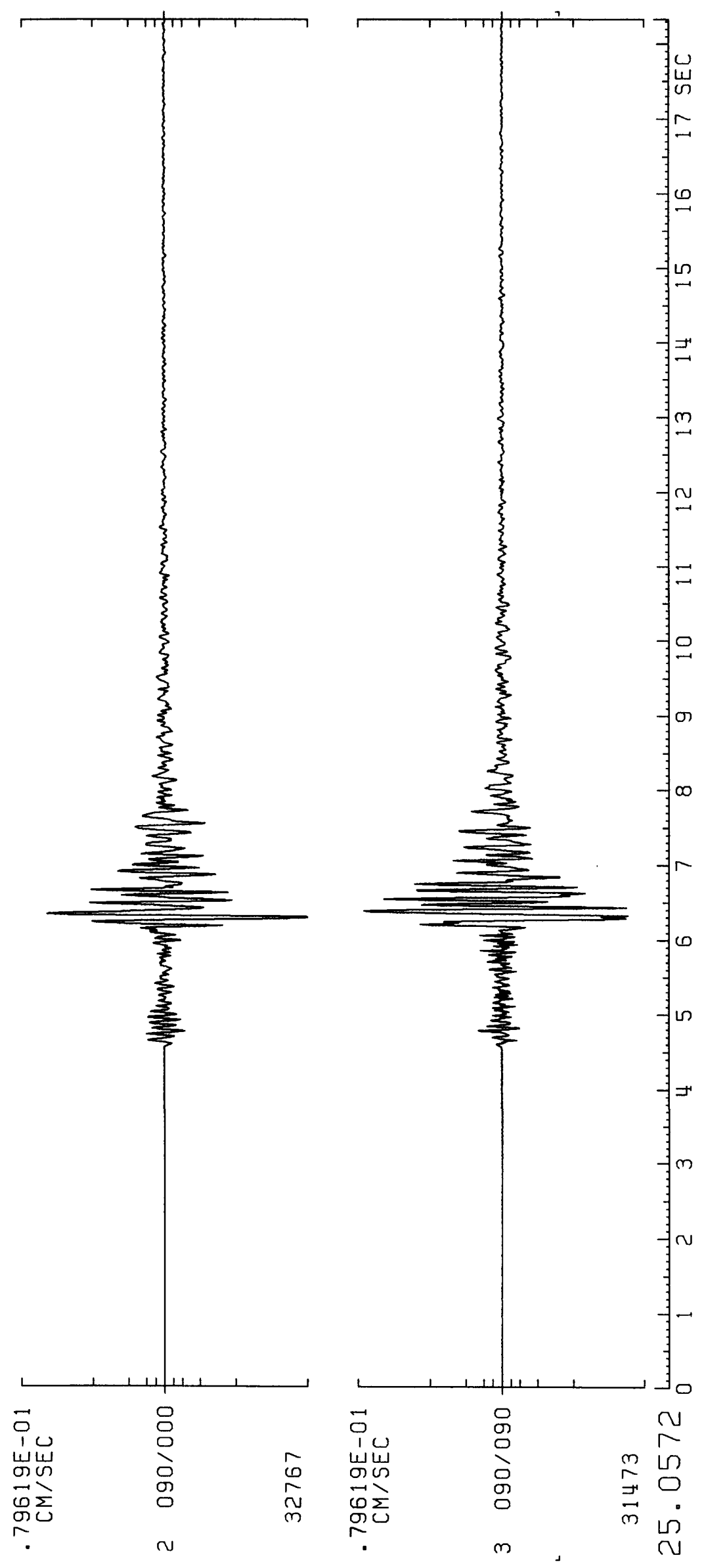


161

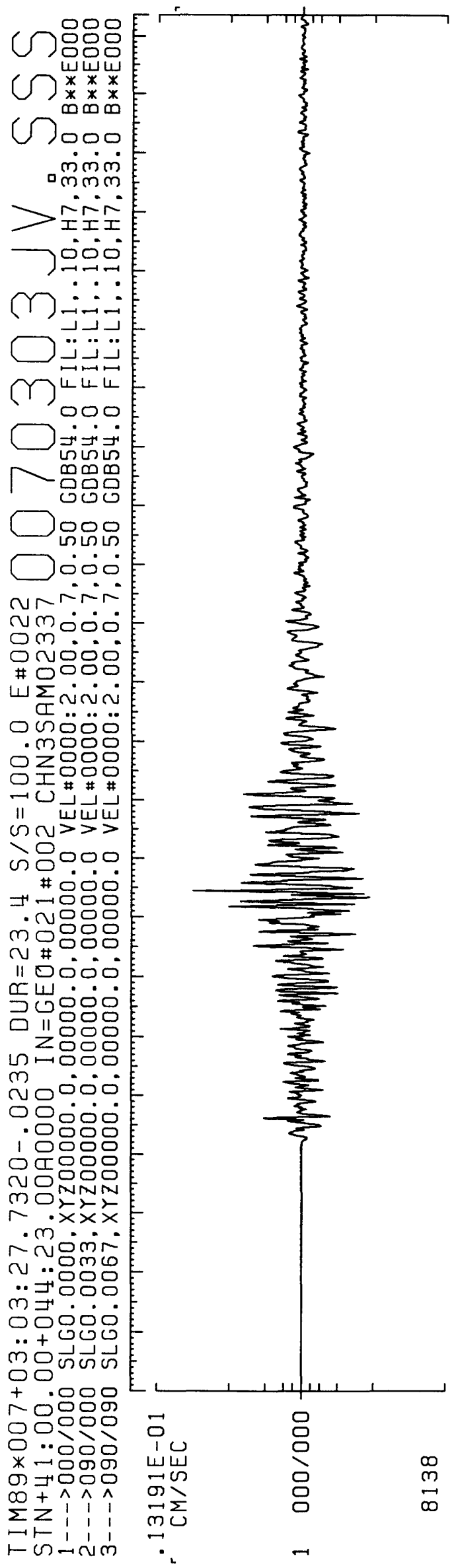

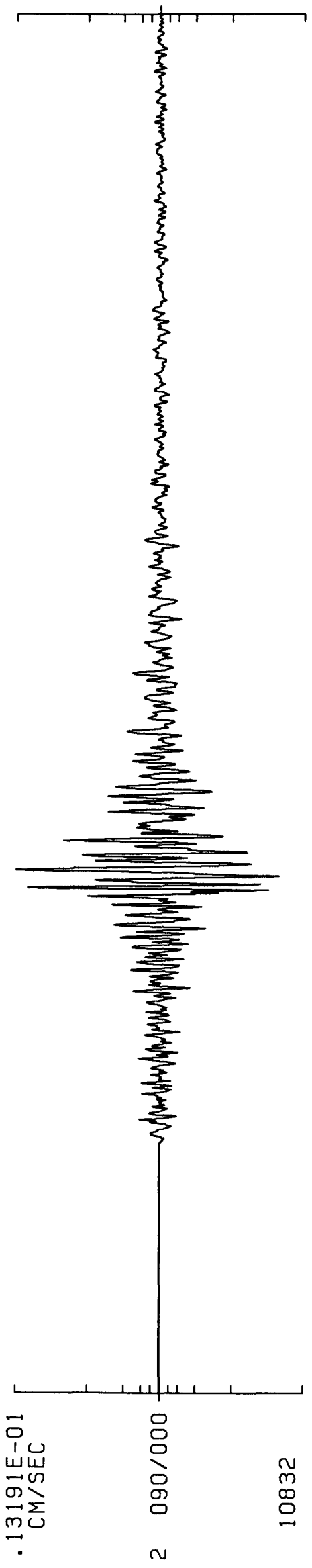

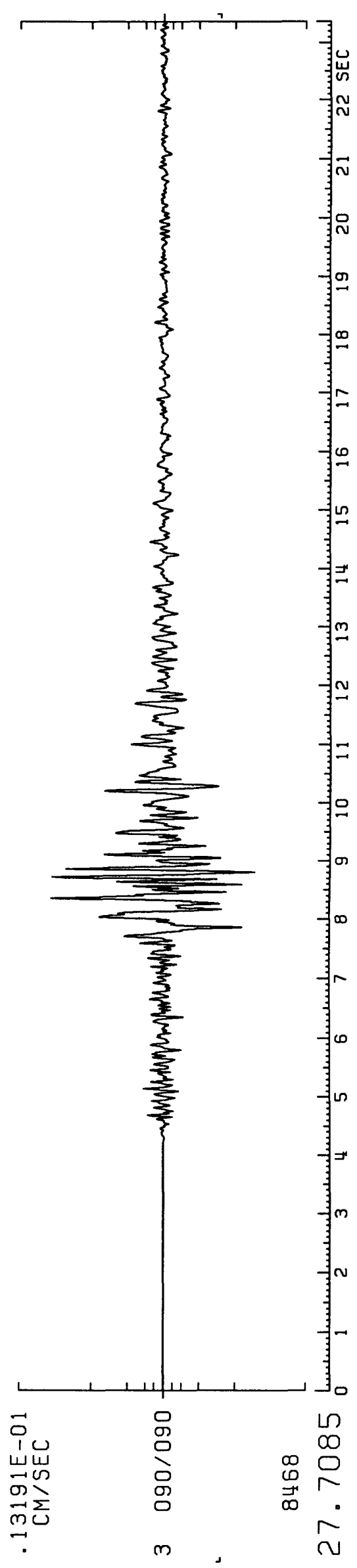


162

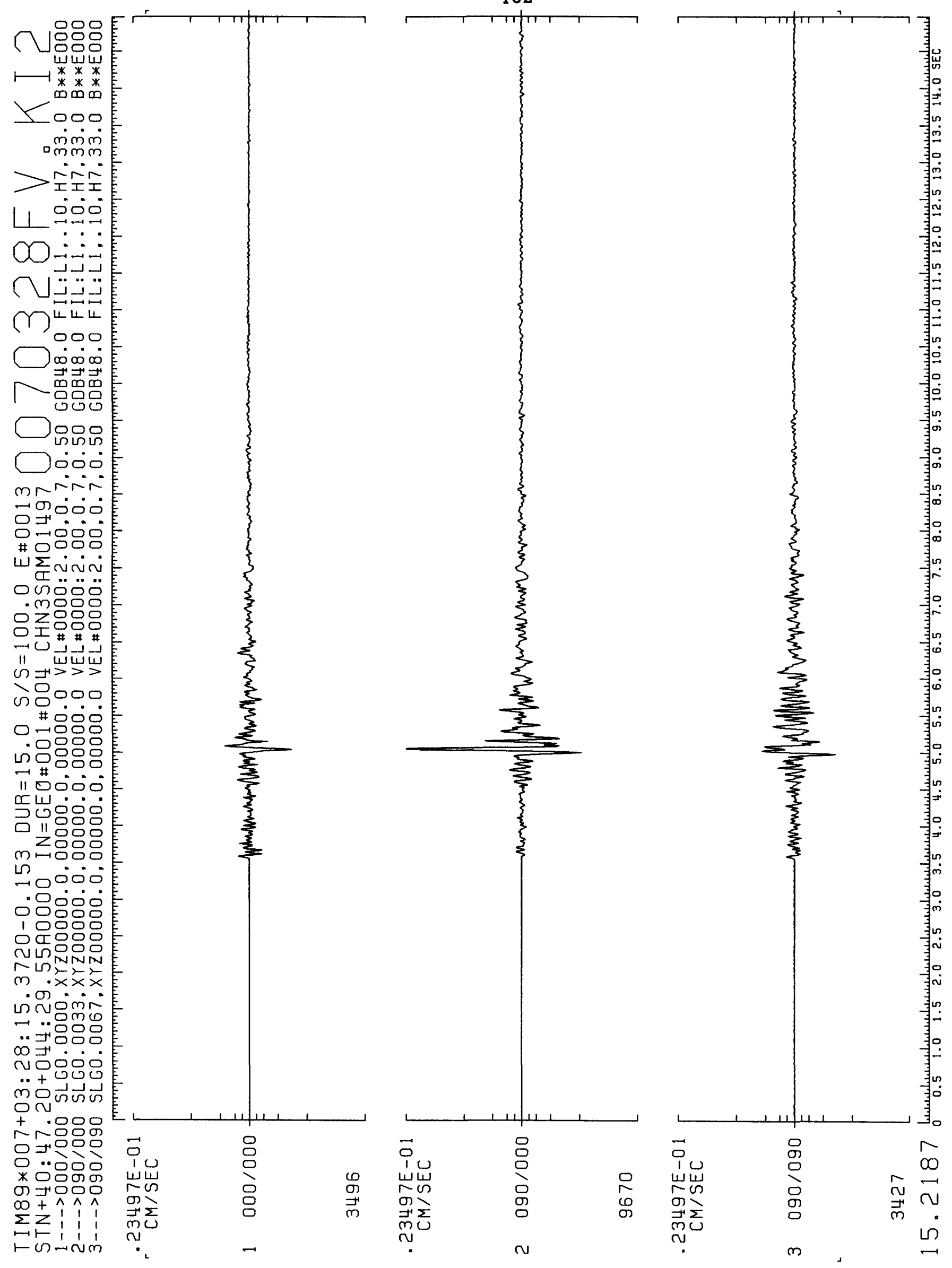




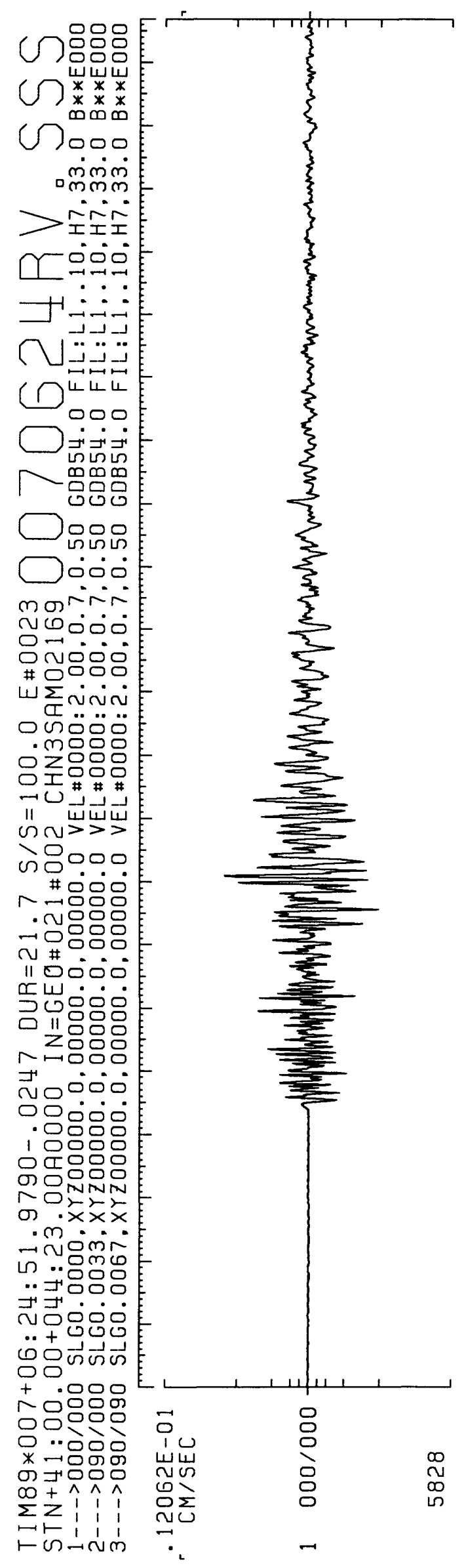

163

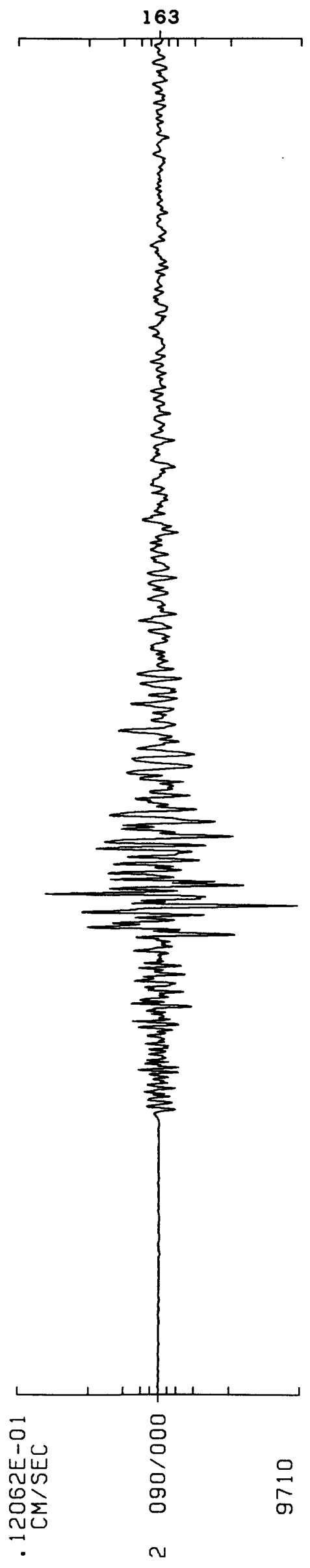

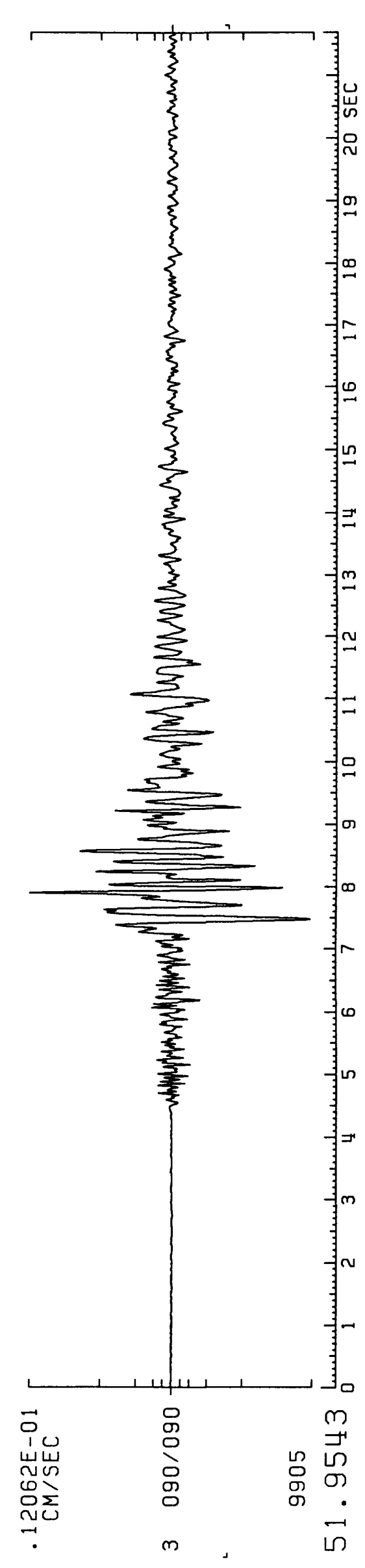


164

$\checkmark$ 응ㅇㅁㅇㅁㅇ

(口)

Tooo $\dot{m} \dot{m} \dot{m}$ mmm iñ

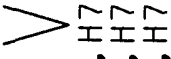

(1)무음

$\checkmark \cap \therefore:$

凷 ニコニ

(口uk 000

$\square$ نं.0

下品品品 जo 으우 $\square$ ○ं० च借

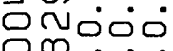
\# ए $\sum_{\pi}$ iñ ○ज品 mㅡㅇㅇㅇㅇㅁㅇ 오뮴유 一艺 \#\# ॥ ヘゴメ के ज०

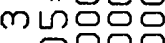
m응ㅁㅇㅁㅇ mo 구유. 띵요 ○ z응ㅇㅇ mm응ㅇㅁ ص००00 응요 - 0 응으 $+0000$ ○匹이임 J" JNNNN $\infty$.xx . $m \times x$

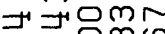
○ ..ㅇํㅇㅁㅜ

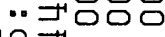
엉요

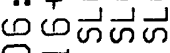
+ $+\infty$ 응응

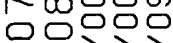
0 ..ㄷㅎㅇ * 임

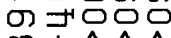
$\infty+\wedge \hat{\imath}$ $\sum_{-1} Z \begin{aligned} & 0 \\ & 1\end{aligned}$ 㦴先向

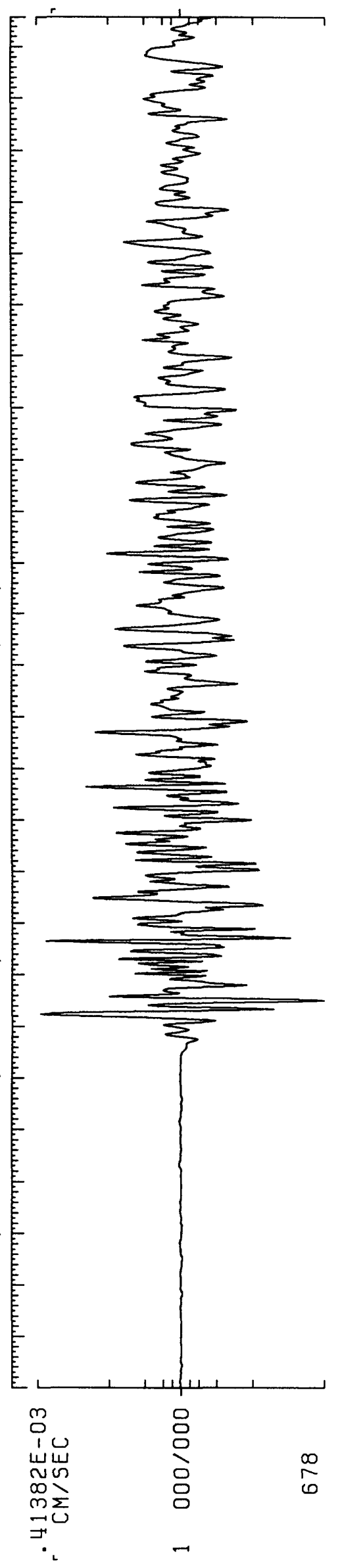

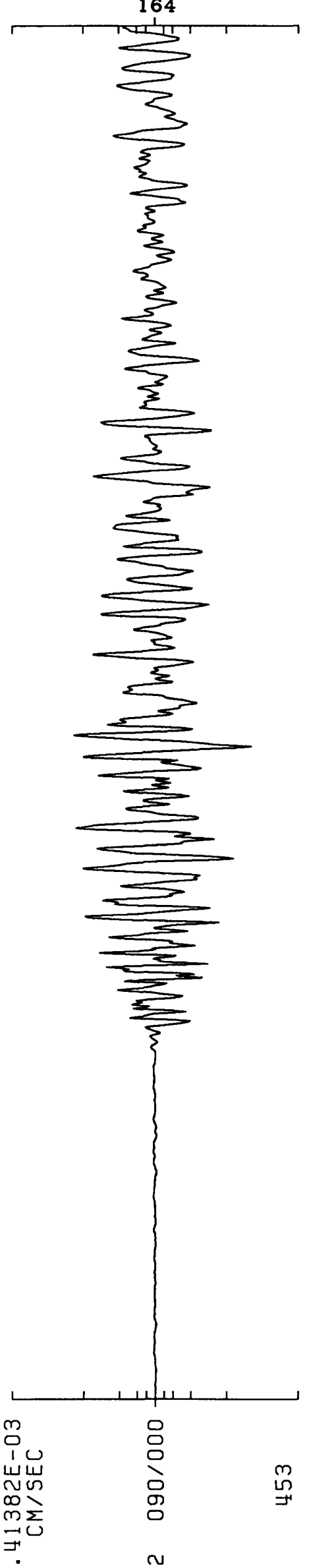

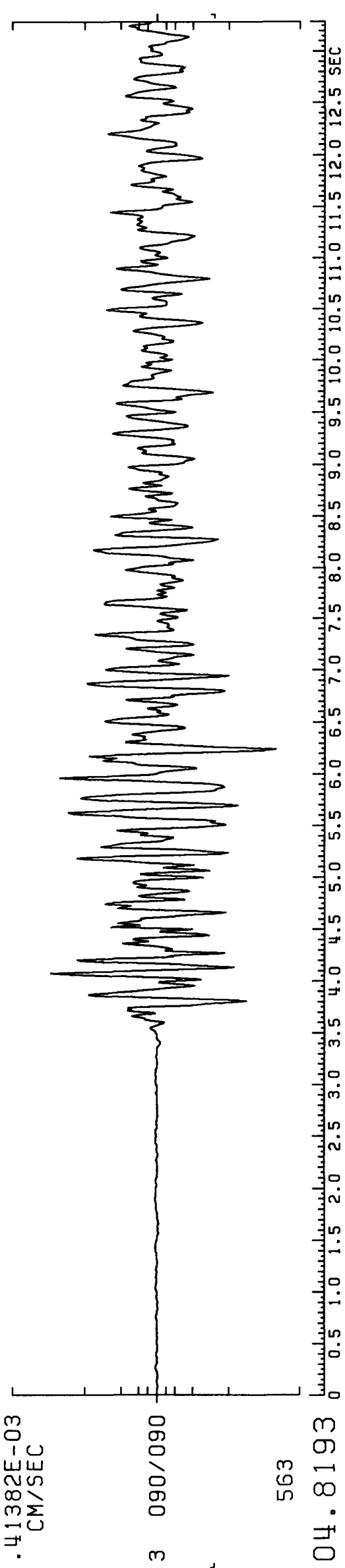




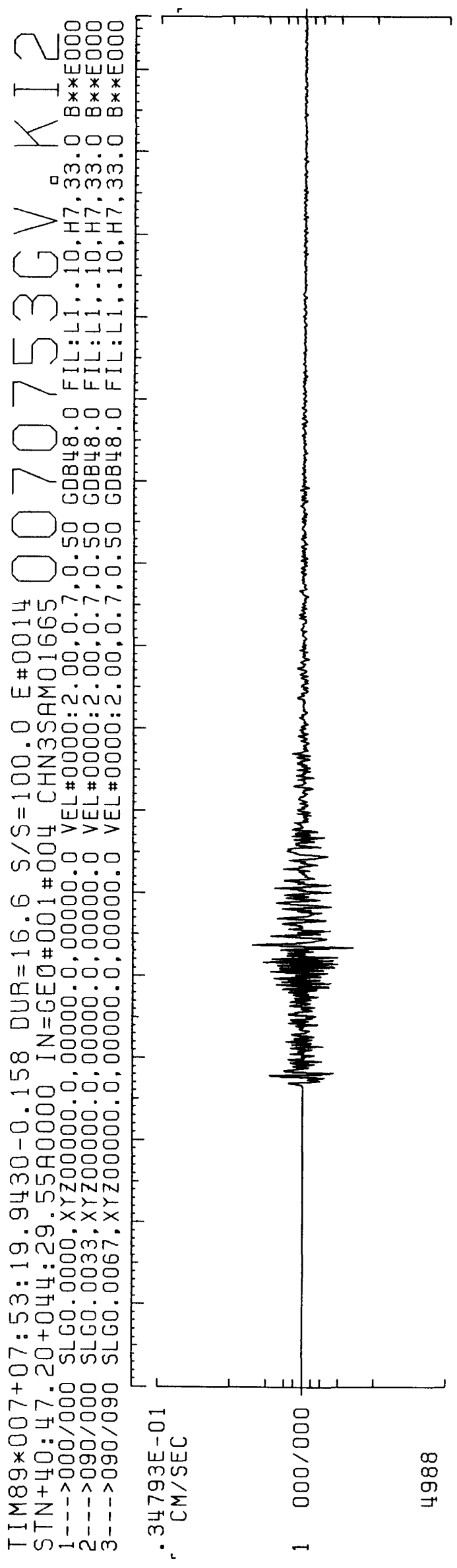

165

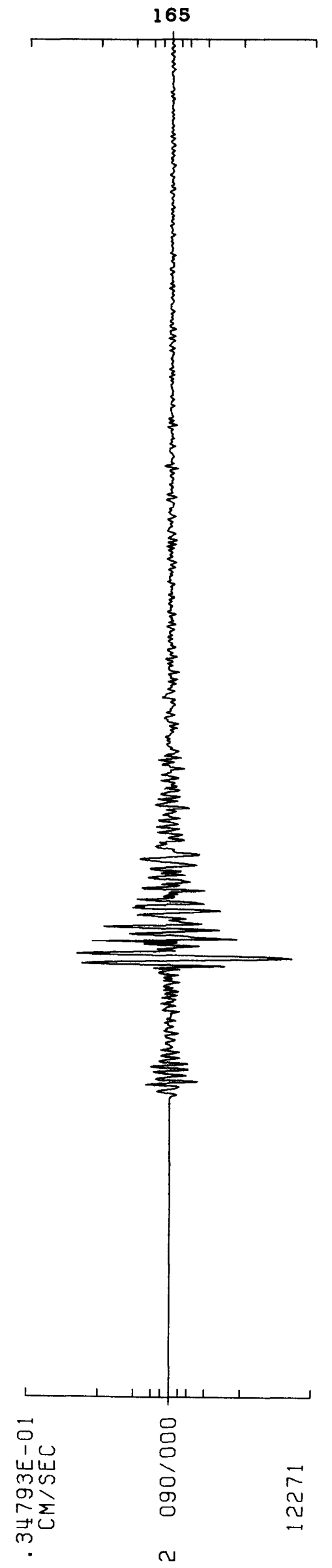

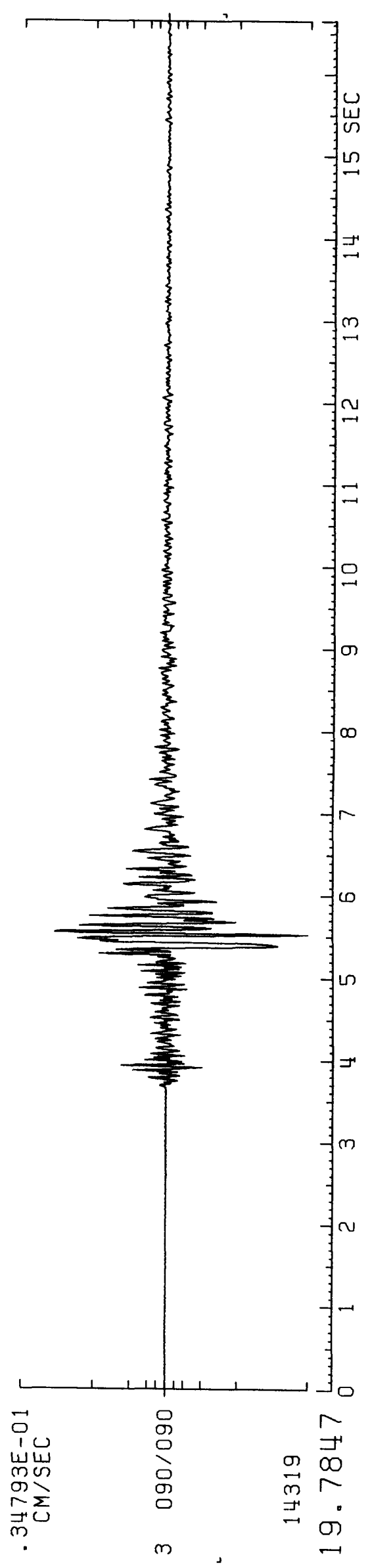



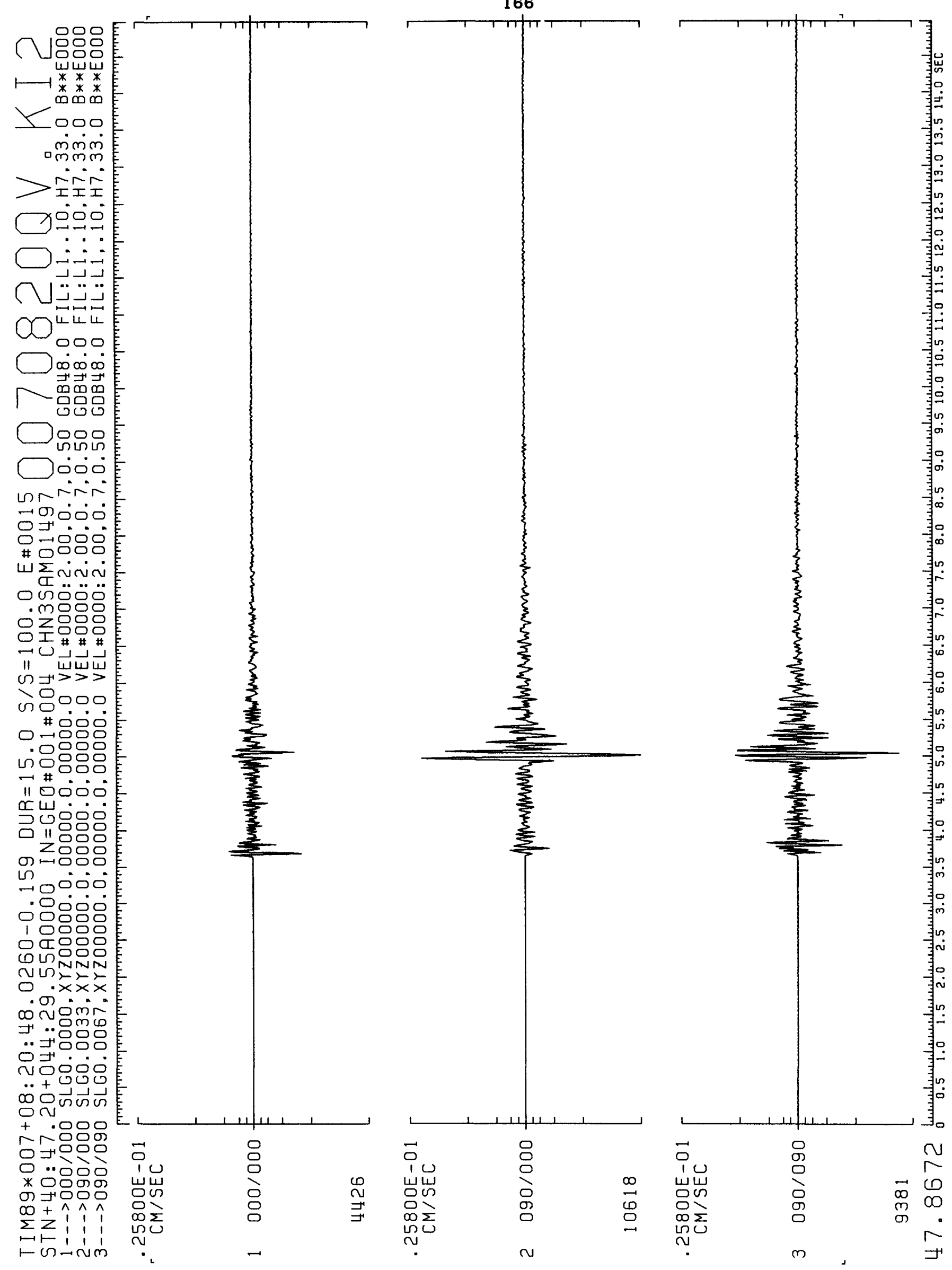
167

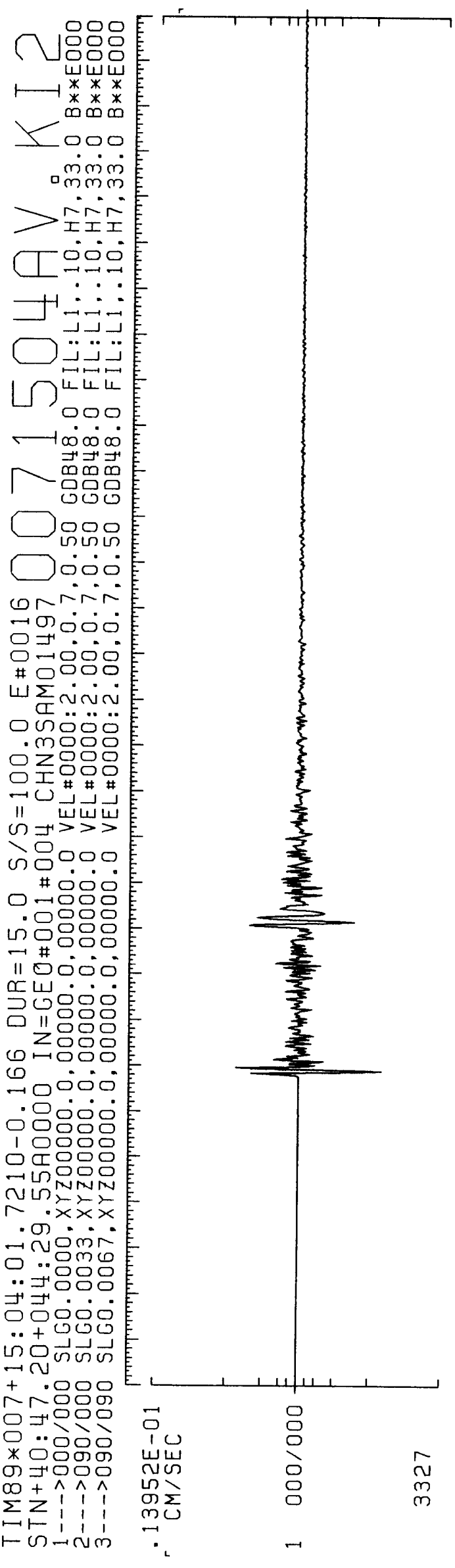

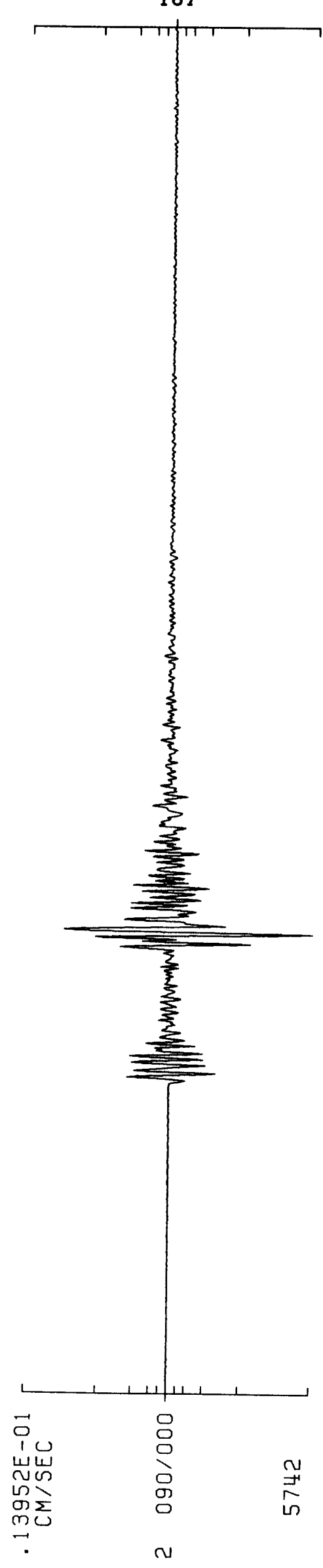

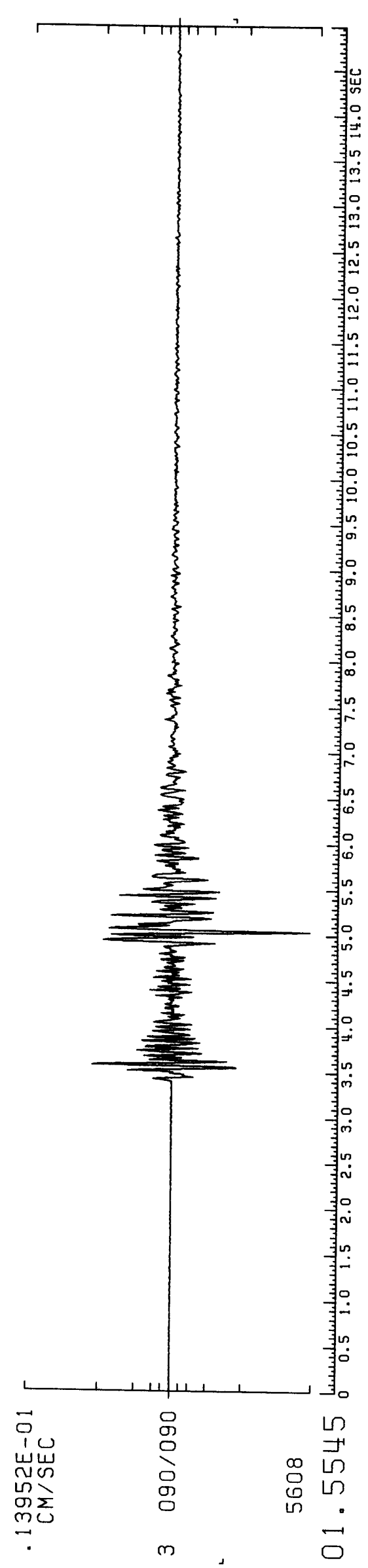


168

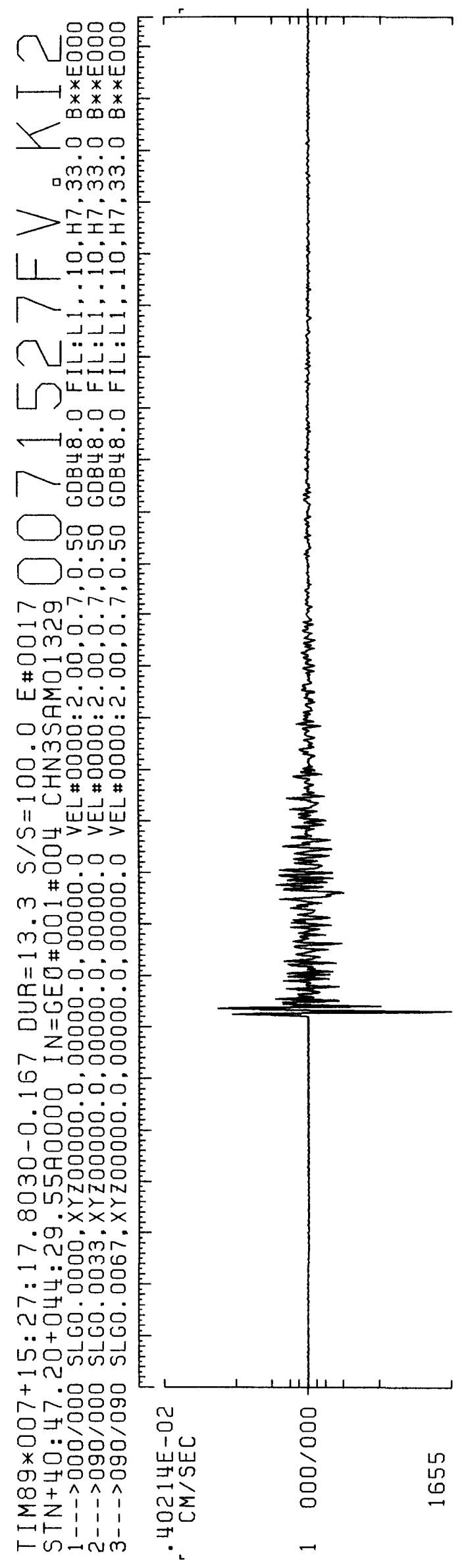

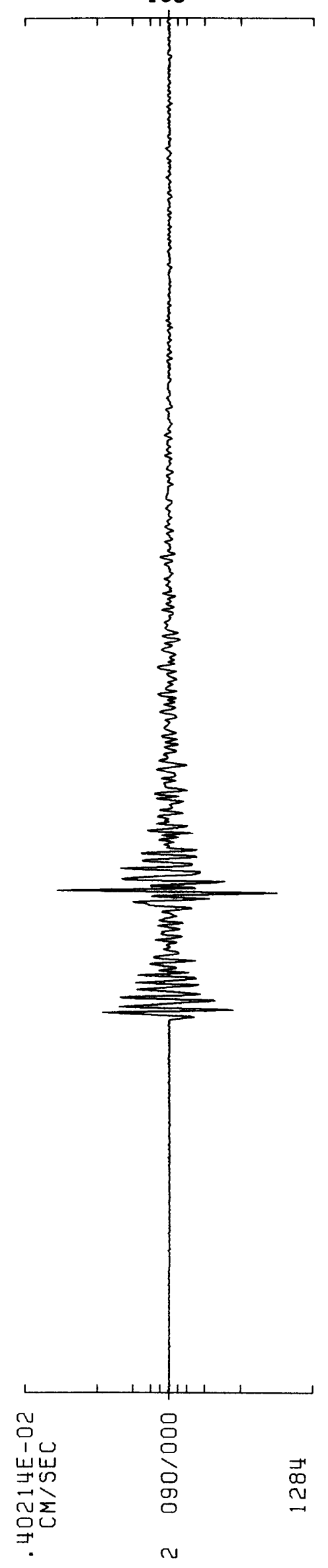

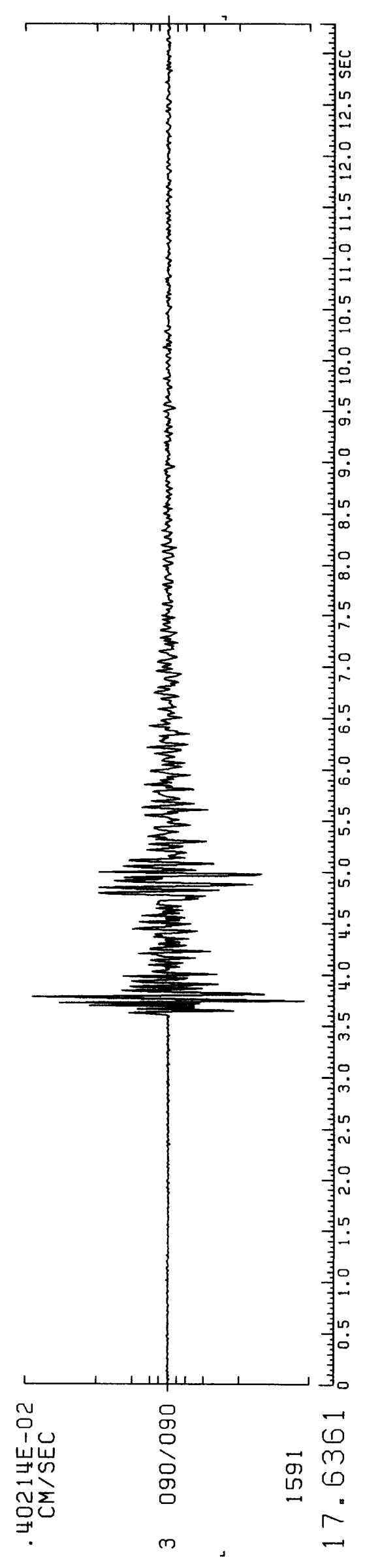


169

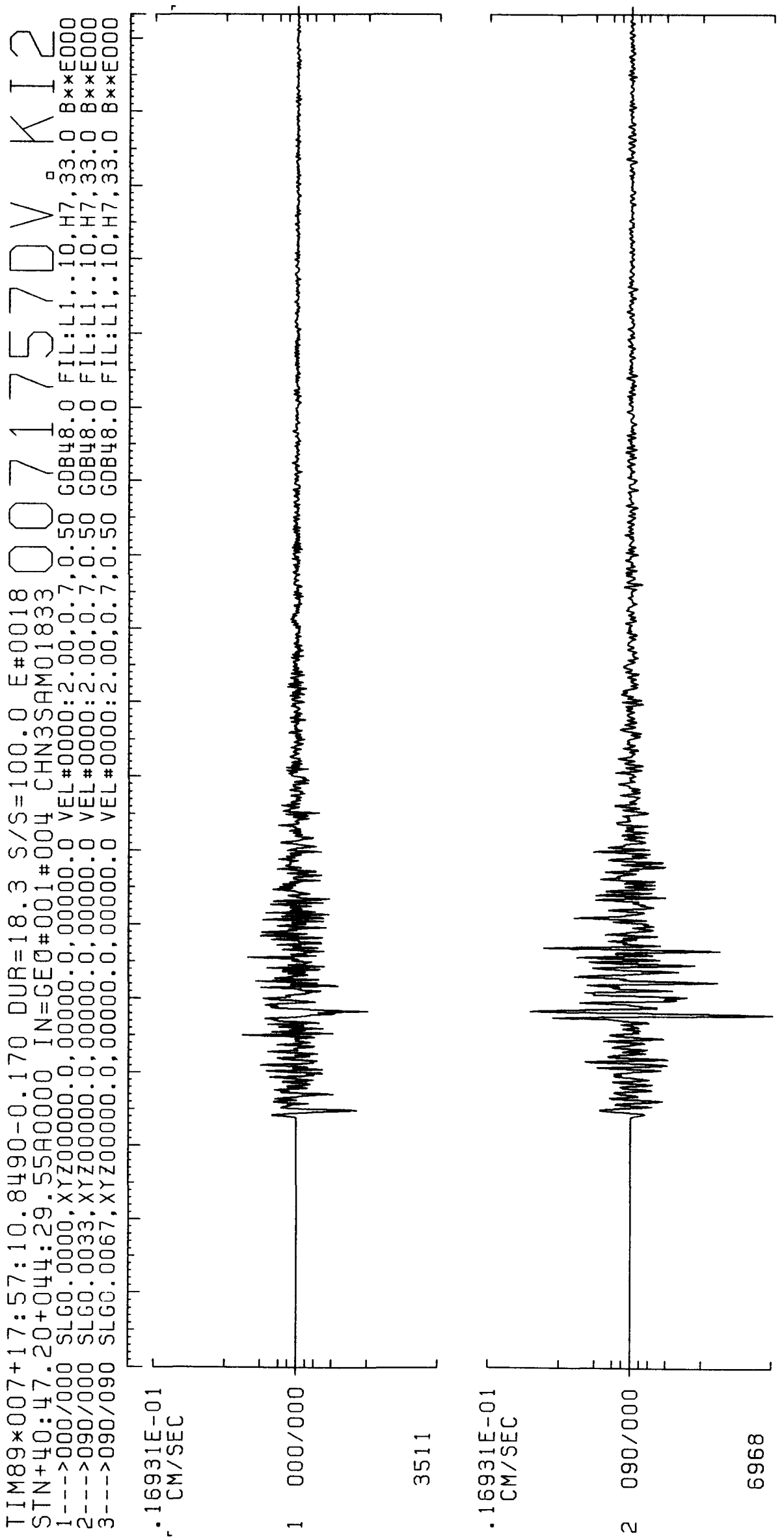

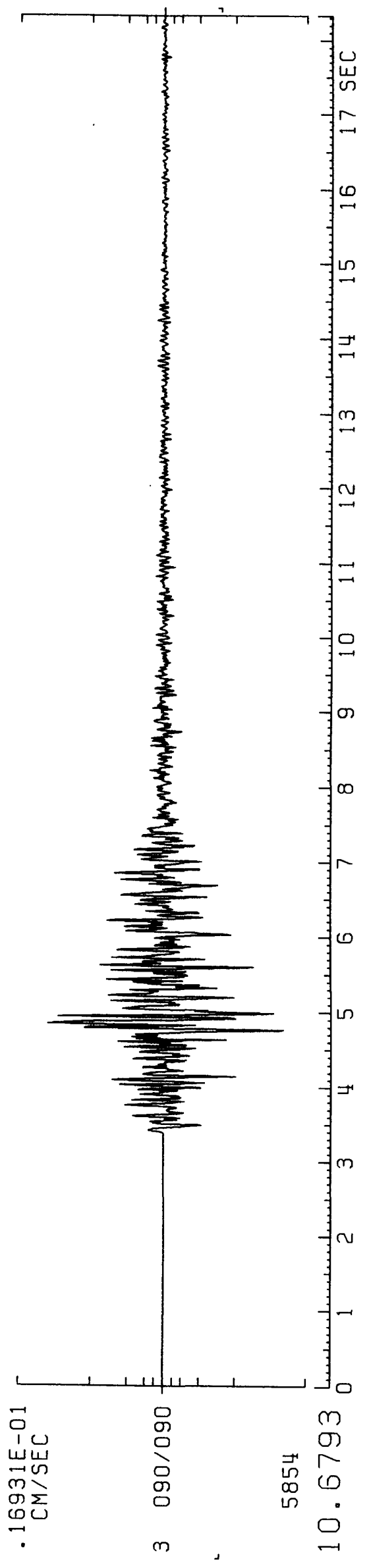


170

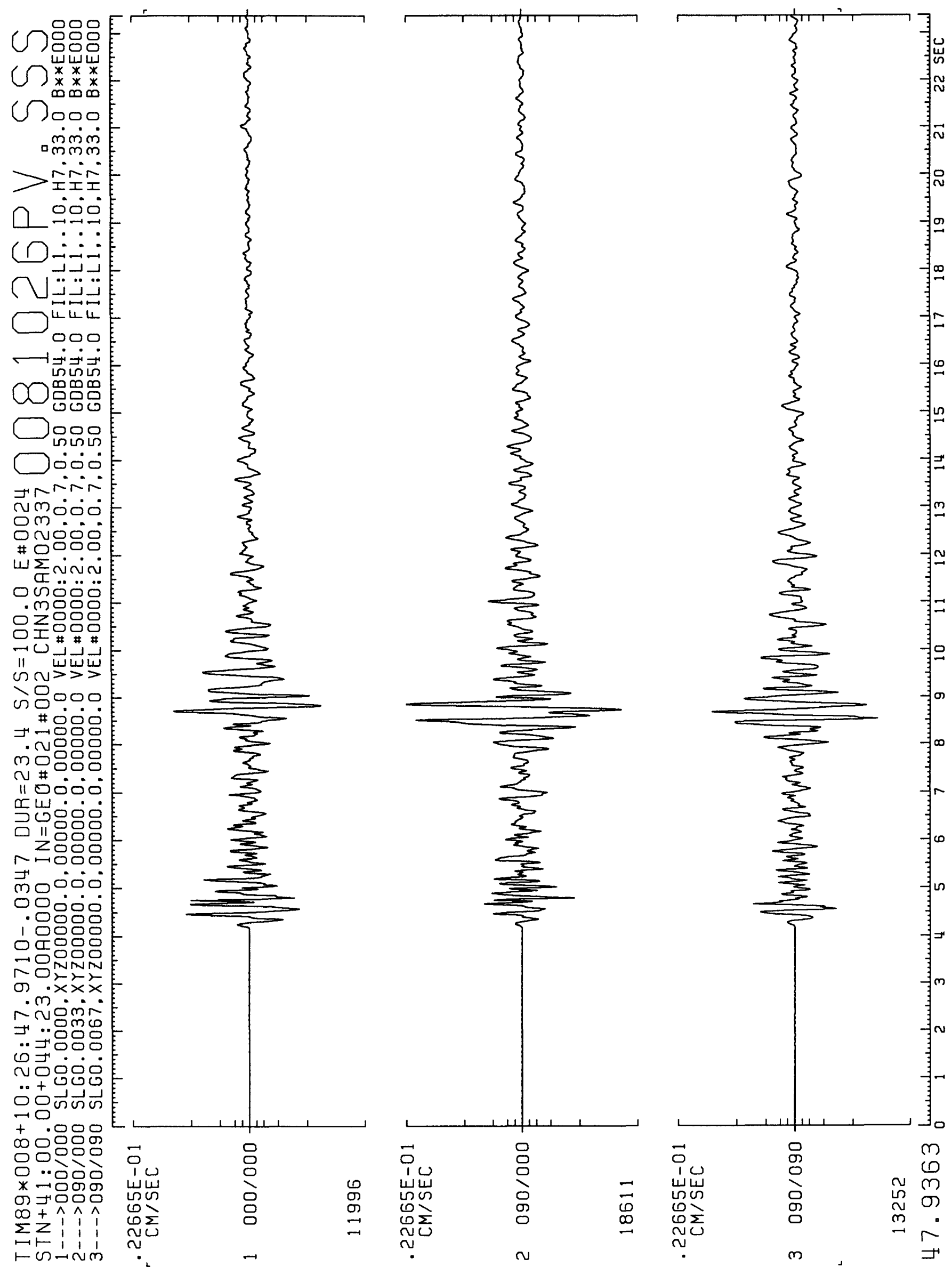


171

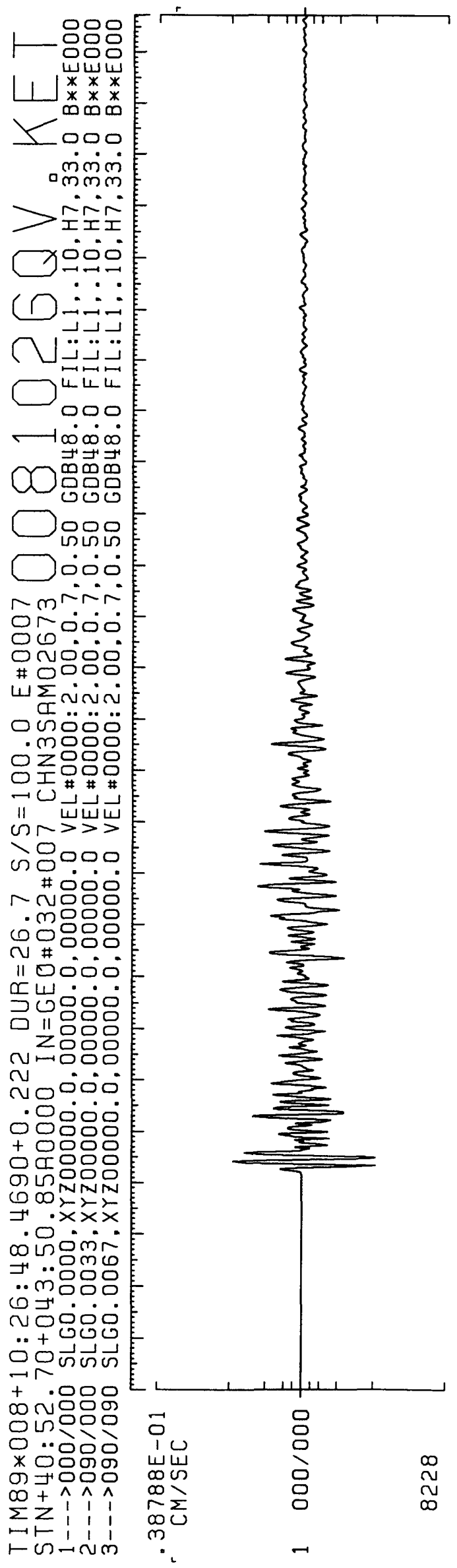

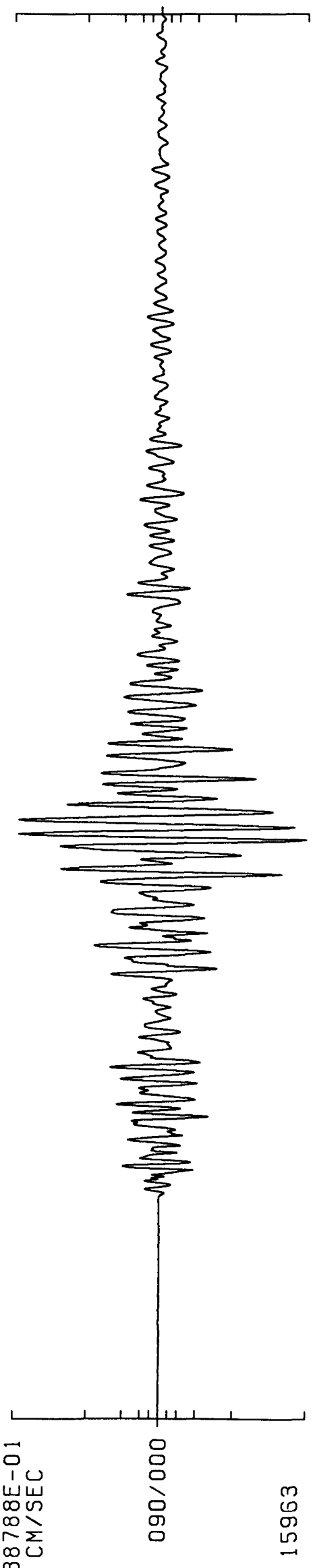

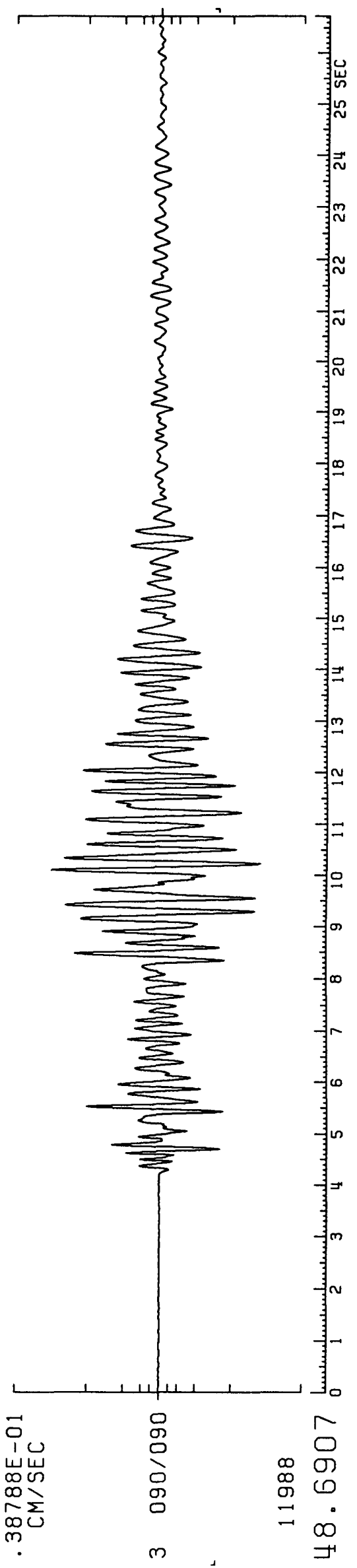


172

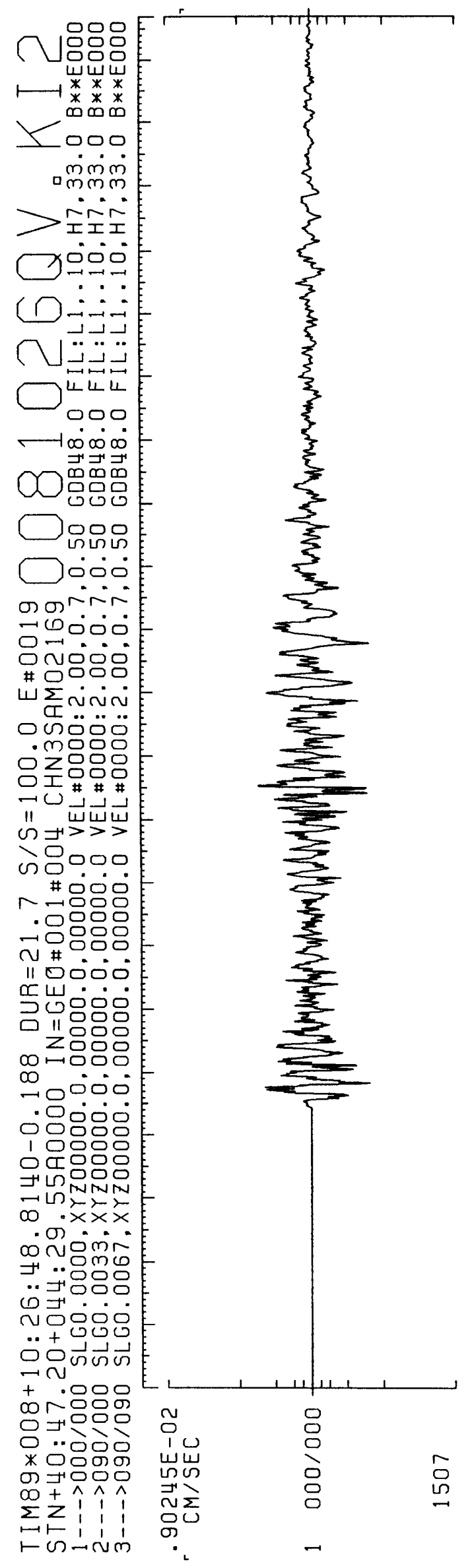

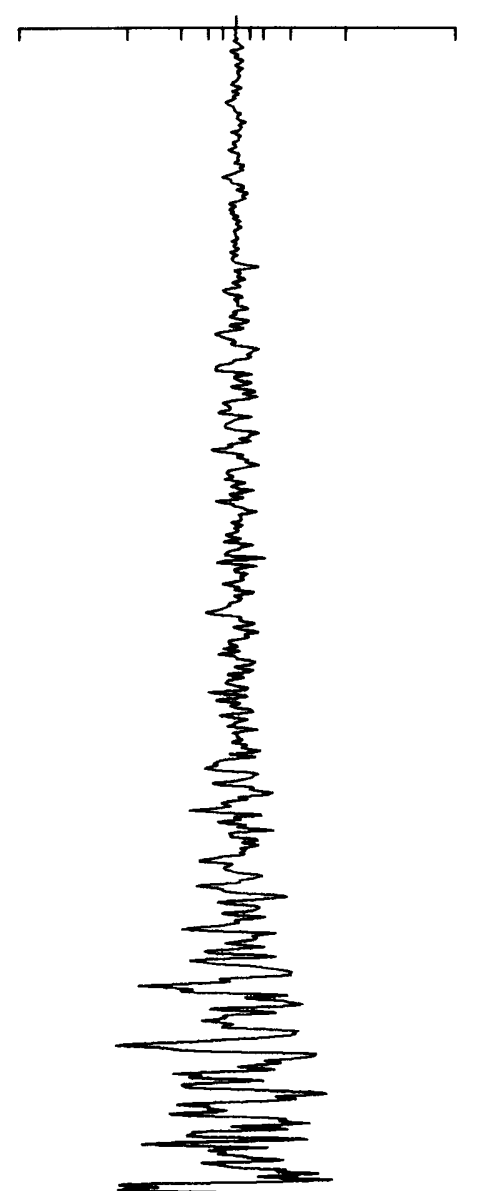
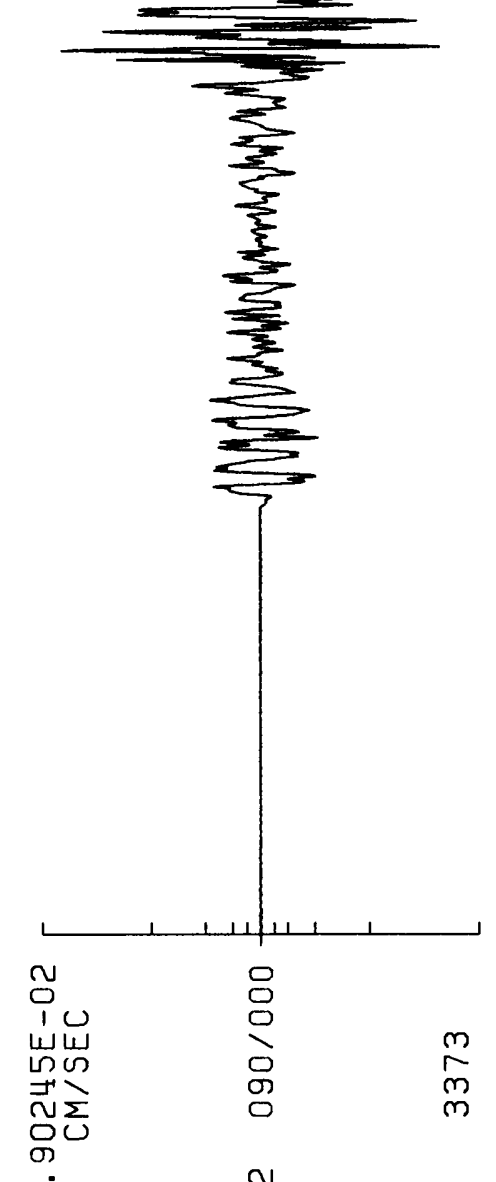

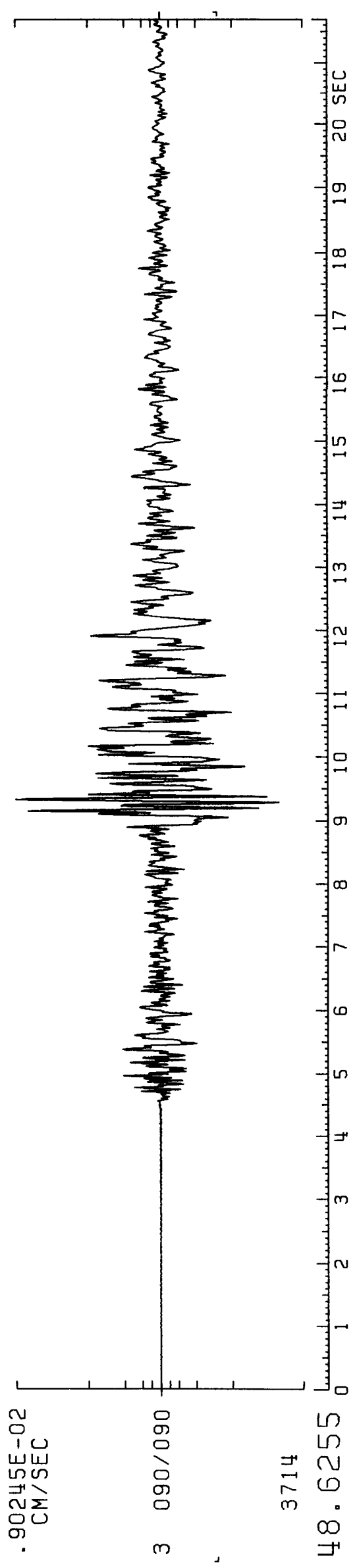


173

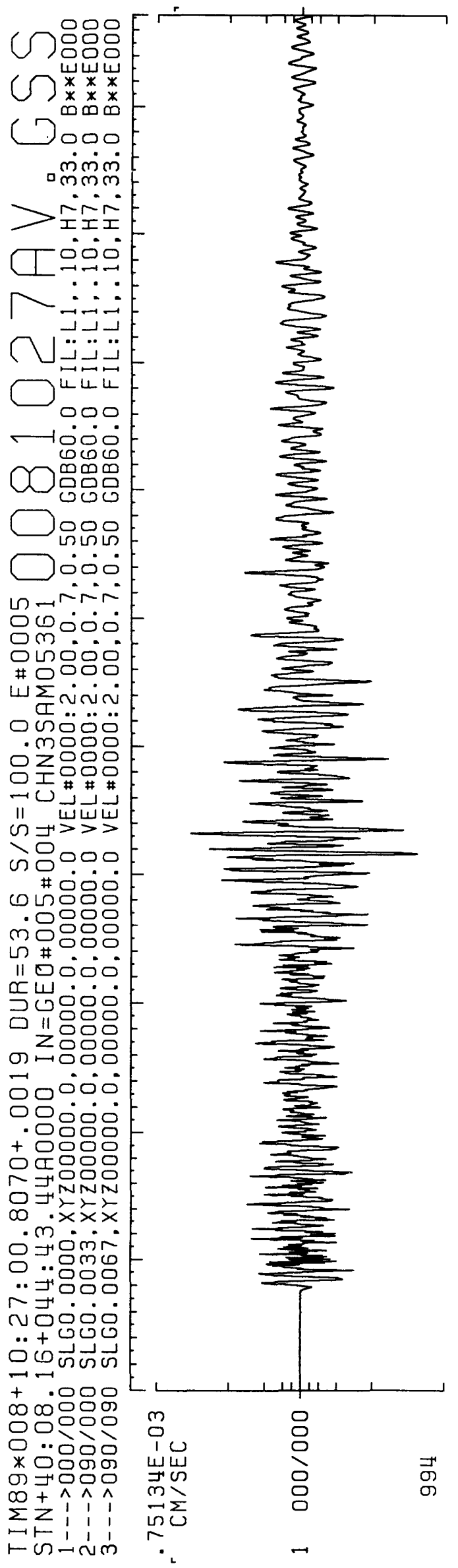

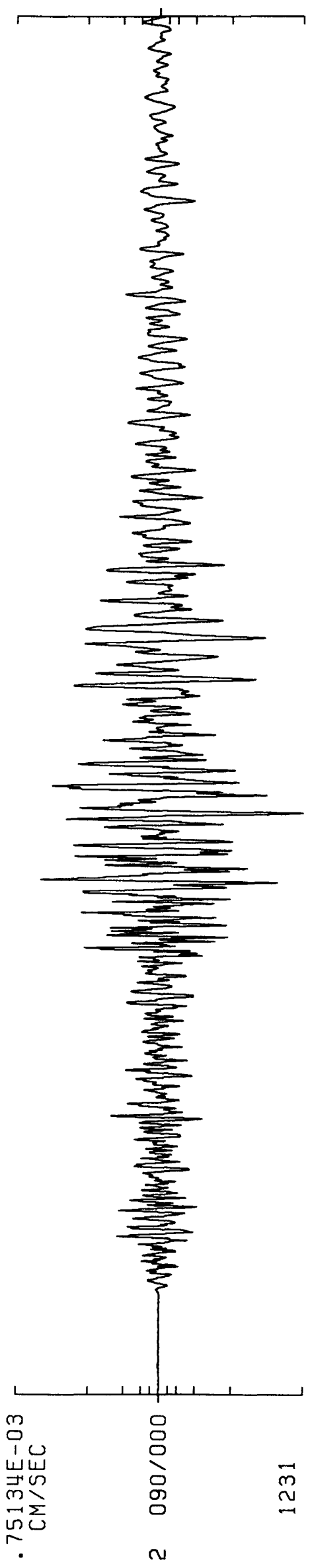

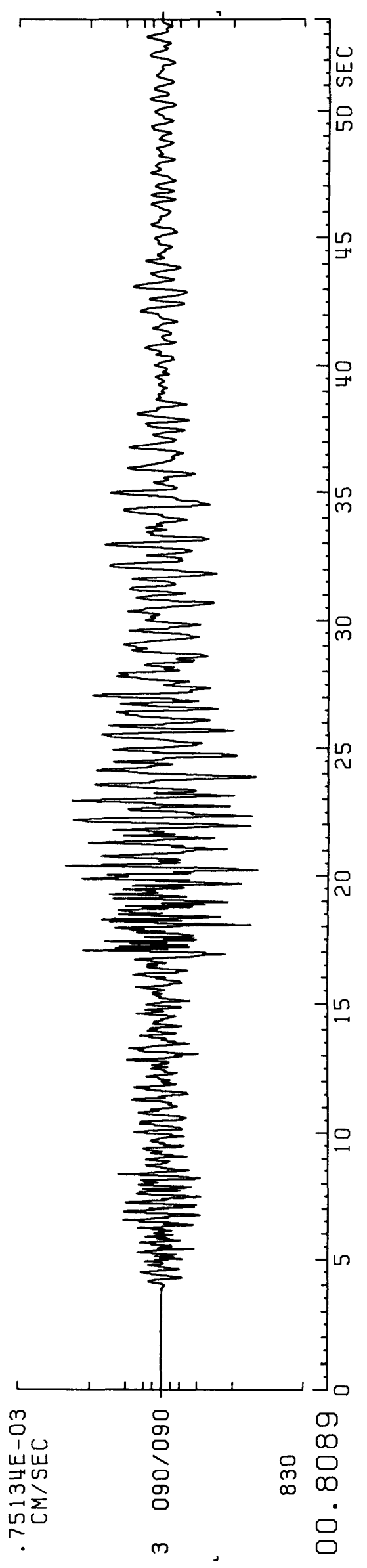


174

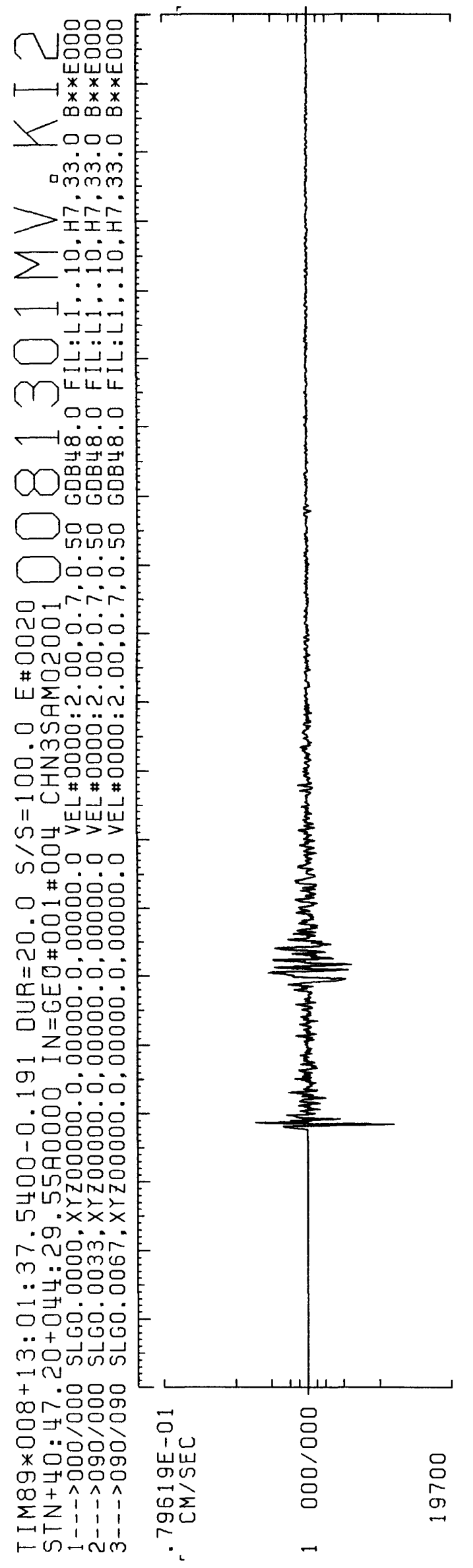

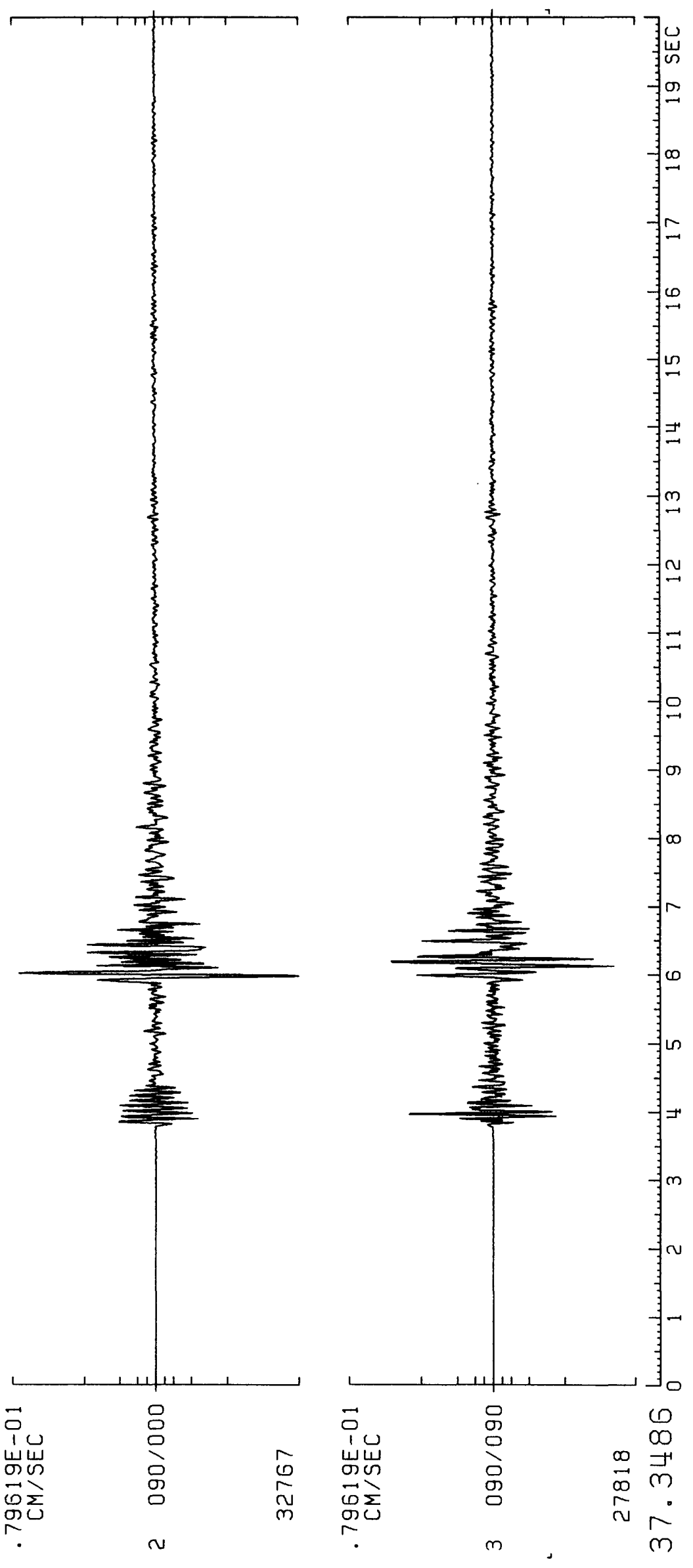


175

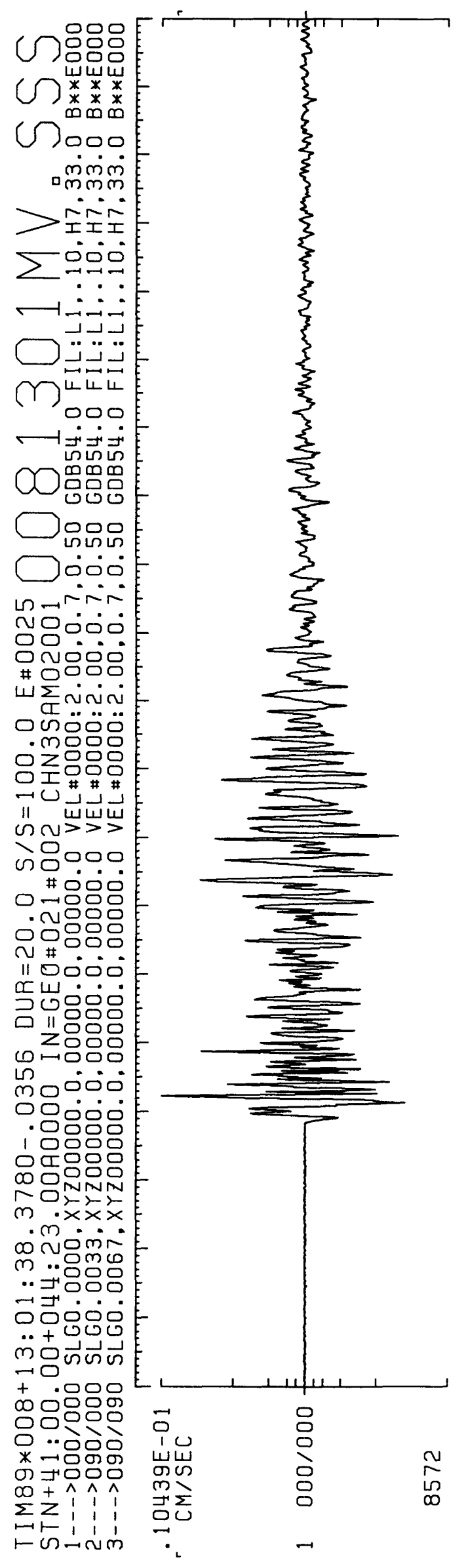

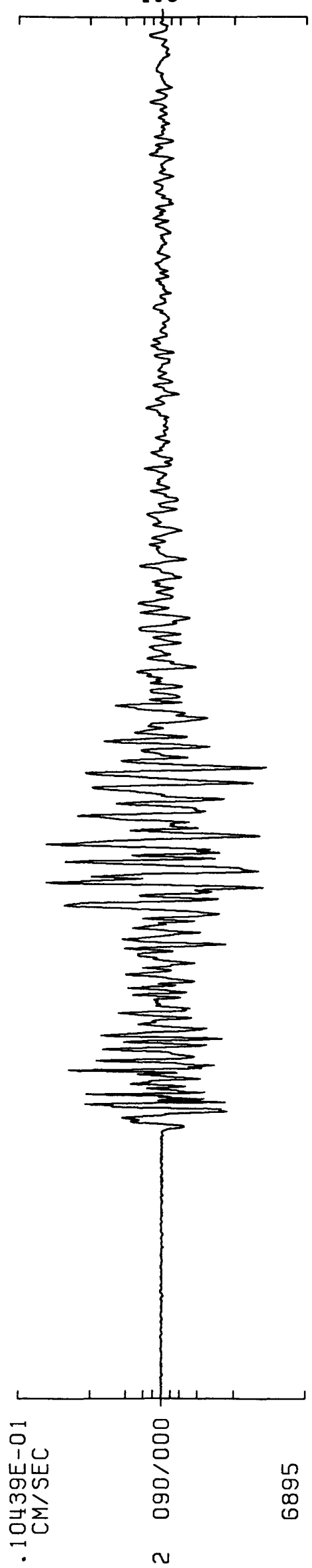

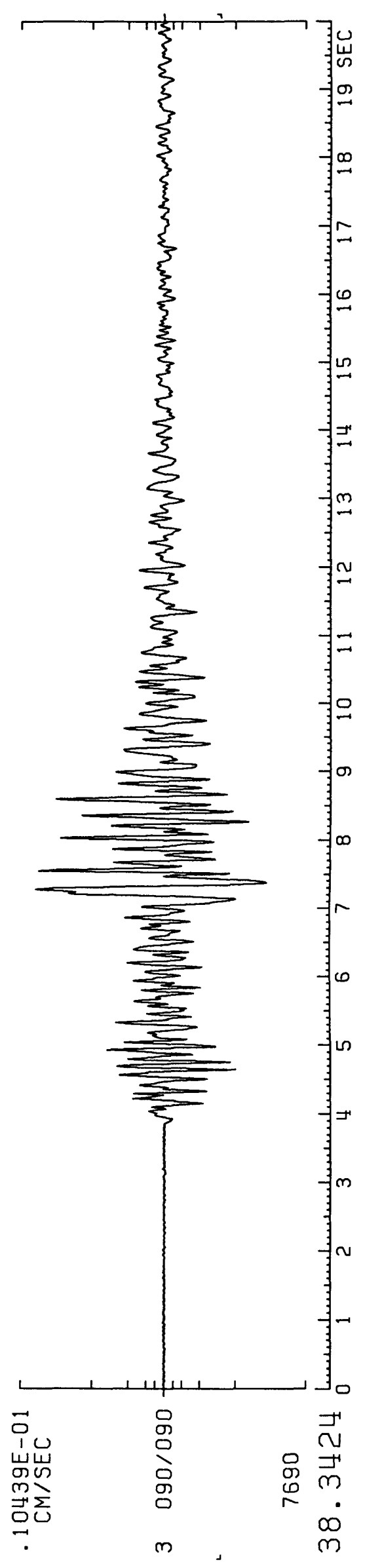


176

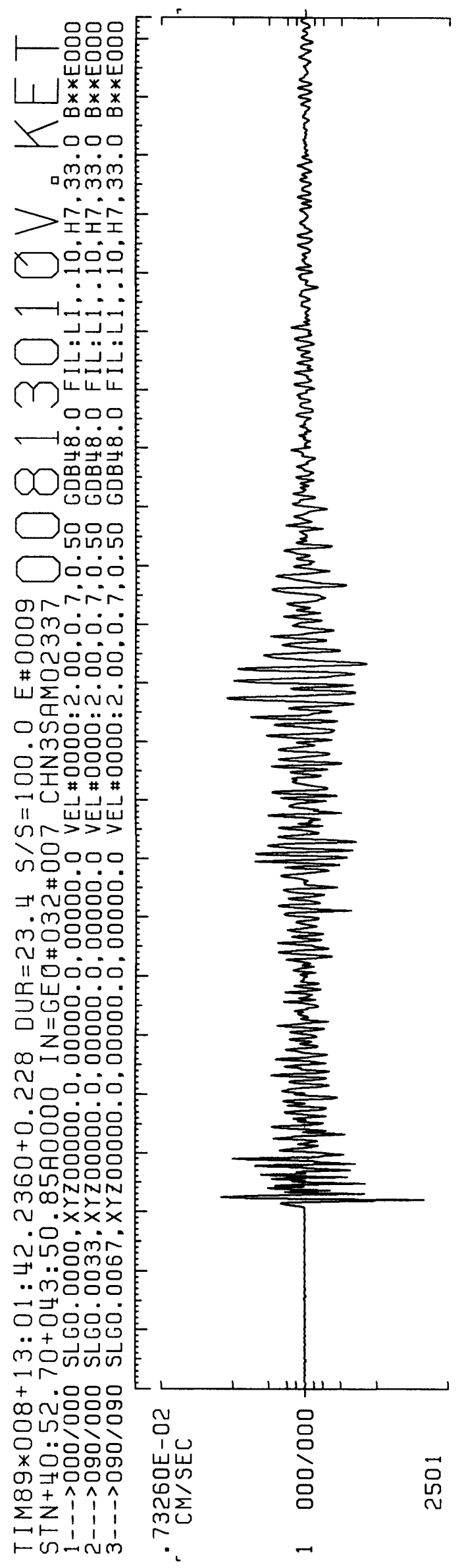

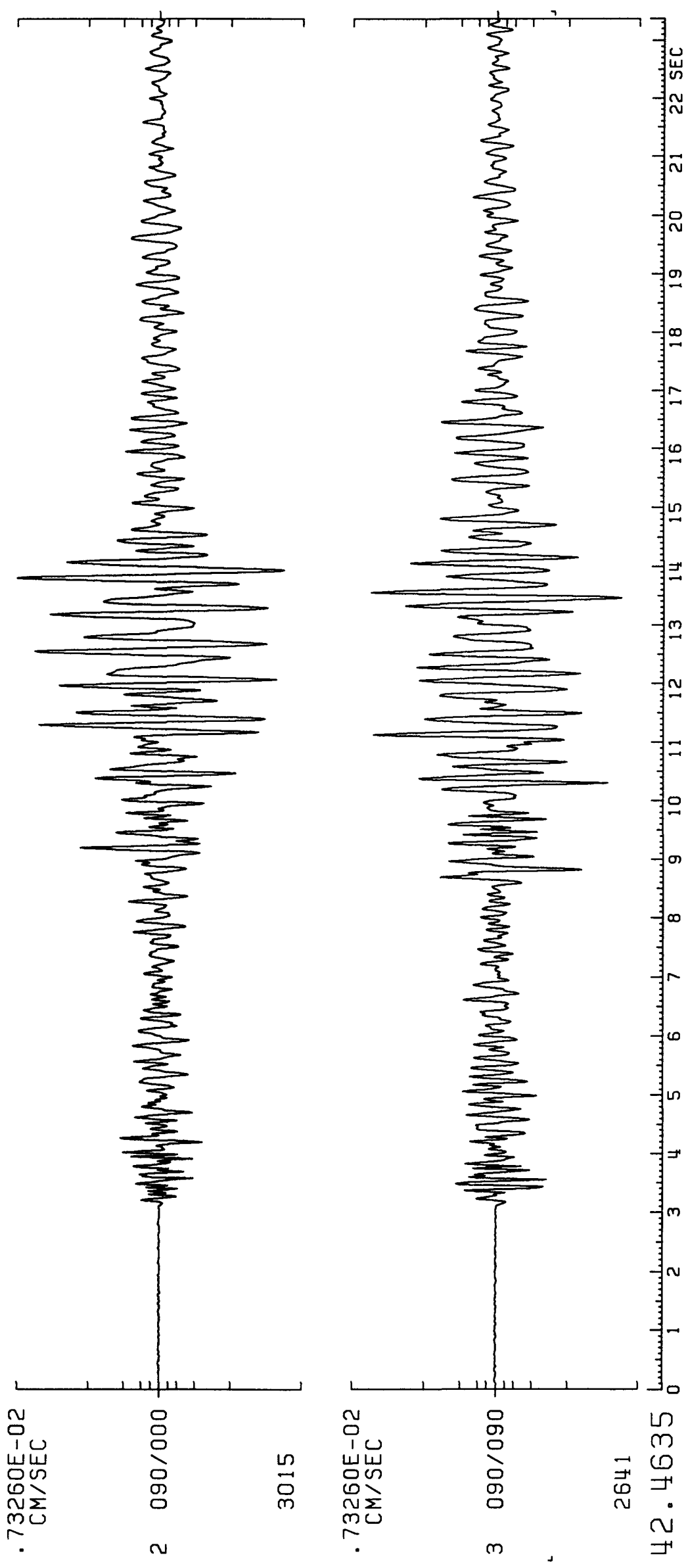


177
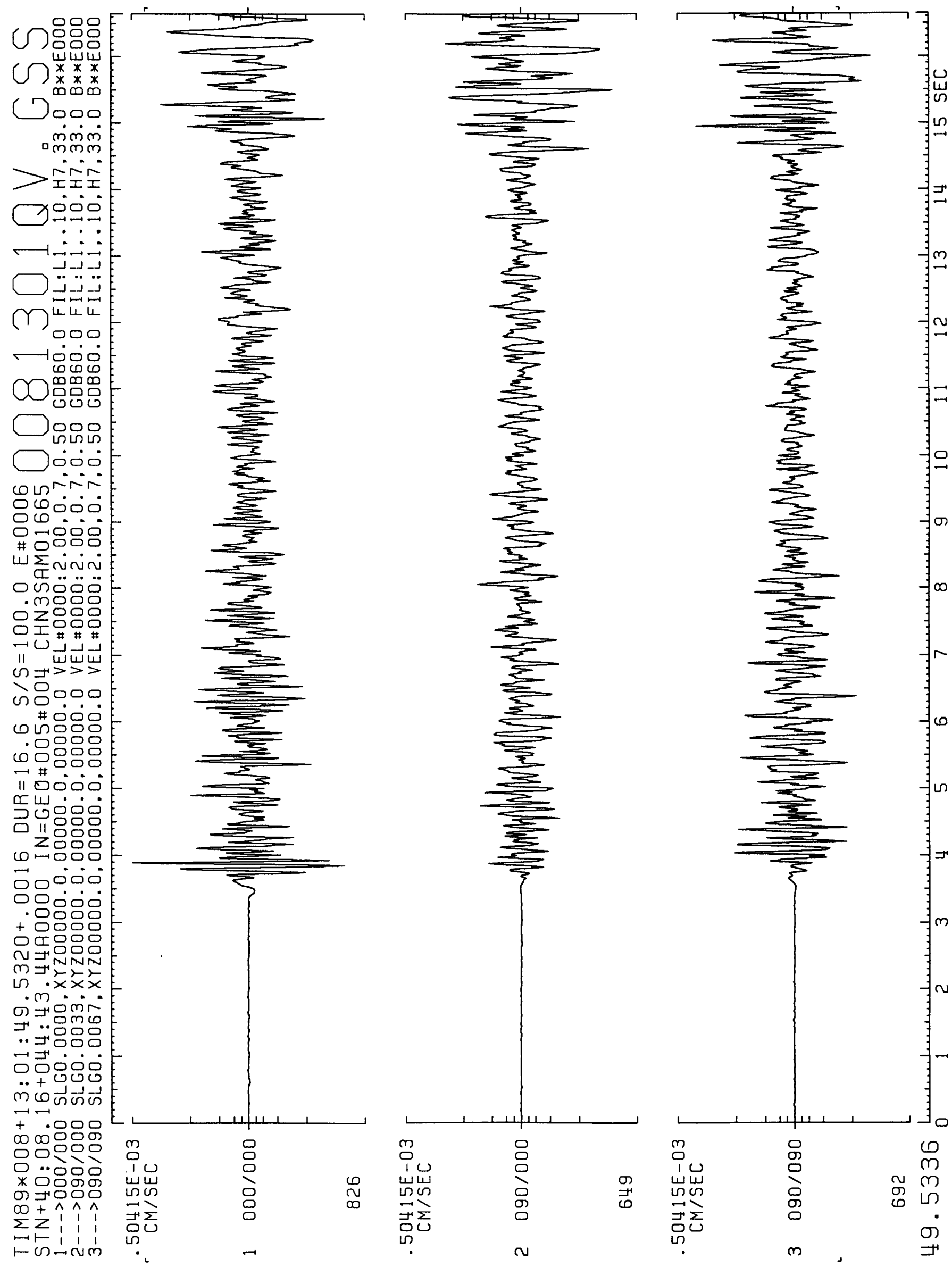


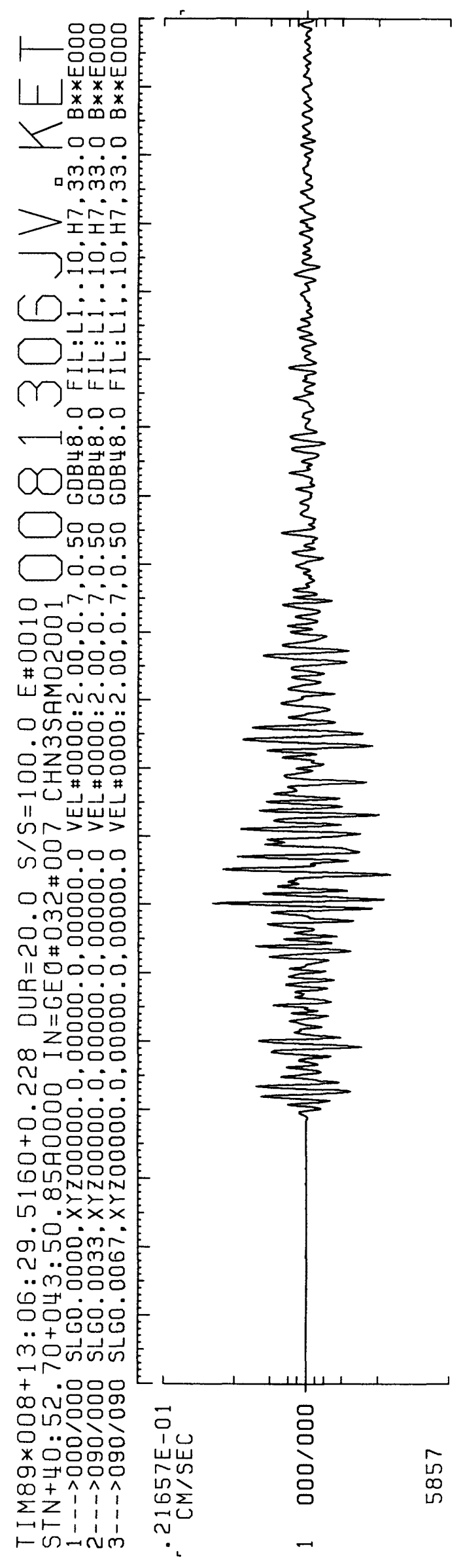

178

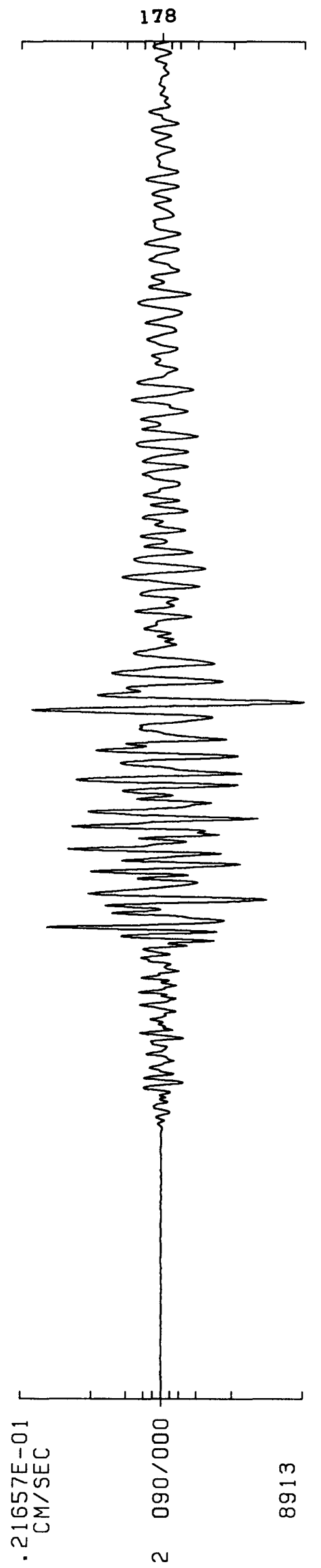

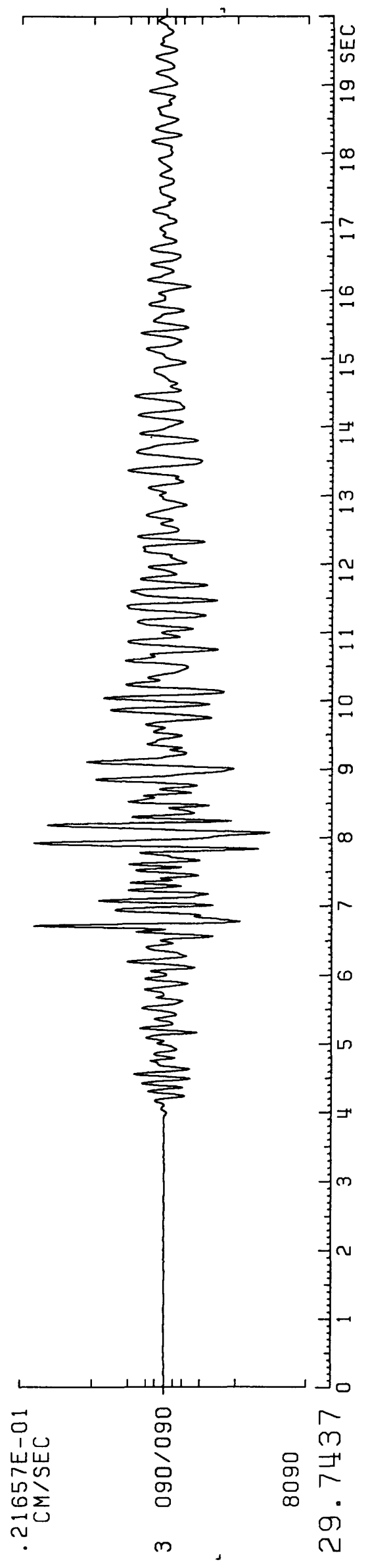



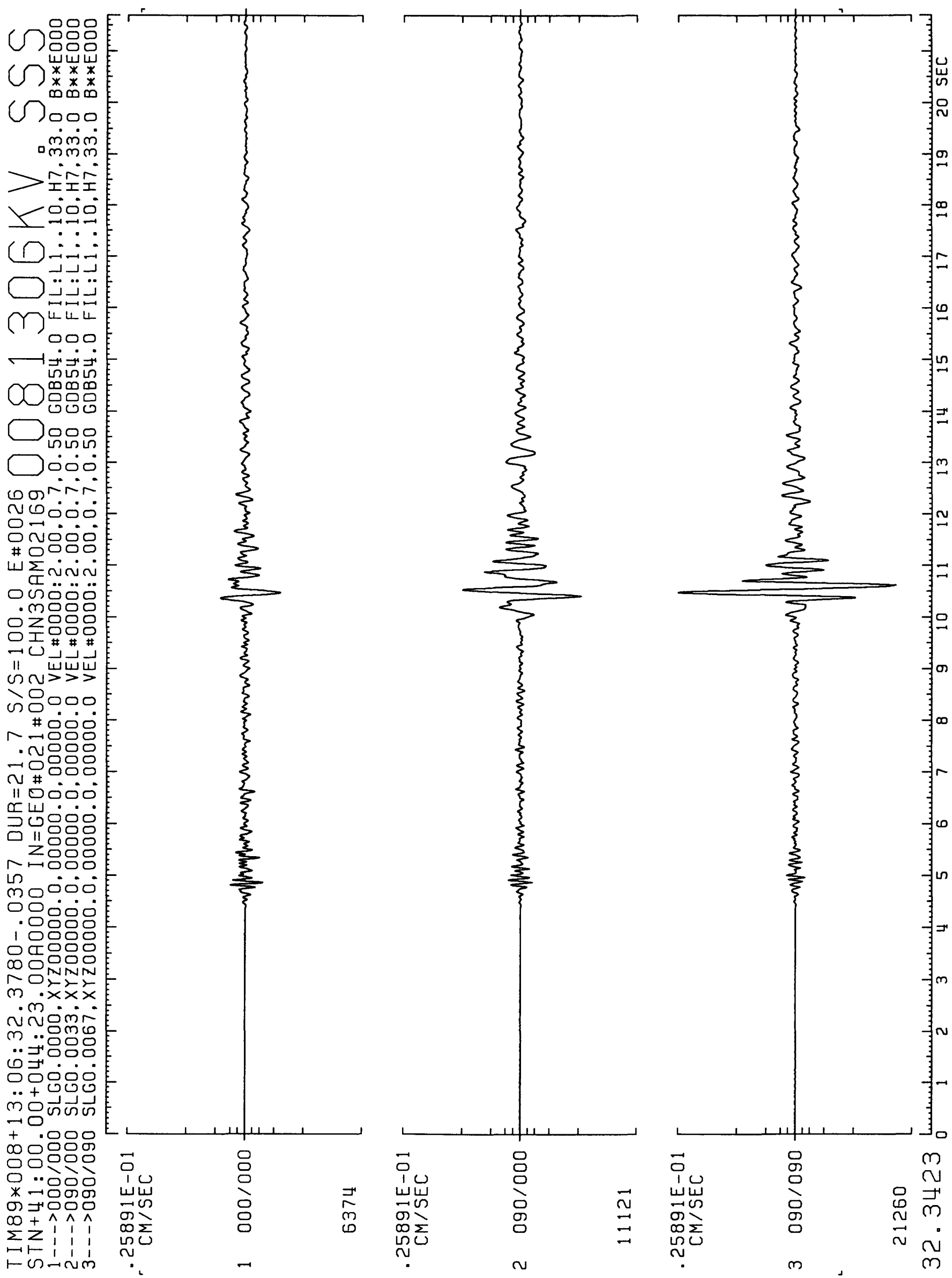
180
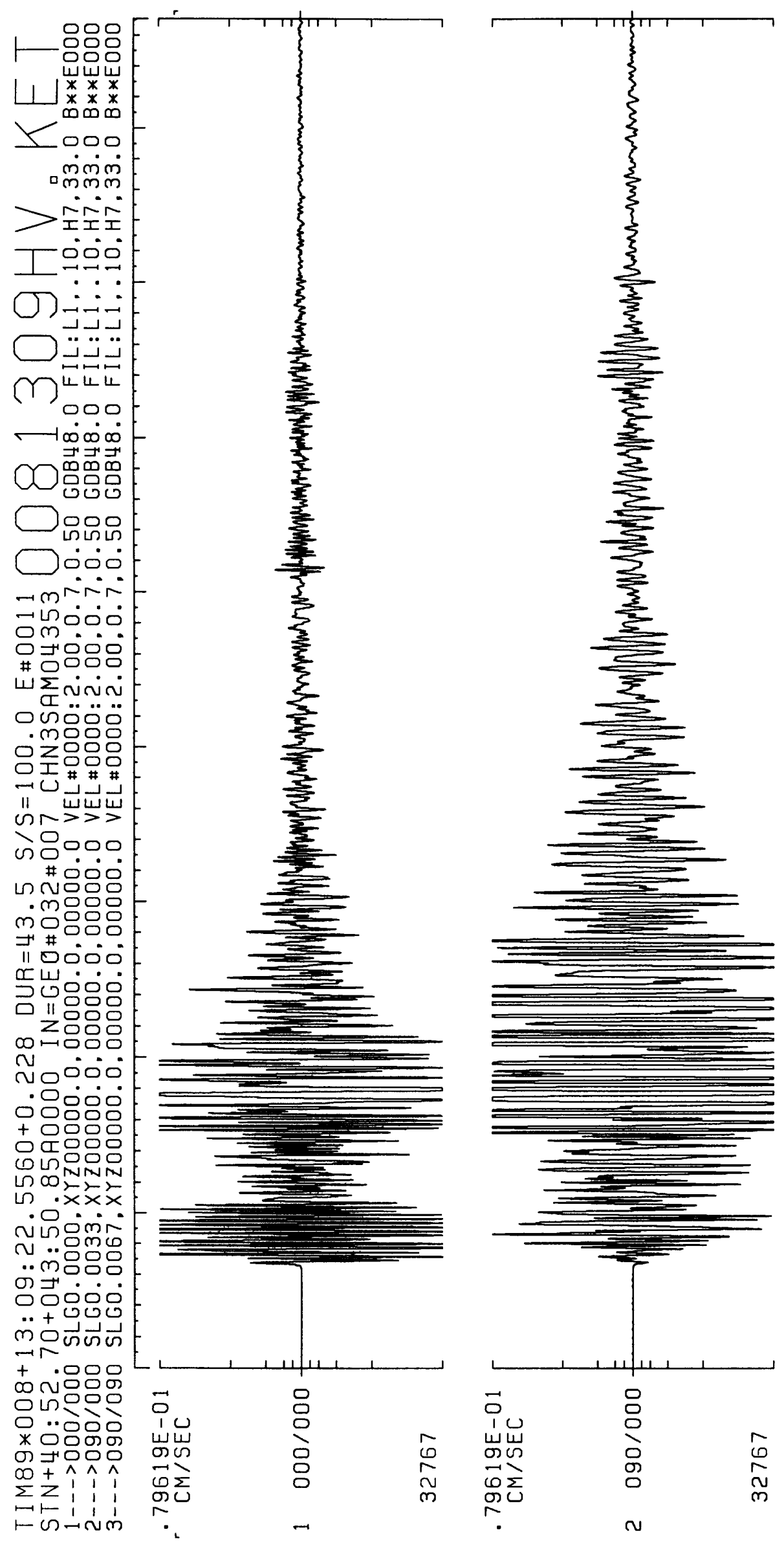

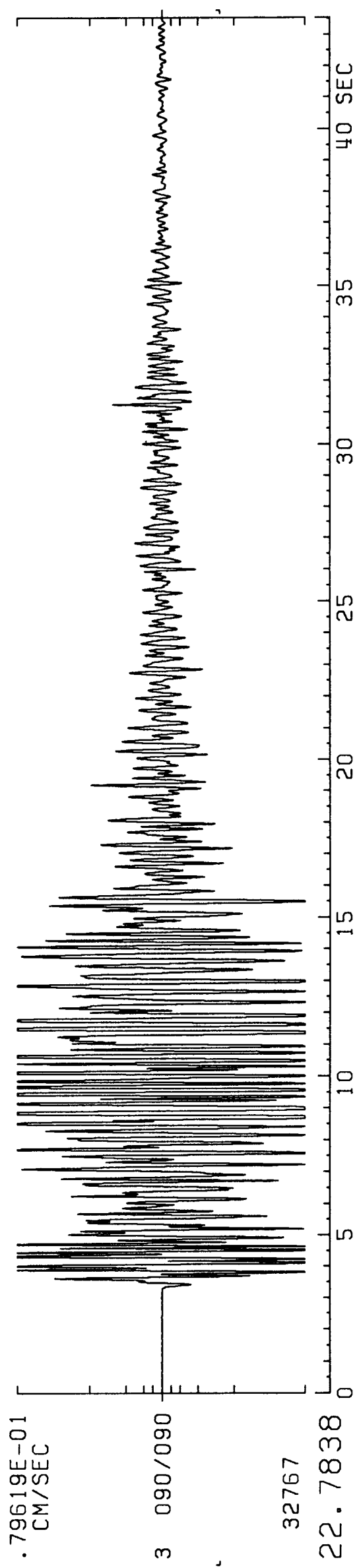


181
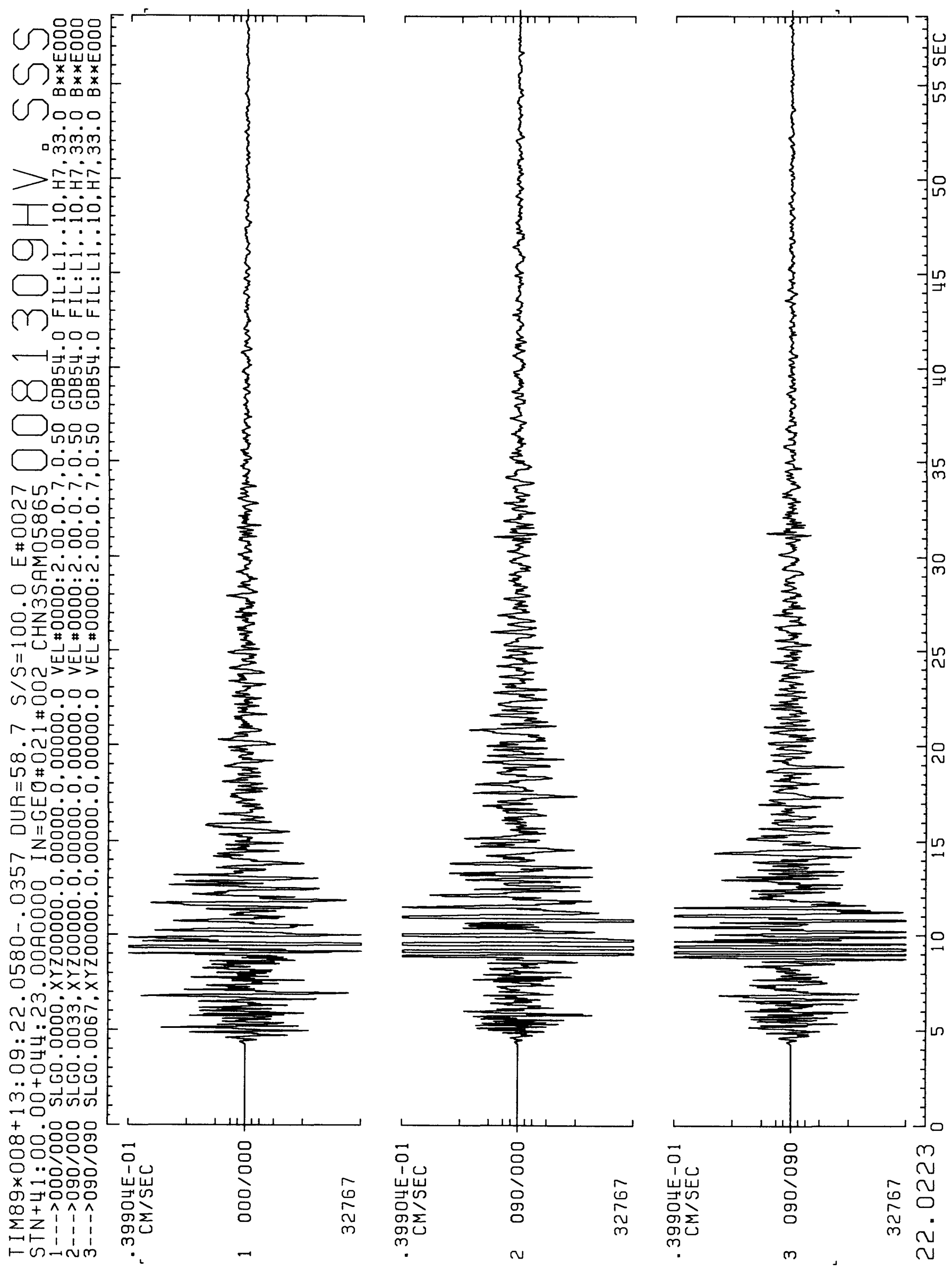
182
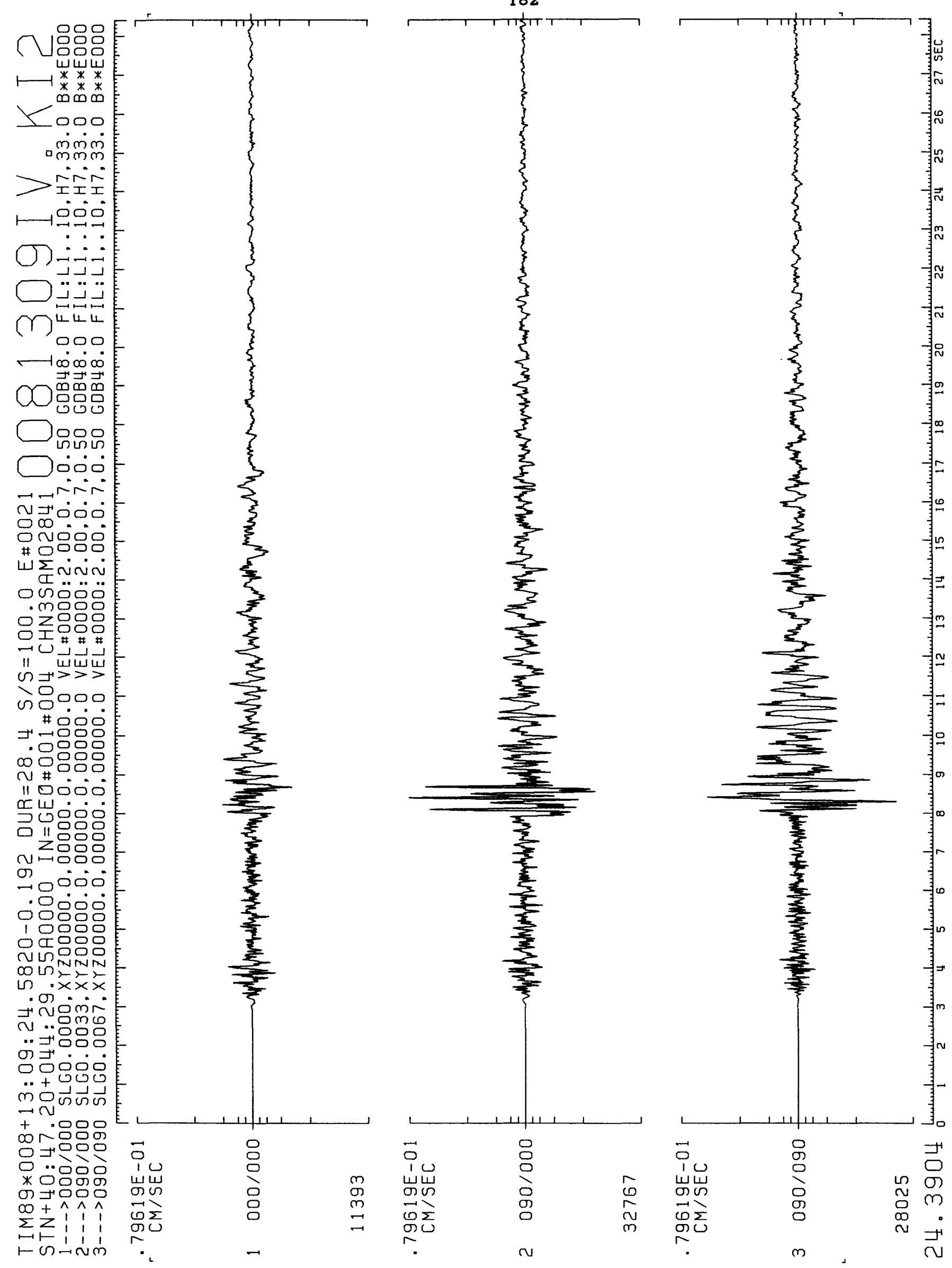
183
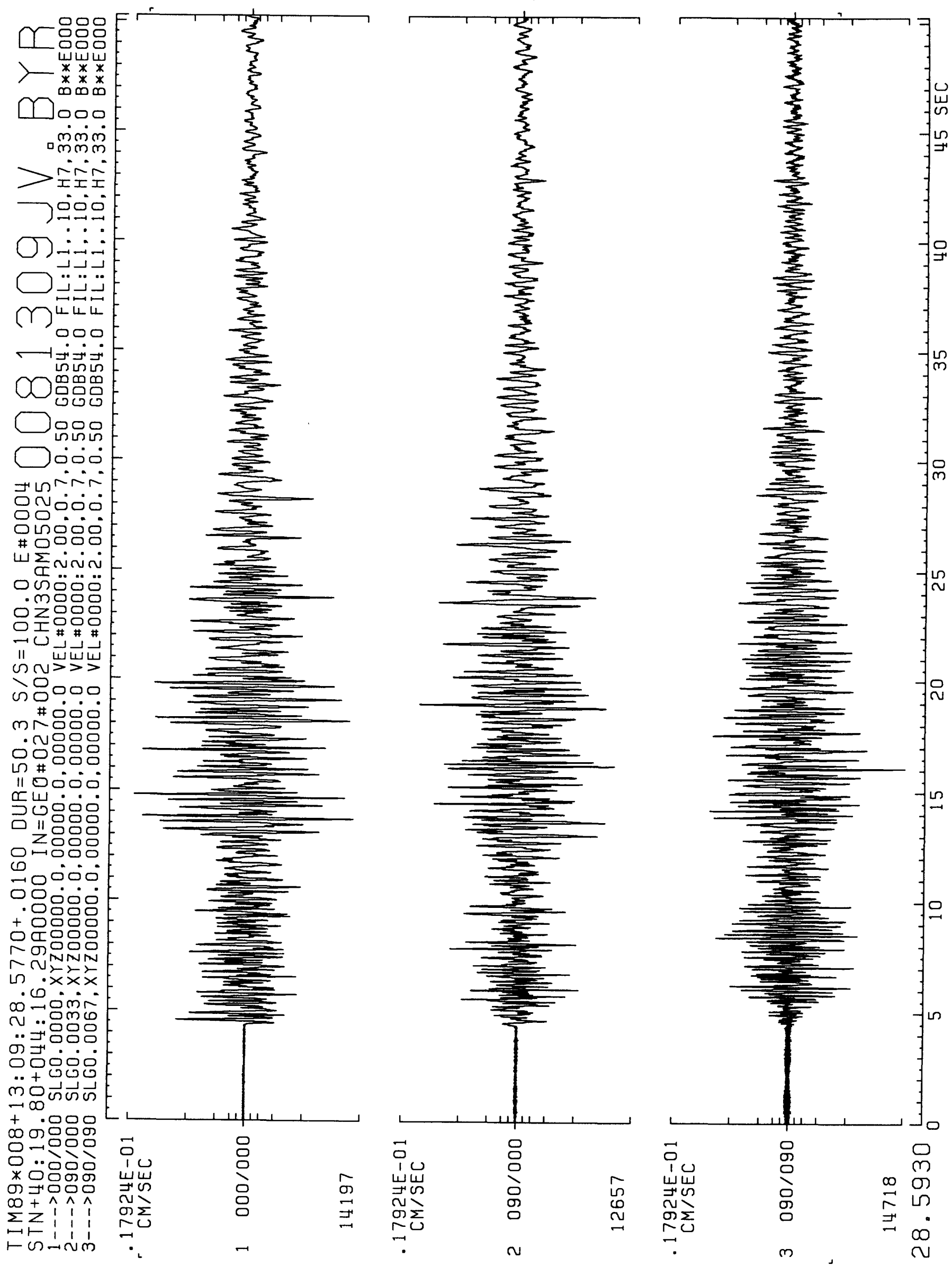
184
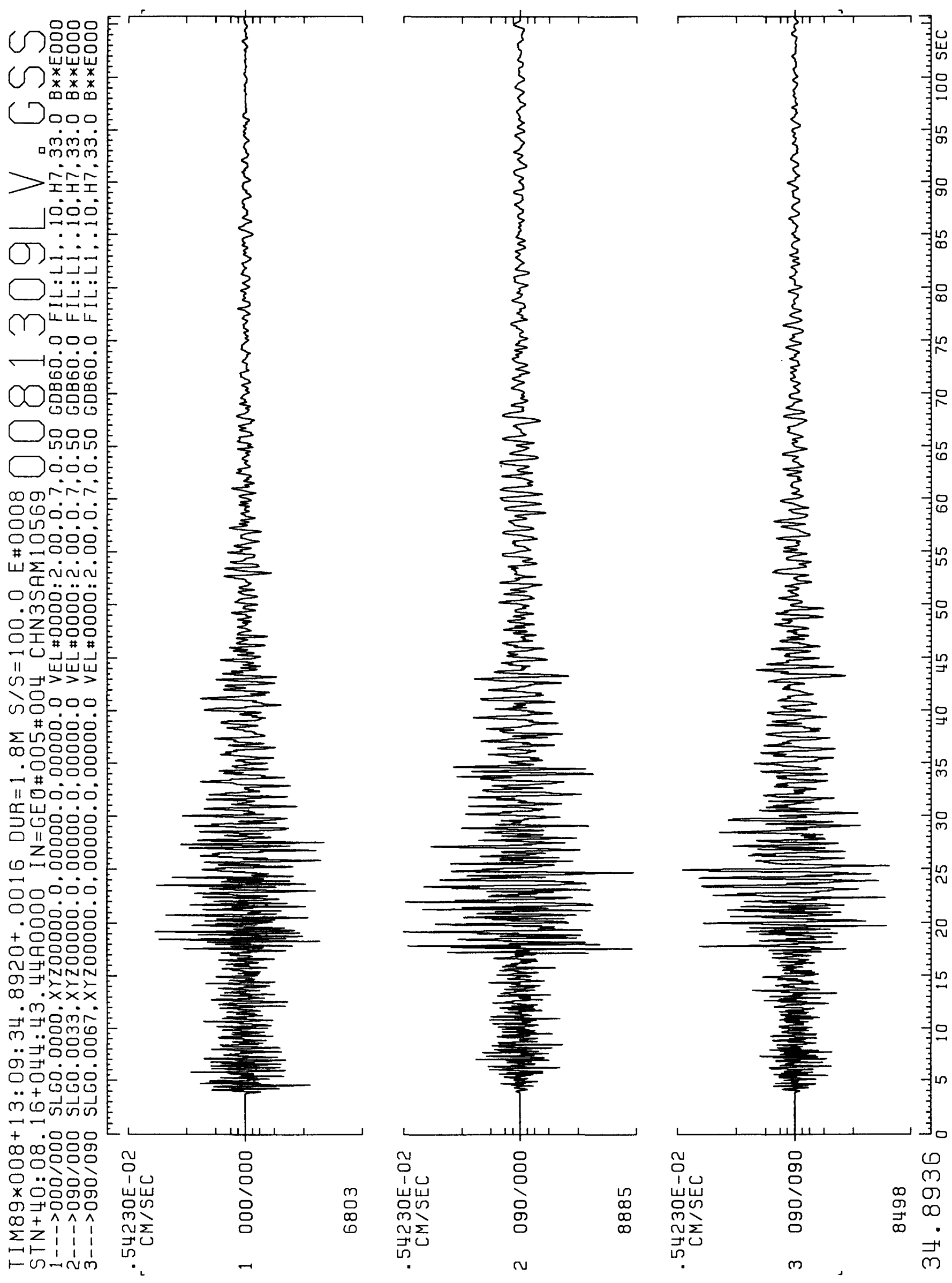


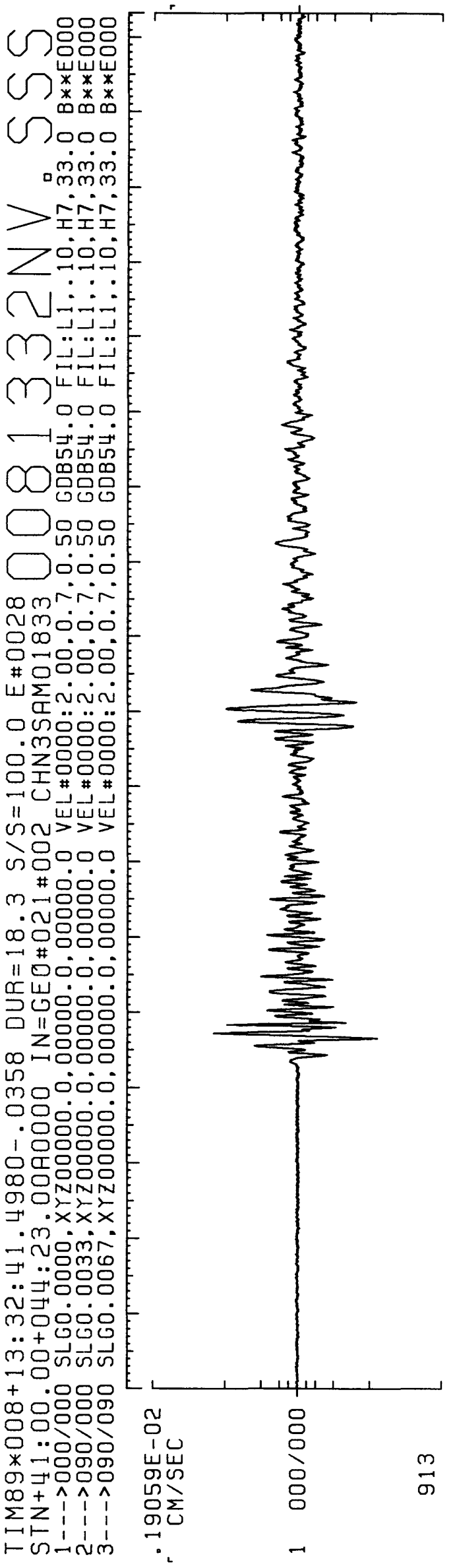

185

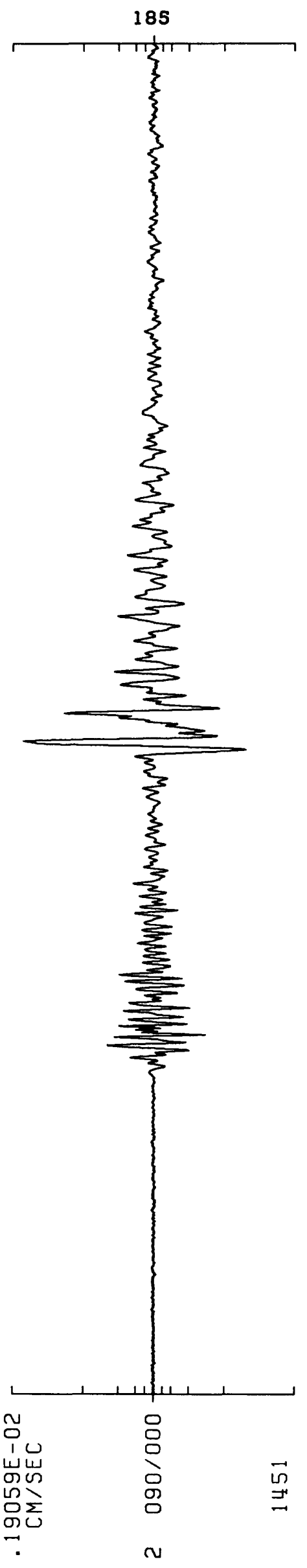

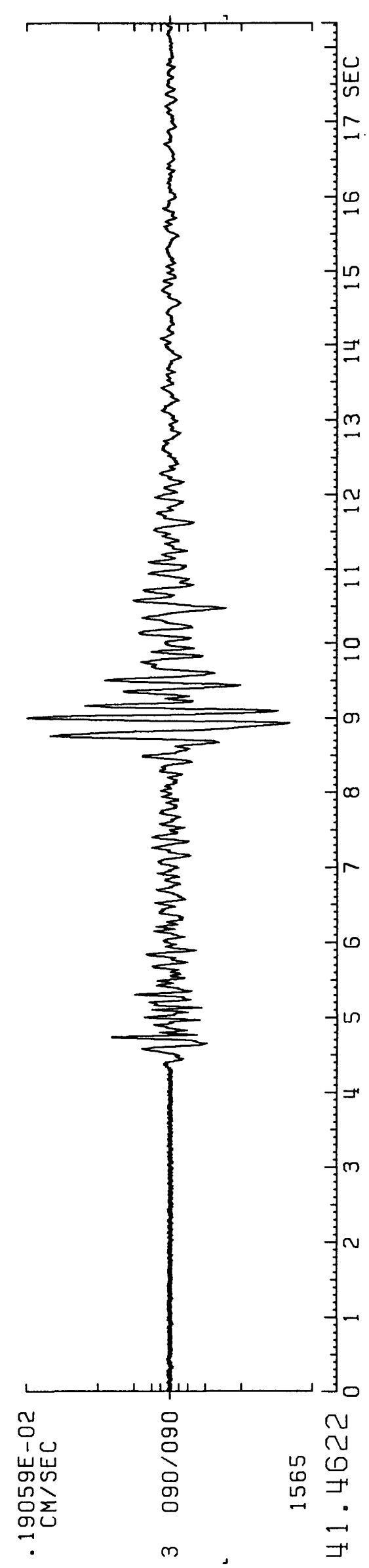



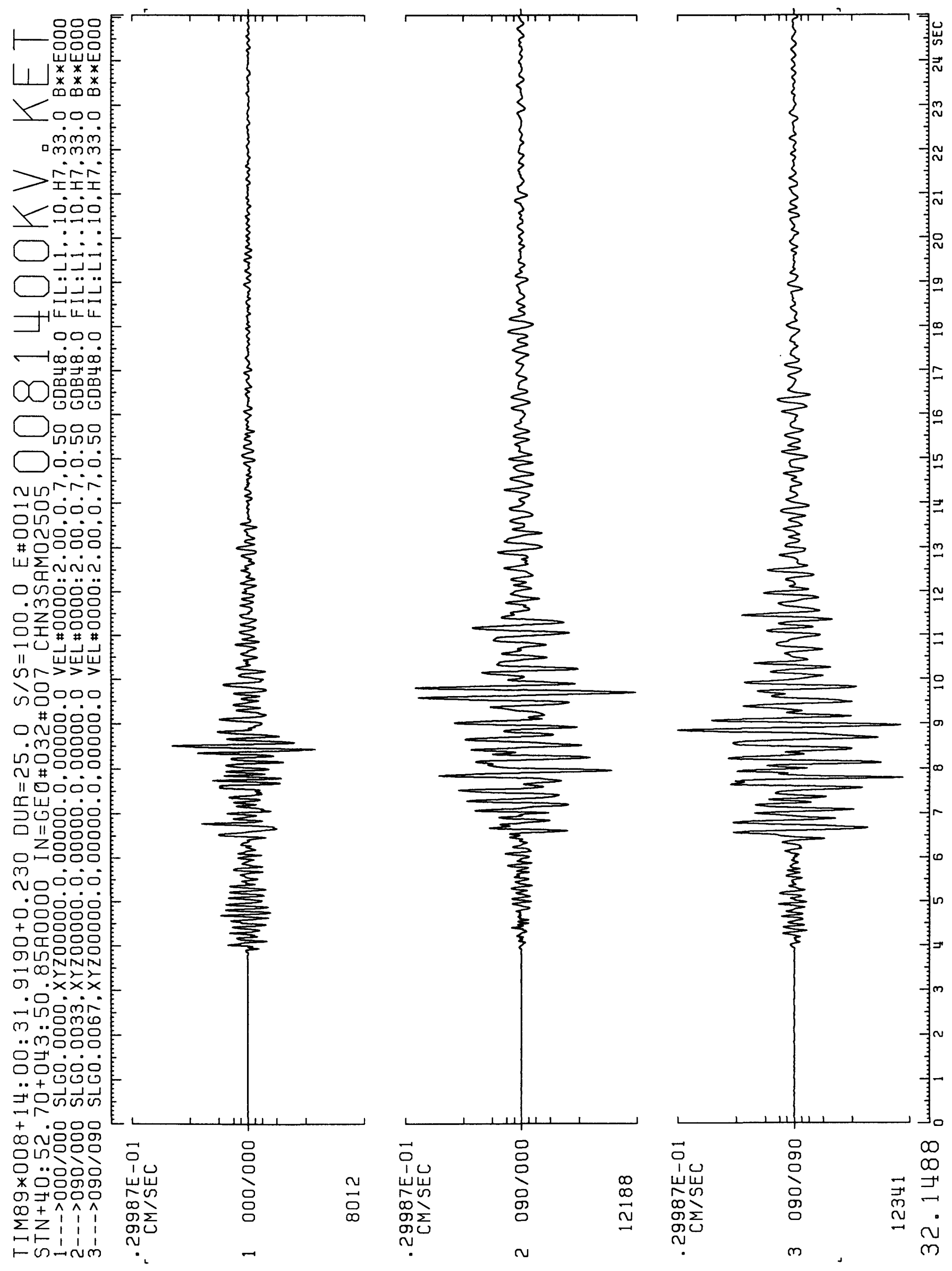

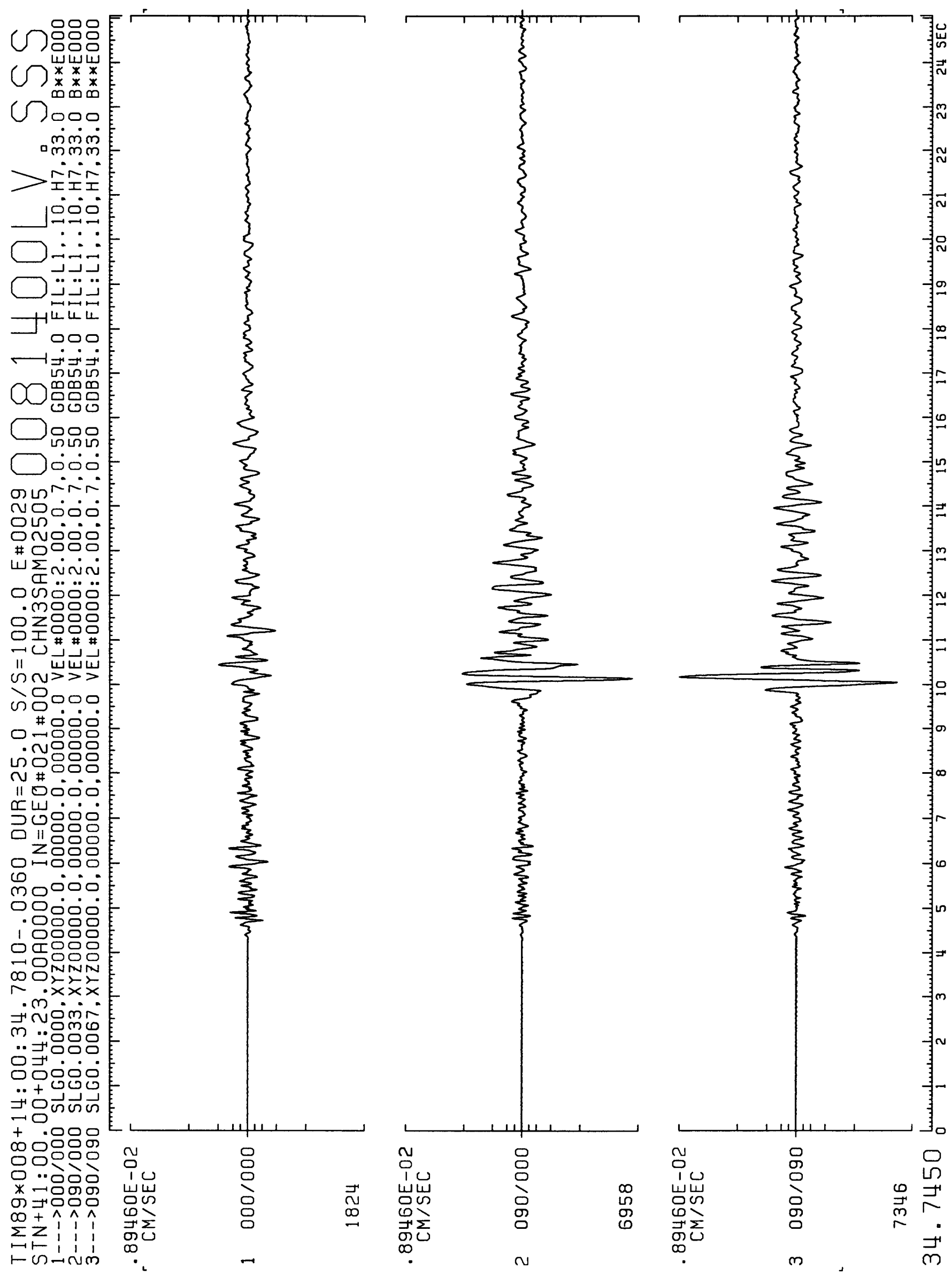
188

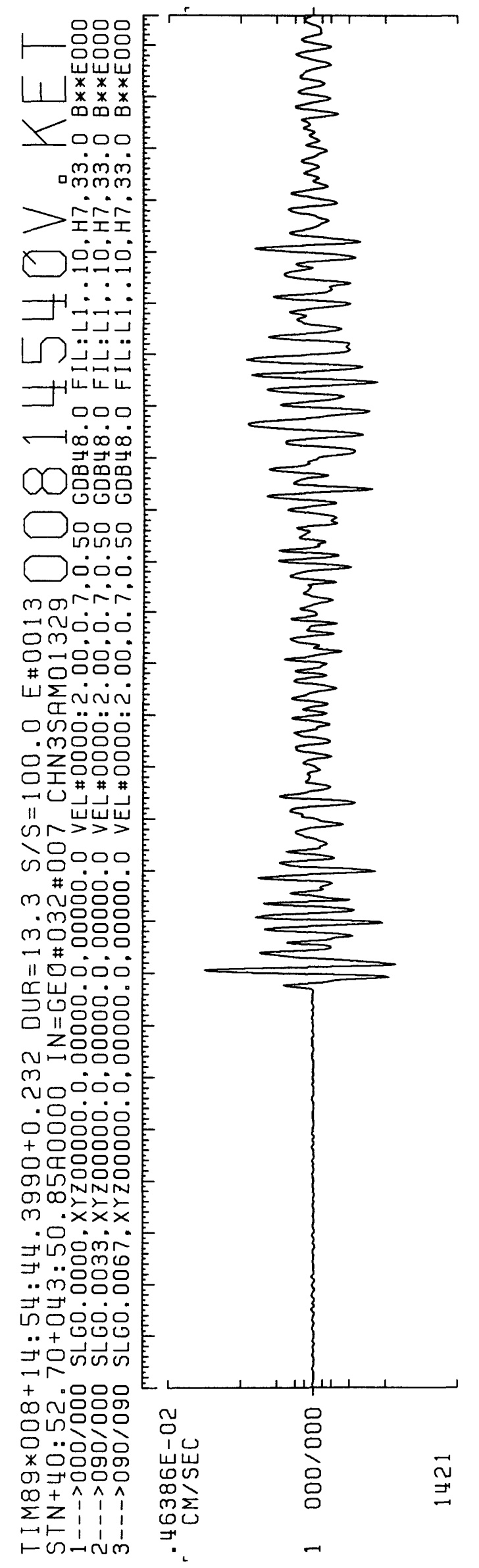

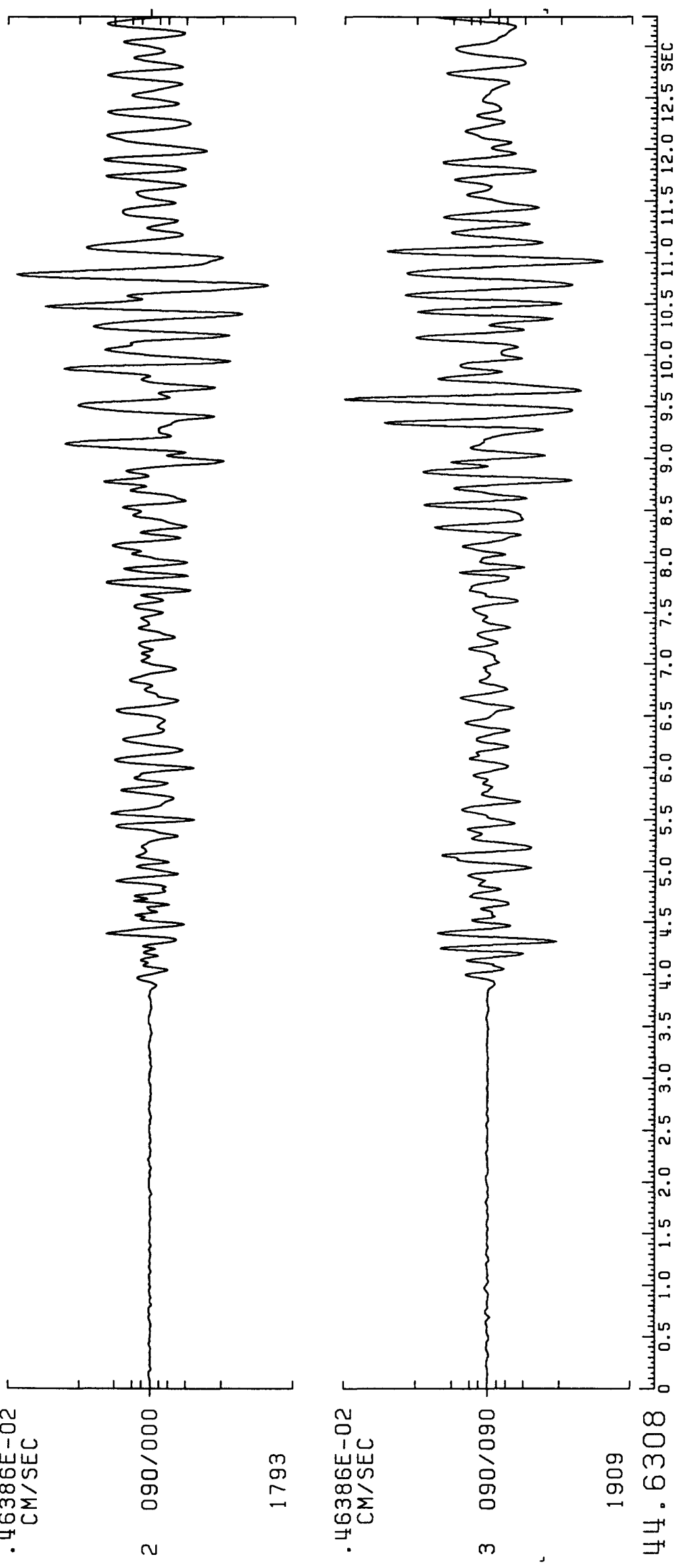


189

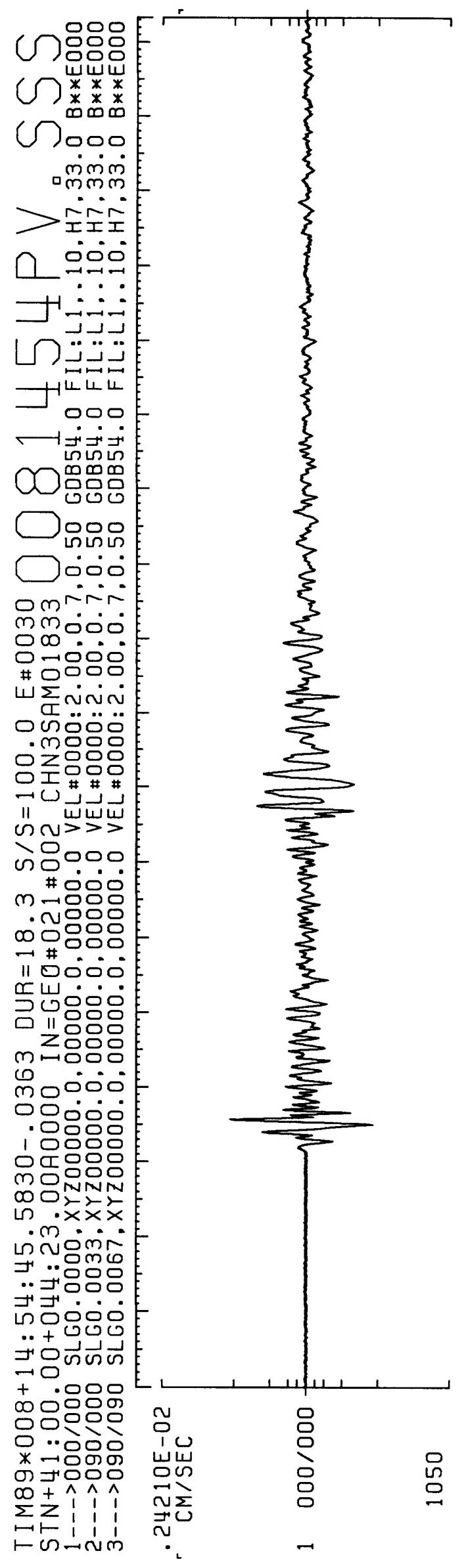

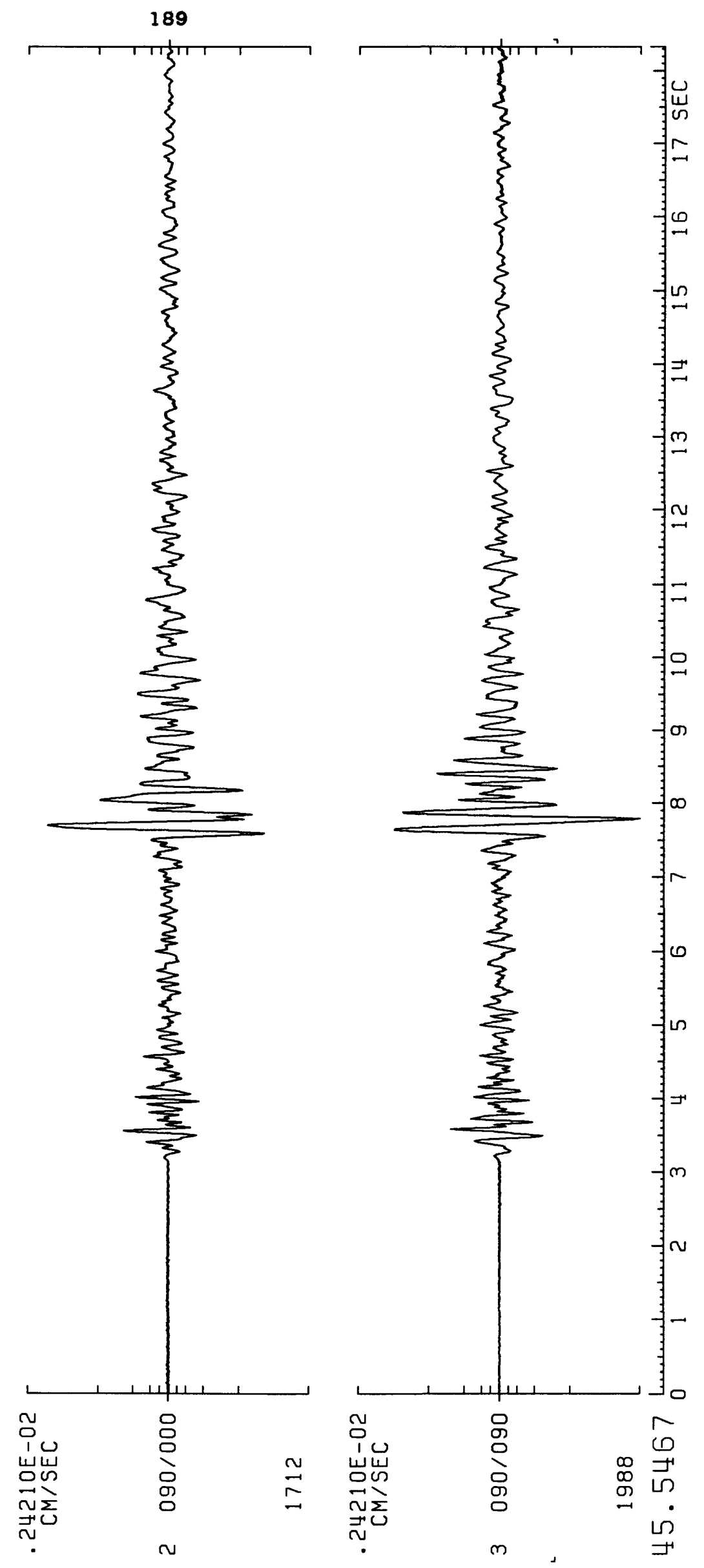


190
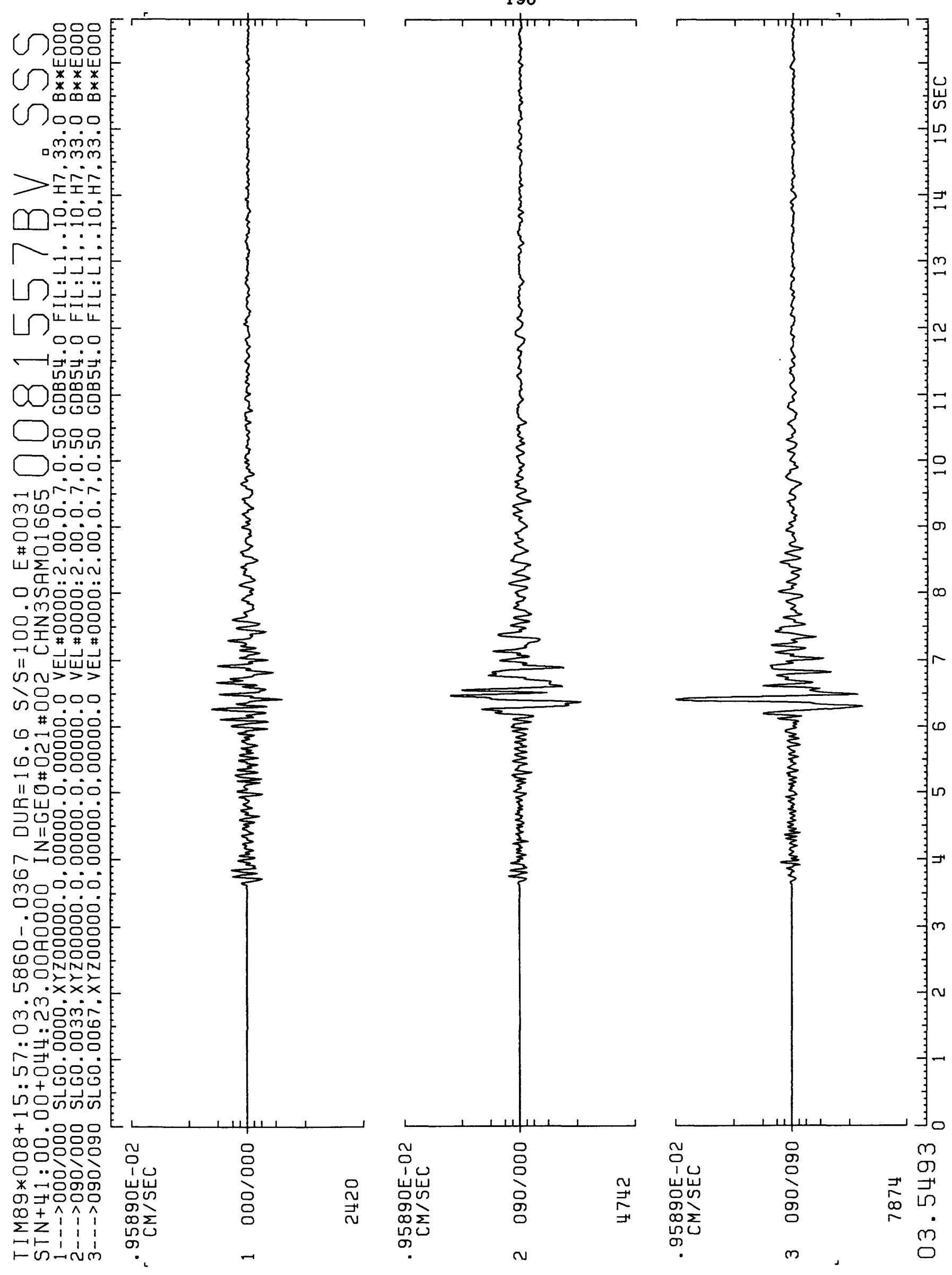
191

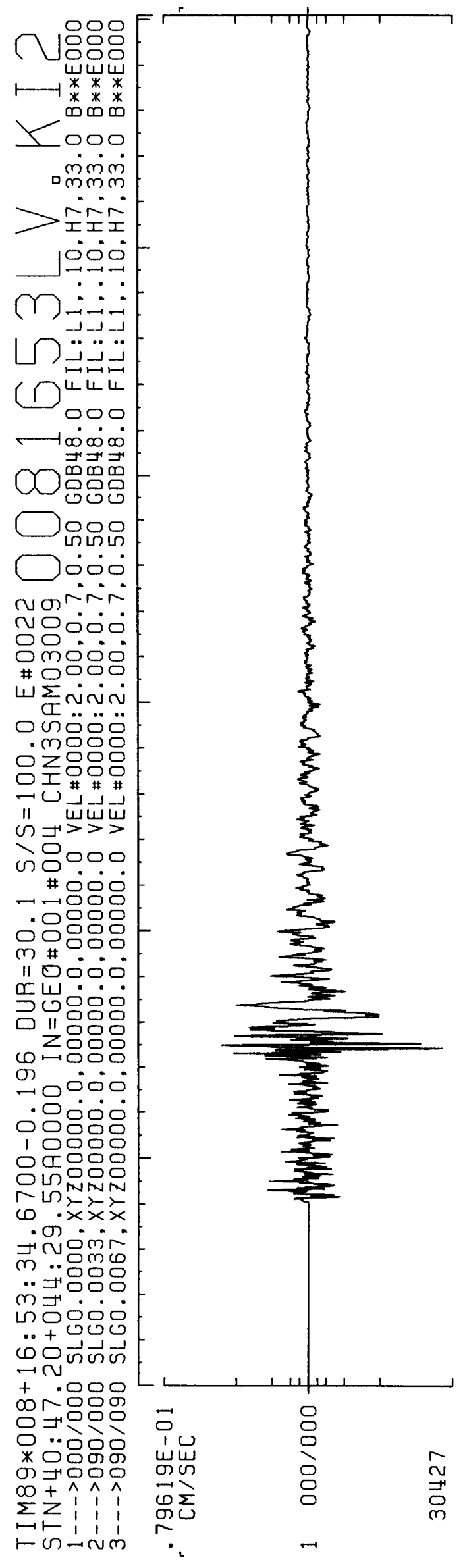

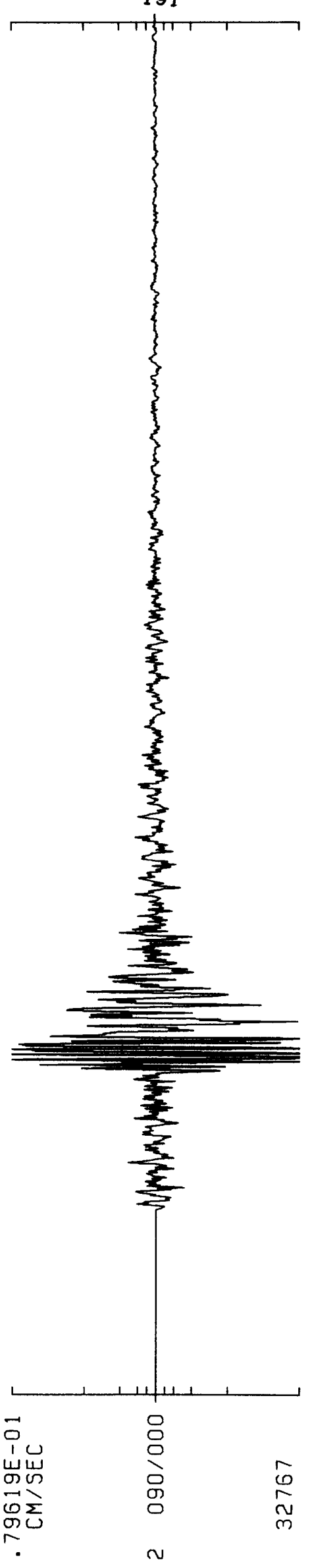

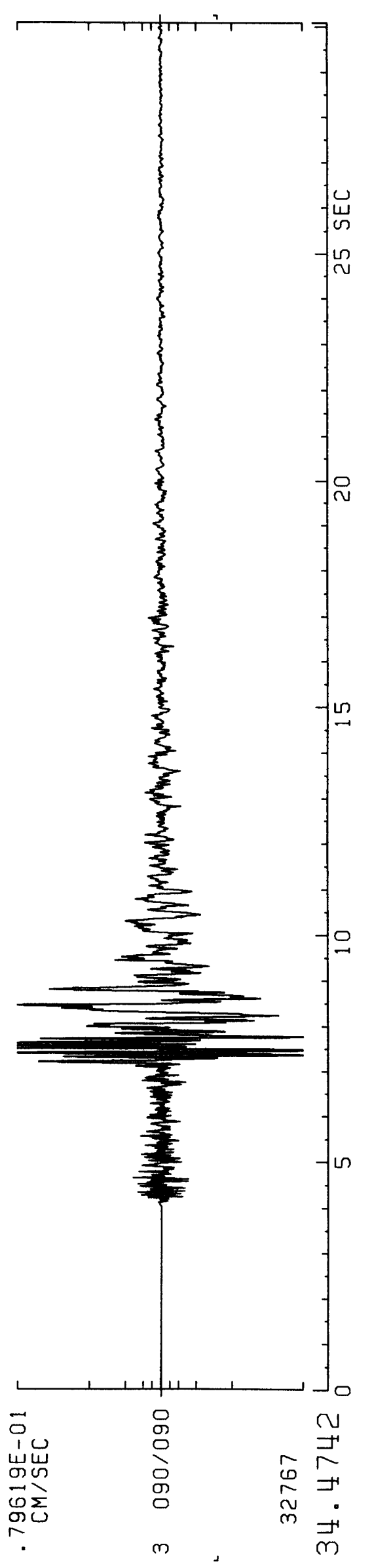



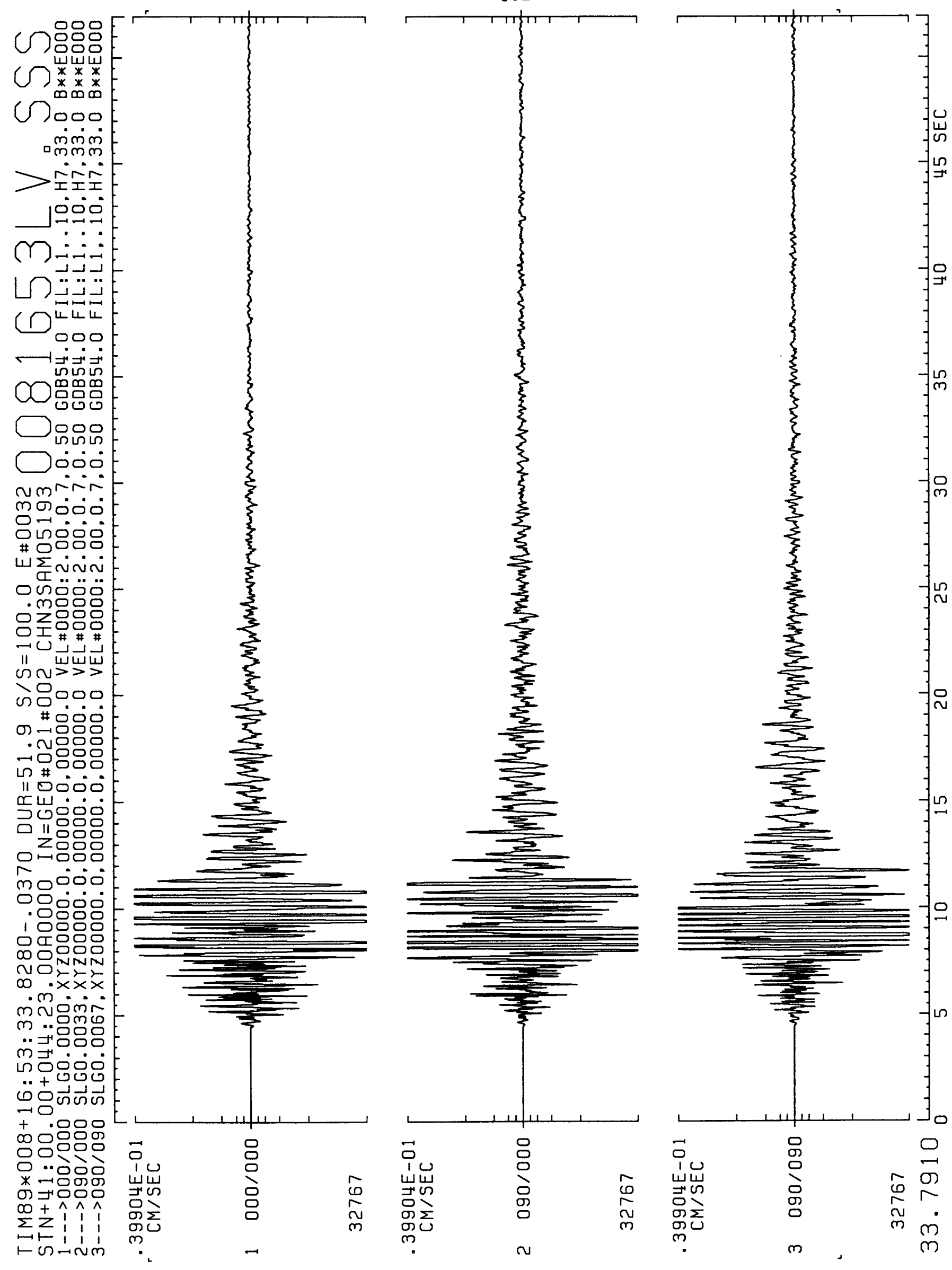
193

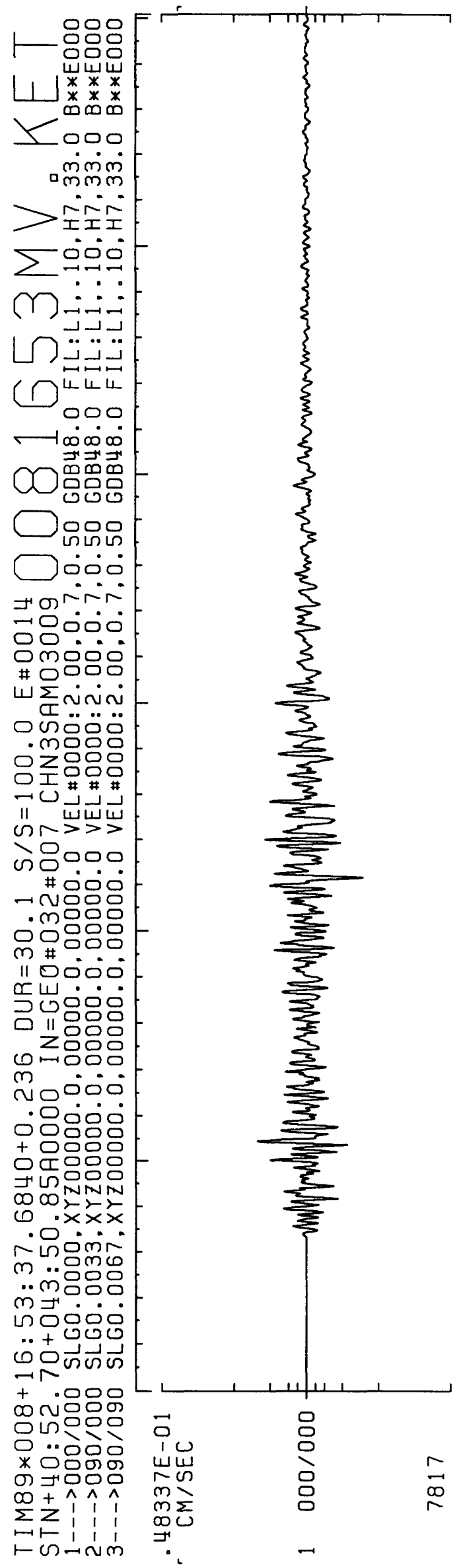

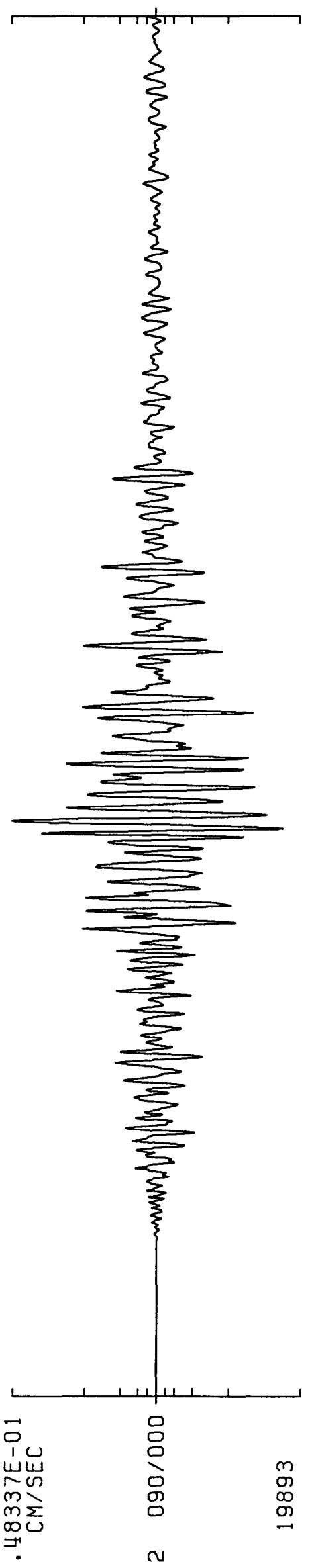

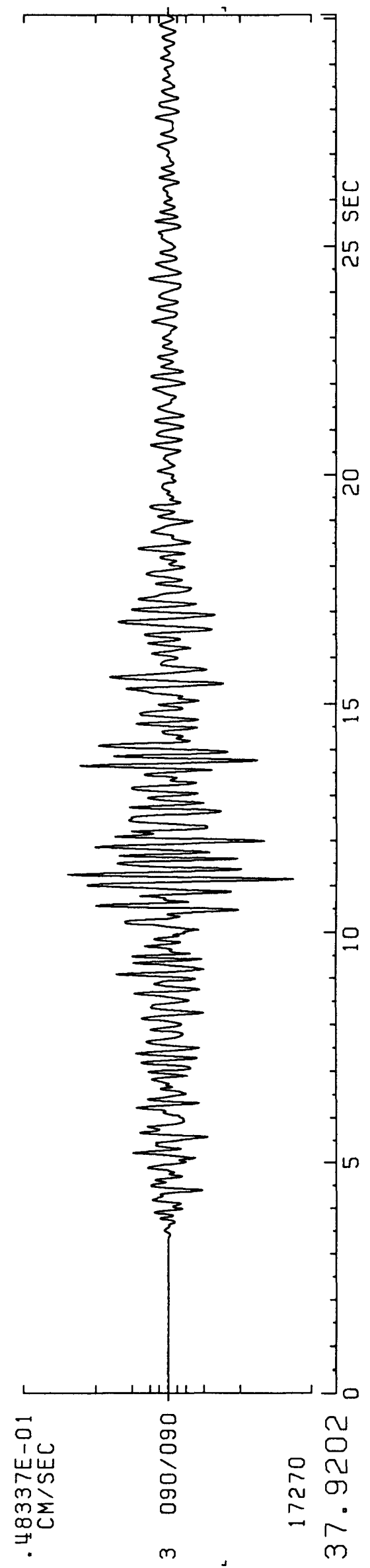


194
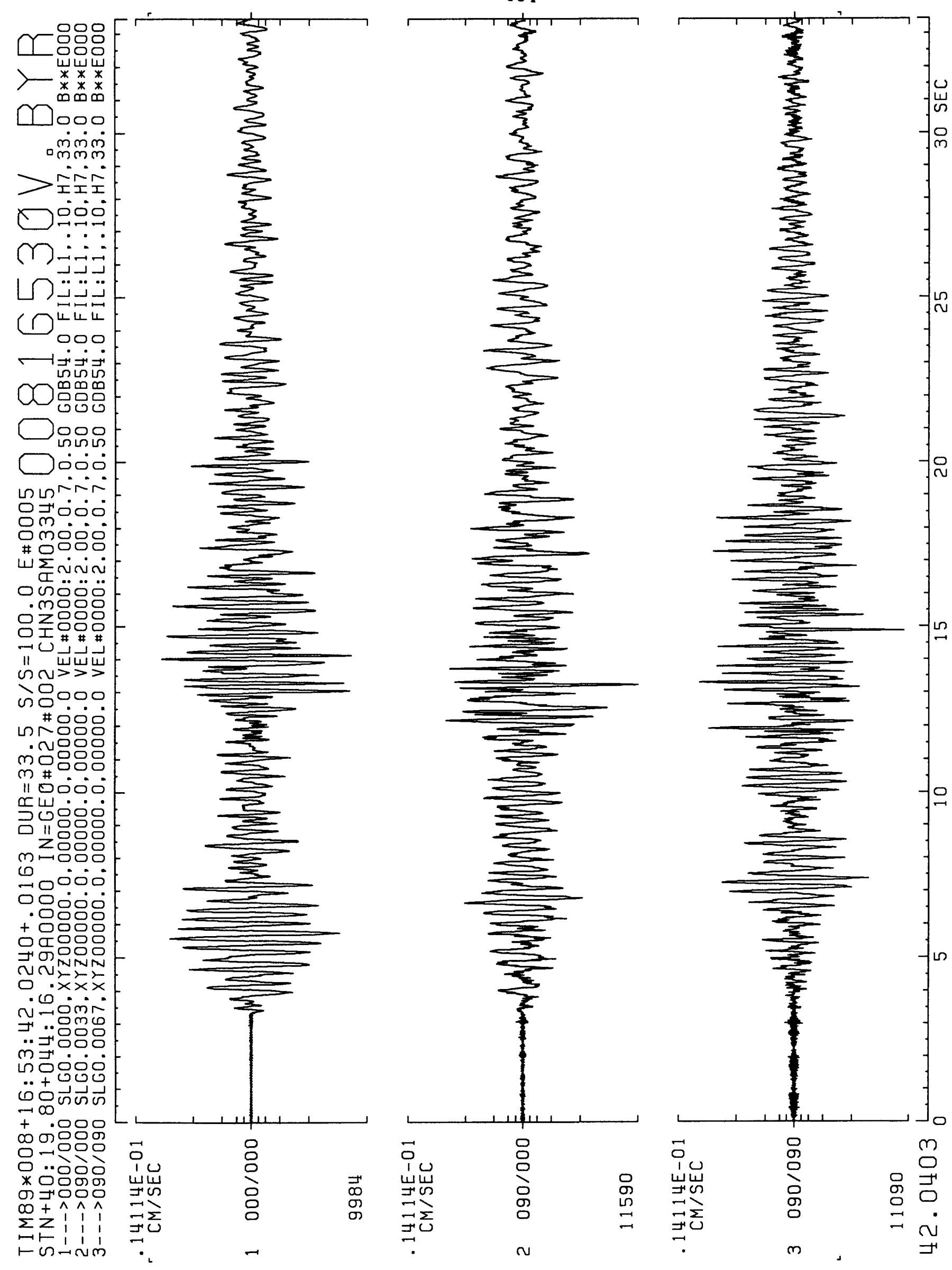


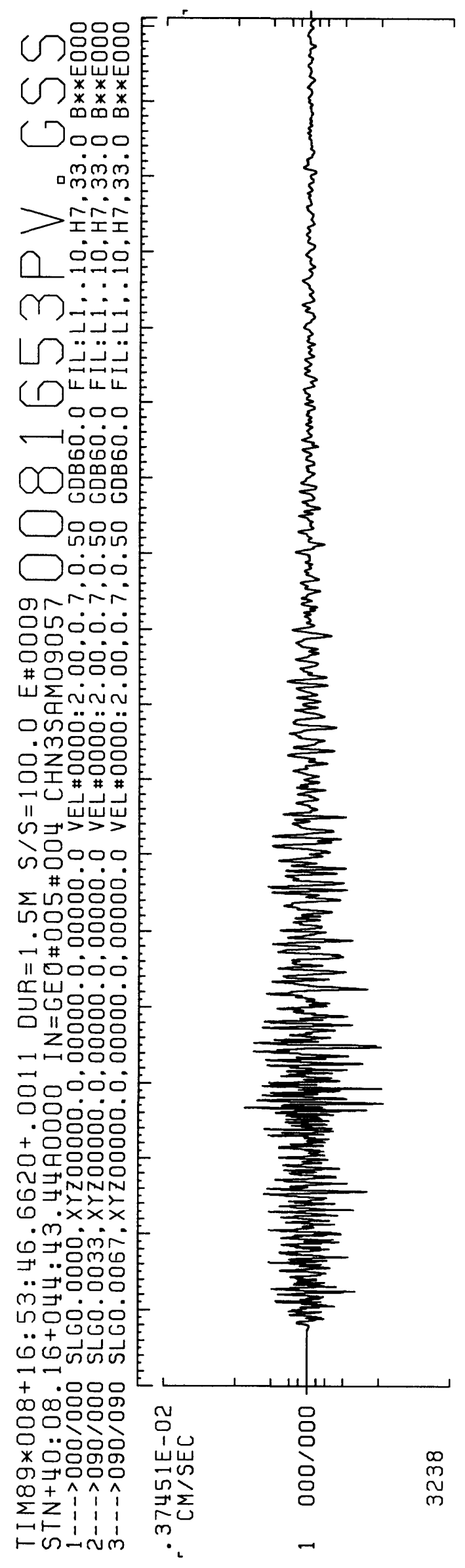

195

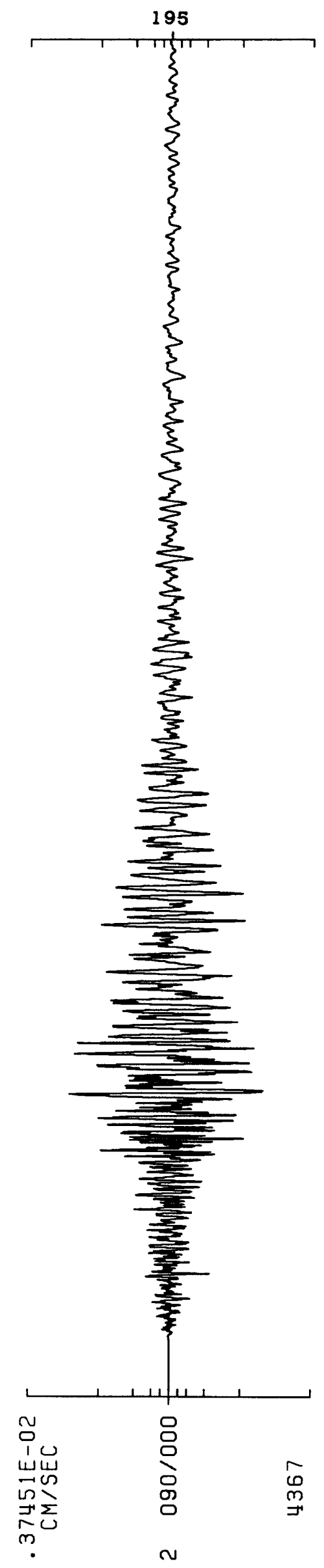

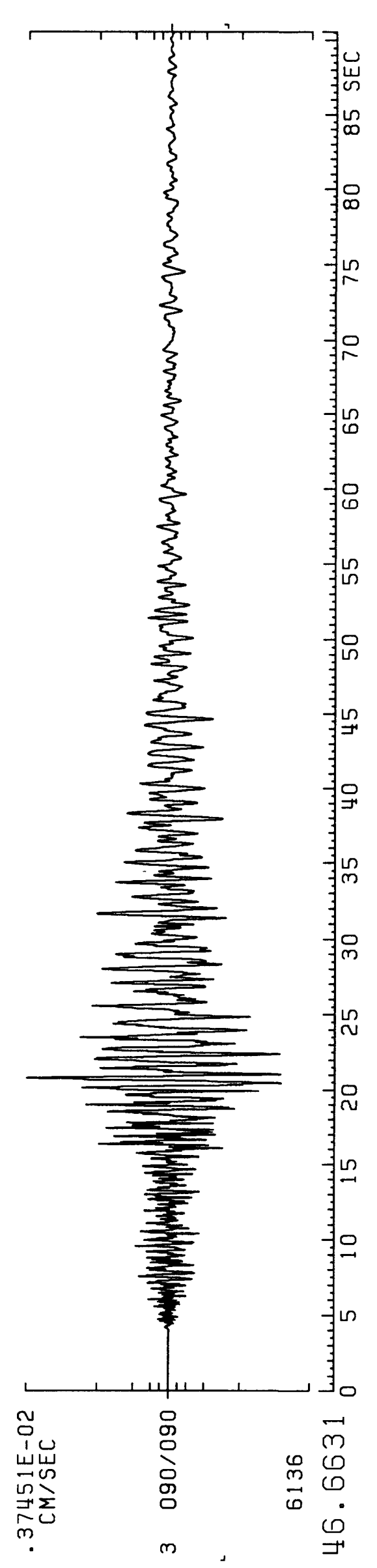


196

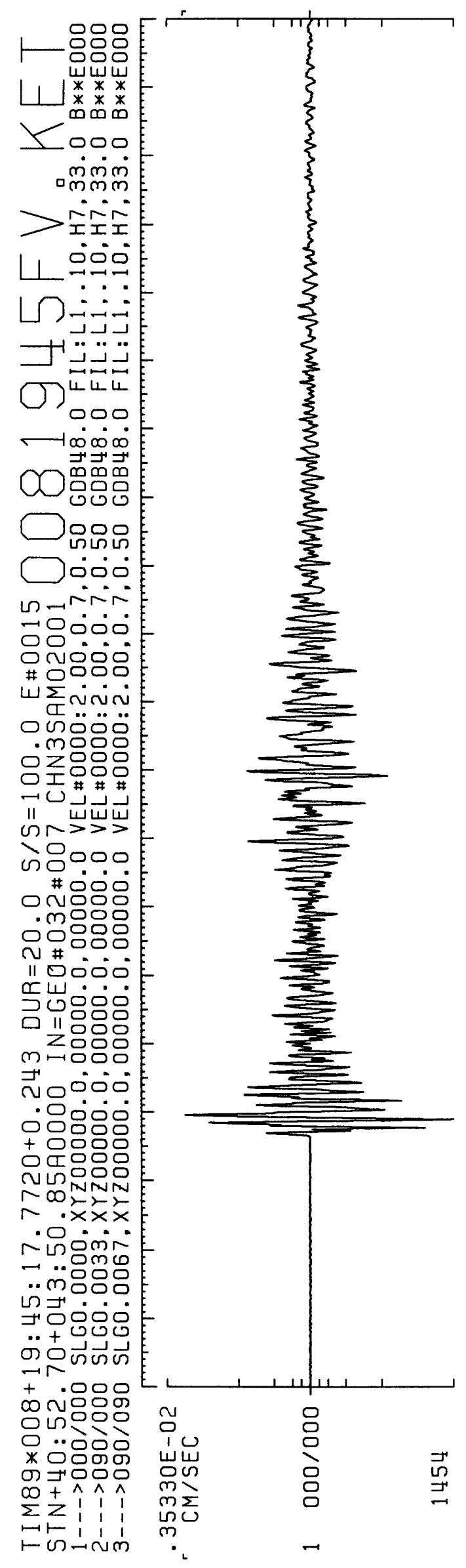

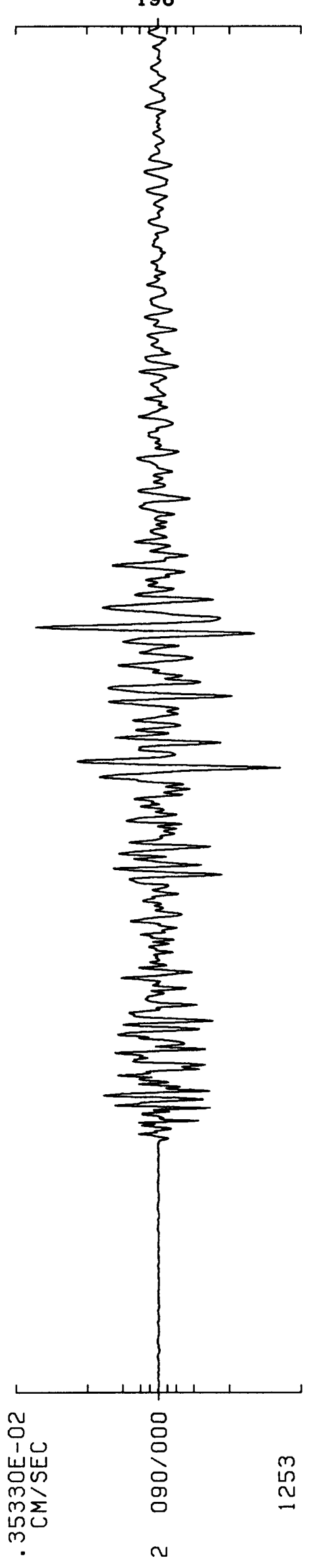

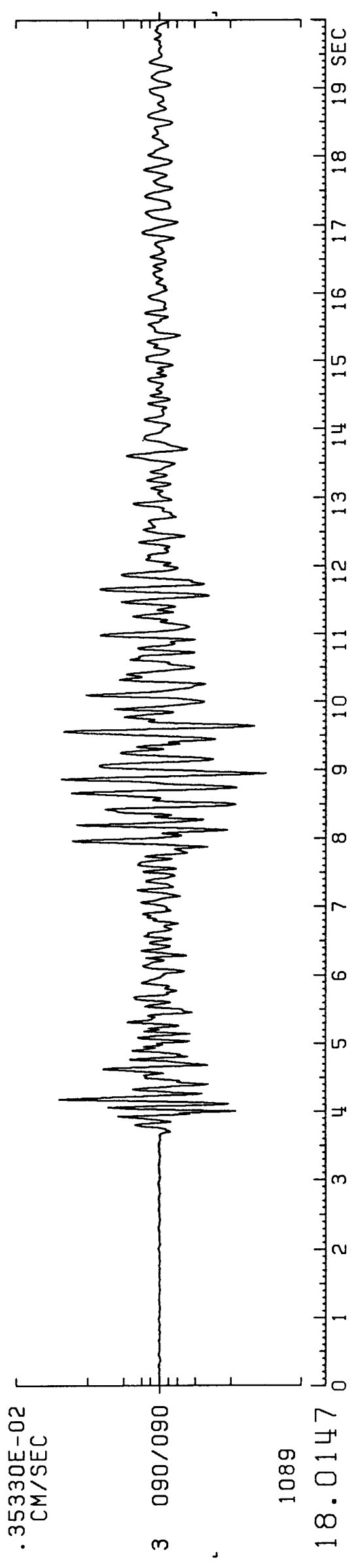



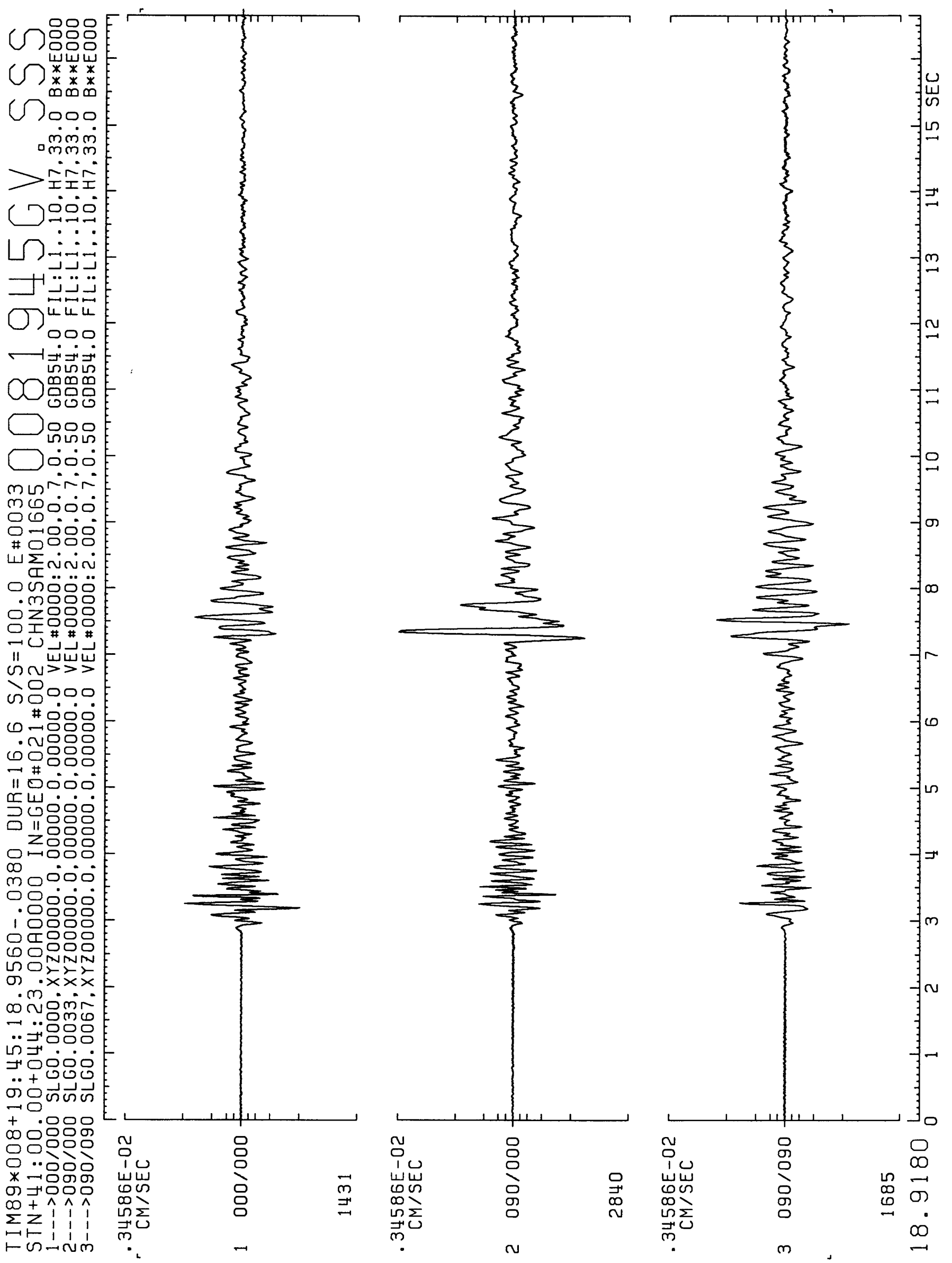


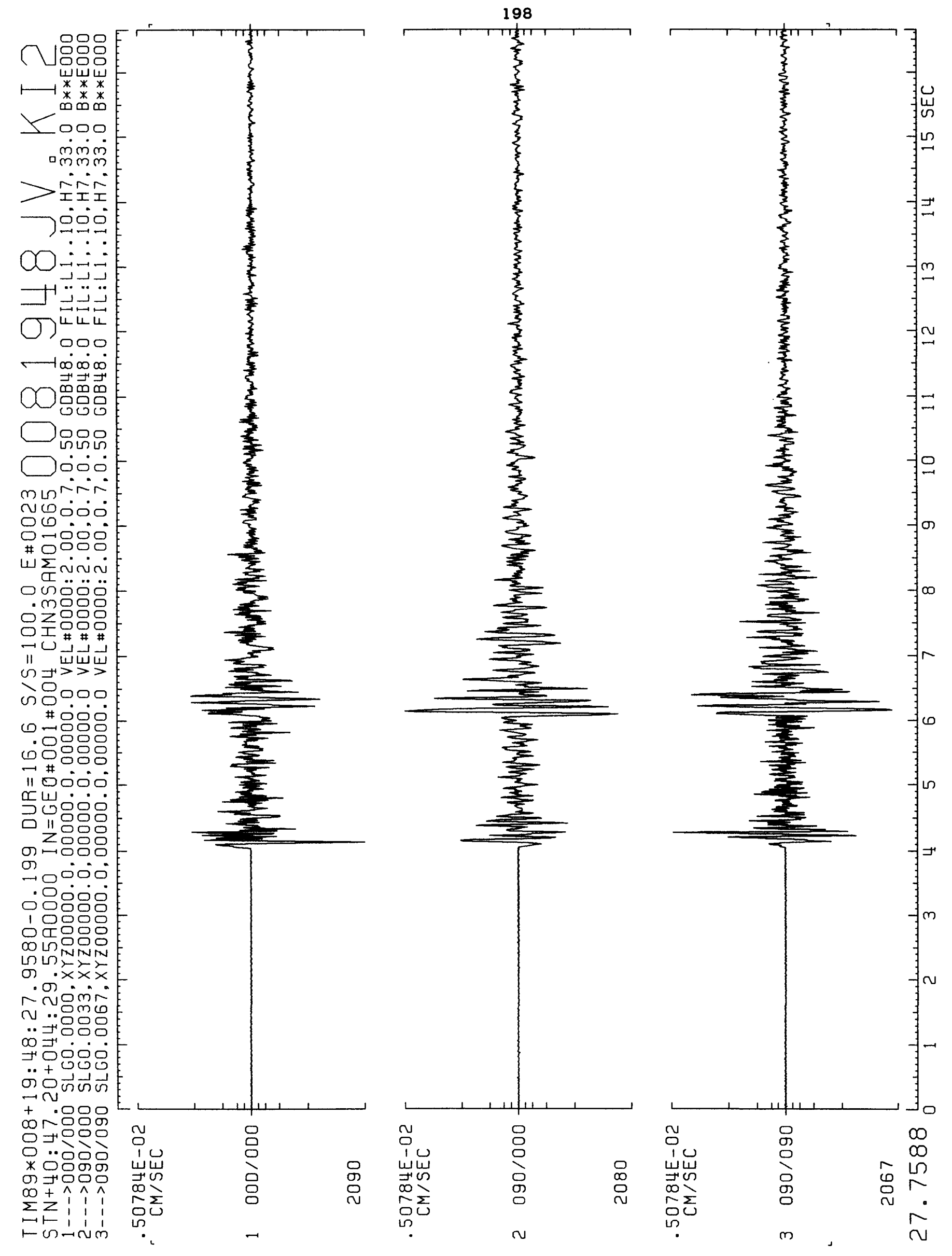



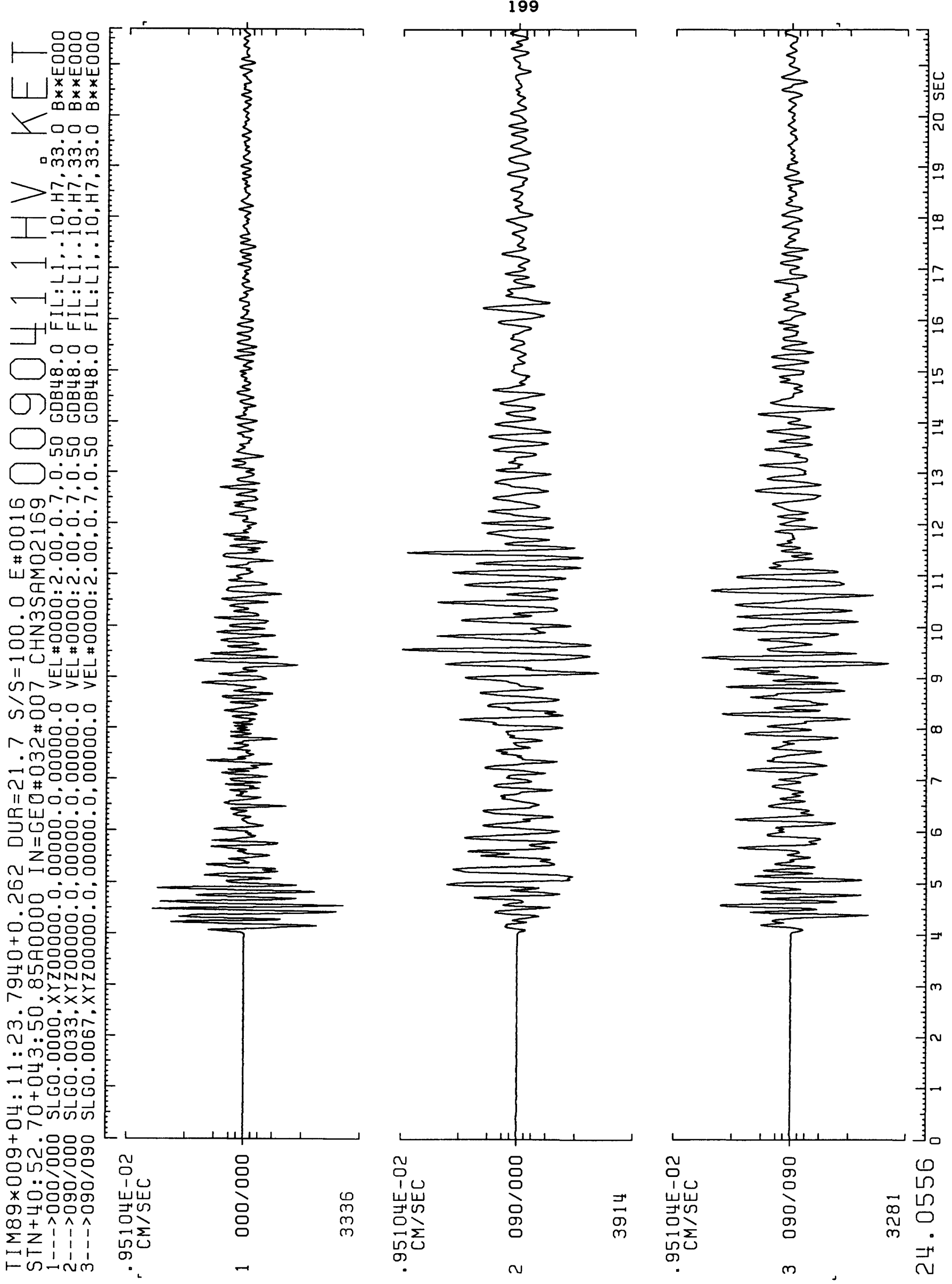


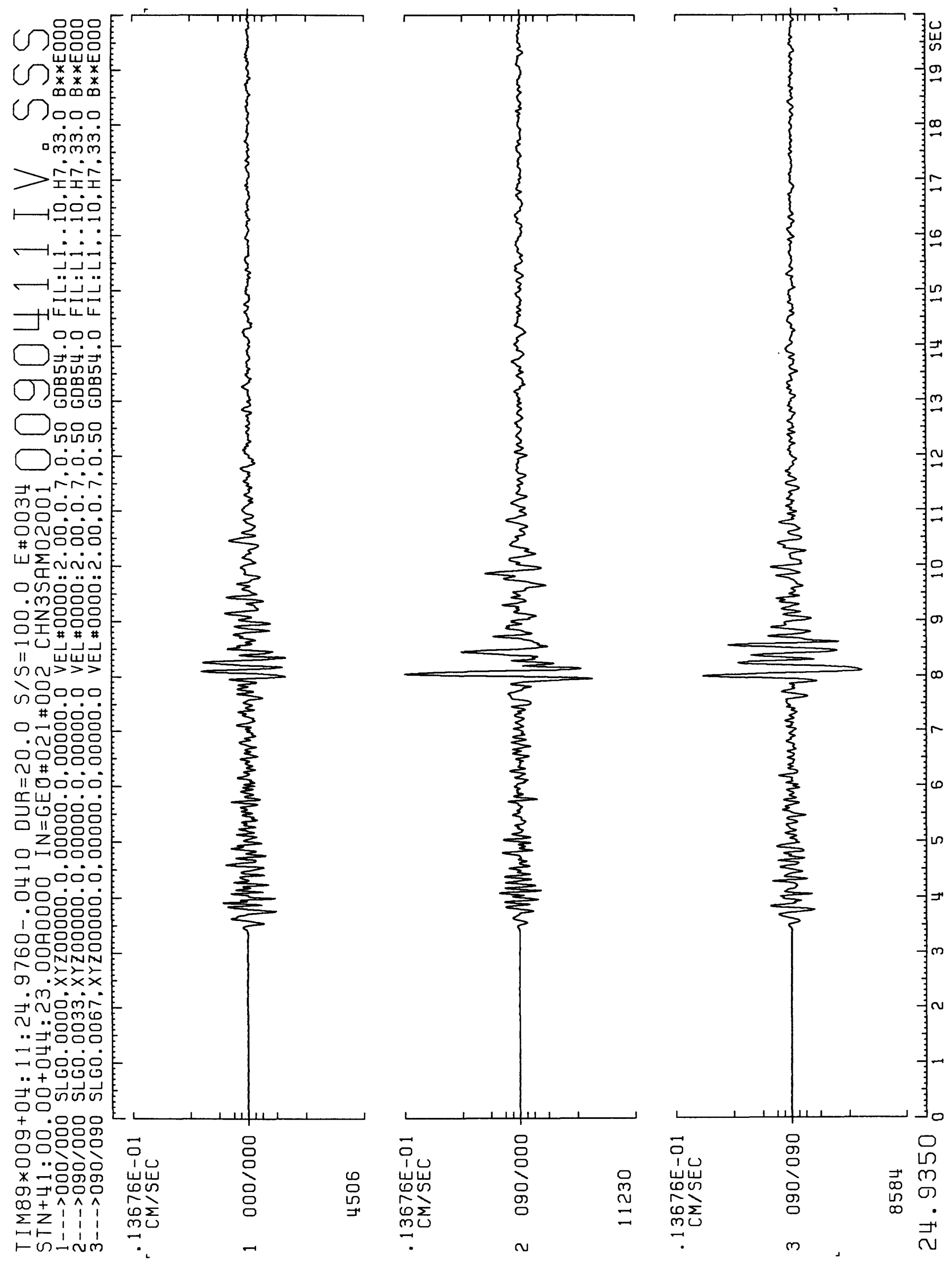


01
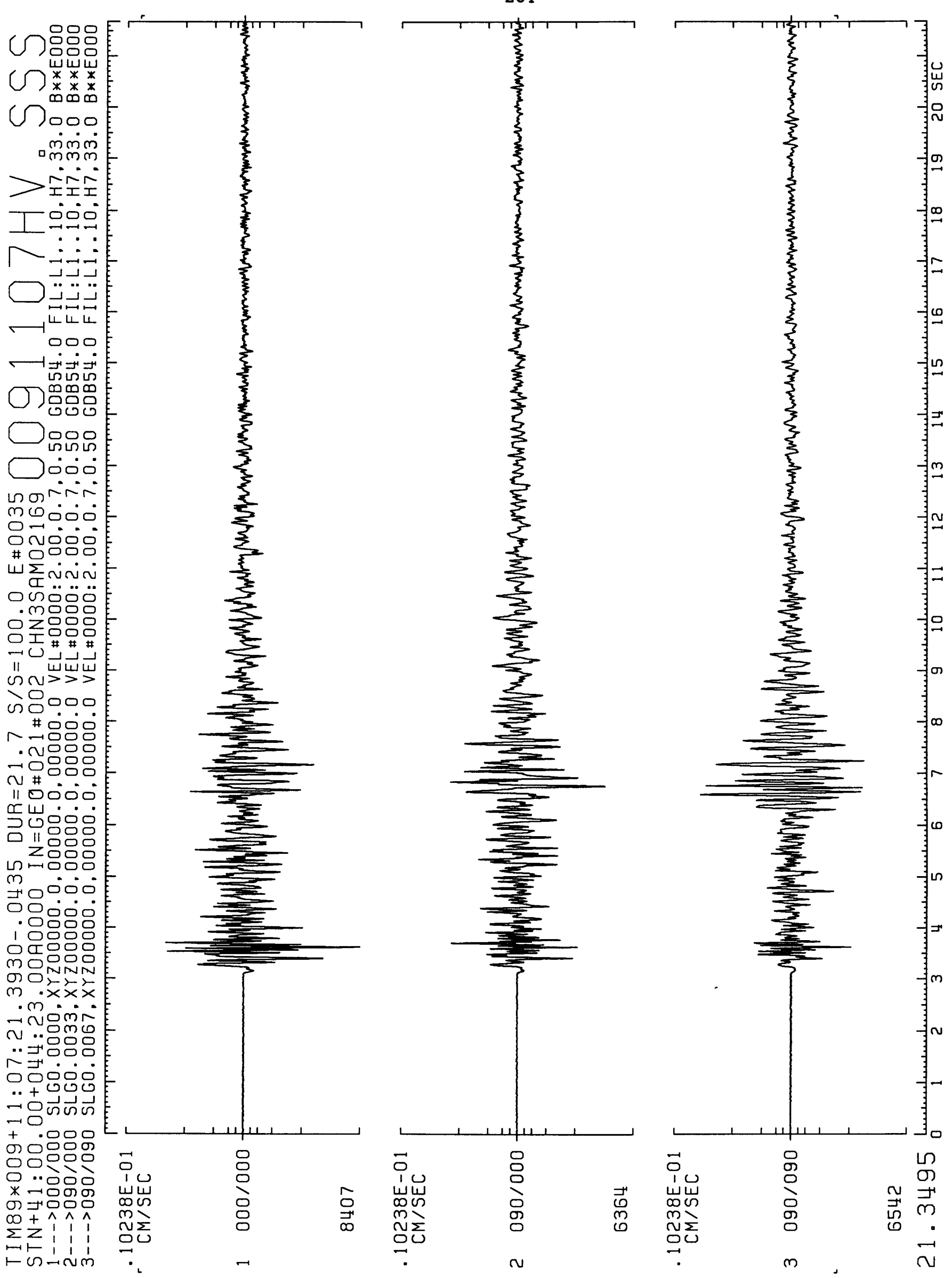
202
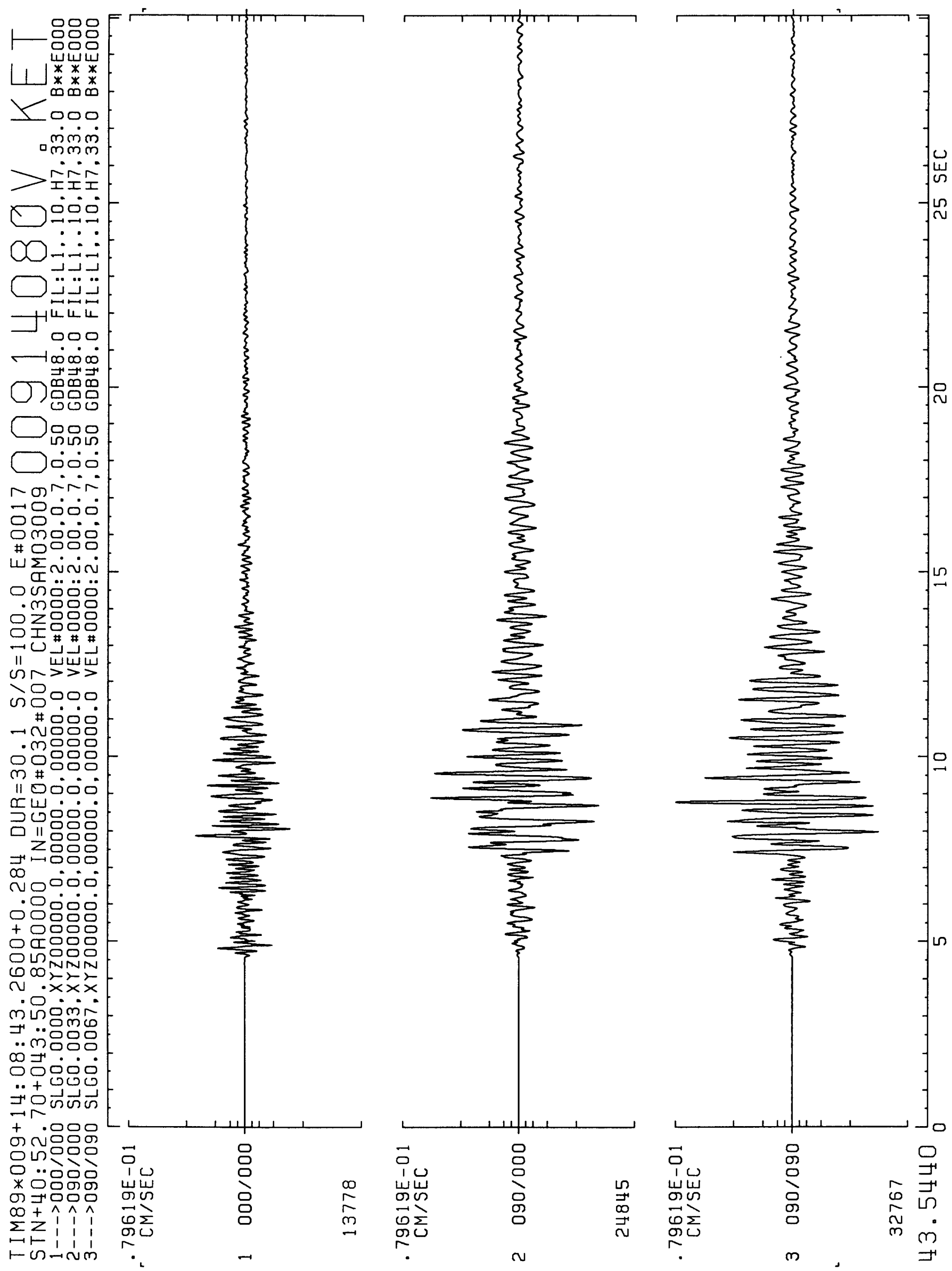
203
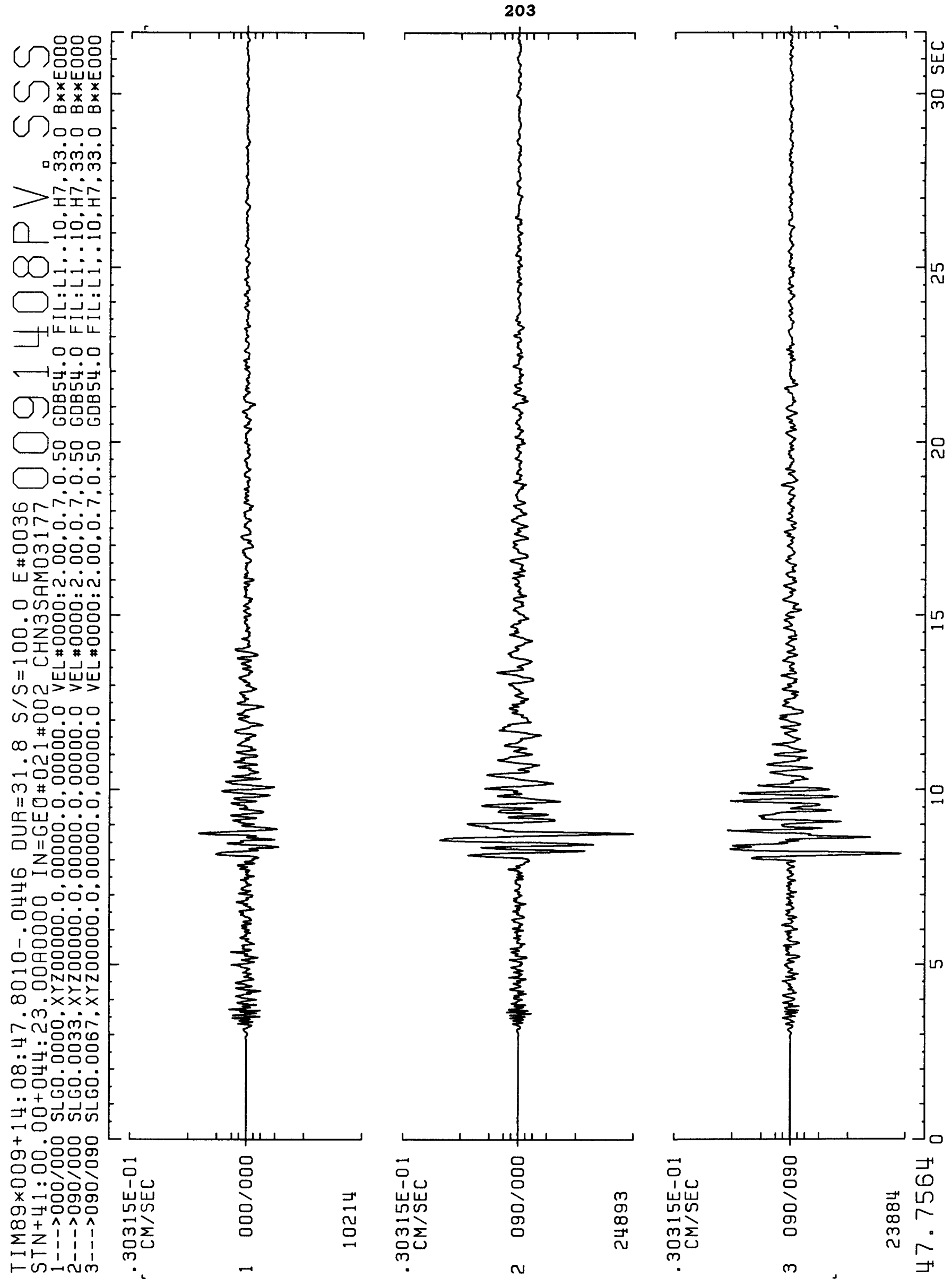

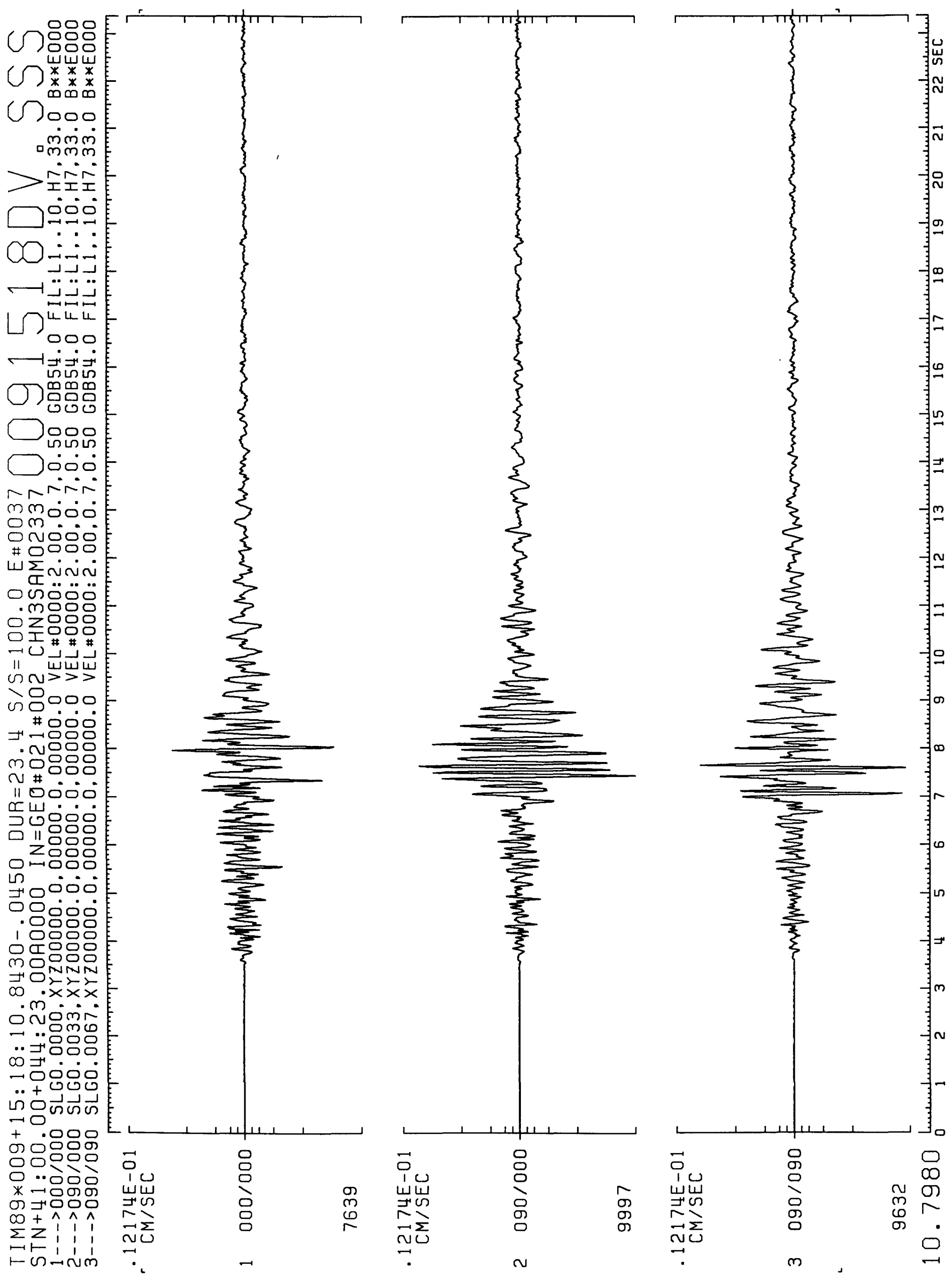


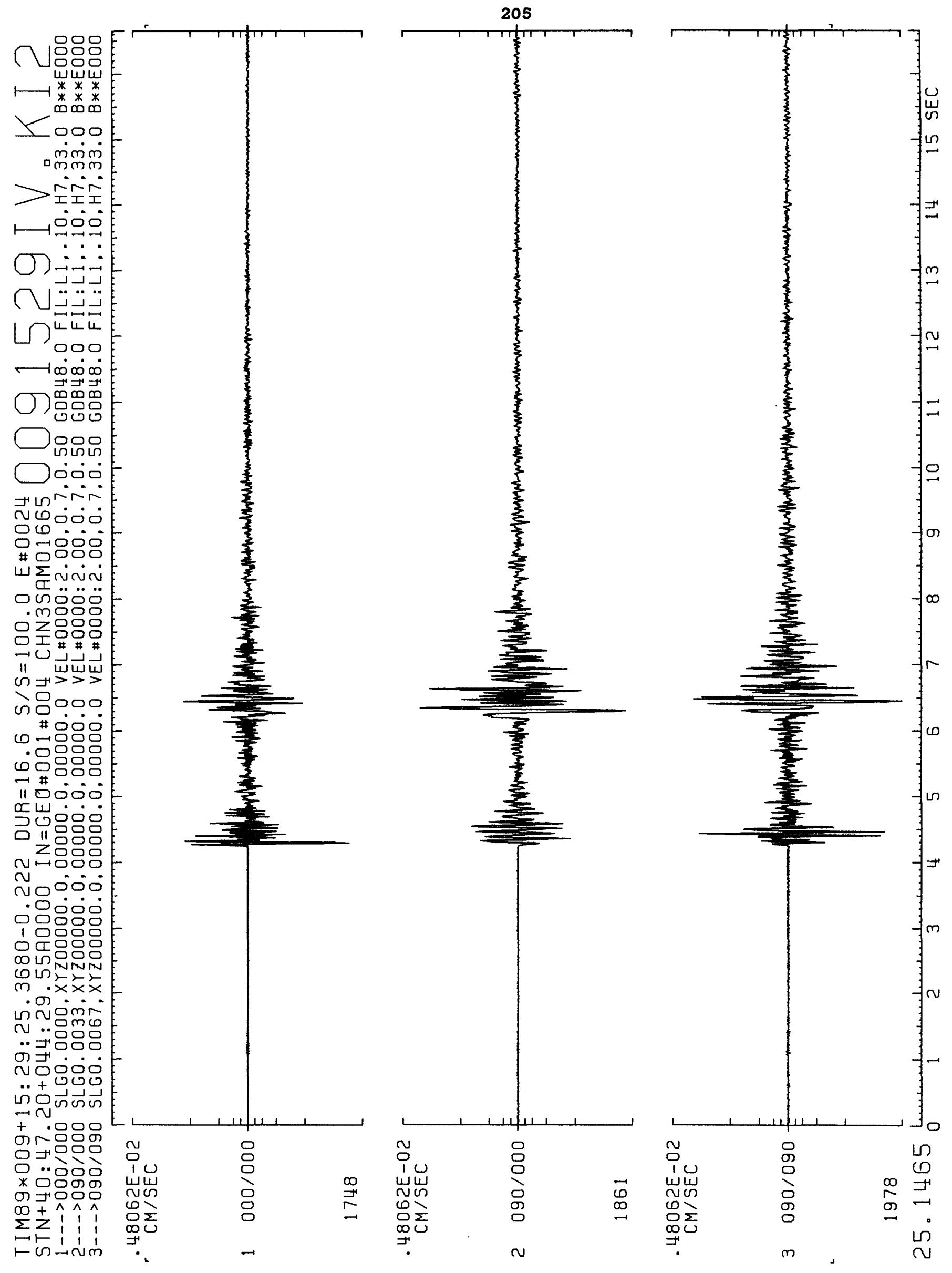



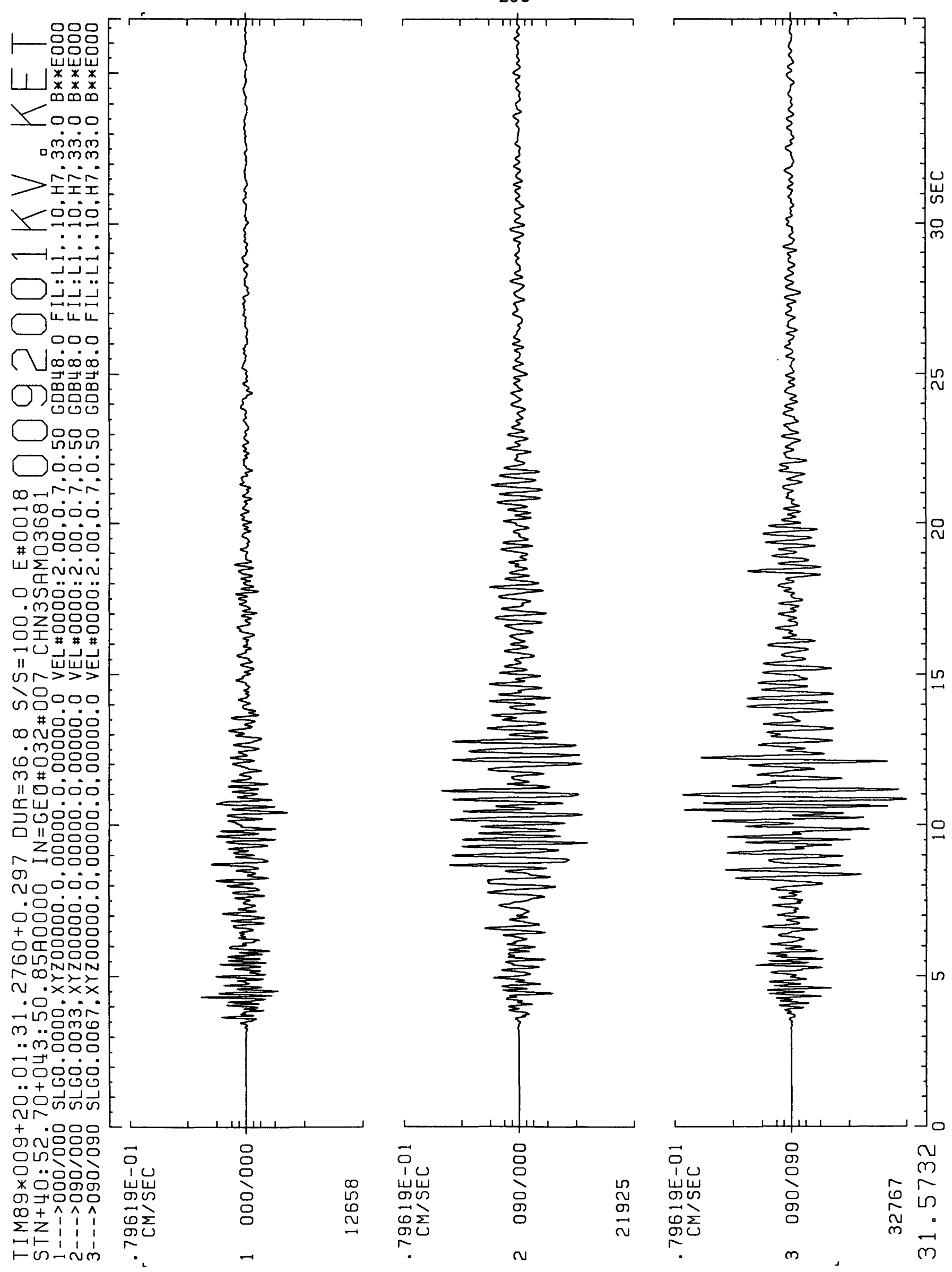


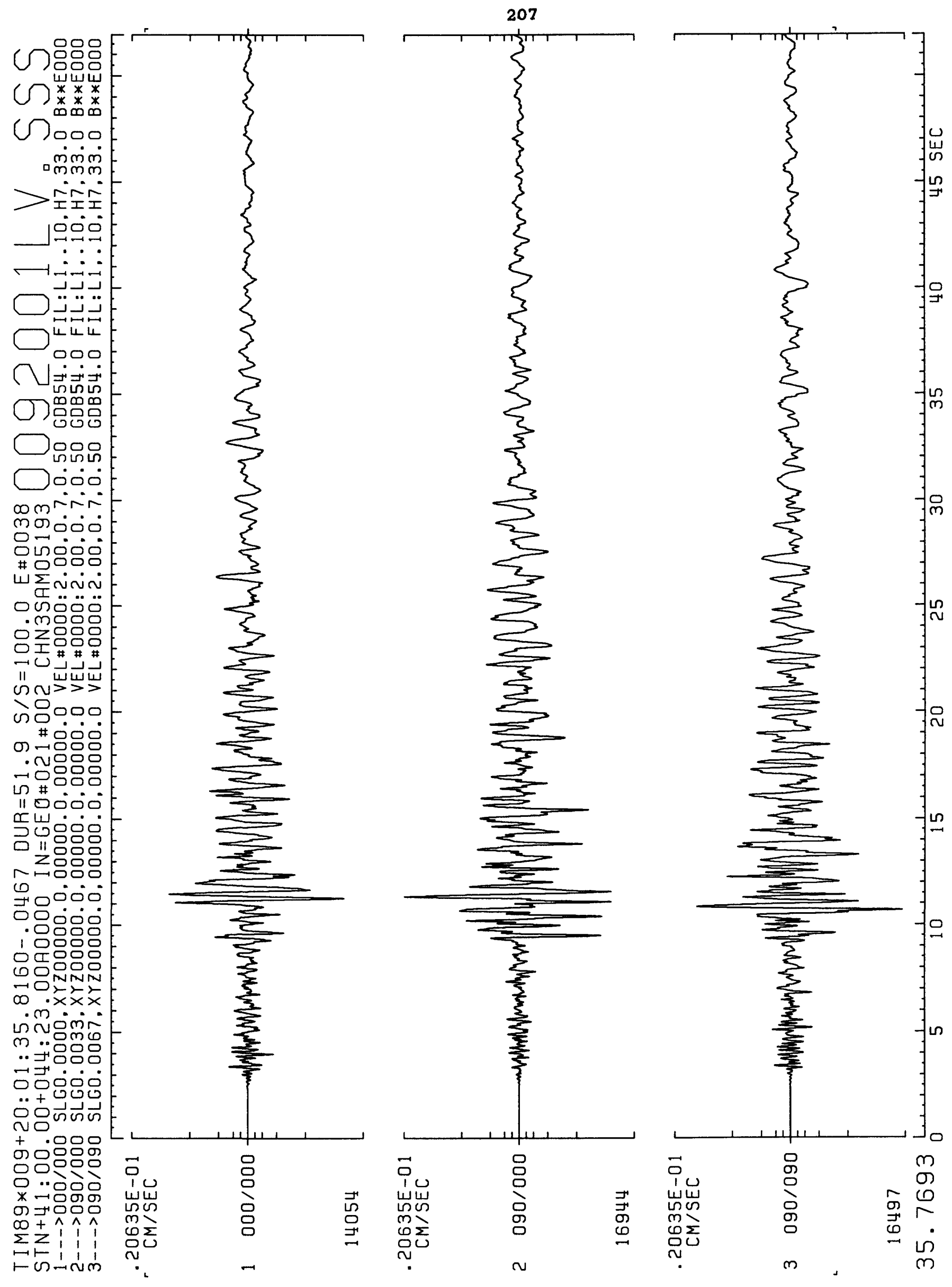


208

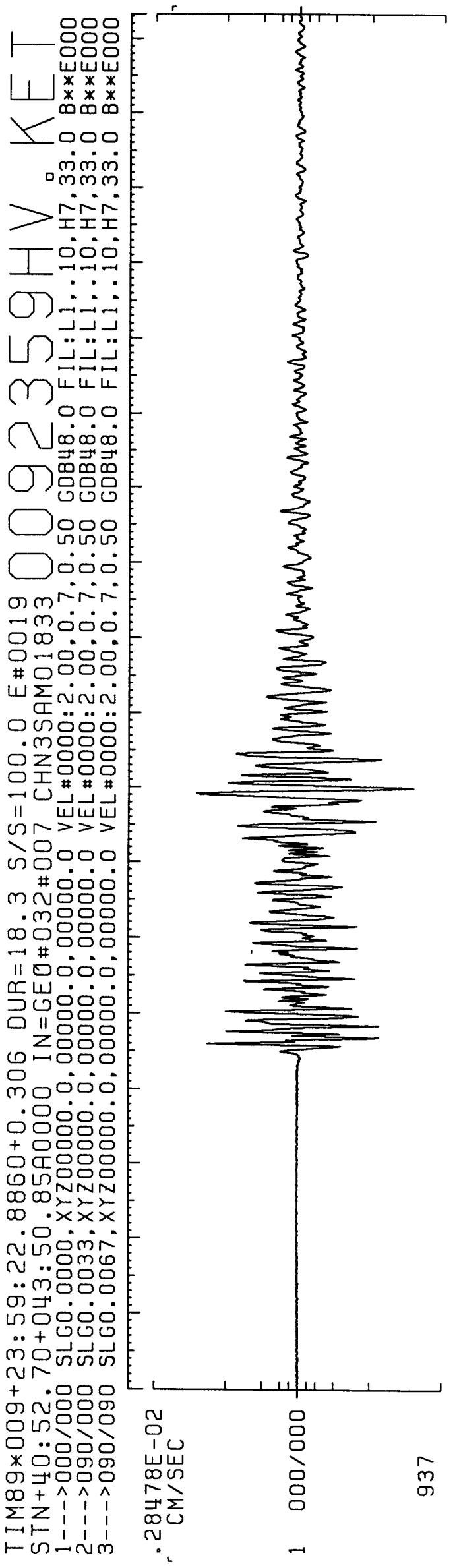

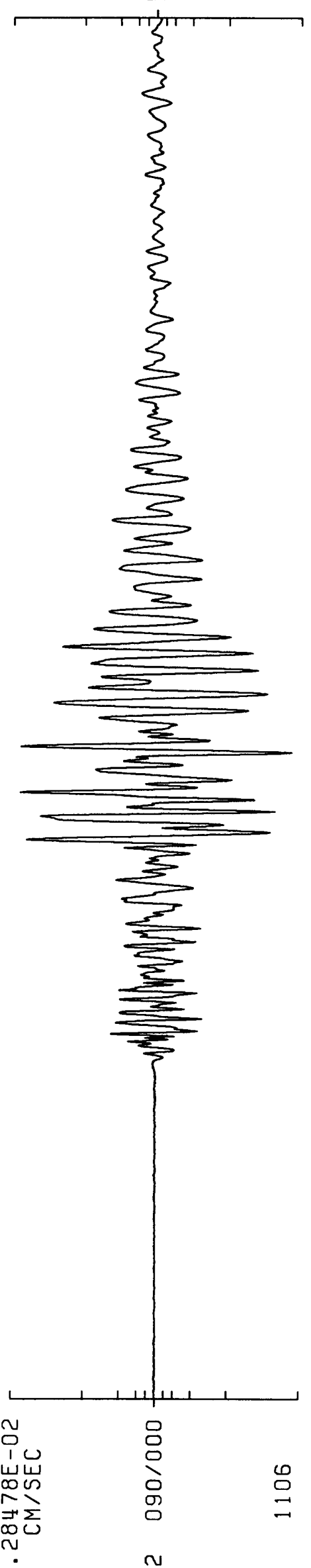

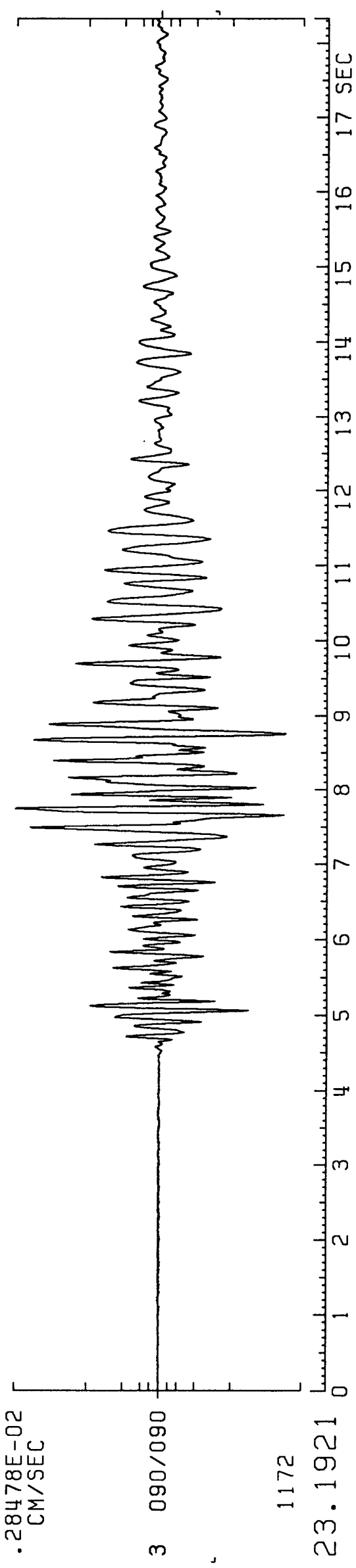



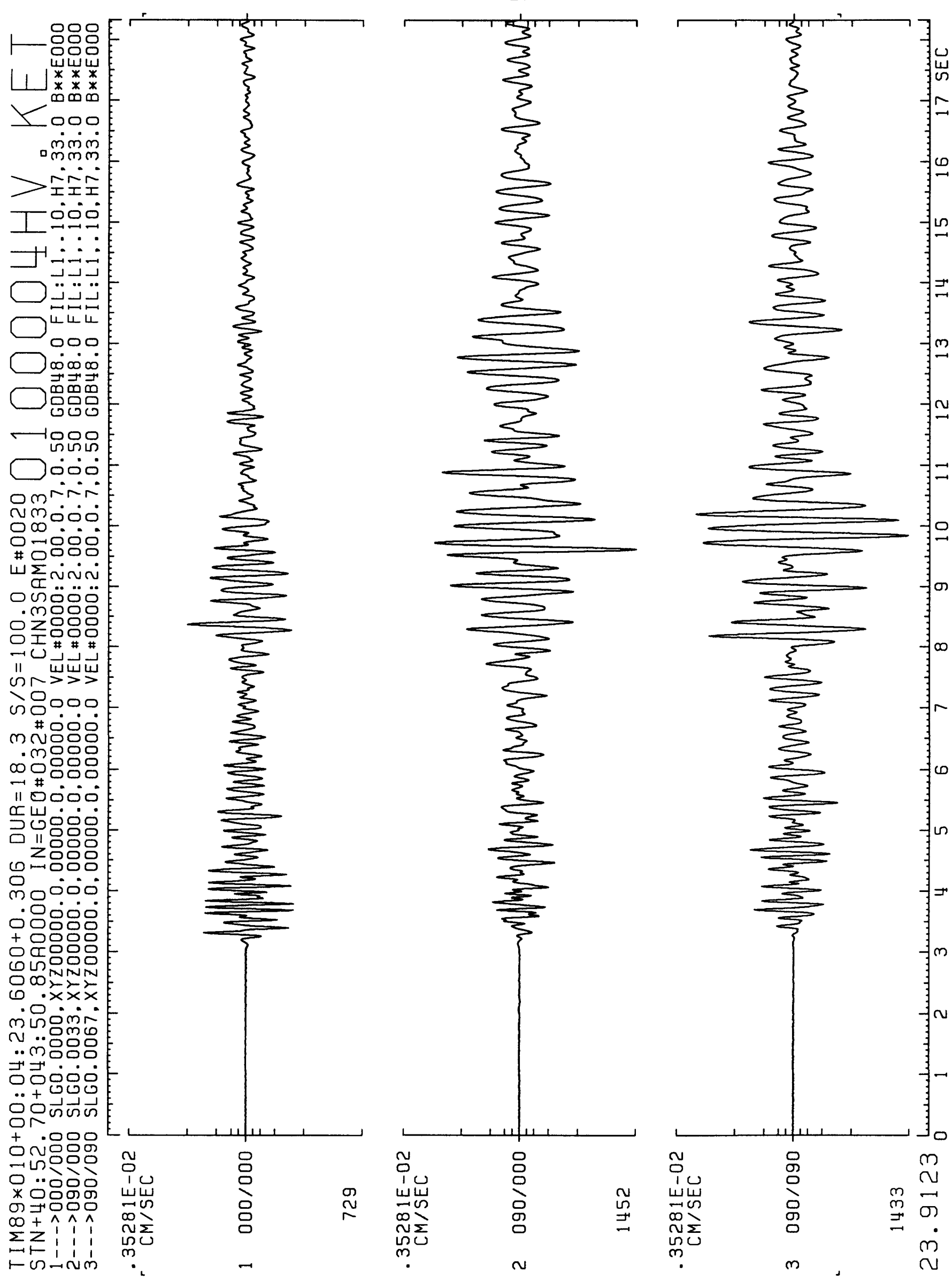
210

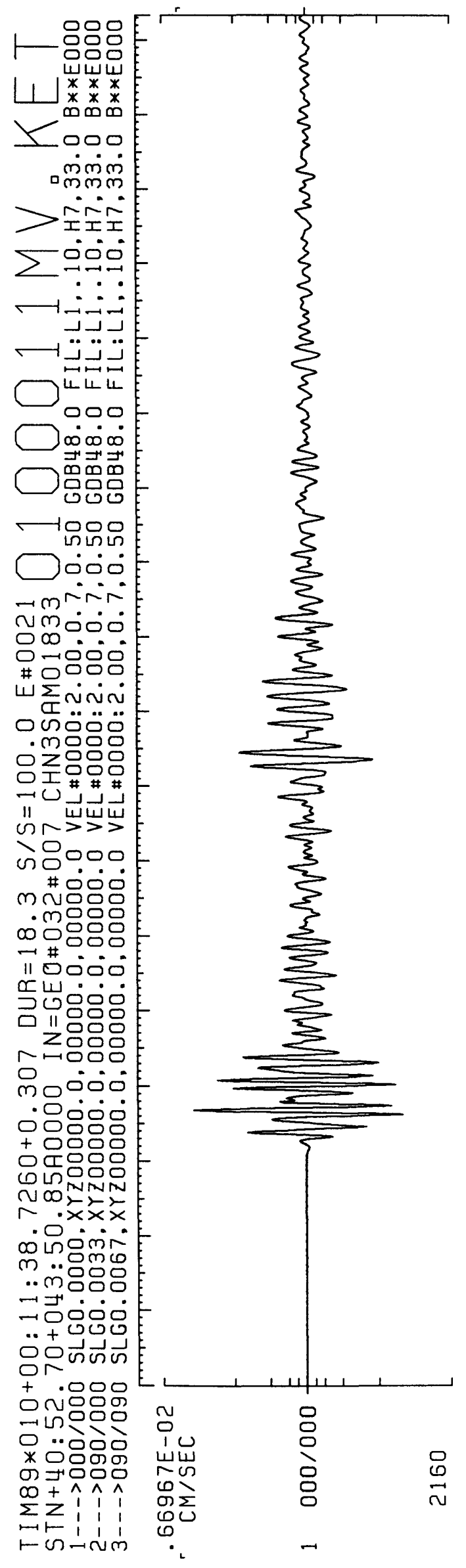

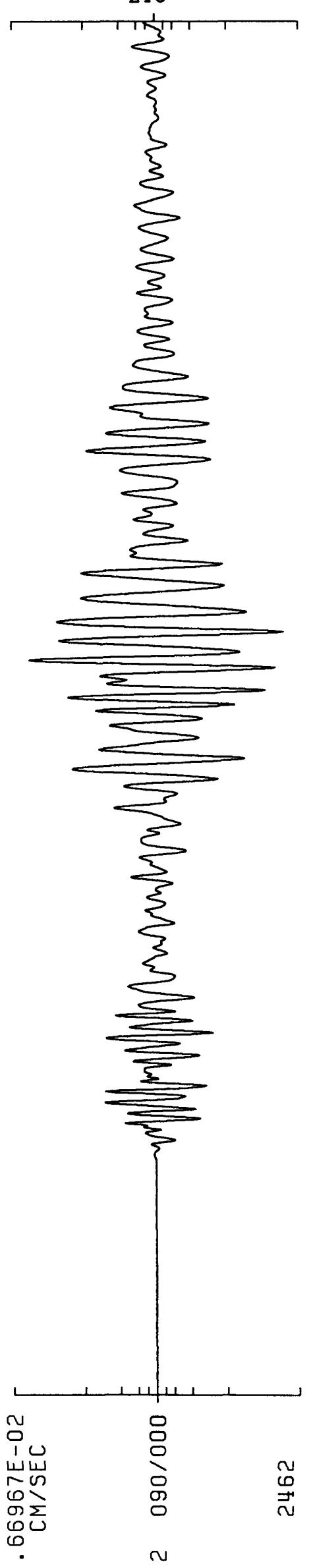

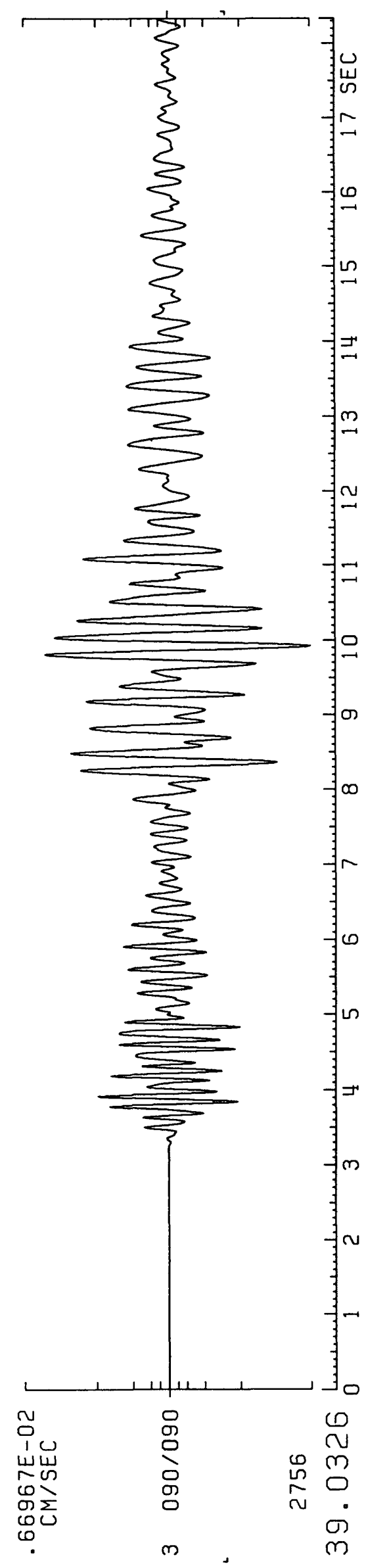


211

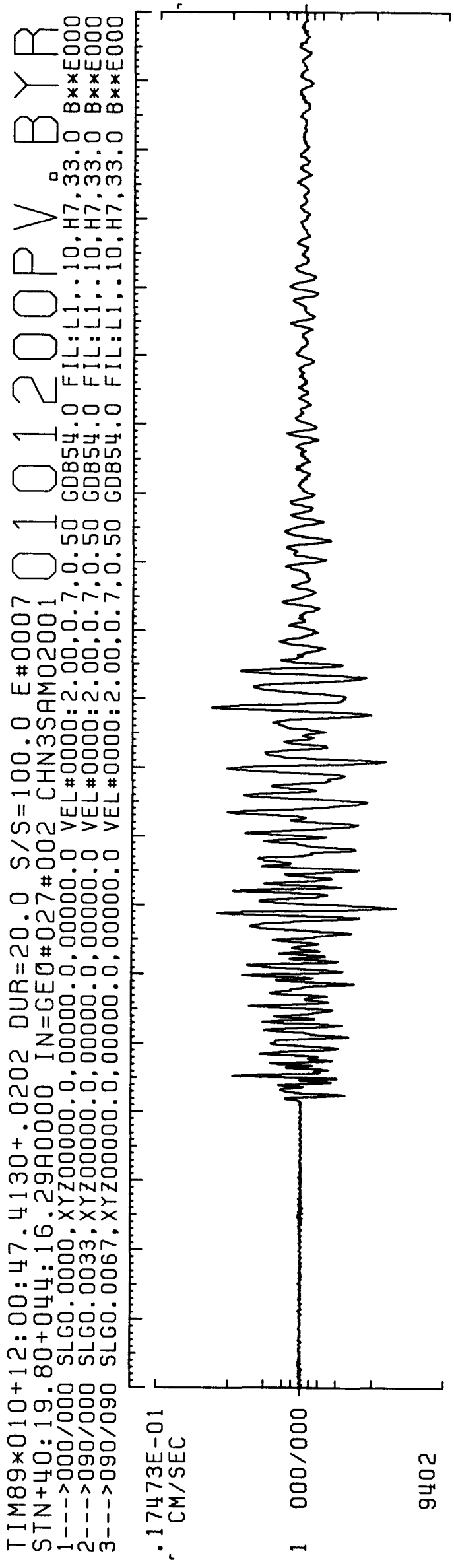

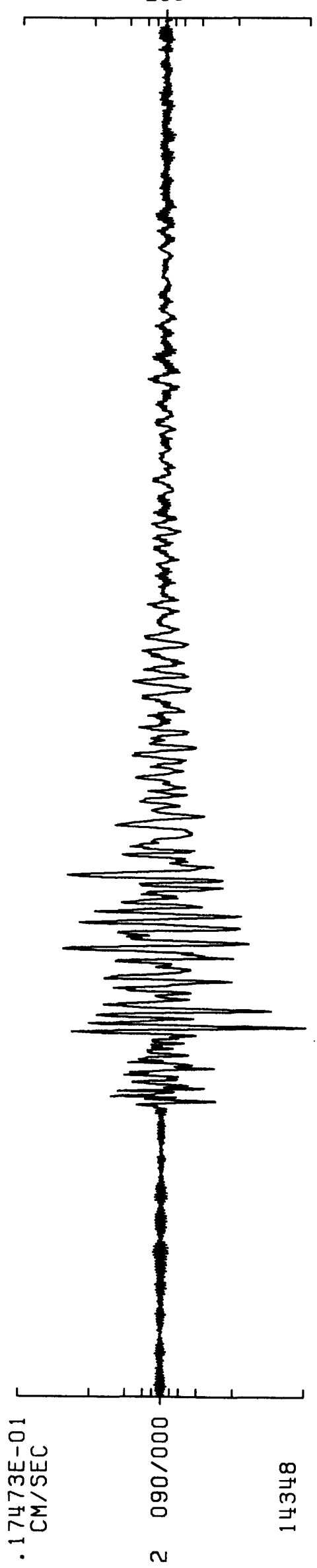

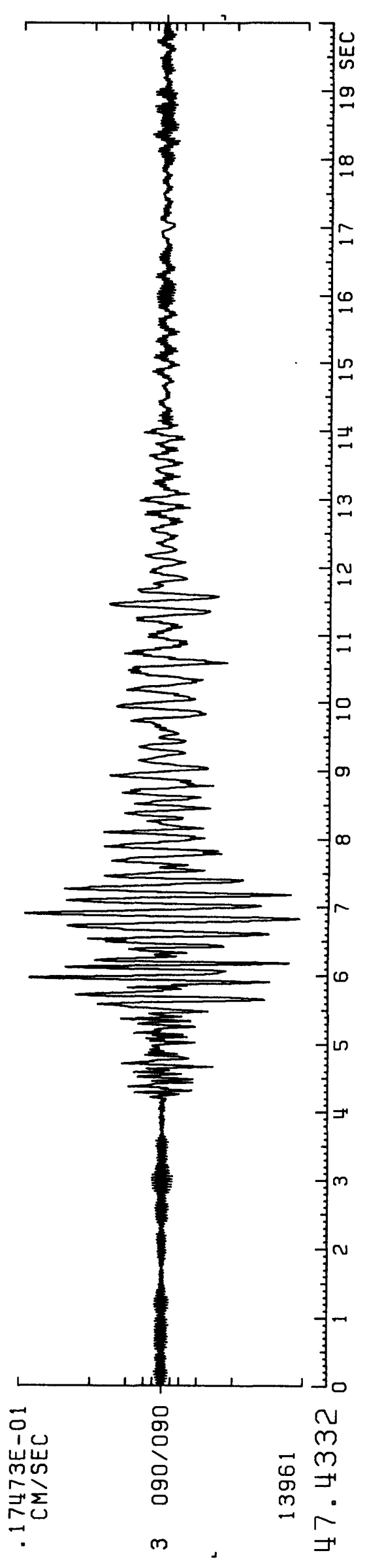


212
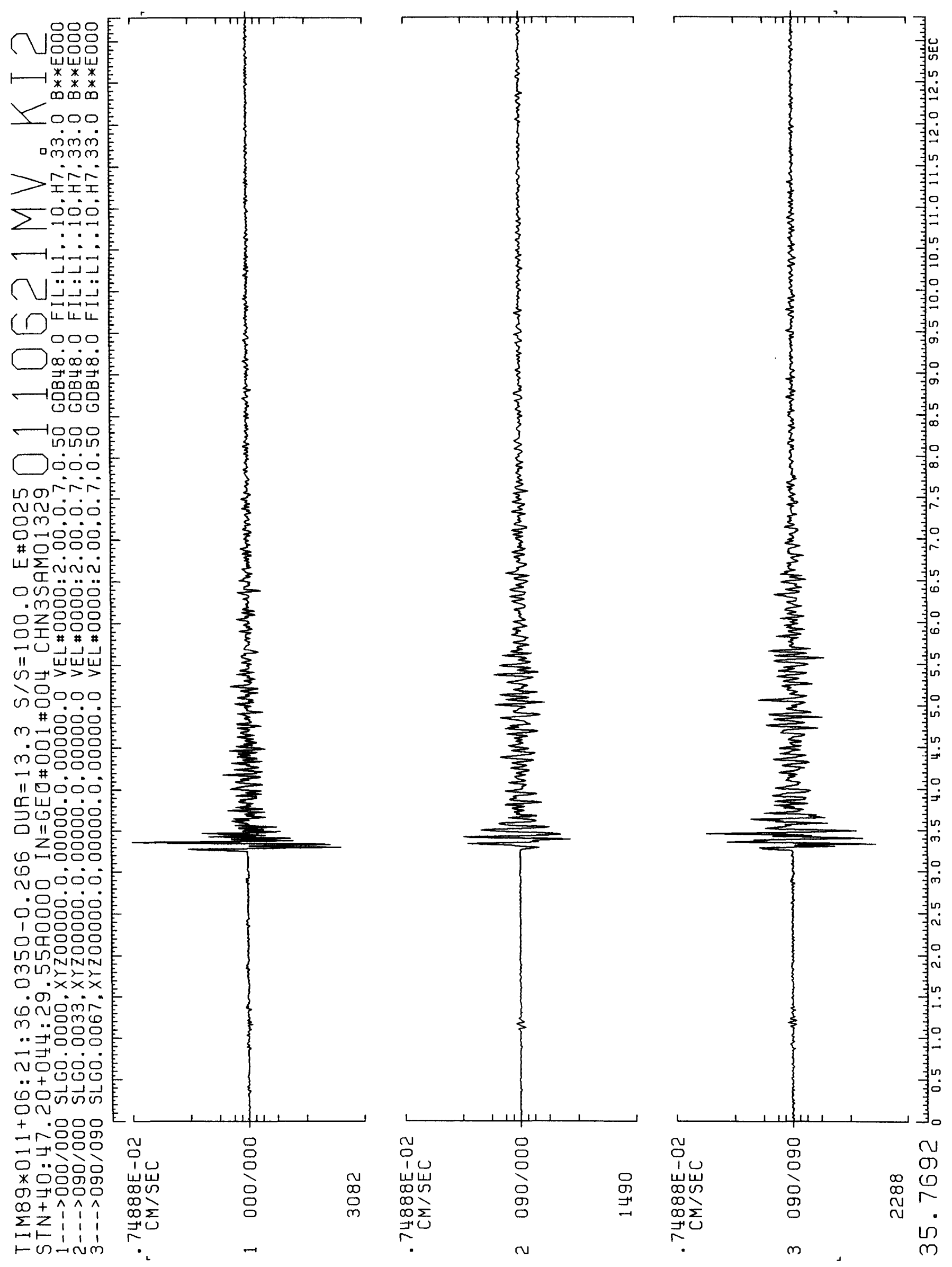


\section{3}
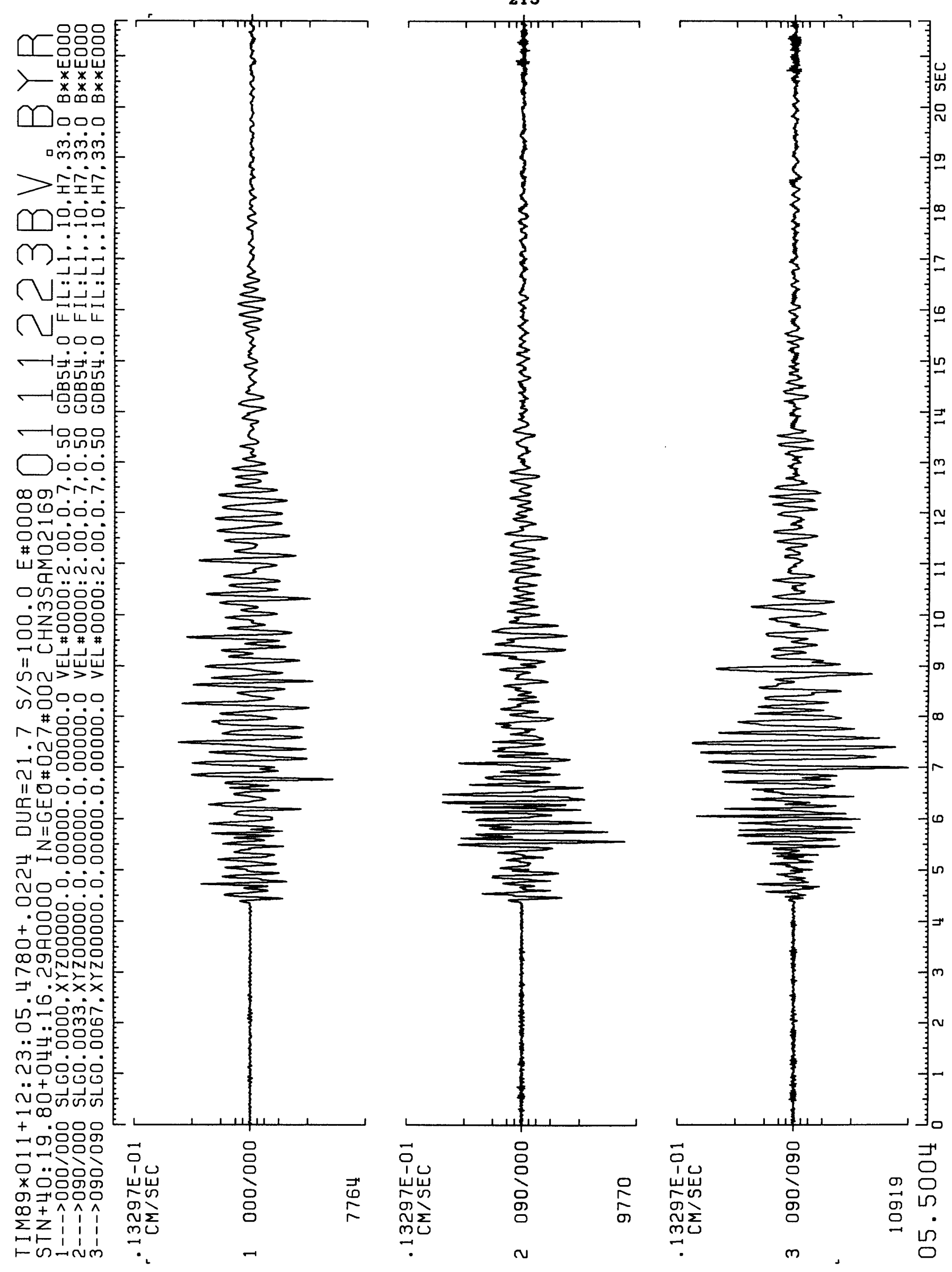


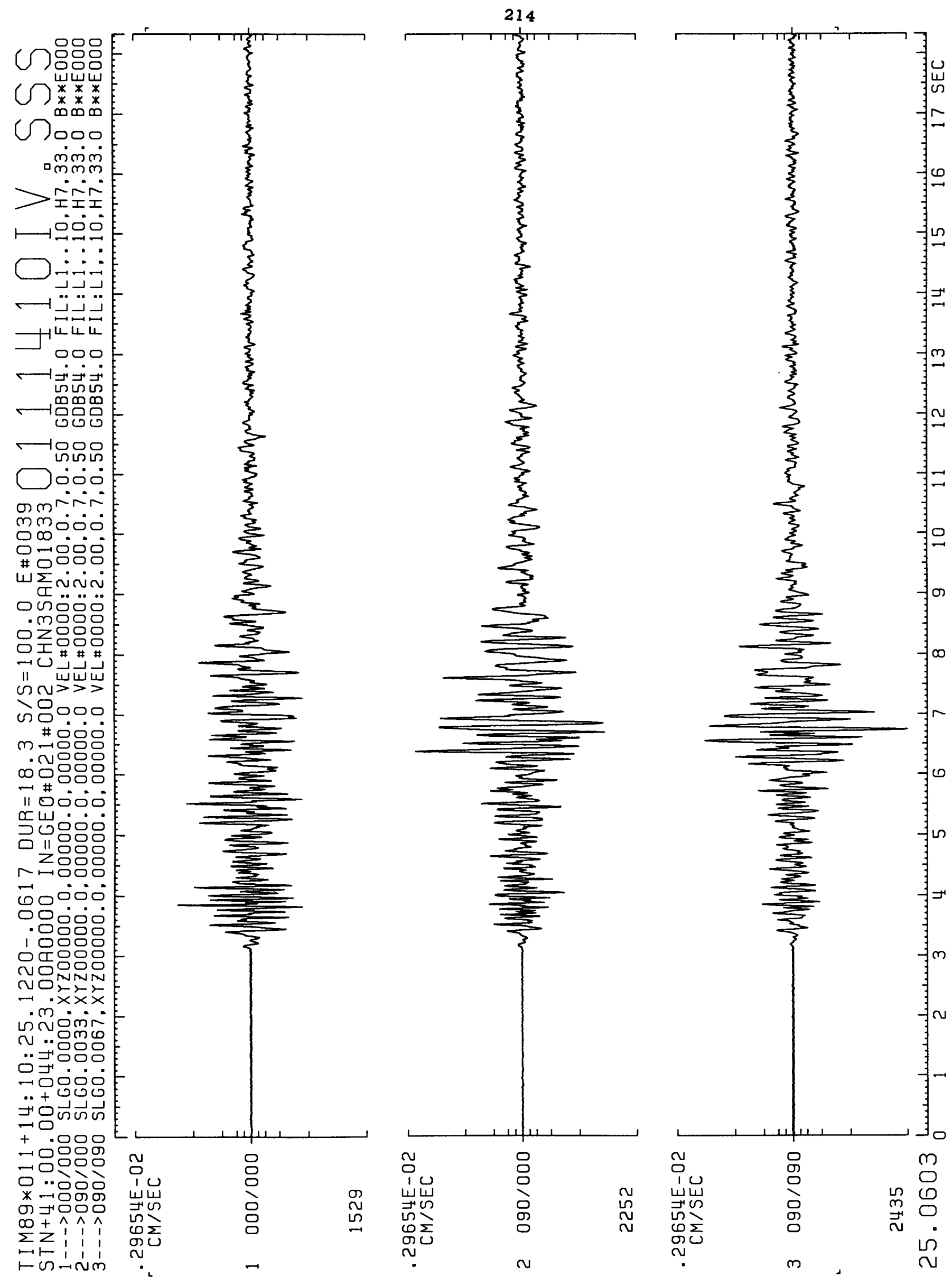



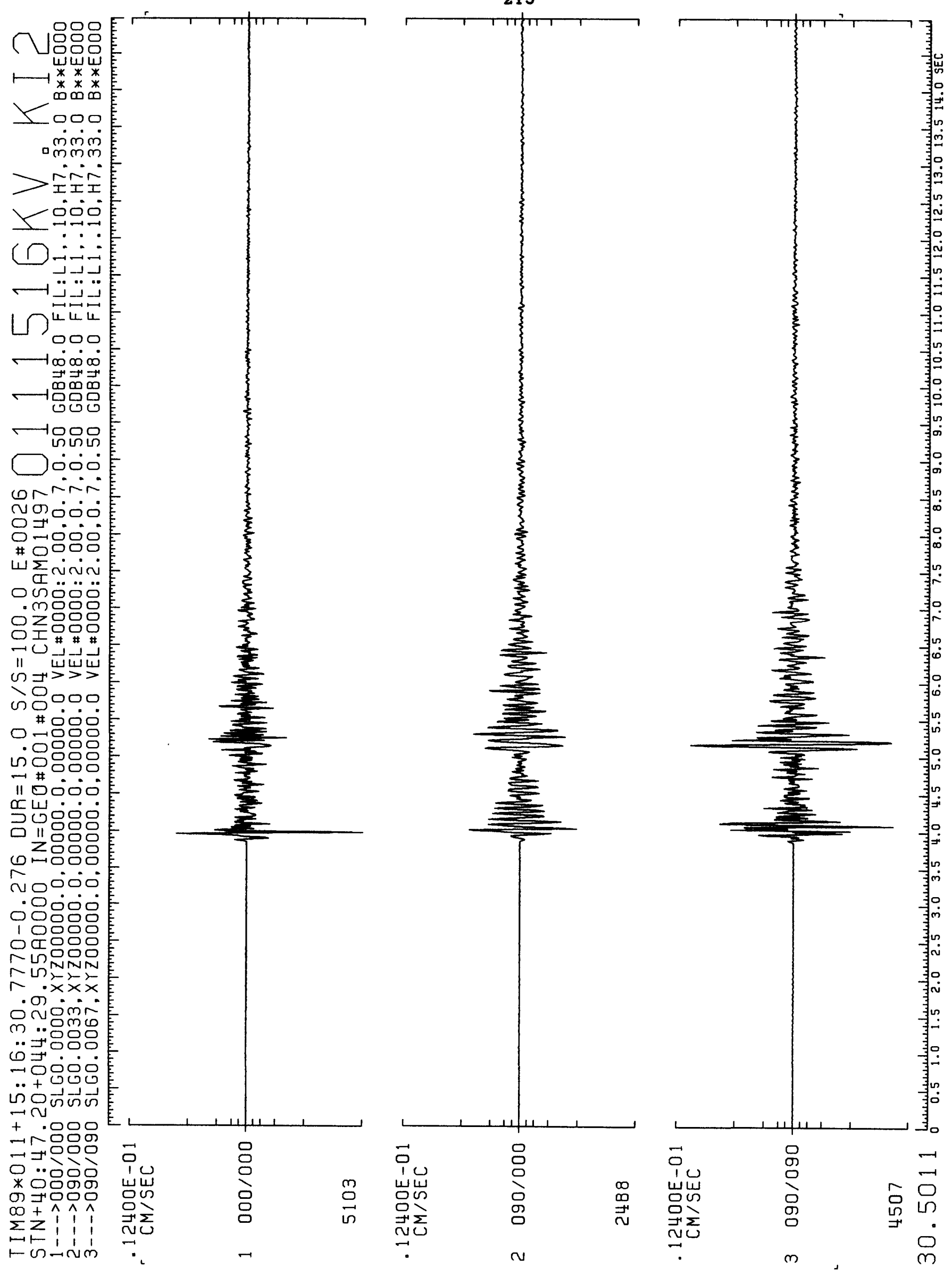


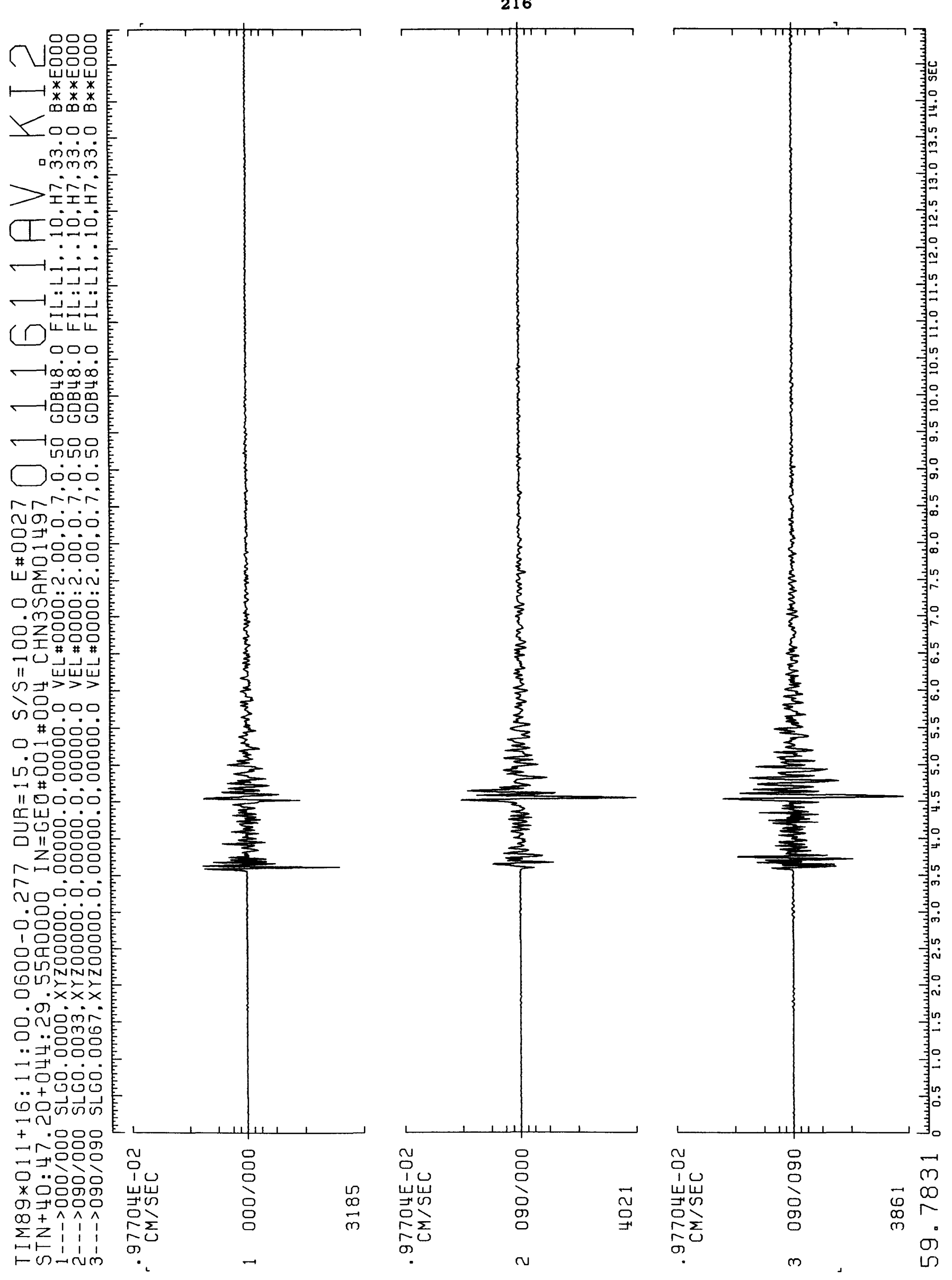



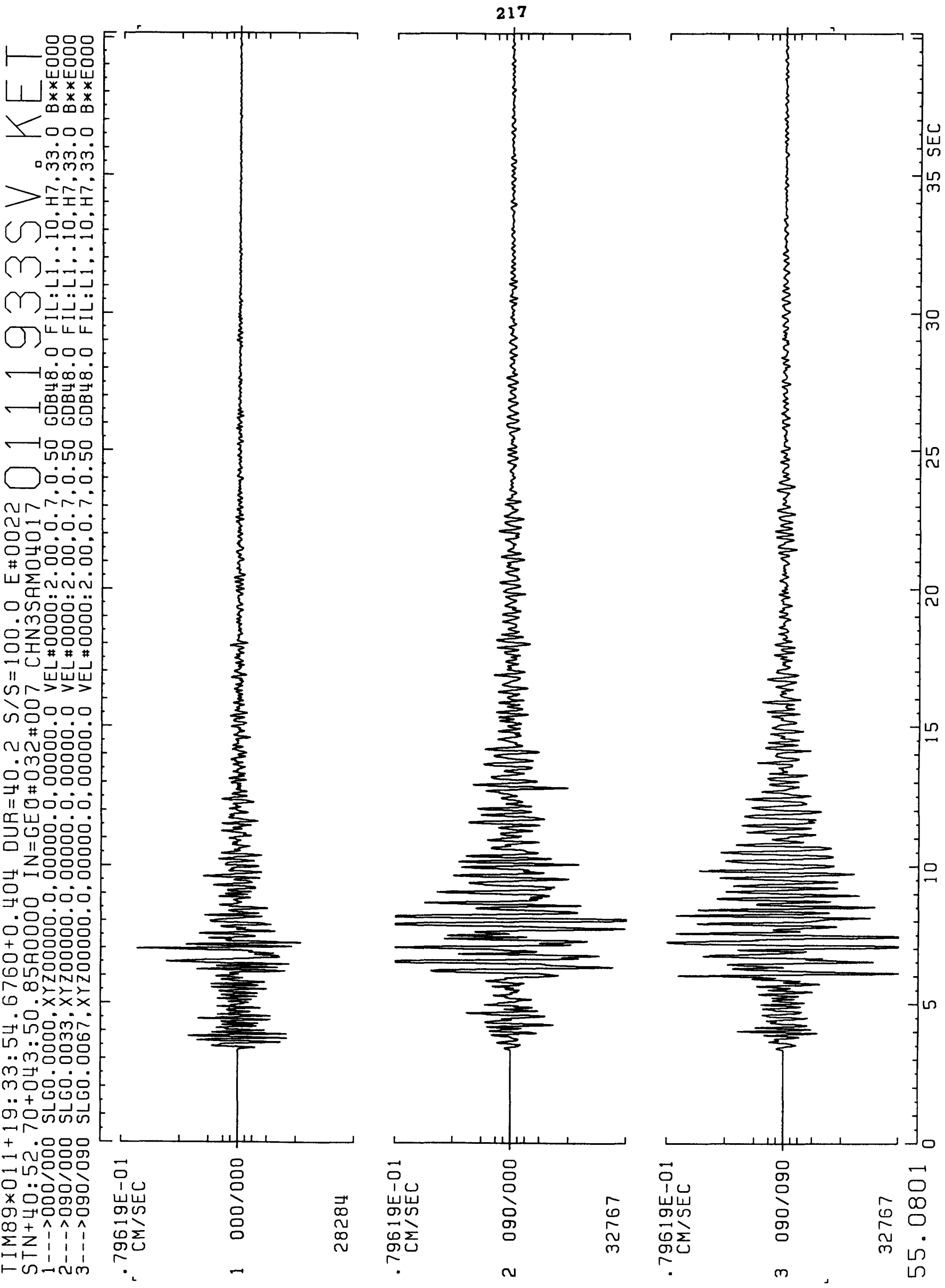
218

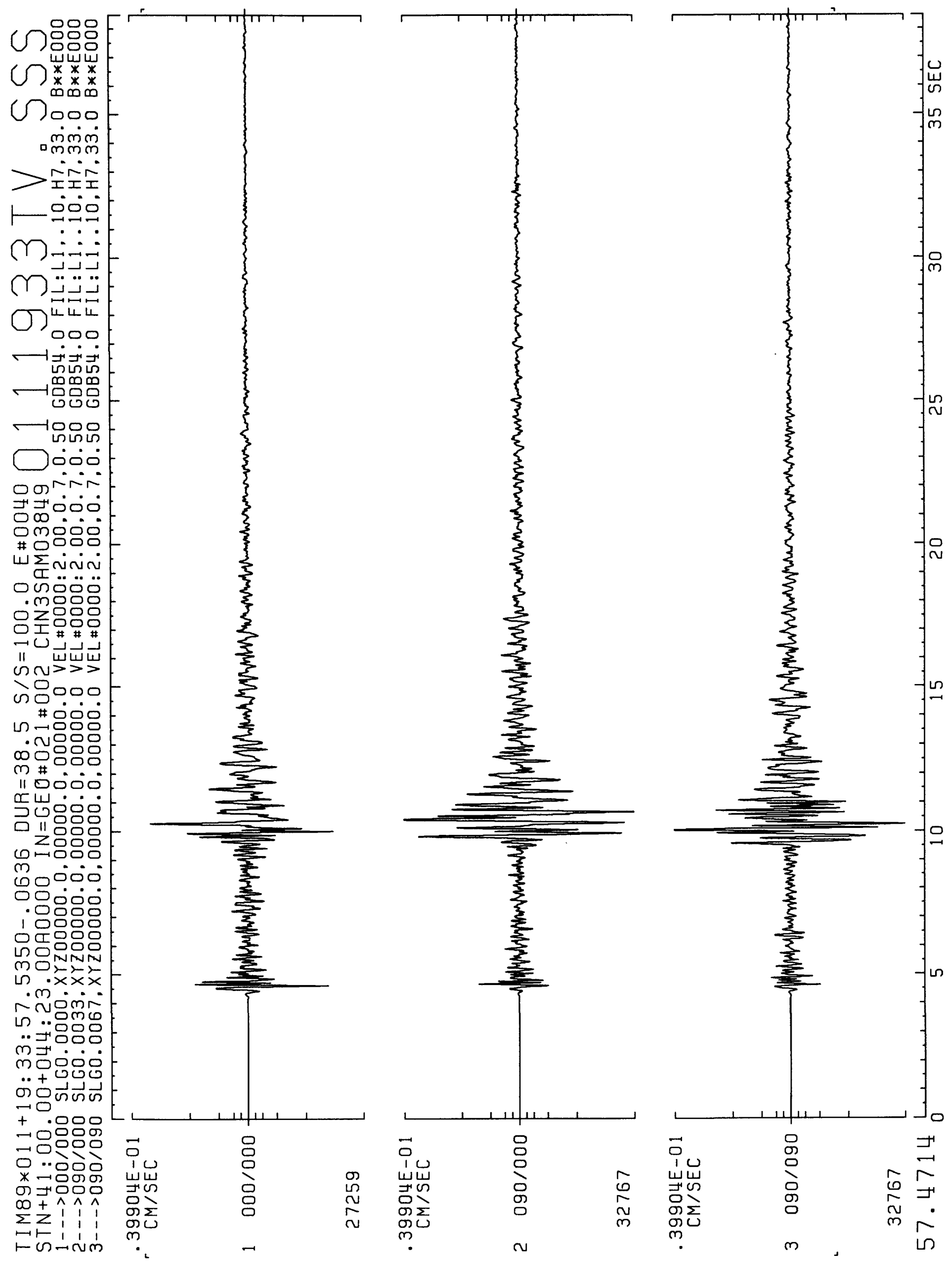



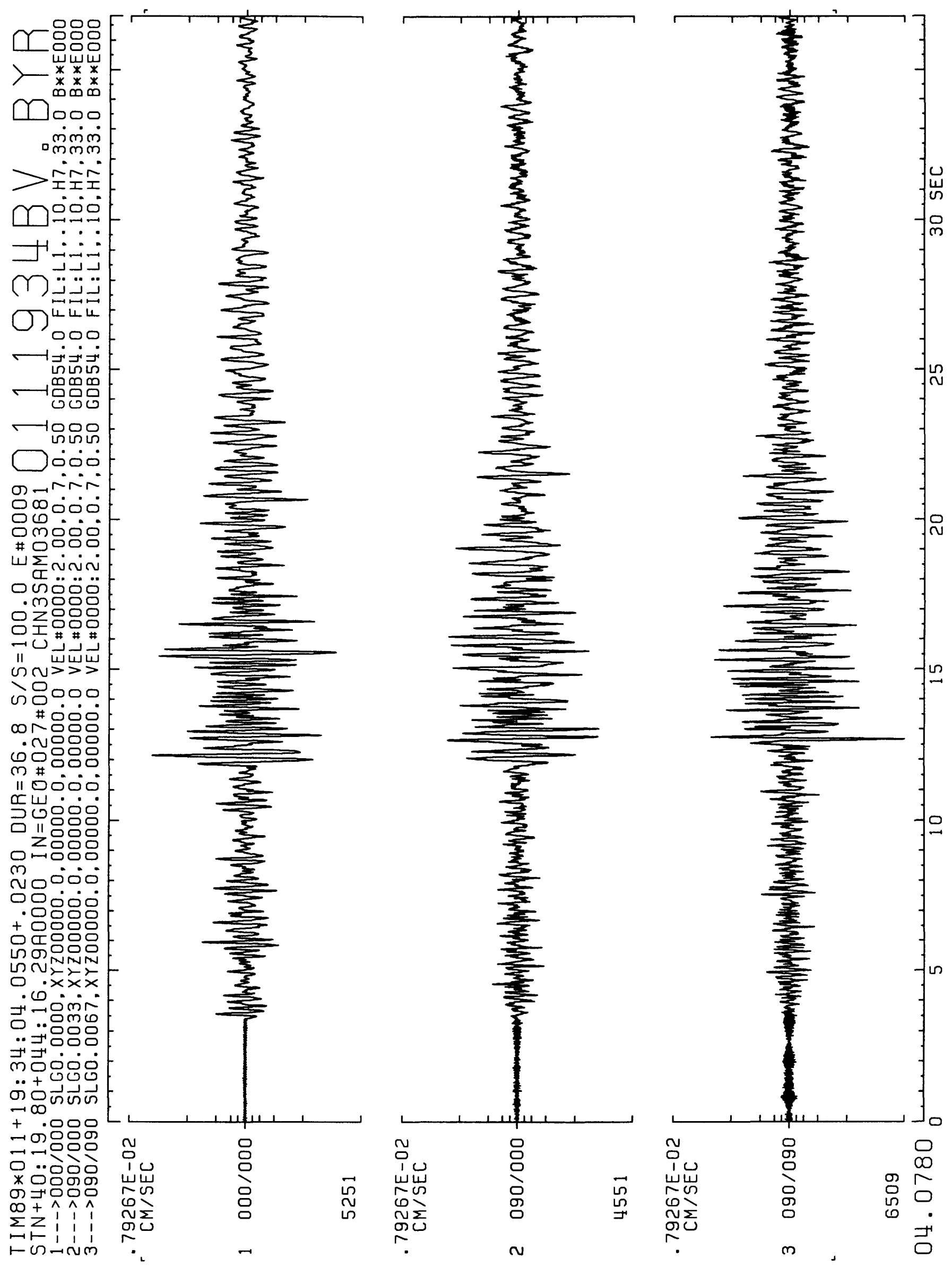
220
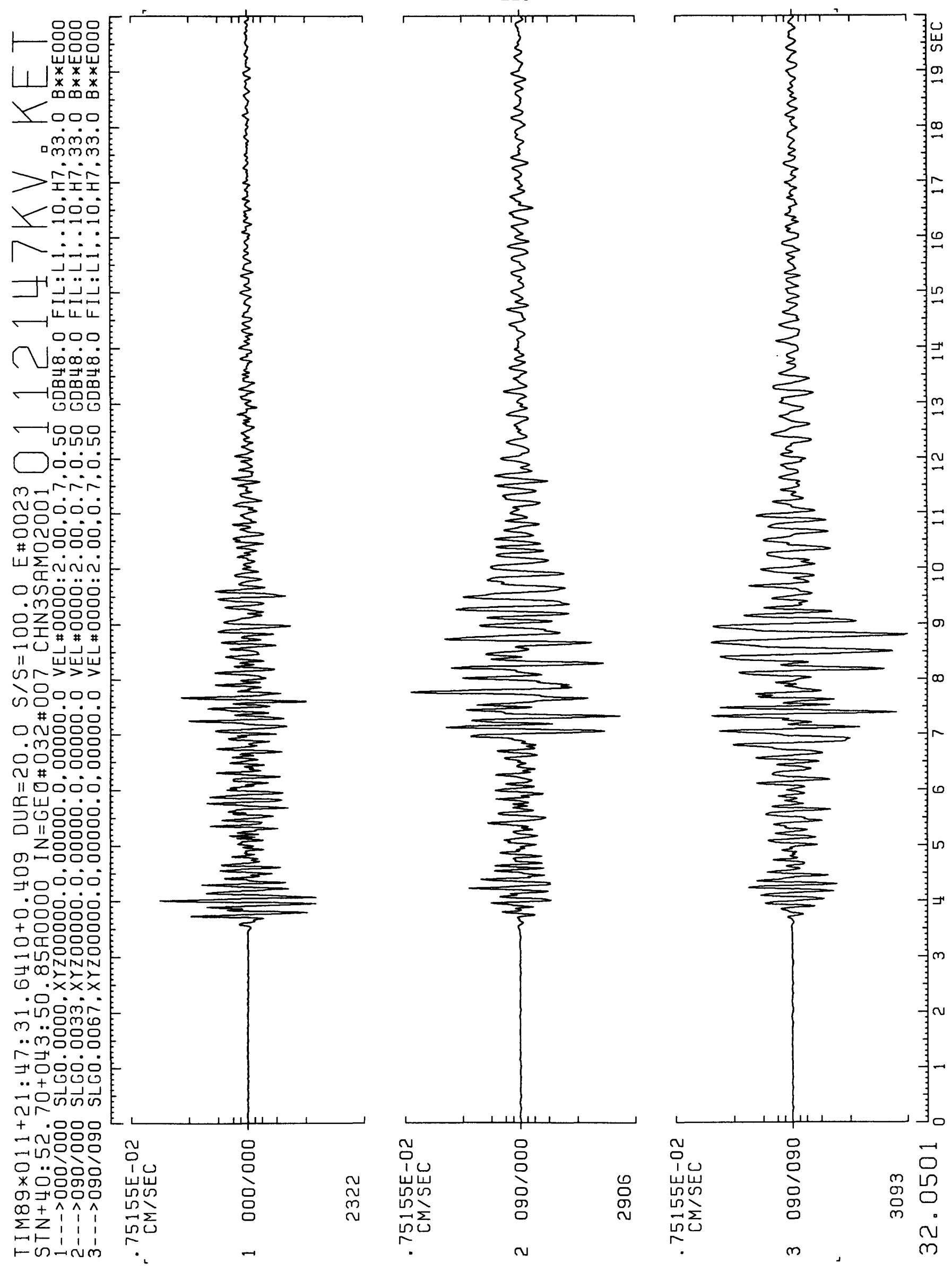

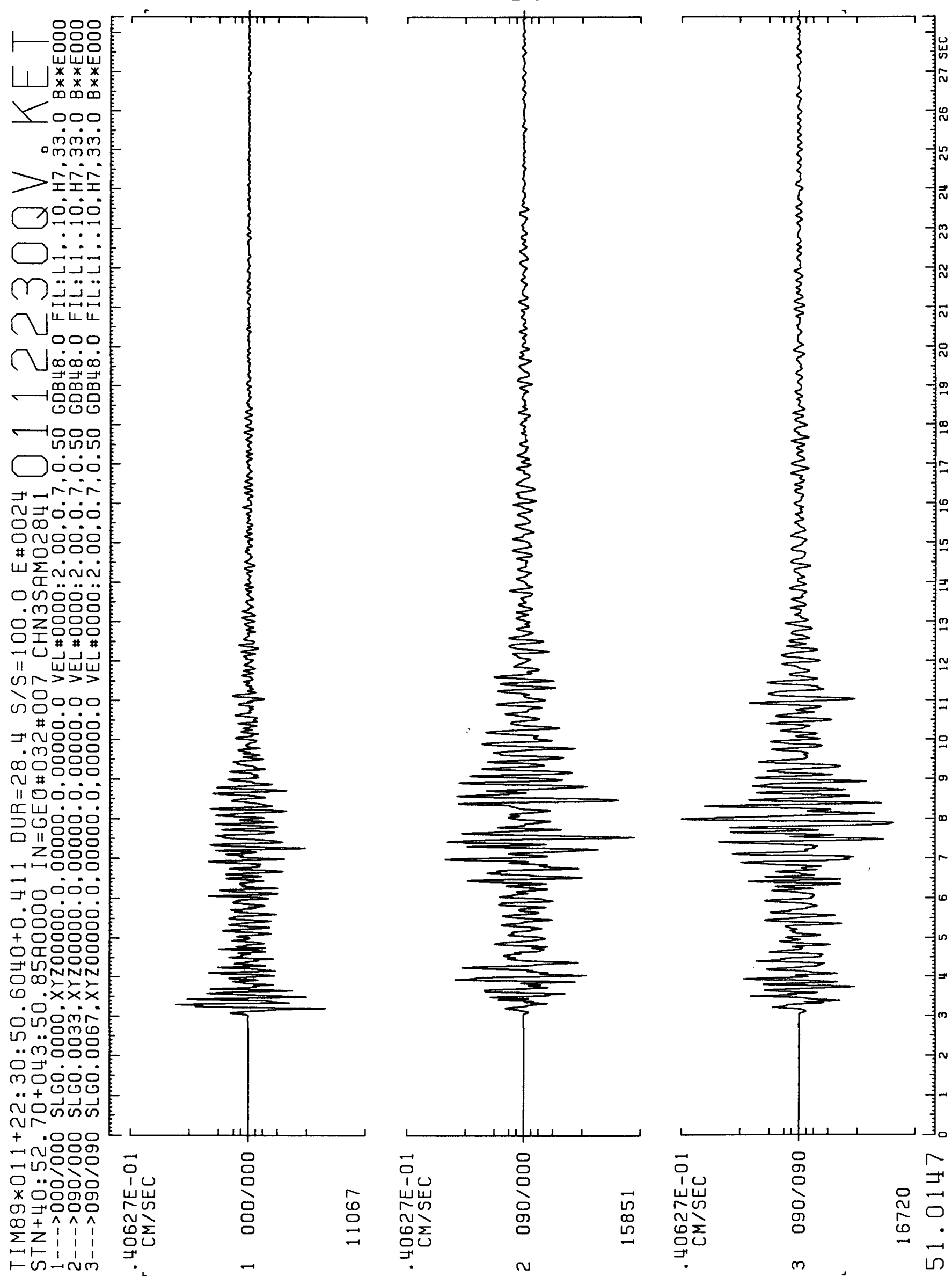


\section{2}
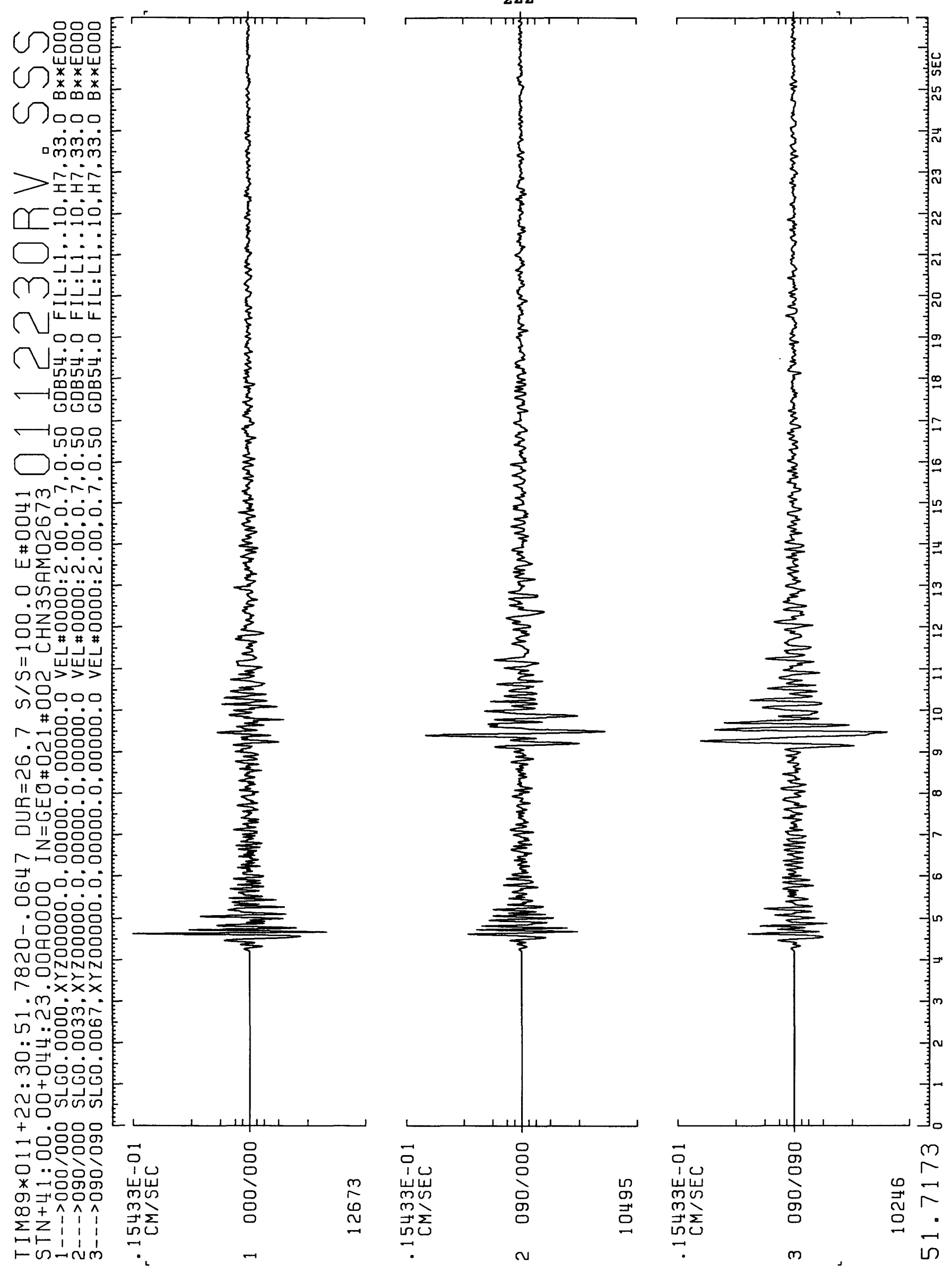


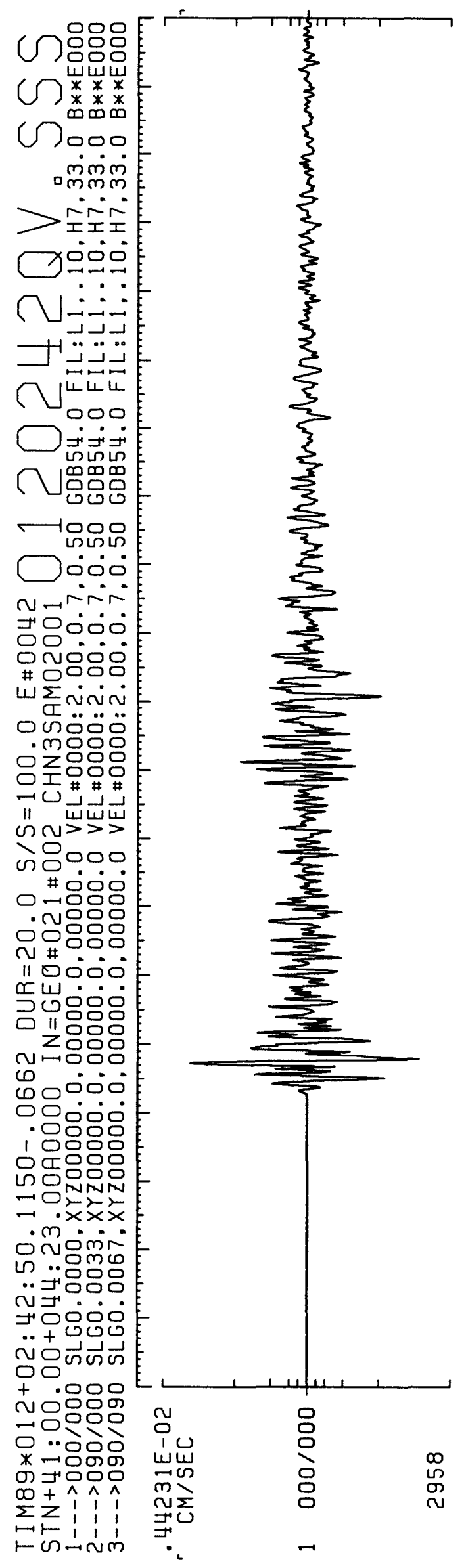

223

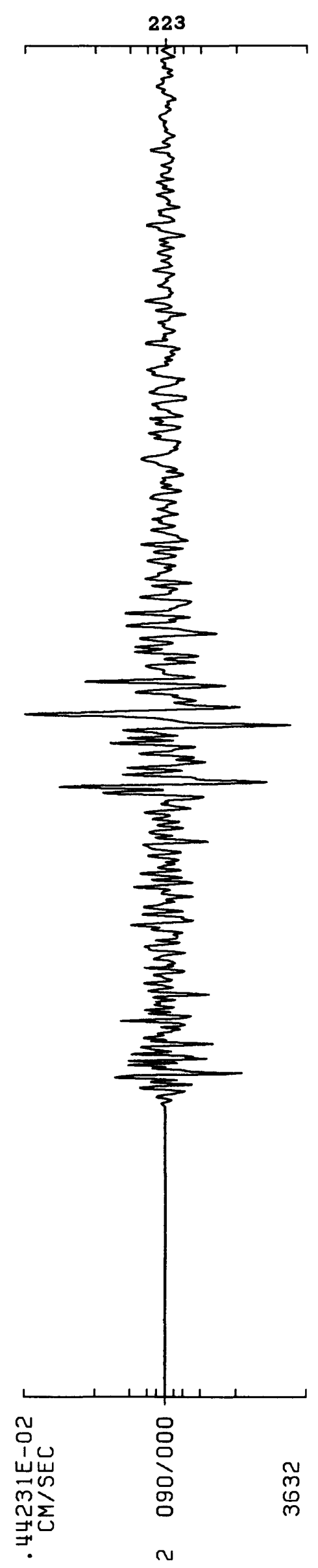

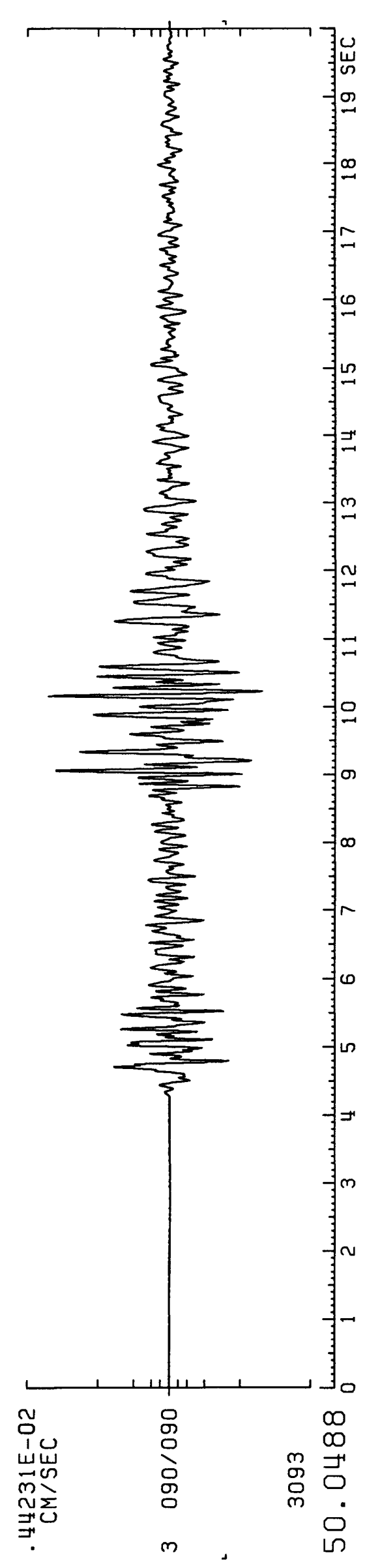



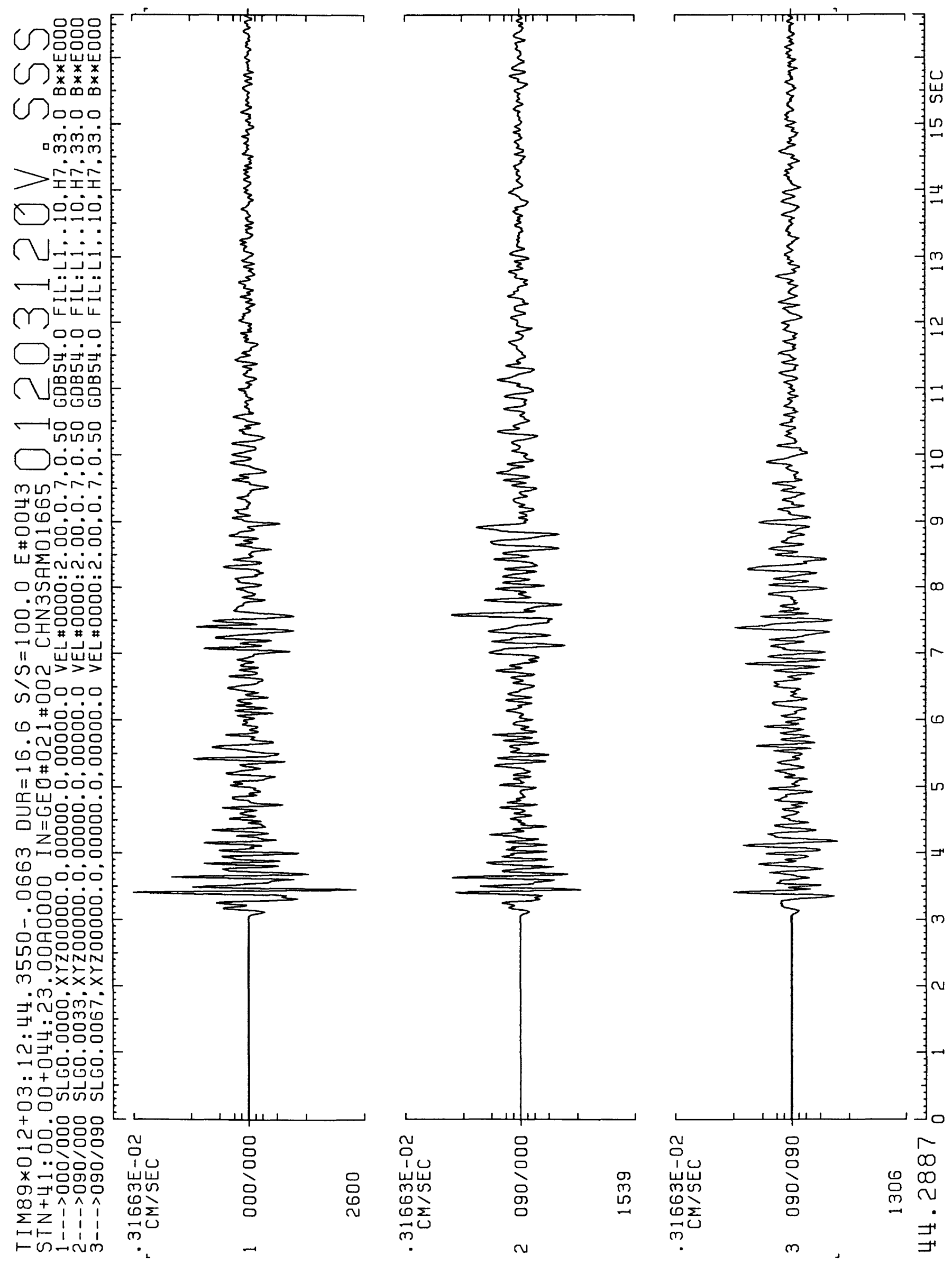


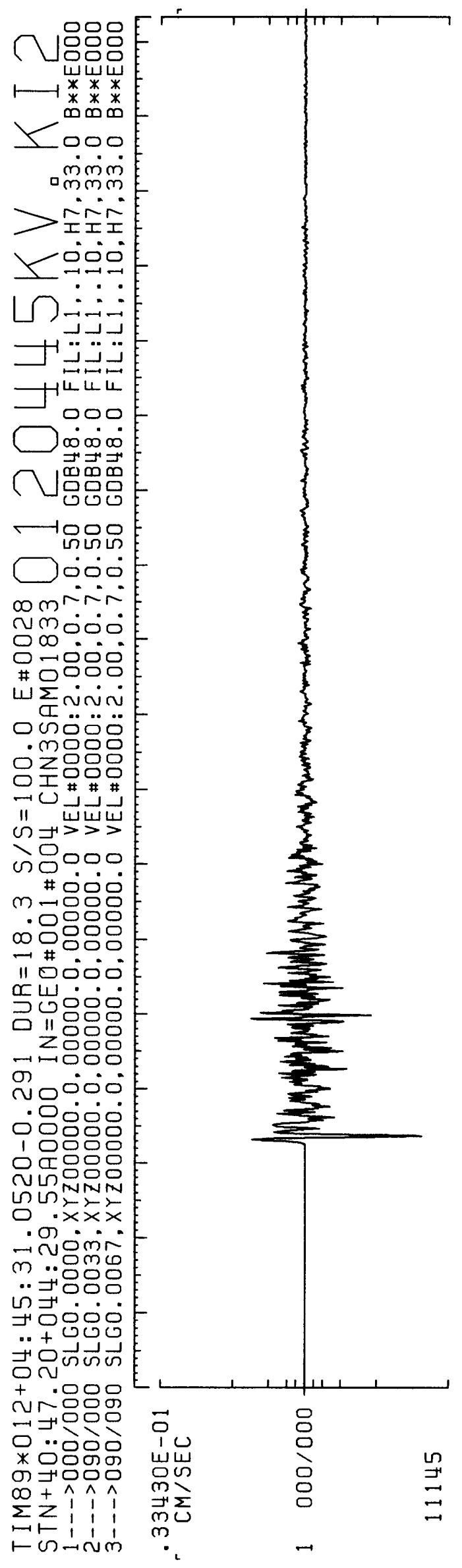

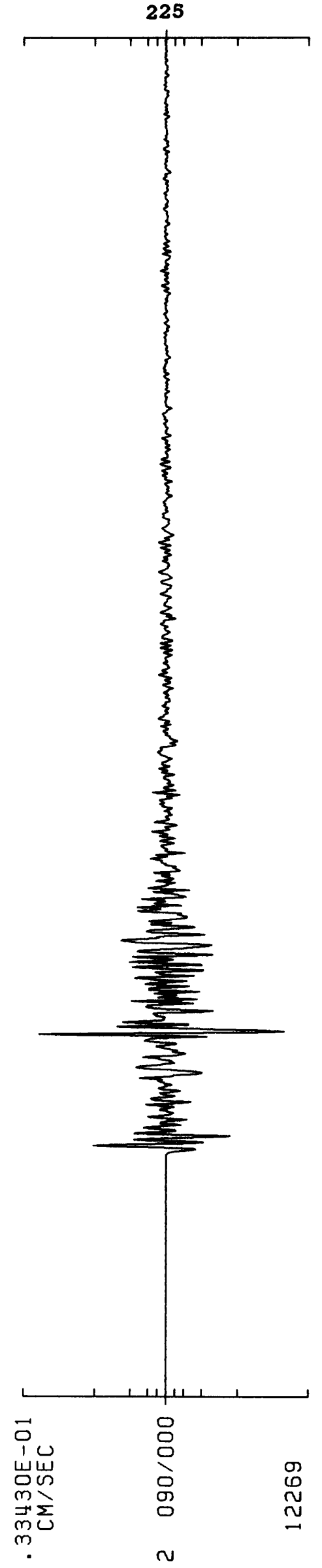

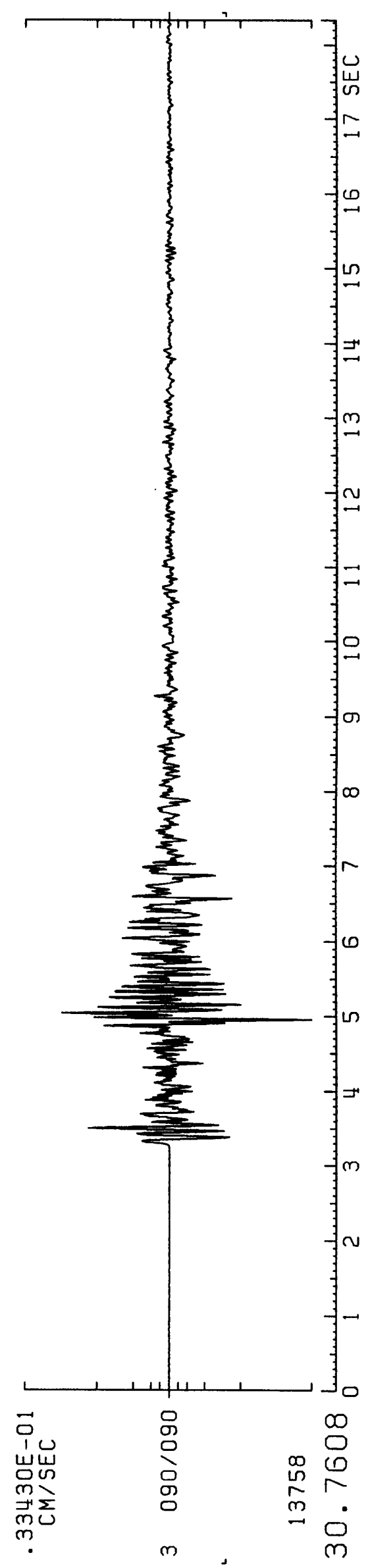




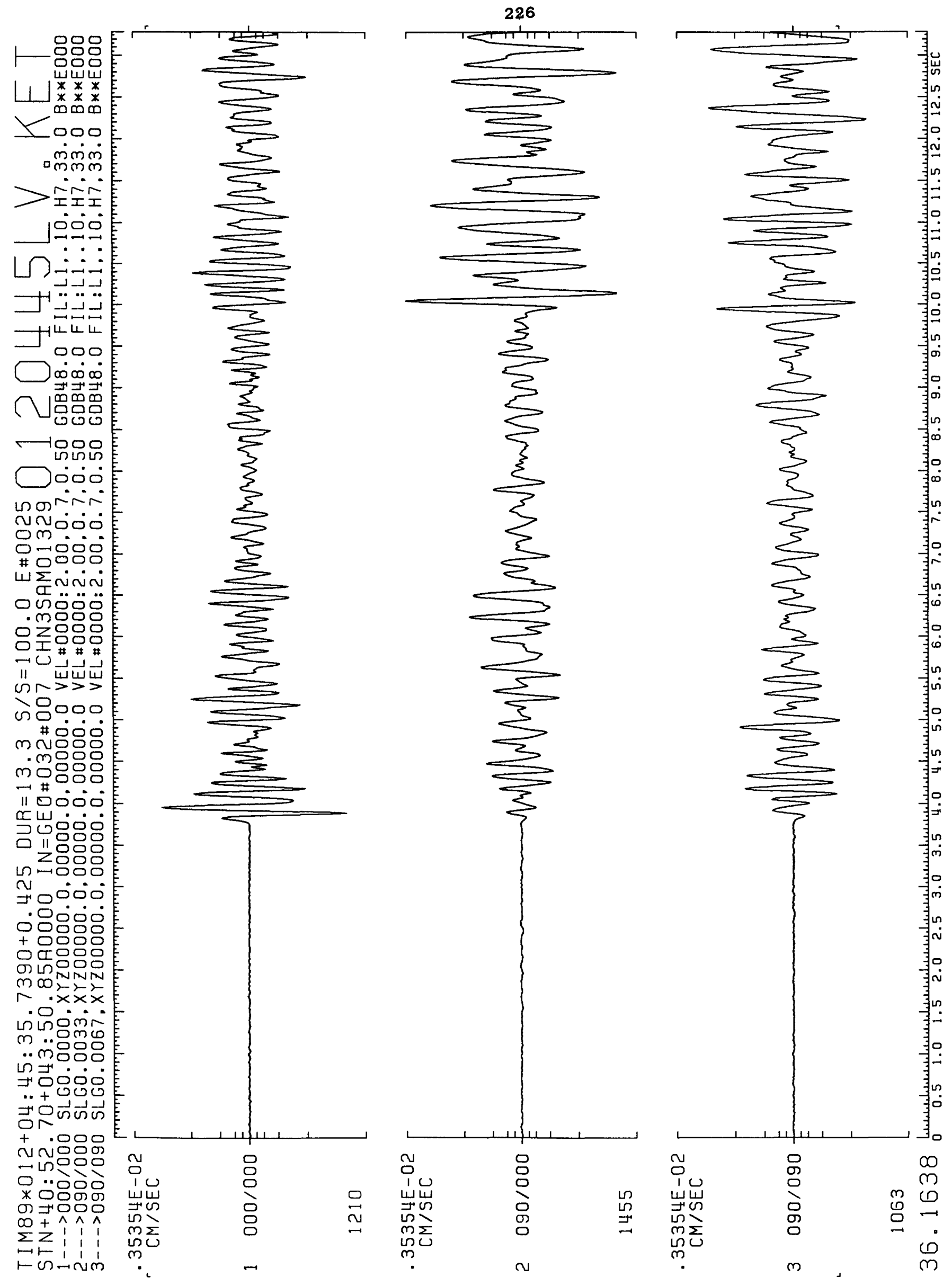




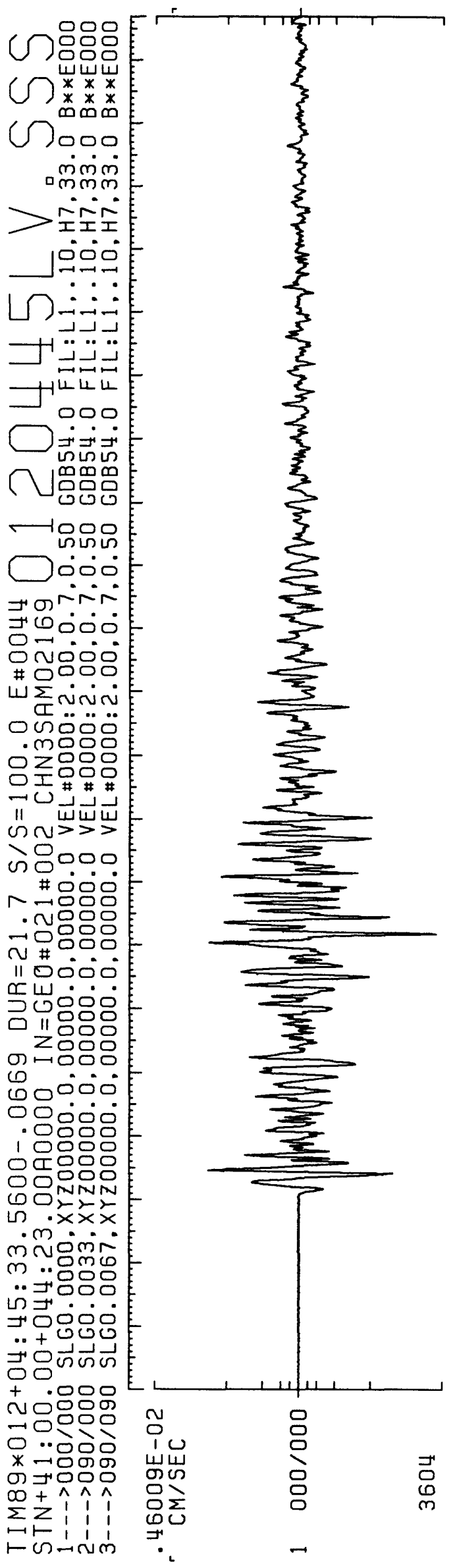

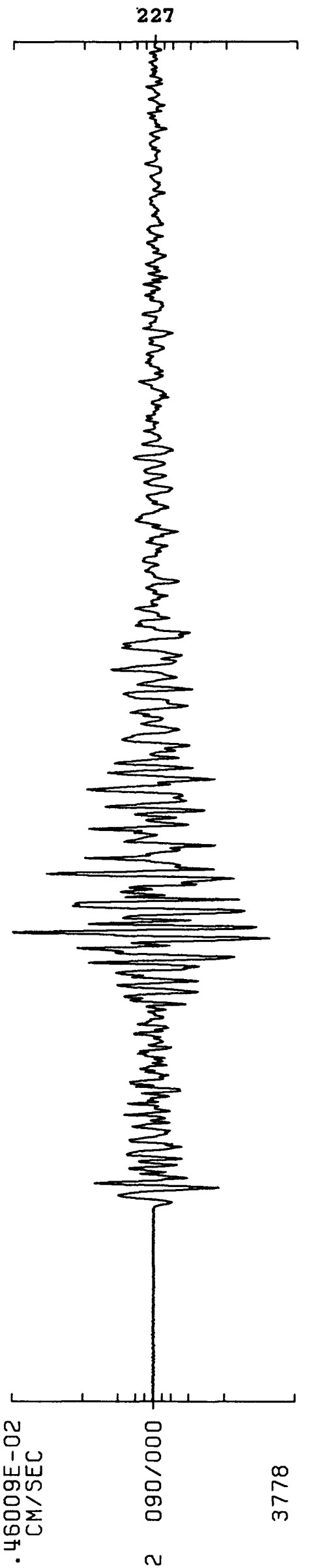

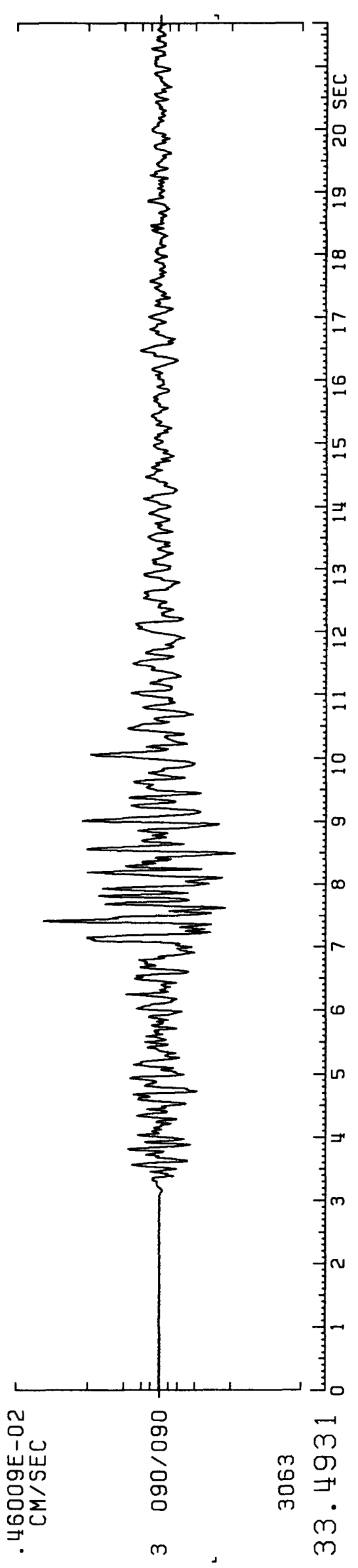




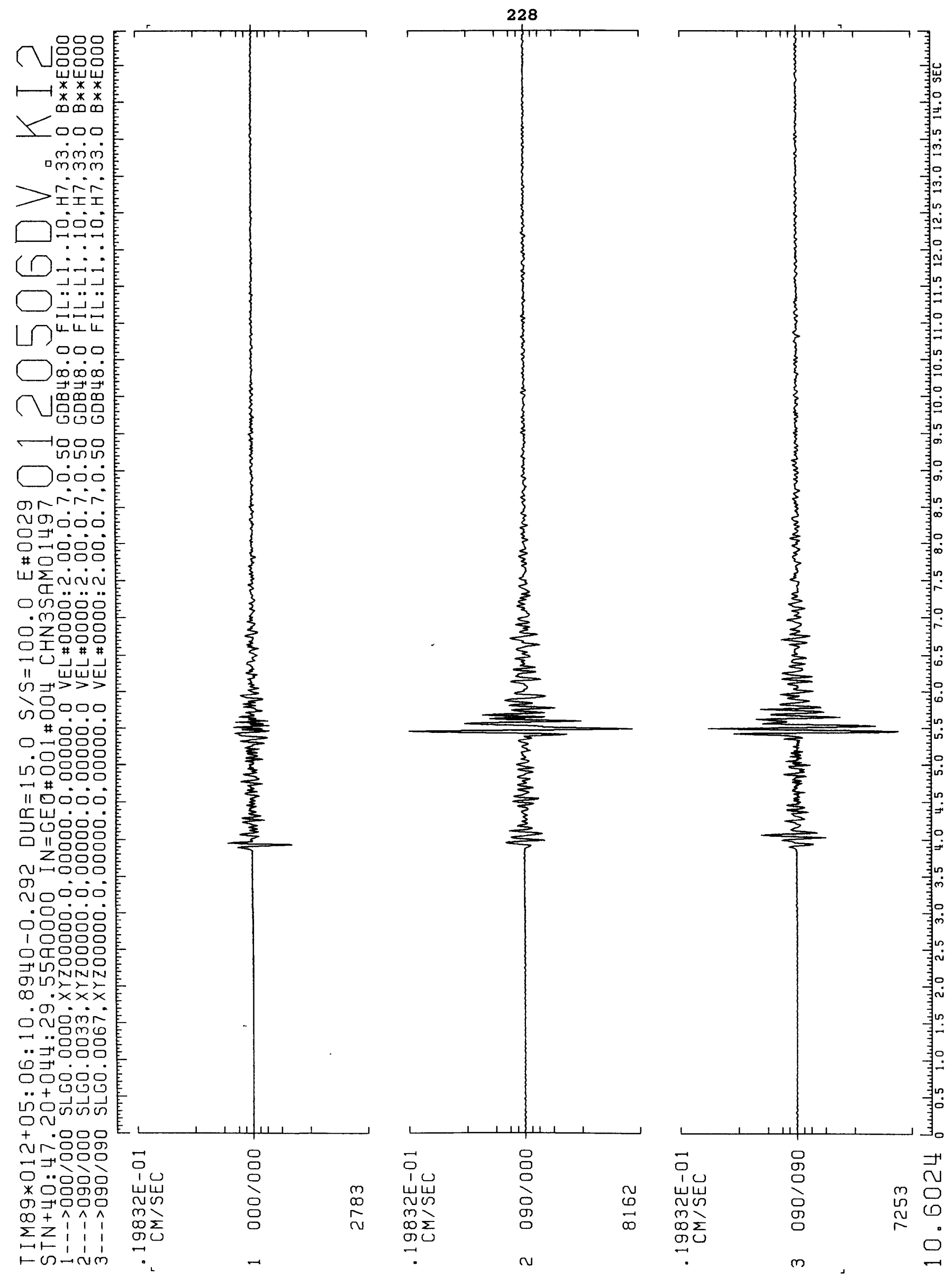




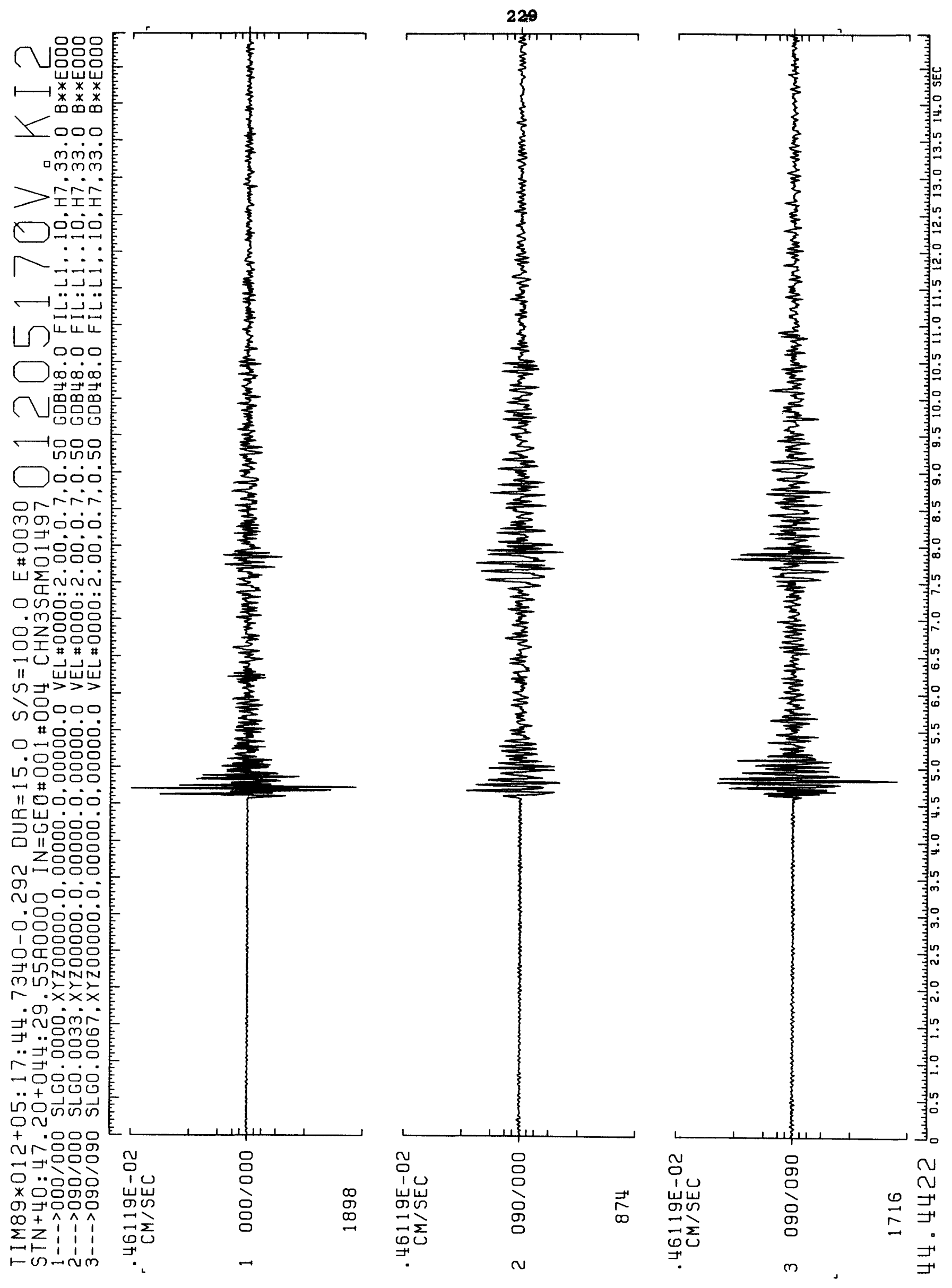



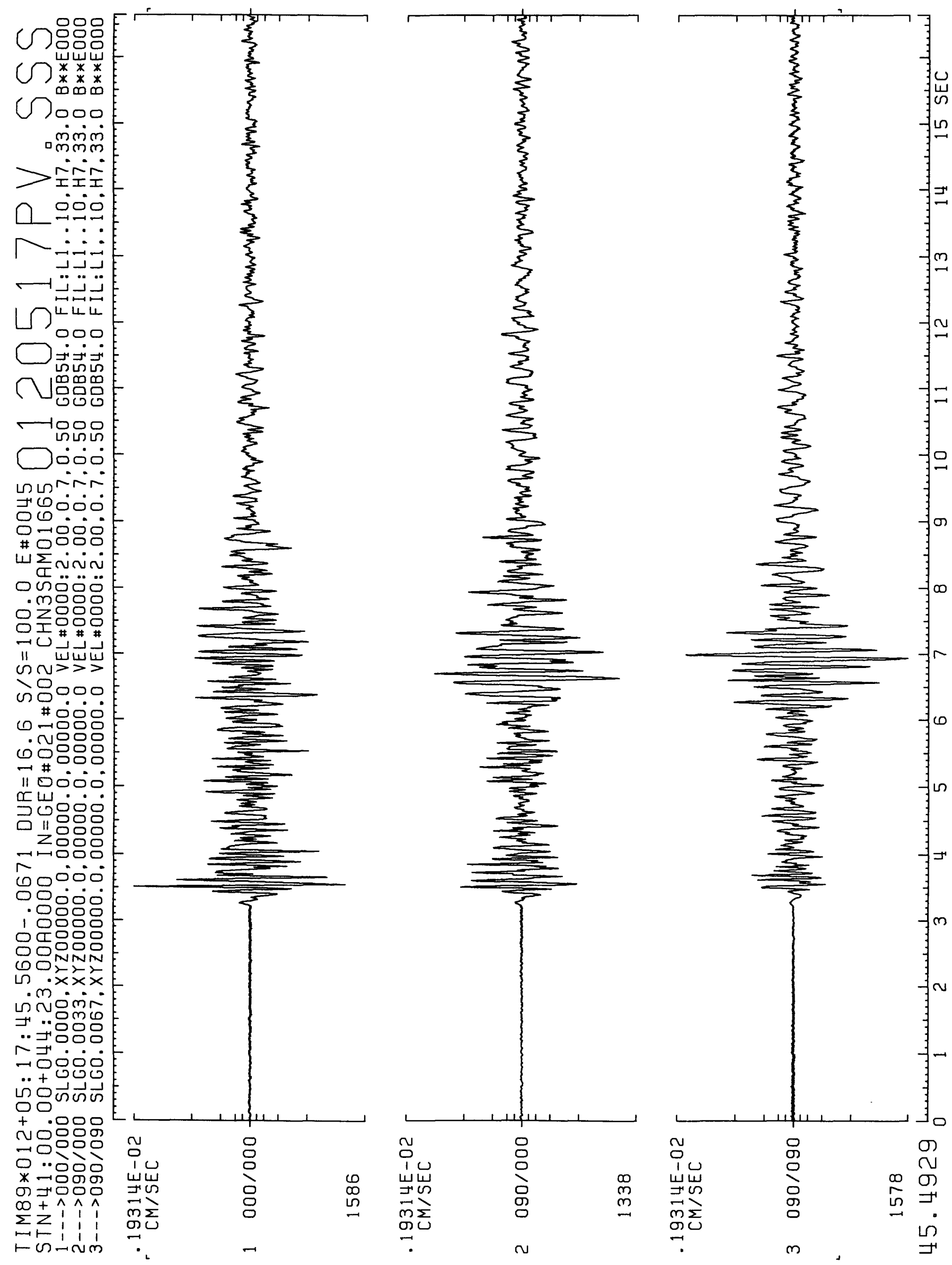
231

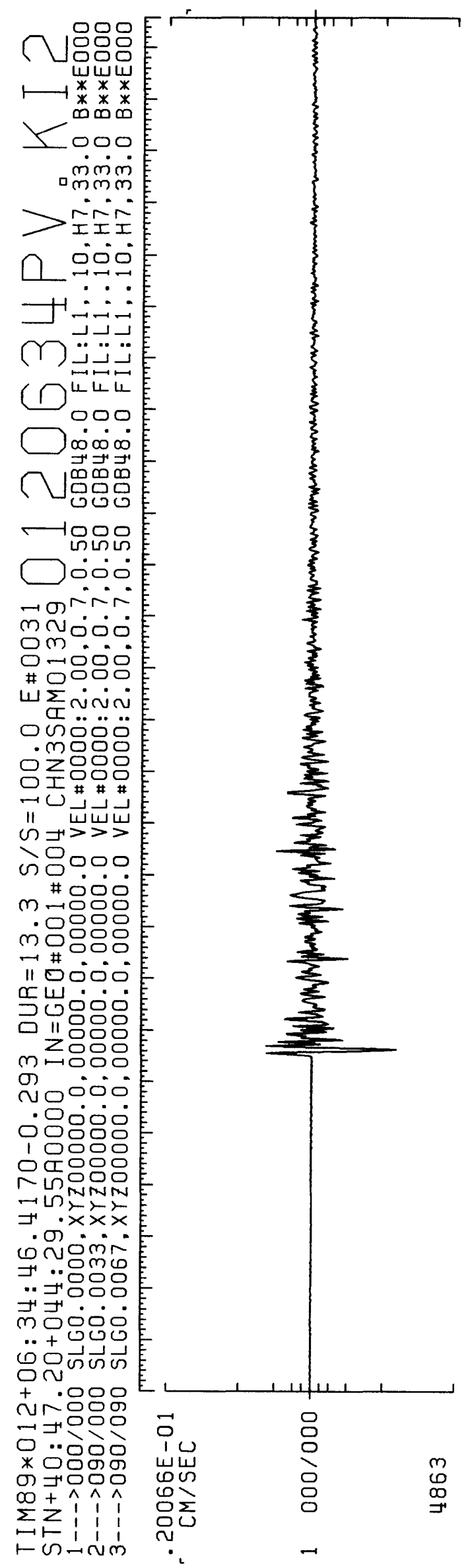

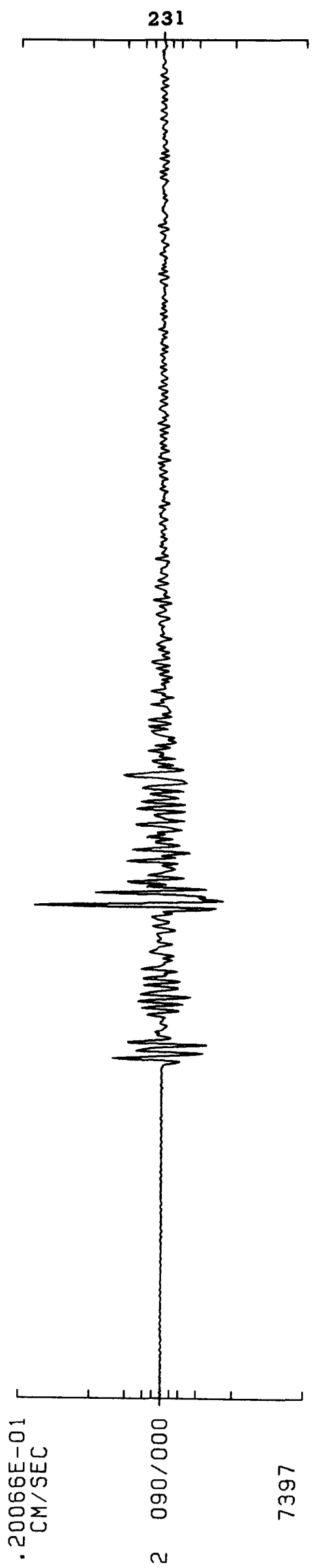

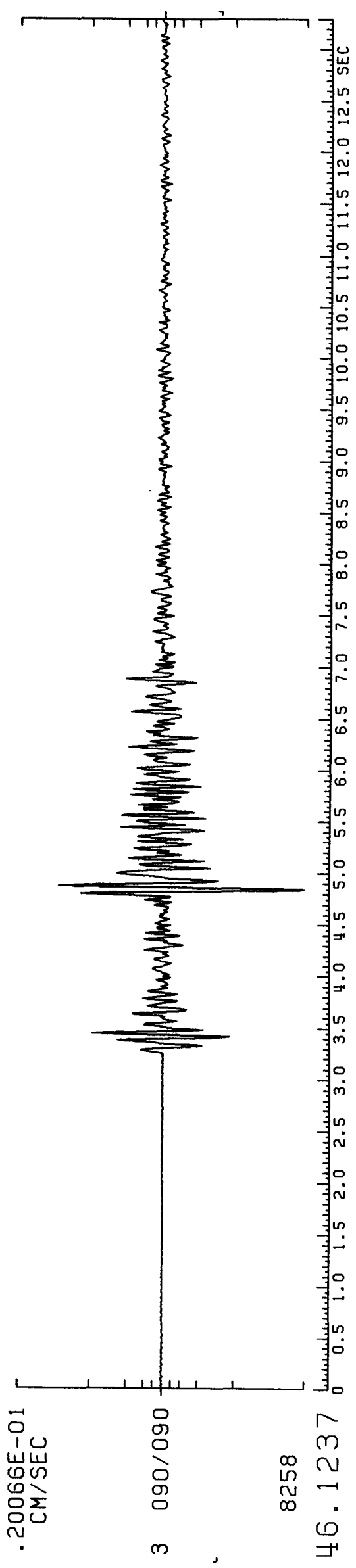



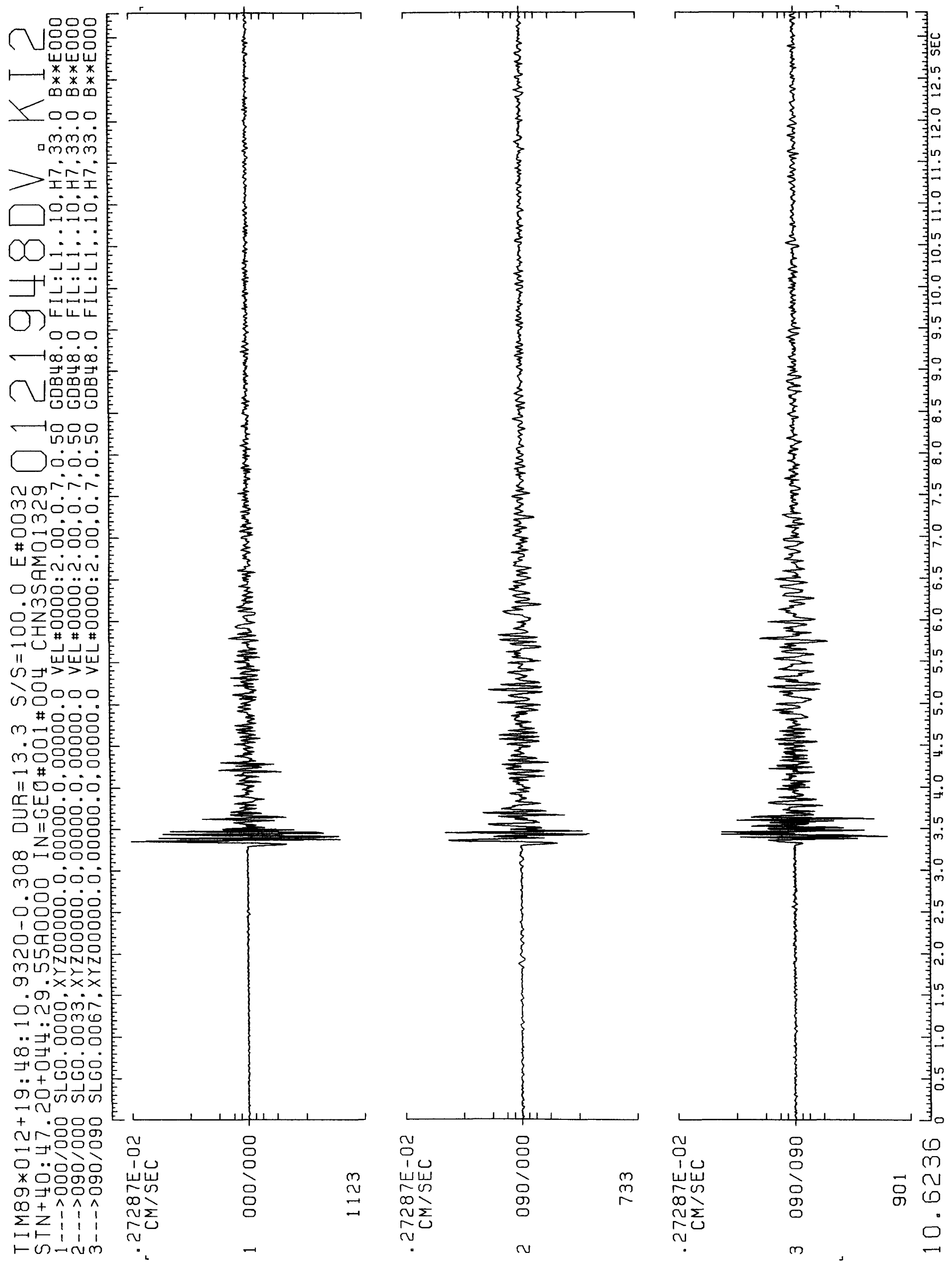


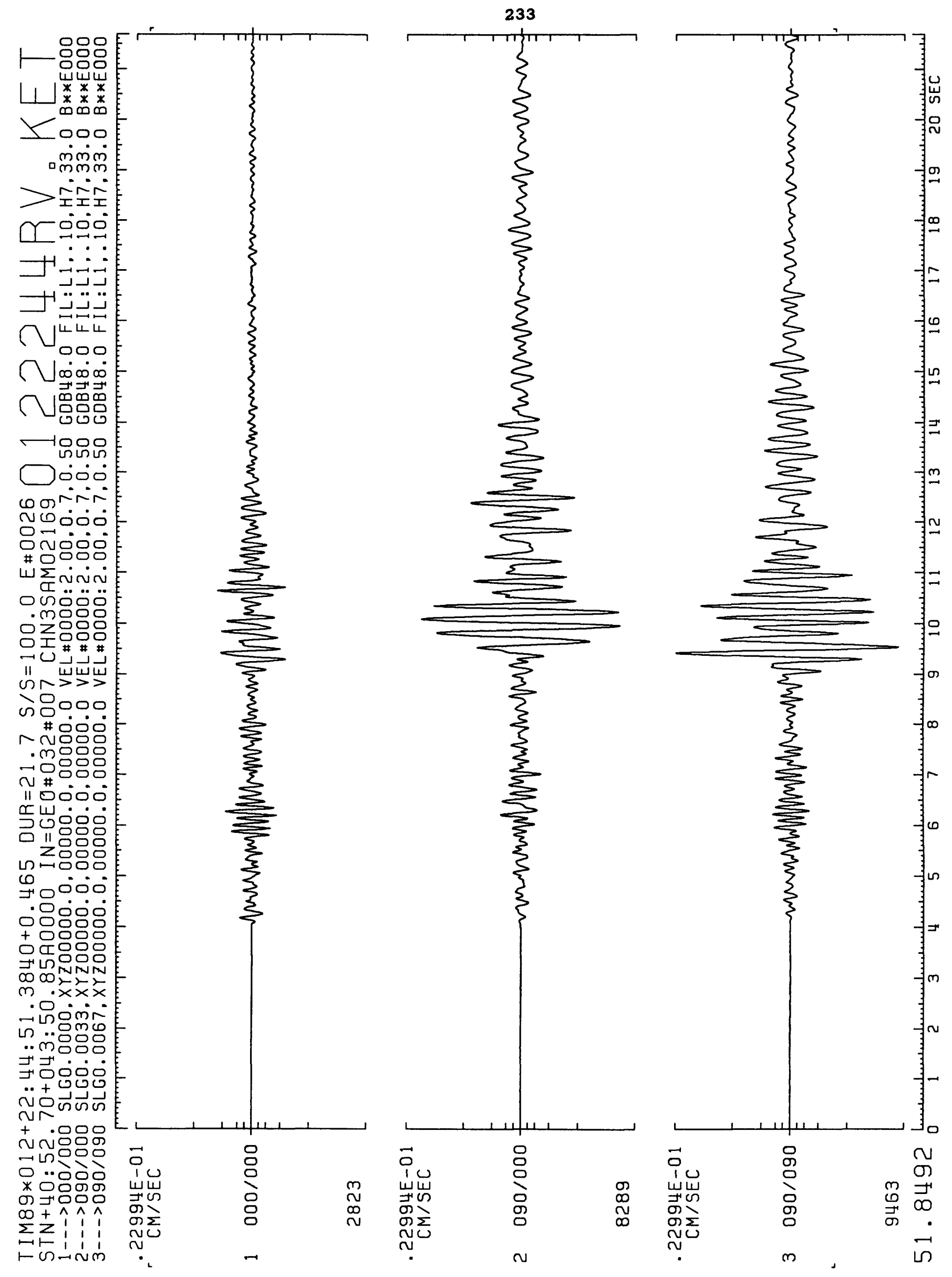


234

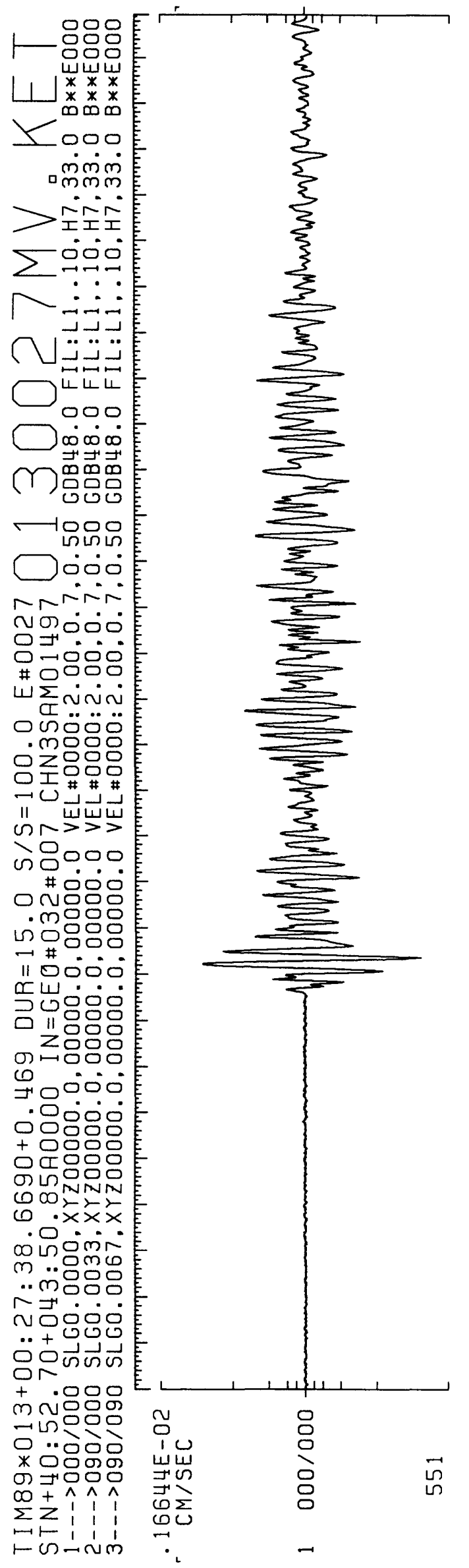

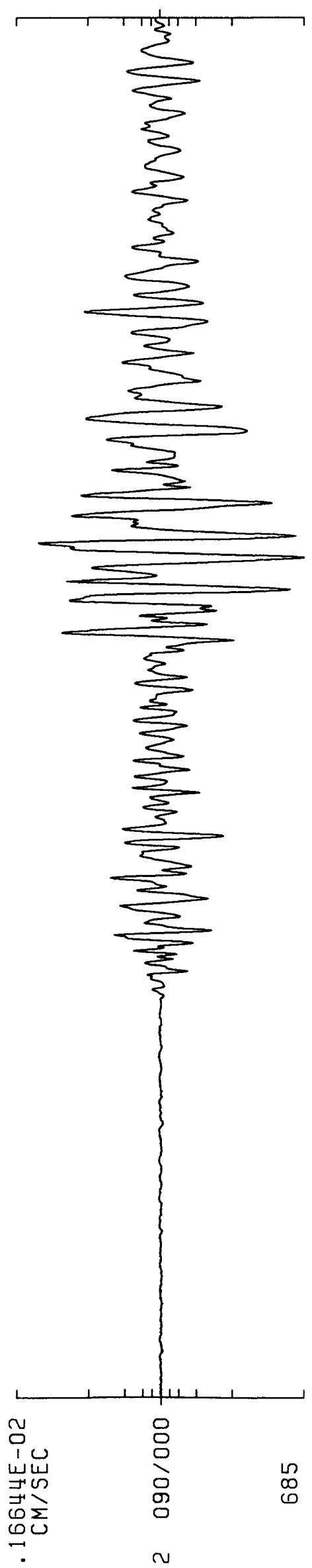

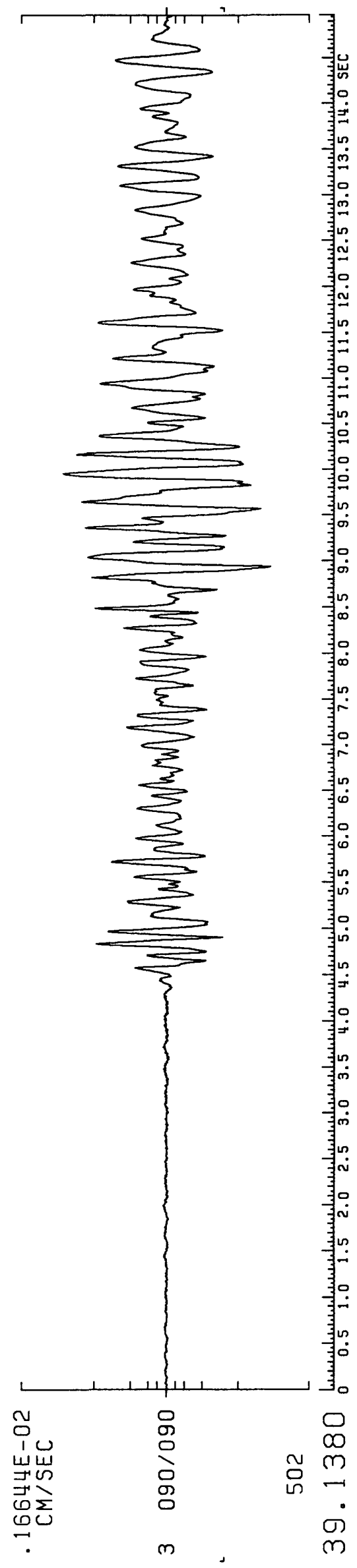



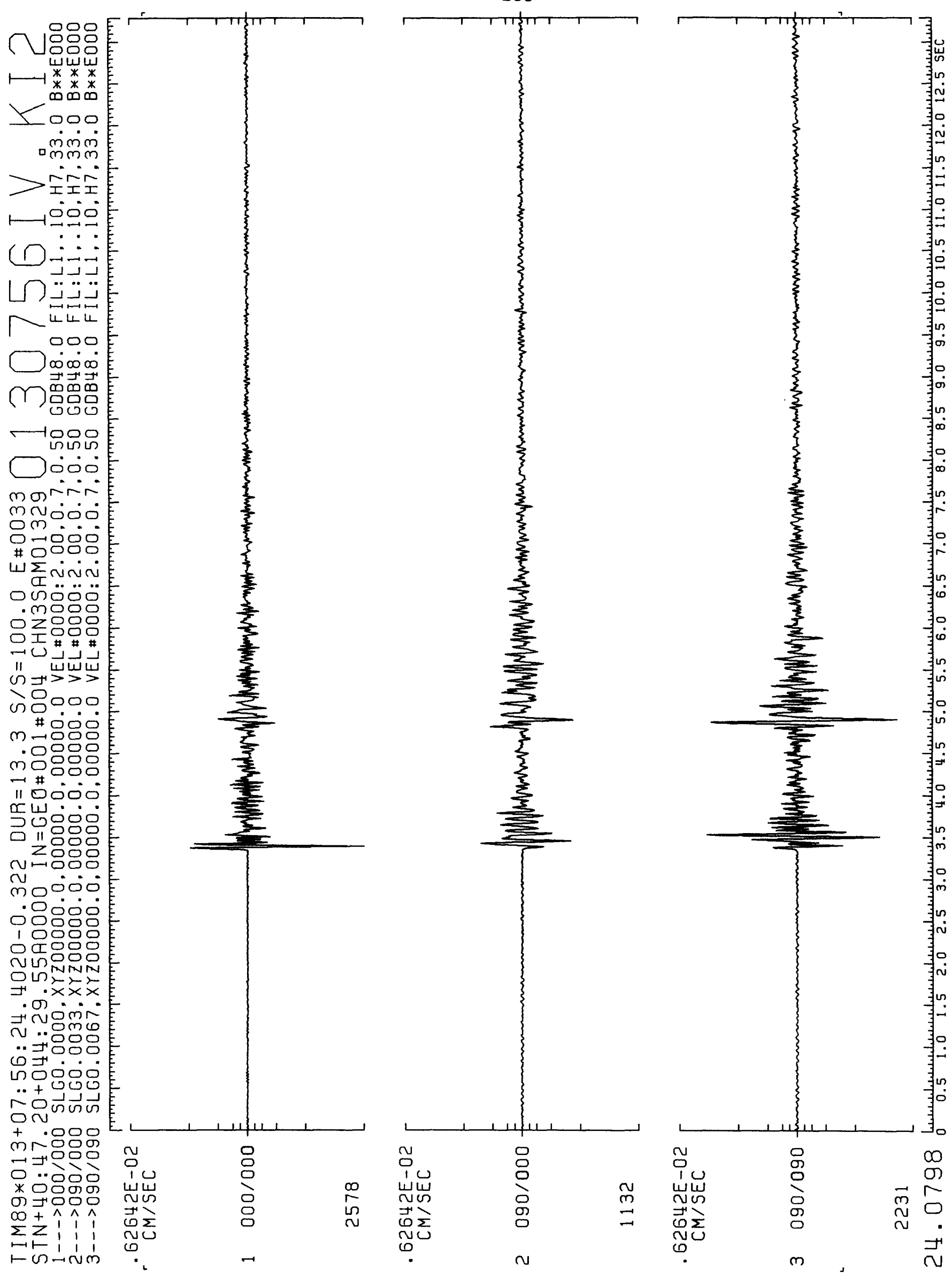


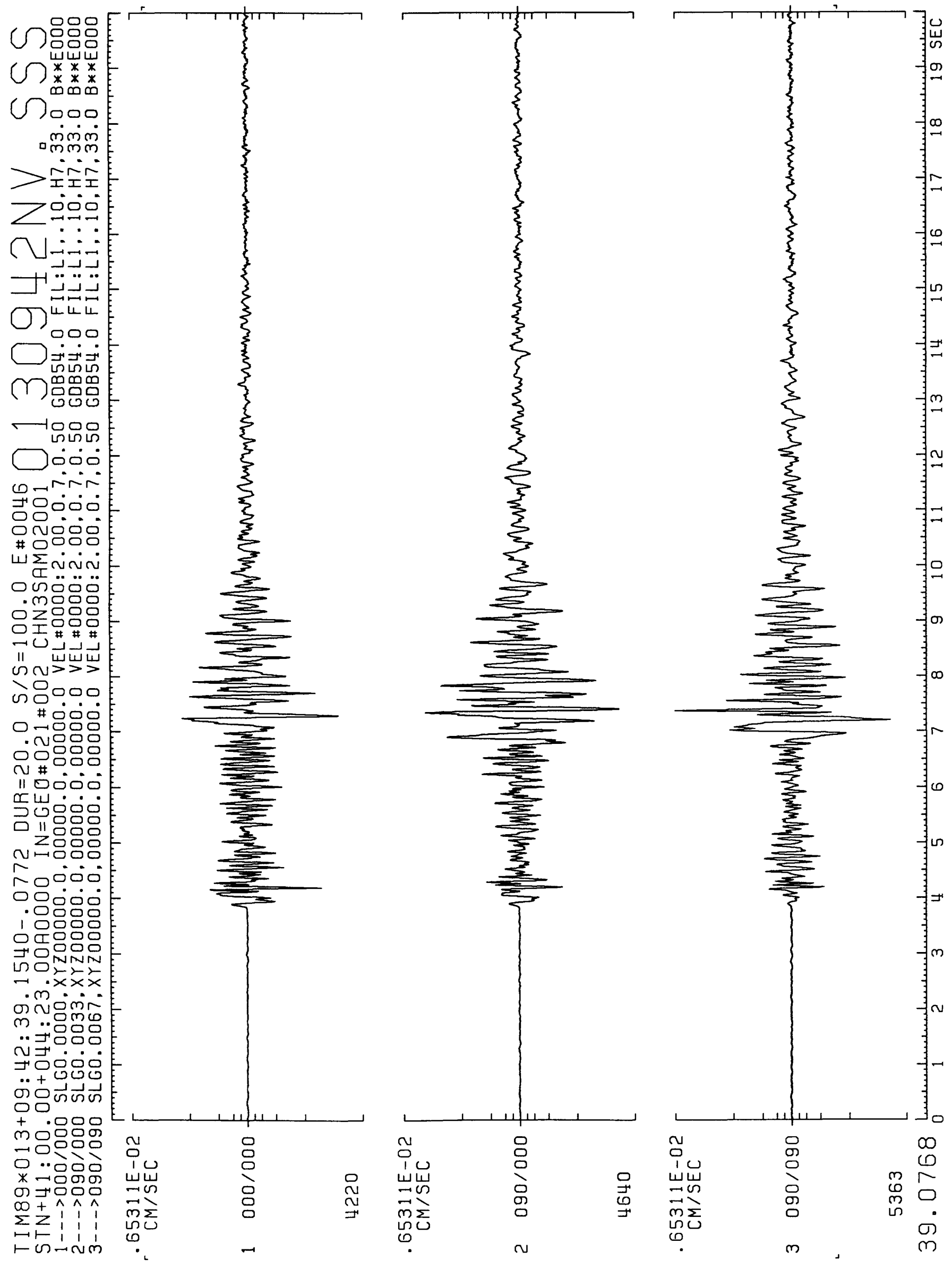




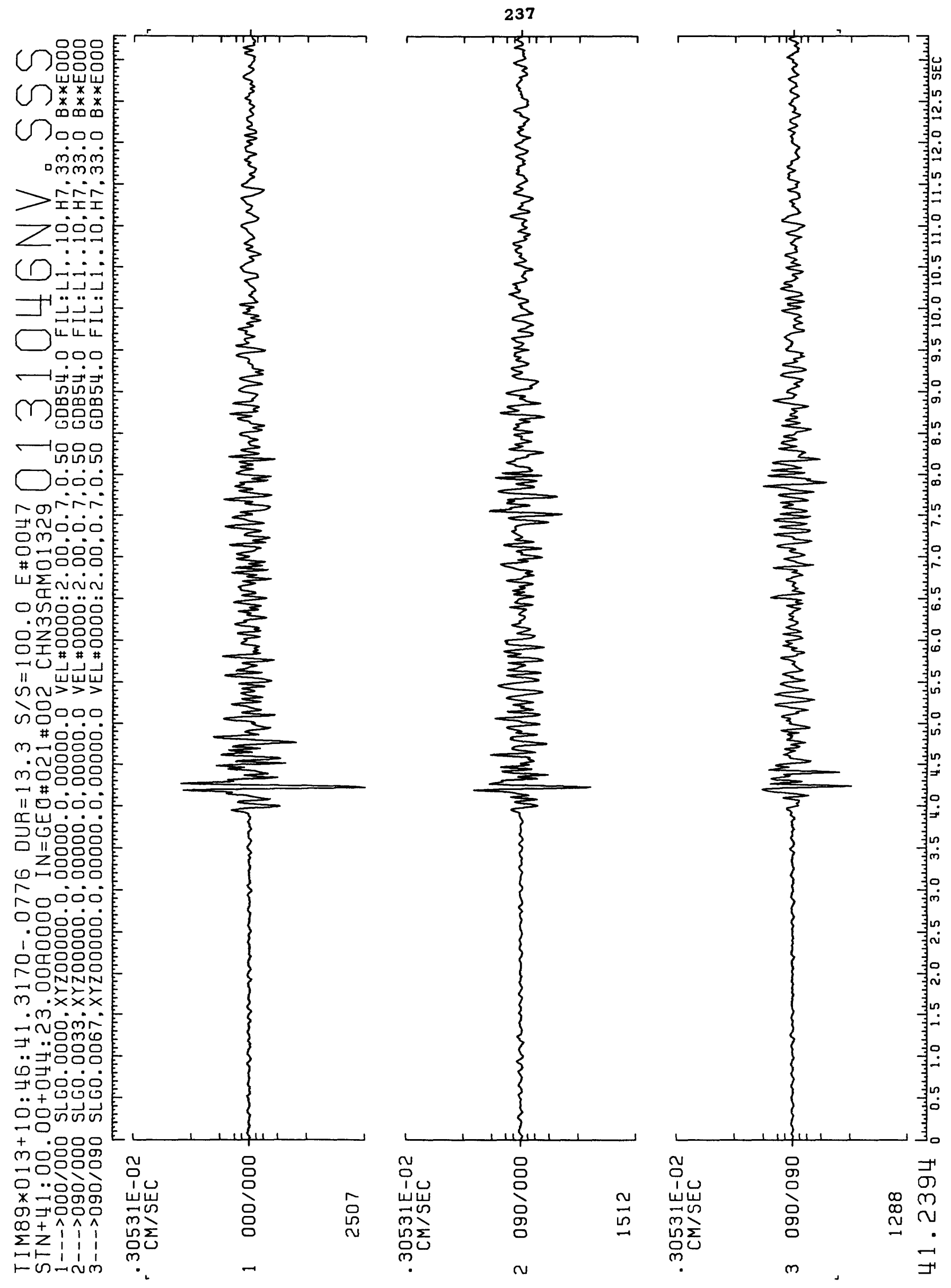




$$
\text { nit }
$$




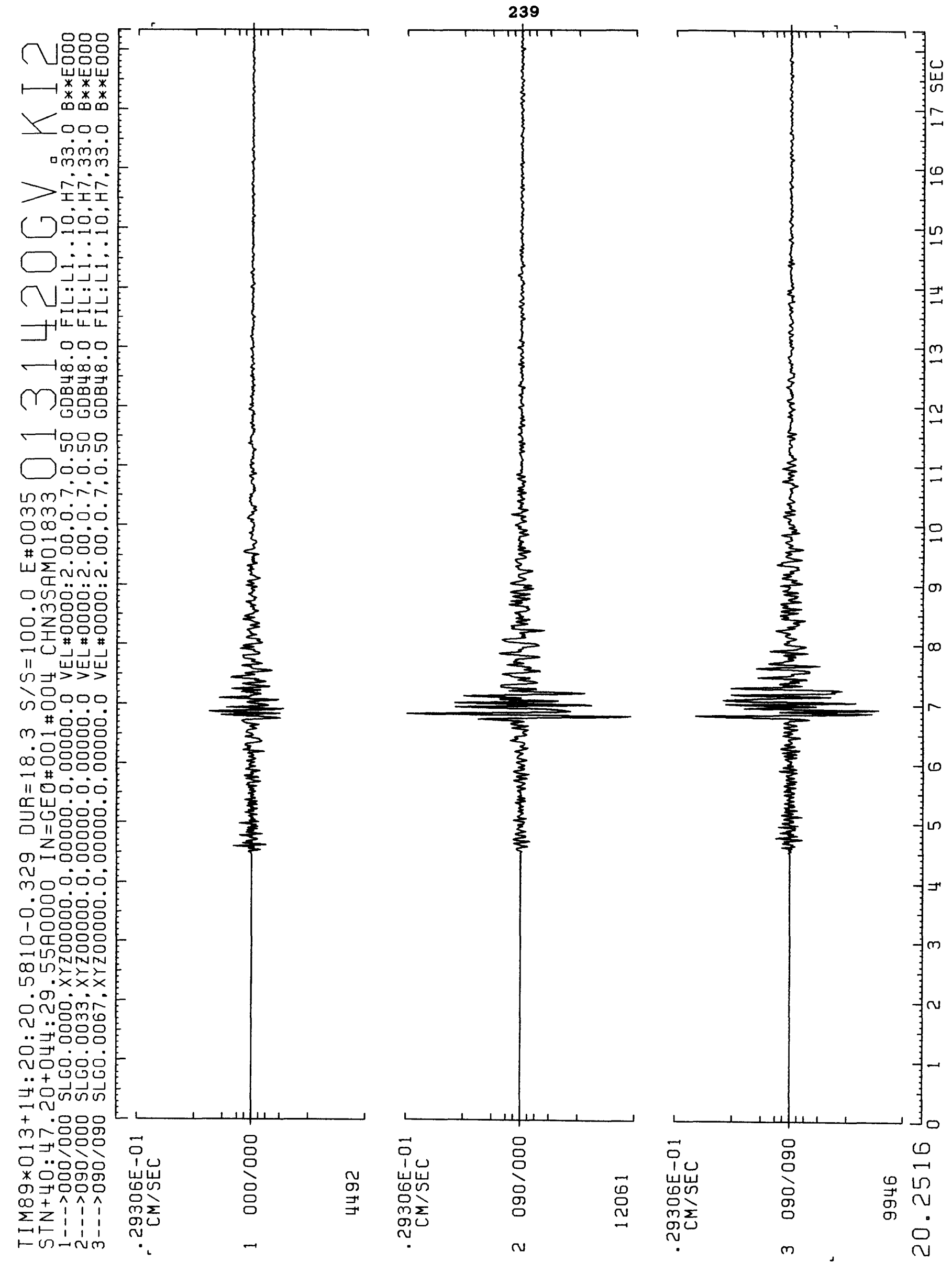



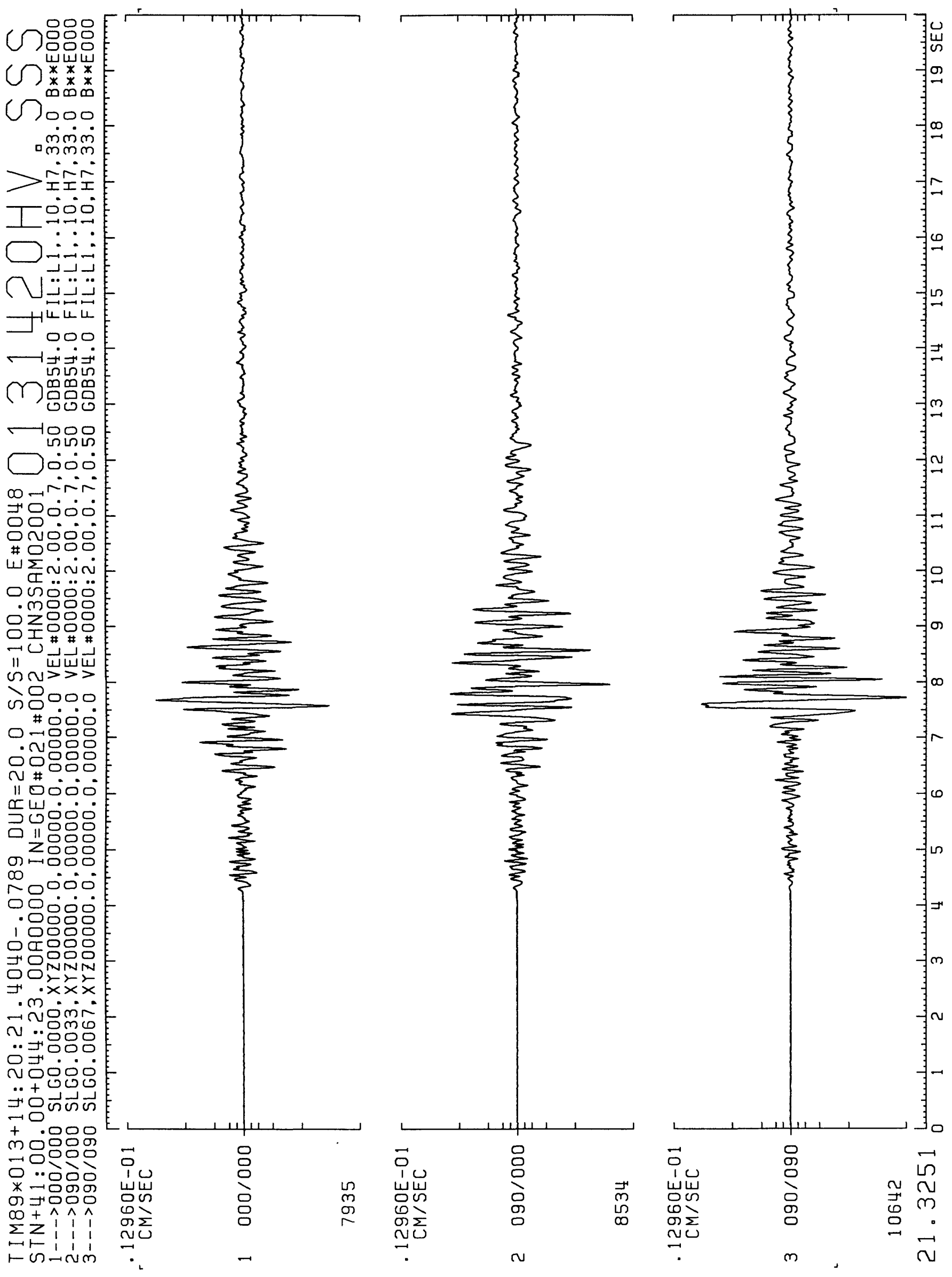
241

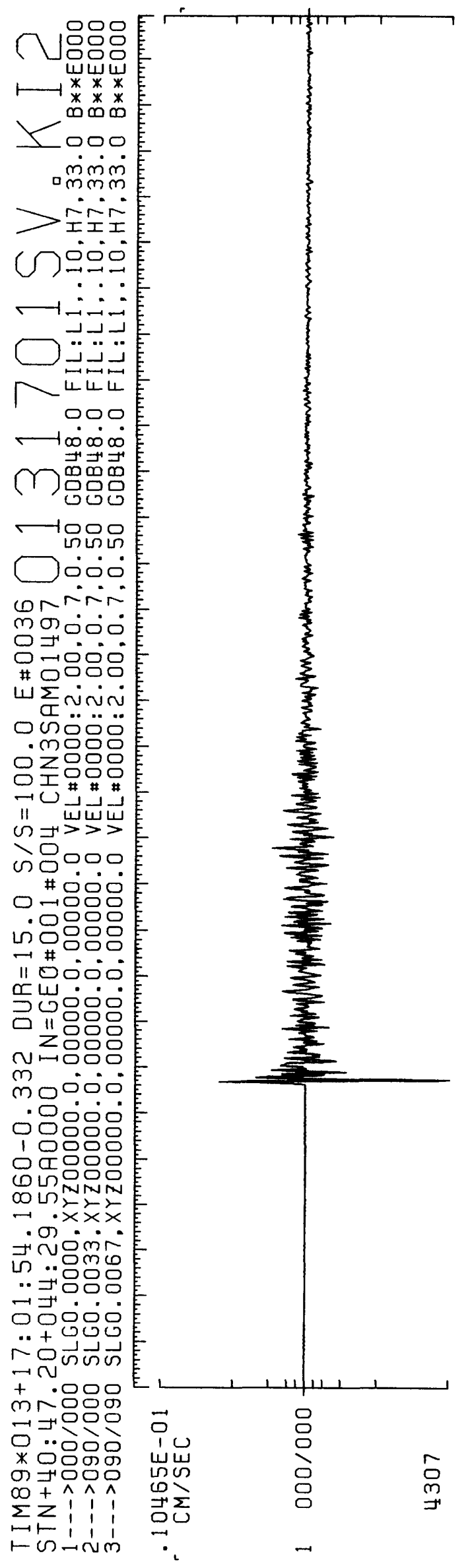

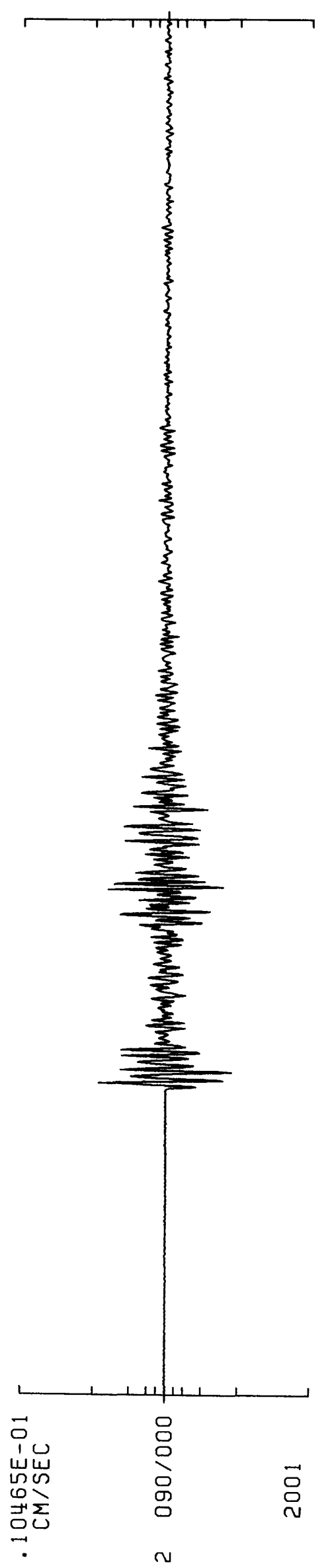

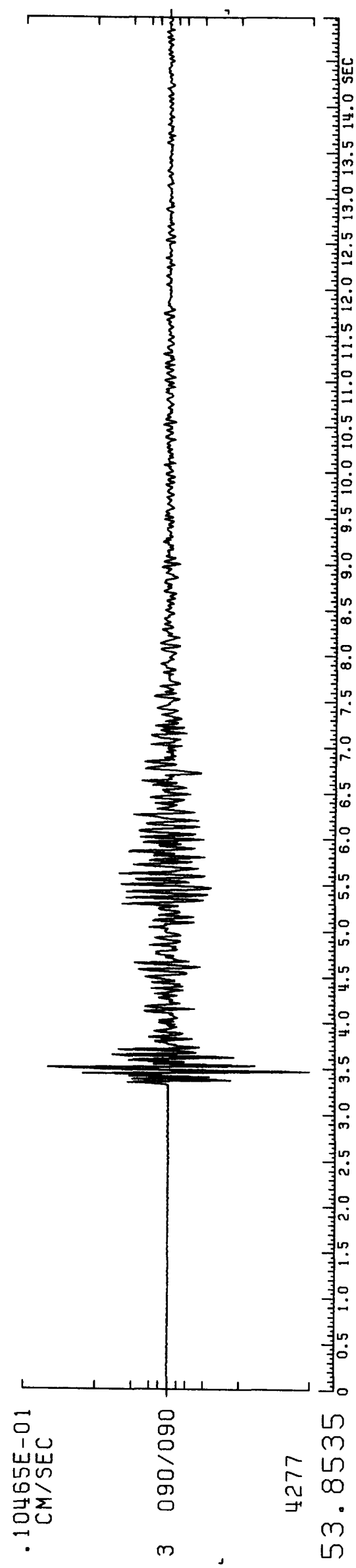




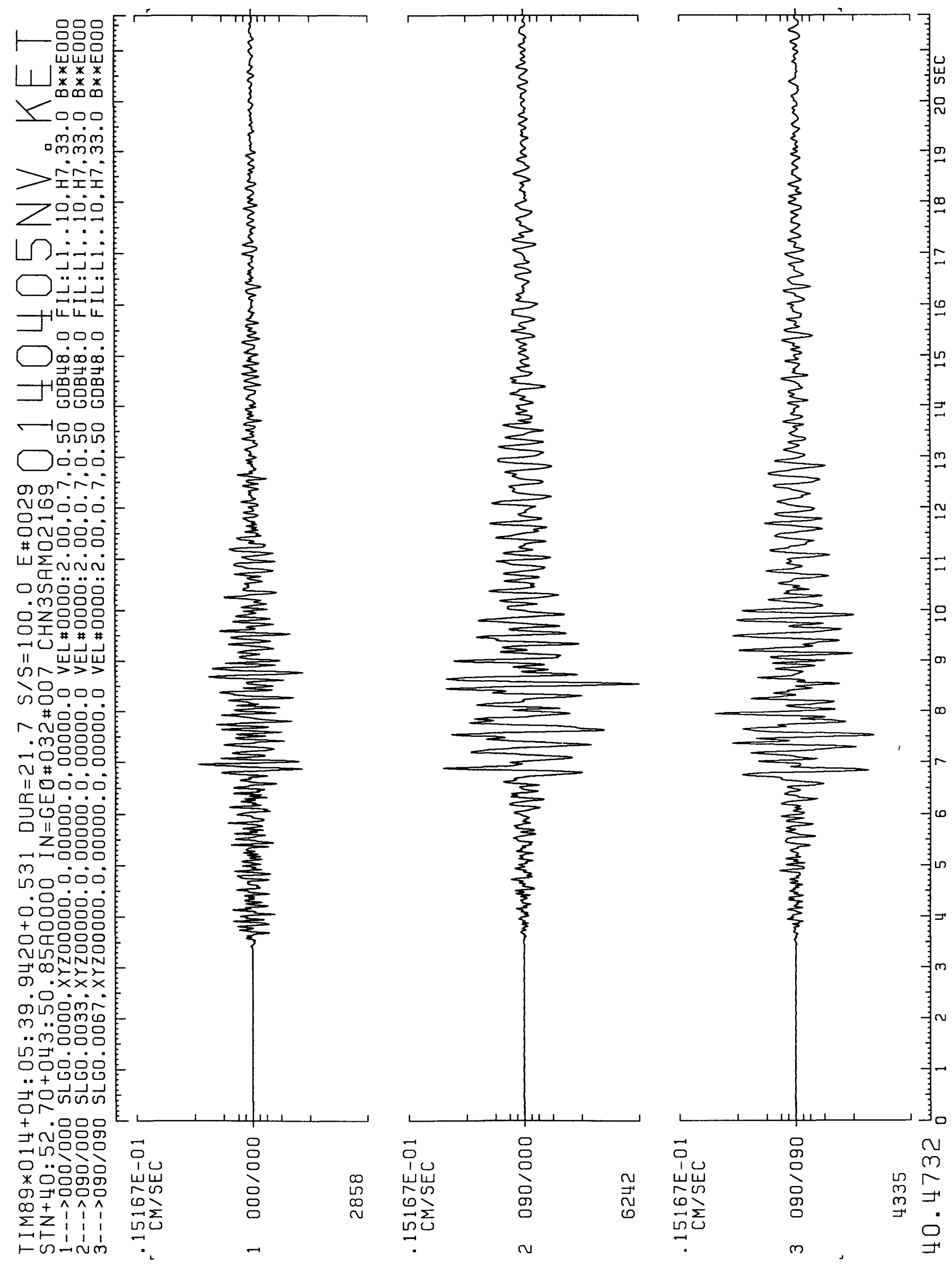


243
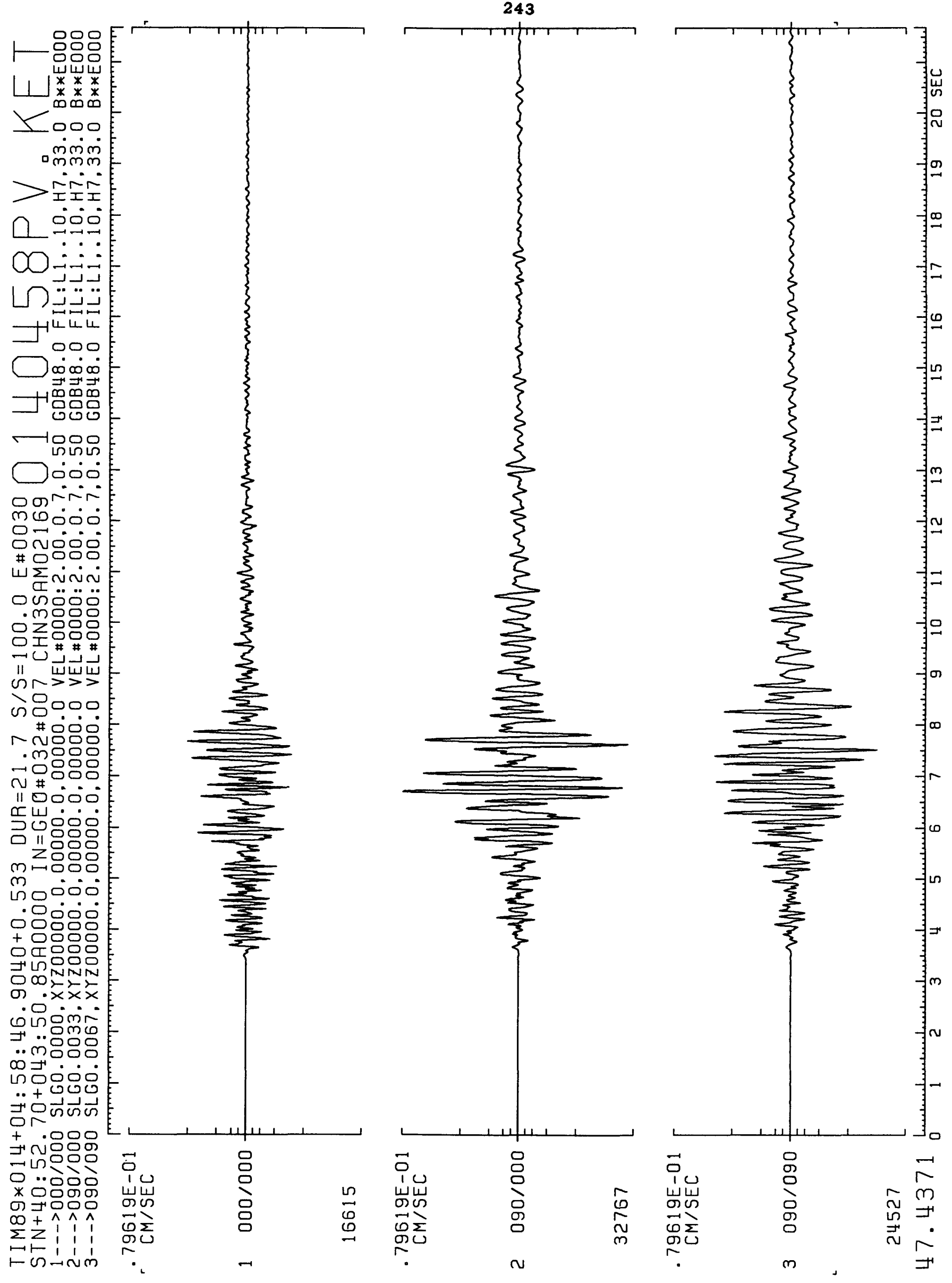
244

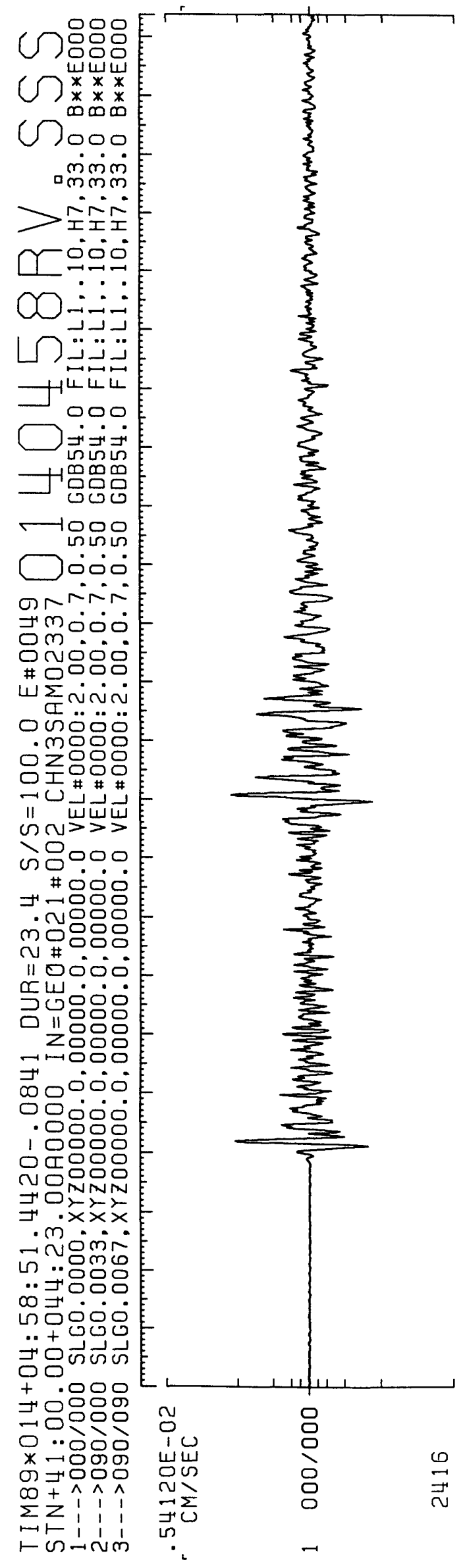

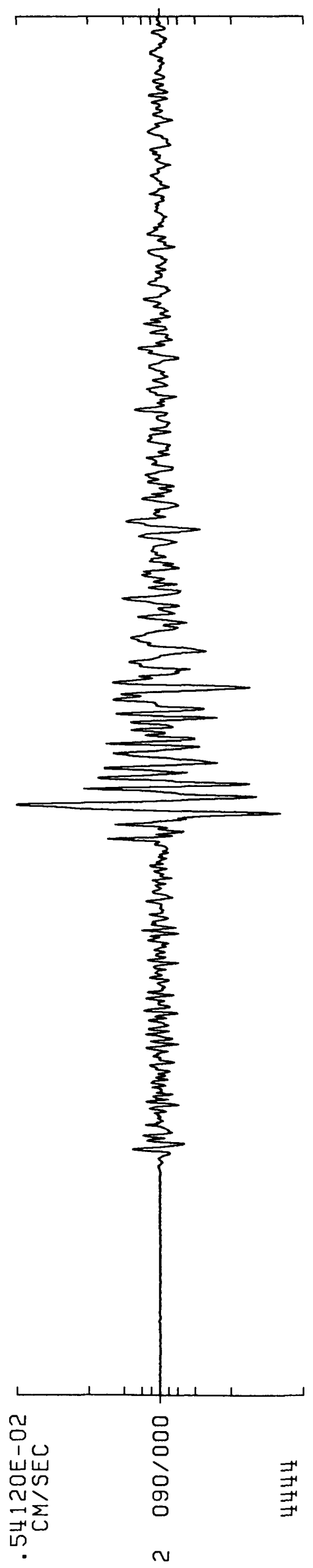

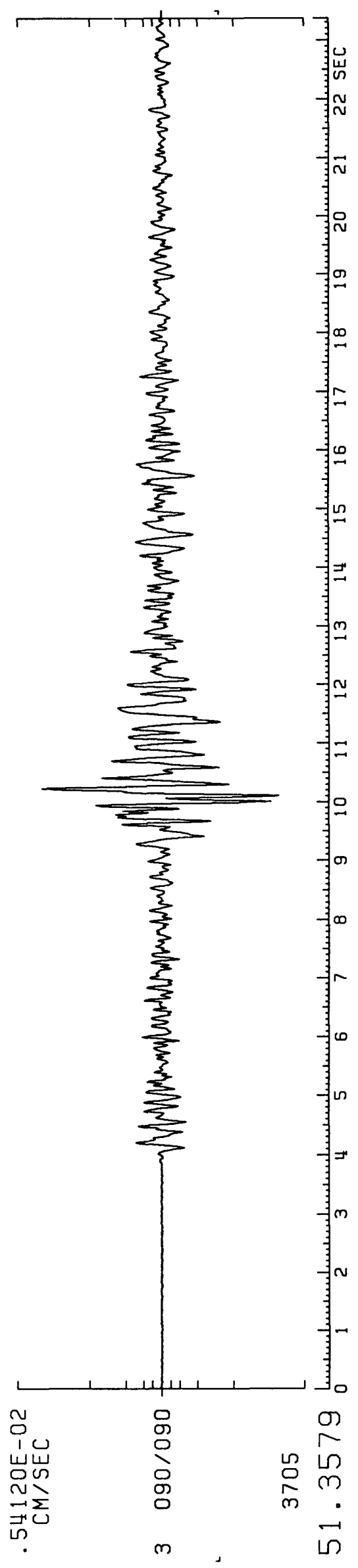




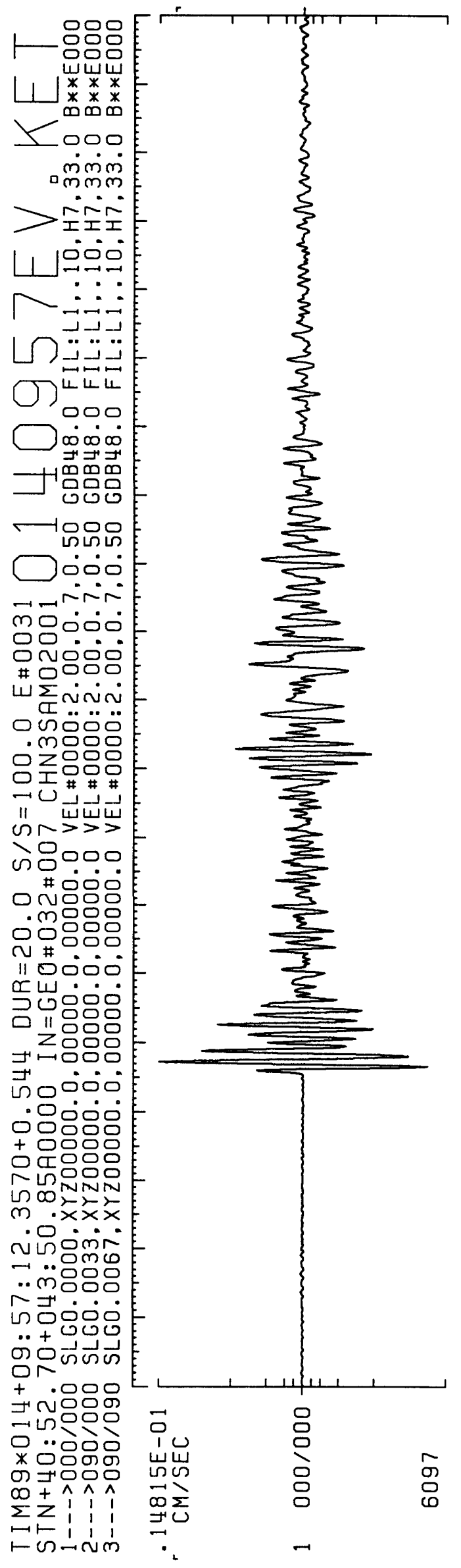

245

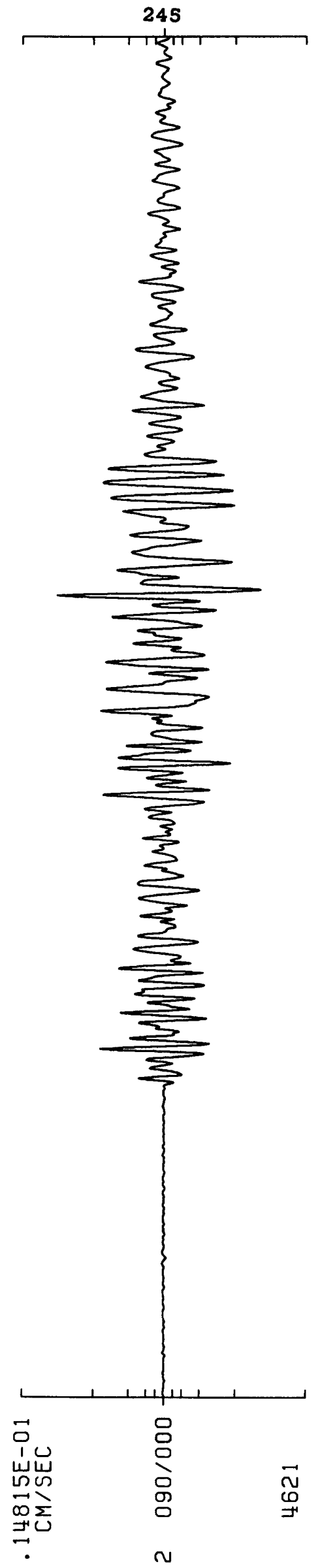

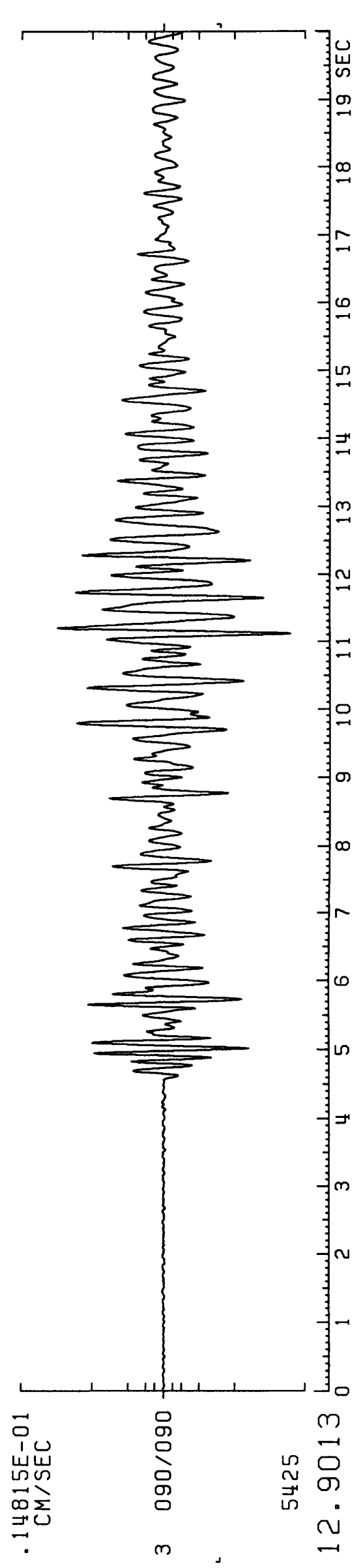



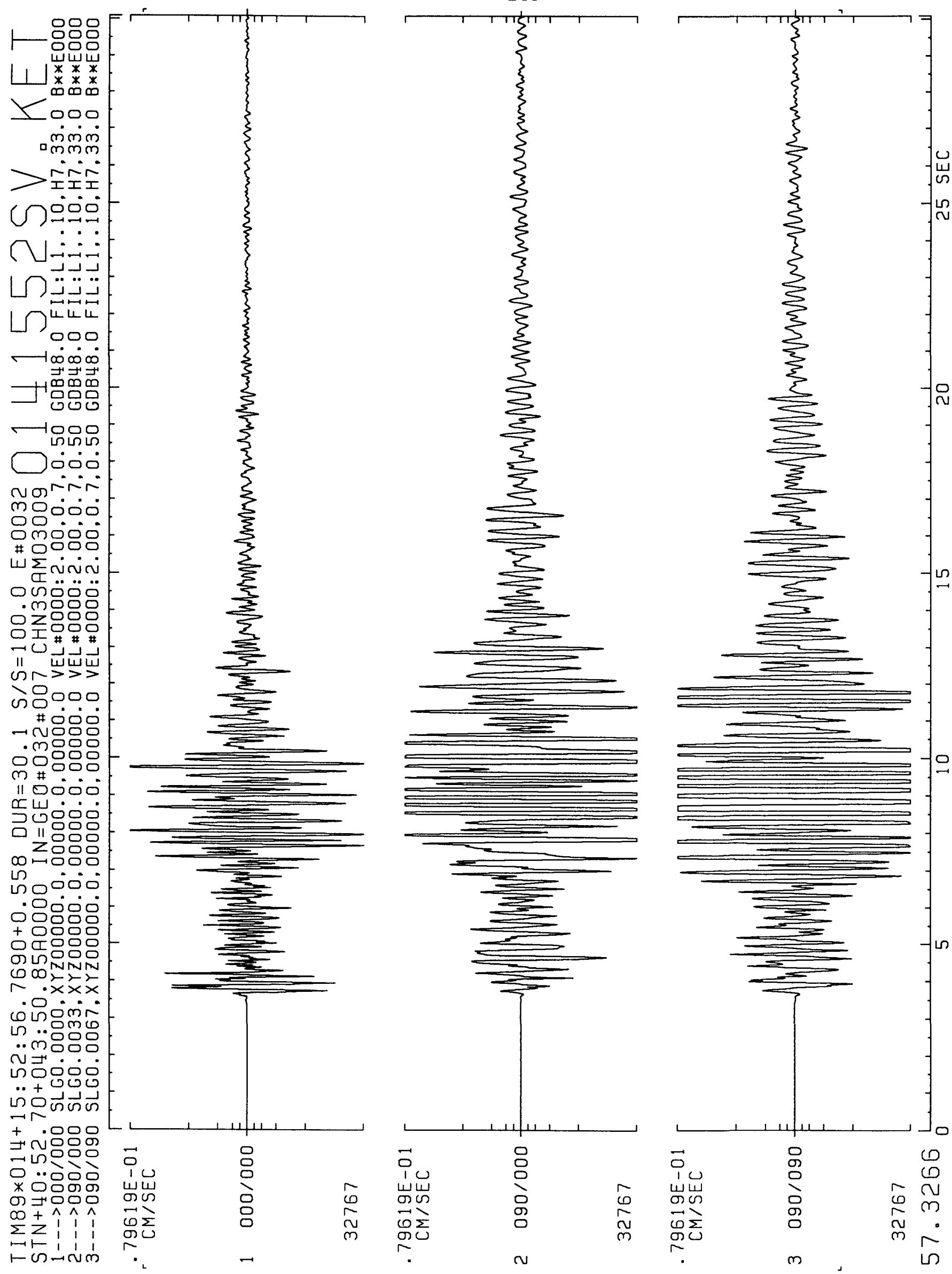


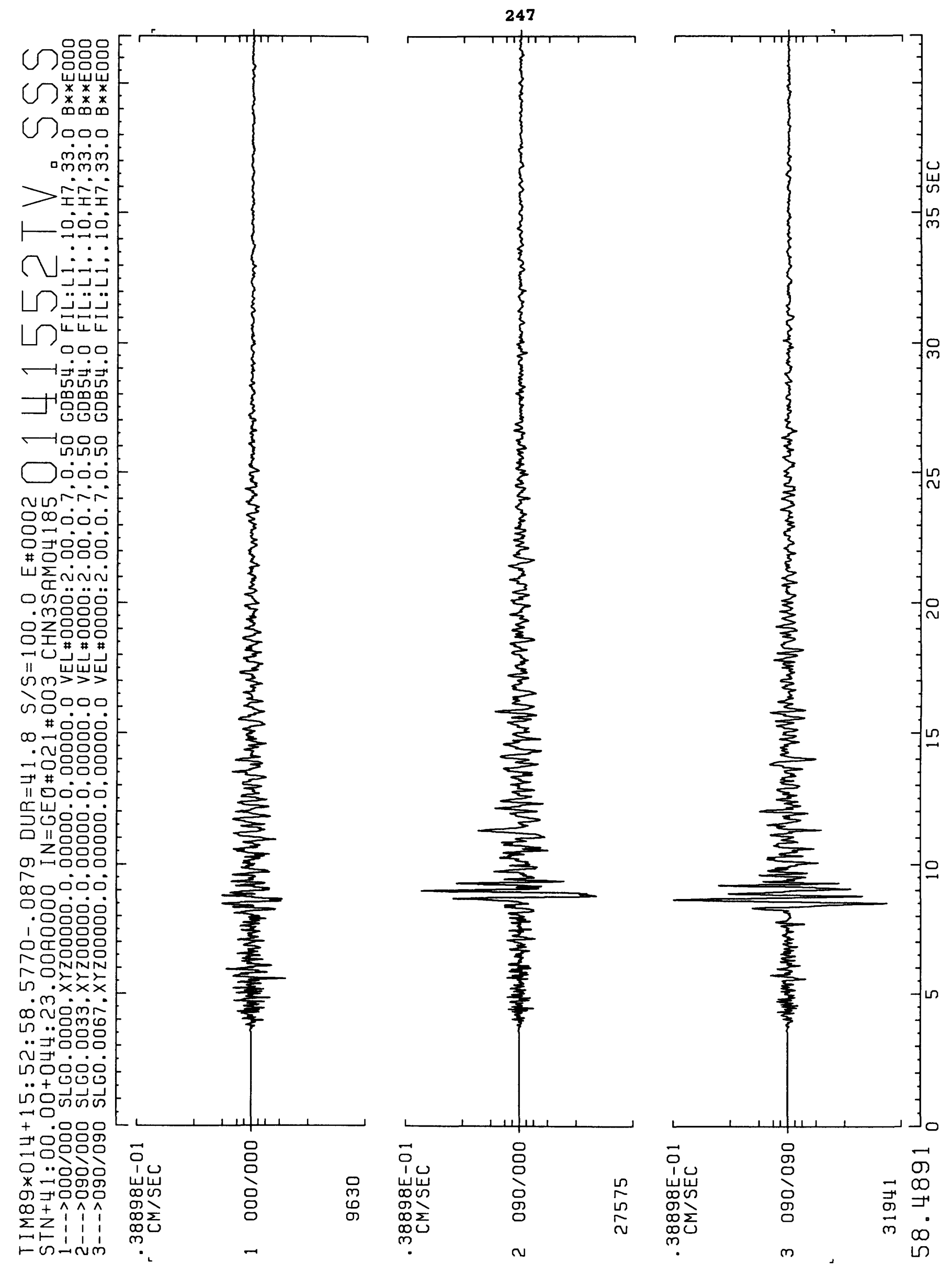


248

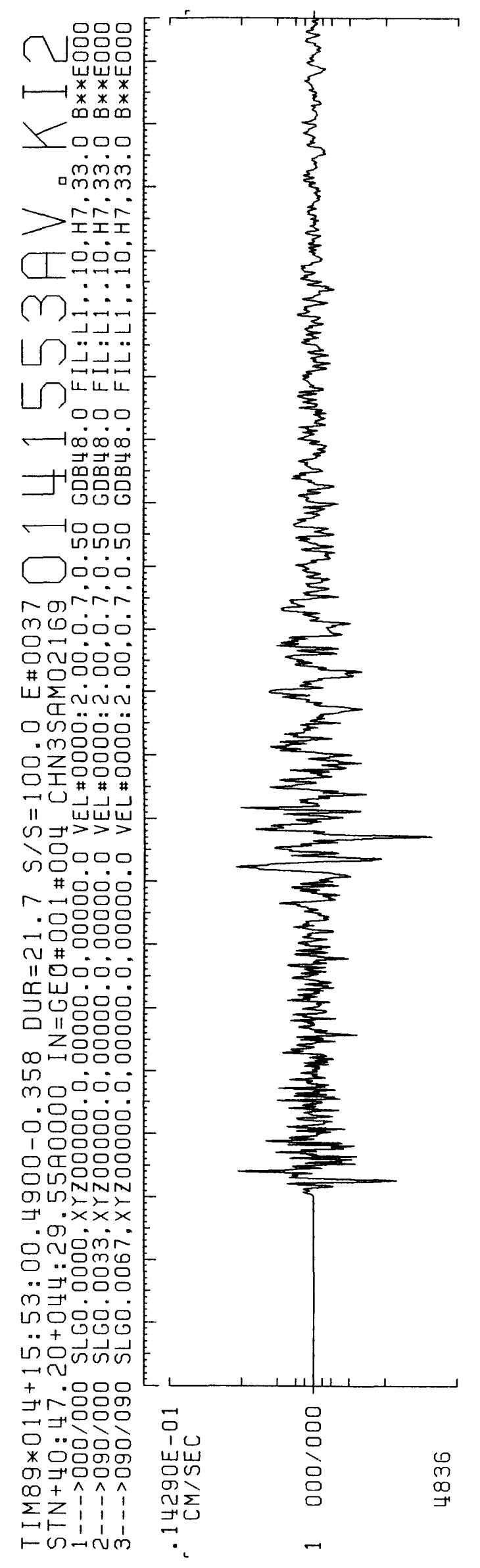

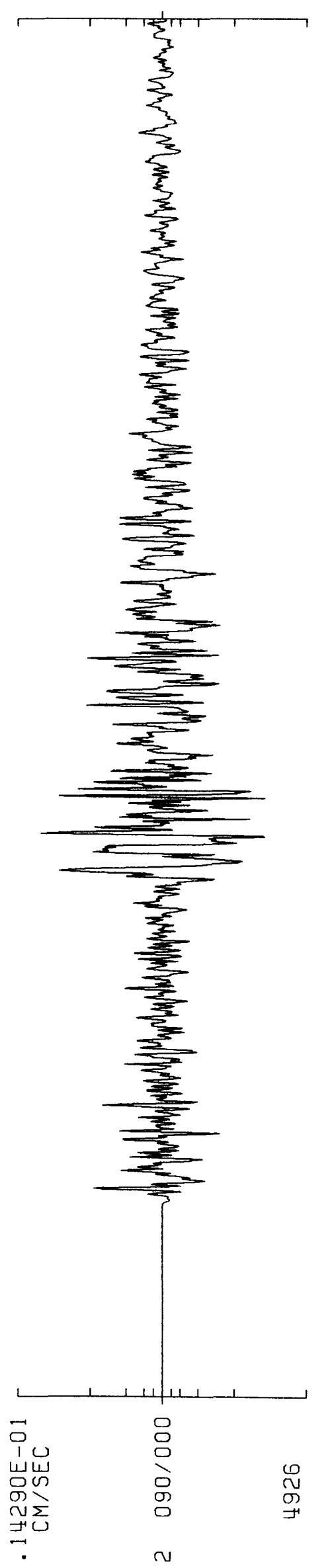

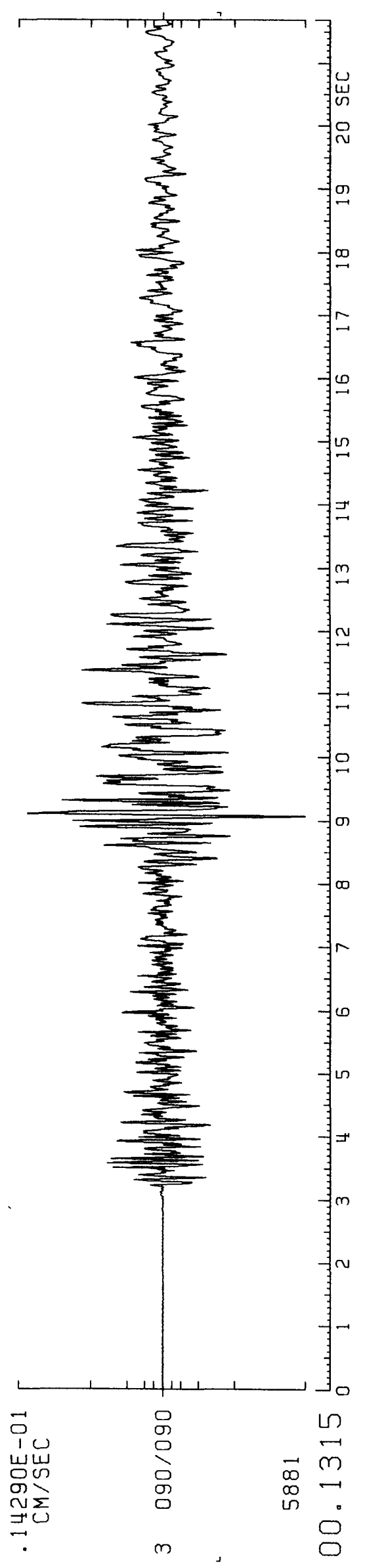



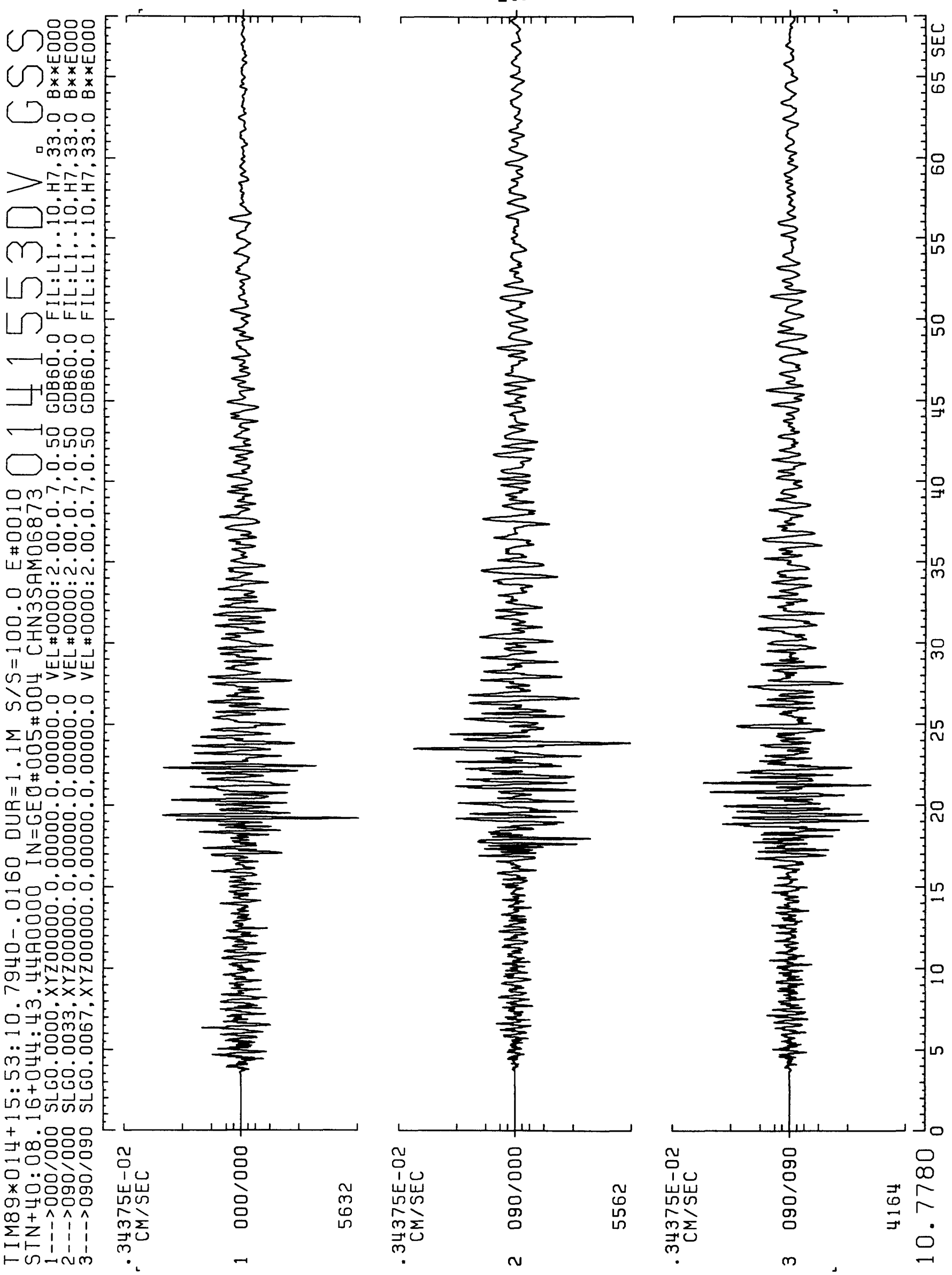

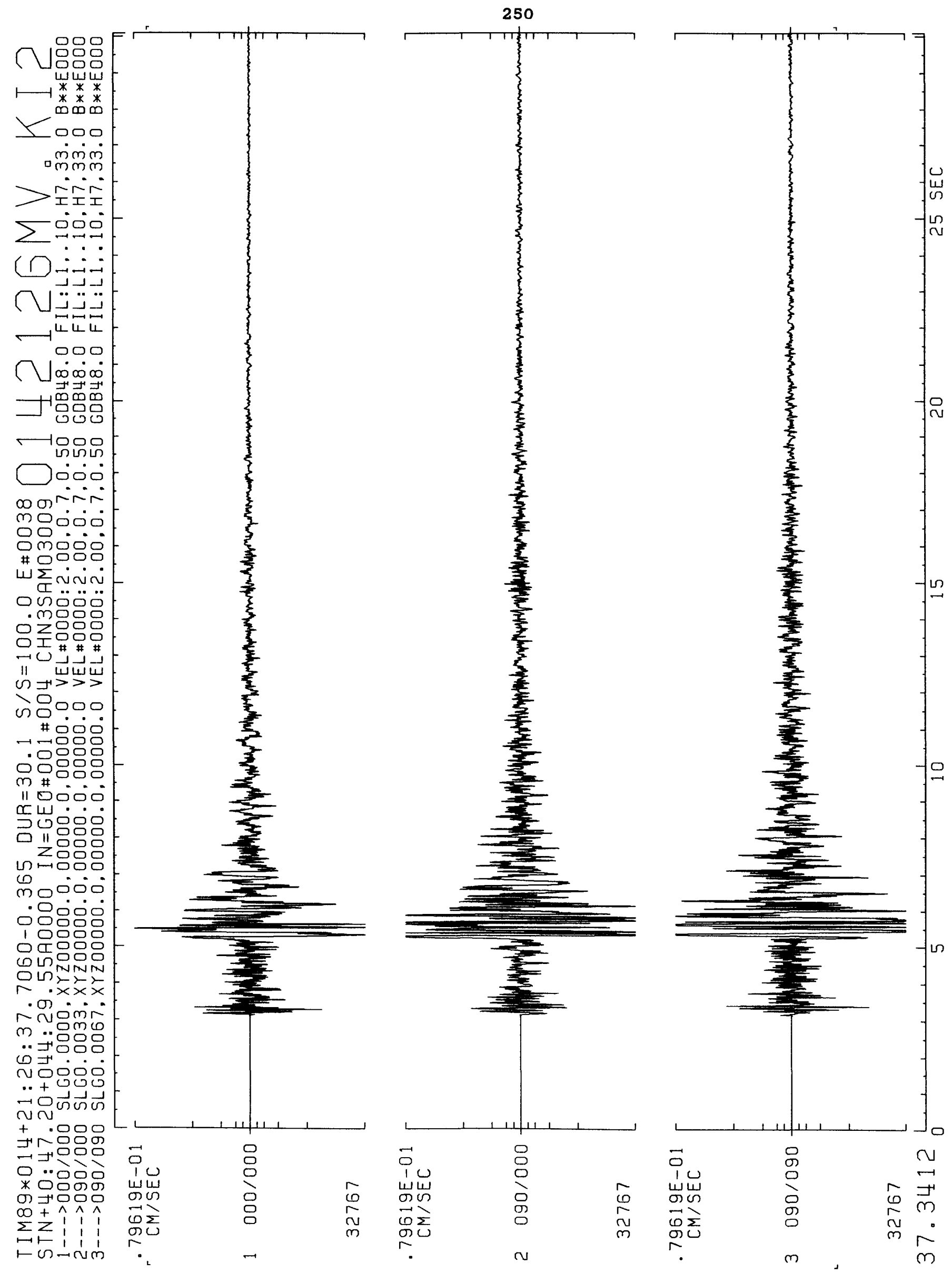
251

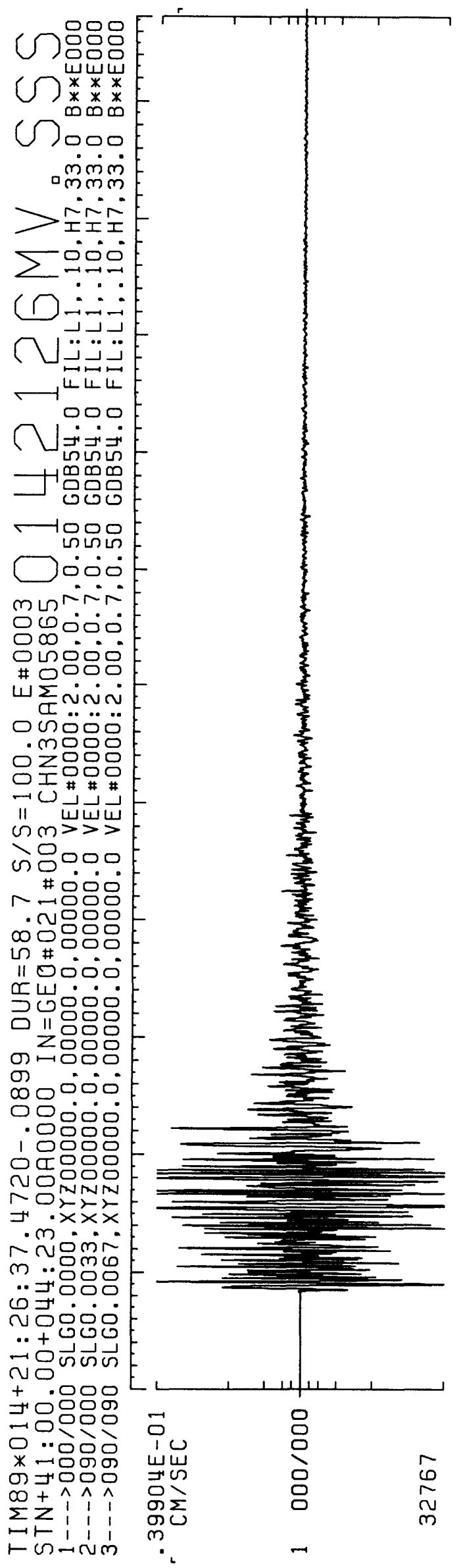

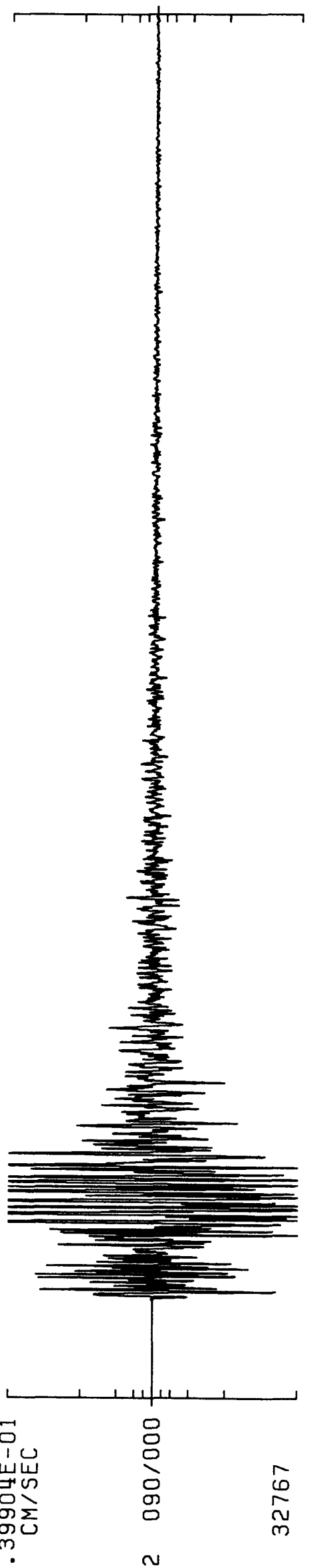

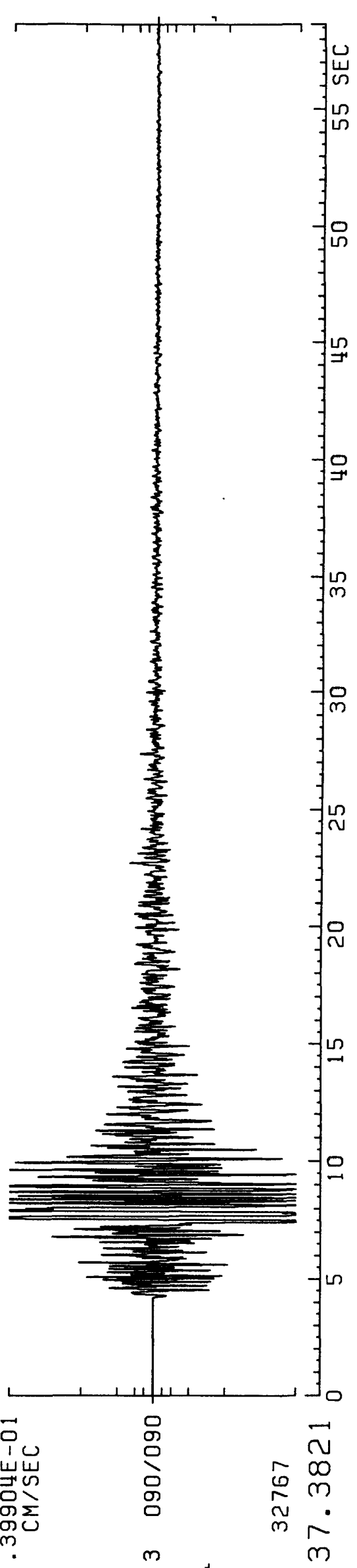



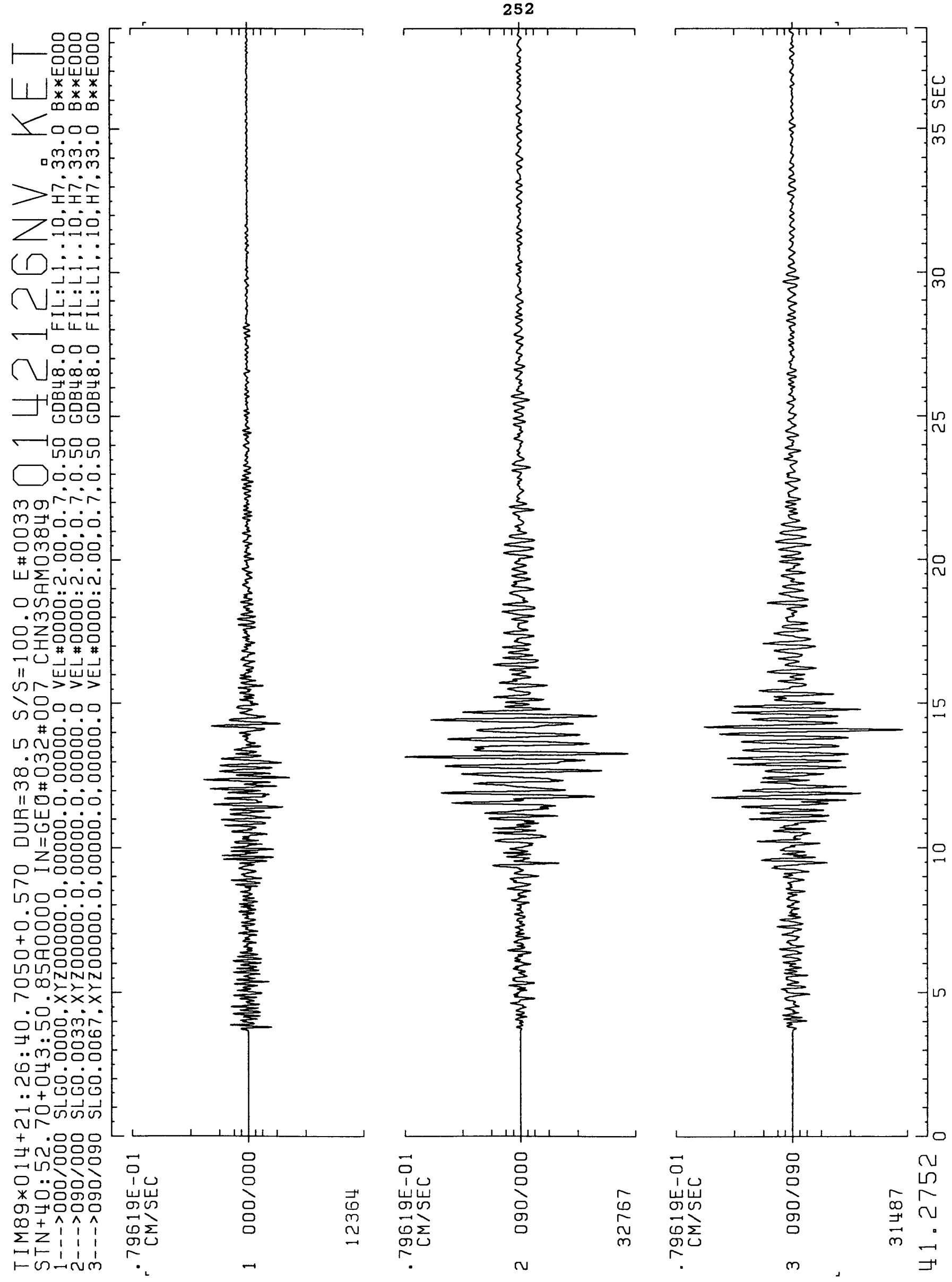
253

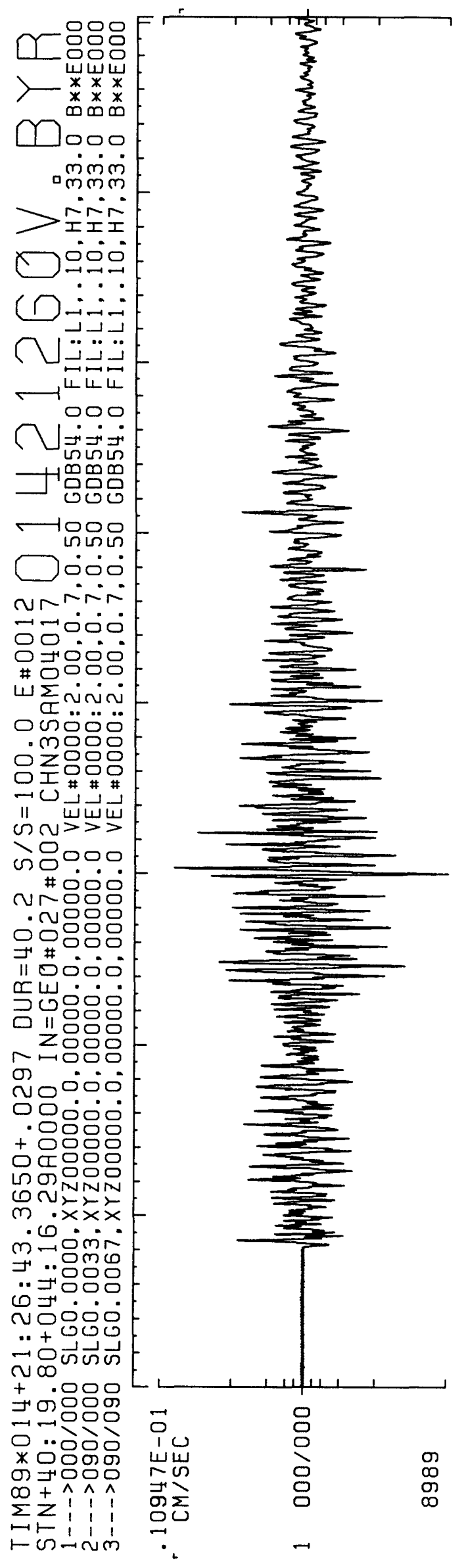

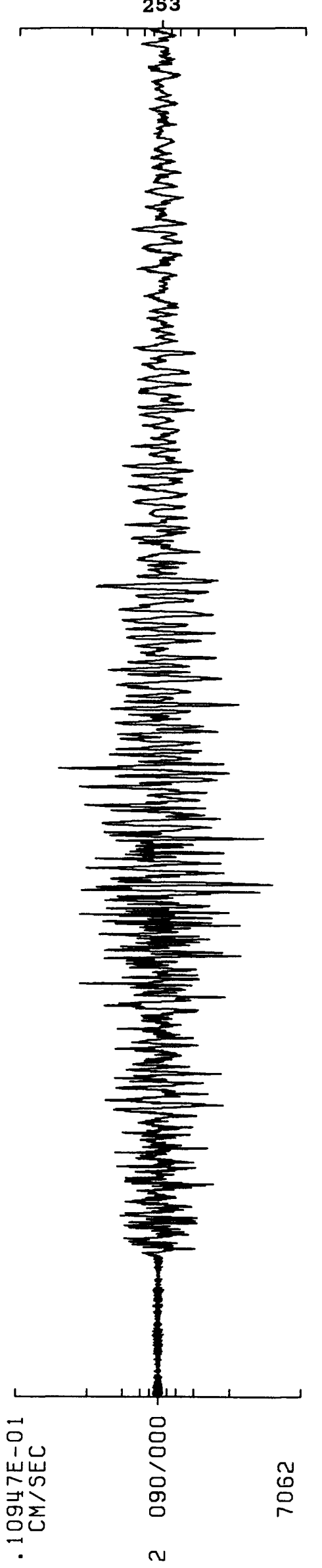

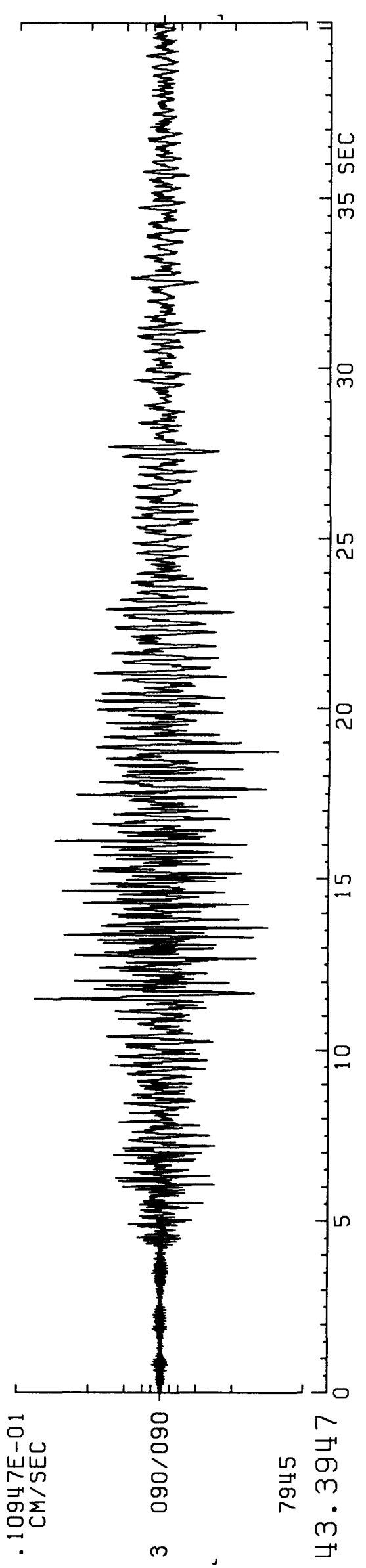




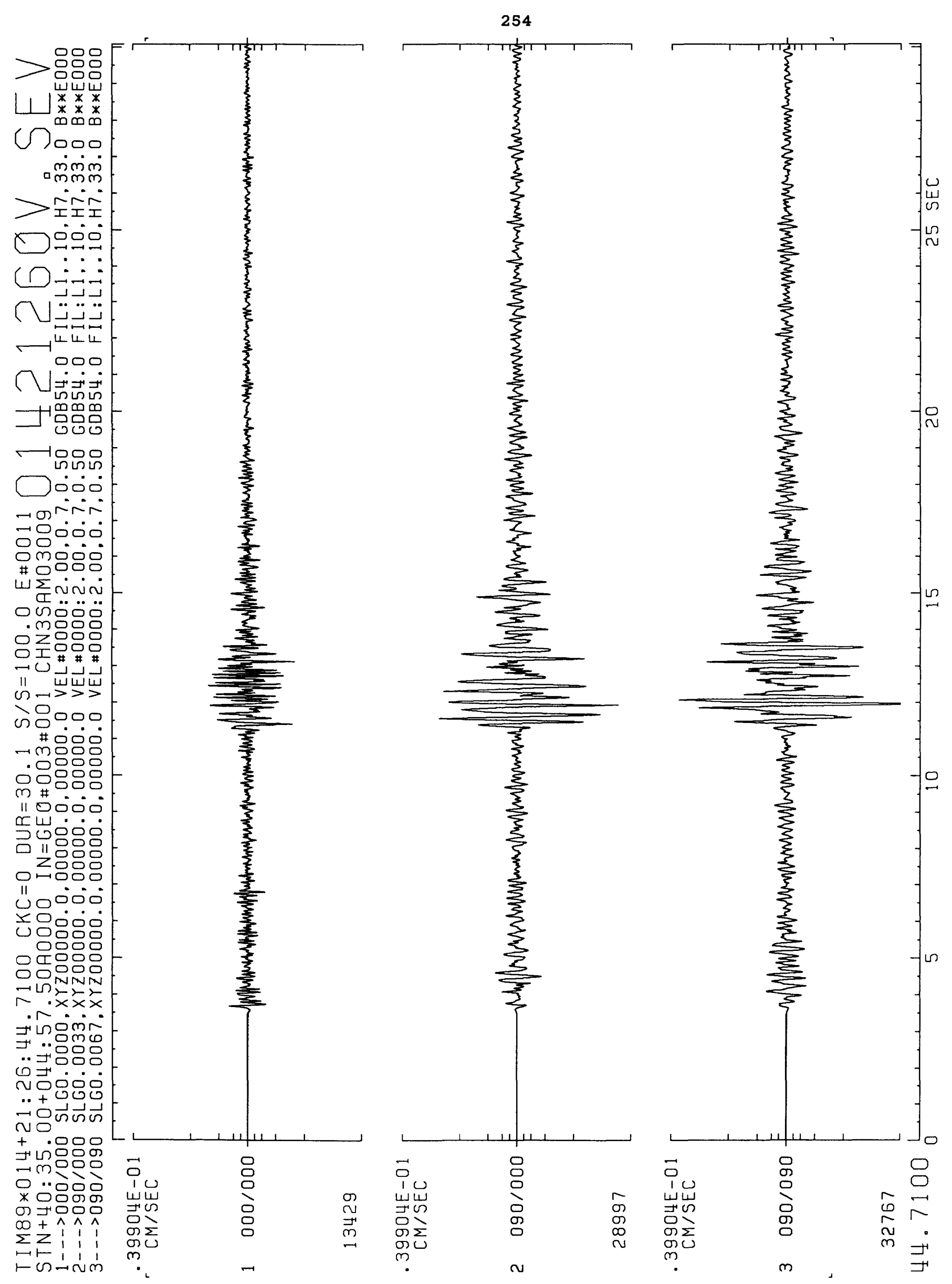



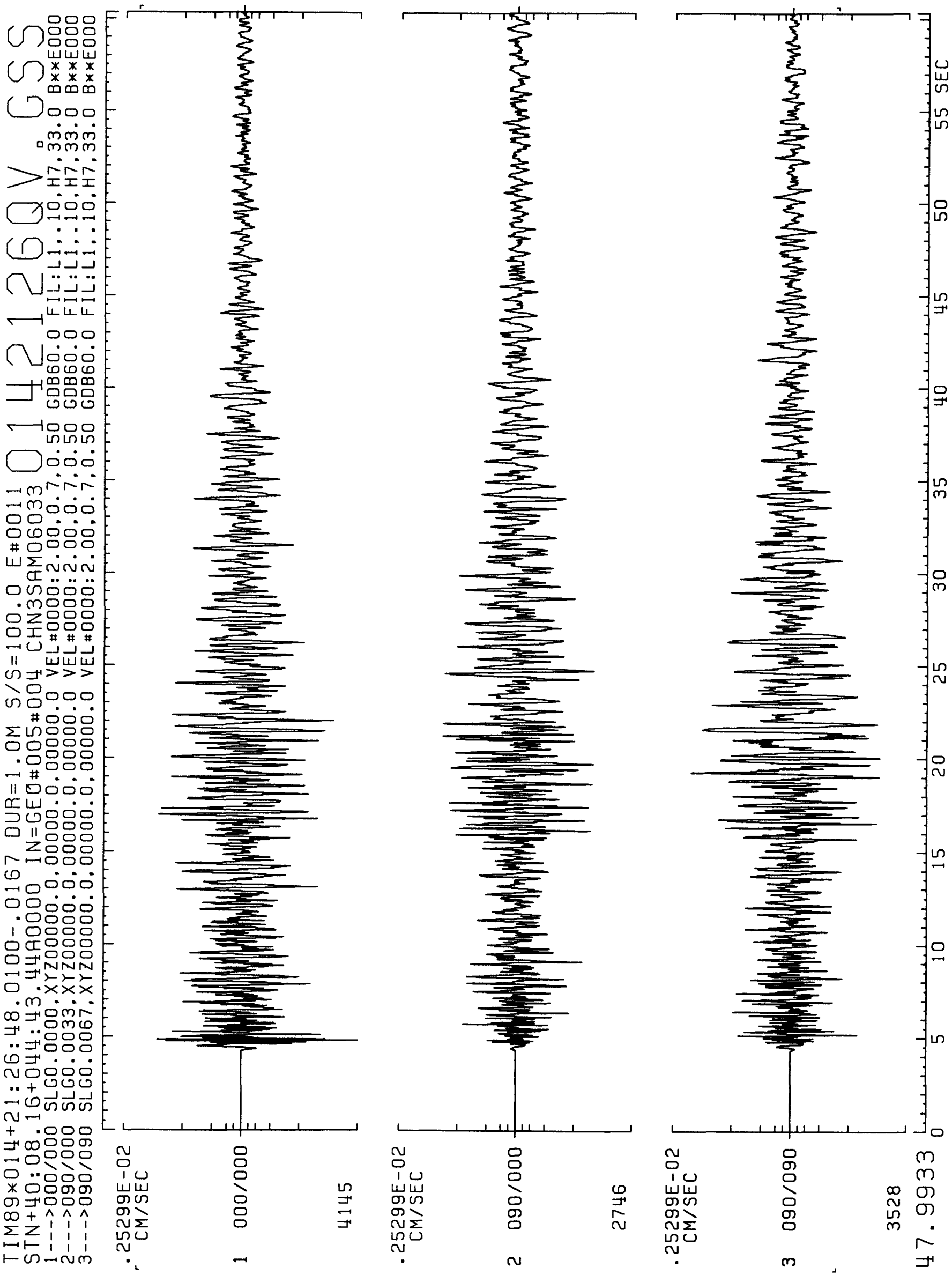

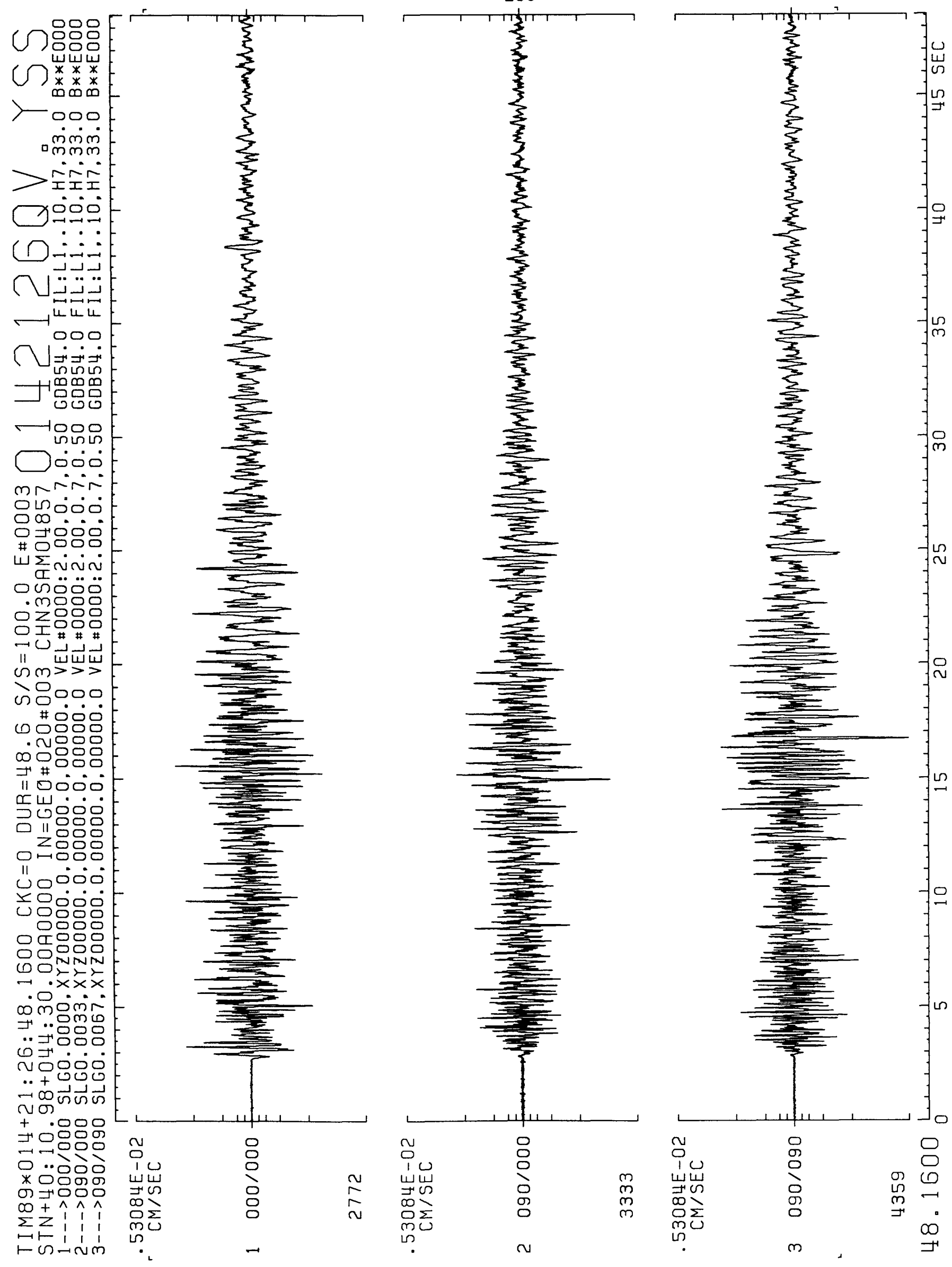

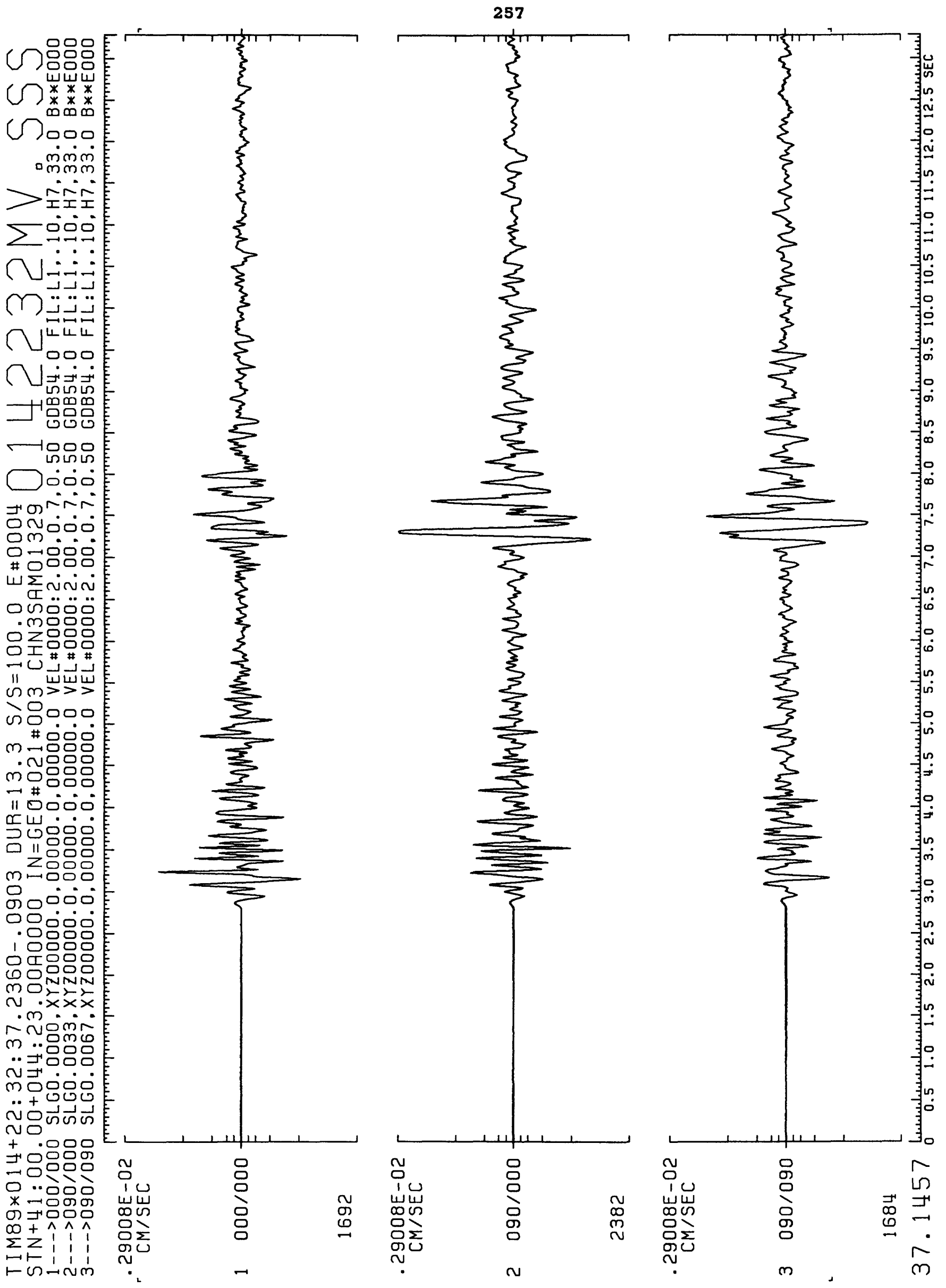
258

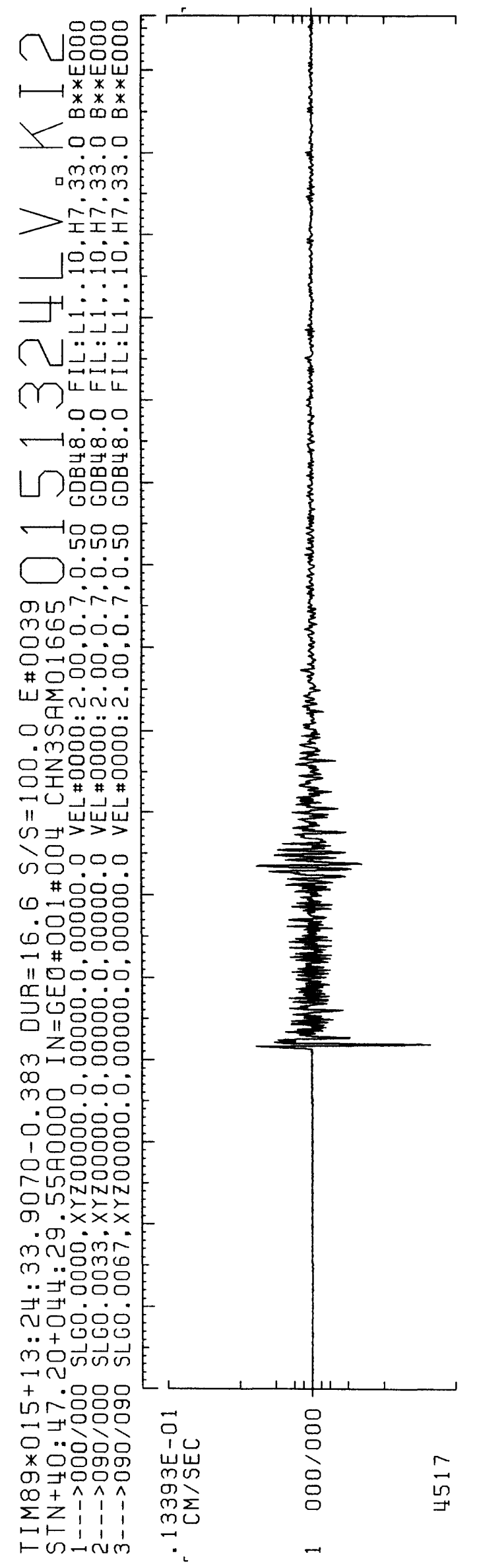

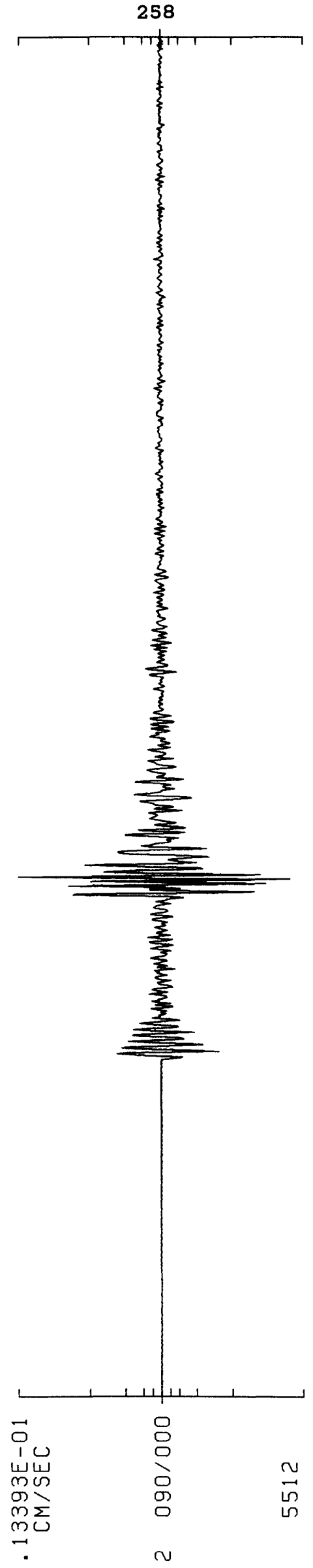

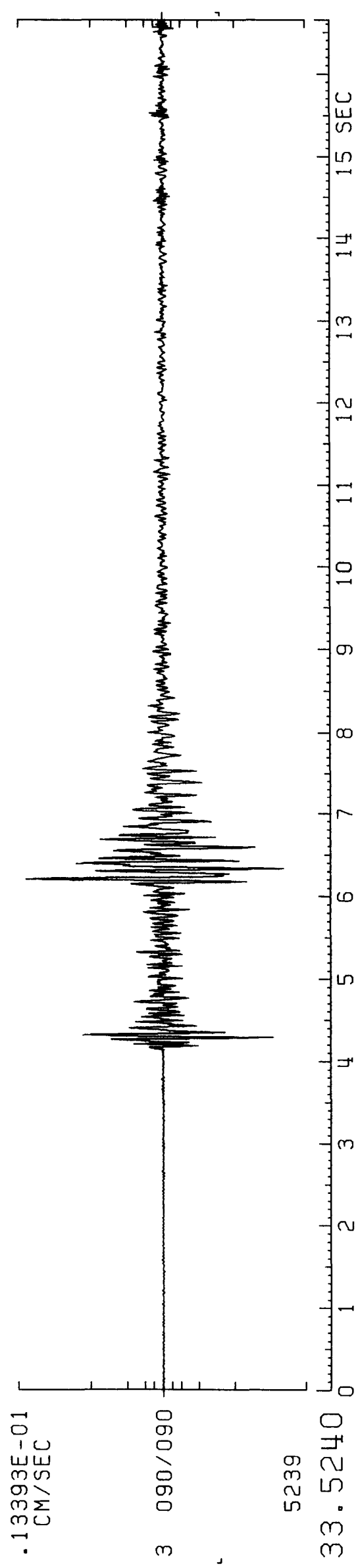



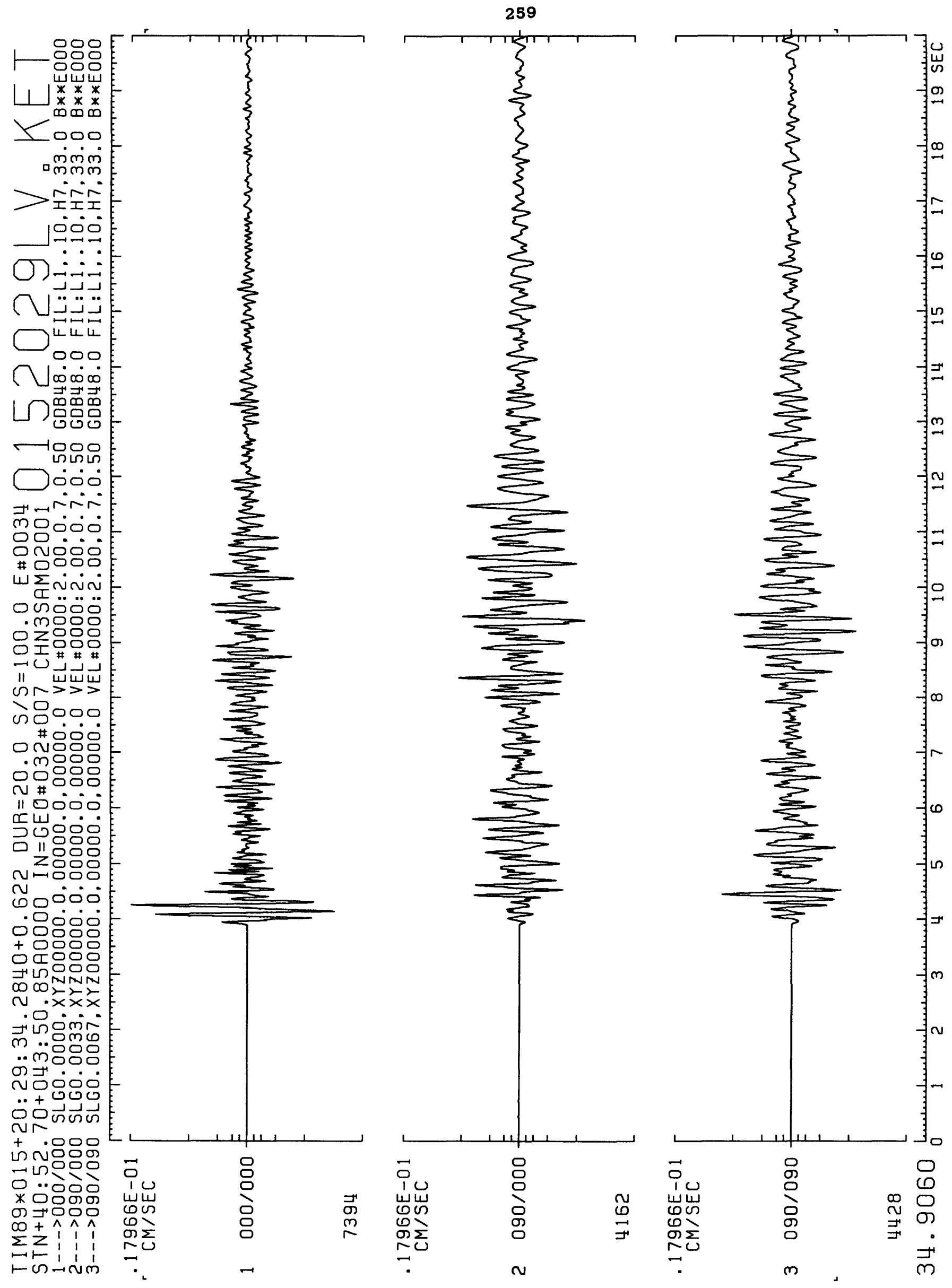


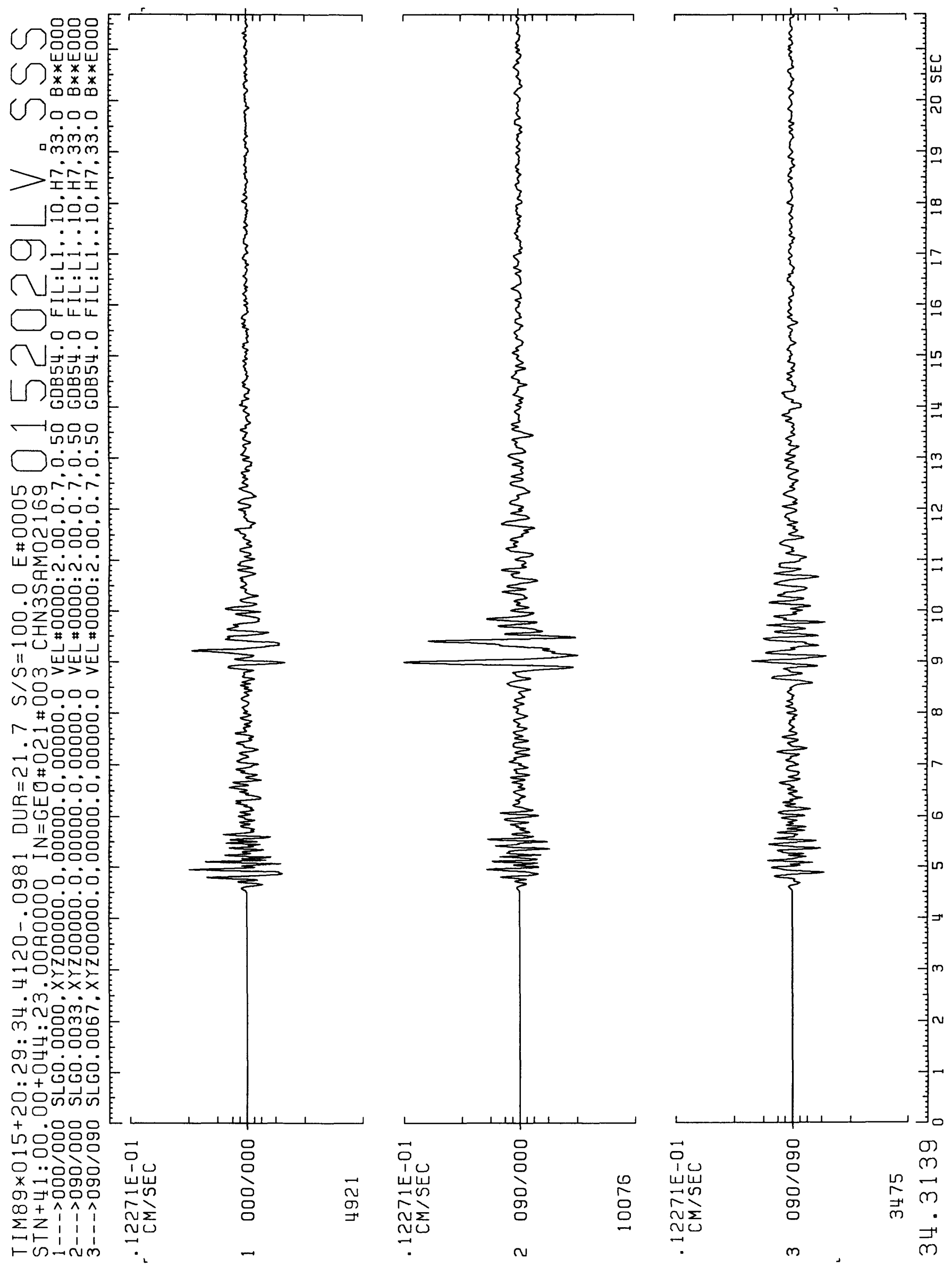




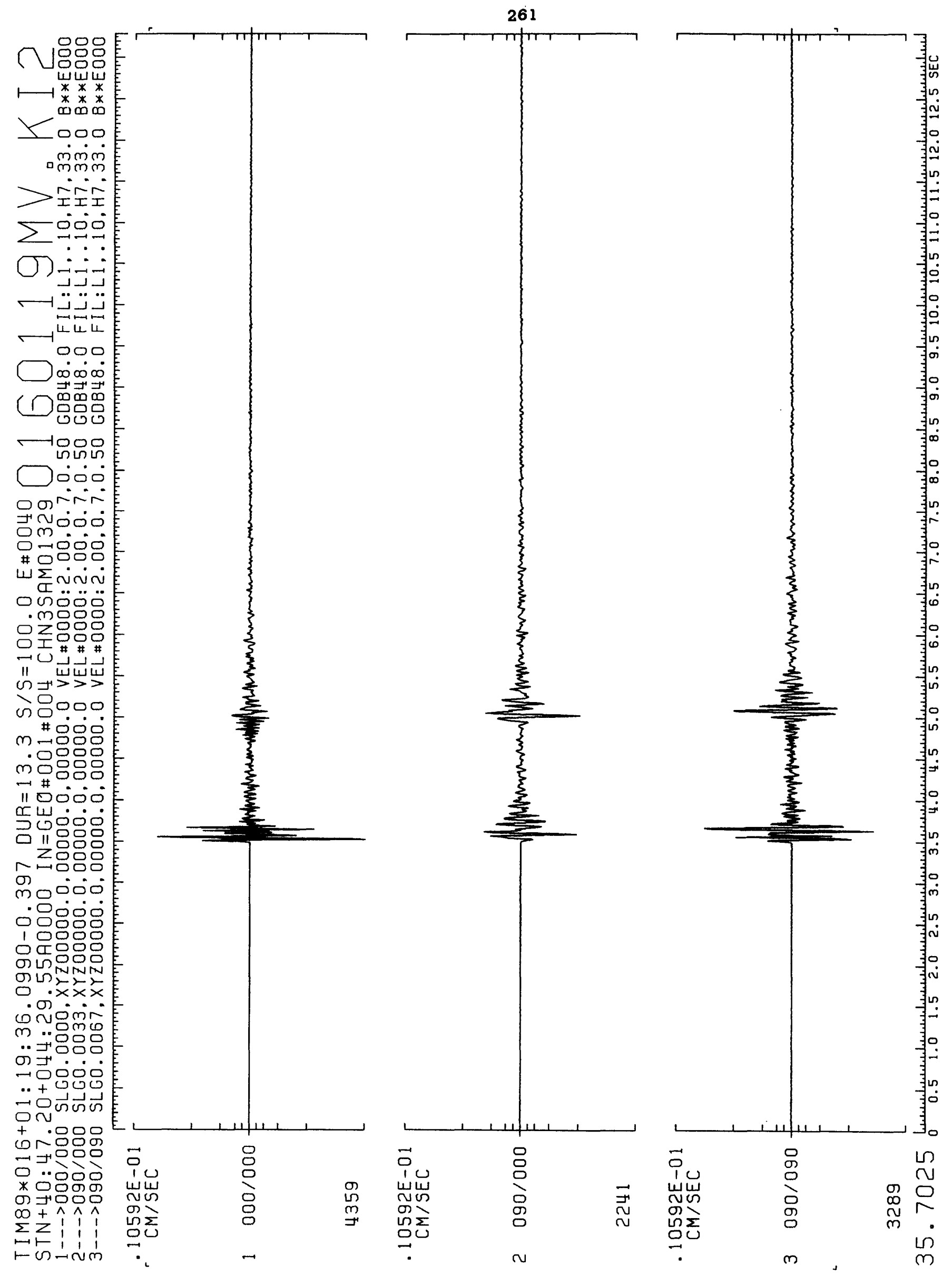


262

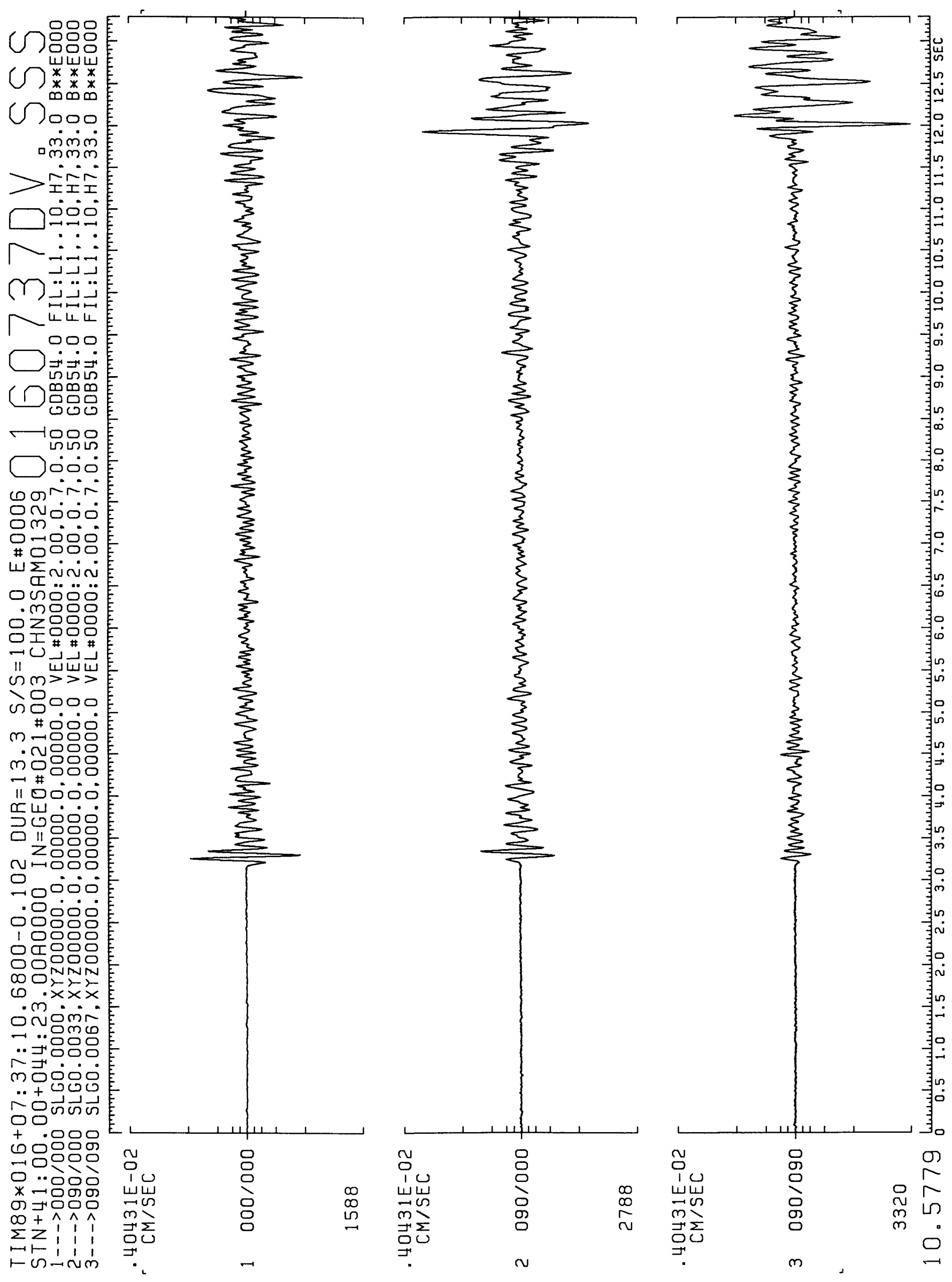



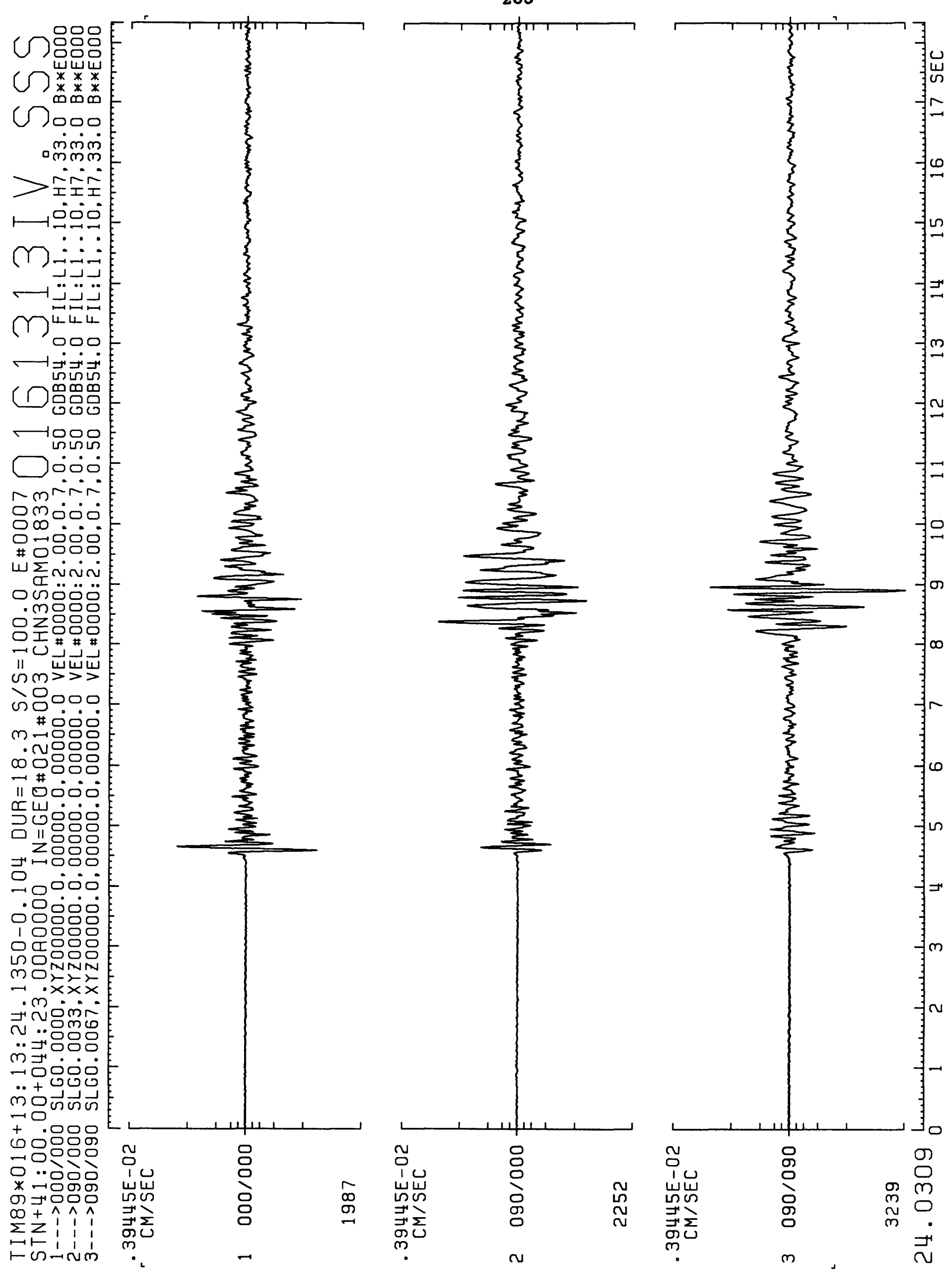

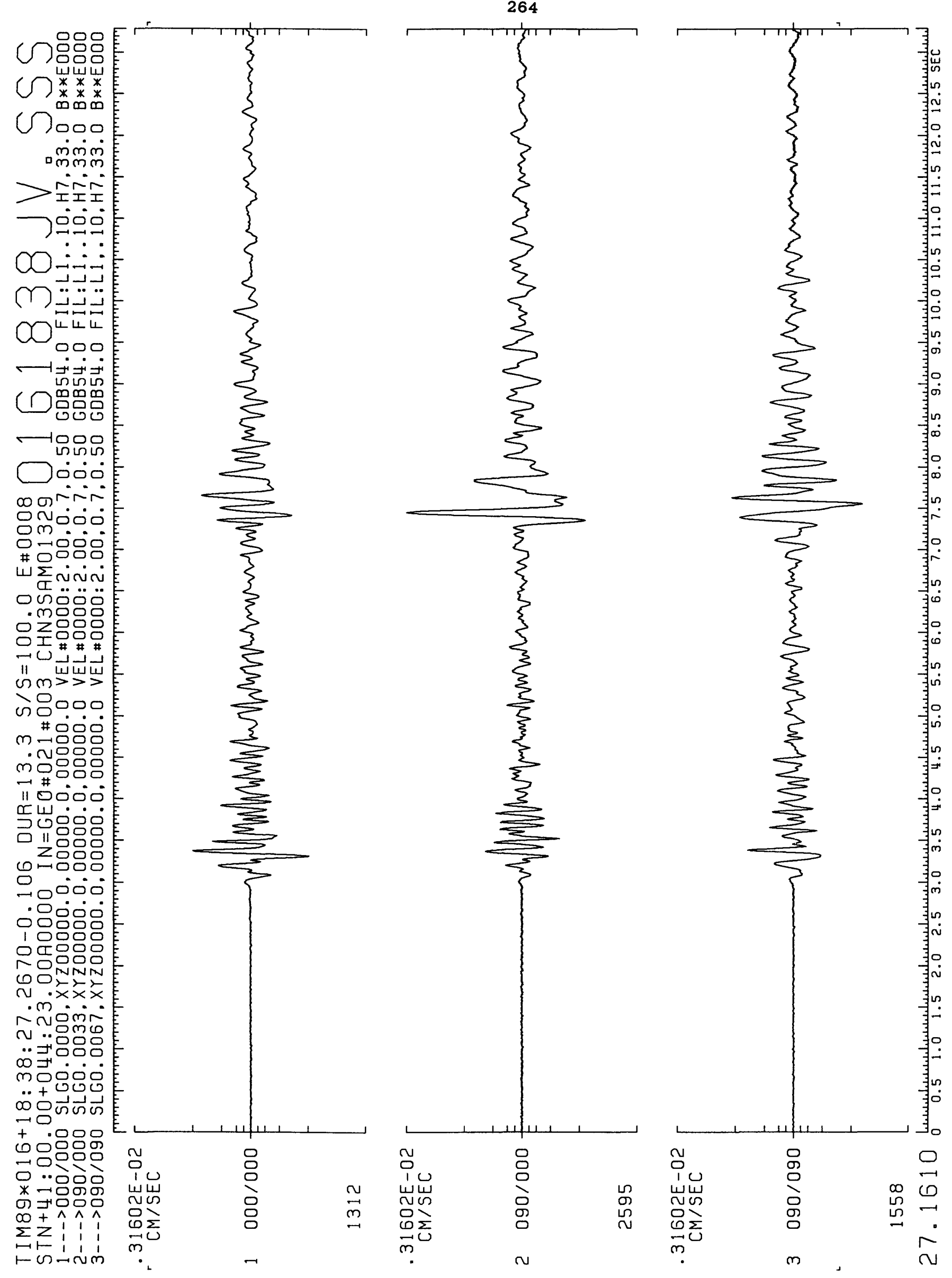


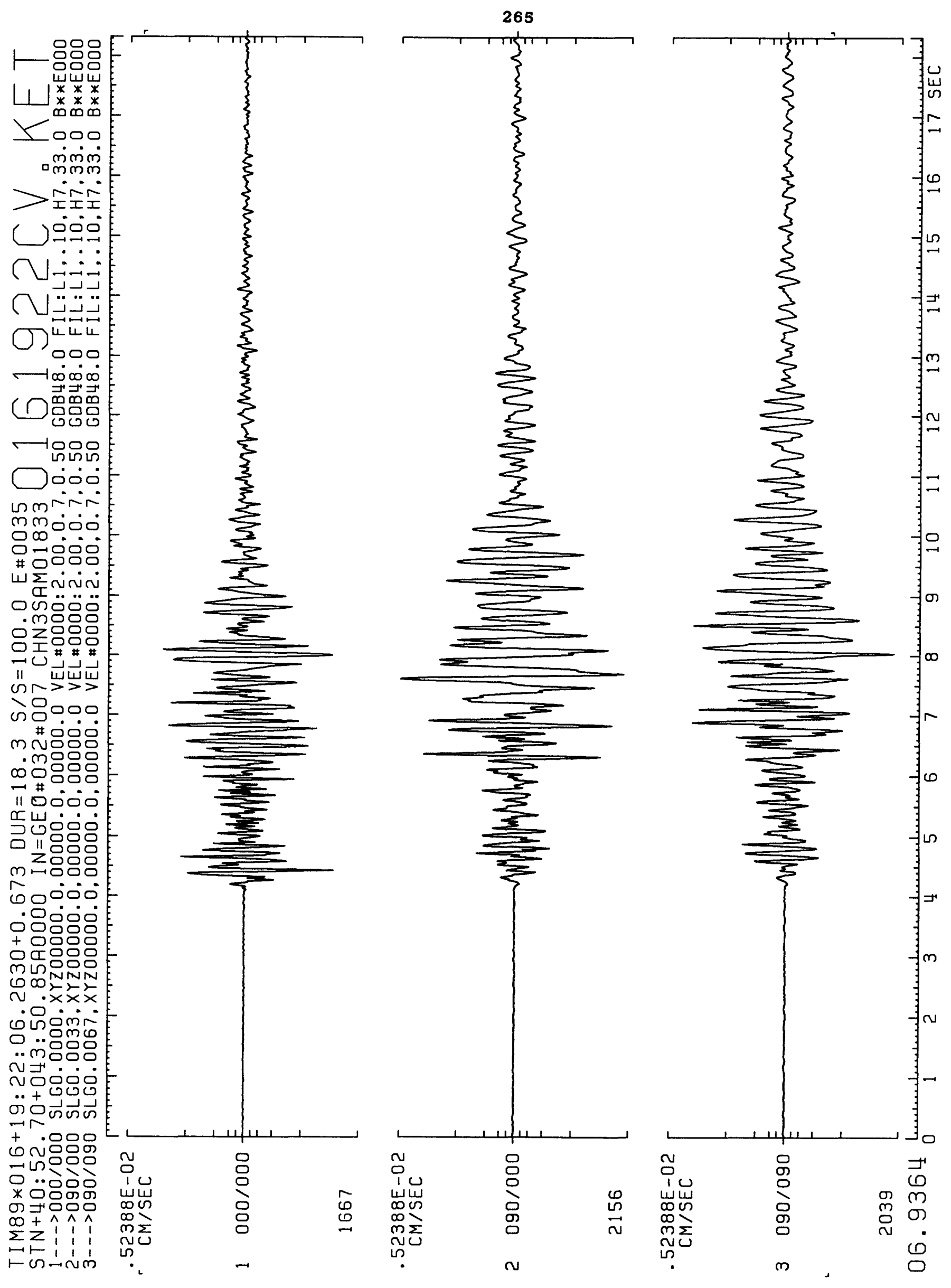


266
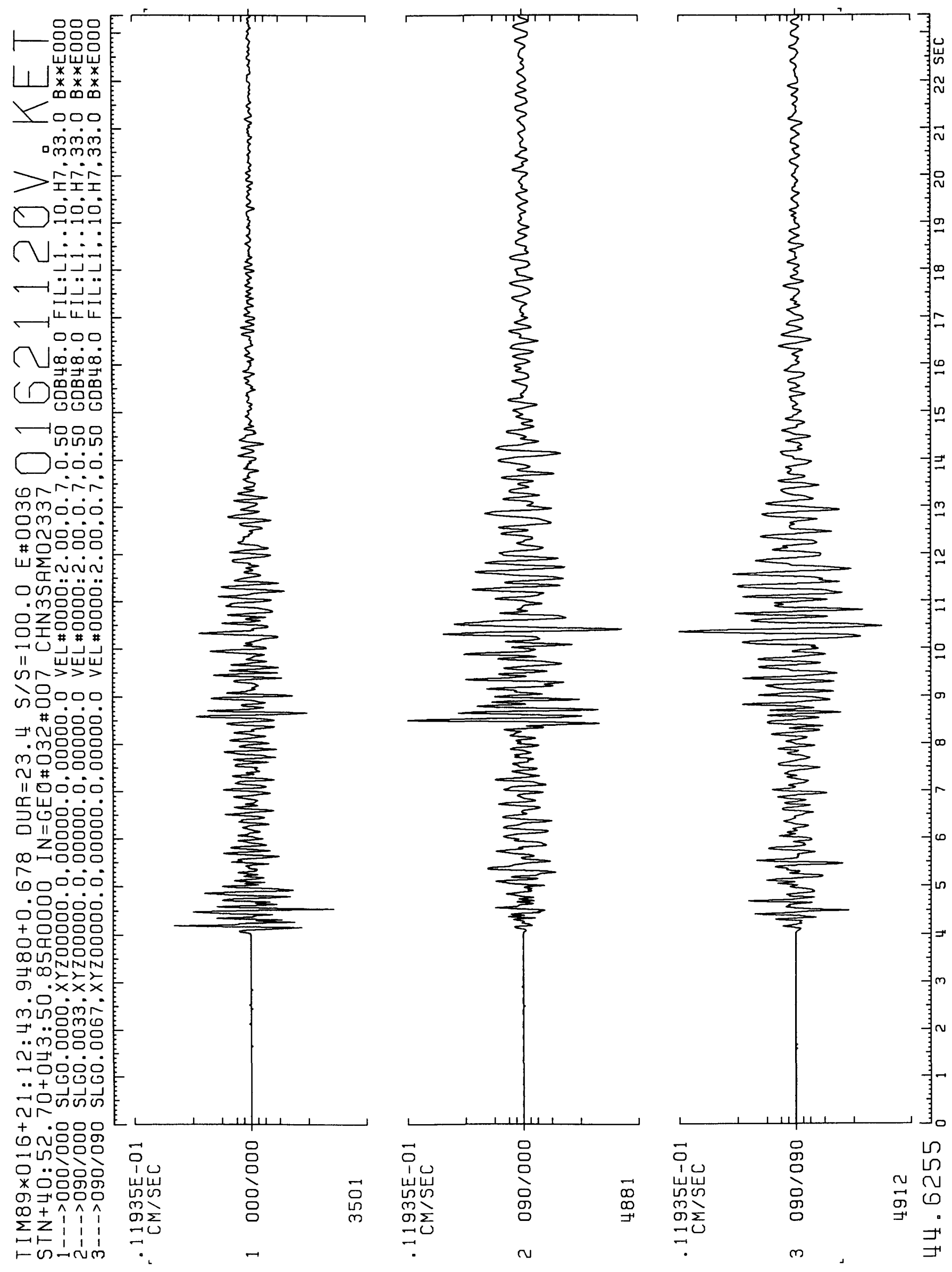


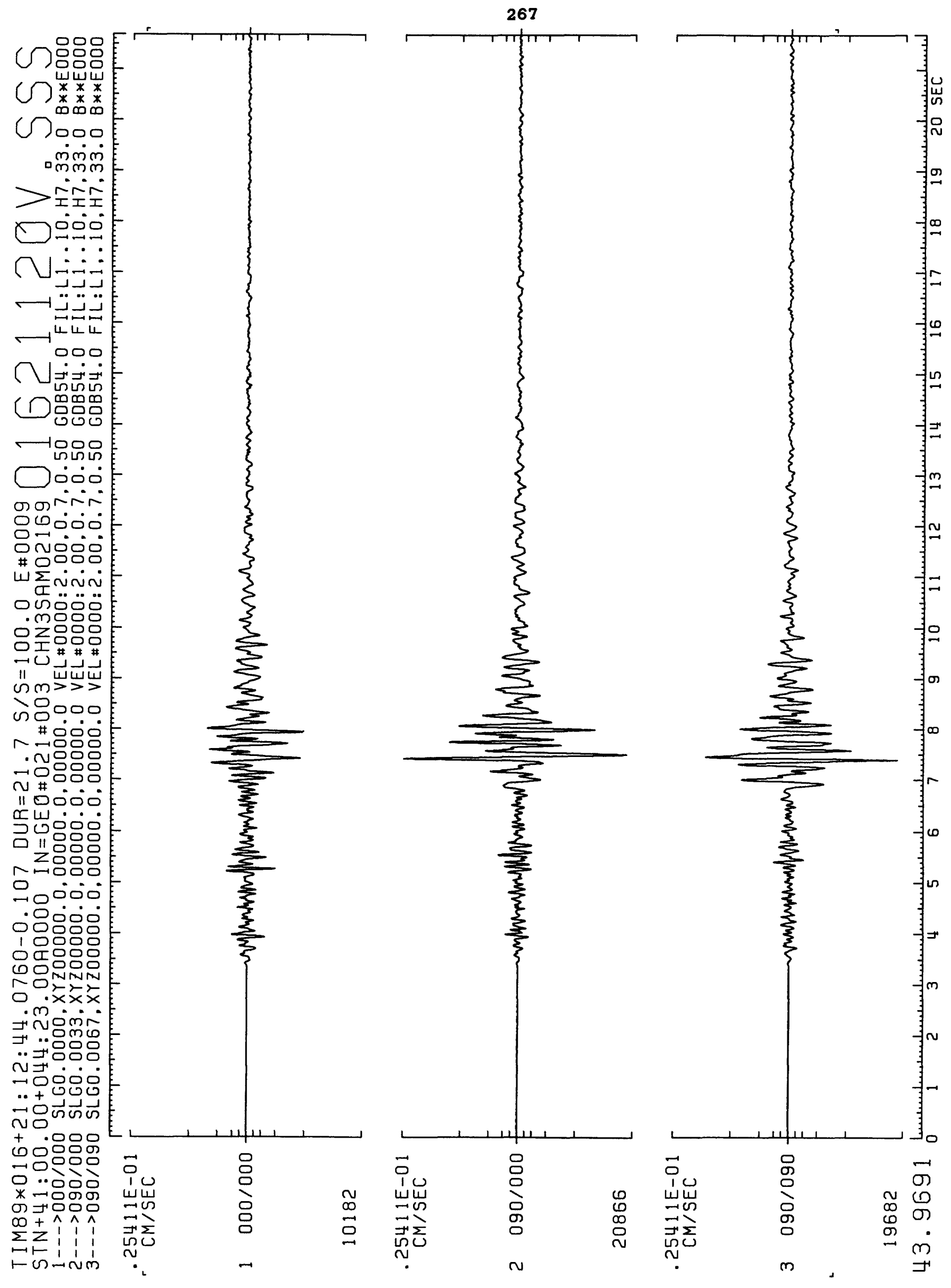




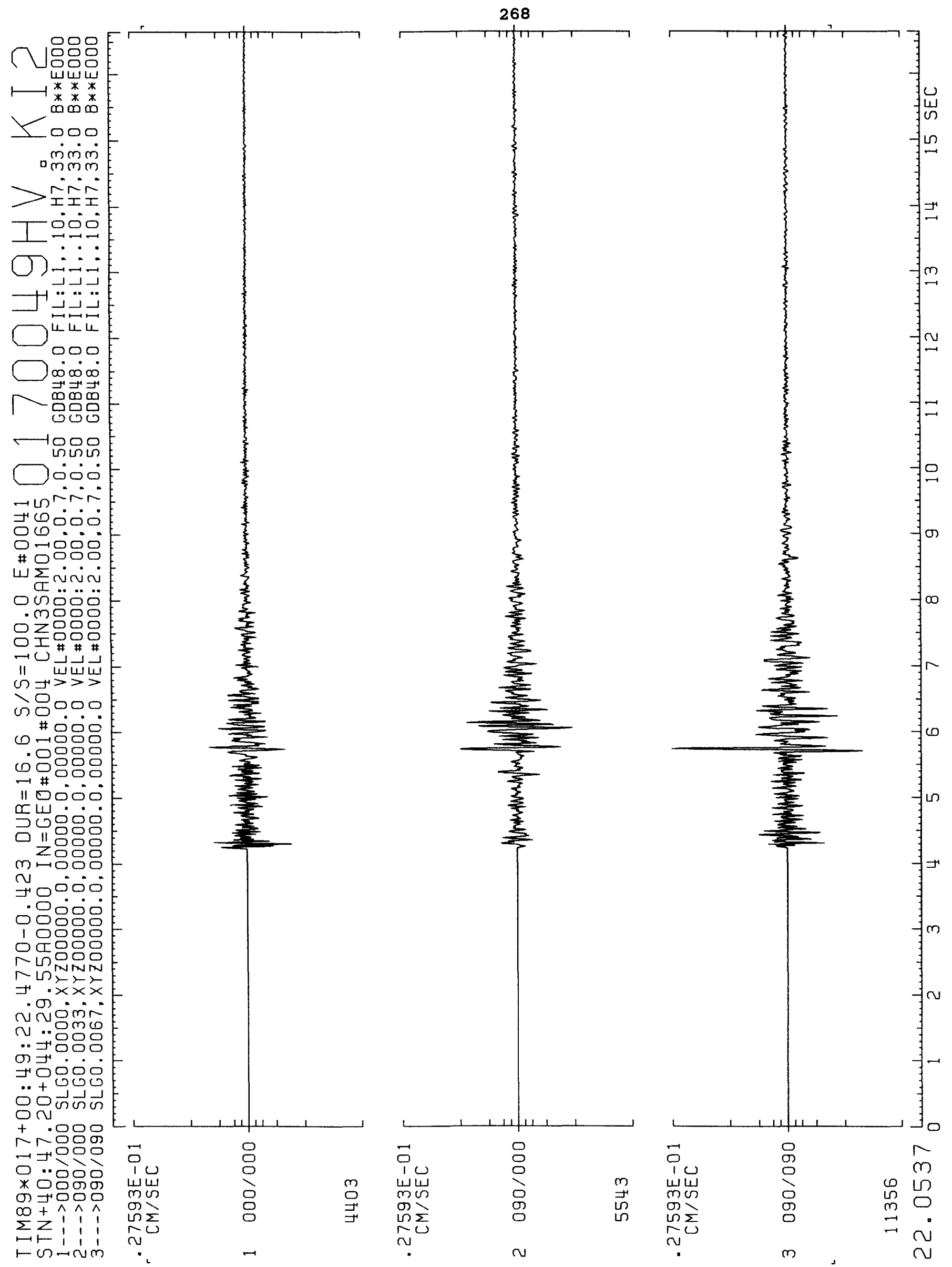


269

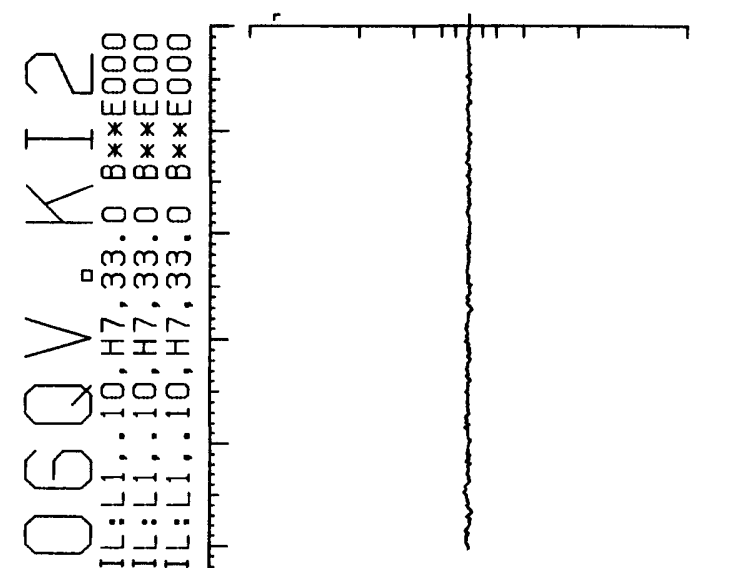
○ाயน

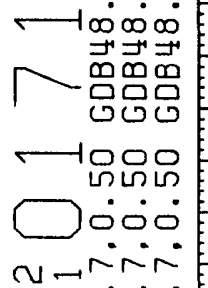

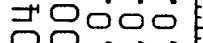
ด ㄷํㅇํํ \#0000 - $\sum_{0} n i n$

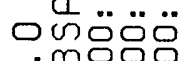
i m응응 口퓨 \# II U जे०००० \# 용 늠ㅇㅁㅇㅇㅁㅇ II ڤं宀 œயن் 밈ㅇㅇㅇ z ๓๓口ㅁㅇㅇㅁㅇ m تصن் 1 음응 ○등ㅇㅁㅇ NUSON OUI, $\rightarrow \cdot x \times x$ iniñ ONO เ . 염 ㅋ.100 ดिं0் in तण्डण + + 응요 ○ ..ㅎํㅇ * 임 ब $\sum \pm \hat{z} \hat{\imath} \hat{\imath}$ $\sum_{\models} Z$

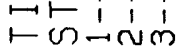
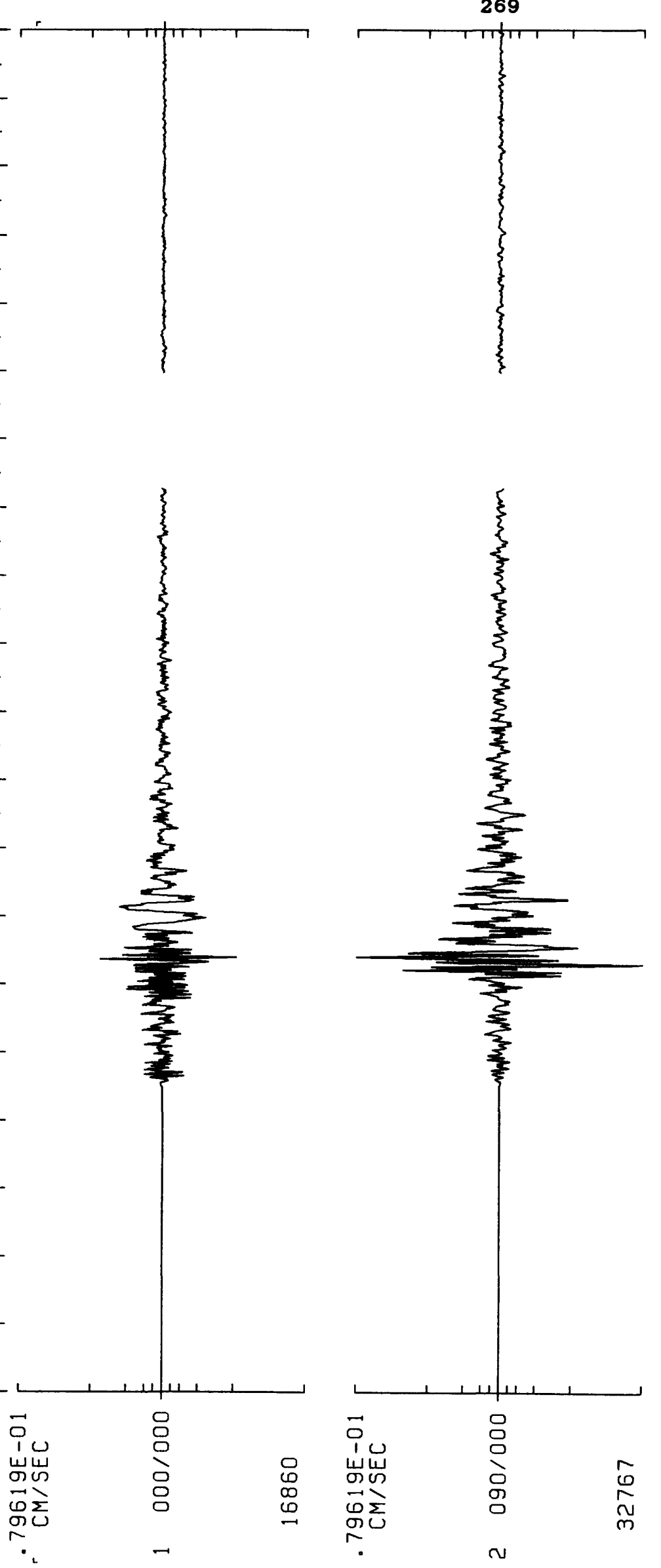

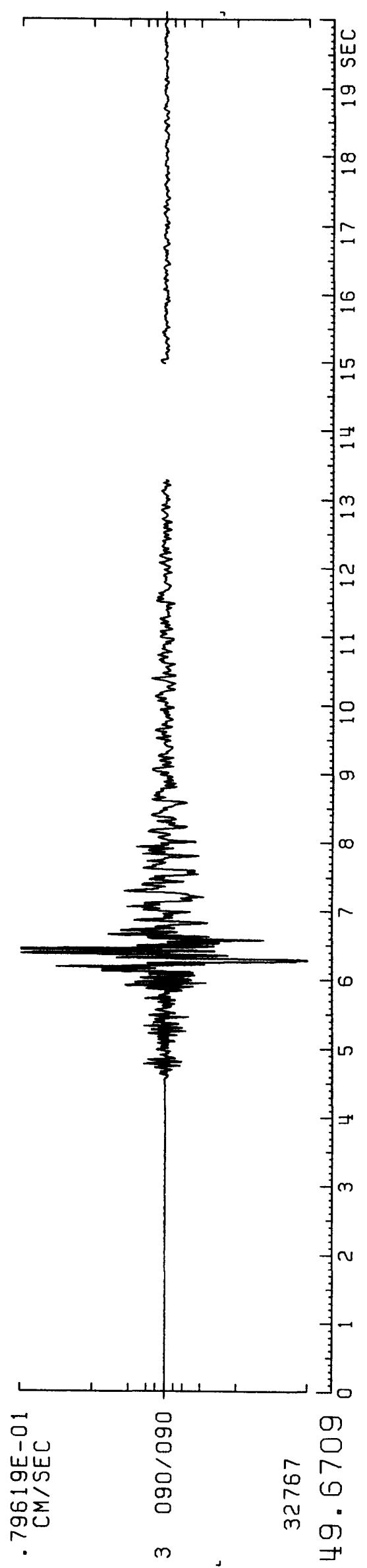




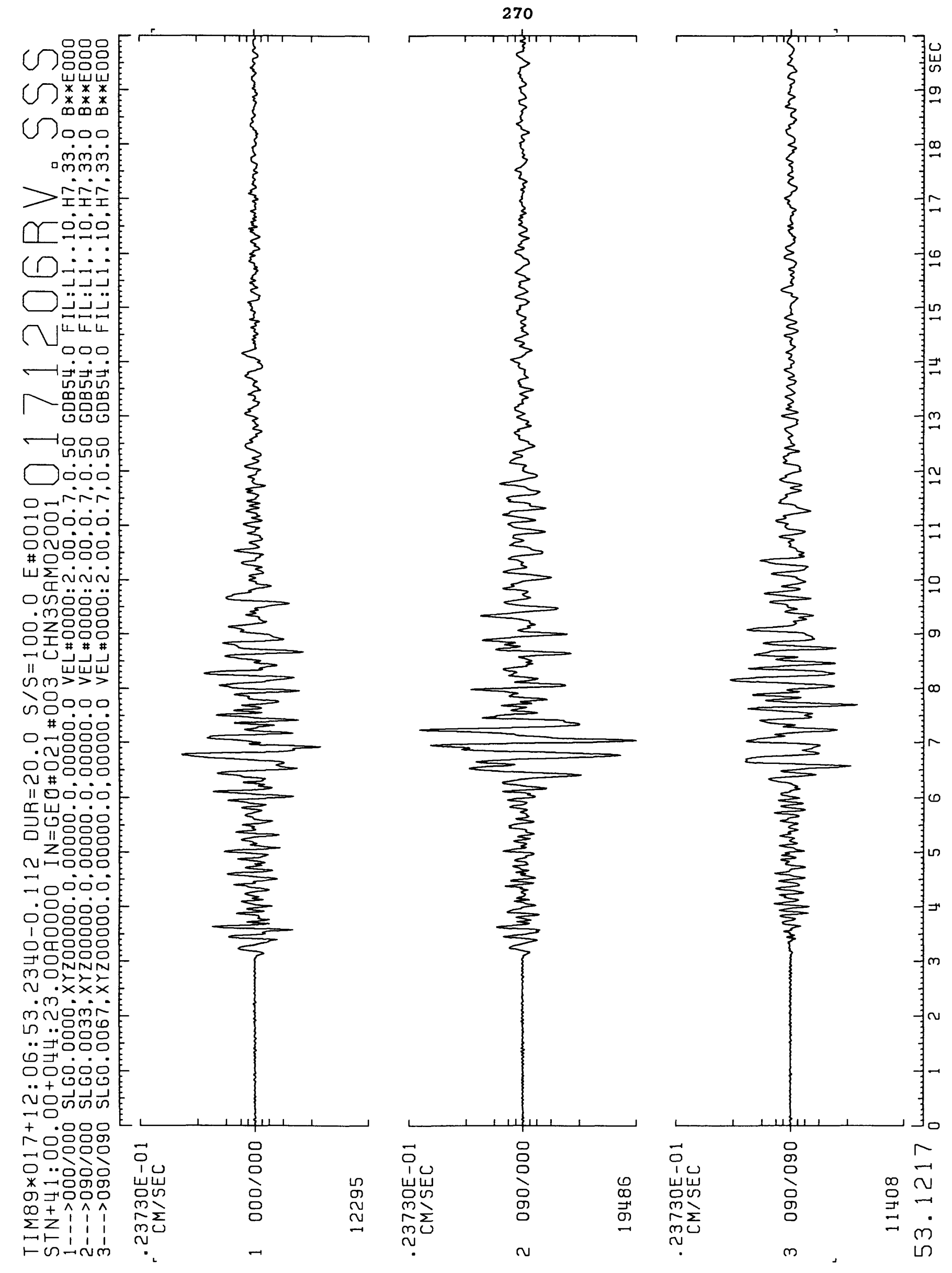



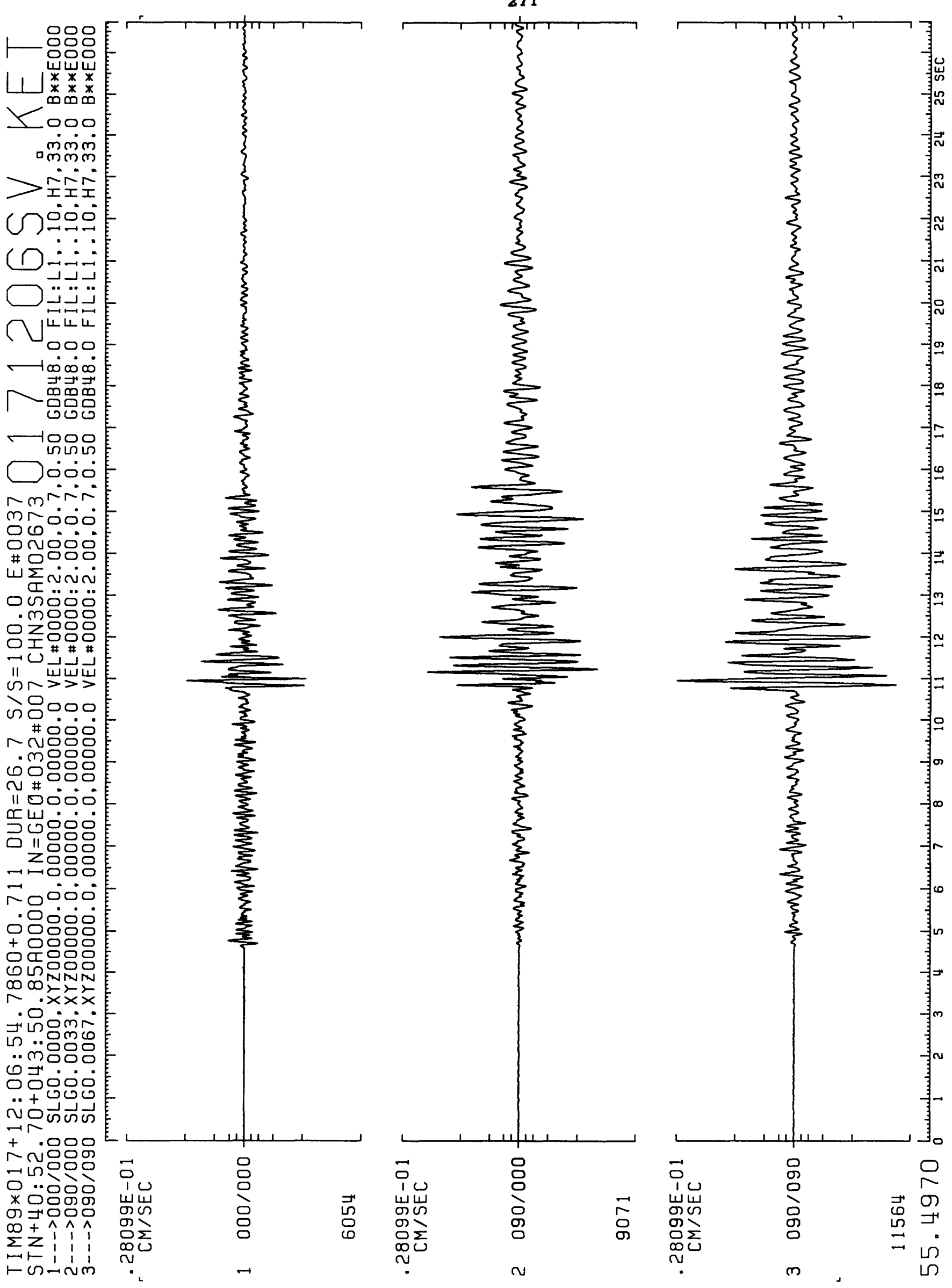
272
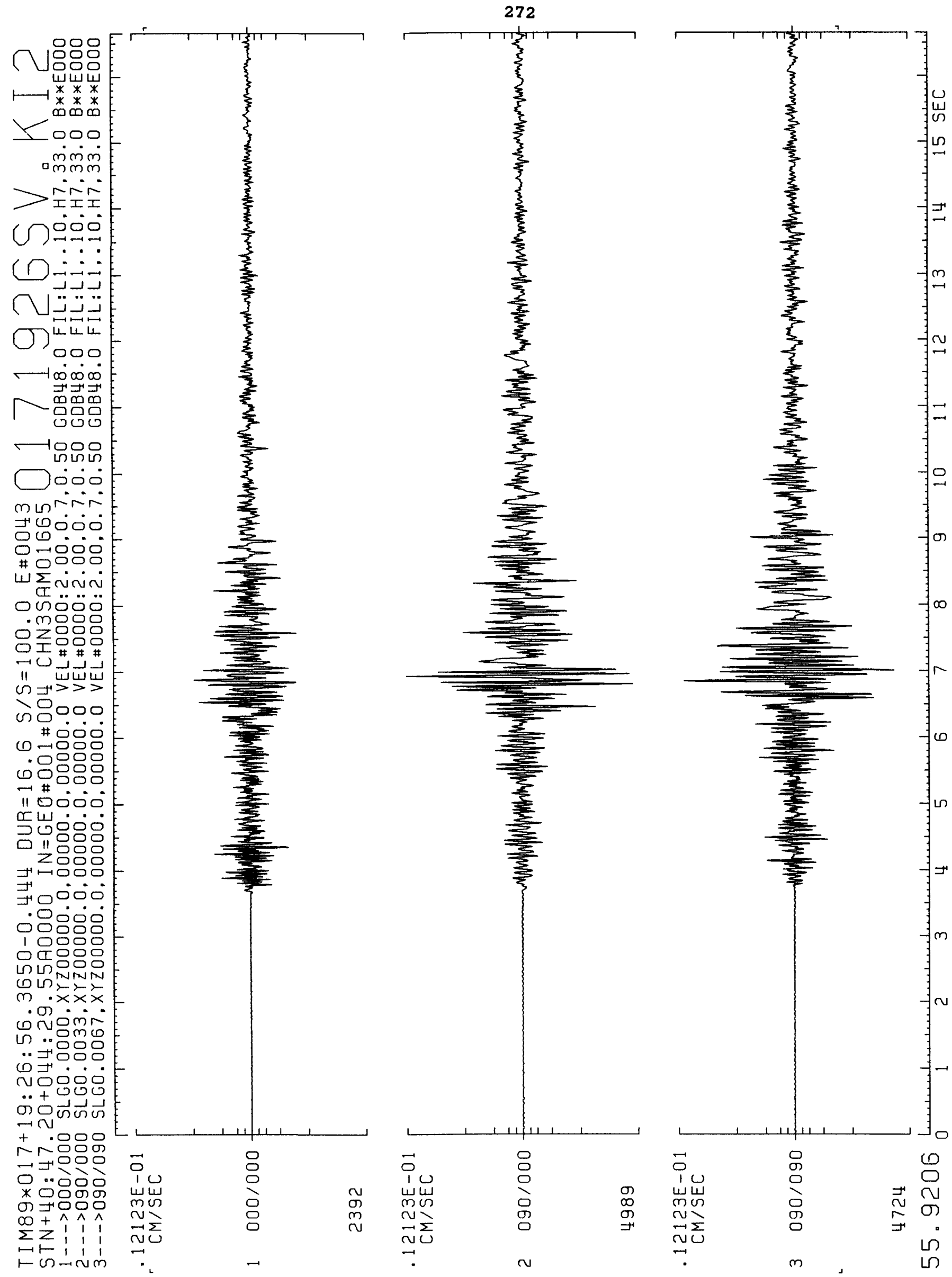
273

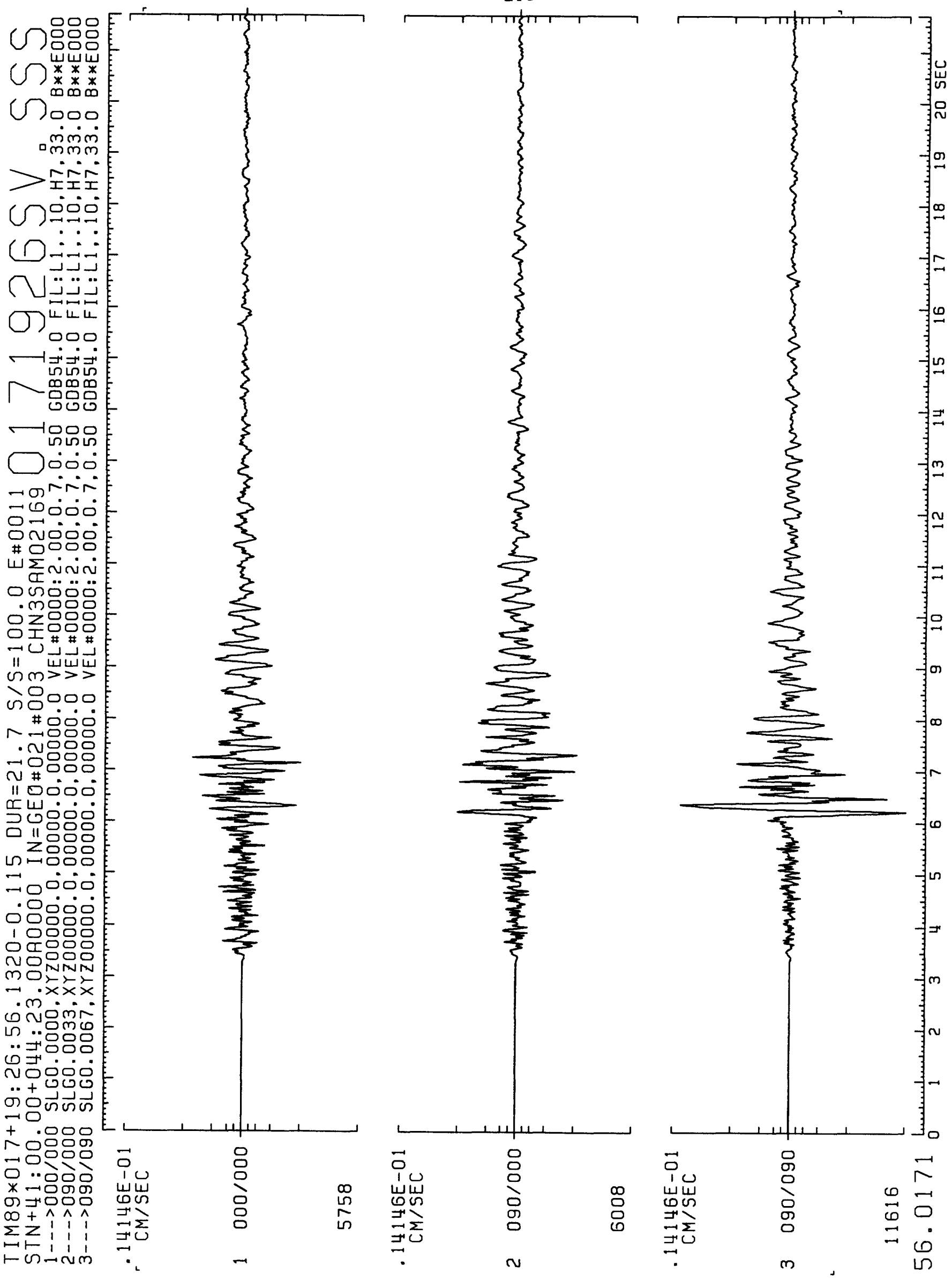




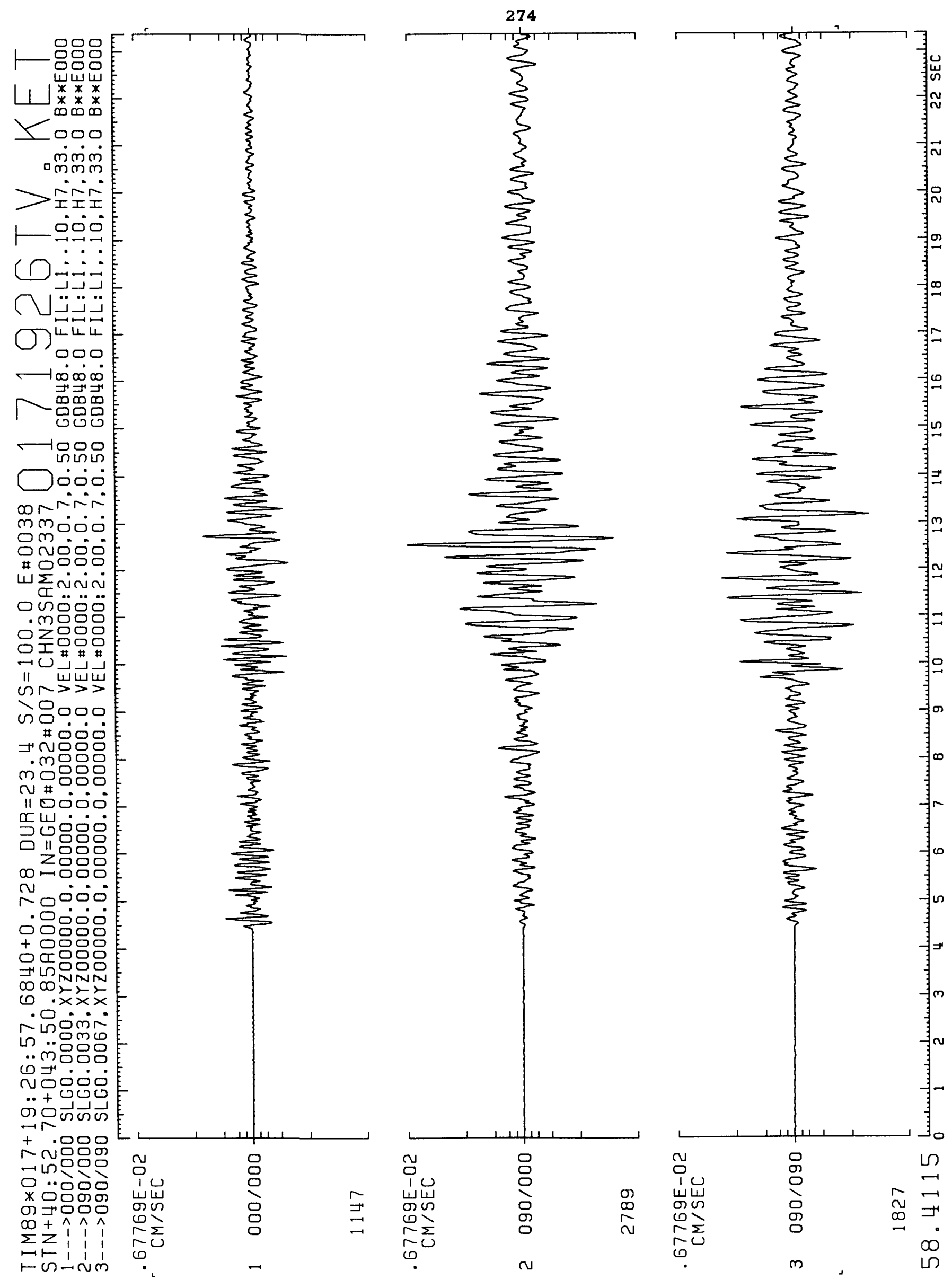




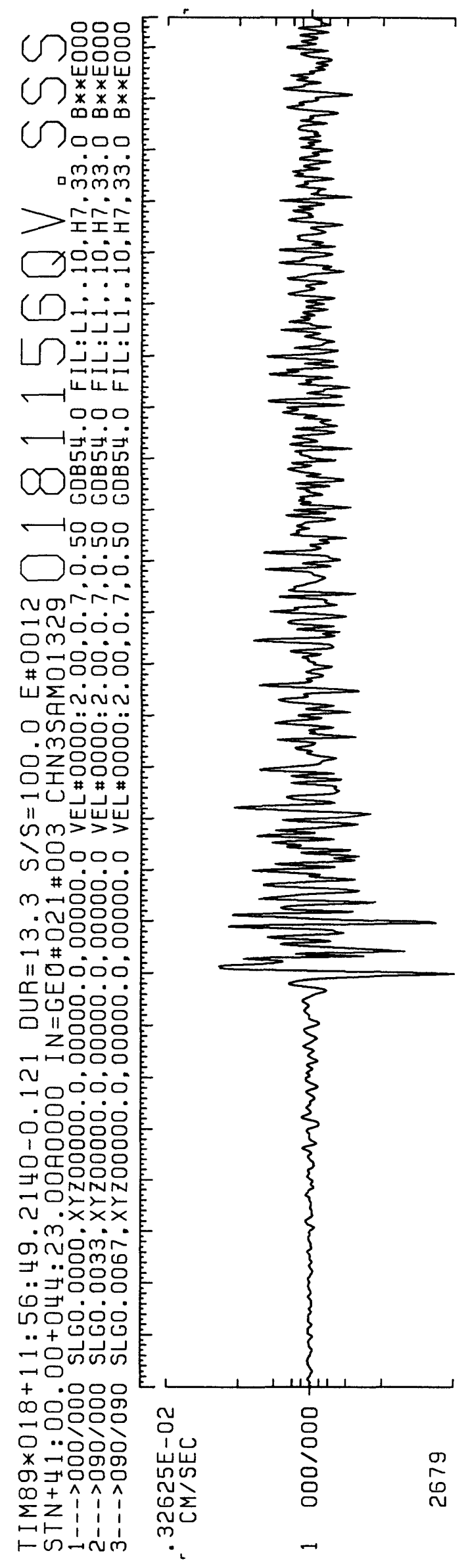

275

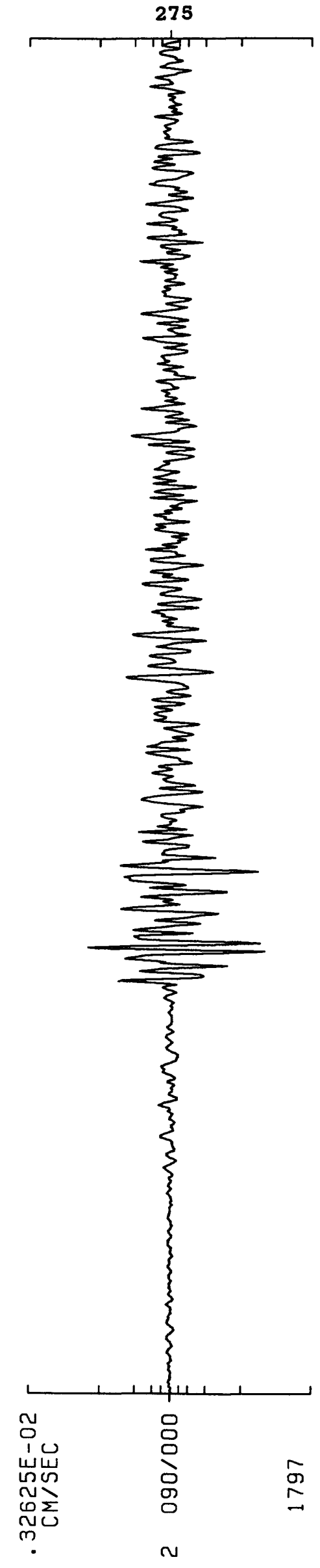

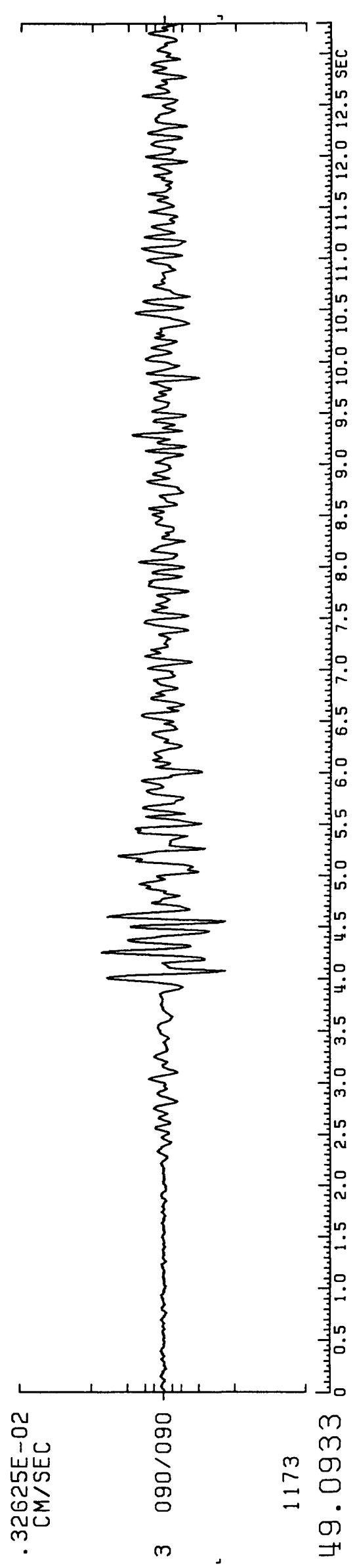



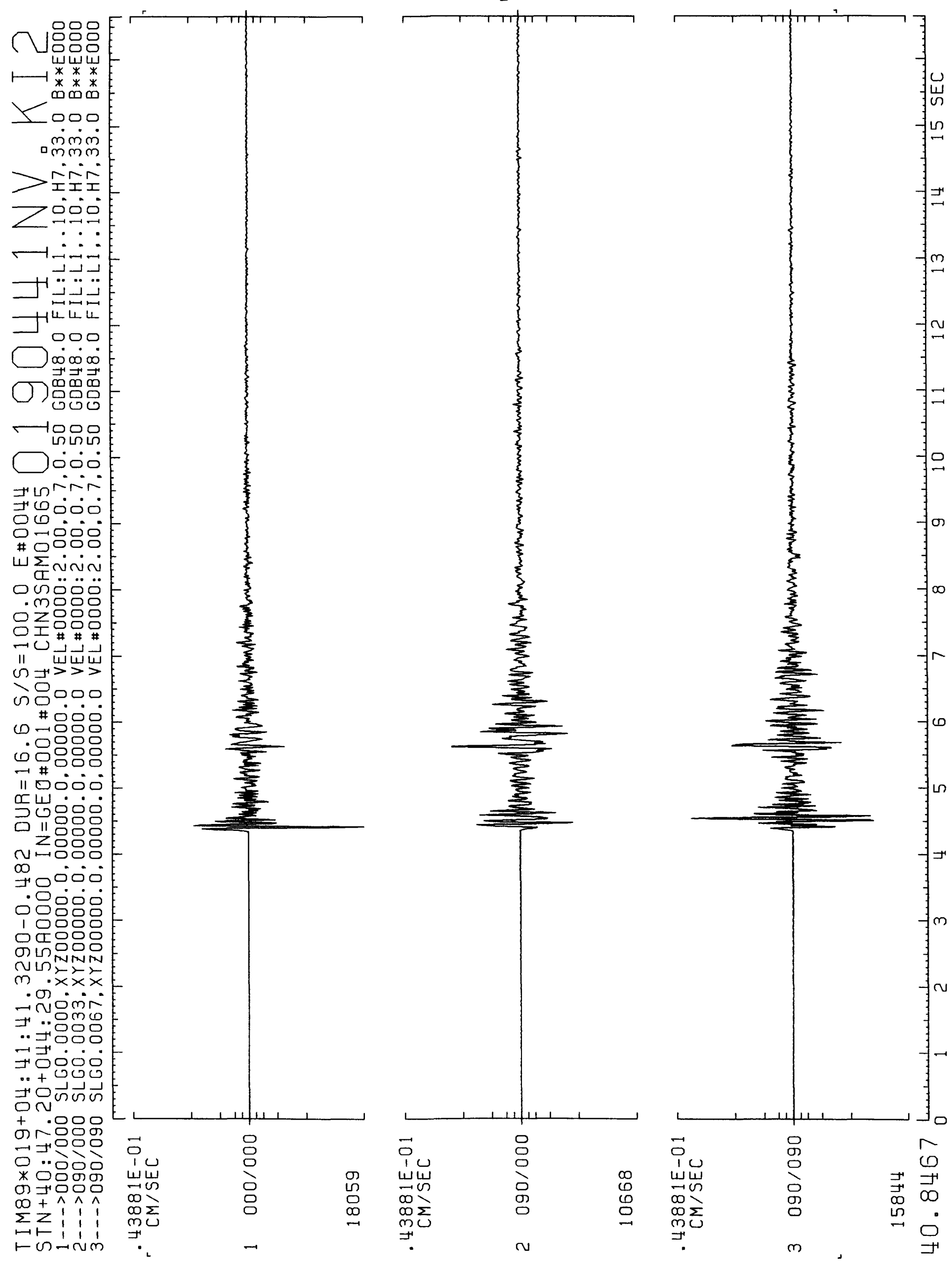


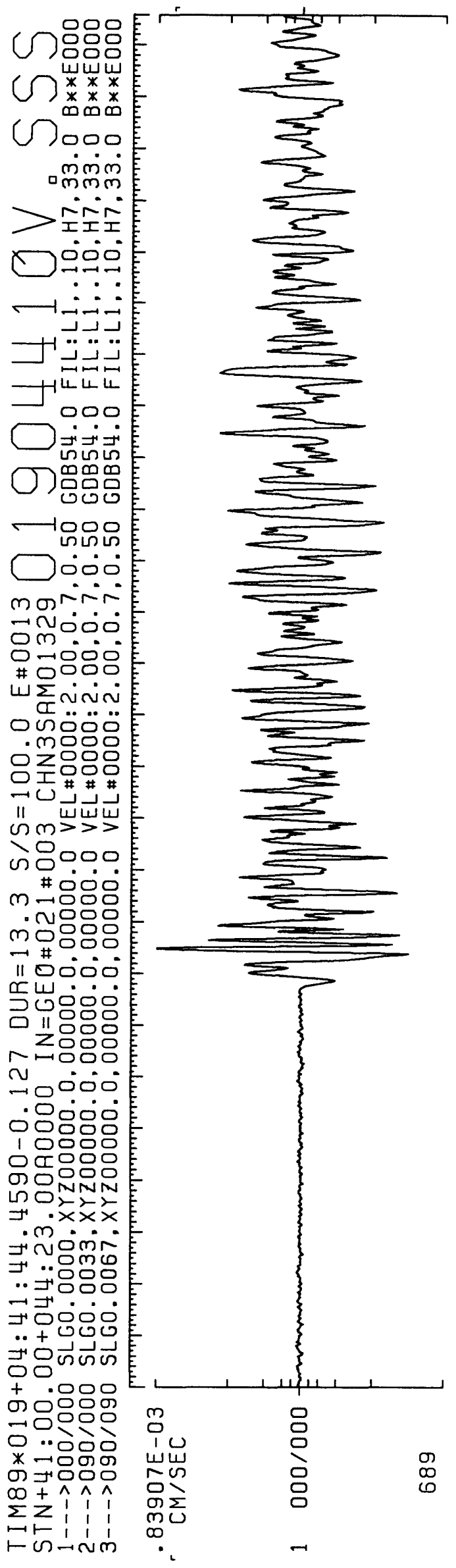

277

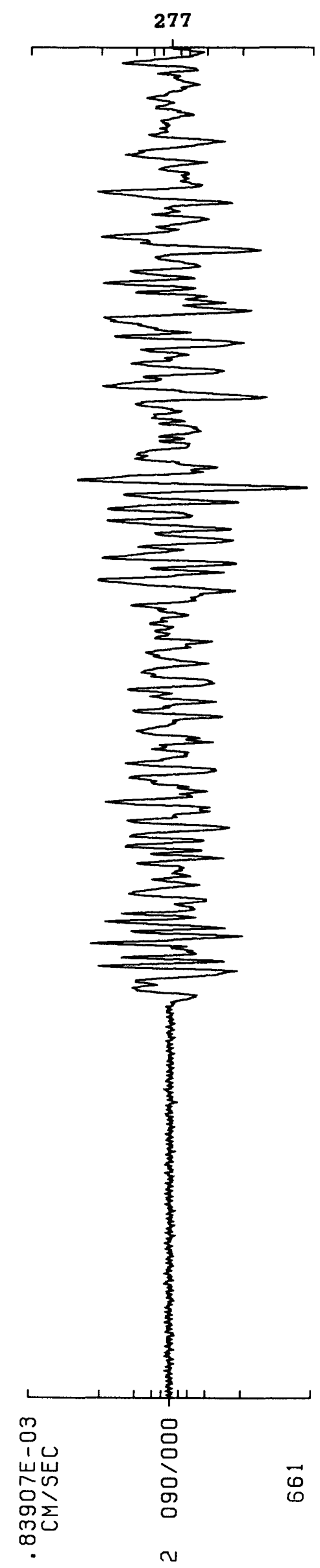

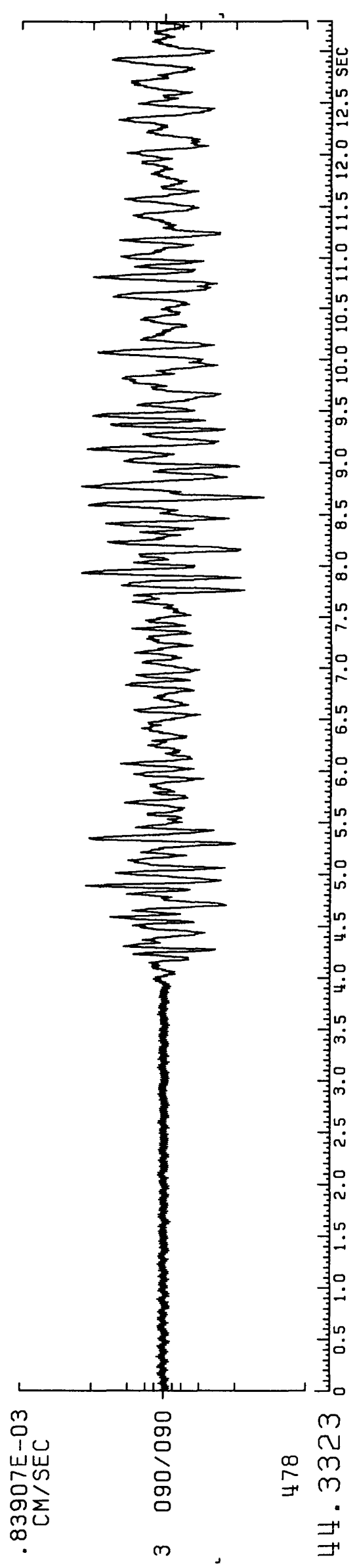




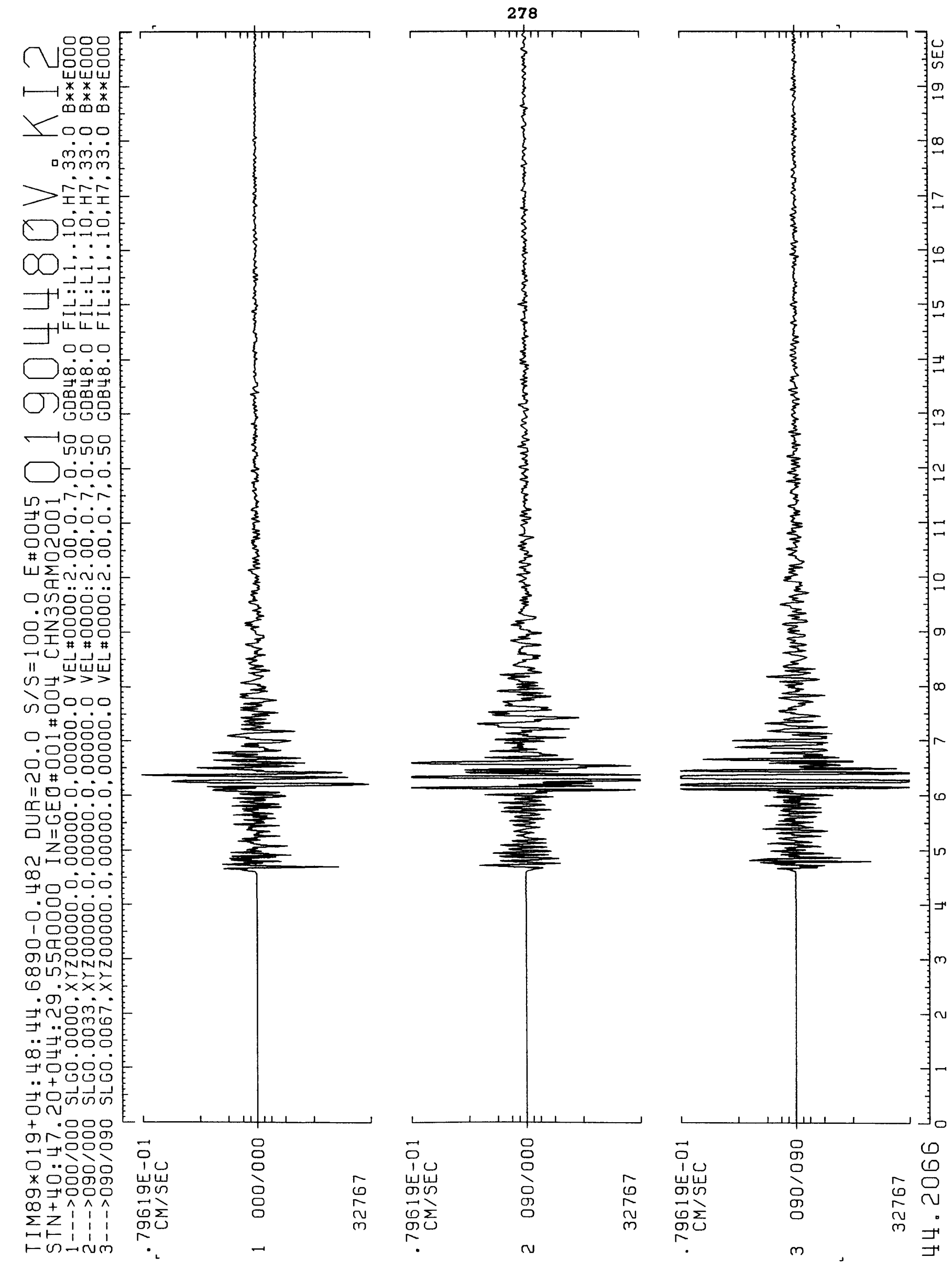




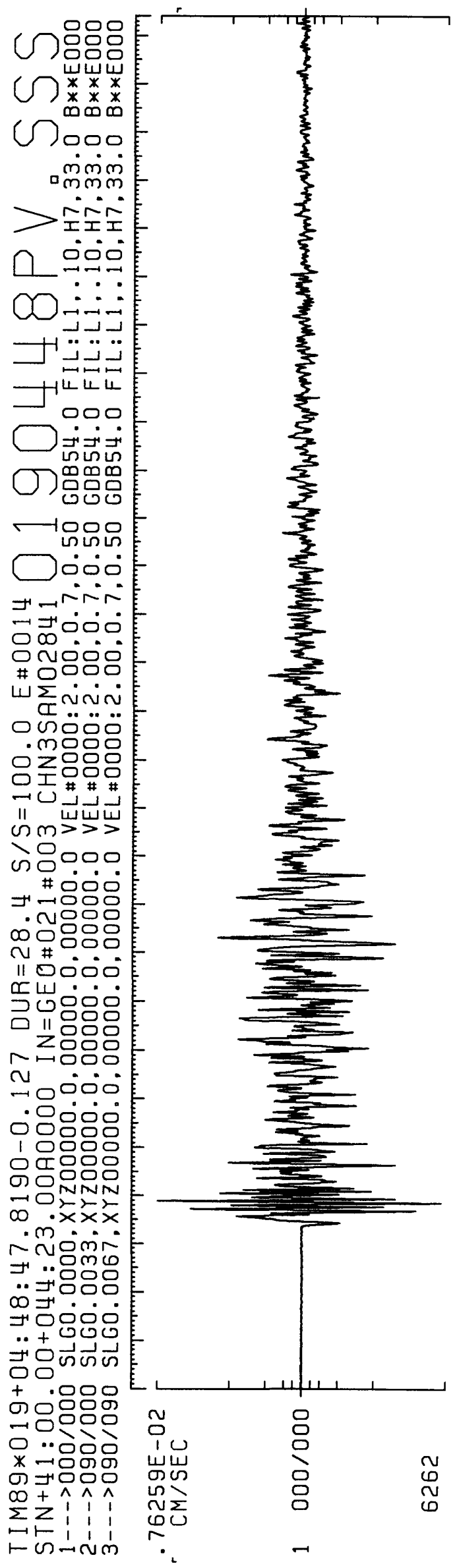

279

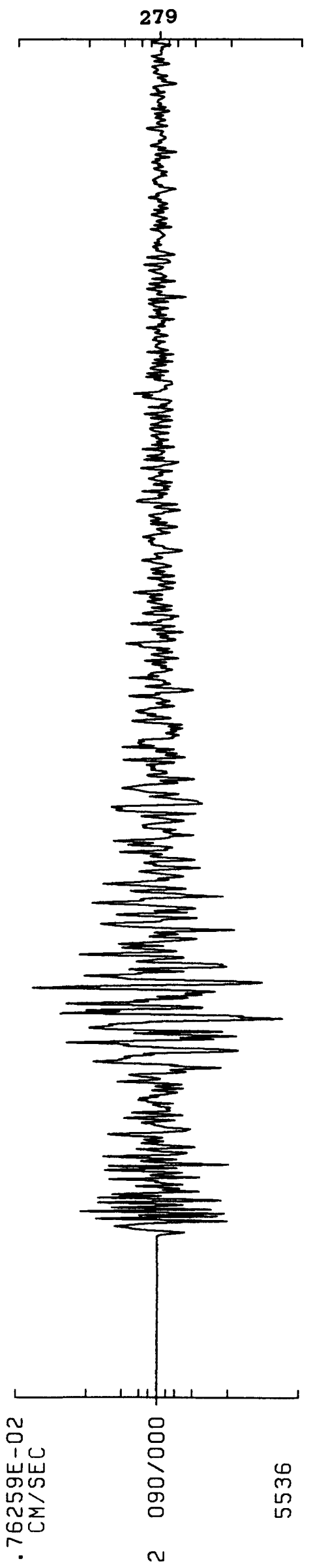

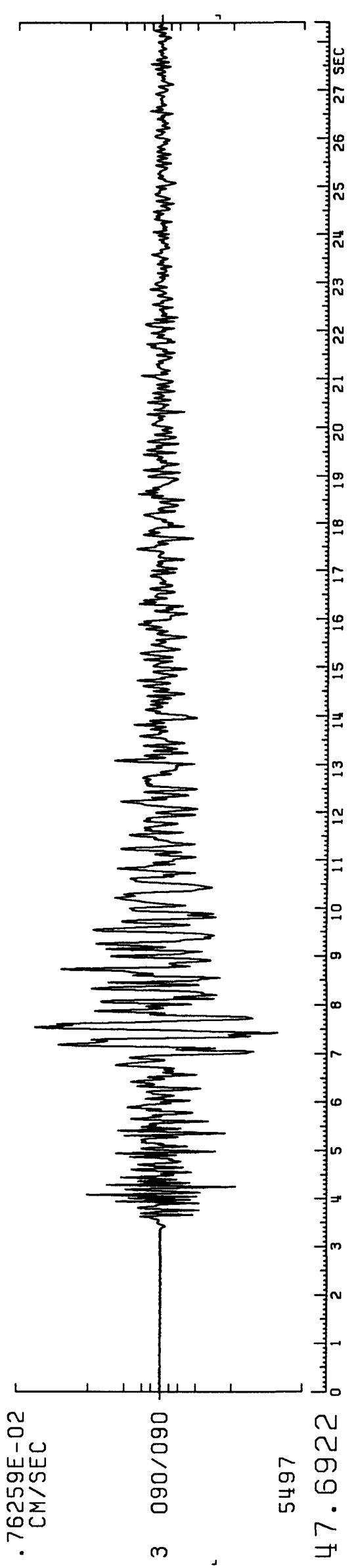


280

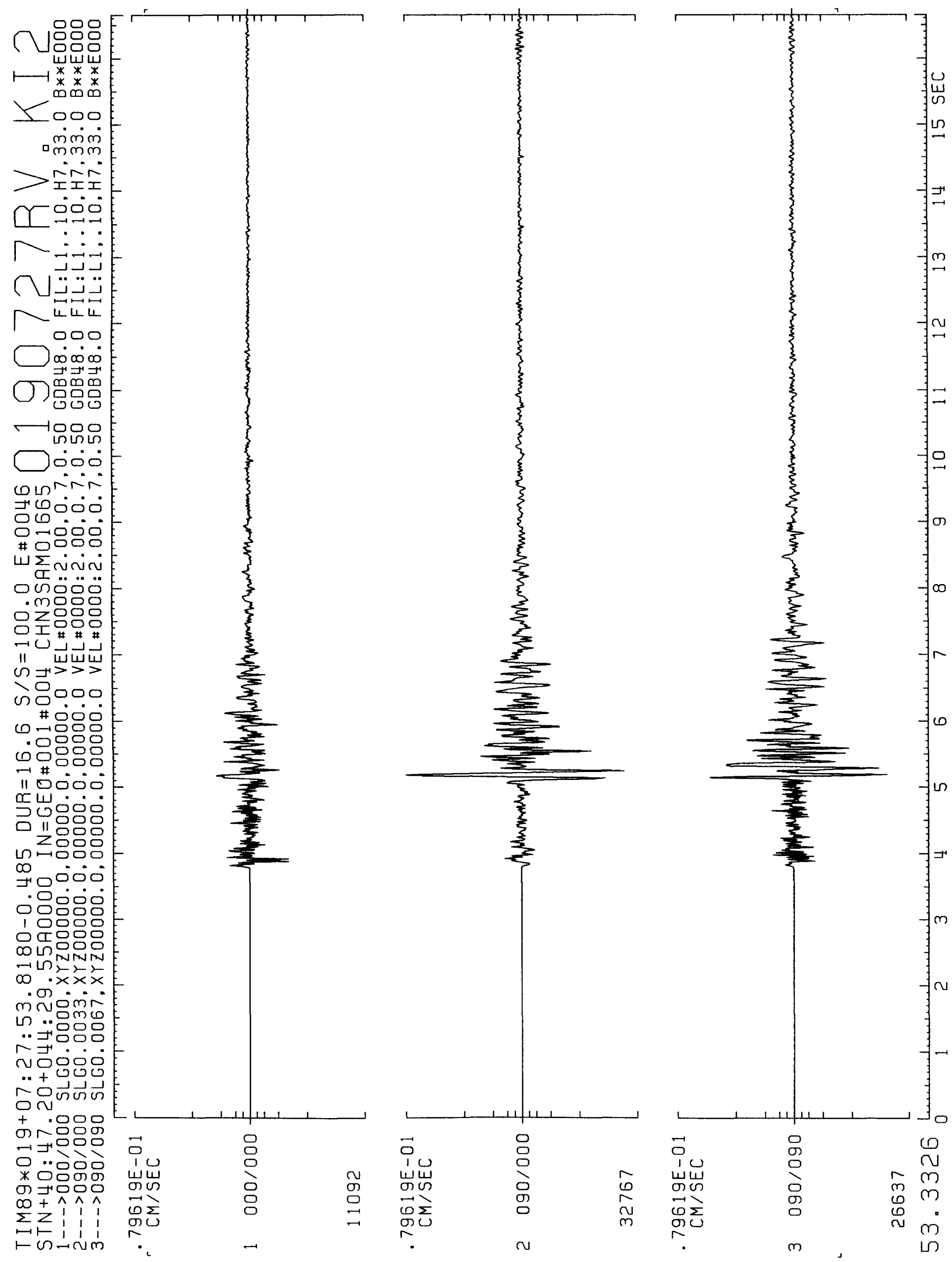


281

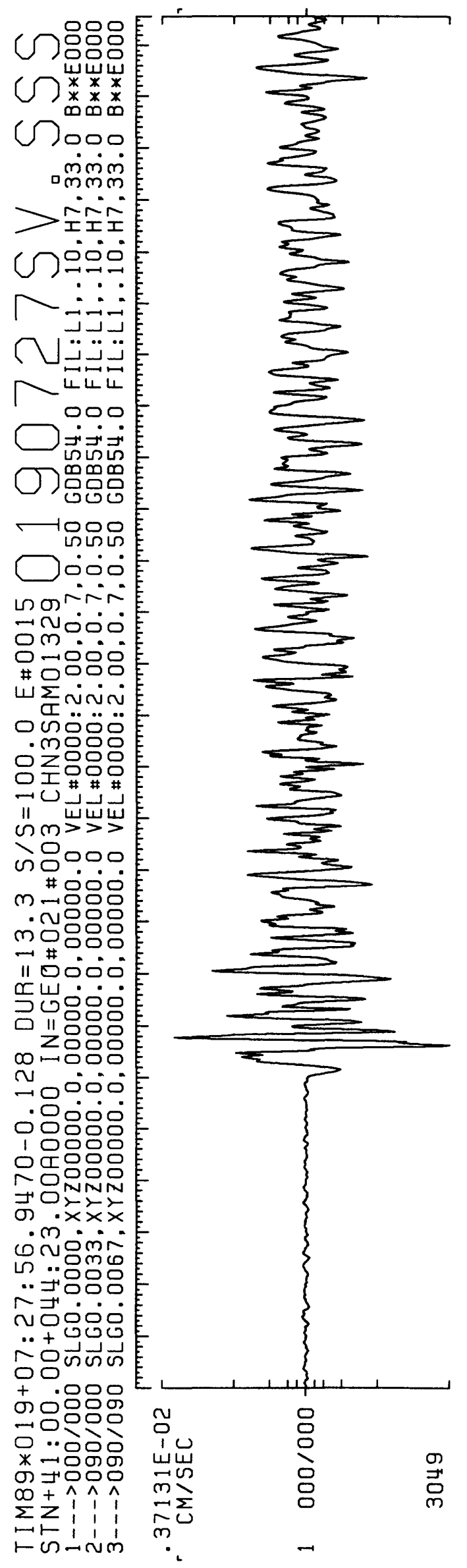

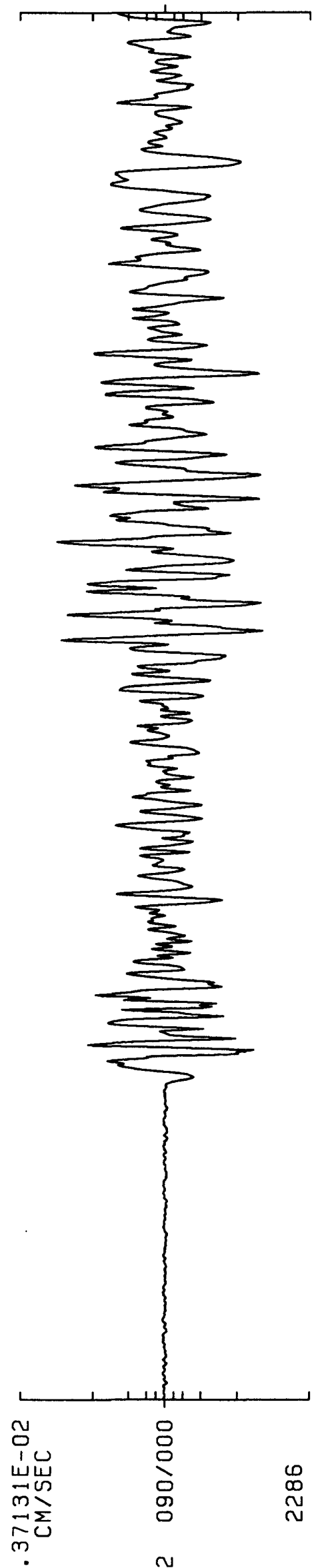

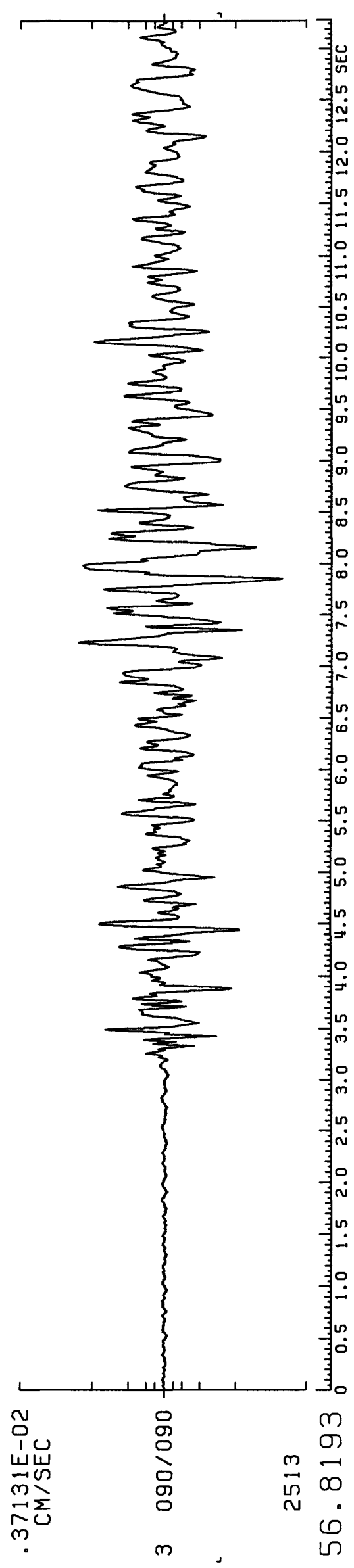




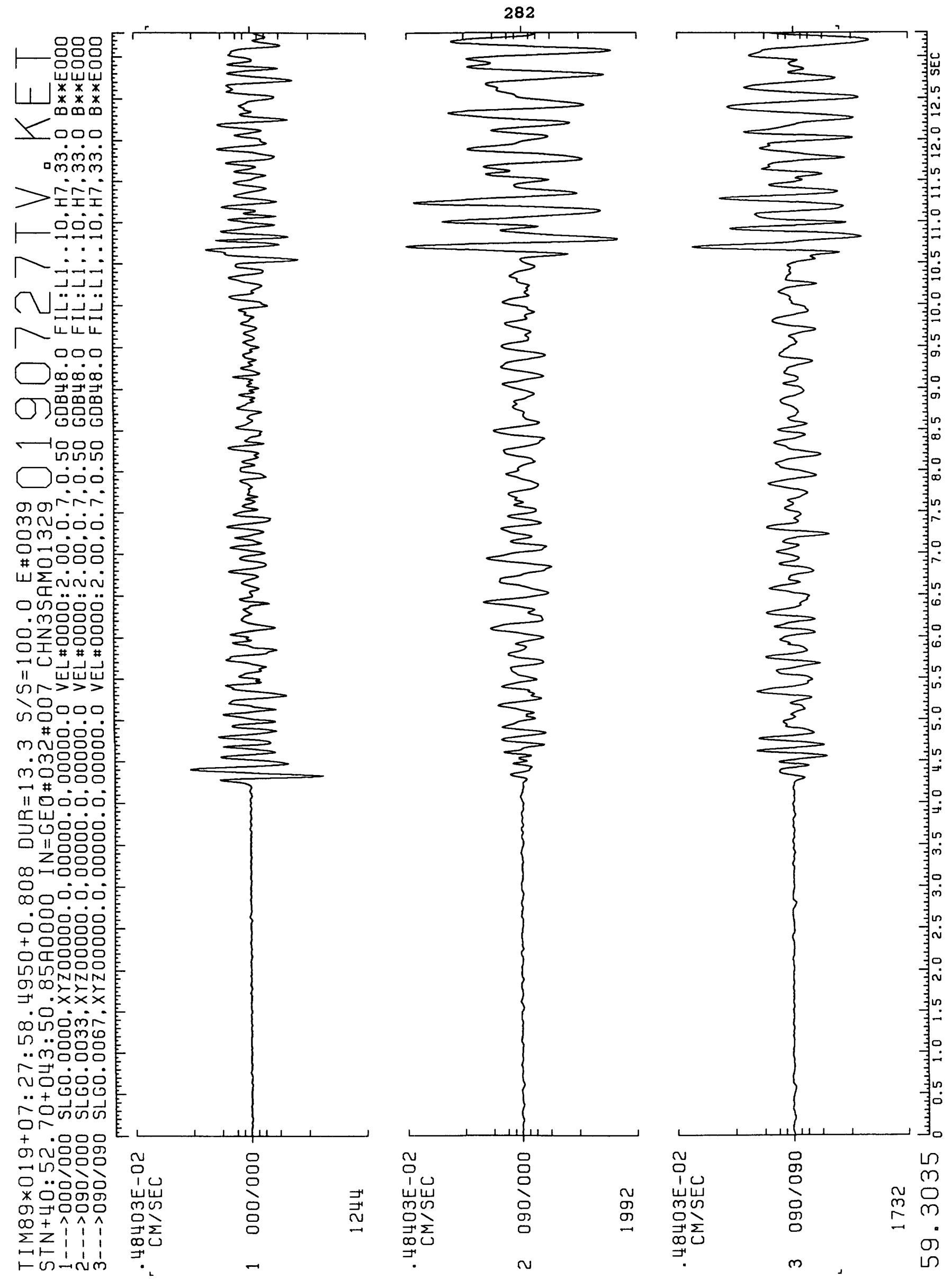


283

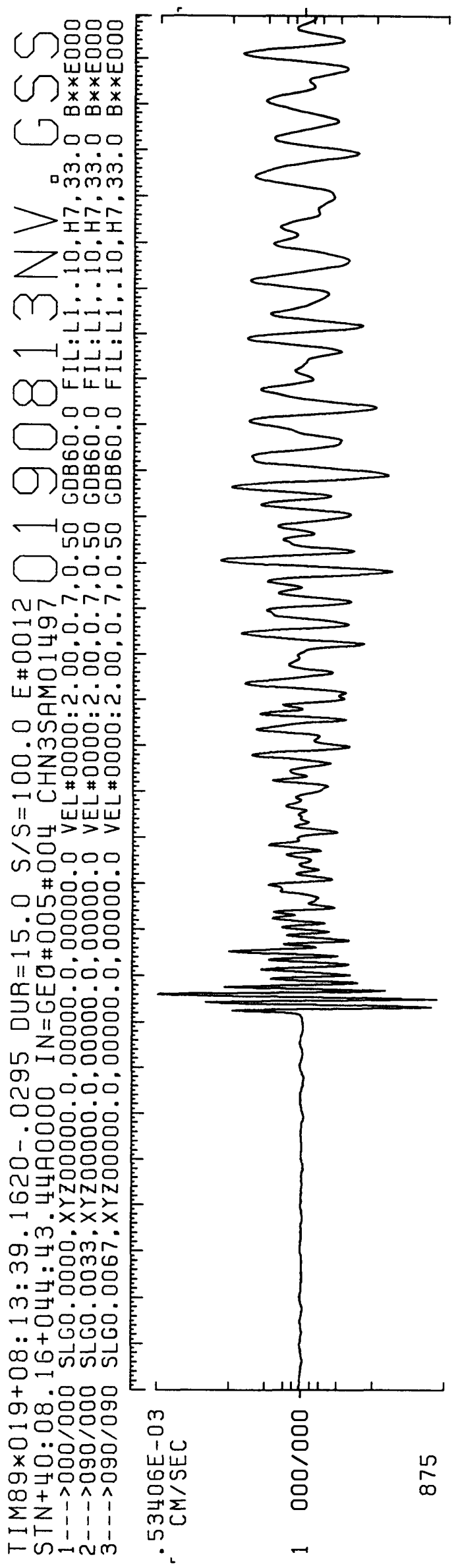

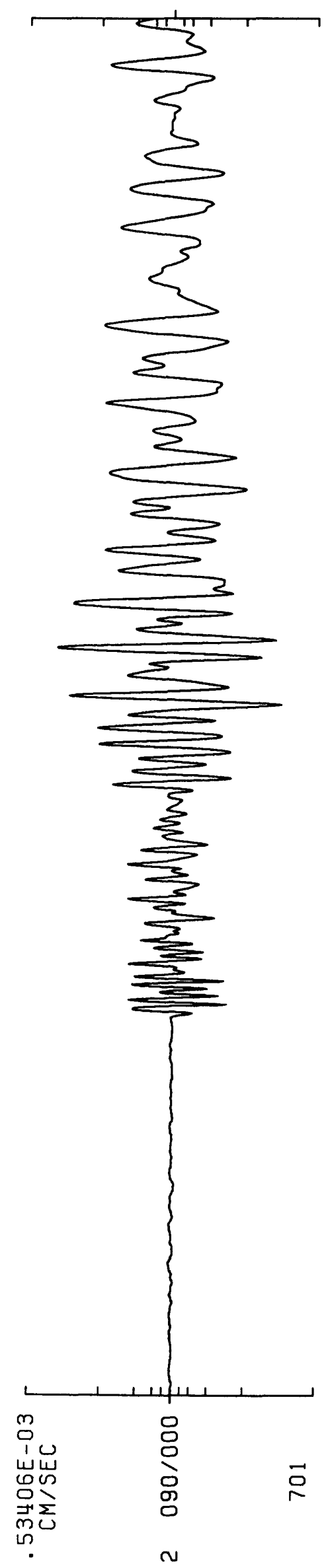

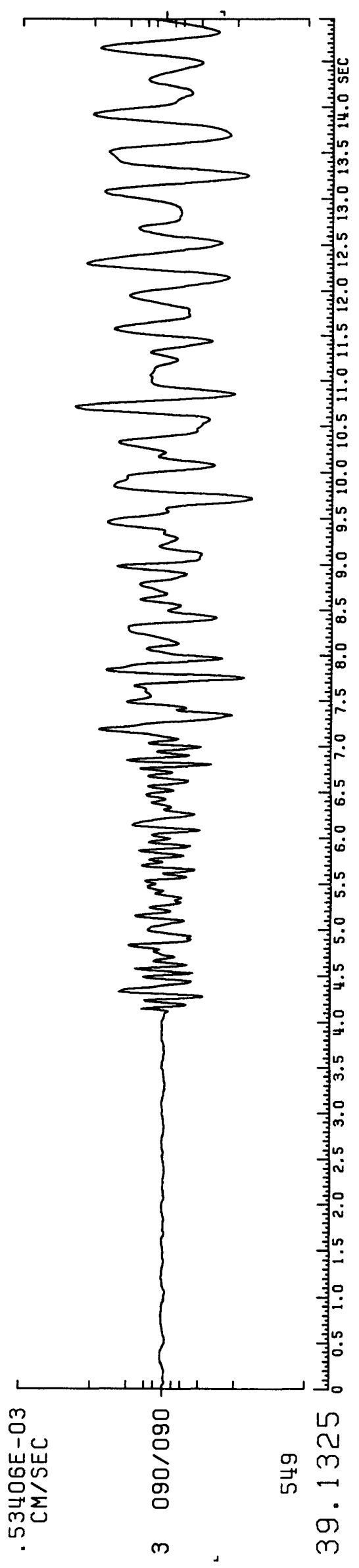




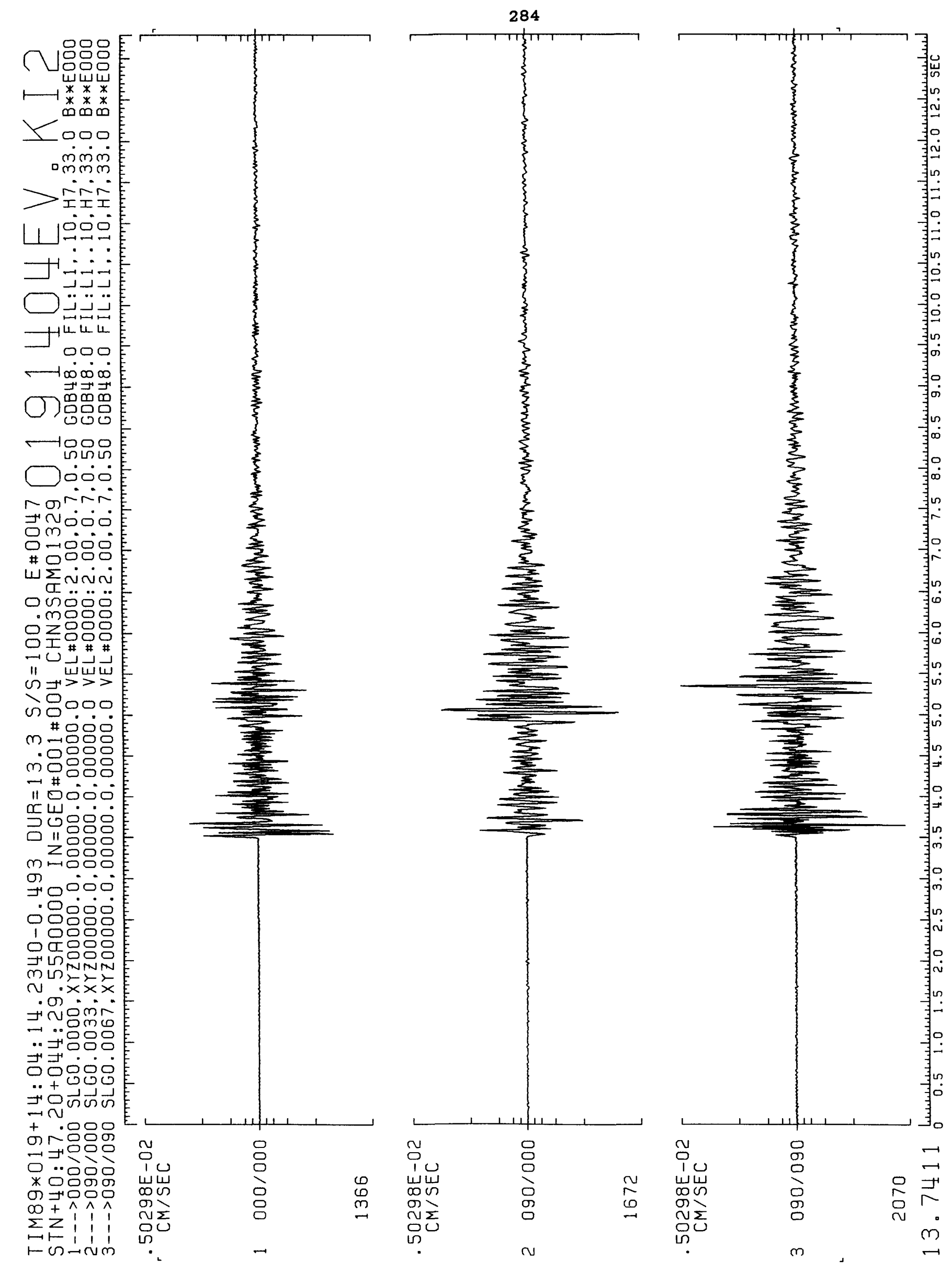




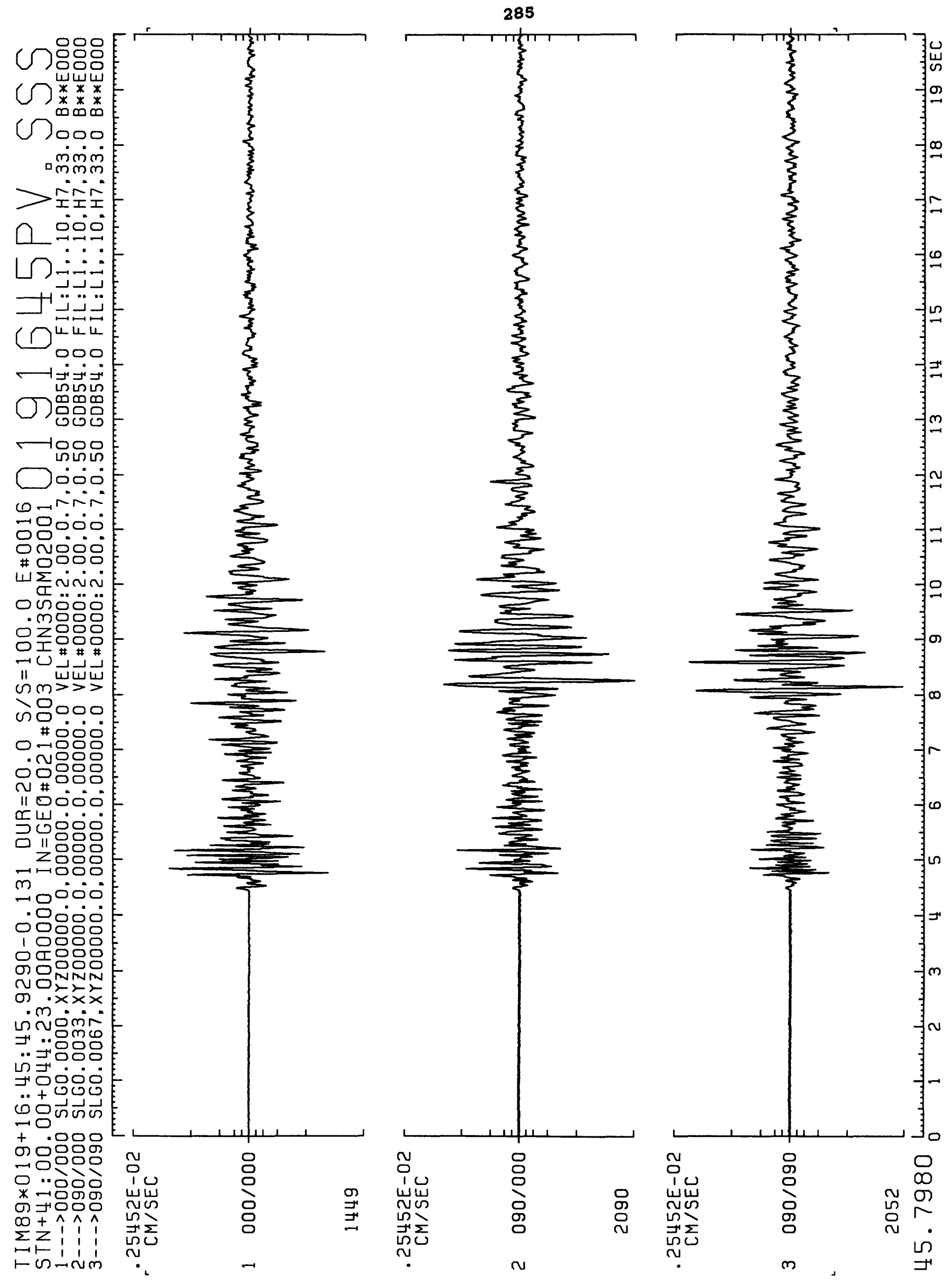




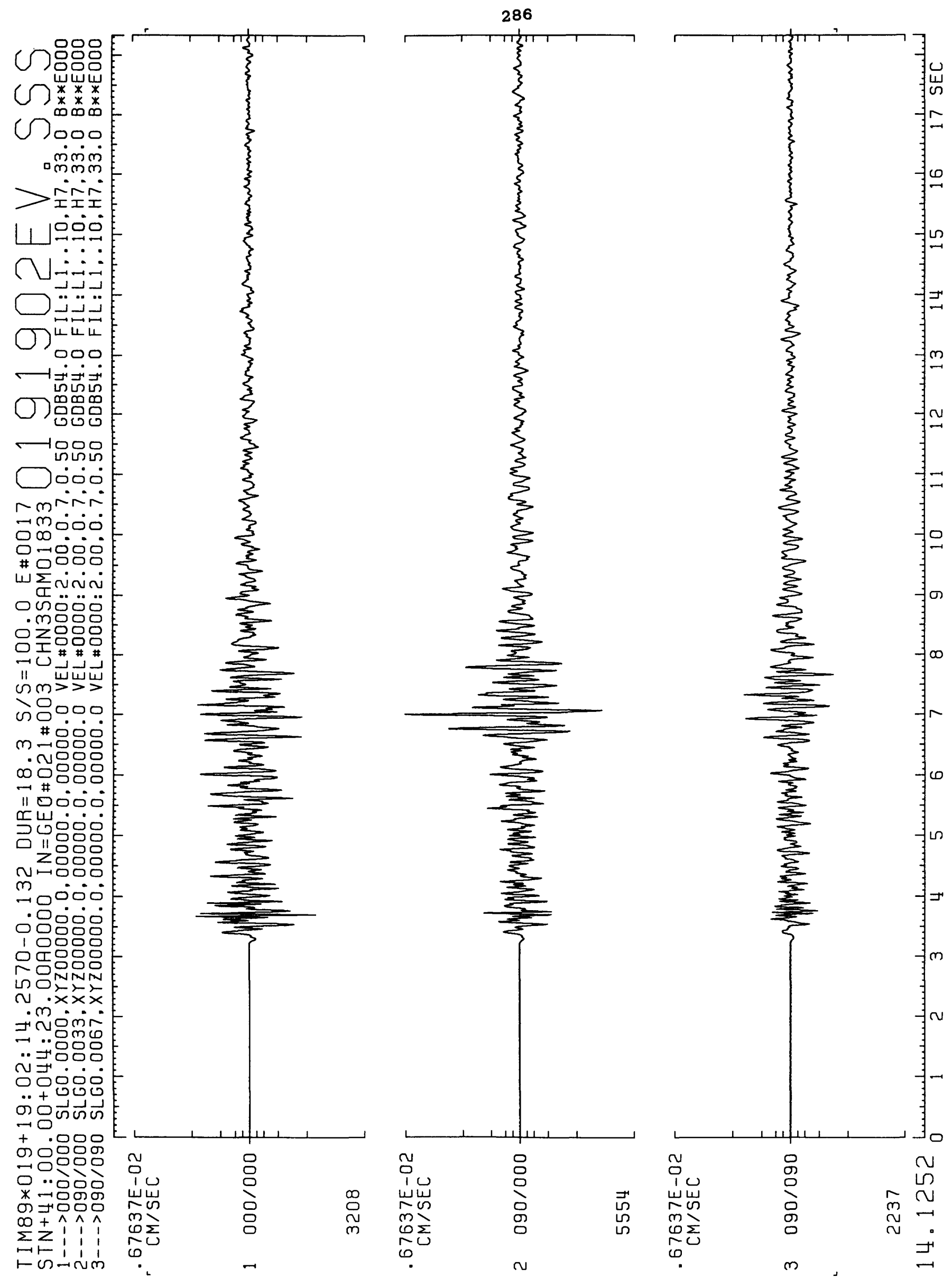




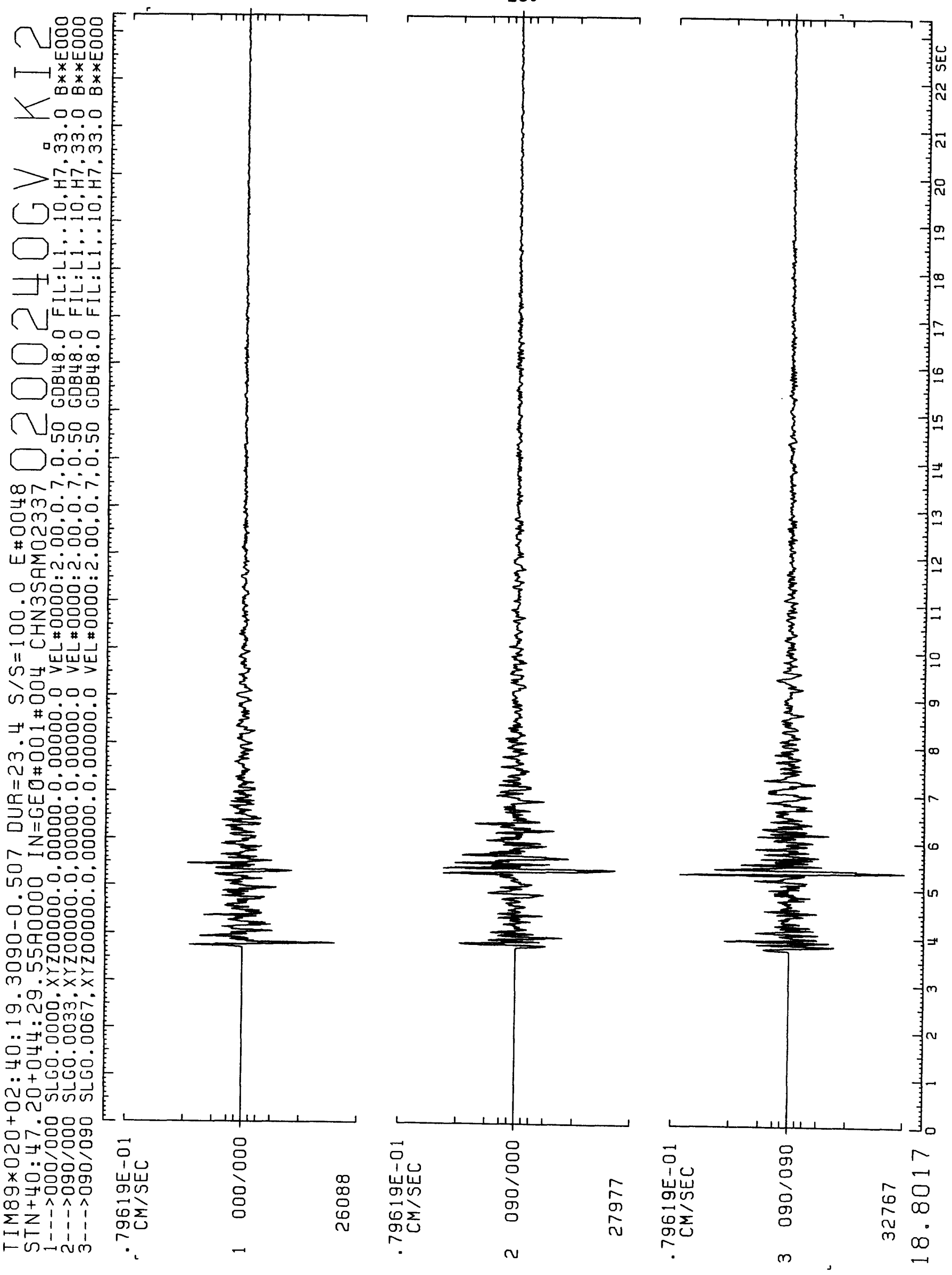




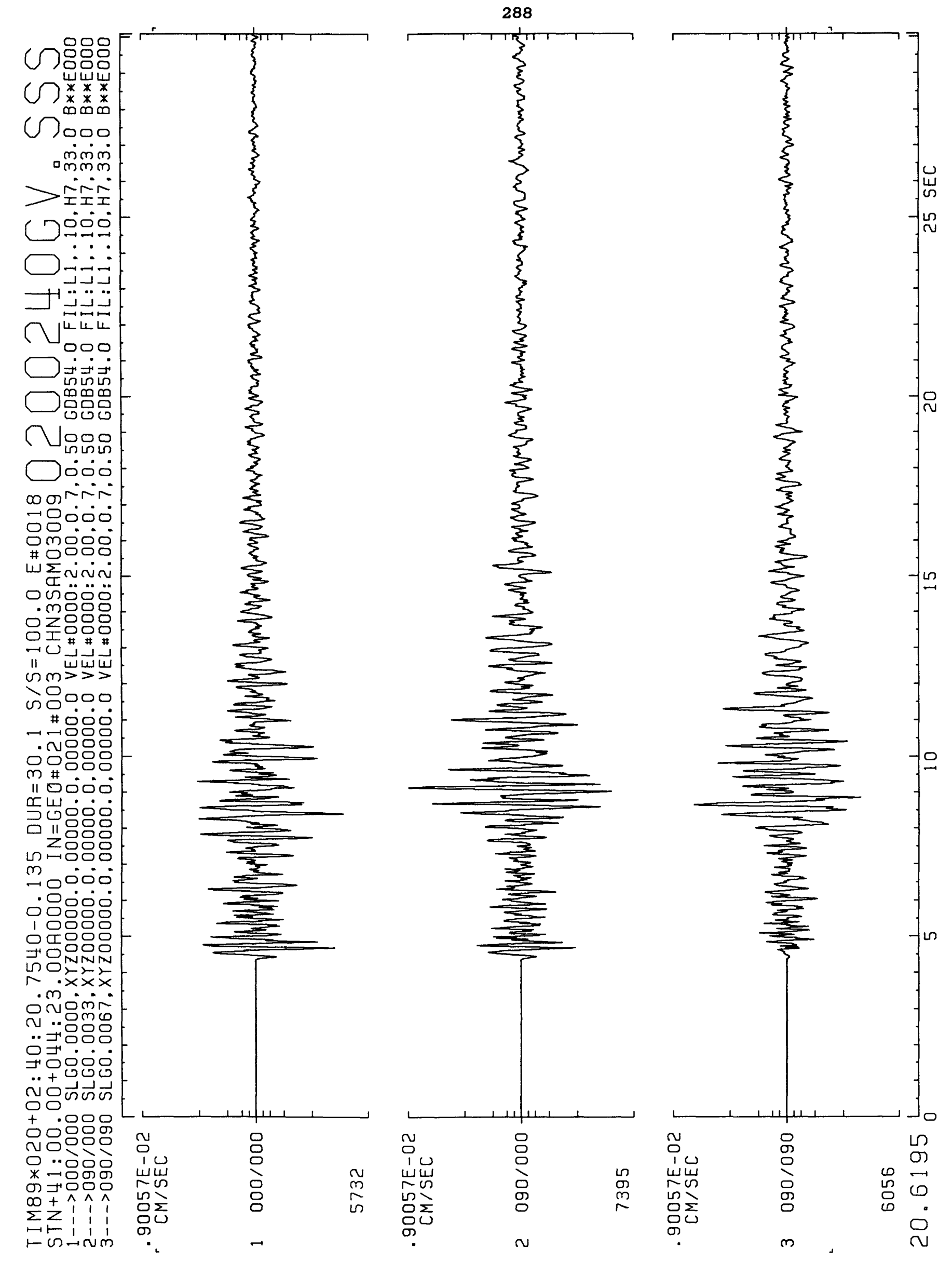



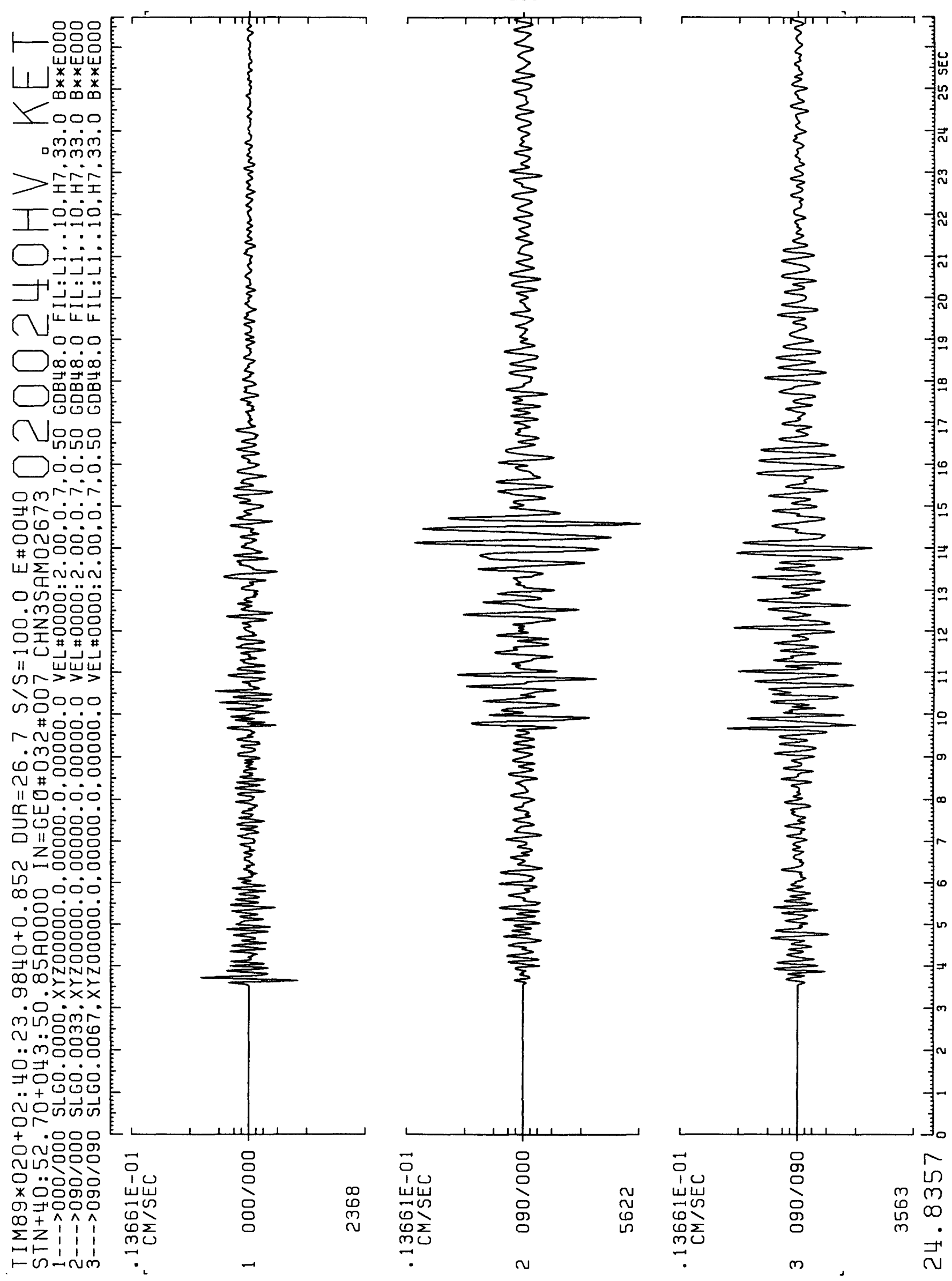
290

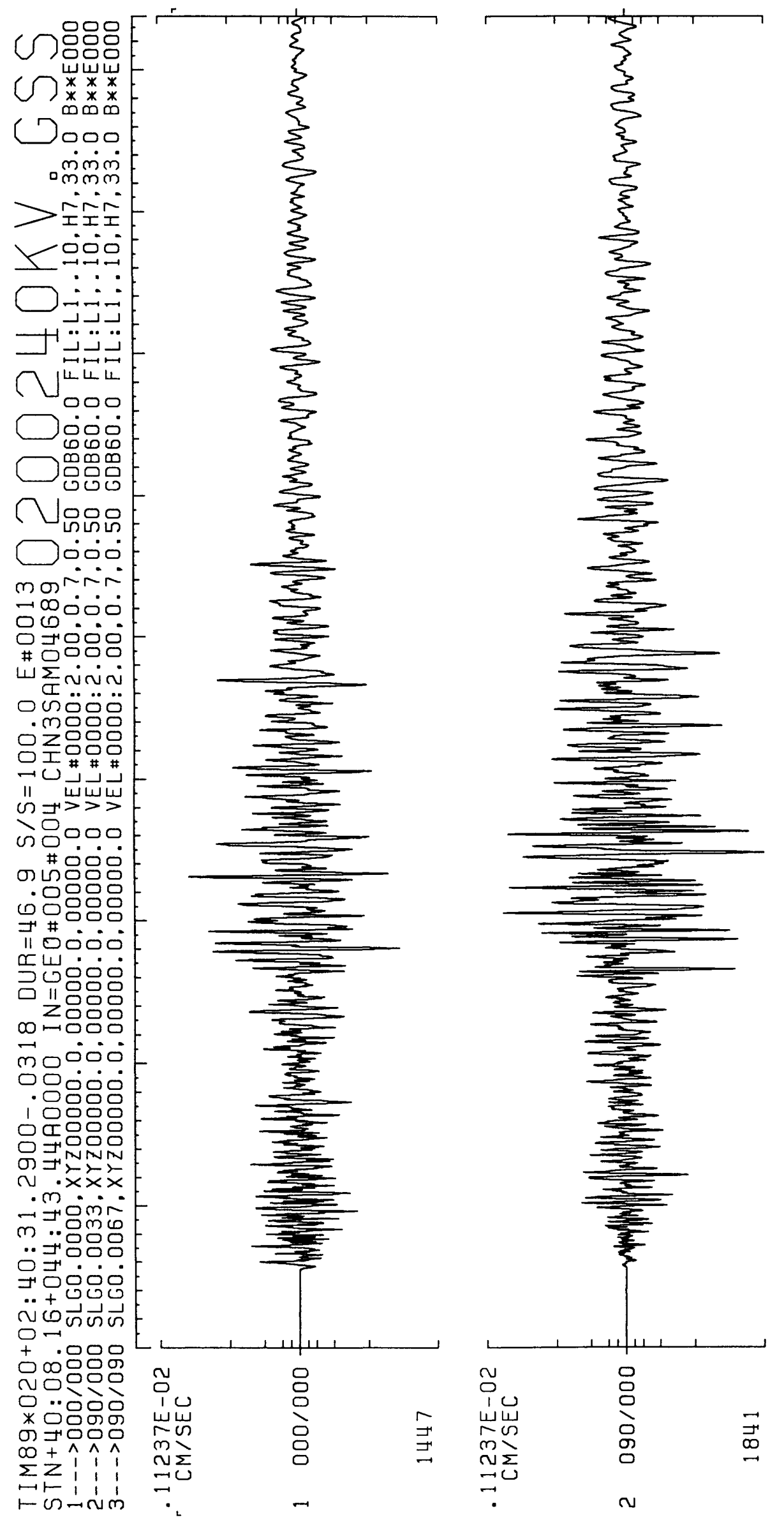

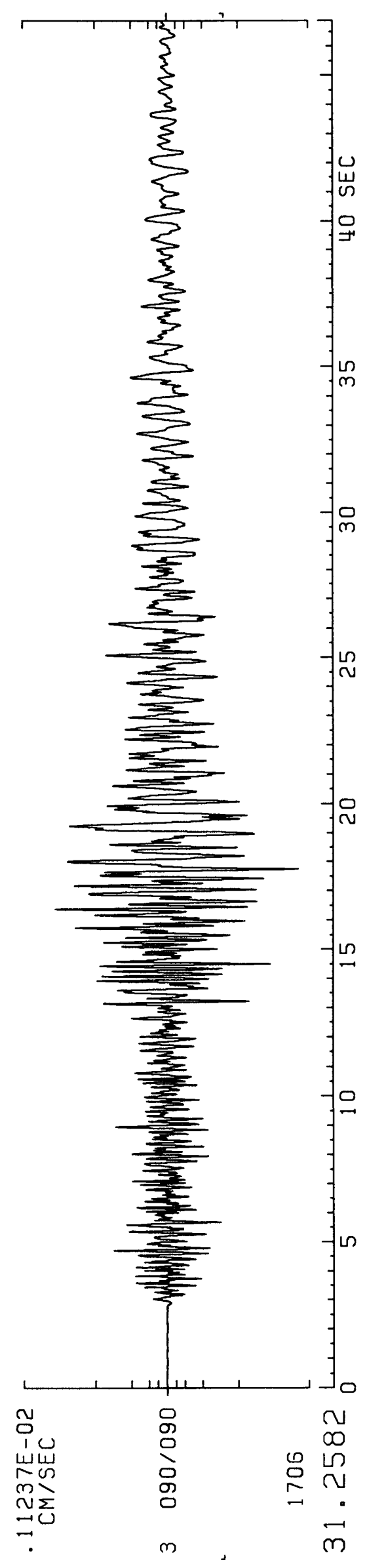




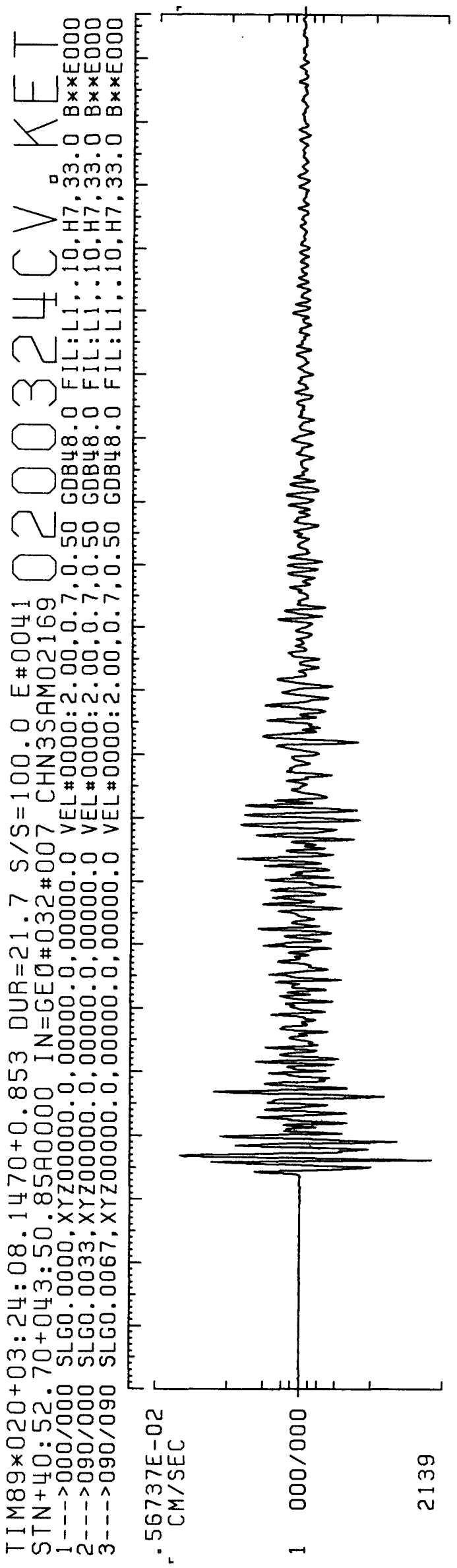

291

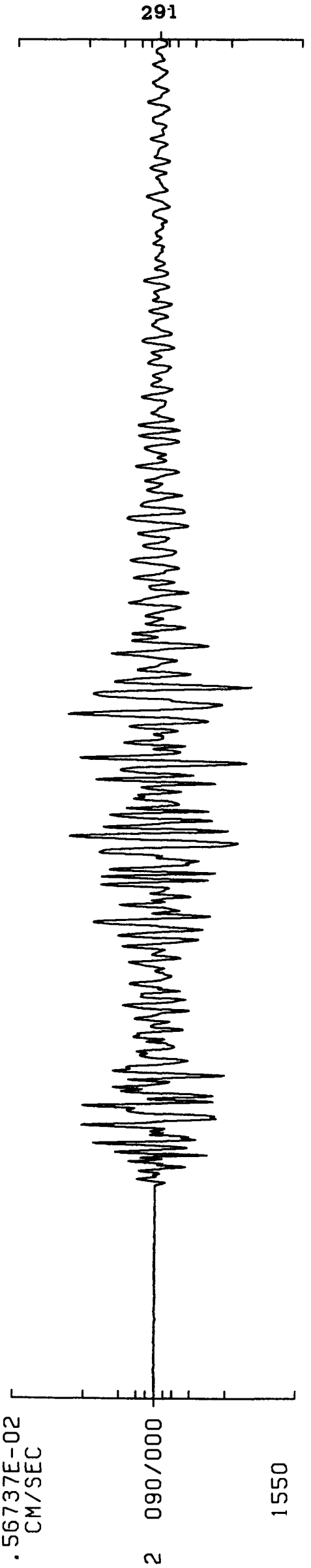

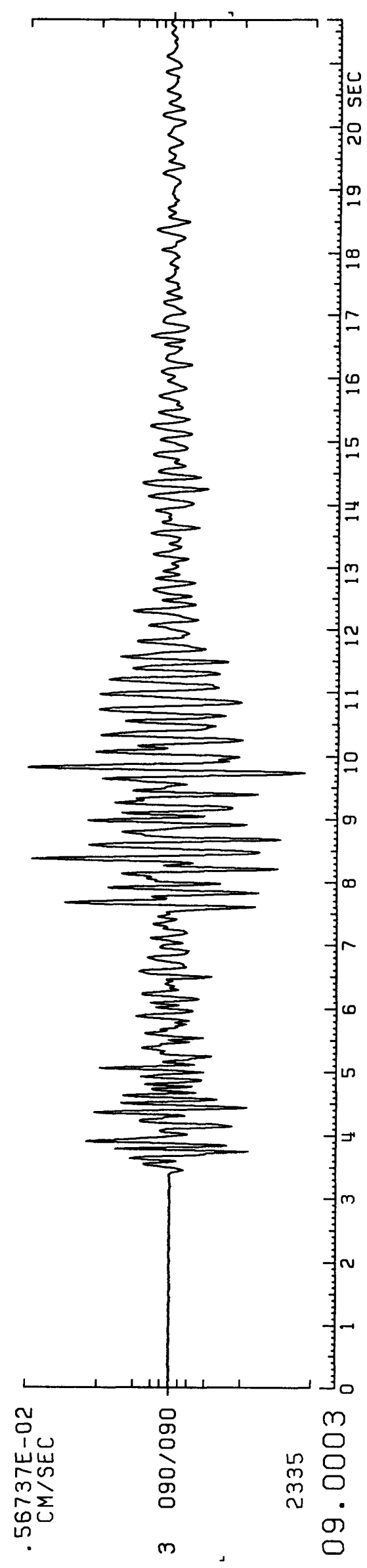




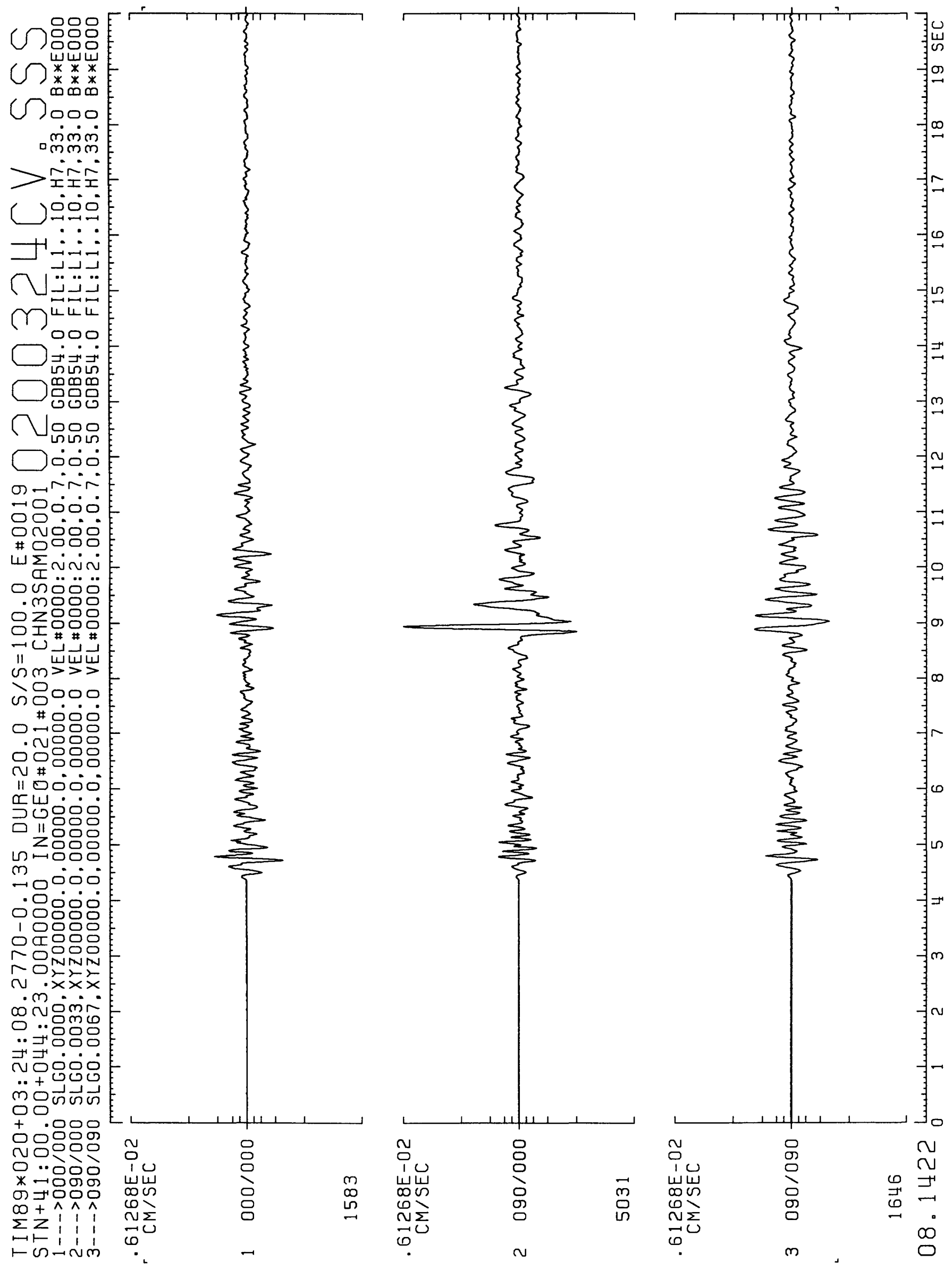




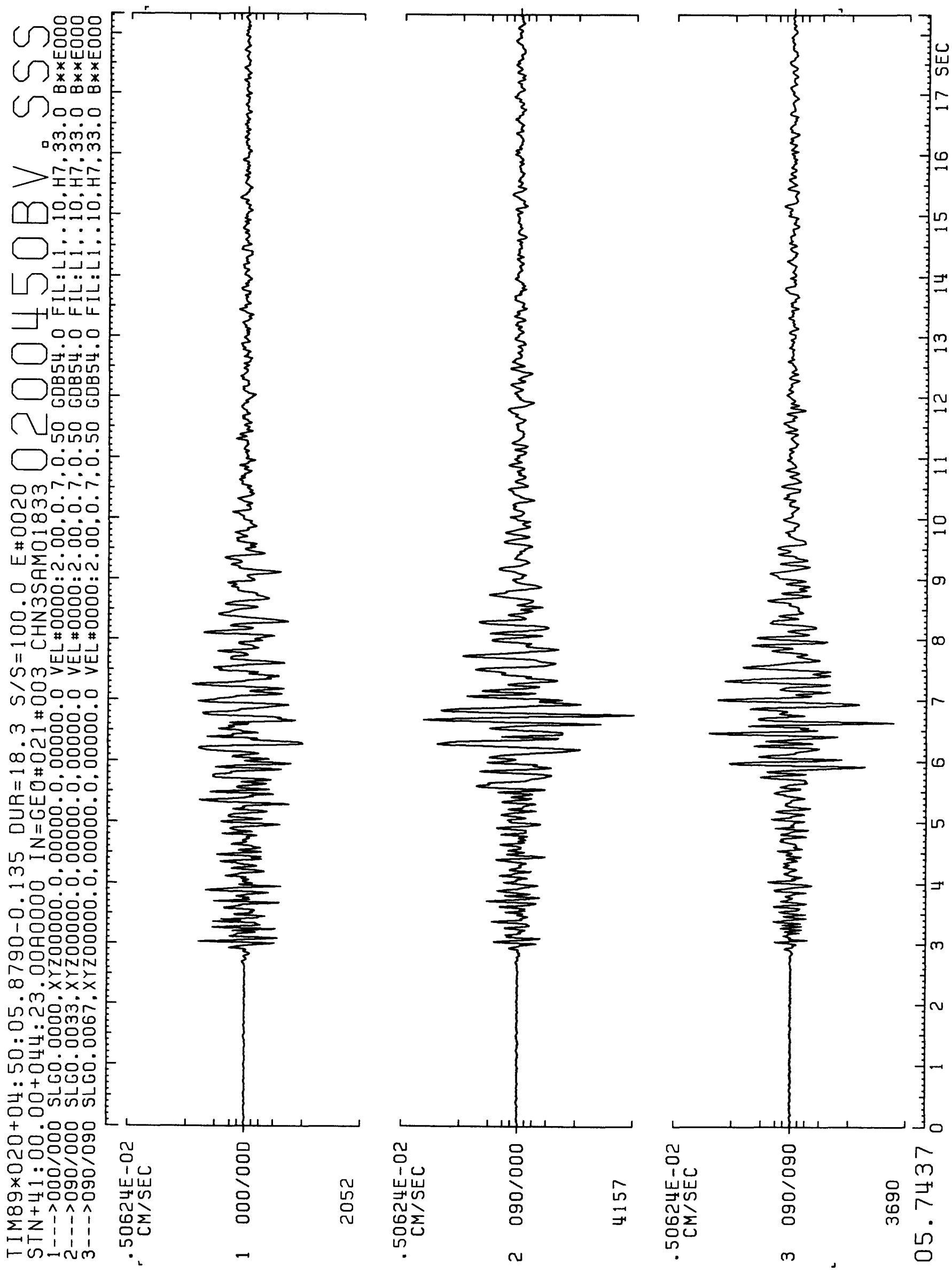




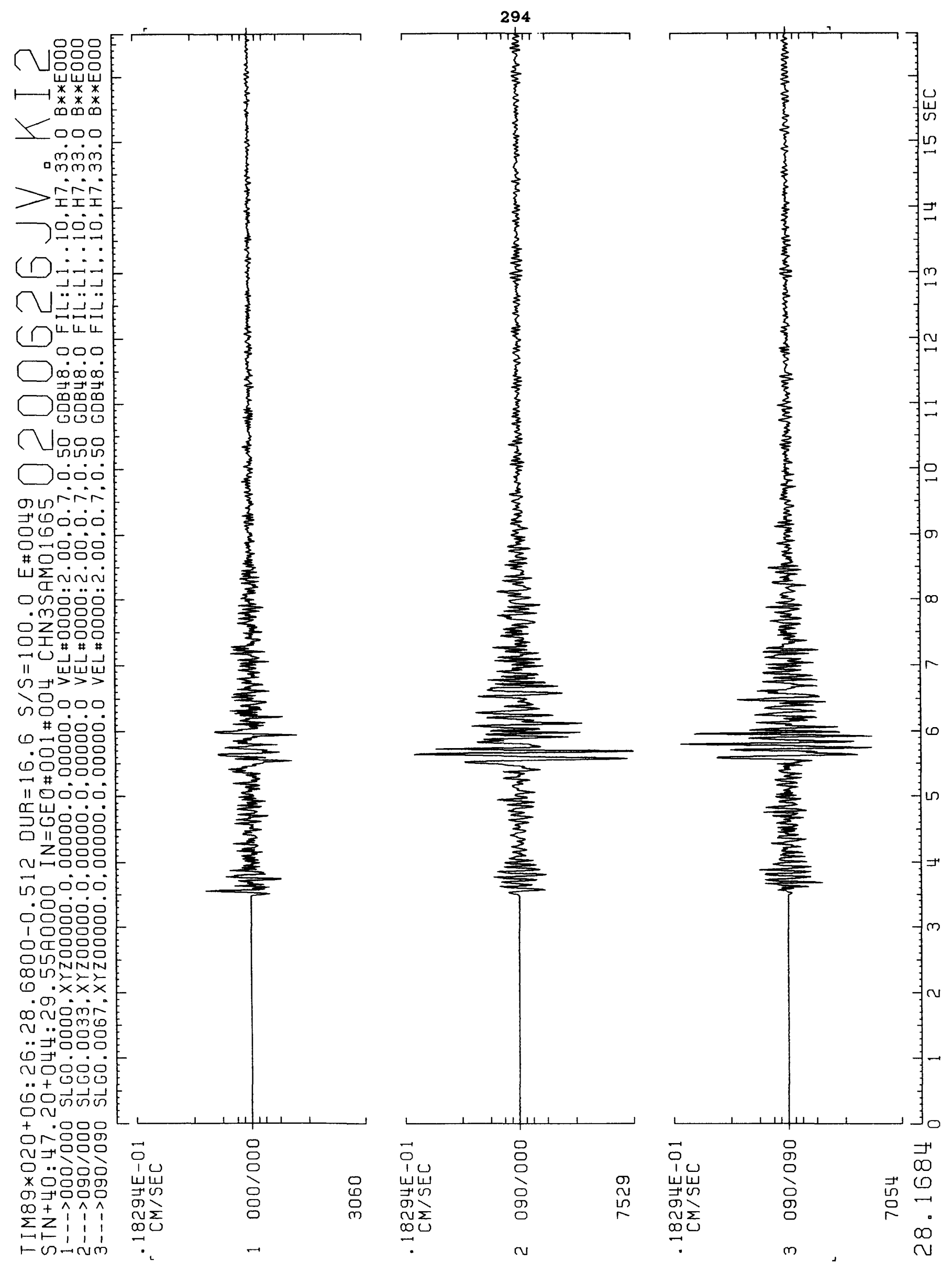



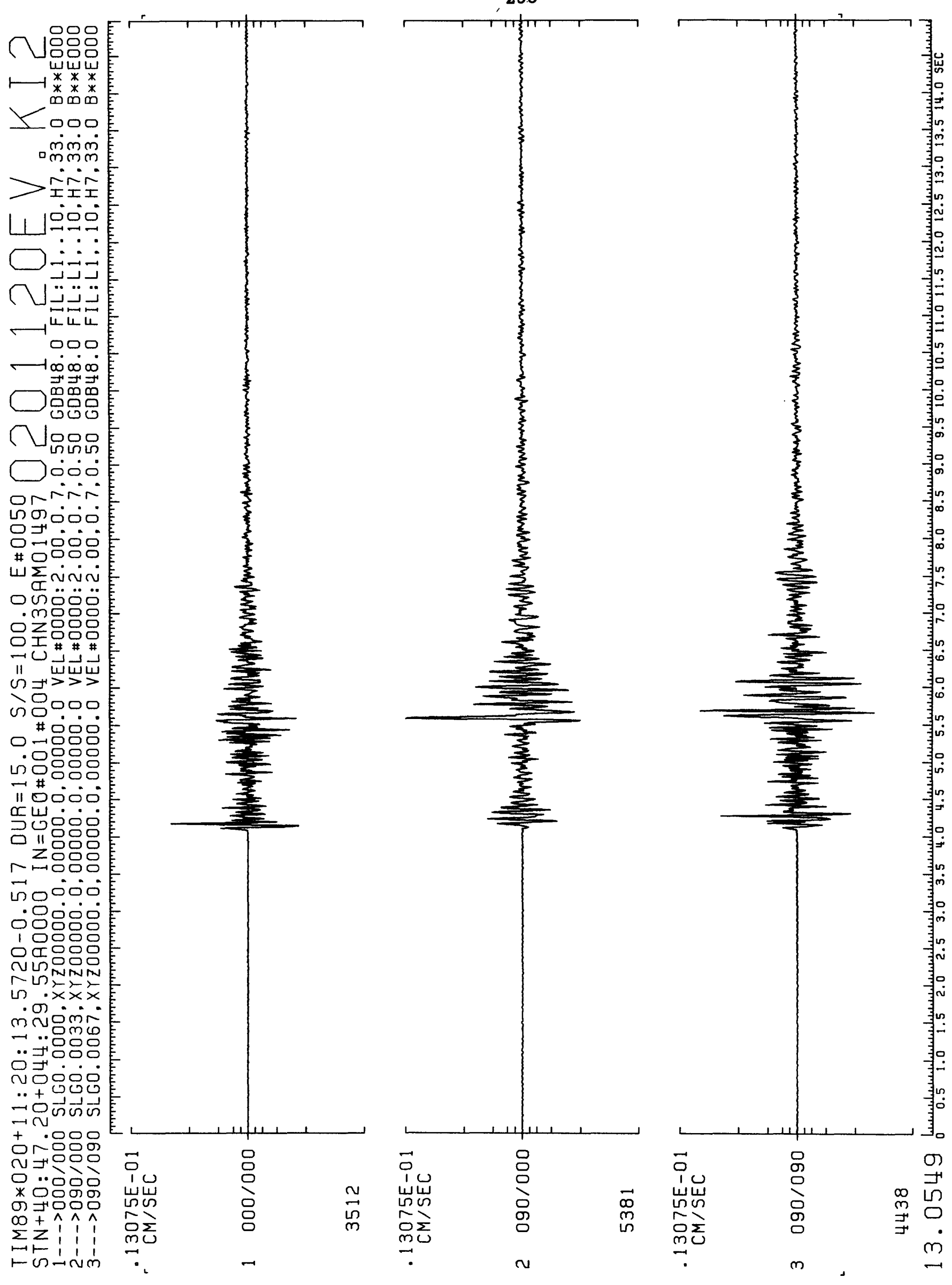

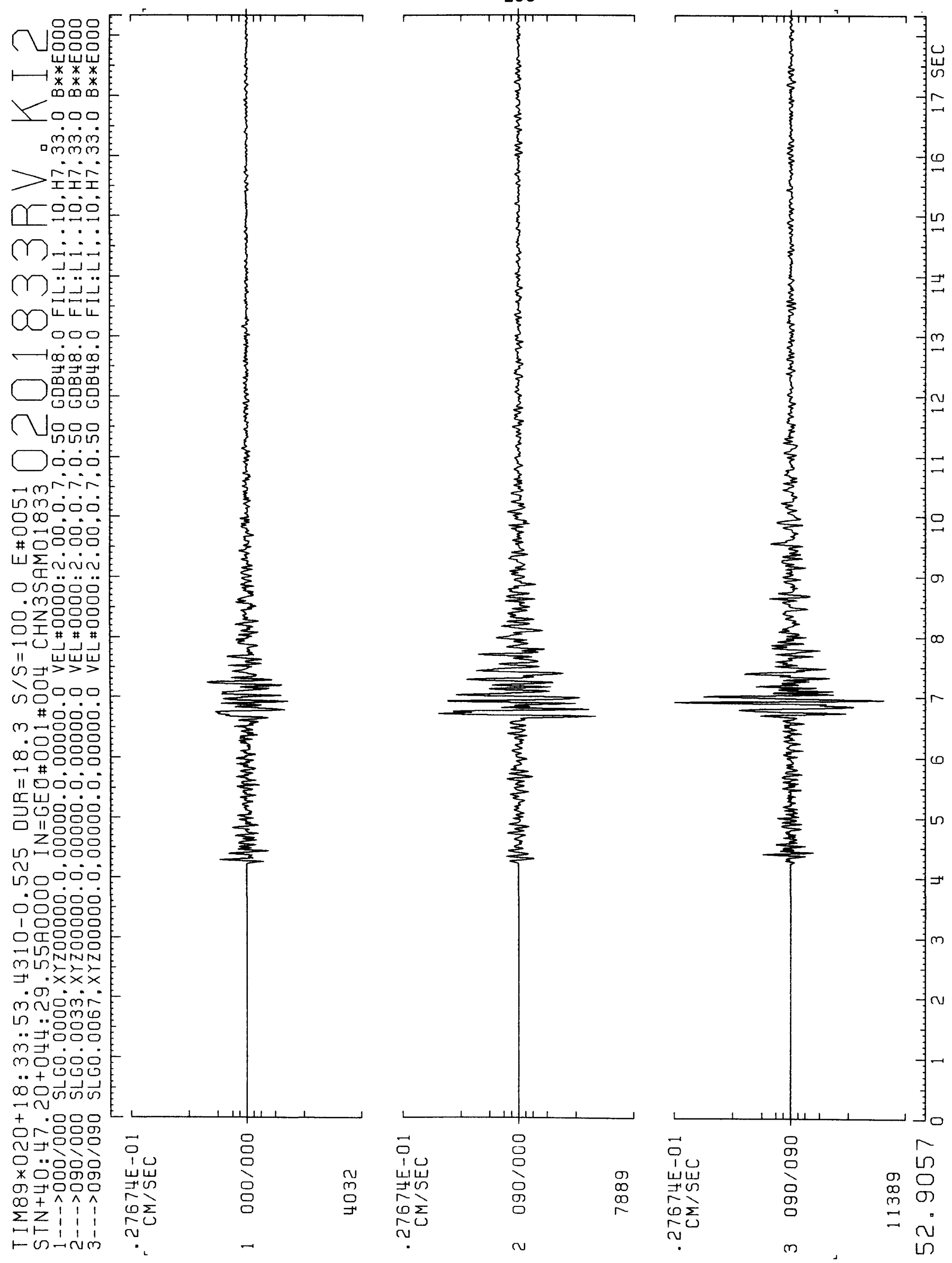


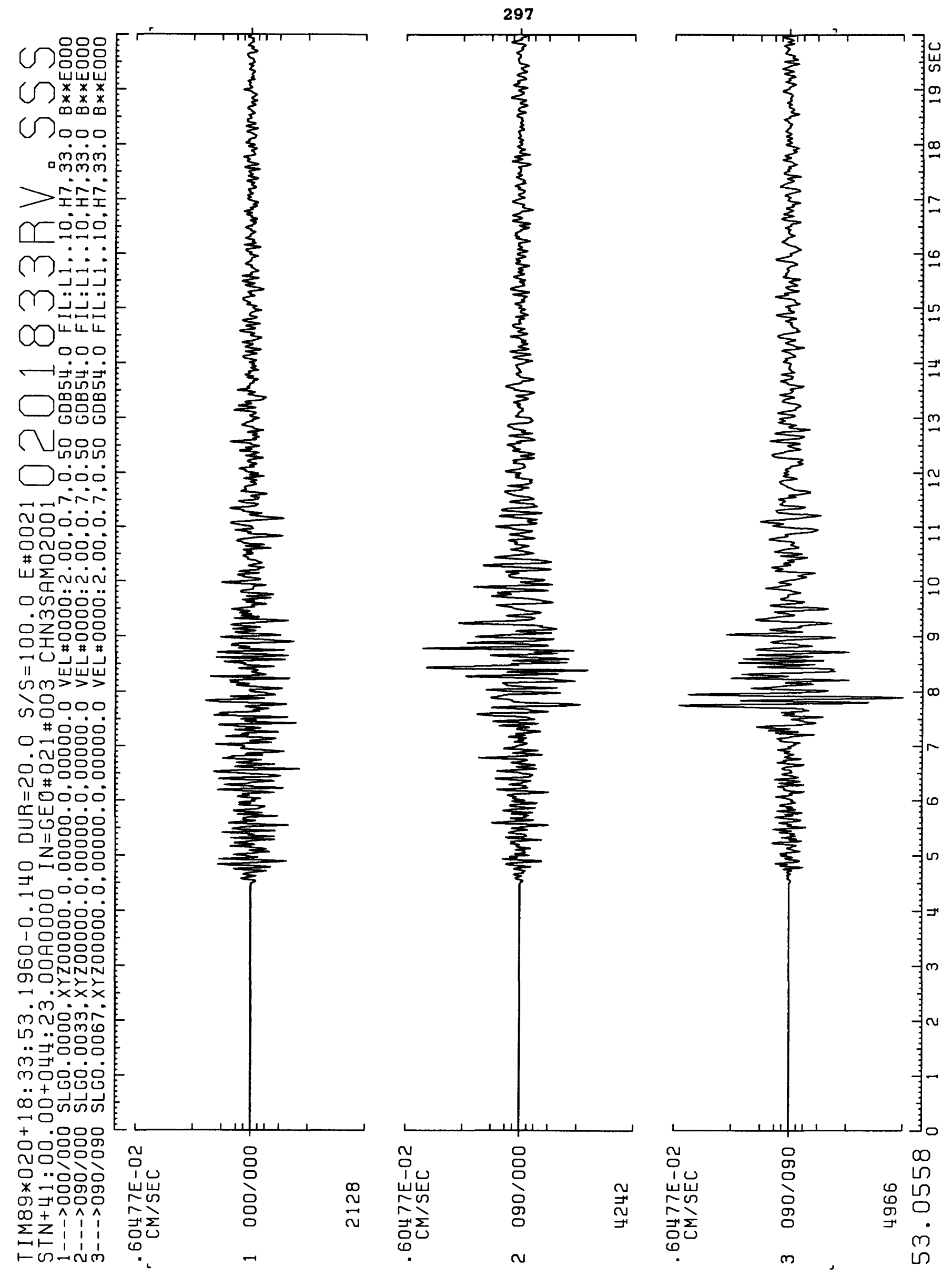


298

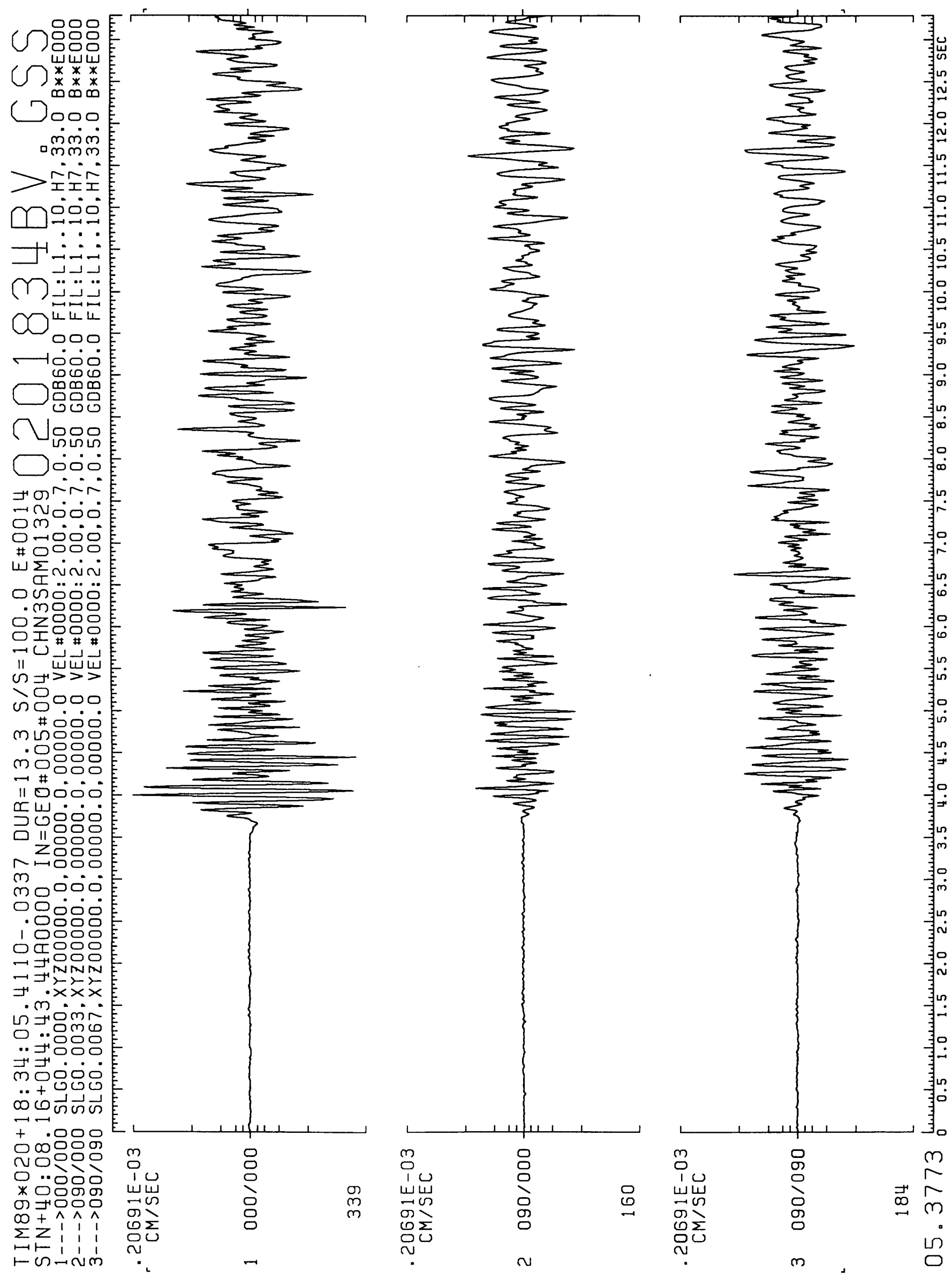




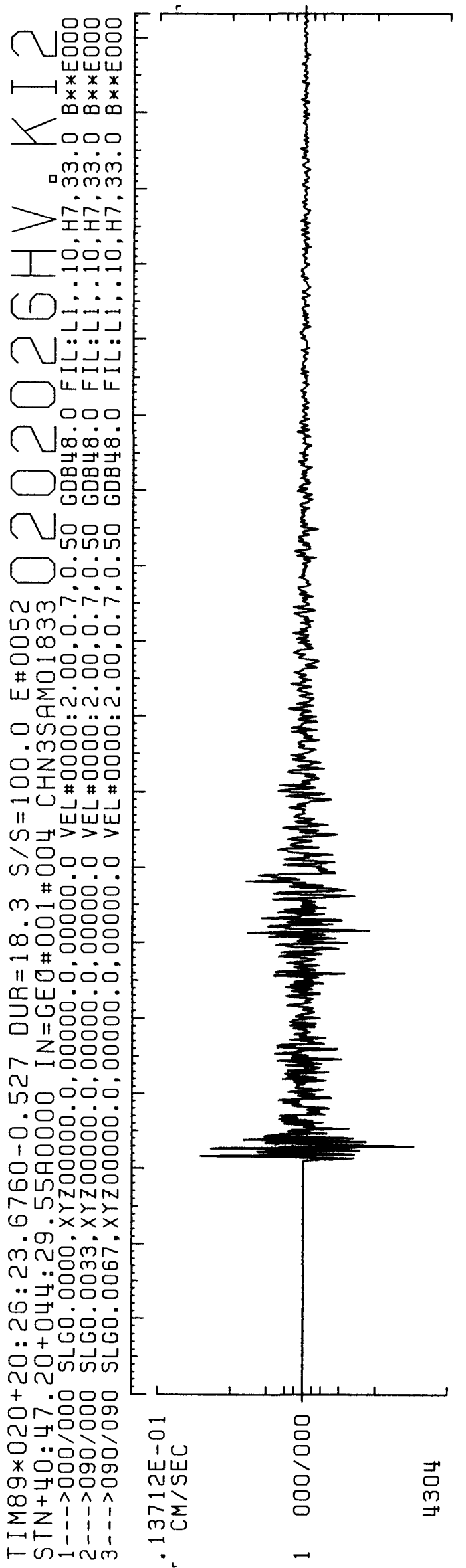

299

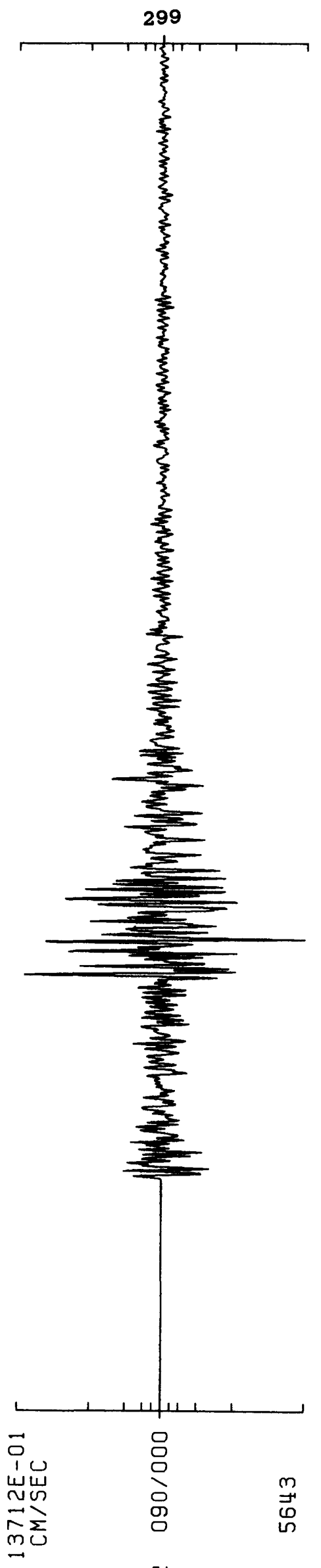

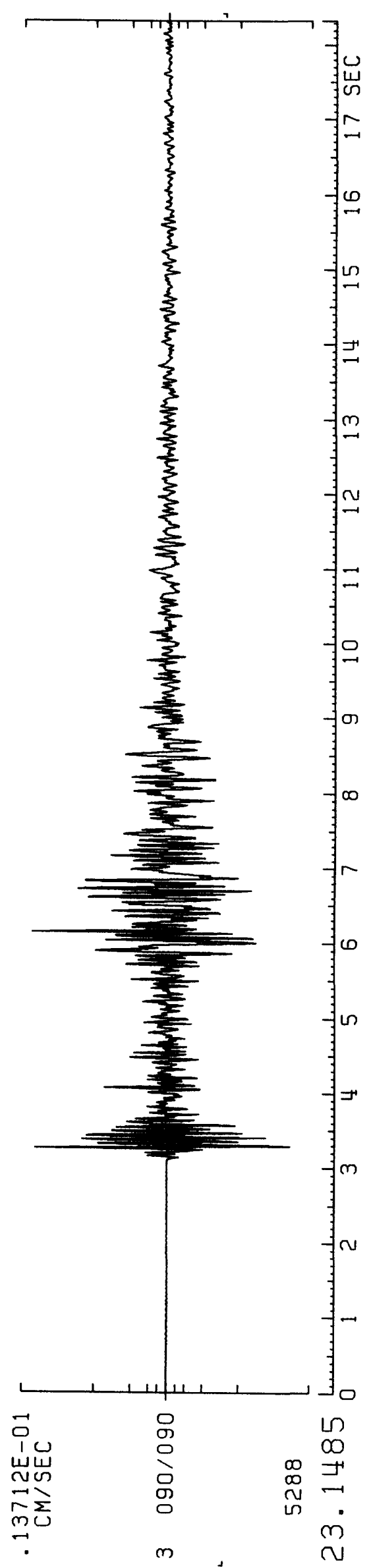




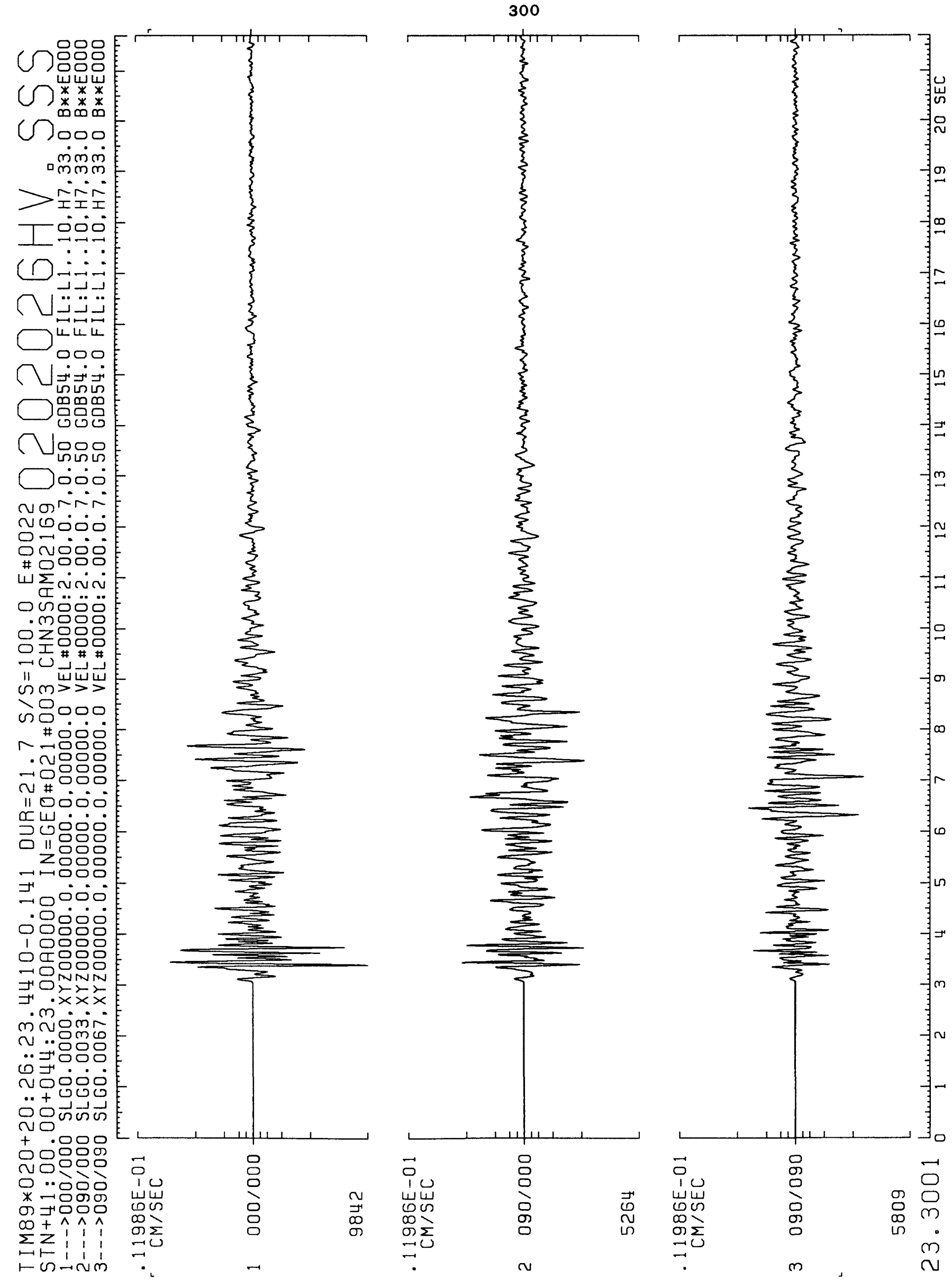




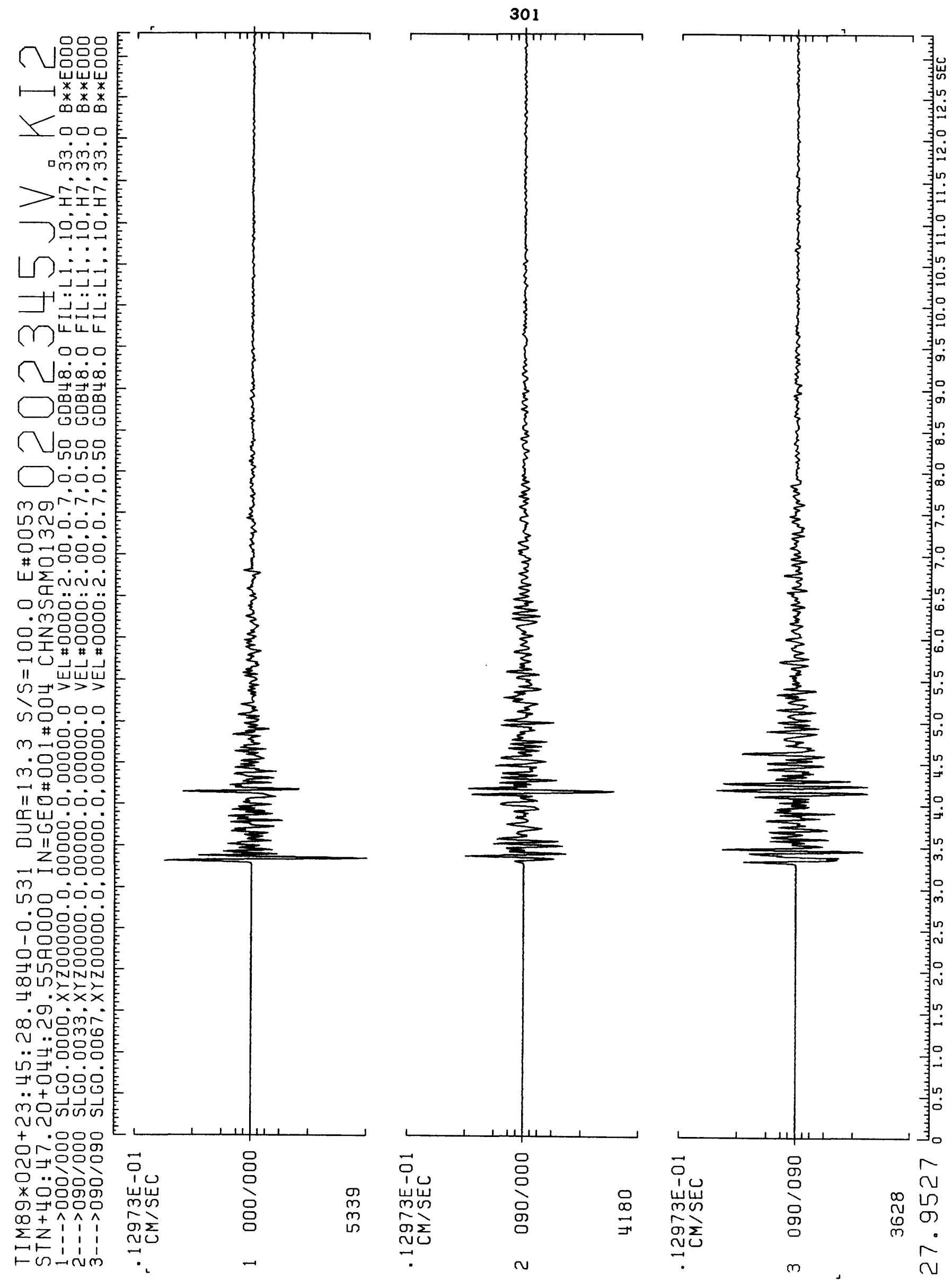




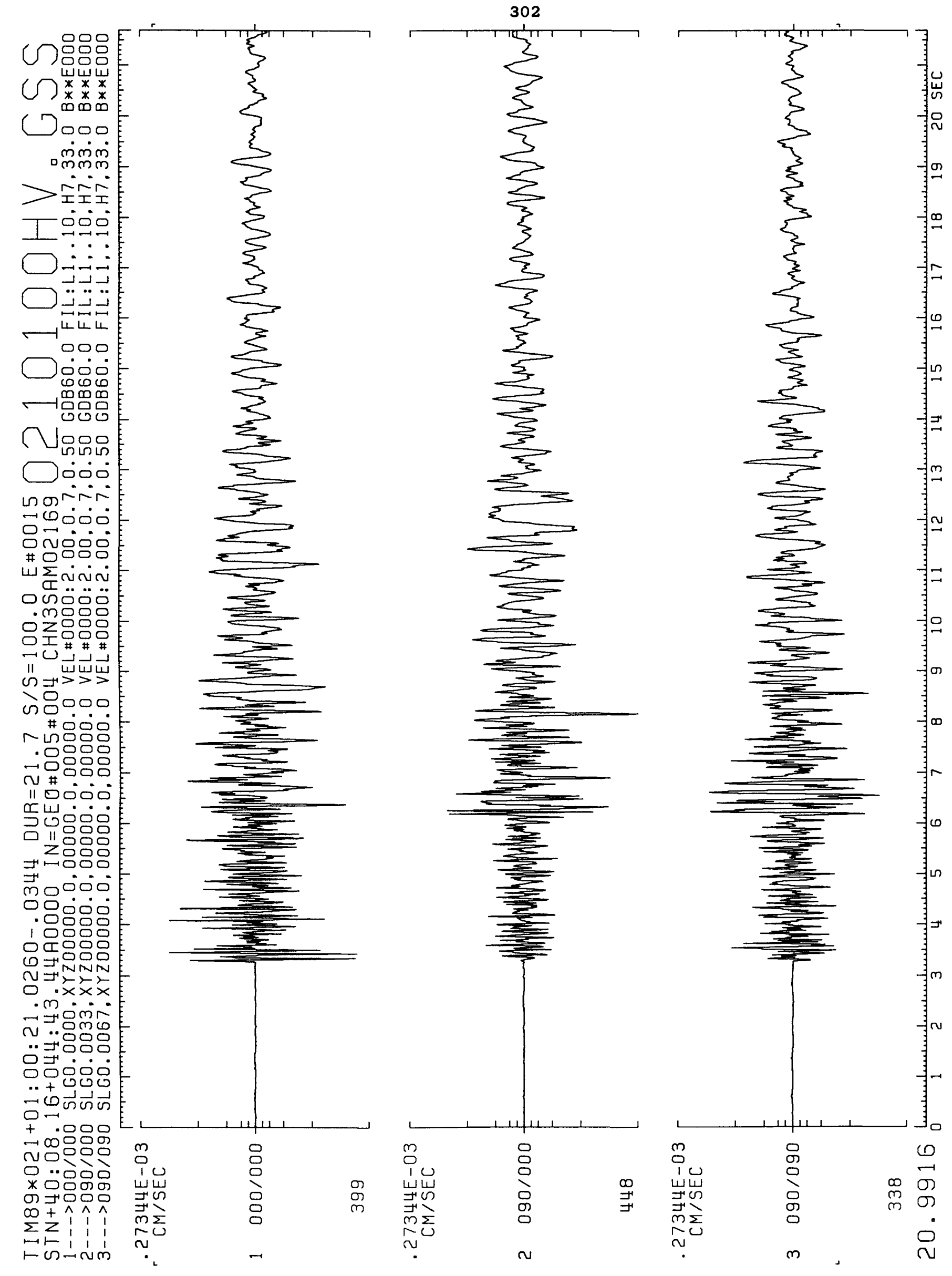




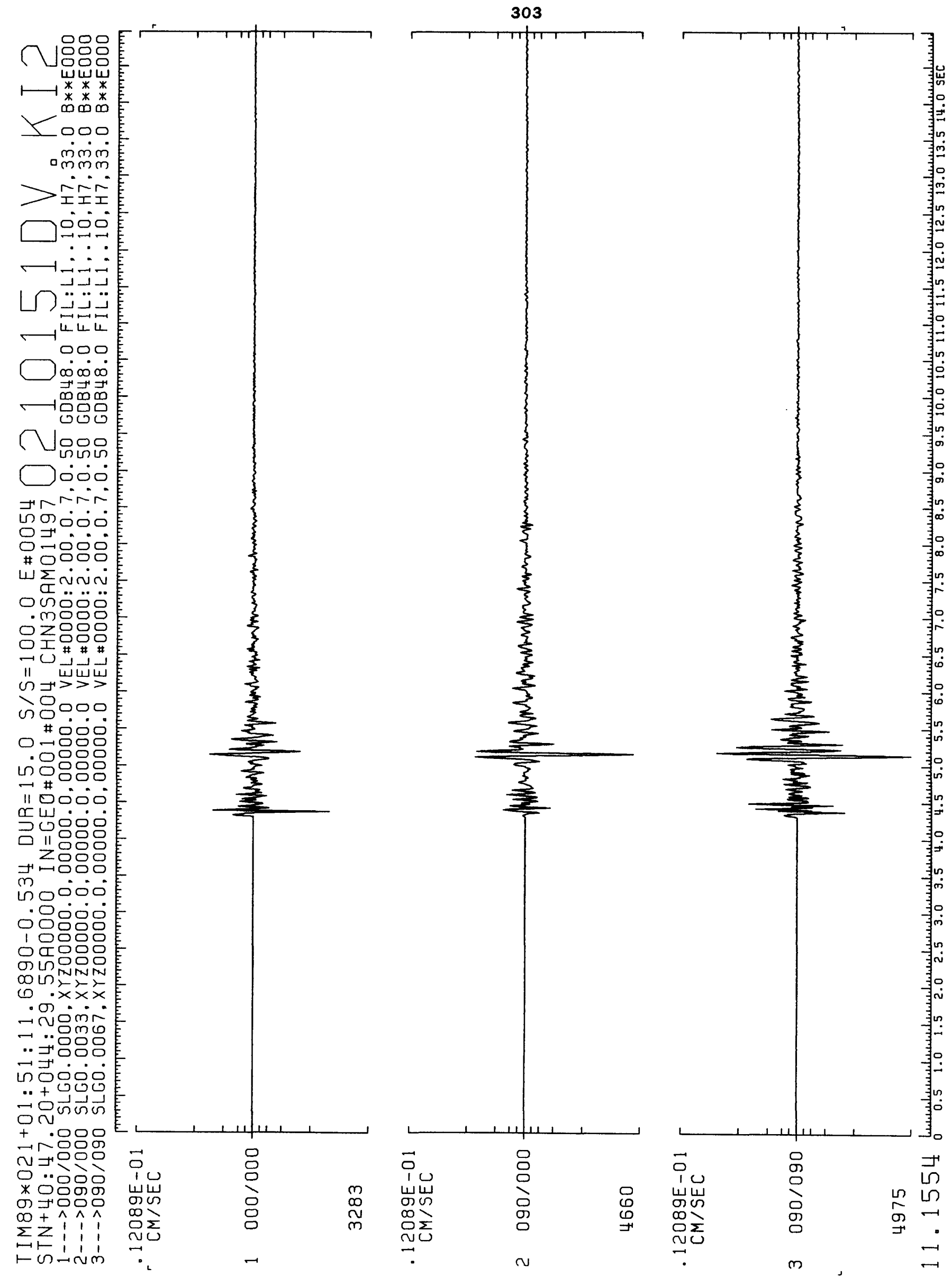




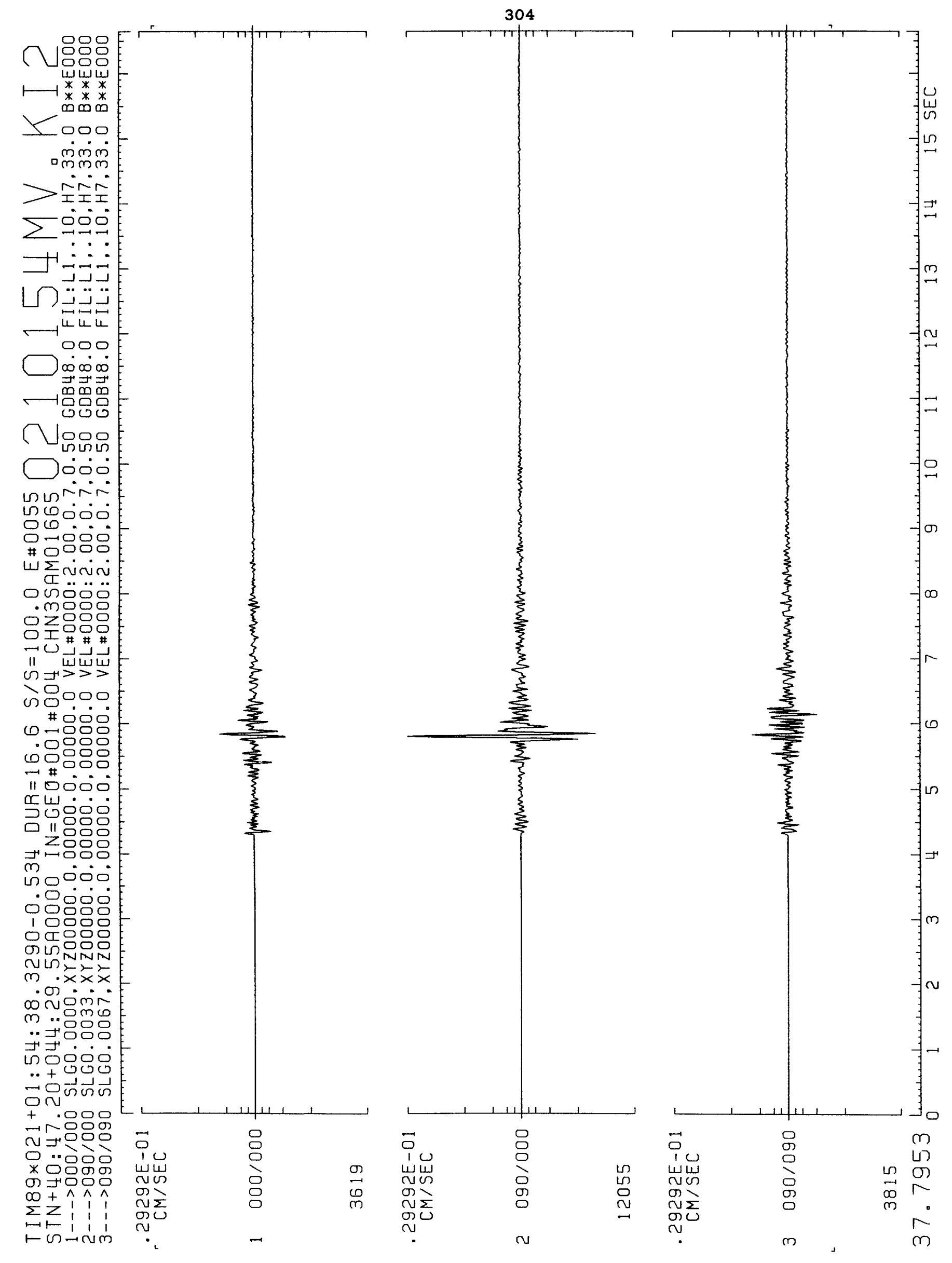



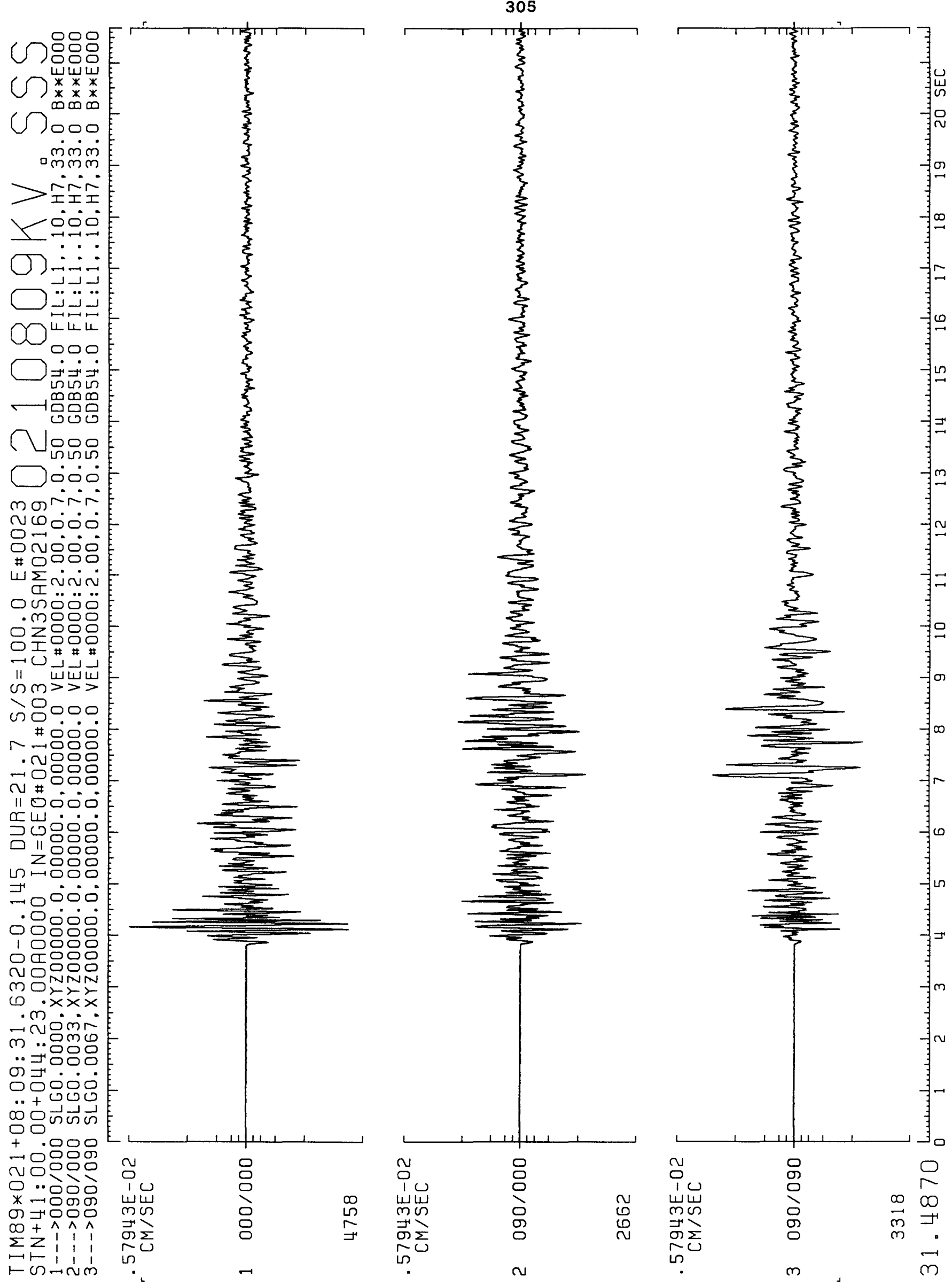

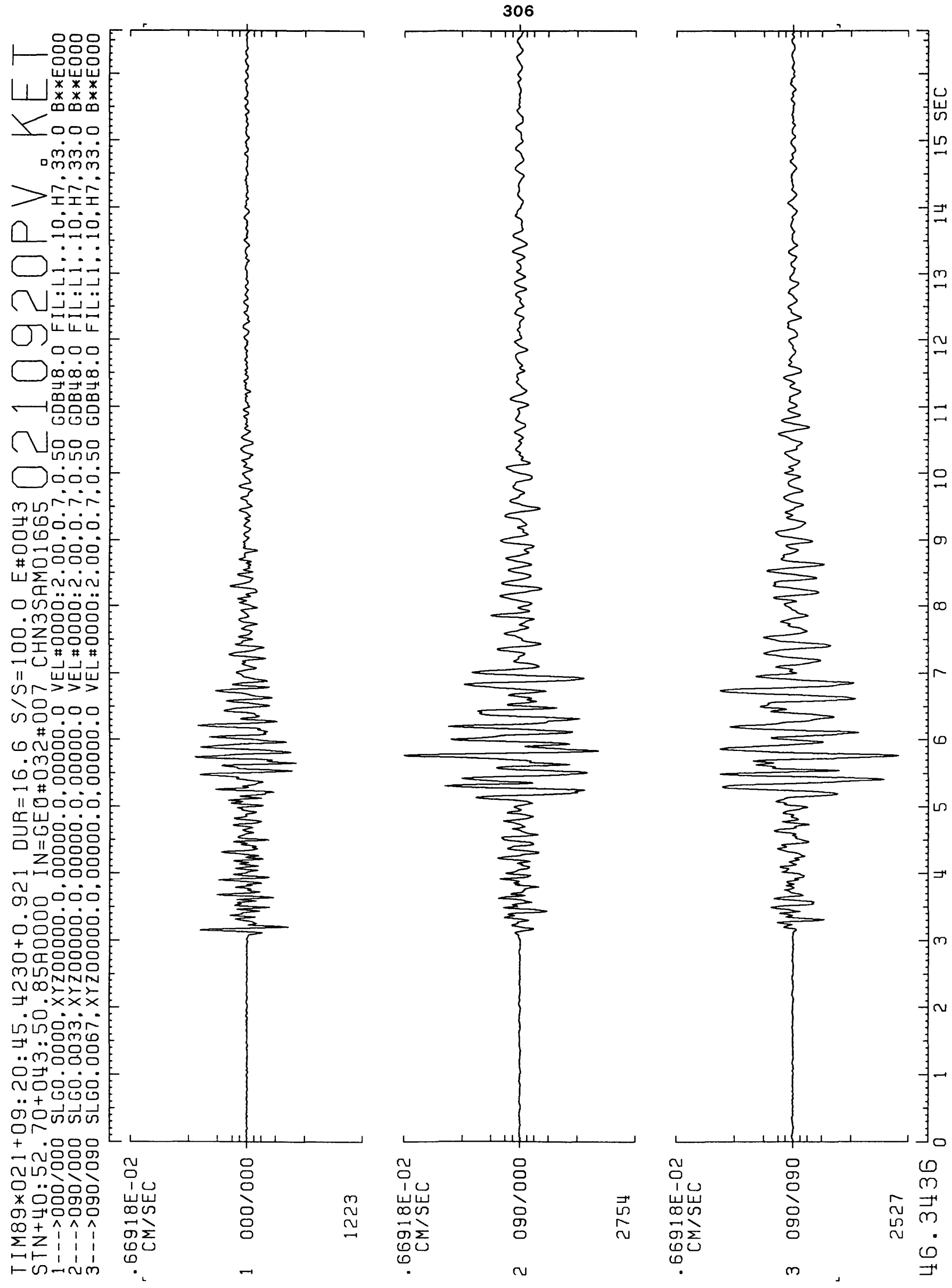


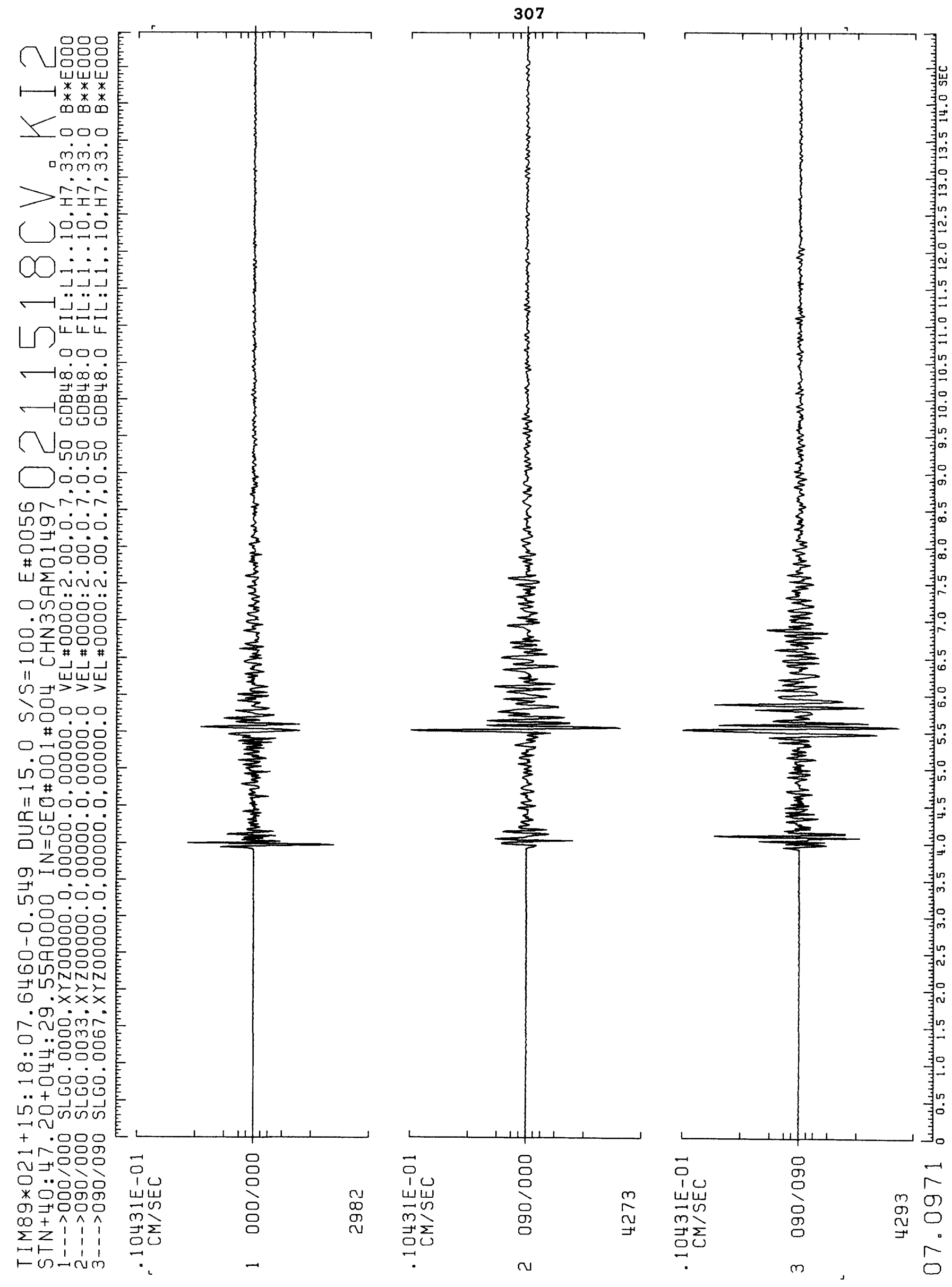




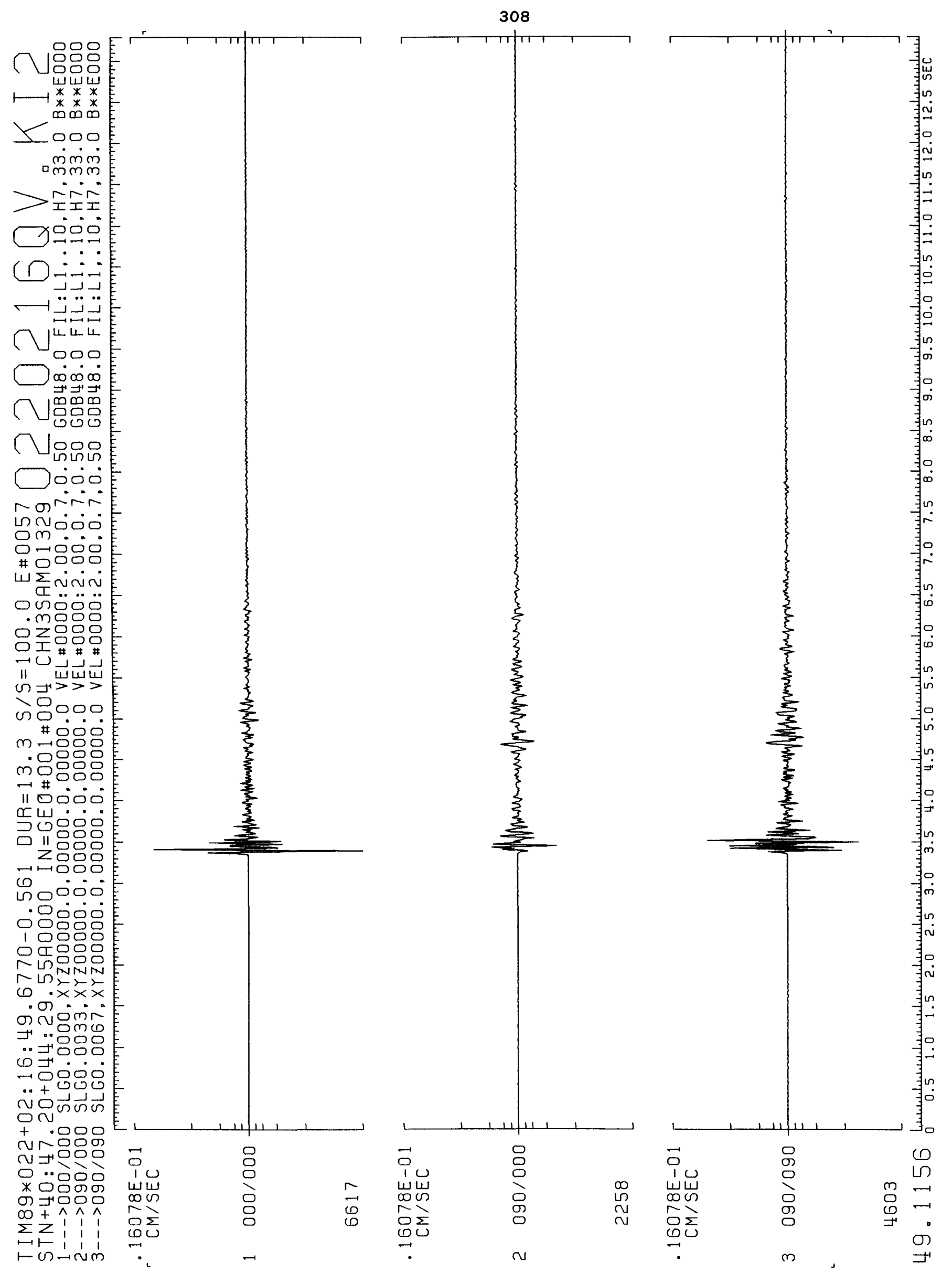


309

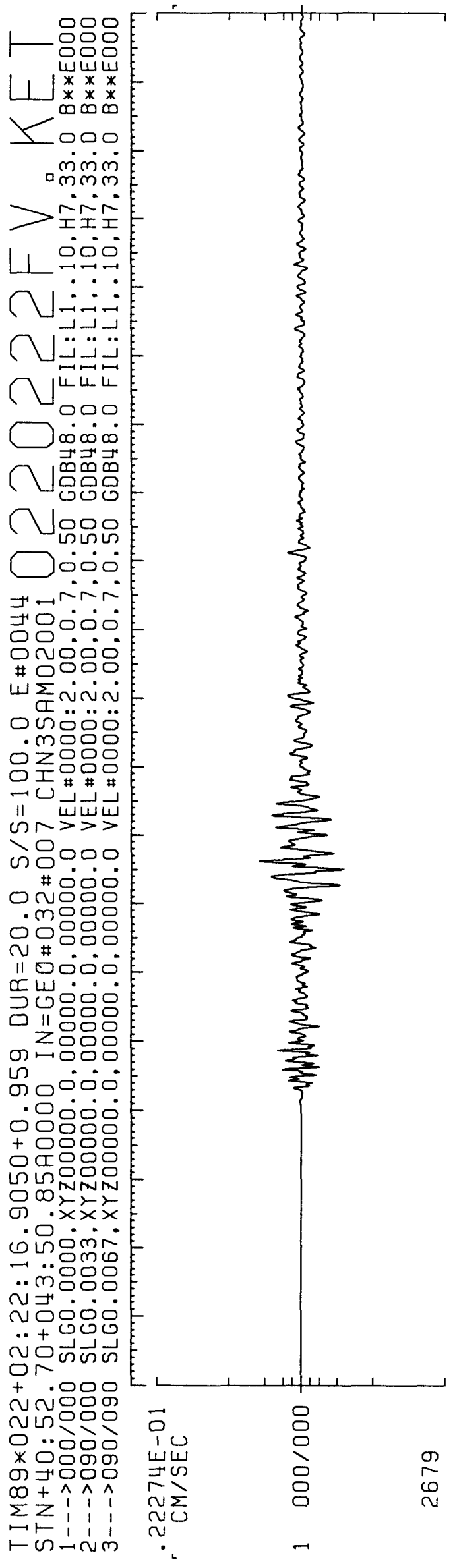

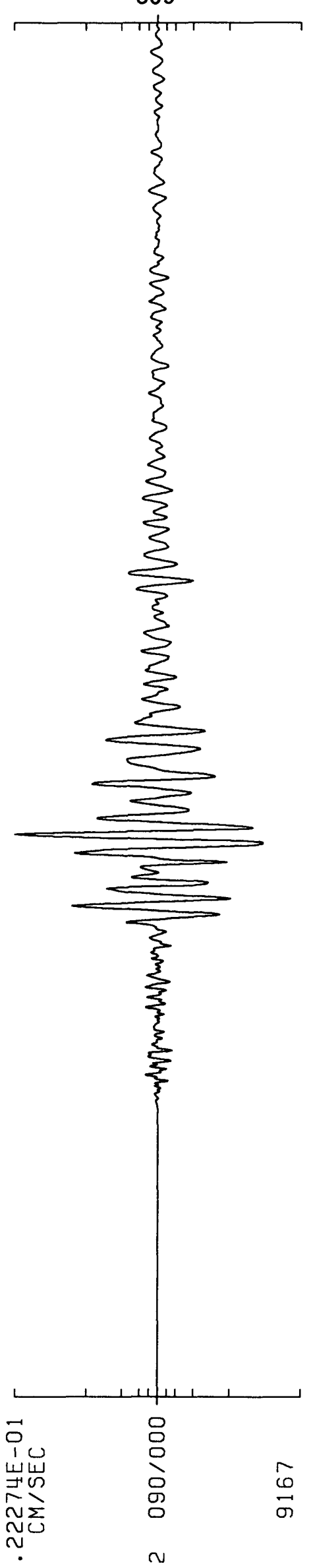

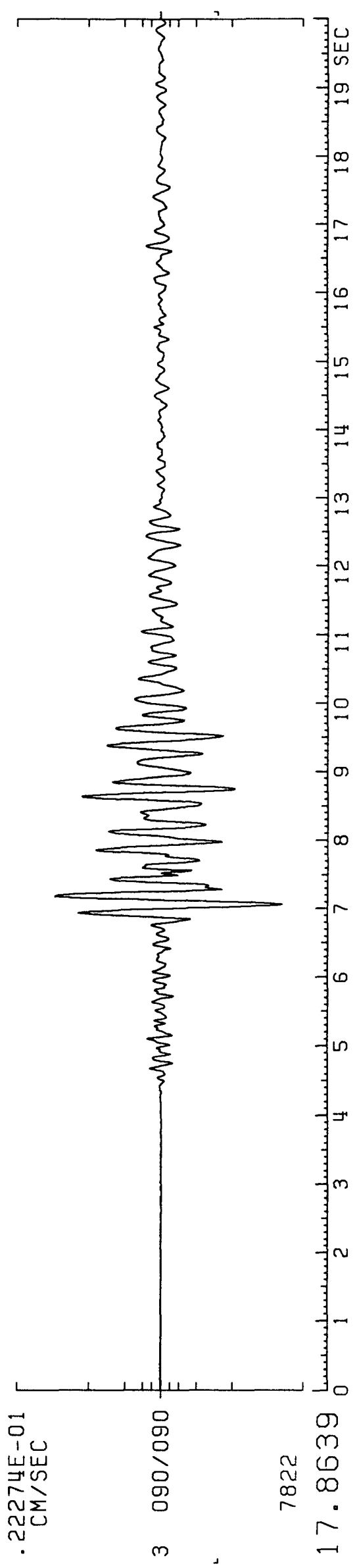


310

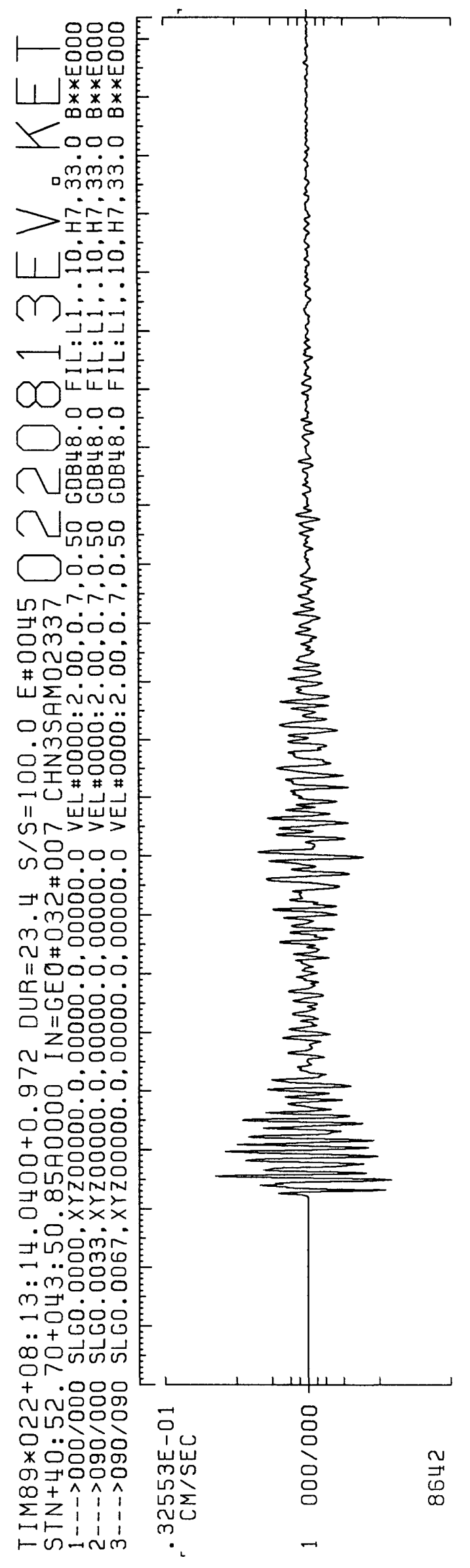

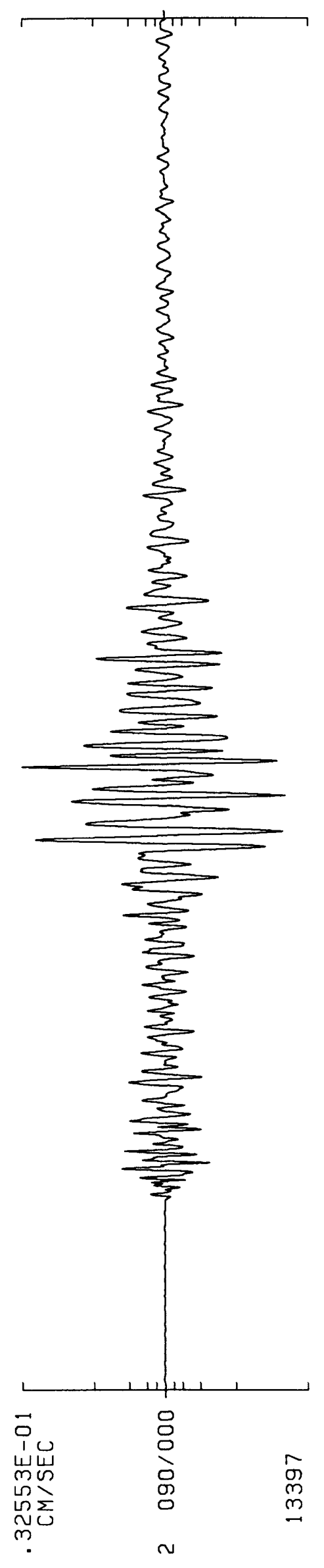

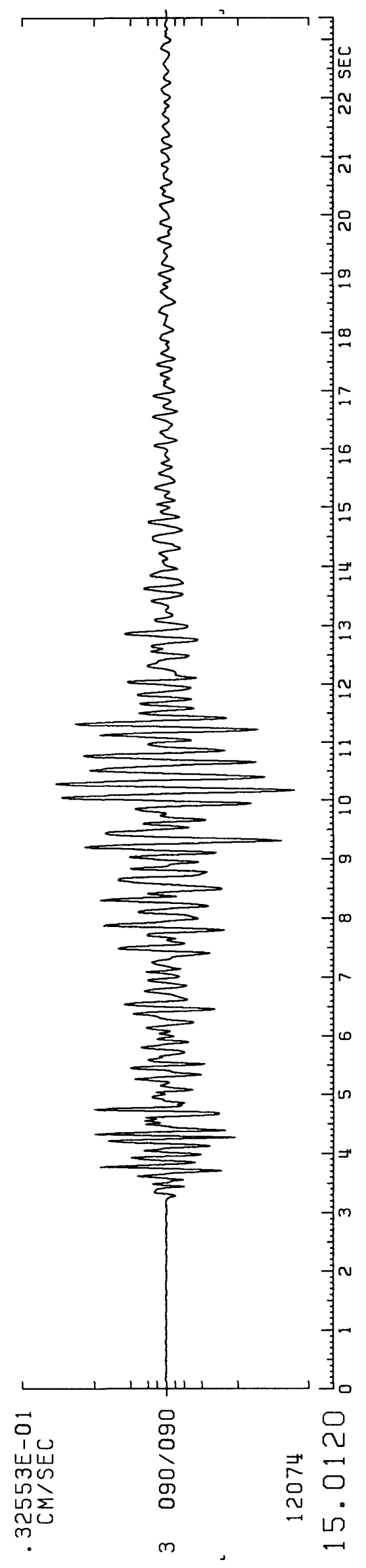


311
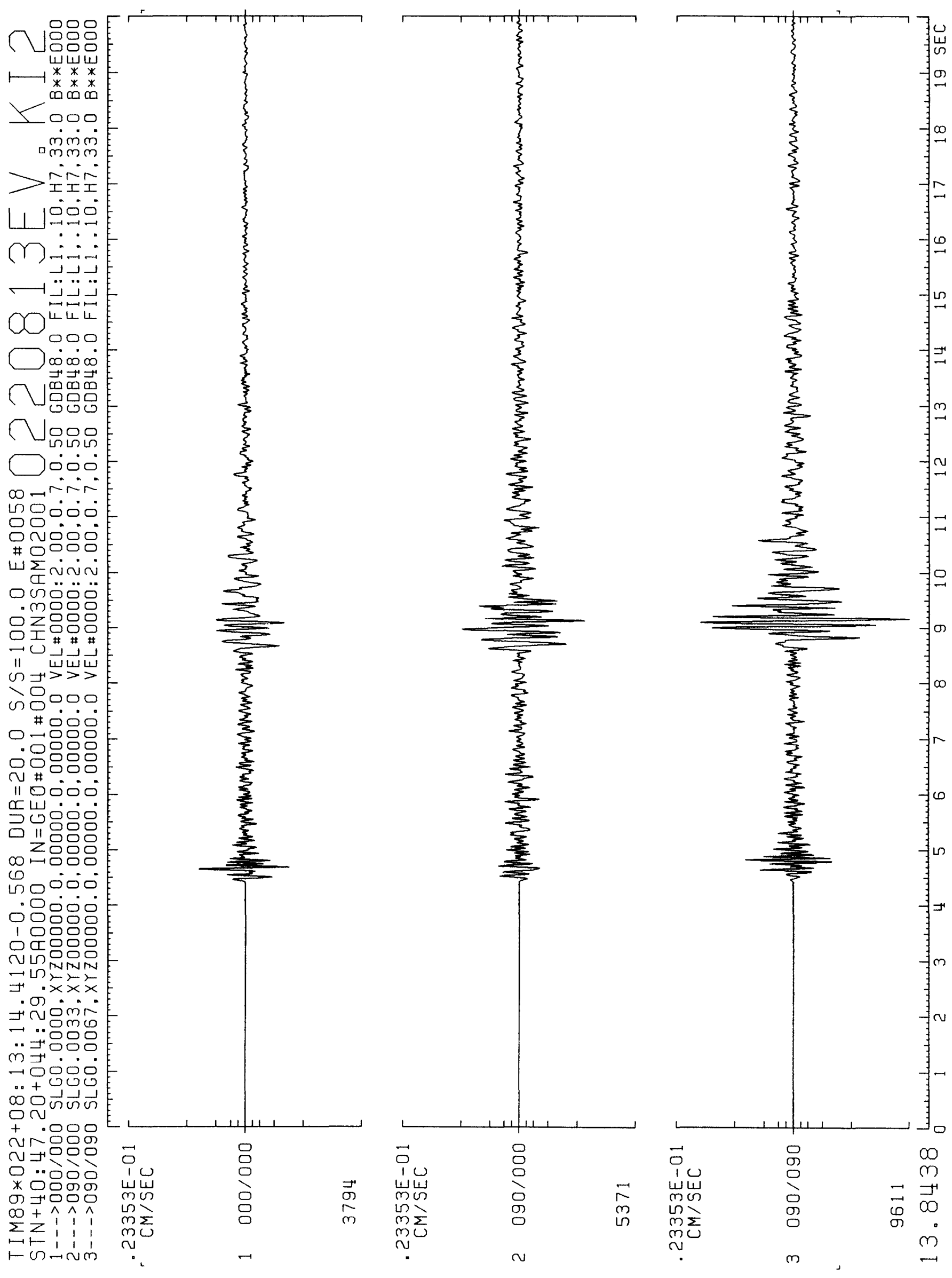
312
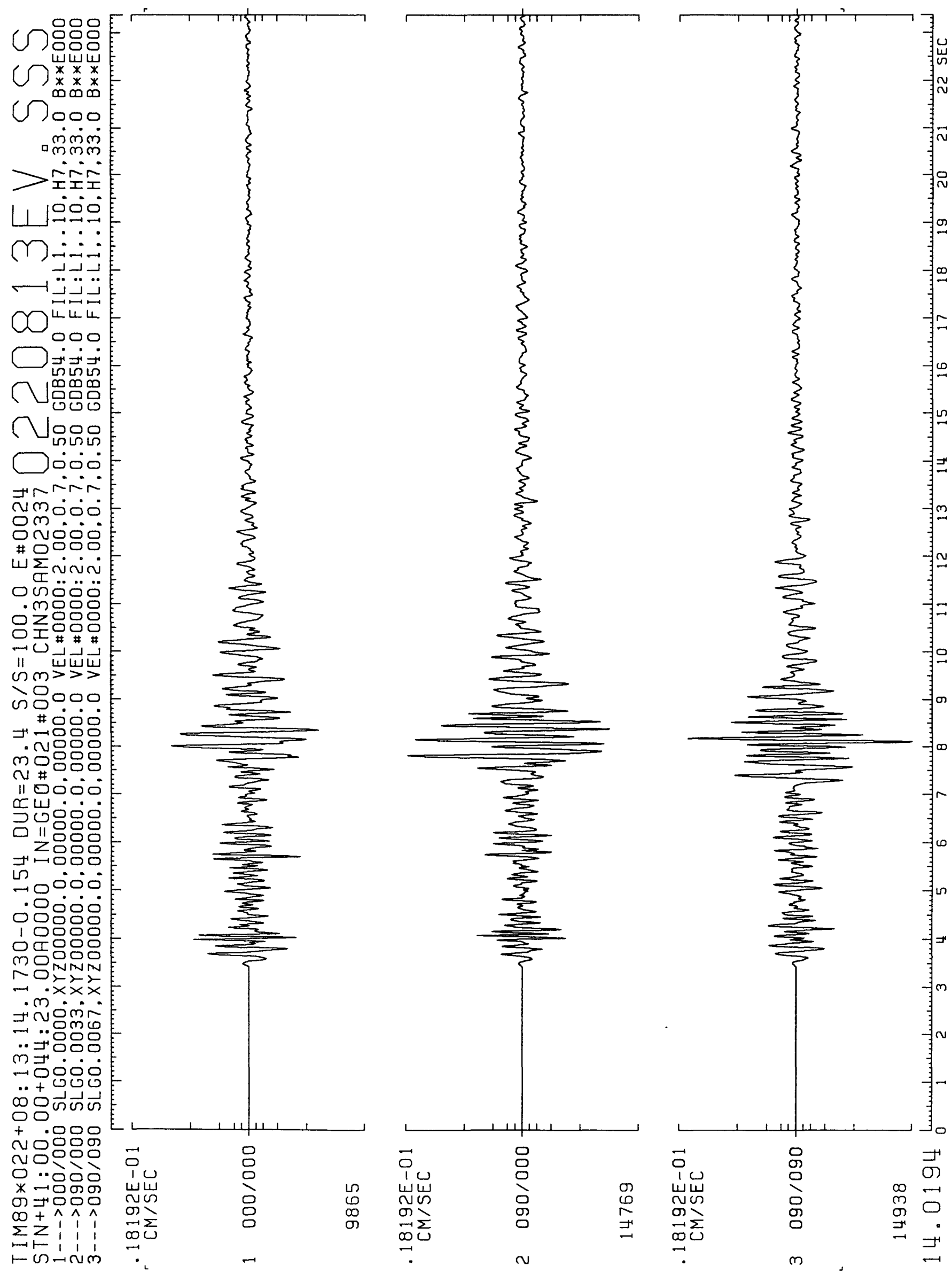


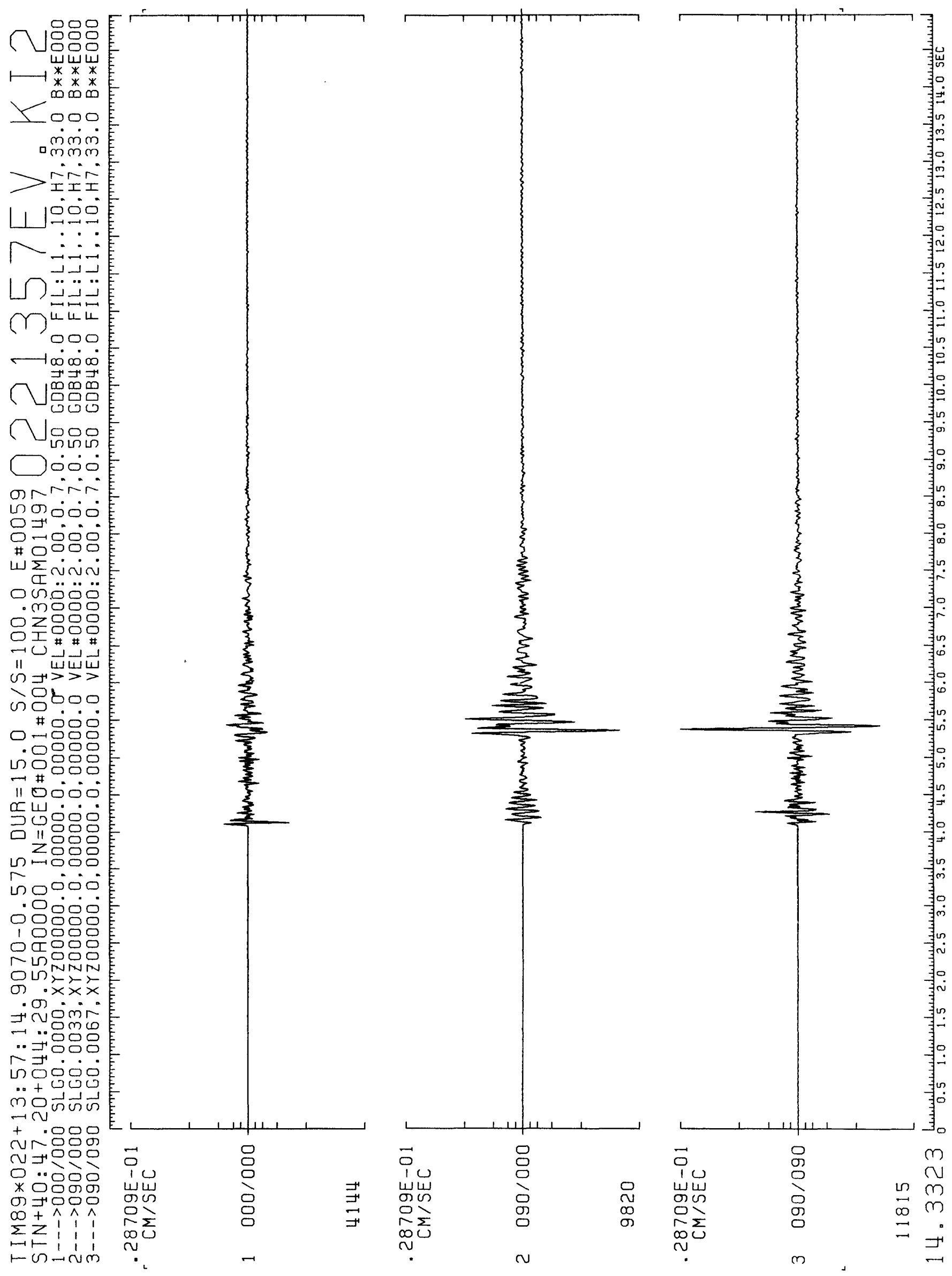


314
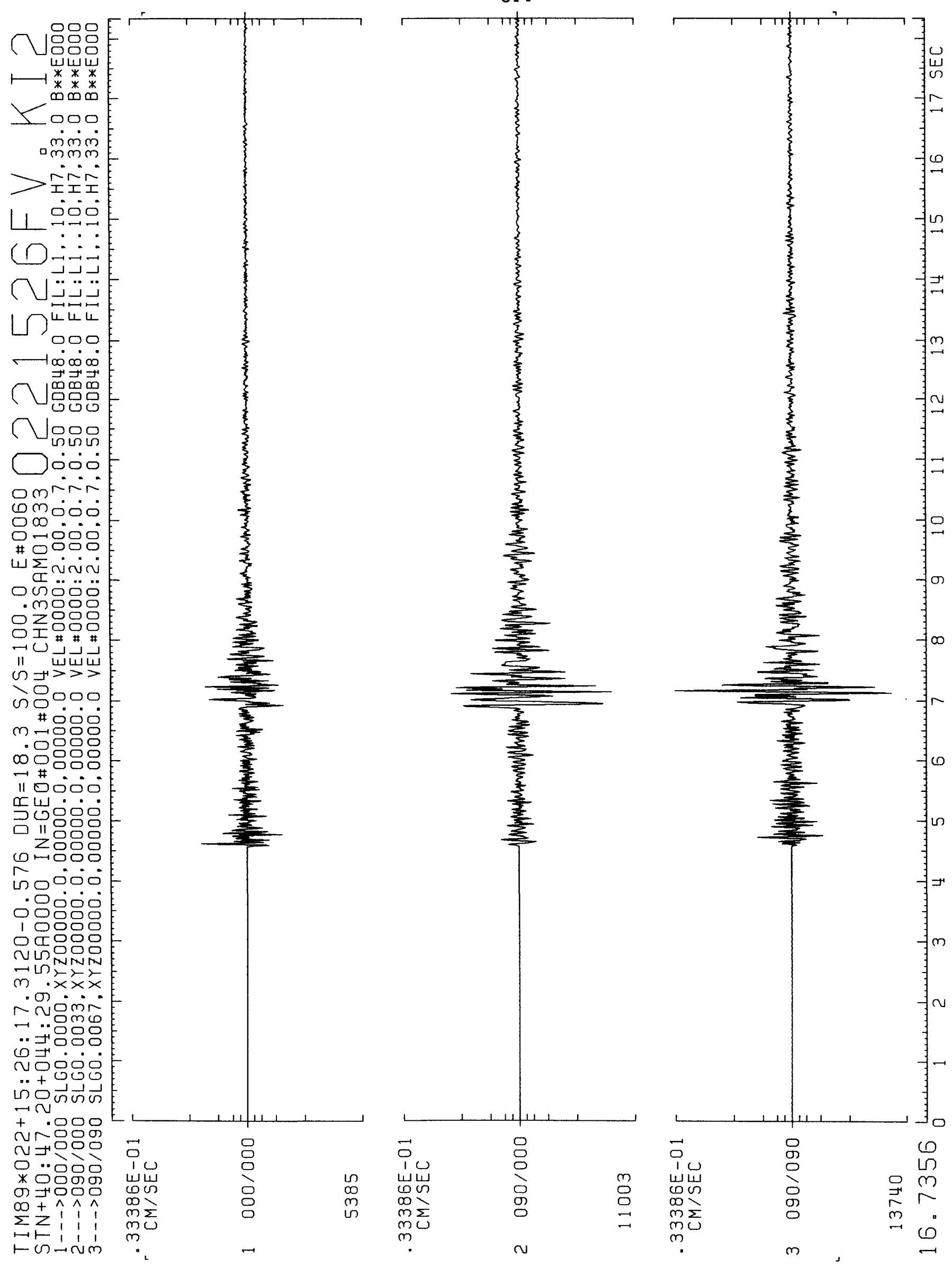
315
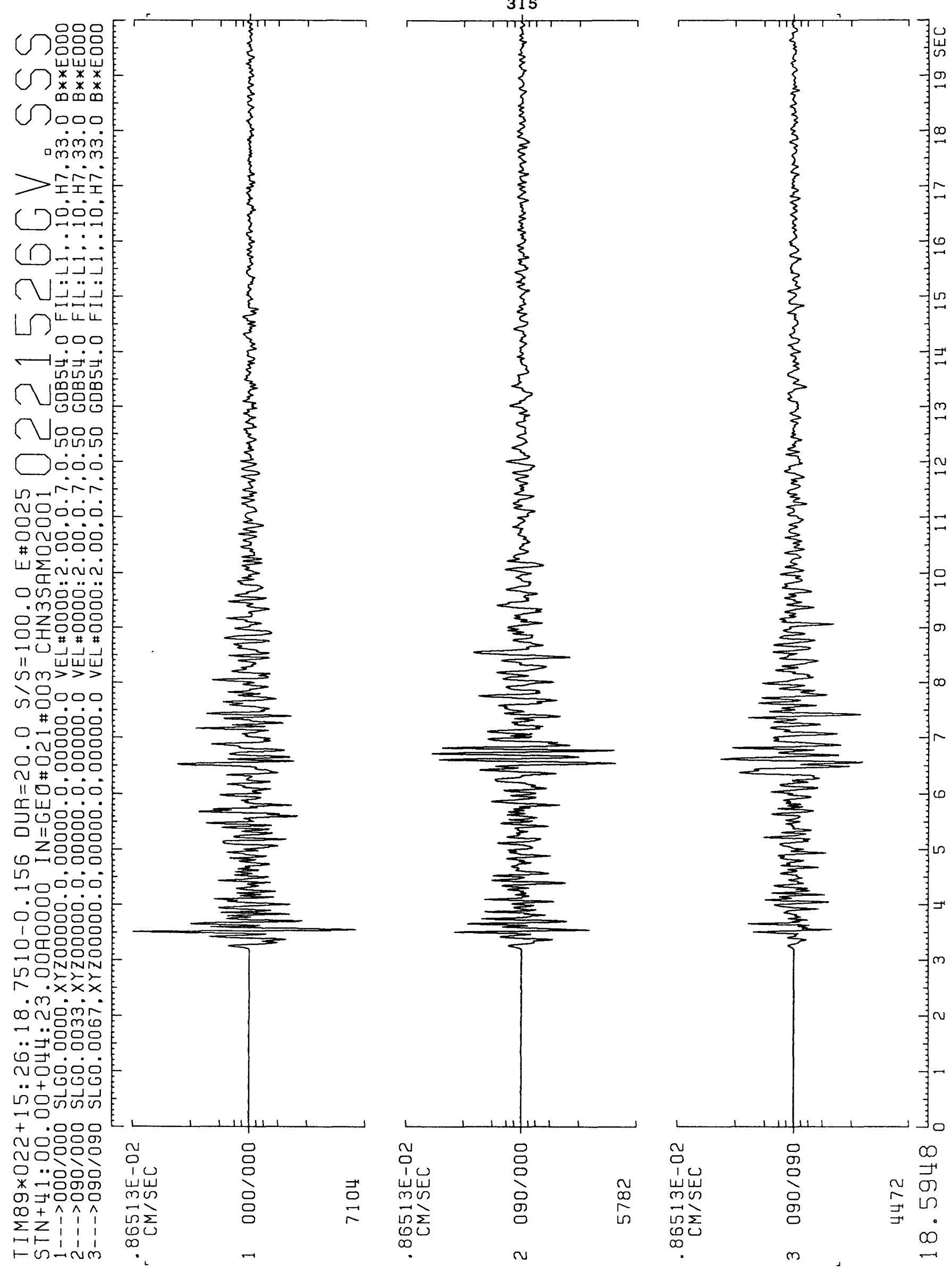
316

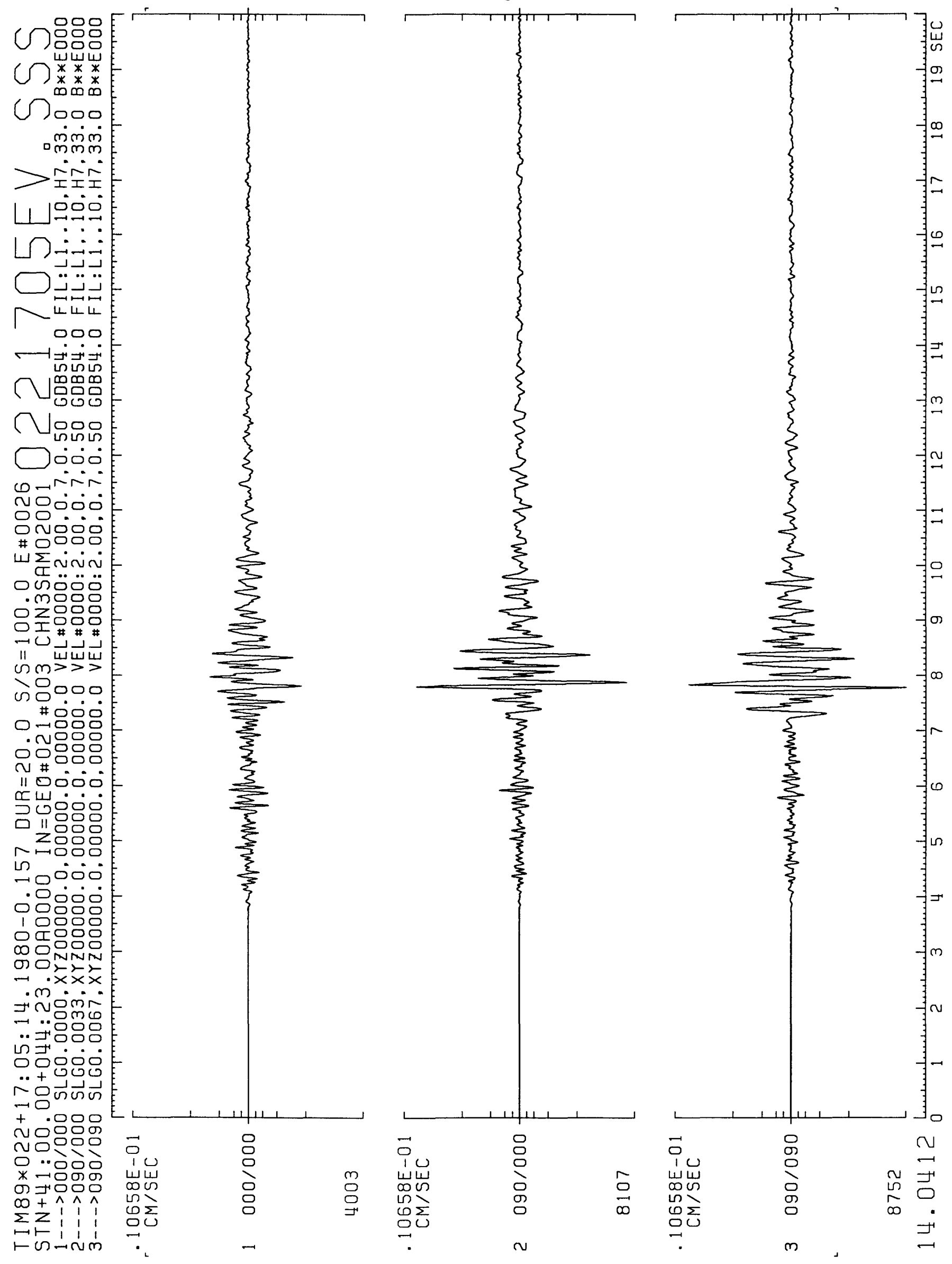


317

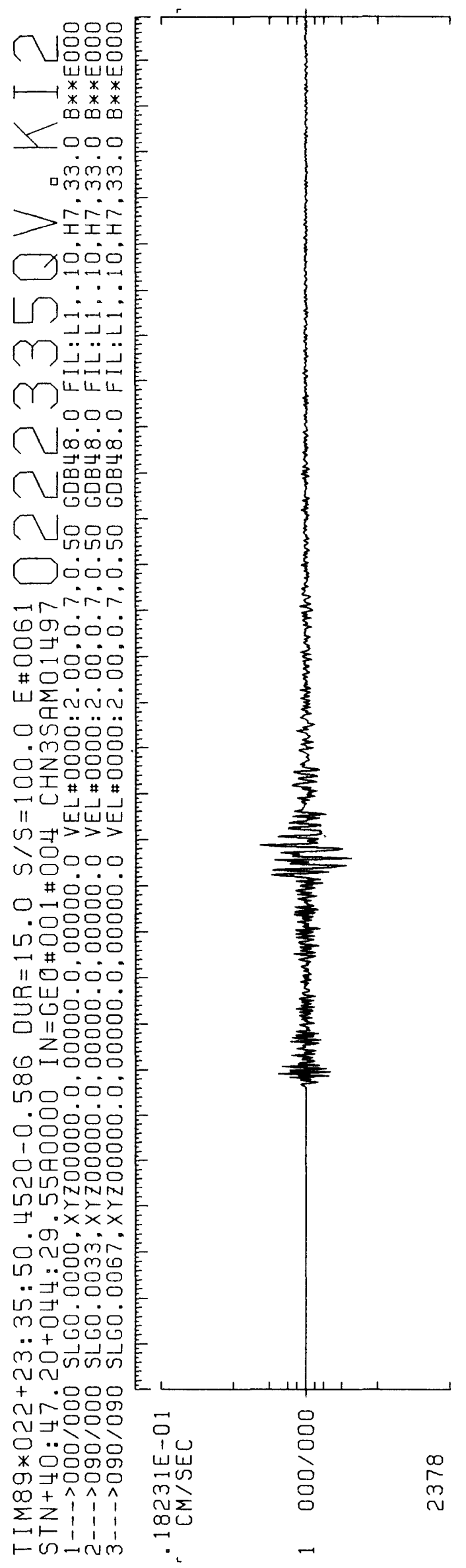

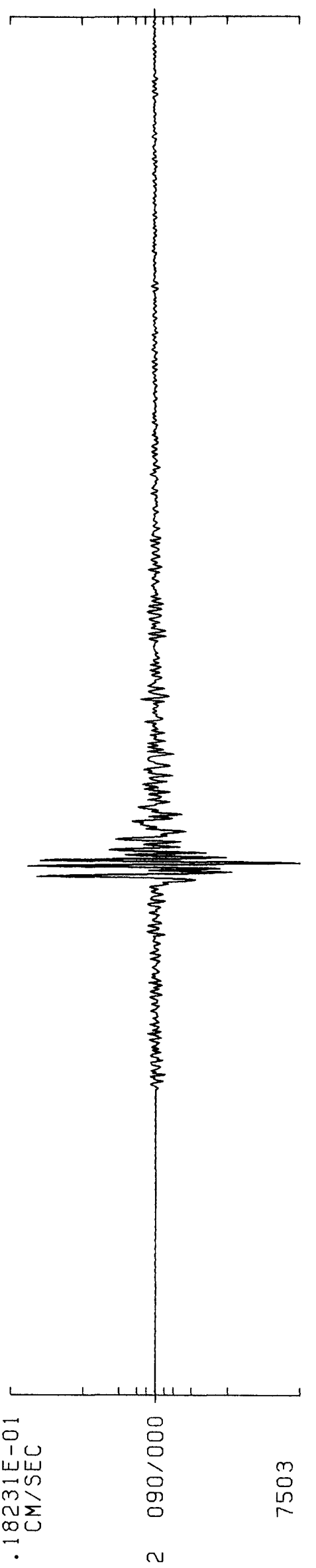

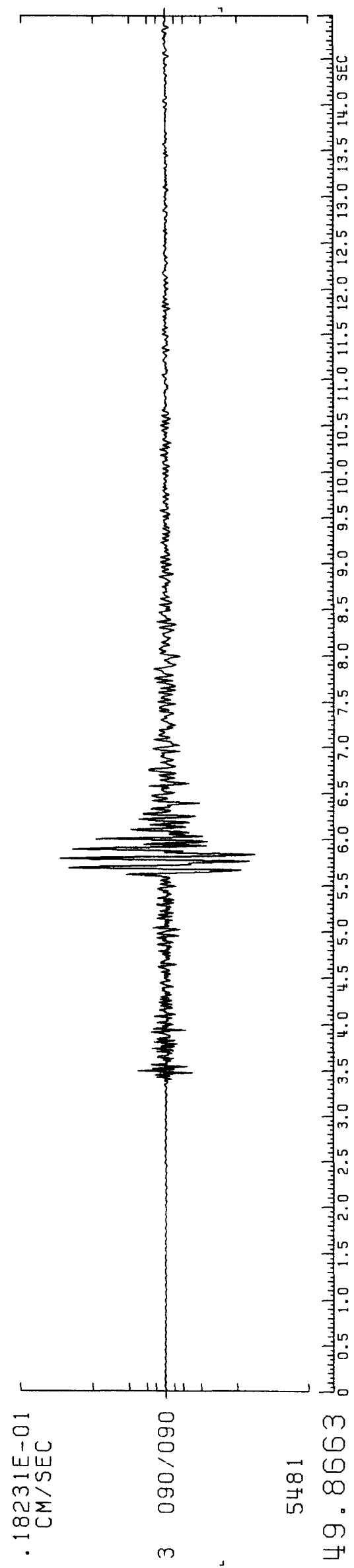


318
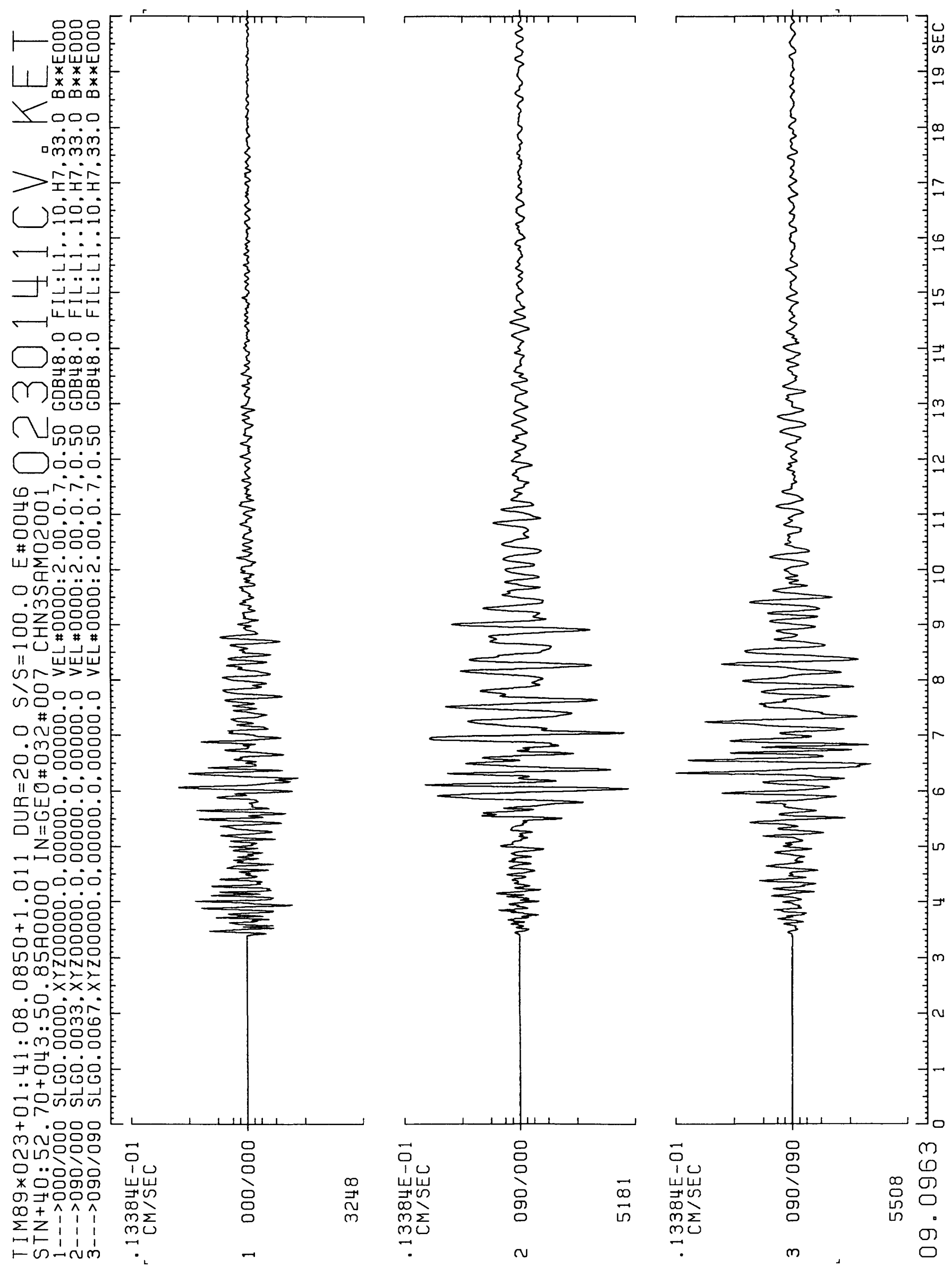
319

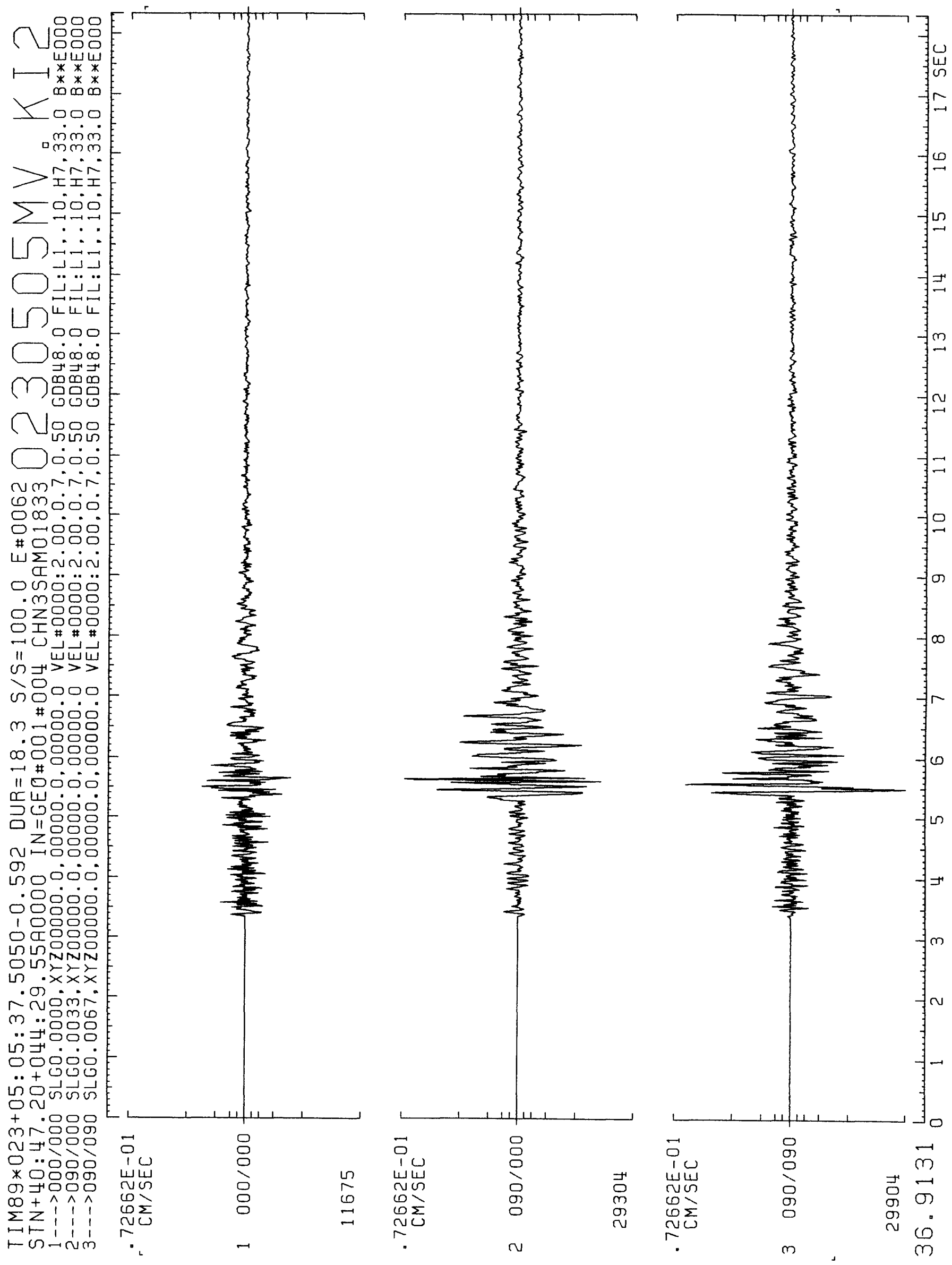



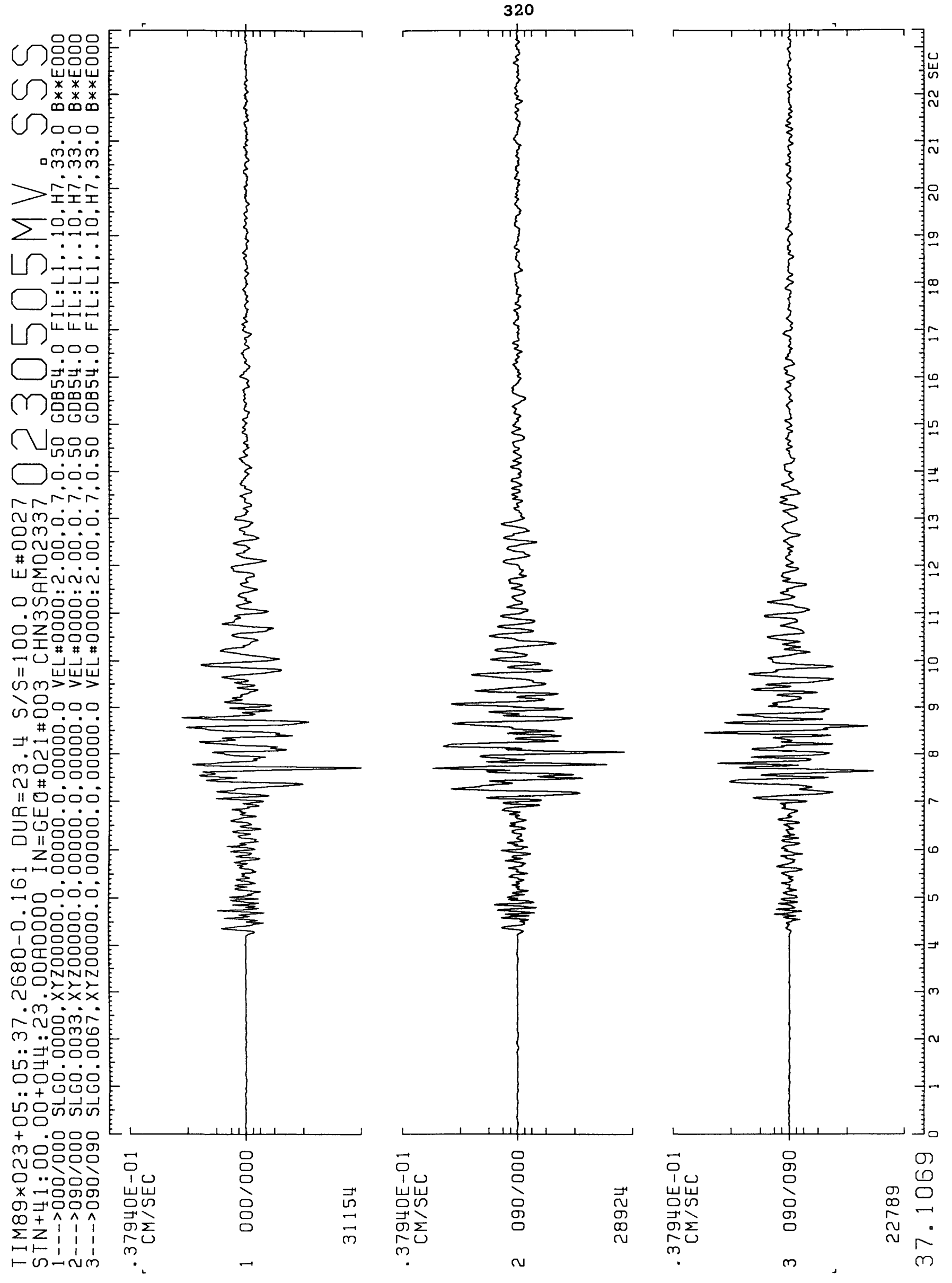
321
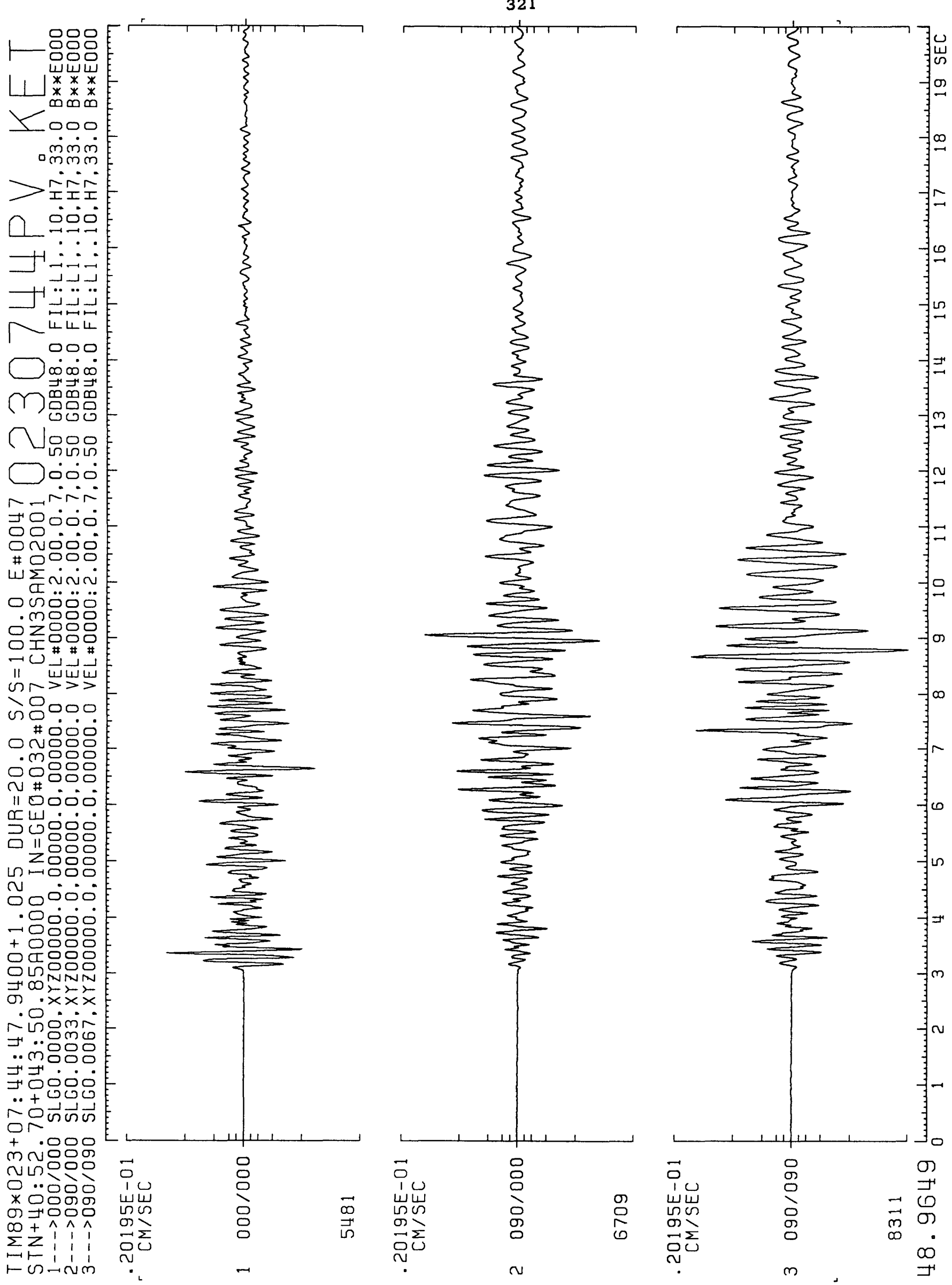


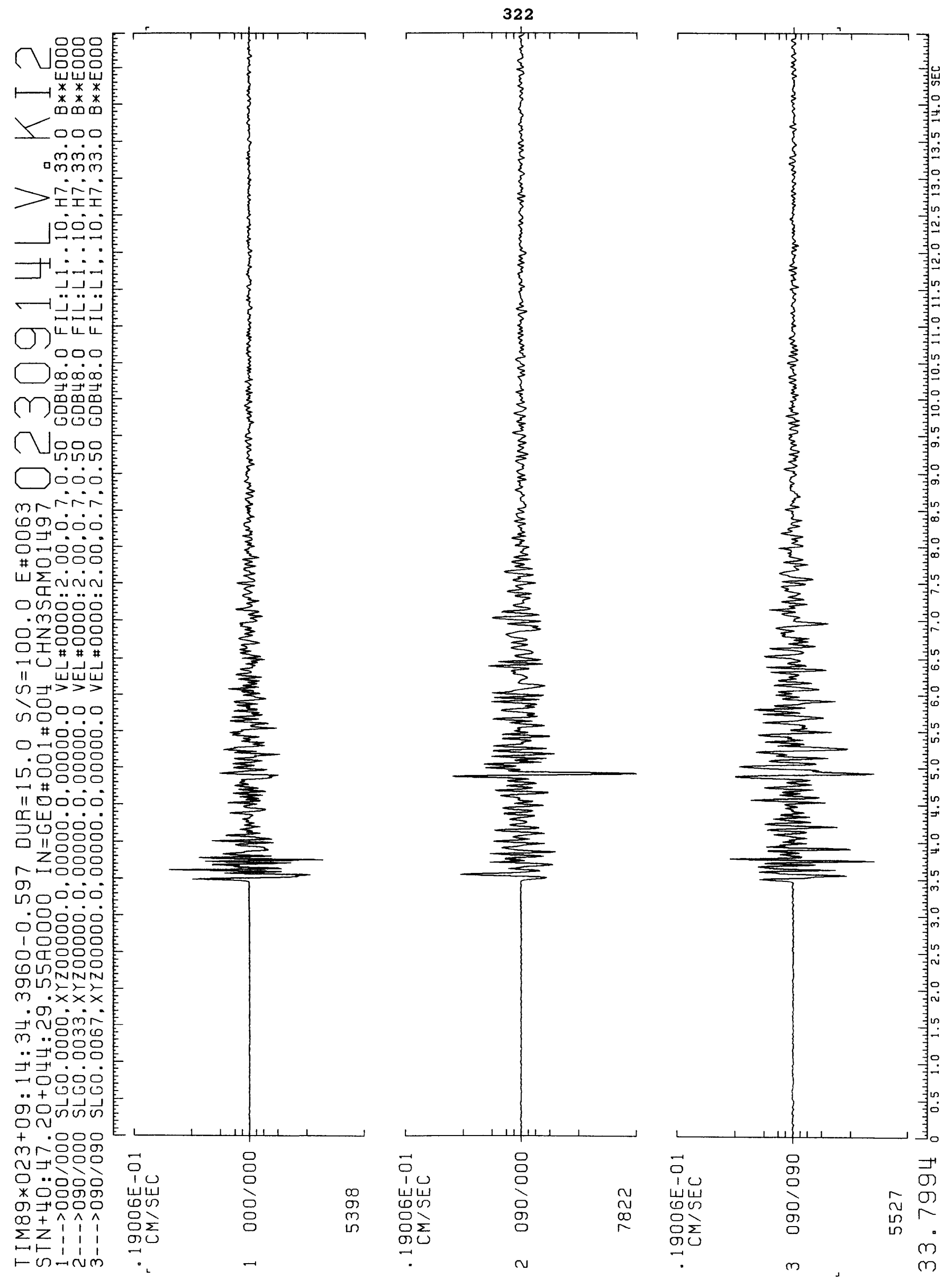




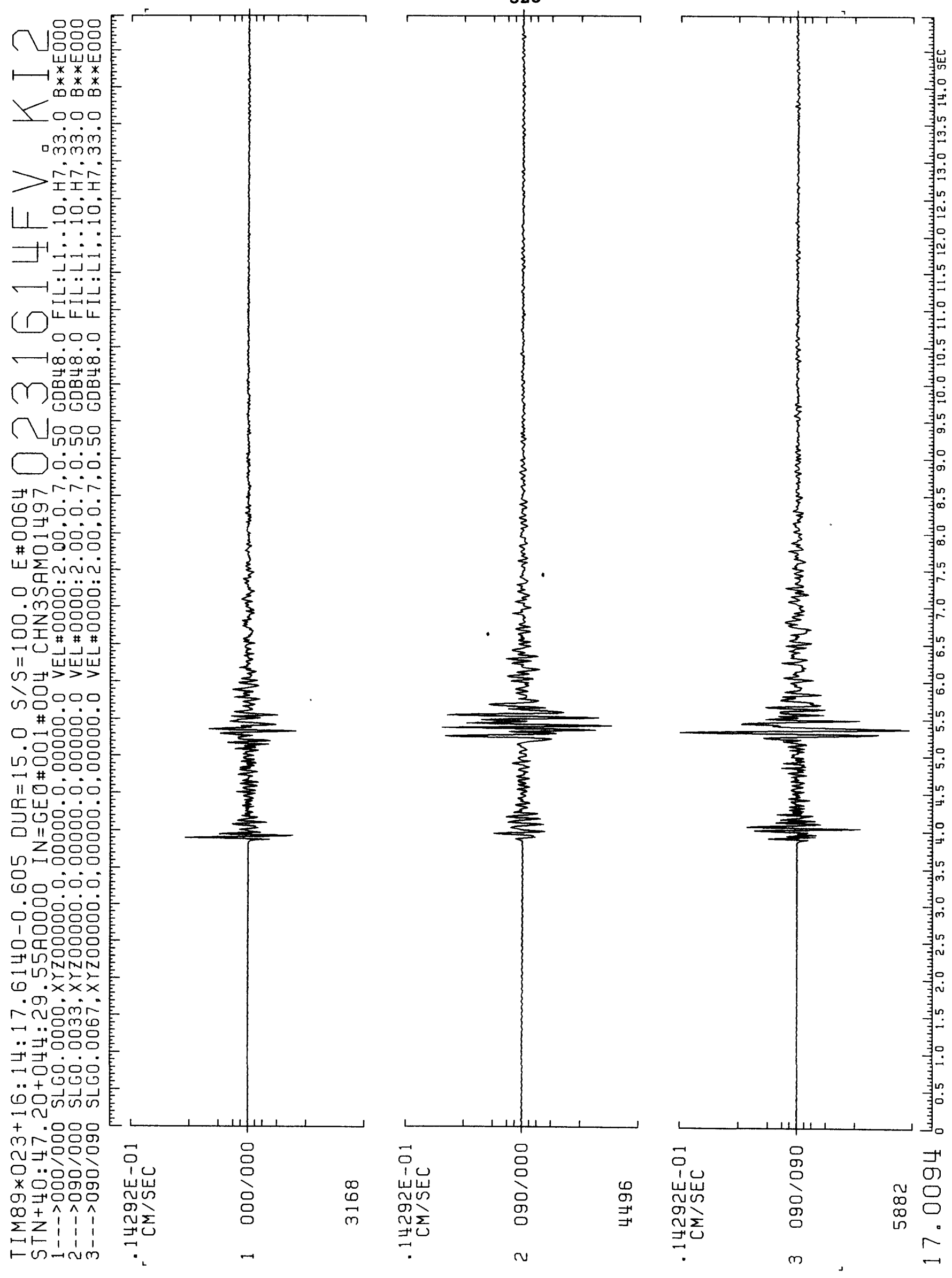


324
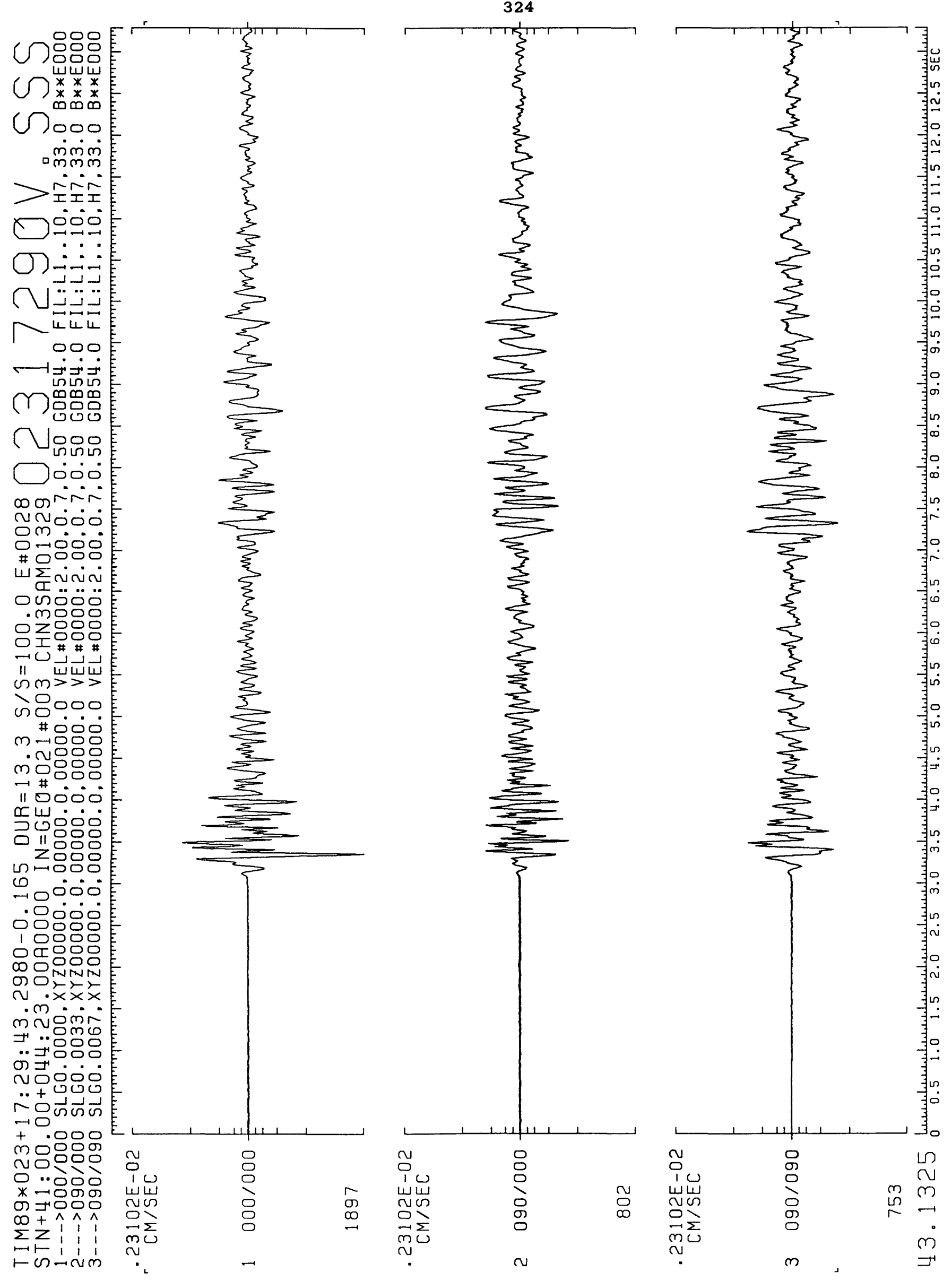

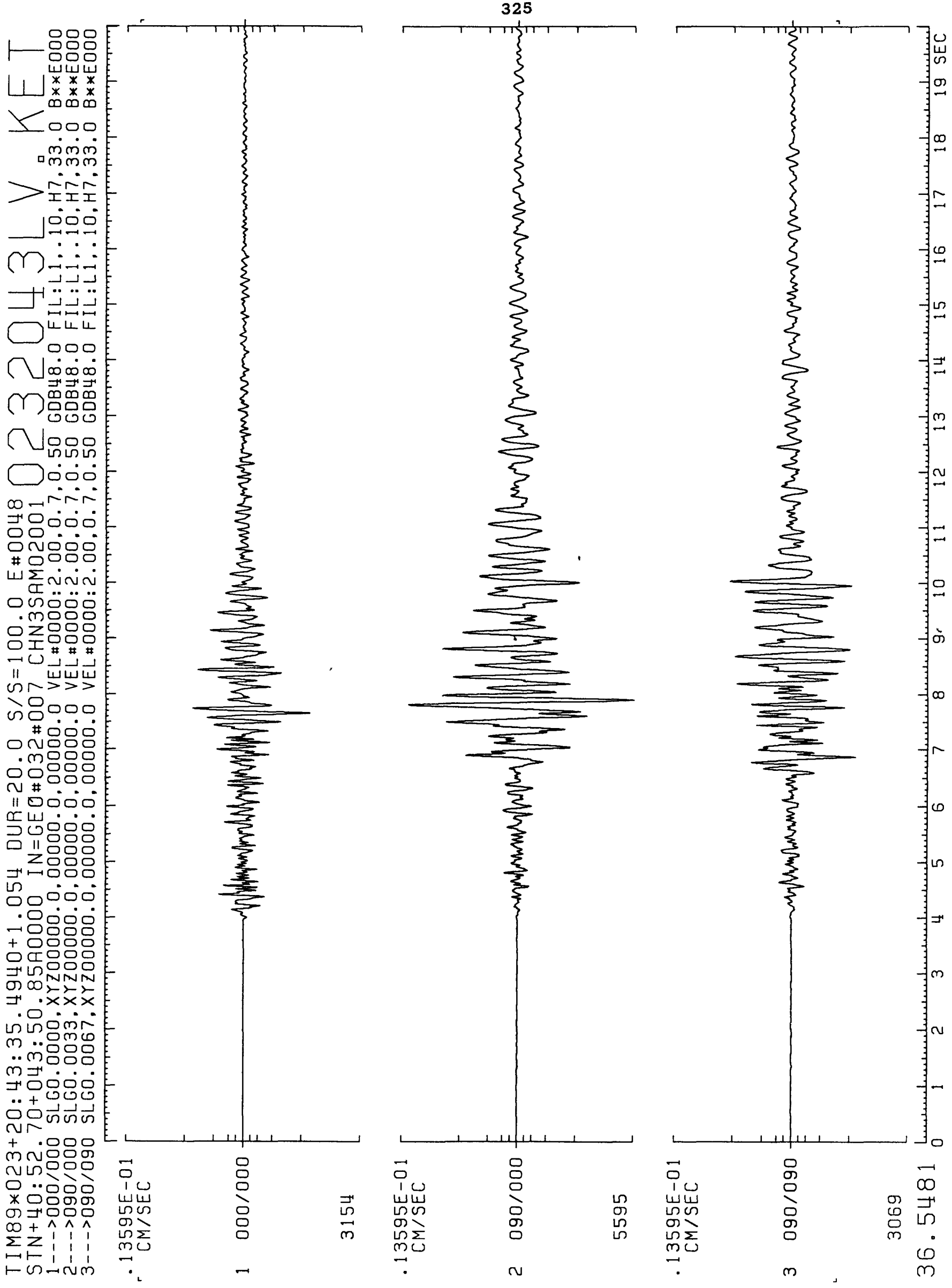


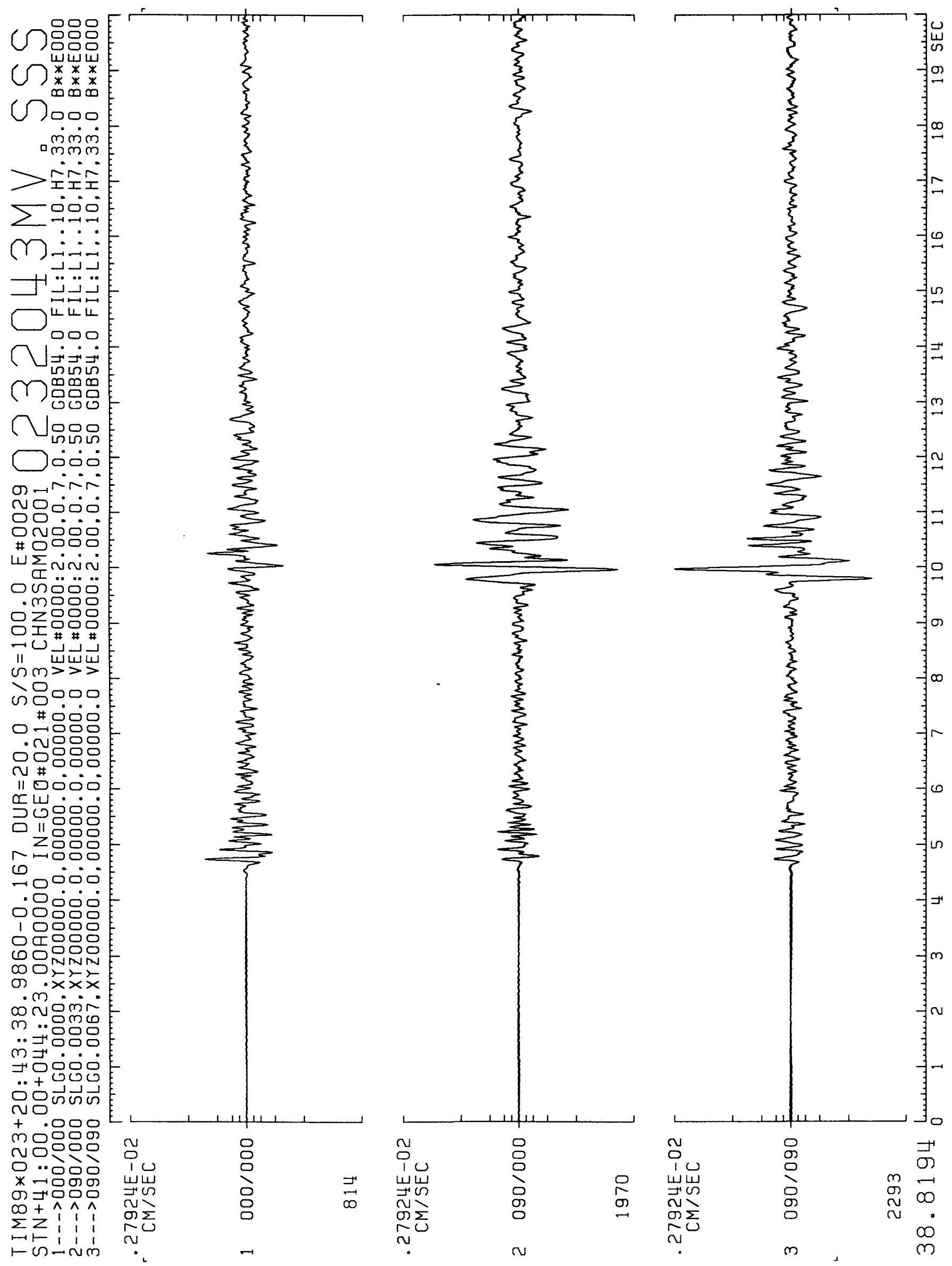



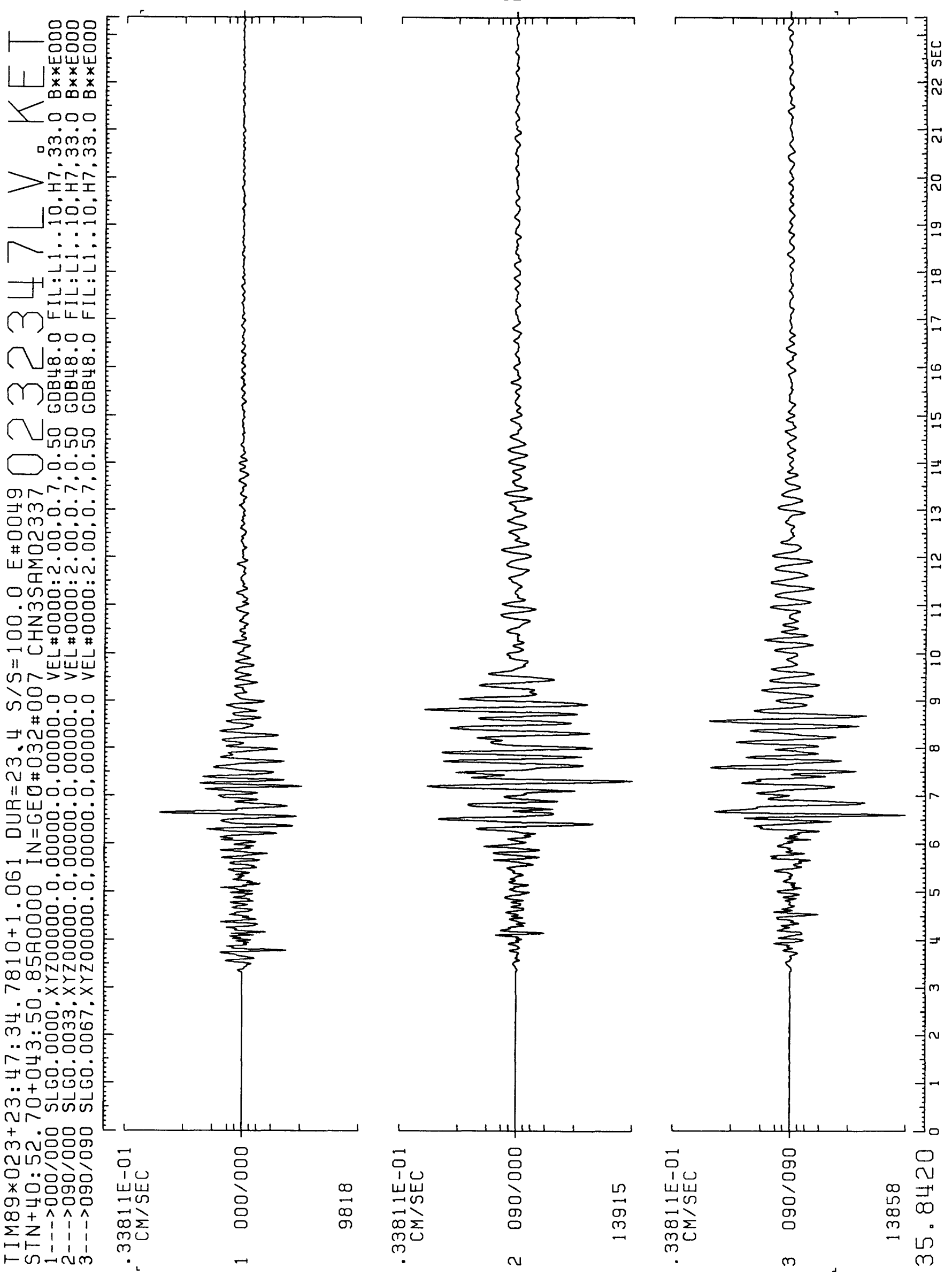


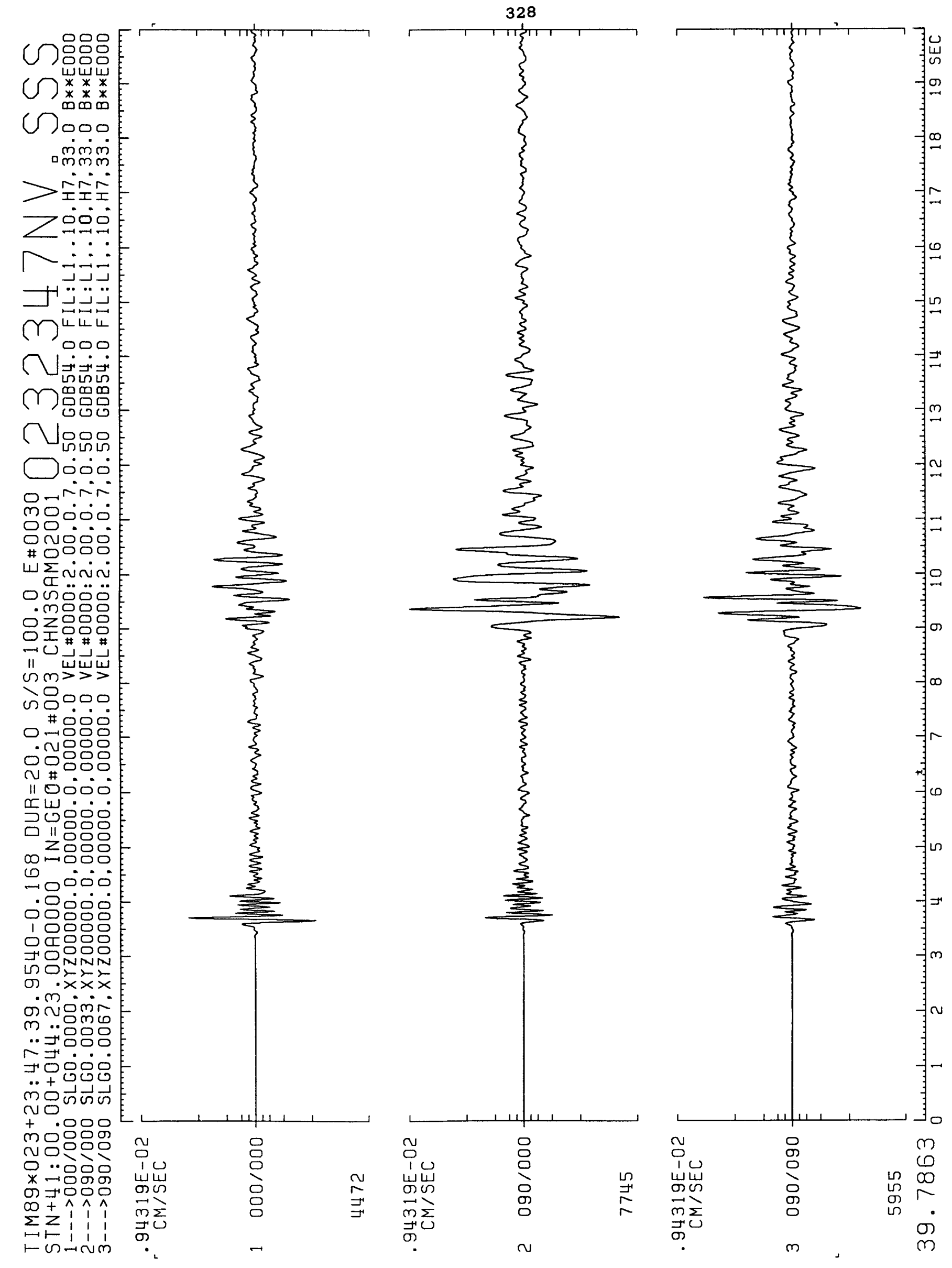




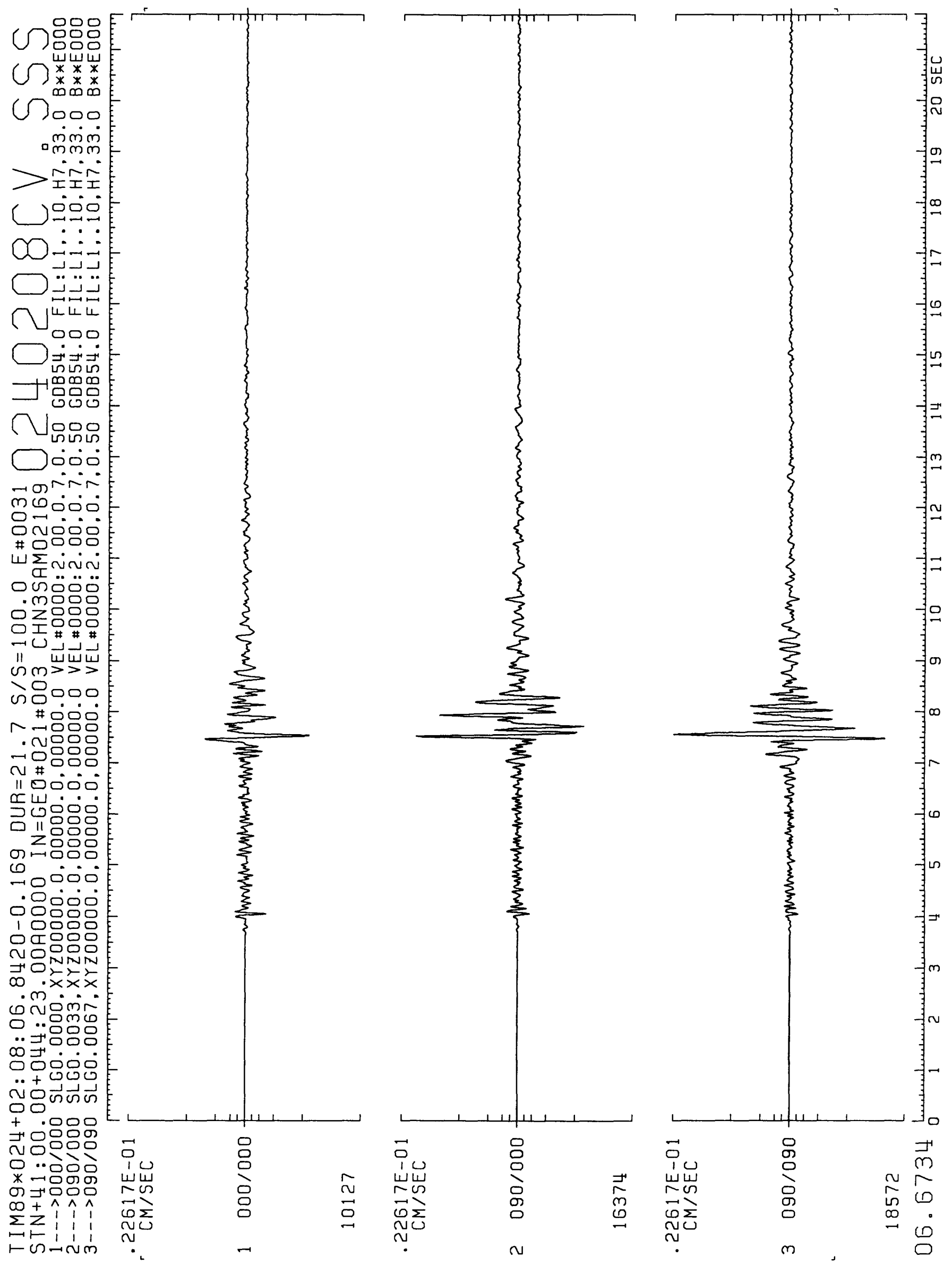



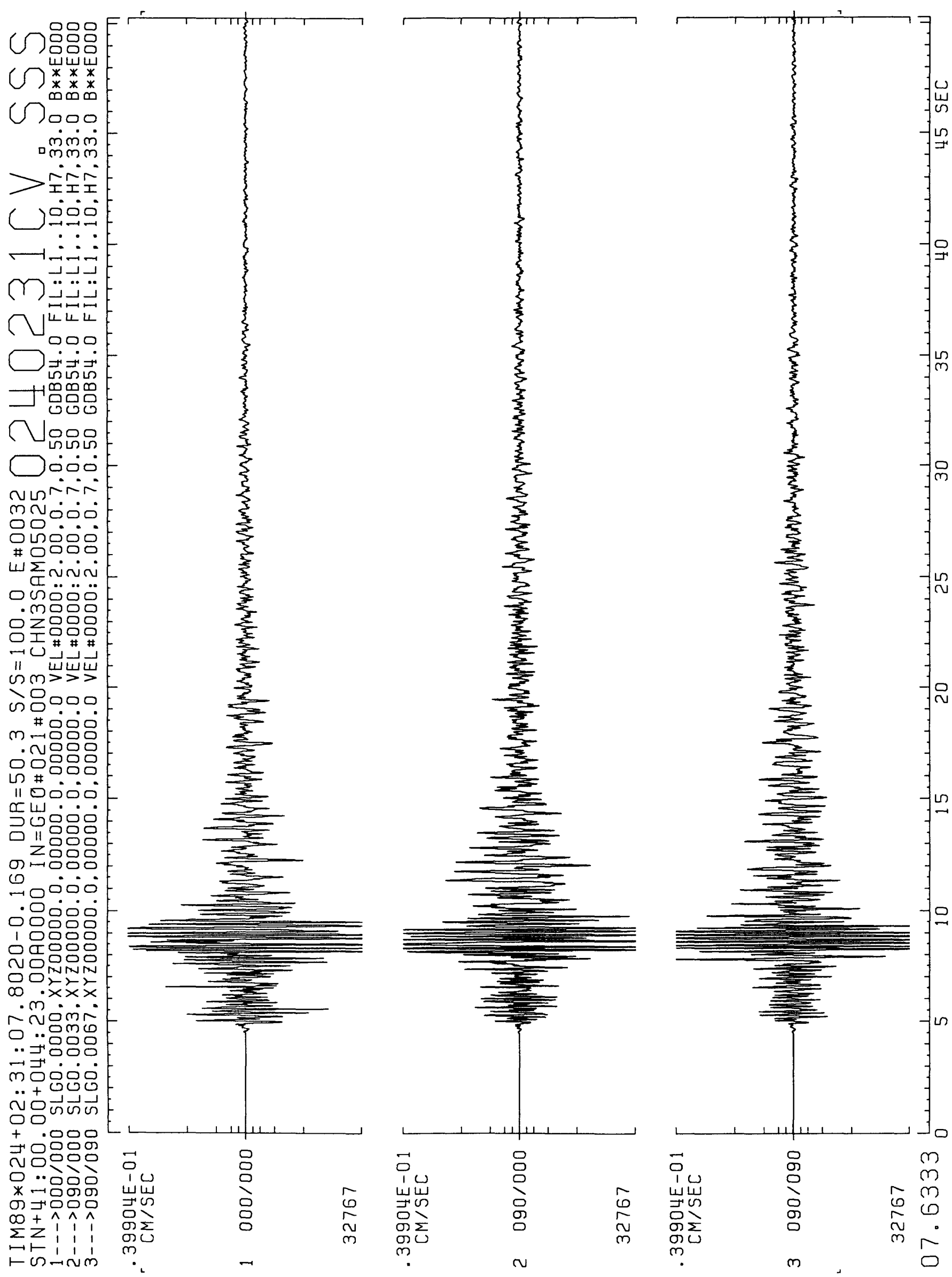

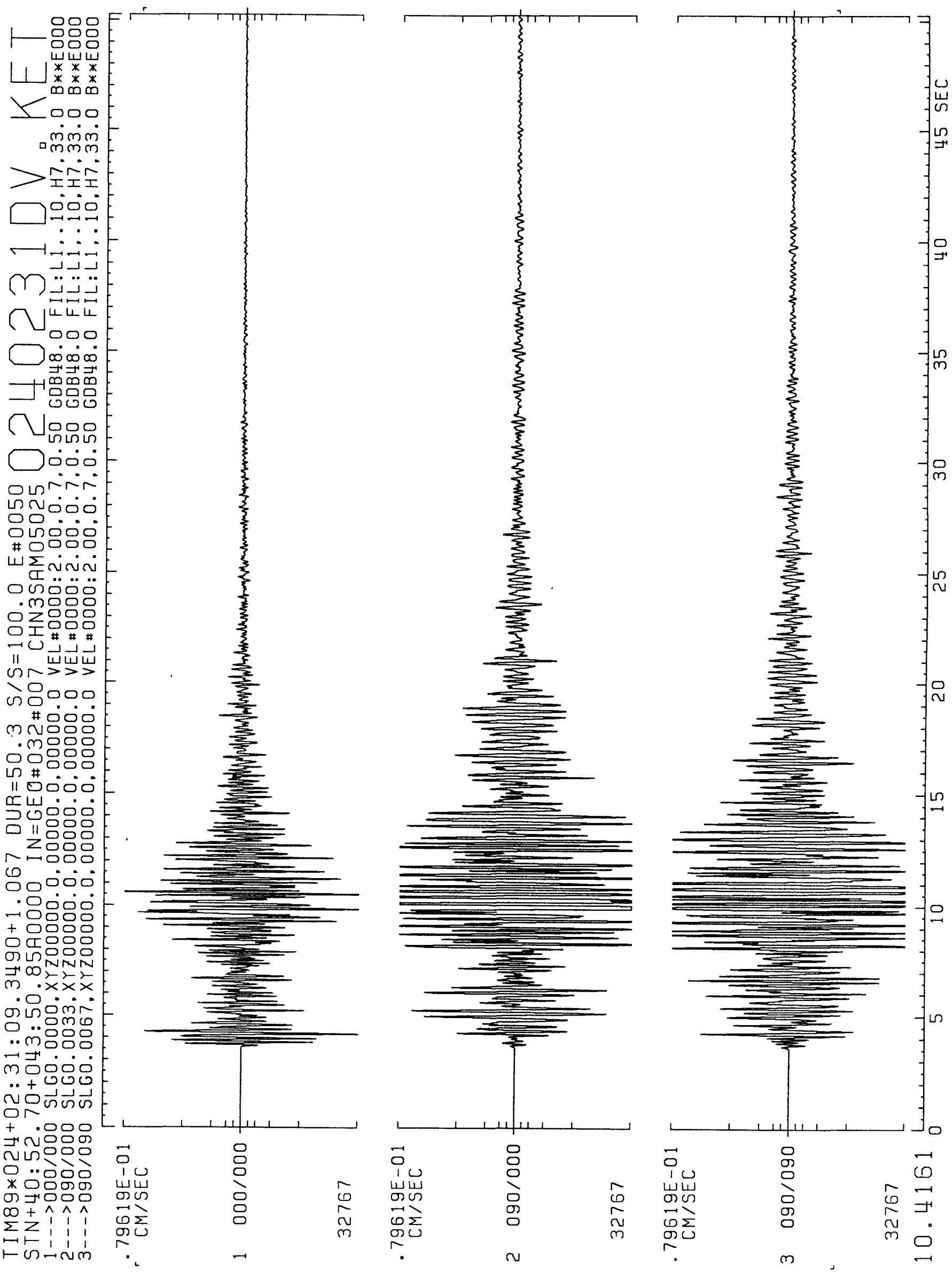

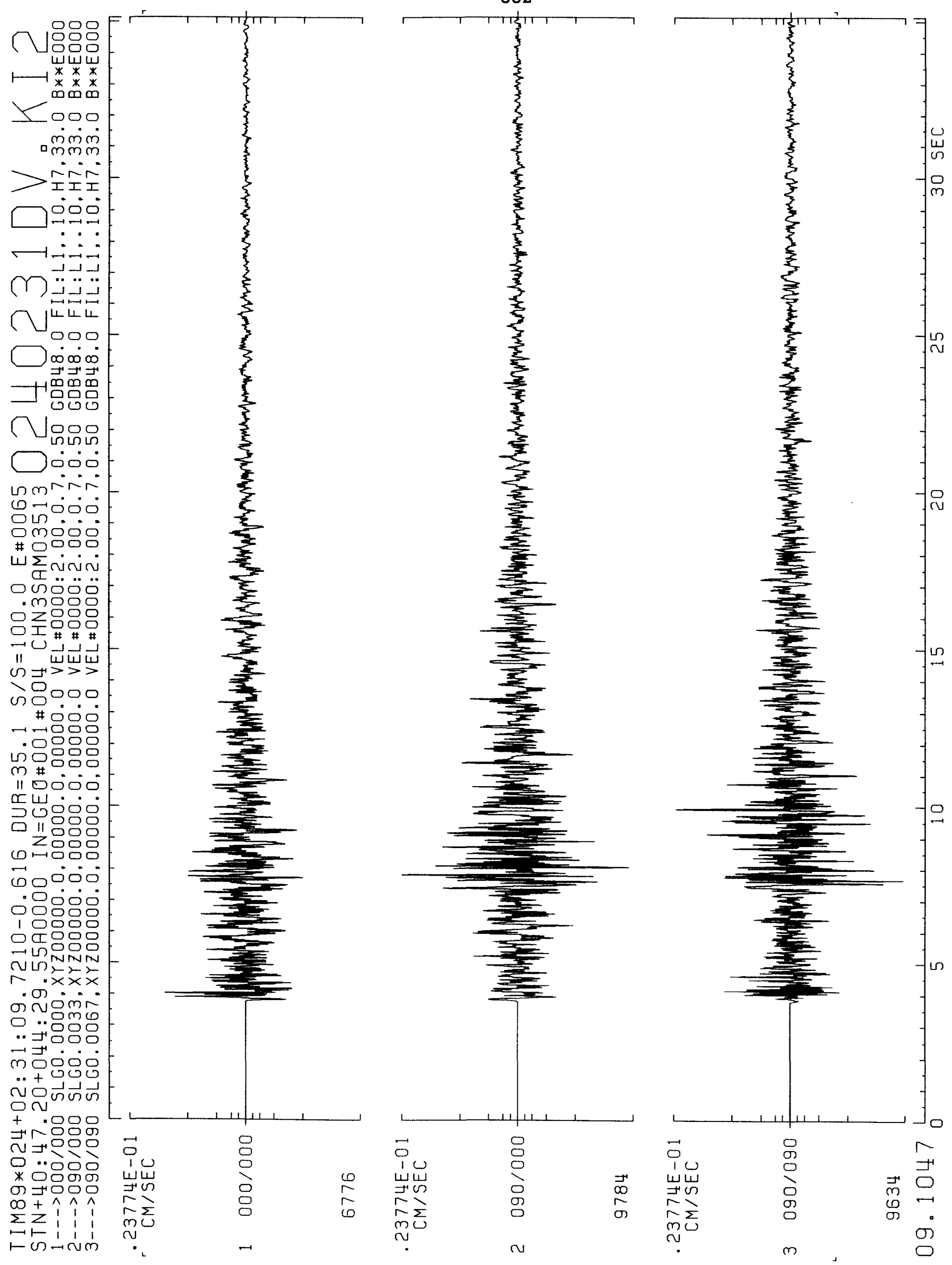
333

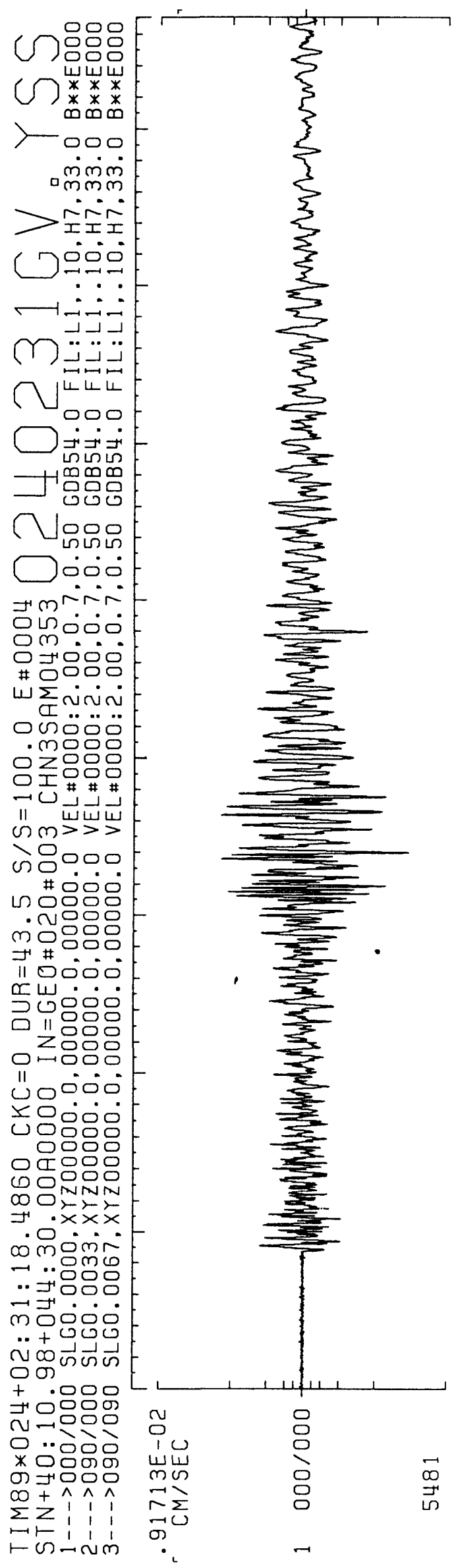

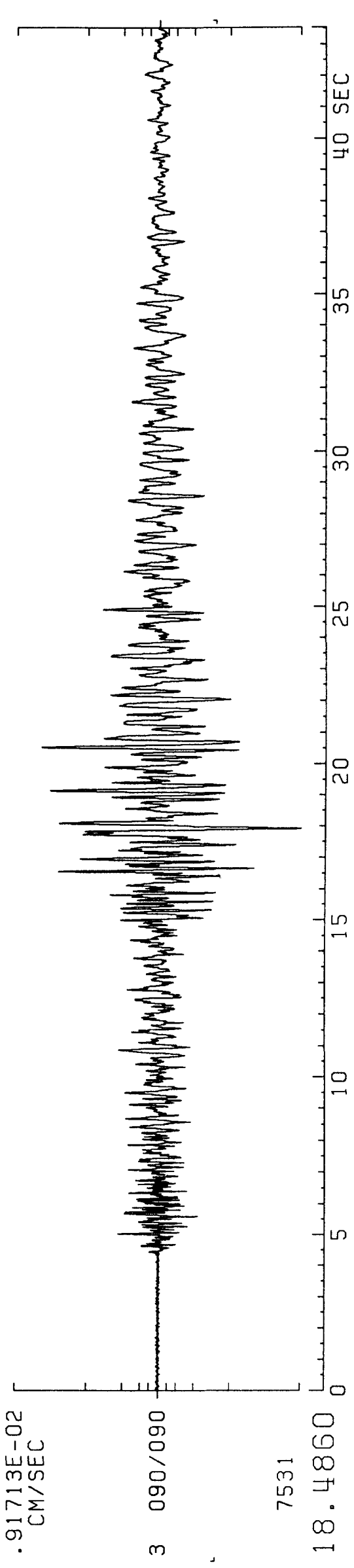


334

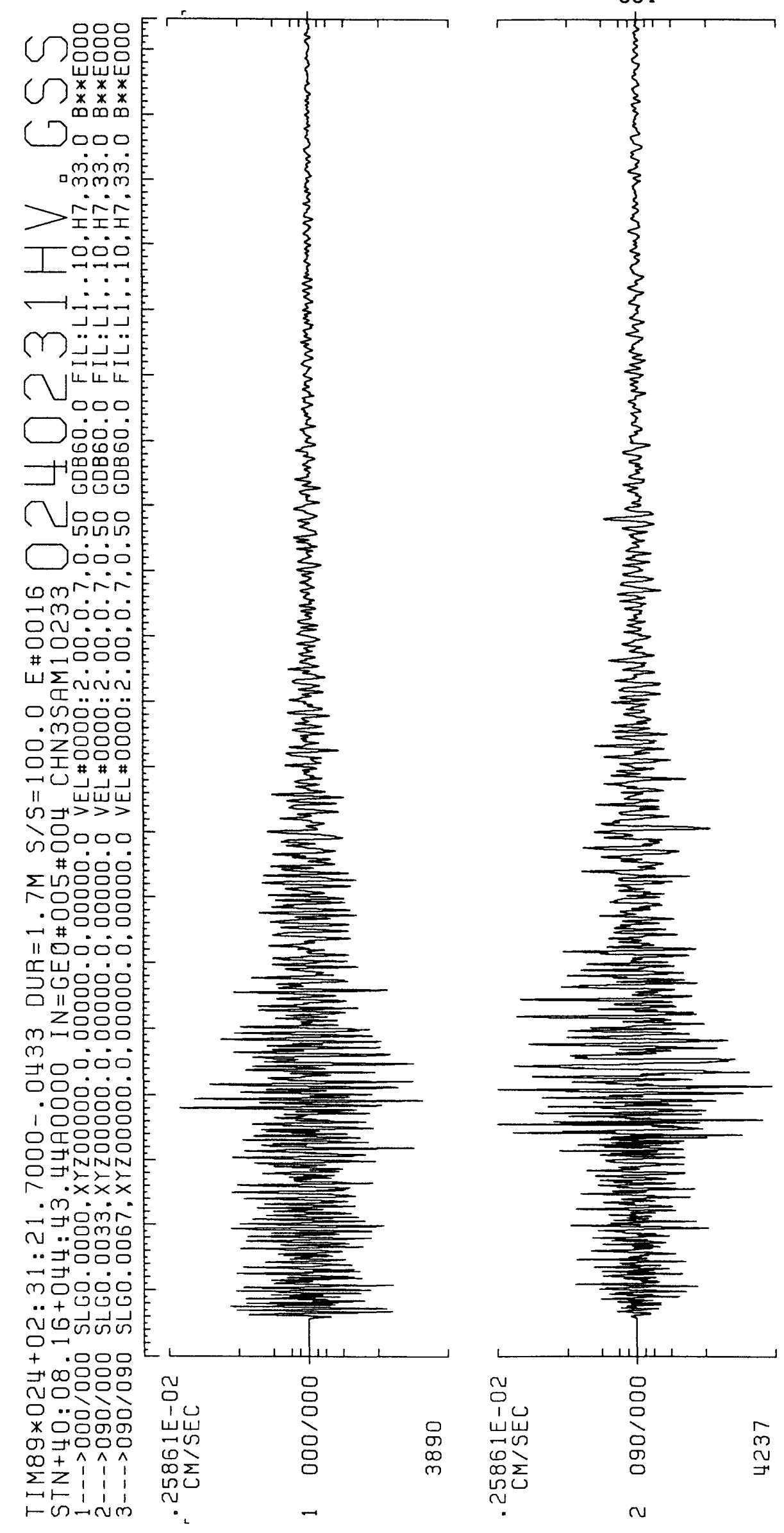

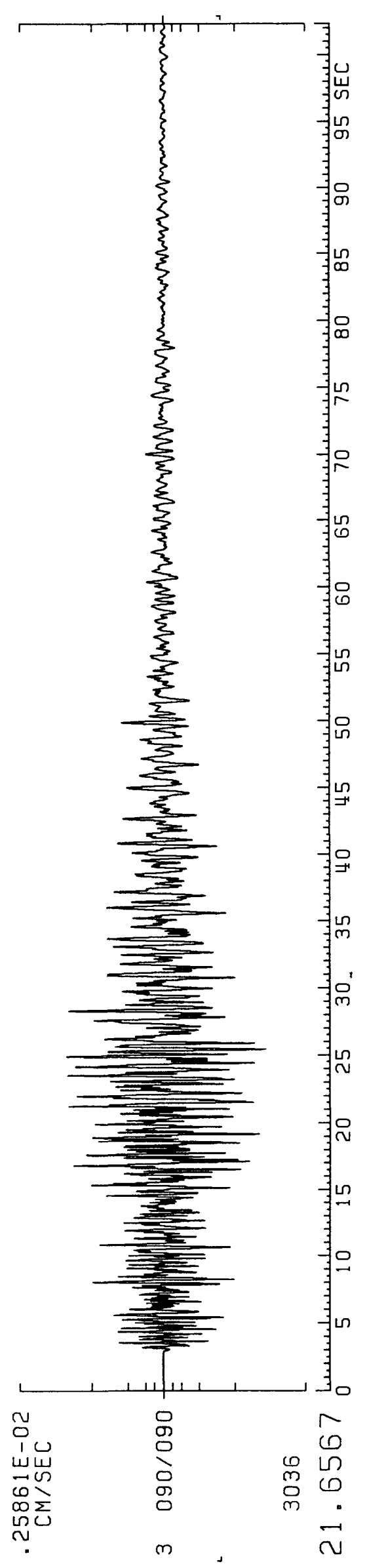




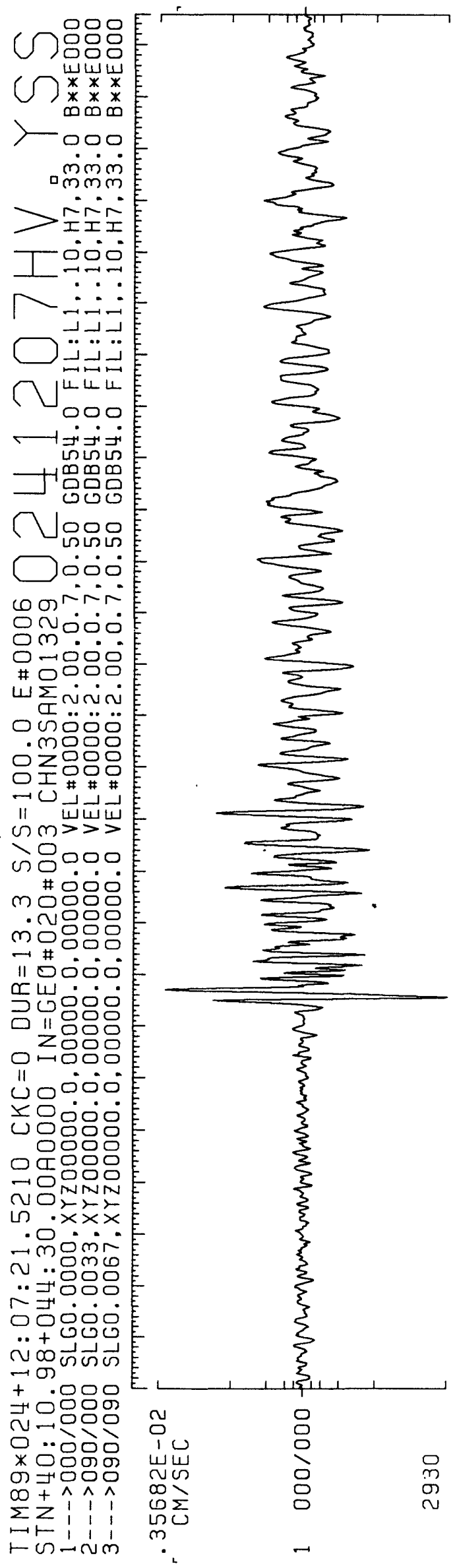

335

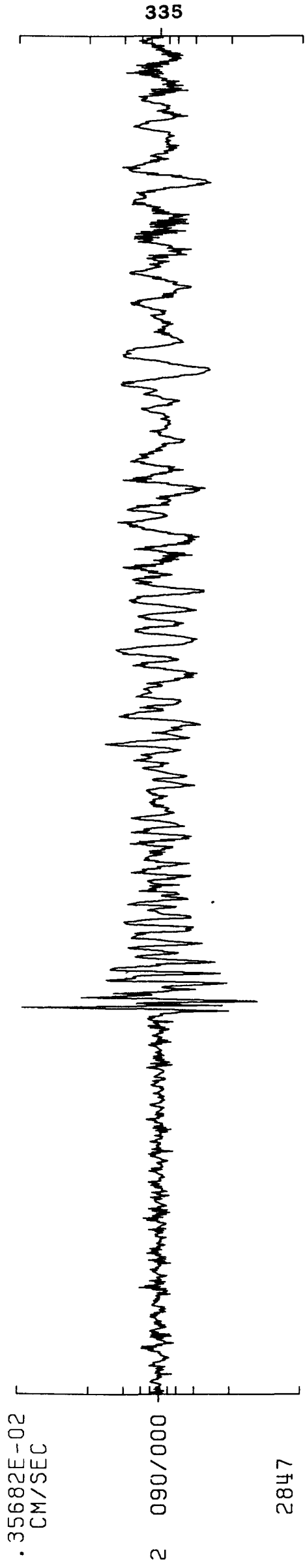

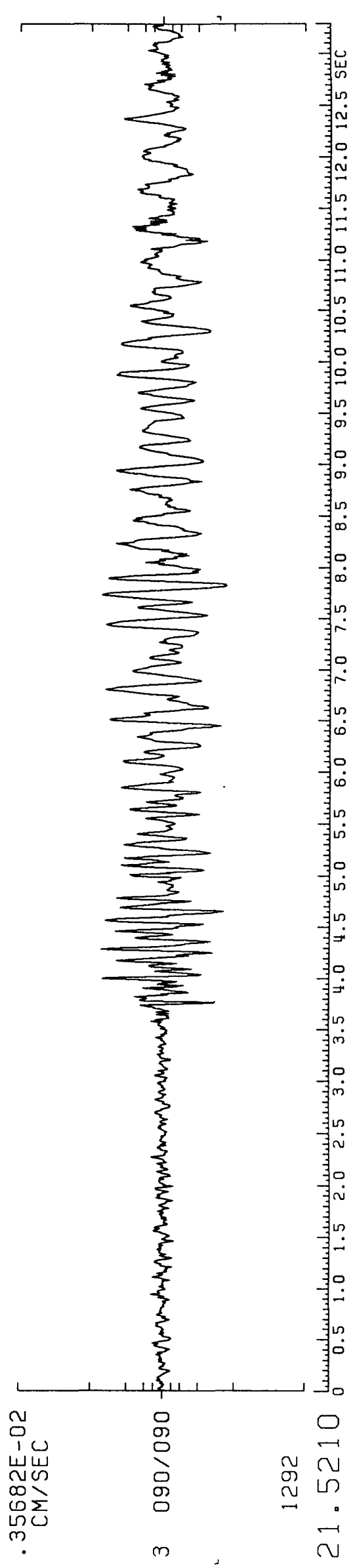




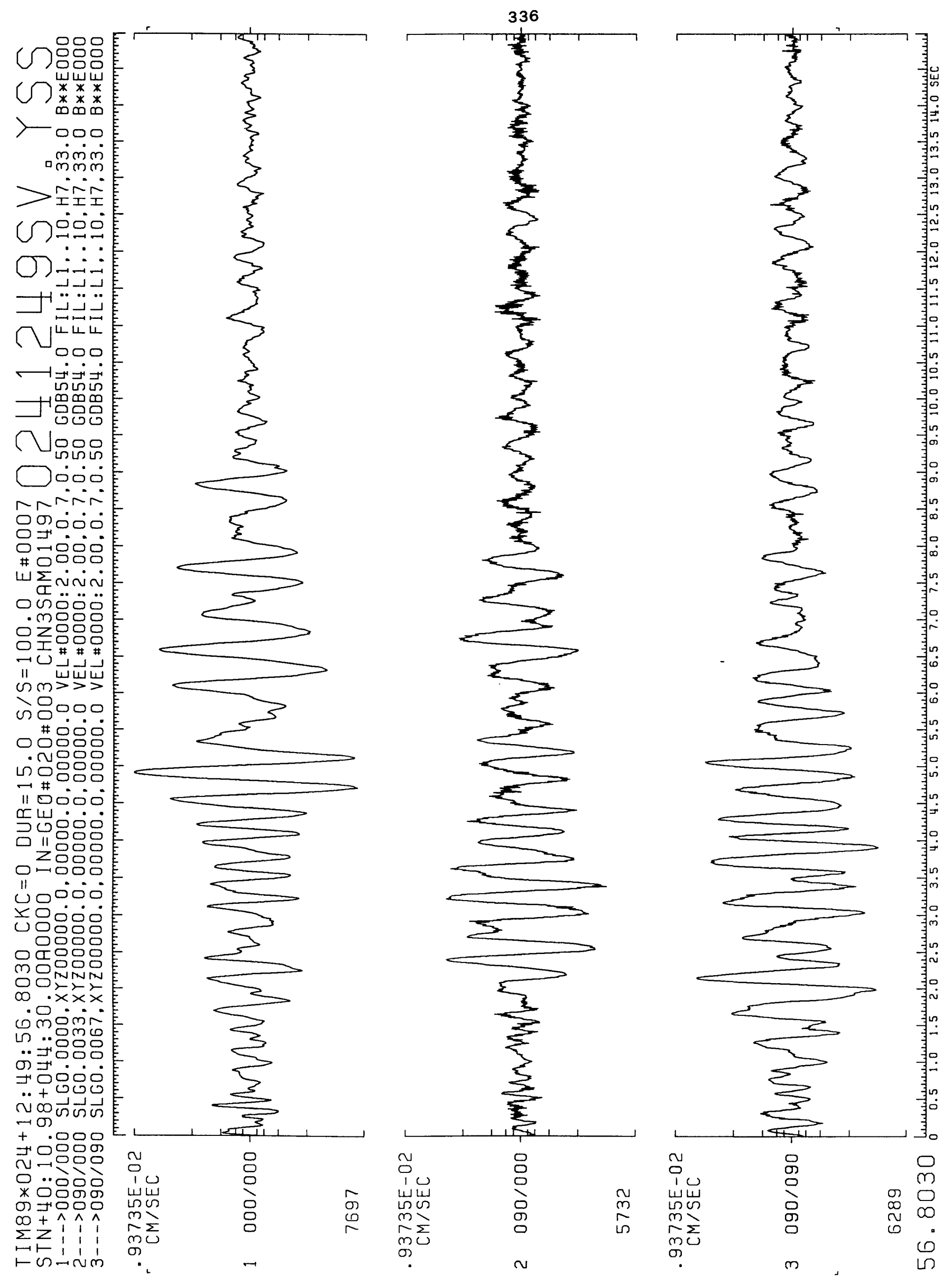




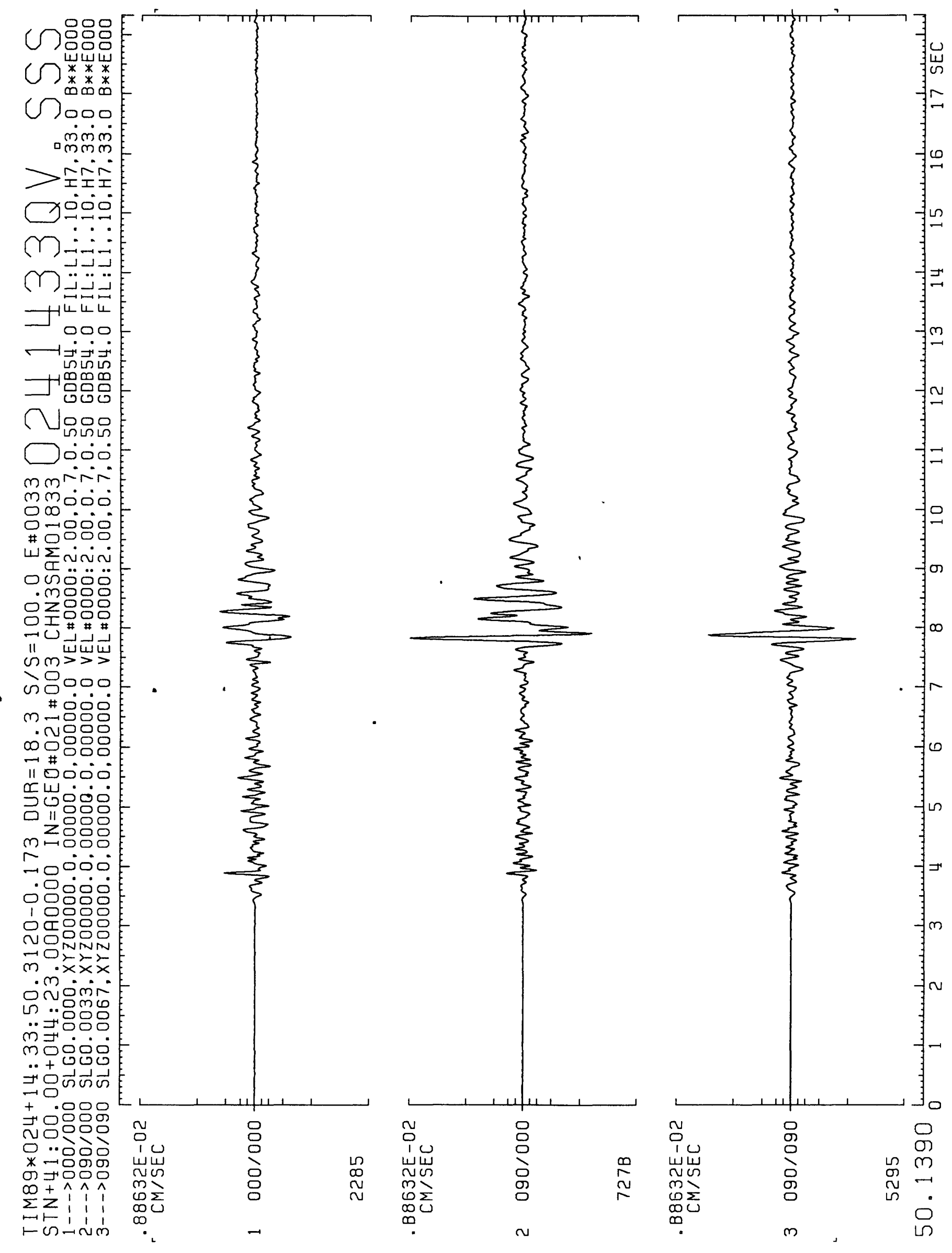


338

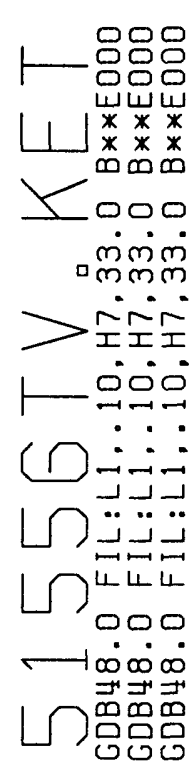

\络品 $\square \circ \dot{0} 0$ trinis

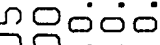
ธกัจ \# एะ oin. - mㅇㅇㅇ 중요 든 "1. य山山 जn>>> in 0000 ऊँ №ด - Mo 00808 落

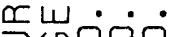
בิำ z咯品 $7-000$ $\rightarrow 0000$ 7000

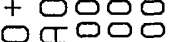

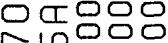

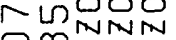
N $x^{\infty} \times x$

- 0 imi I .. .. m⿺辶万

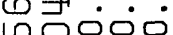
no.000

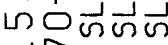
+ +. ए人iogo บino음 …잉 * 잉

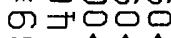
$\infty+\wedge \hat{\wedge}$ $\sum \sum 1$ एल

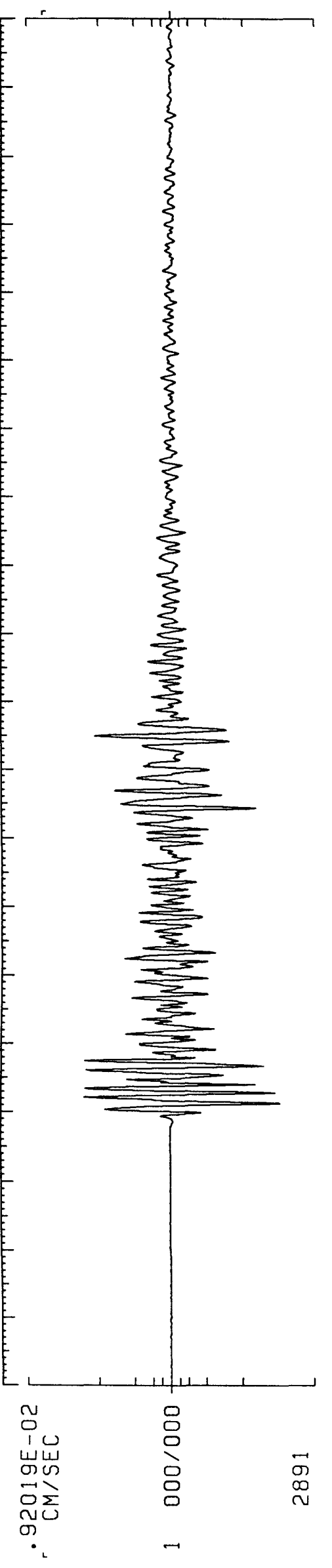

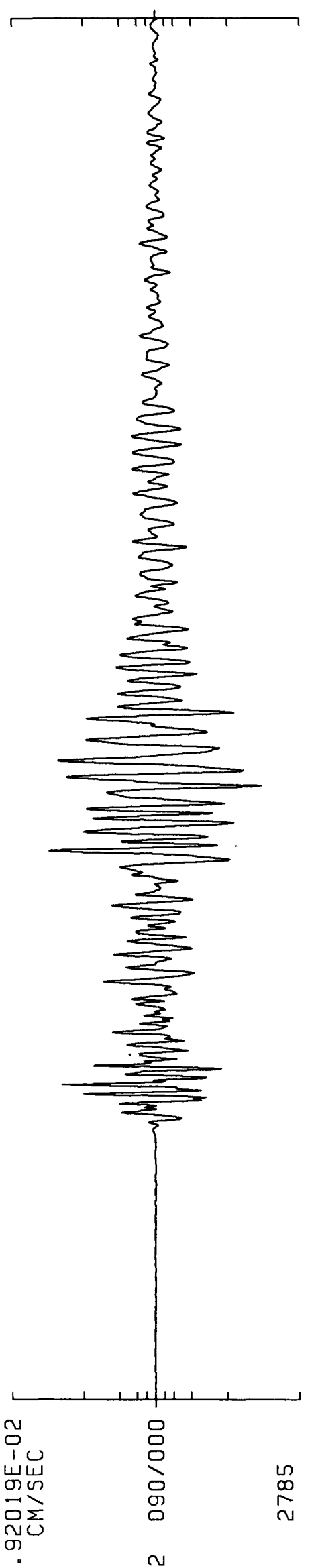

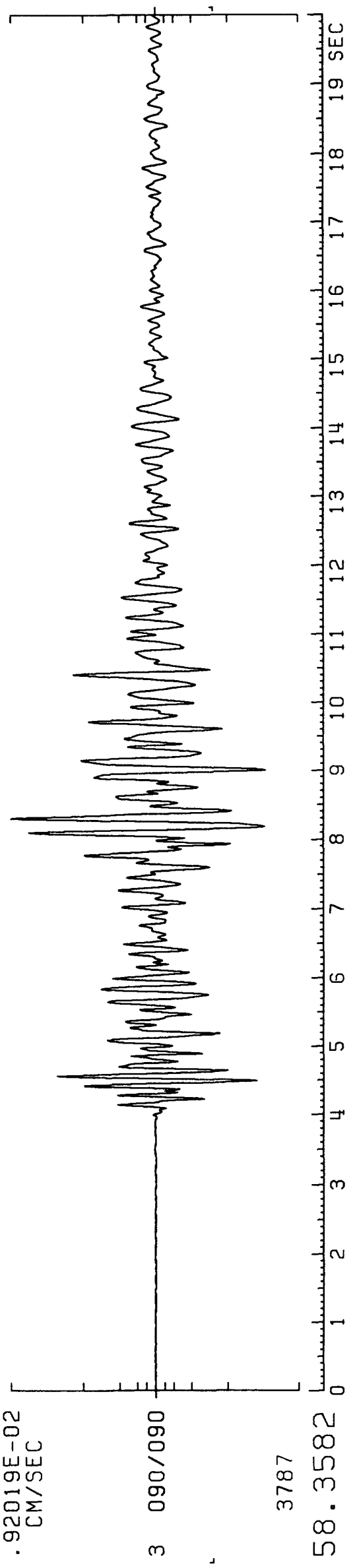


339

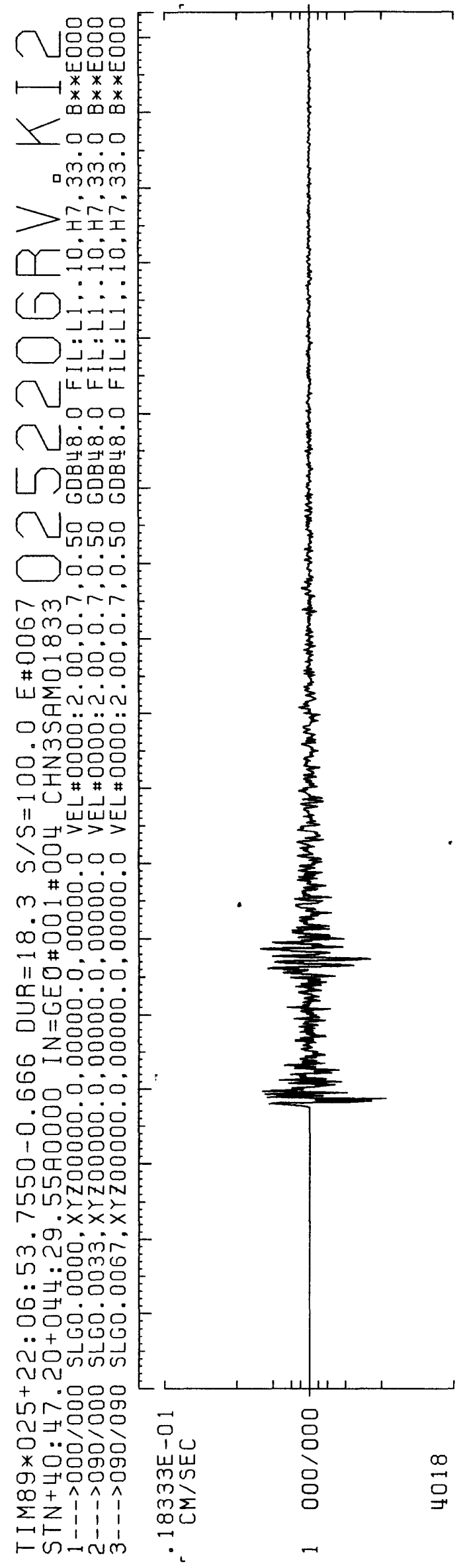

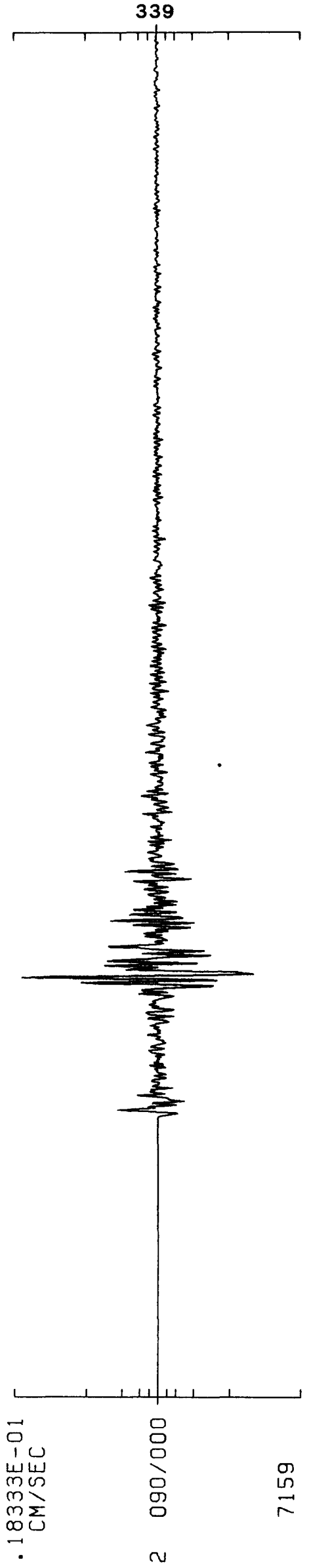

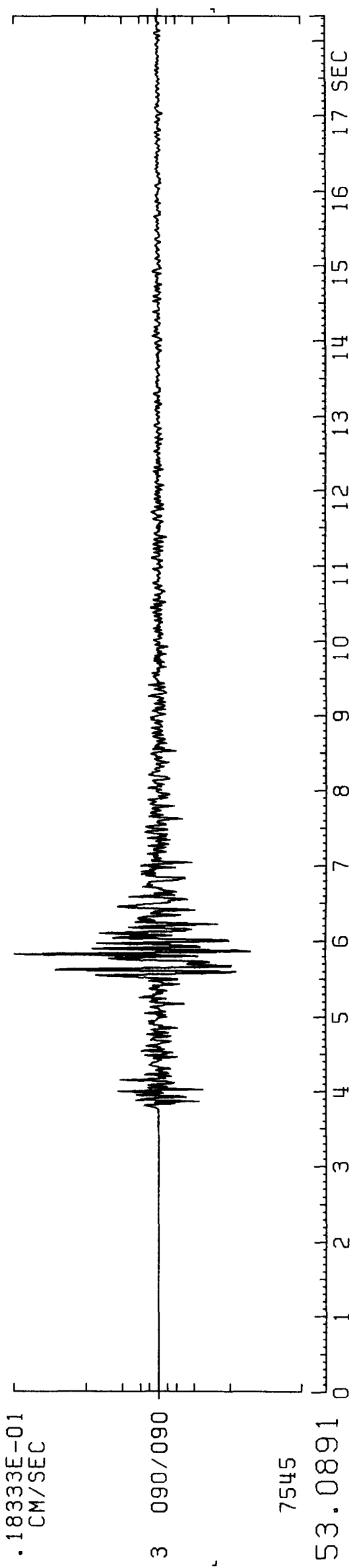




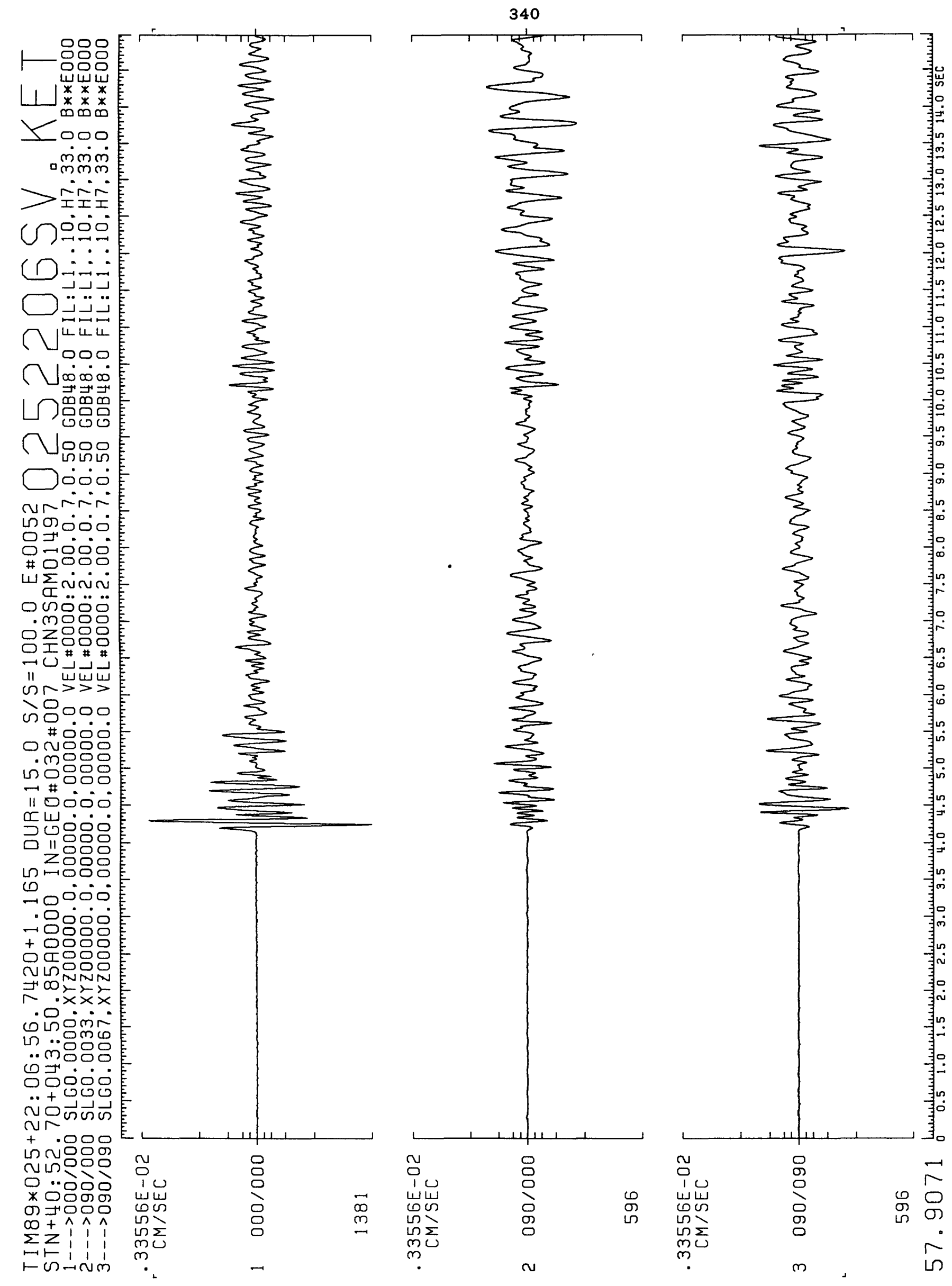


341

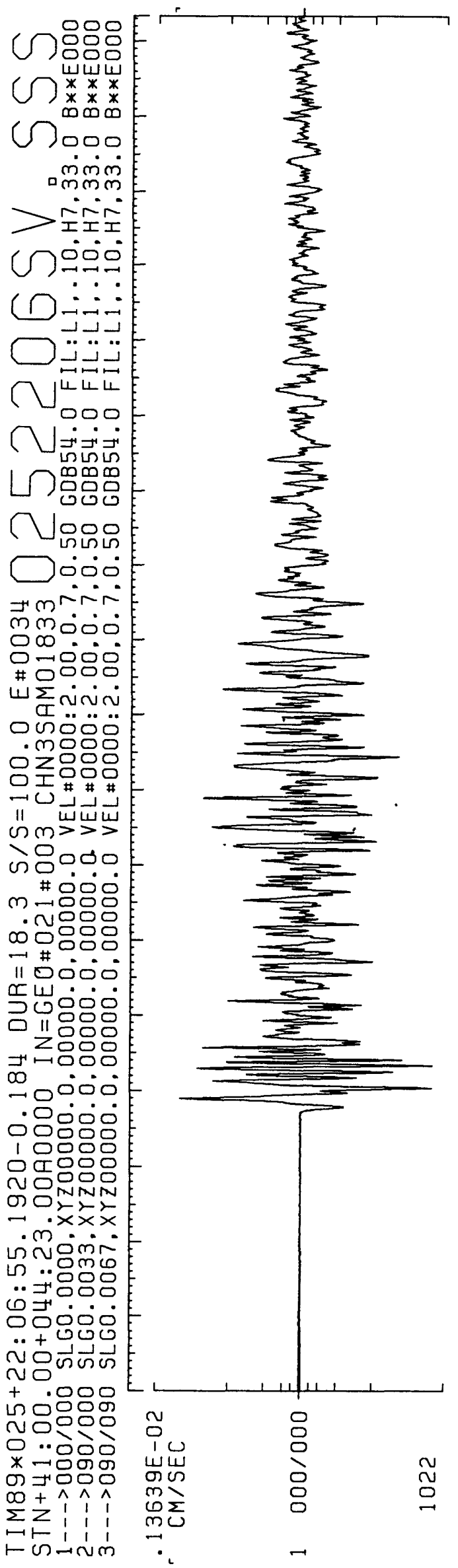

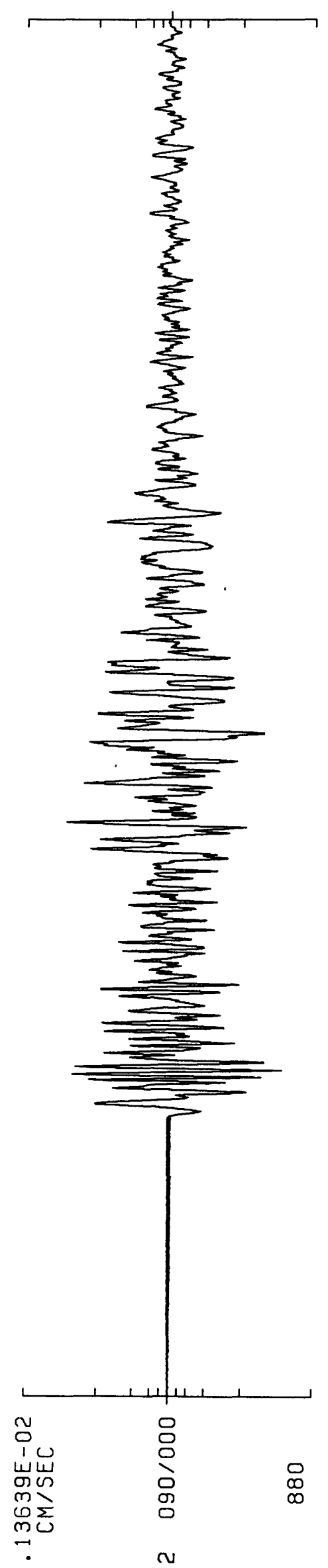

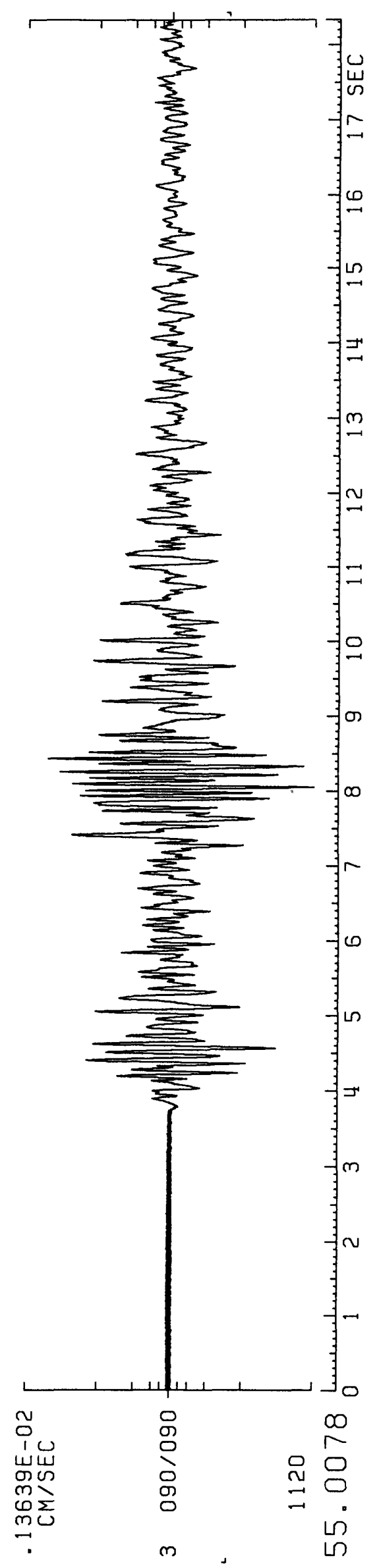




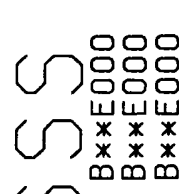

ODo00 mंm. वलm rin III $\circ 0^{\circ}$

O : : 济 … $=-1$

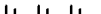
○० . ブゴ (口田田 엉

Vonon  mธं் 웅요 \#

ए $\sum_{n}$ in 등... - moㅇㅇㅇ 긍ㅇㅇㅁ음 ○工巩 可山出 m $m$ जेO000 \# ๑-7ㅇㅁㅇㅇㅁ Nㅣㅇㅇㅇ 음ㅇㅁㅇㅇㅛ $1100^{\circ}$ 뚱ㅇ 깅ㅇㅇㅇㅇㅇ zoㅇㅇ $\infty$ అ๐०० $\infty$ $\rightarrow 000^{\circ}$ - Oீं0்

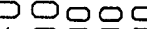
1 응ㅇㅁ음 엉ㅁㅇㅁㅇㅡ. OONNAN o . xx - m تn जom ง ..요요

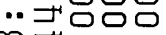

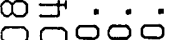
+

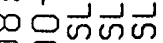
잉ㅇㅇ 당ㅁㅇㅁㅇ กo임 …잉 ต $\infty+\wedge \wedge \wedge$ $\sum z ! !$ キ上

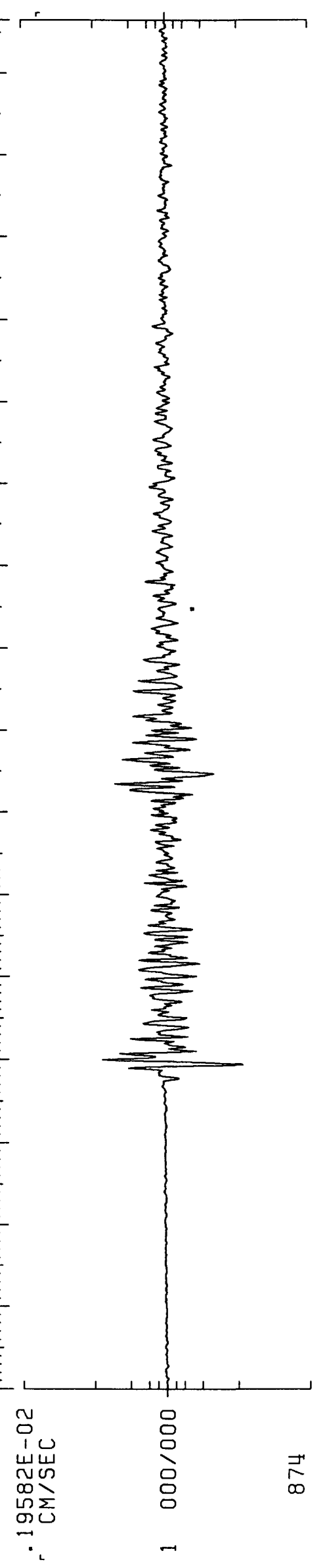

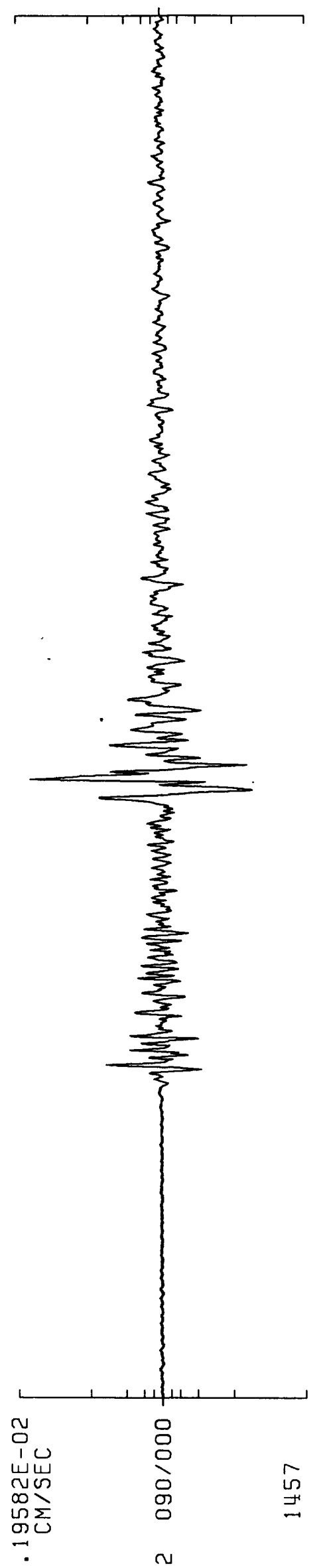

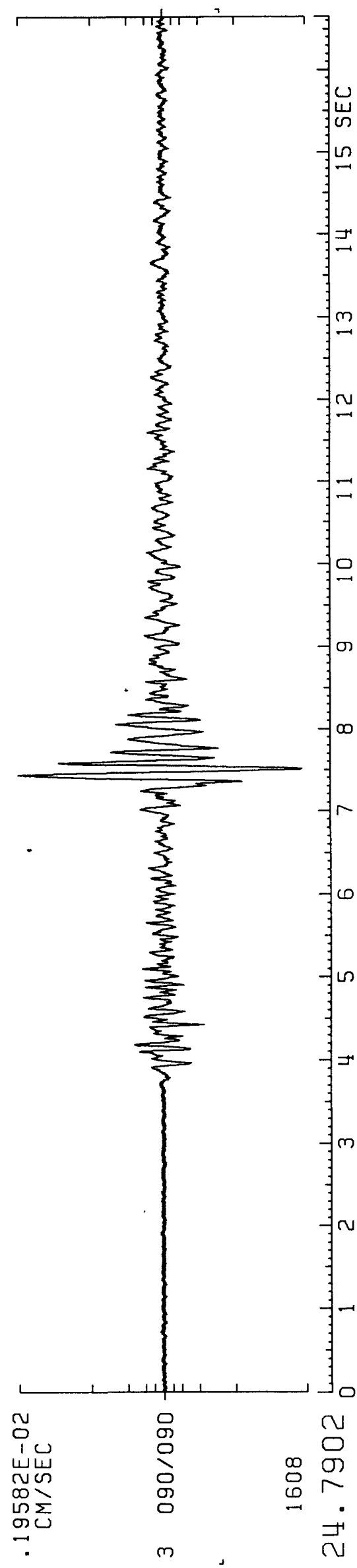


343

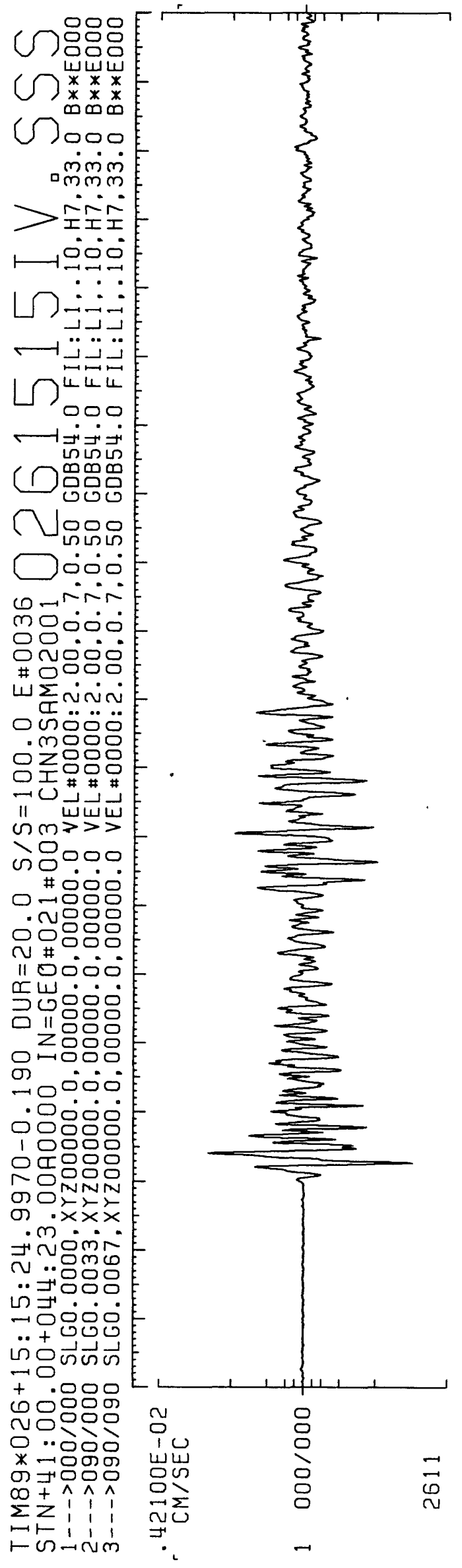

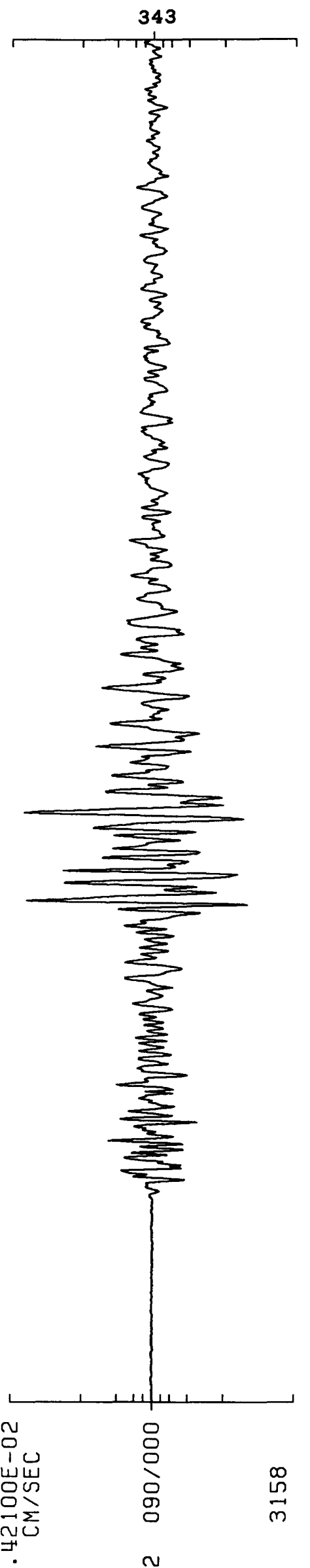

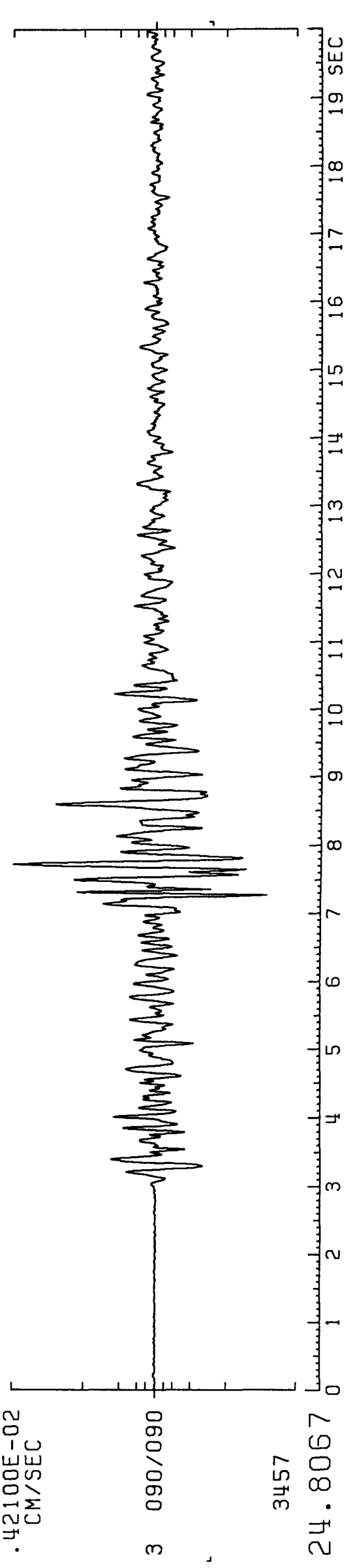




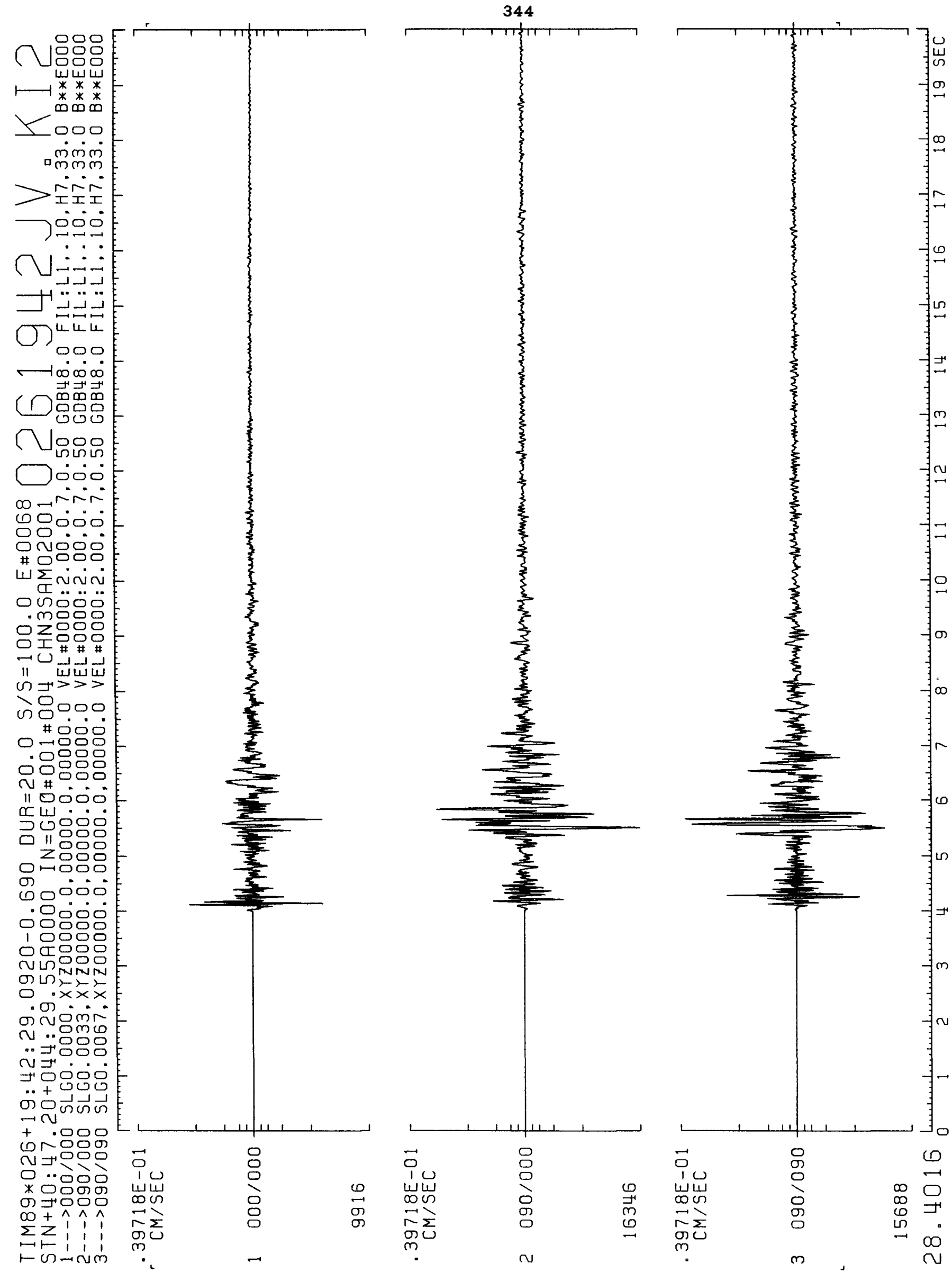



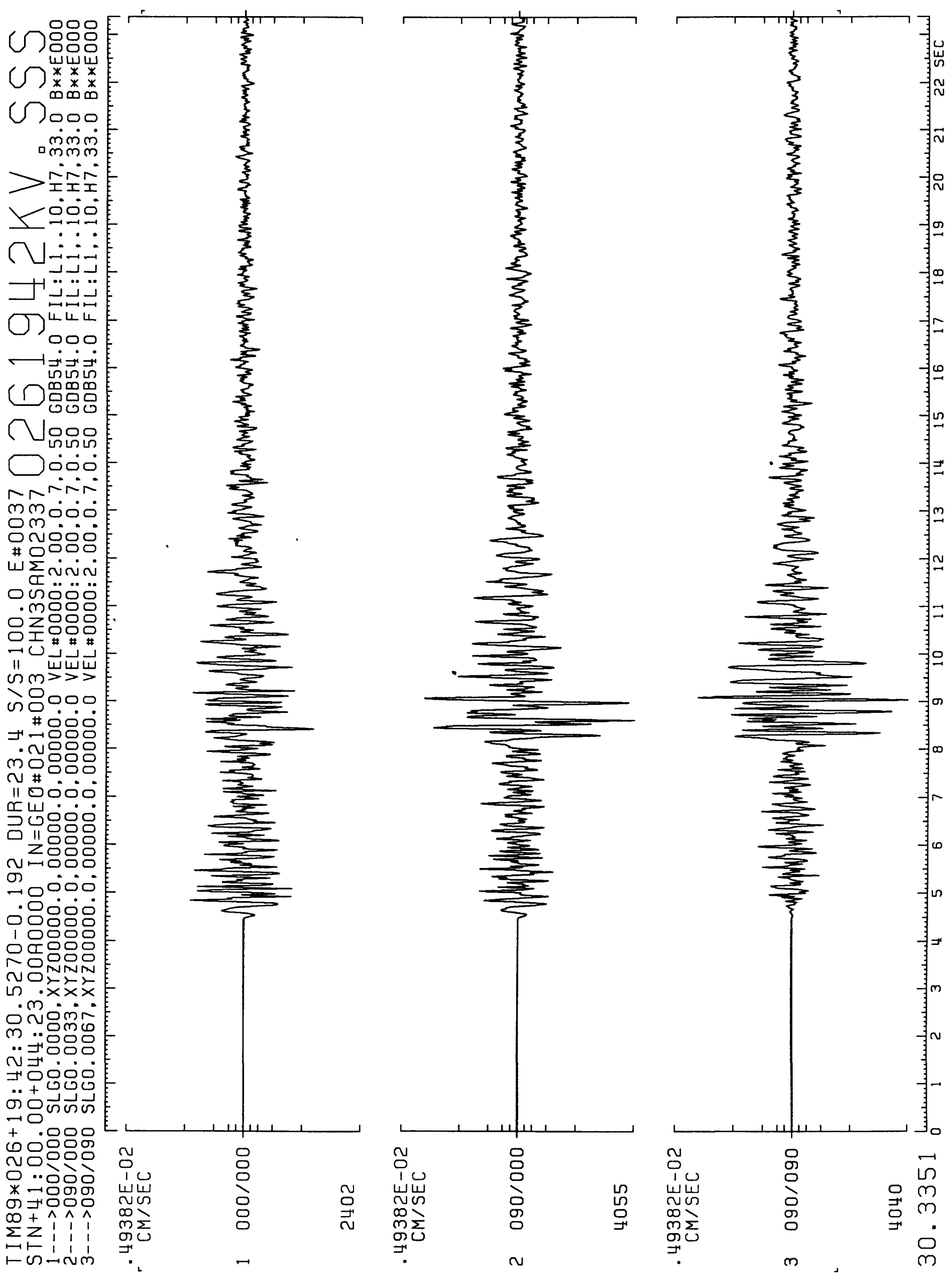


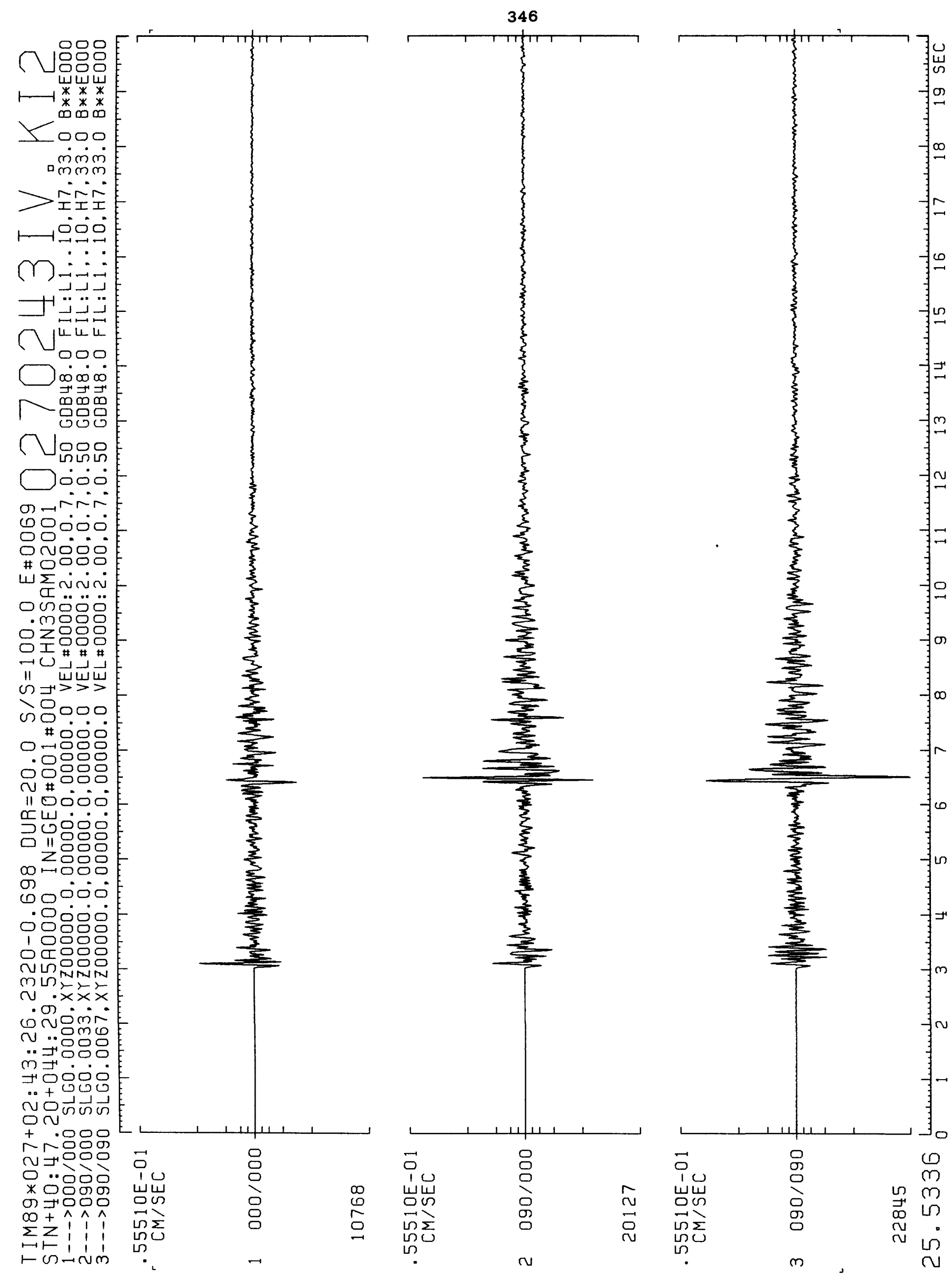




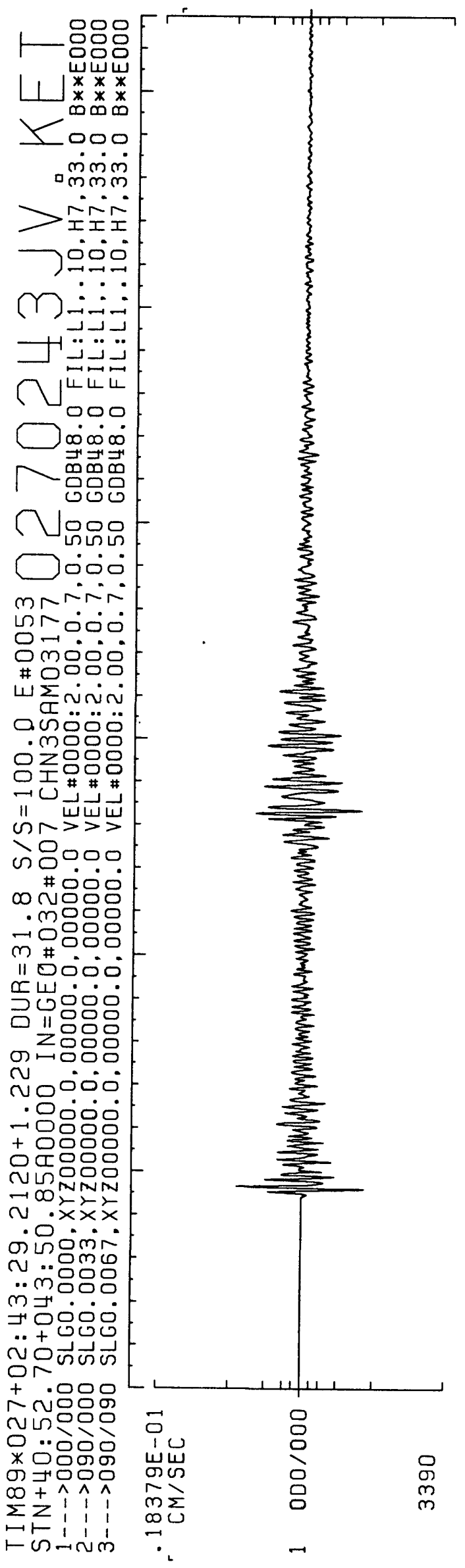

347

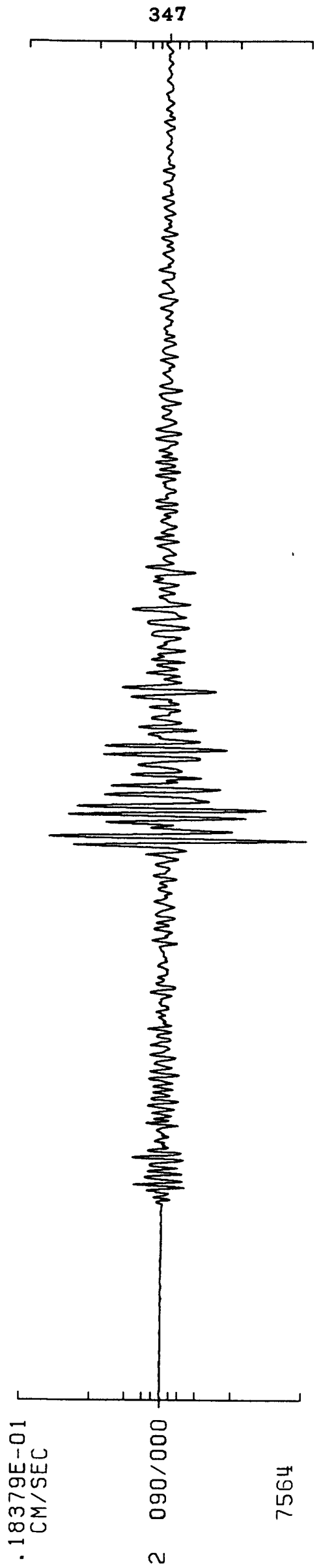

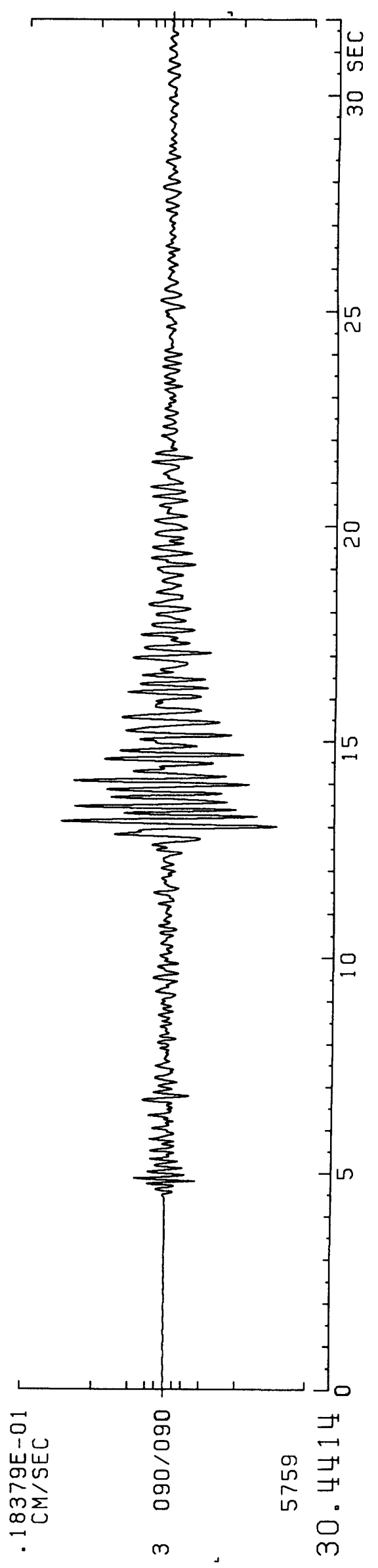



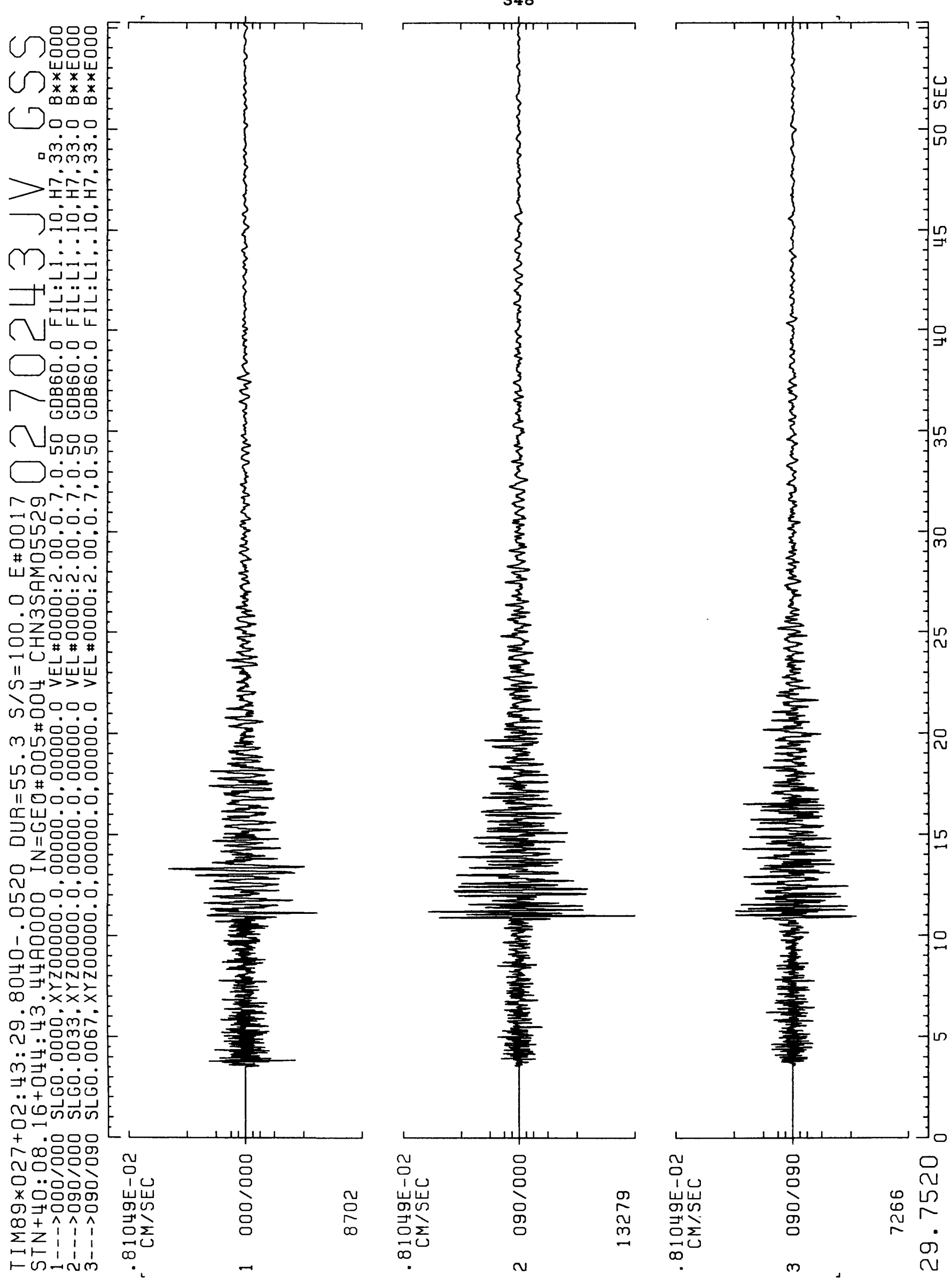
349

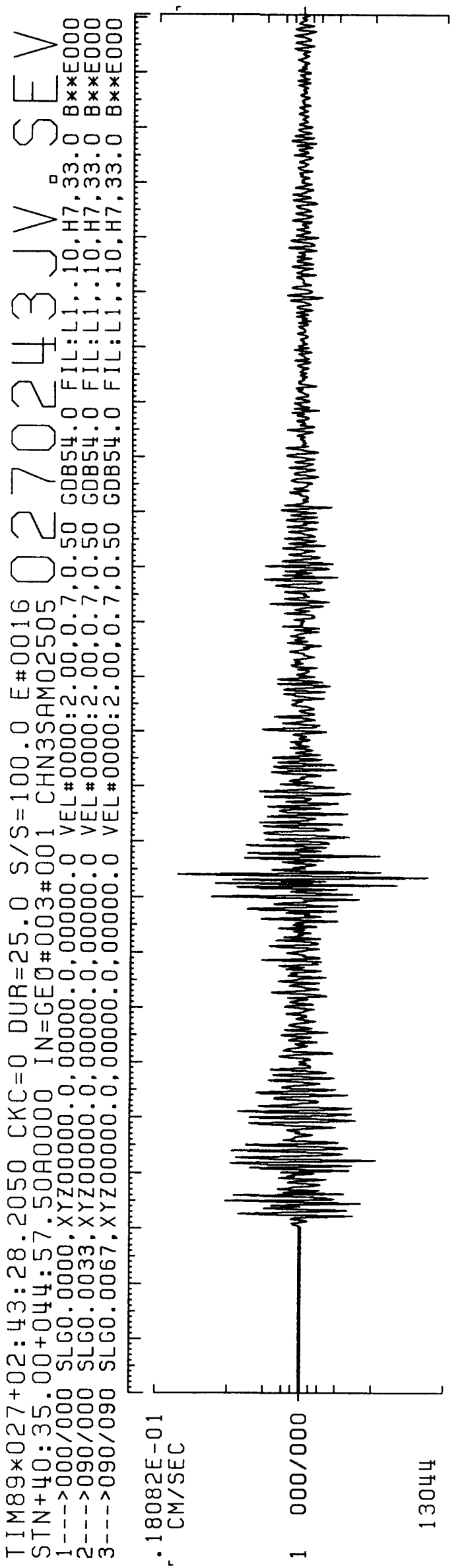

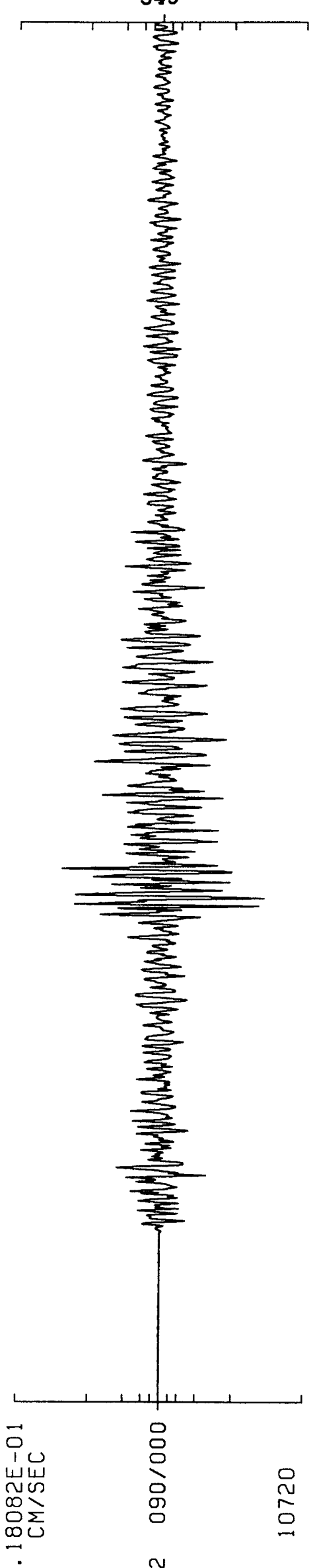

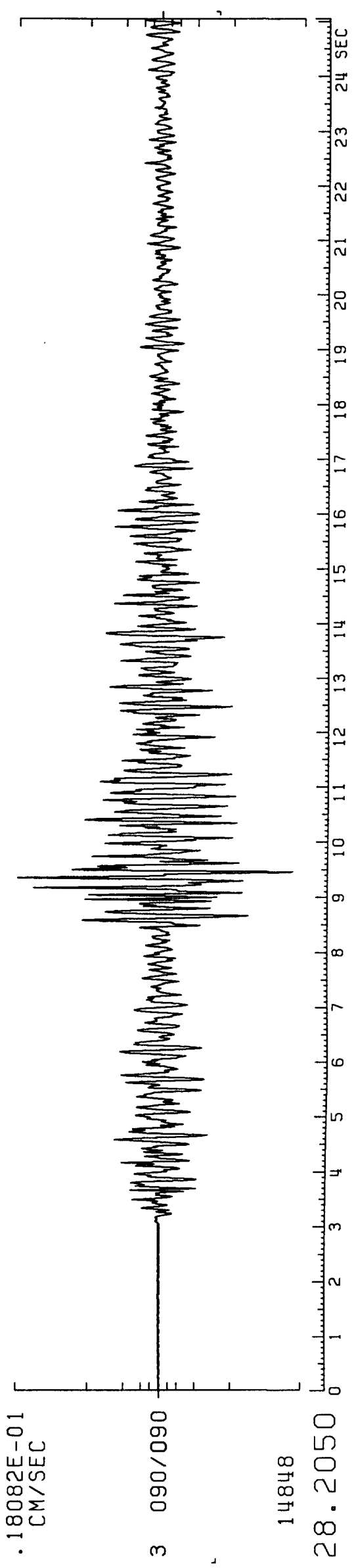




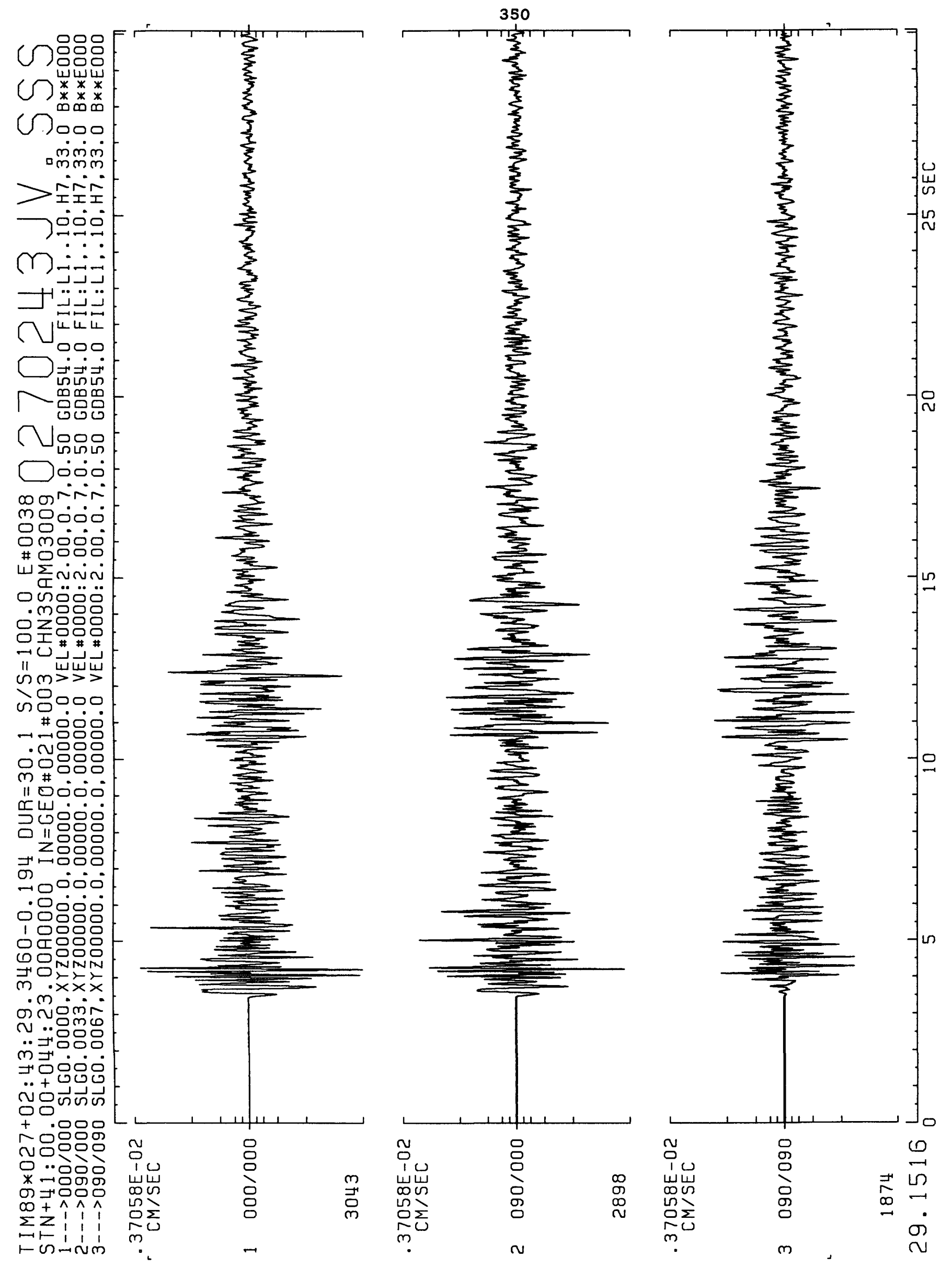


351
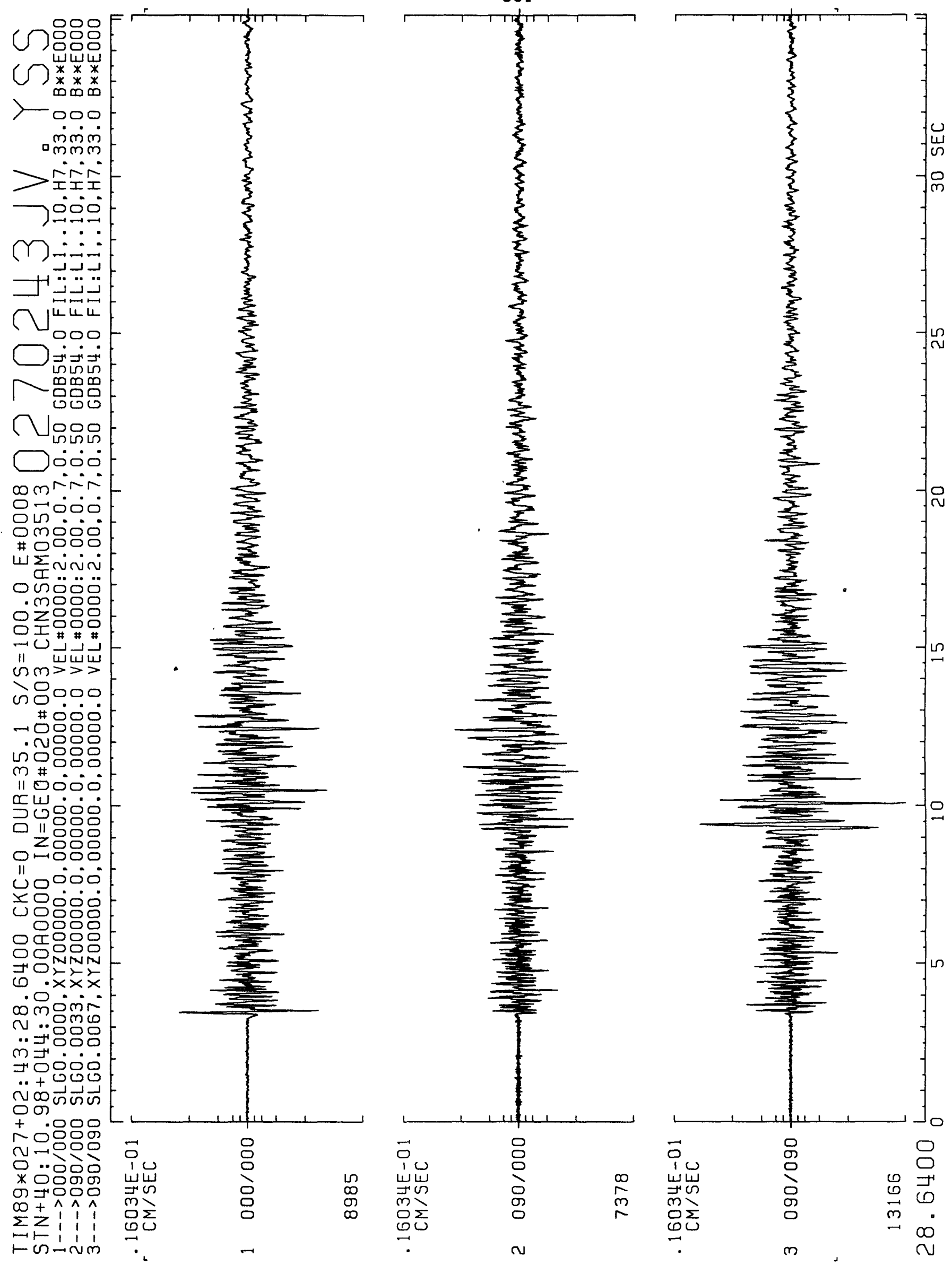
352
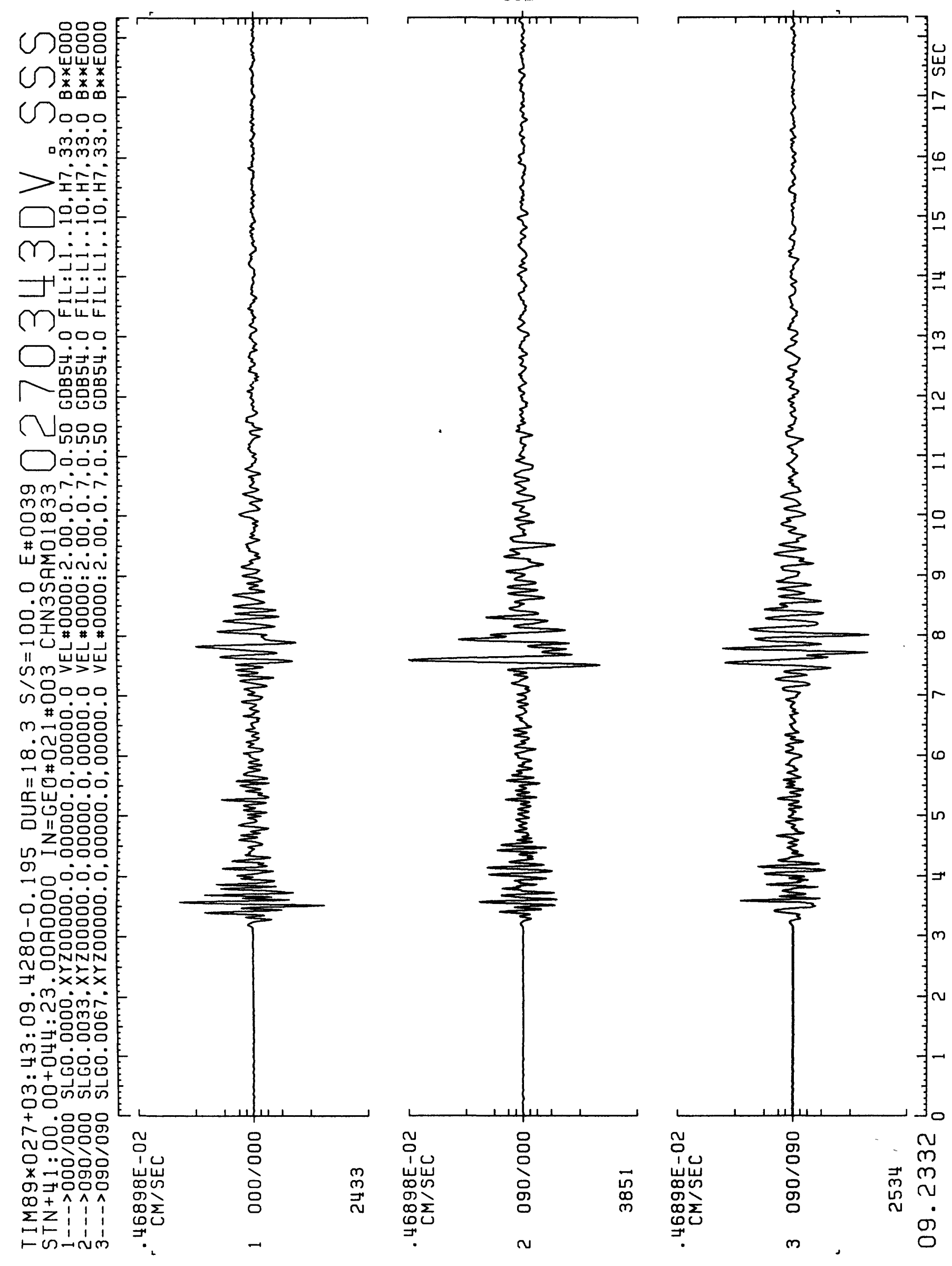
353

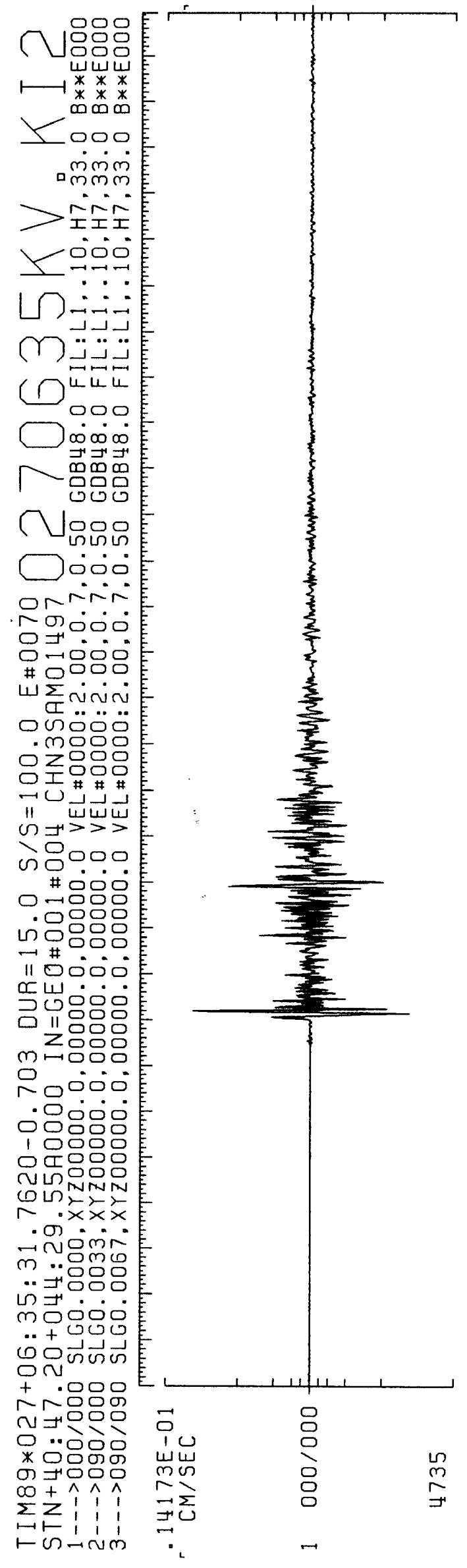

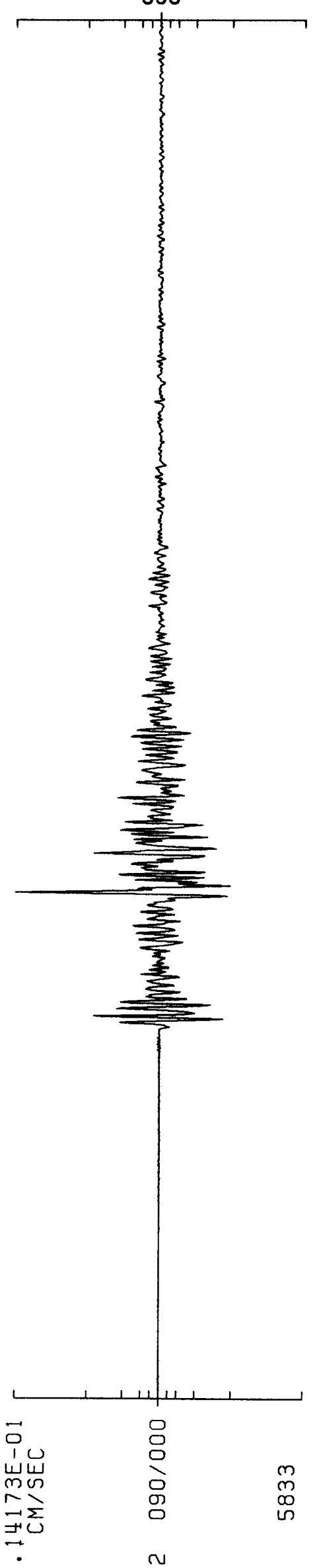

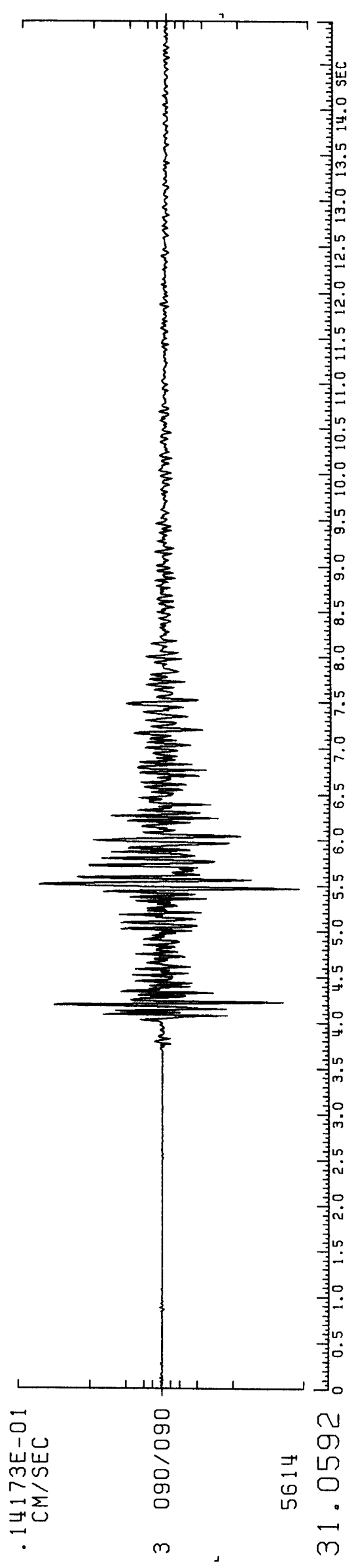




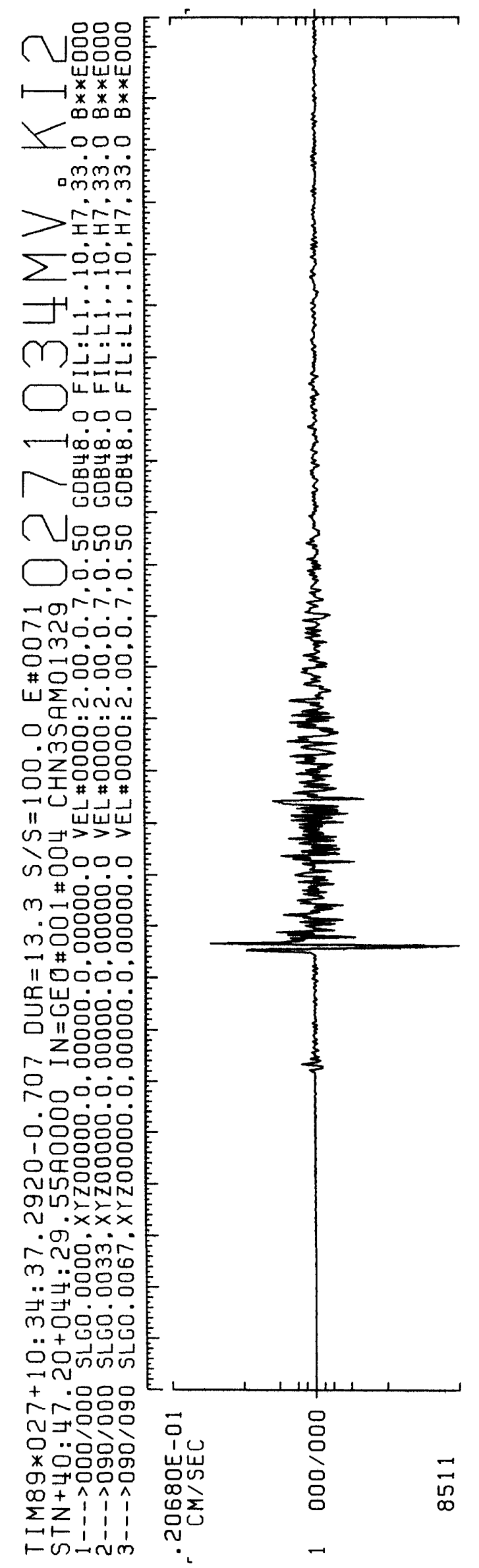

354

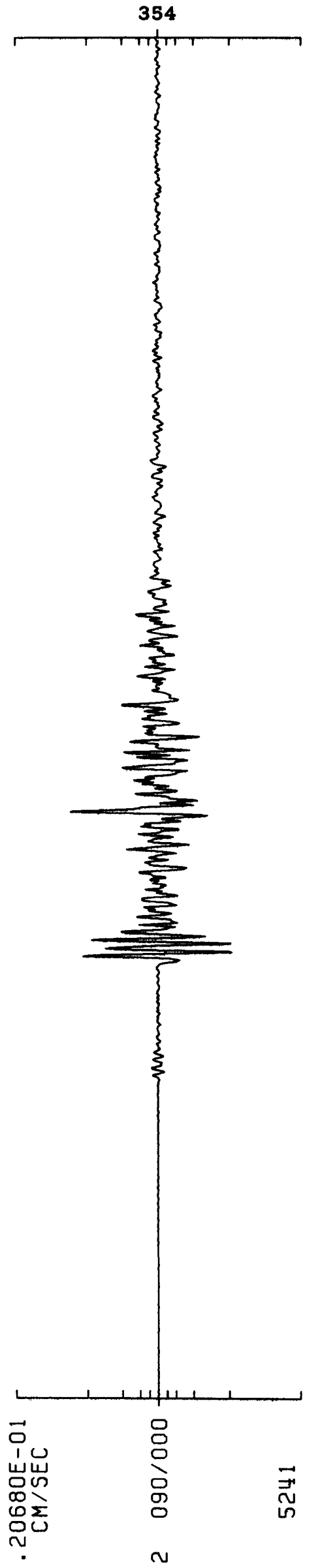

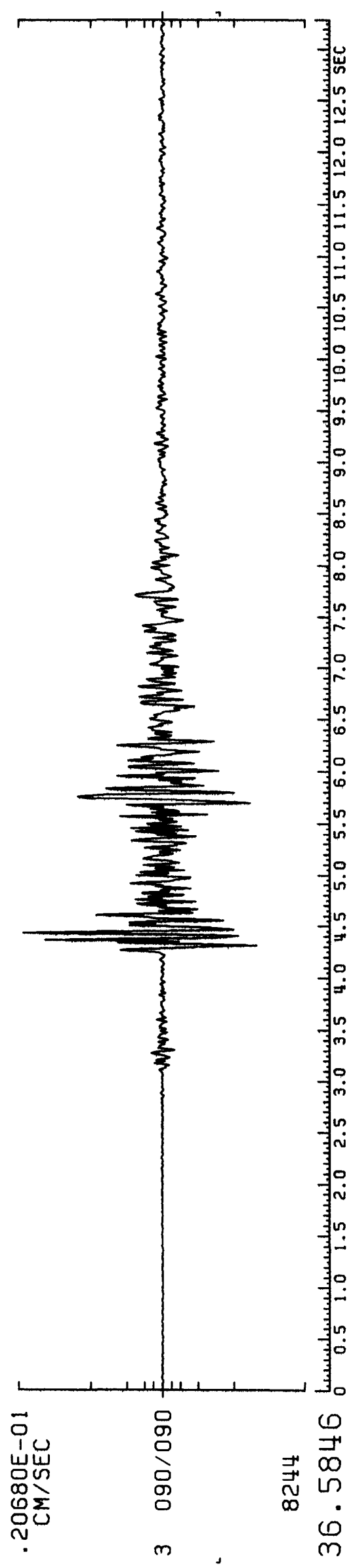


355

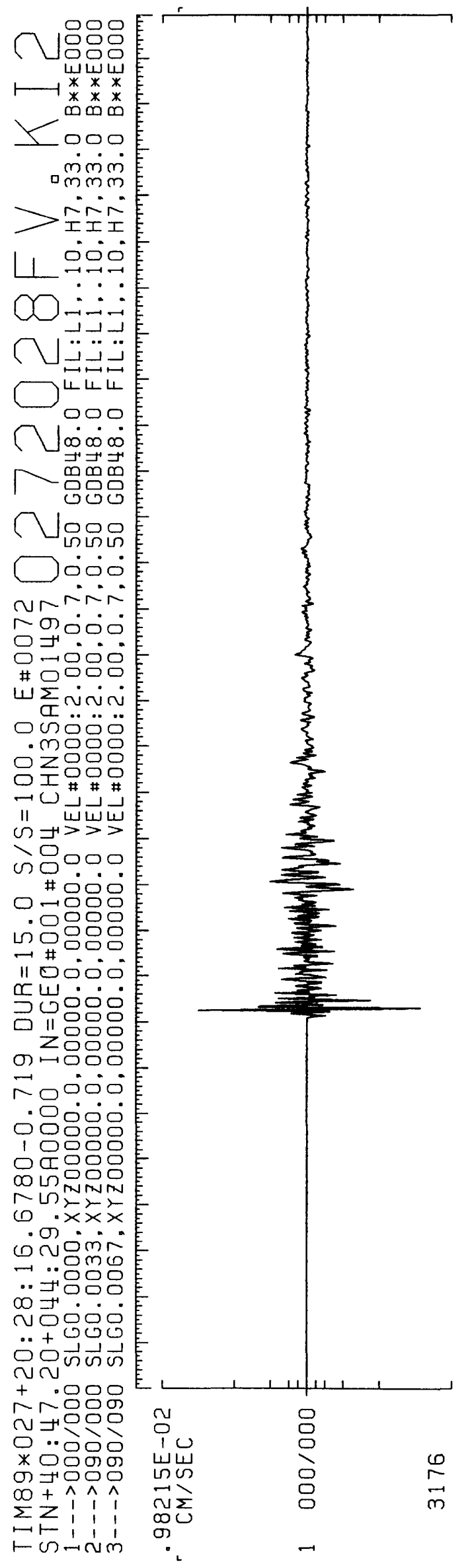

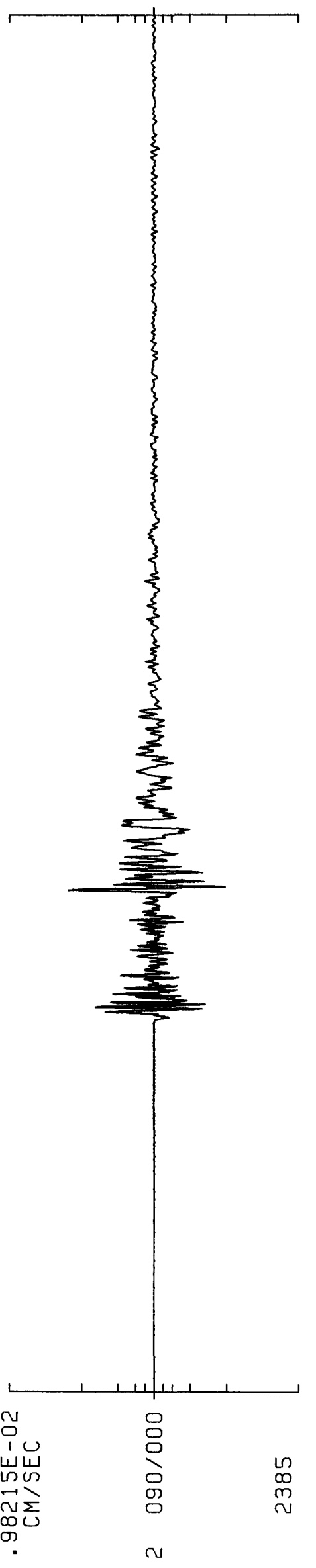

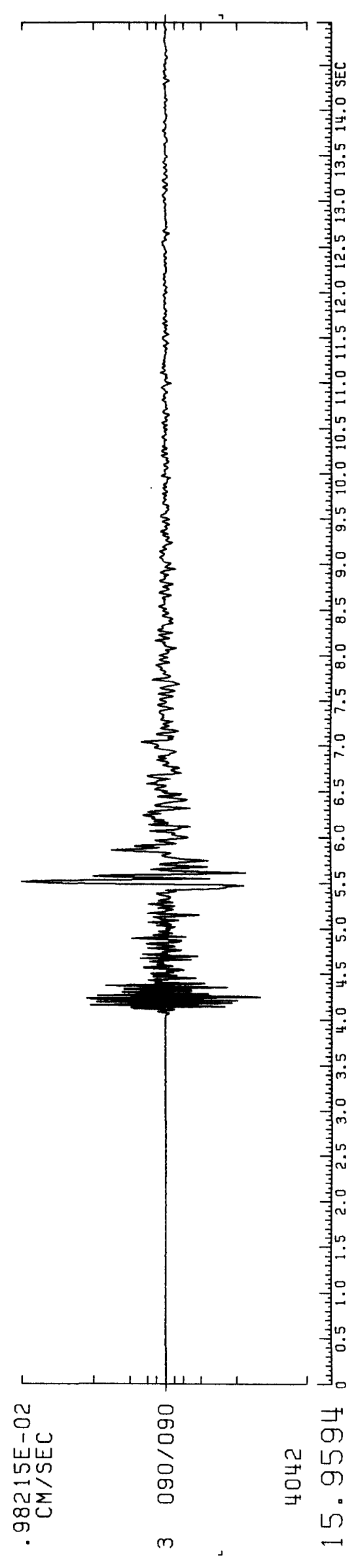


356

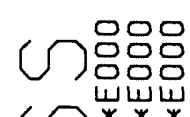

$\int \sqrt{*} x^{*} *$ 苑苂

$\gamma-000$ мंmin व $\mathrm{mm}$ inir III 100 $\checkmark \cap$ : (Y) ترتّت டோ 000 \ت゙ت゙ت゙ ๓⿴囗十 $\Gamma$ 品品 \ooo • $\square 00^{\circ}$ onrir ด

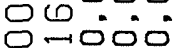
\# 잉ㅇㅁㅇㅛ 山 $\sum_{\text {Tini }}$ 更..... - mo응 ○z응응 오뮴유 问 जm $\gg$ iो \# ט음음 Nㅜ응응 2 \# II $00^{\circ} 0^{\circ}$

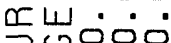

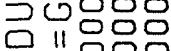

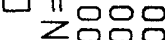
믐ㅇㅇ un Yo

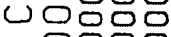
응ㅇㅁ음 modoo MONNN $\rightarrow \cdot \times x \times$ $\infty$ momi ت十... .. 几 m엉엉 त กัजजज + .응요 ㄷㅇㅇㅇㅁㅇ

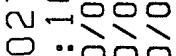
* 붕음ㅇ * 음요 क커융 $\sum z 1$

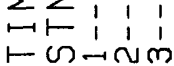

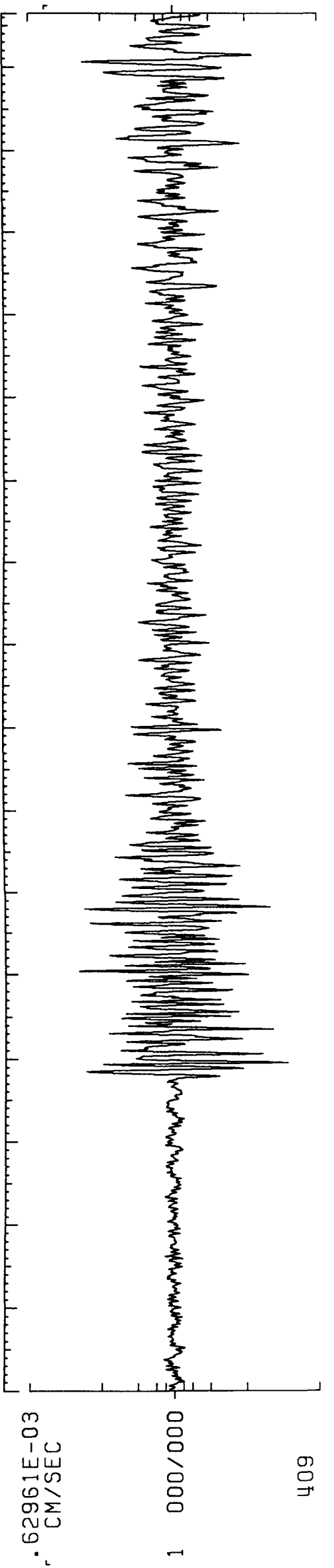

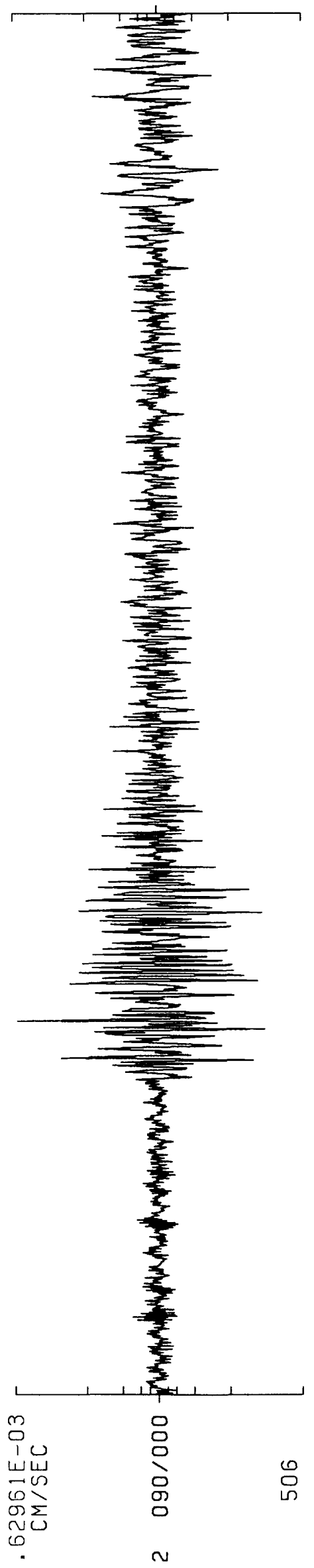

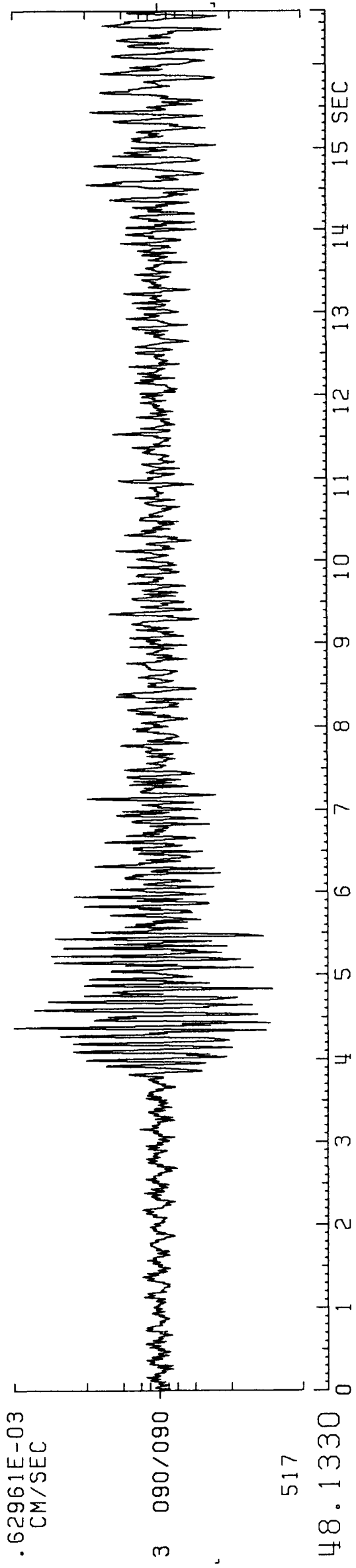




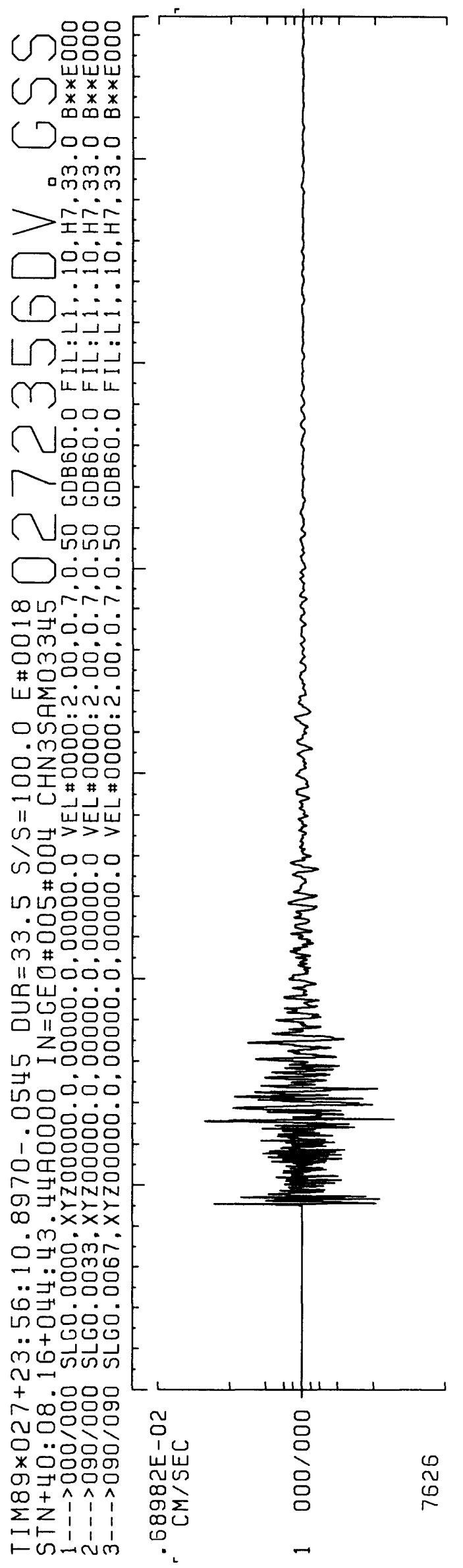

357

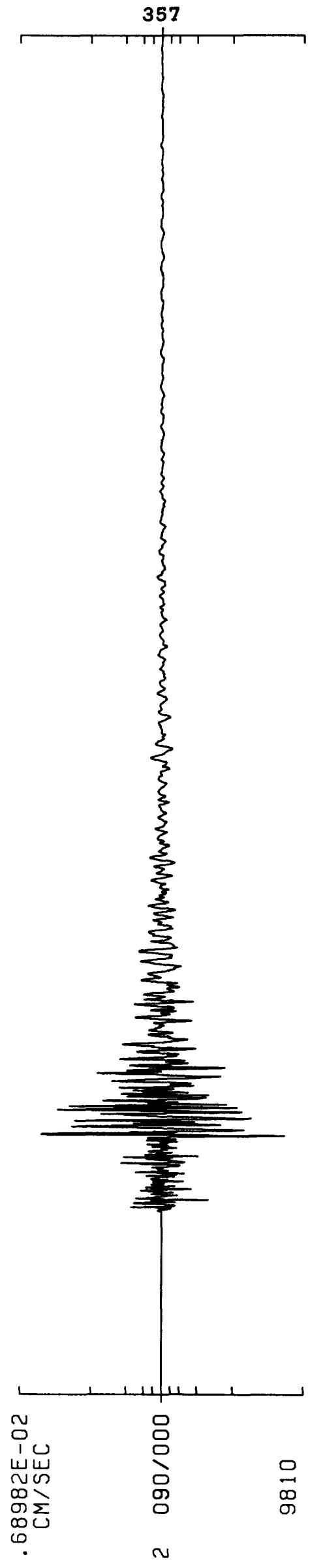


358

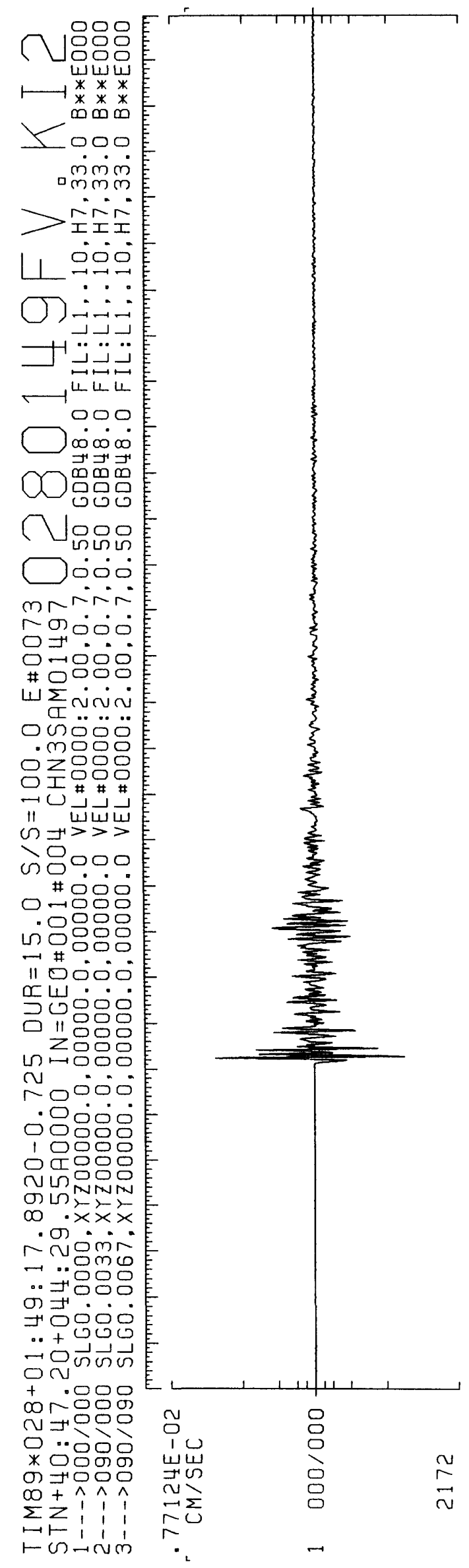

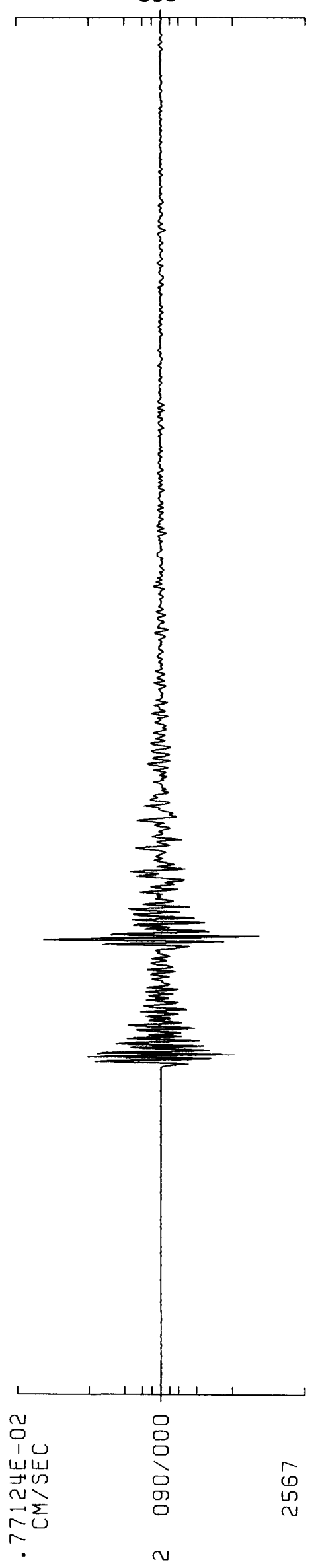

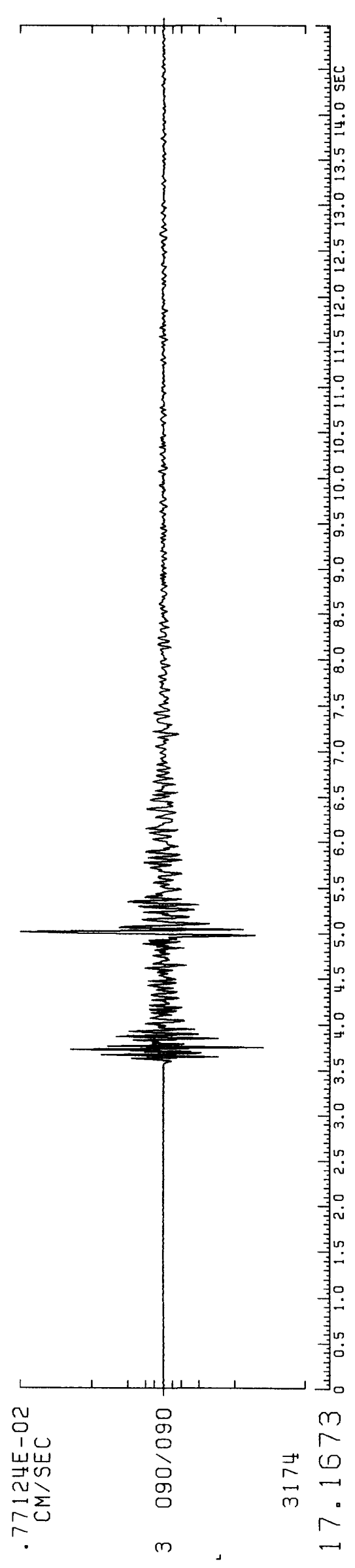




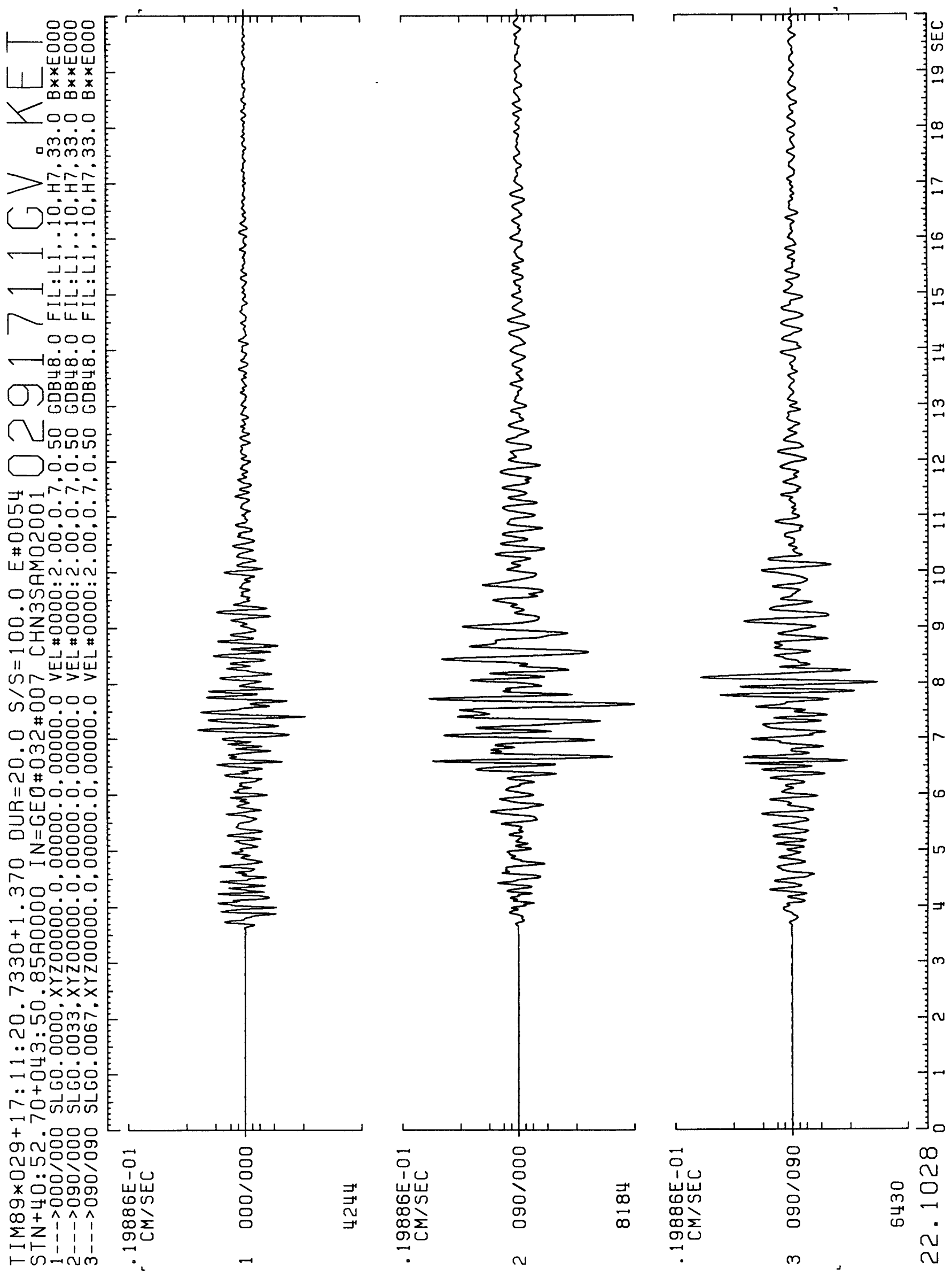




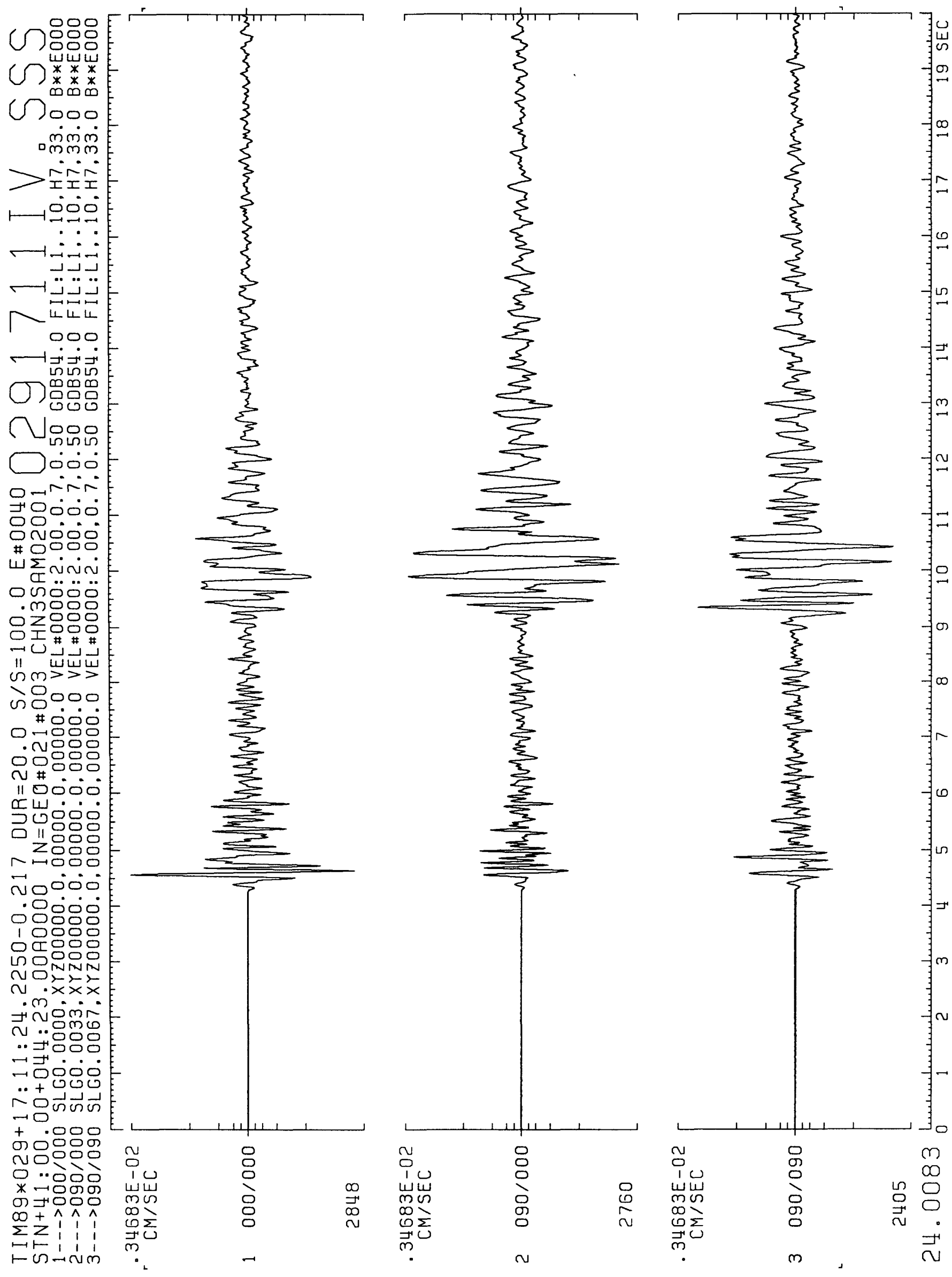


361

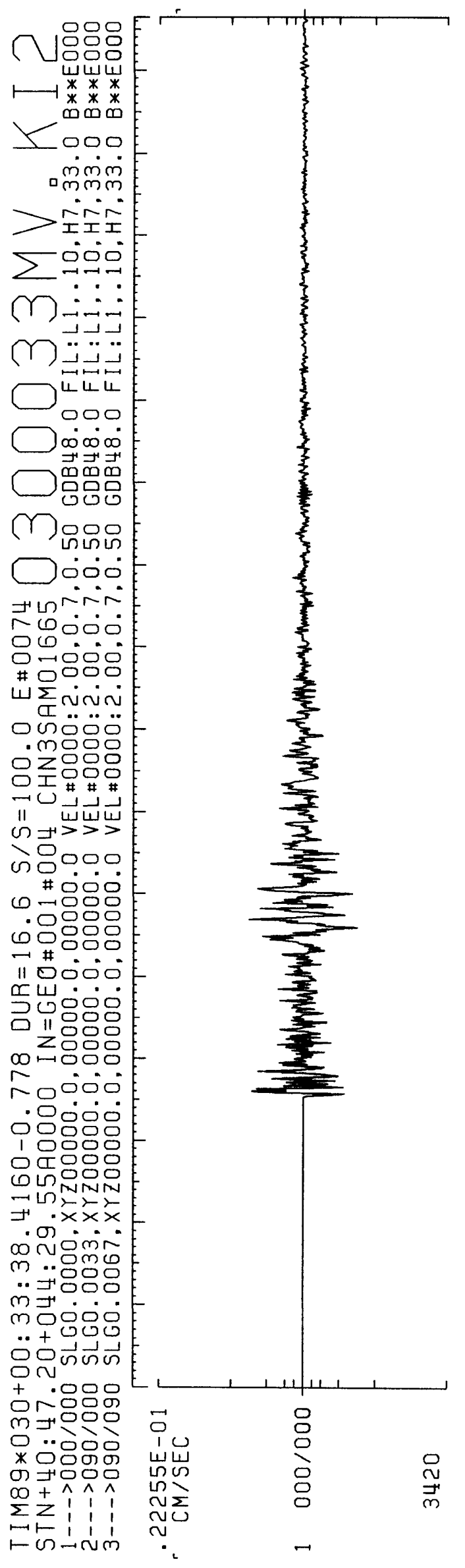

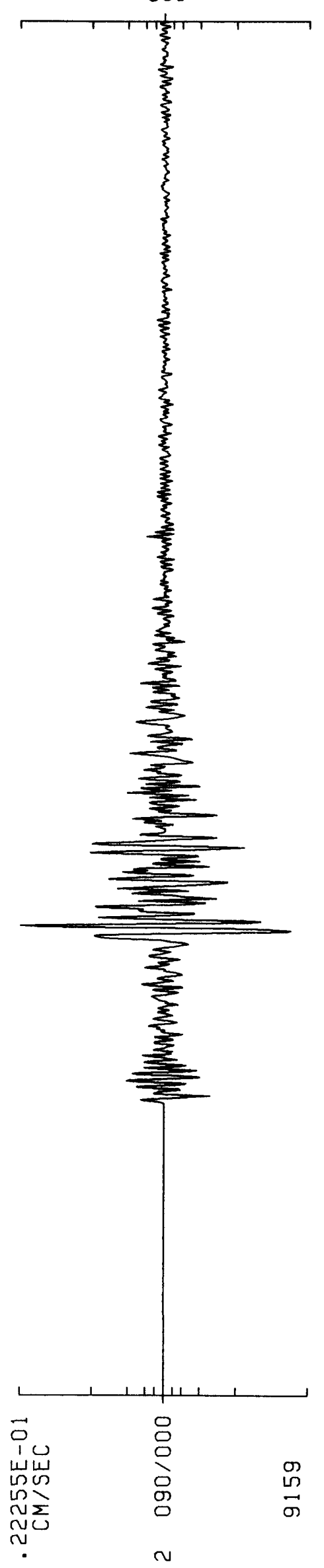

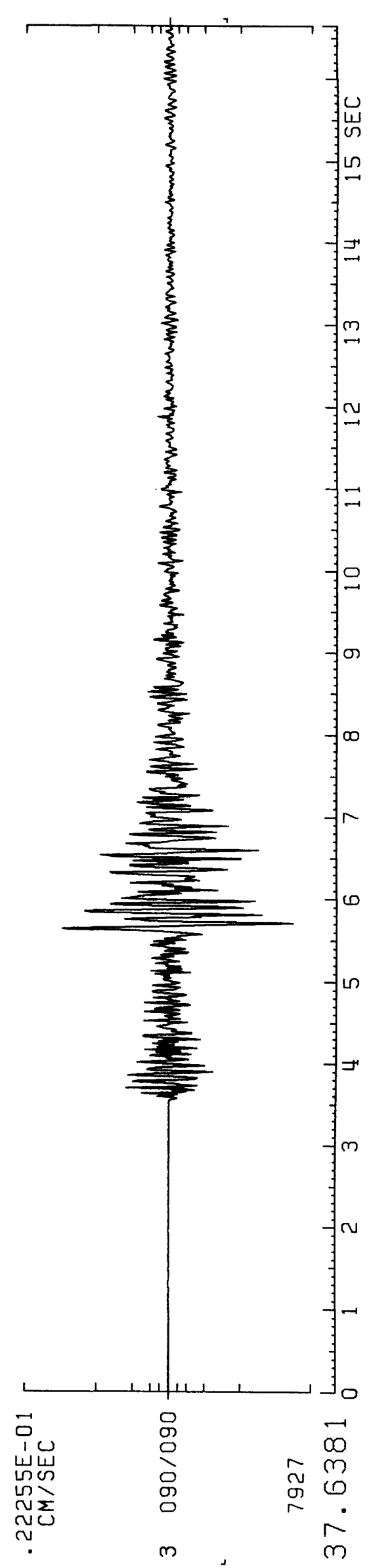


362

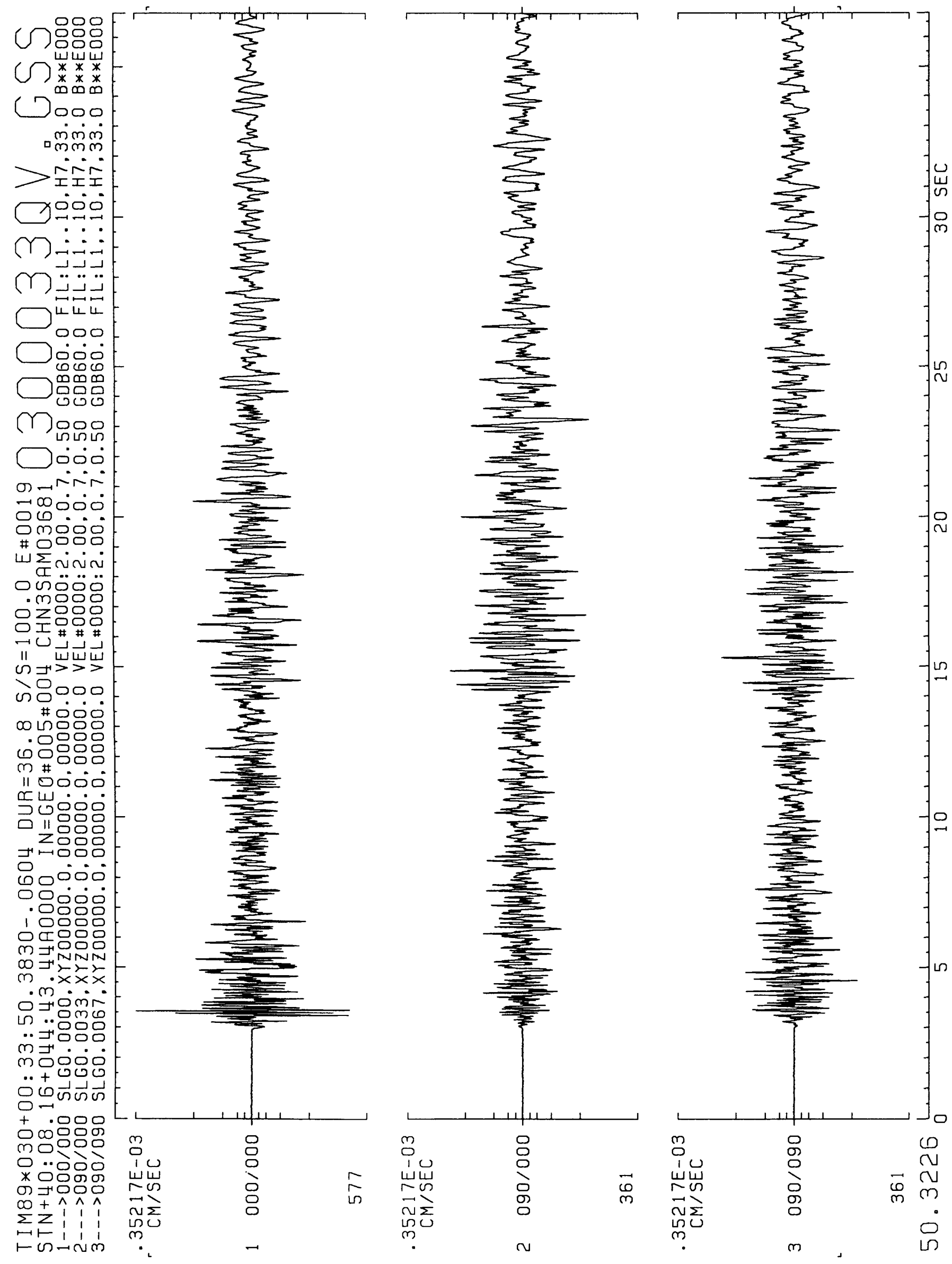


363

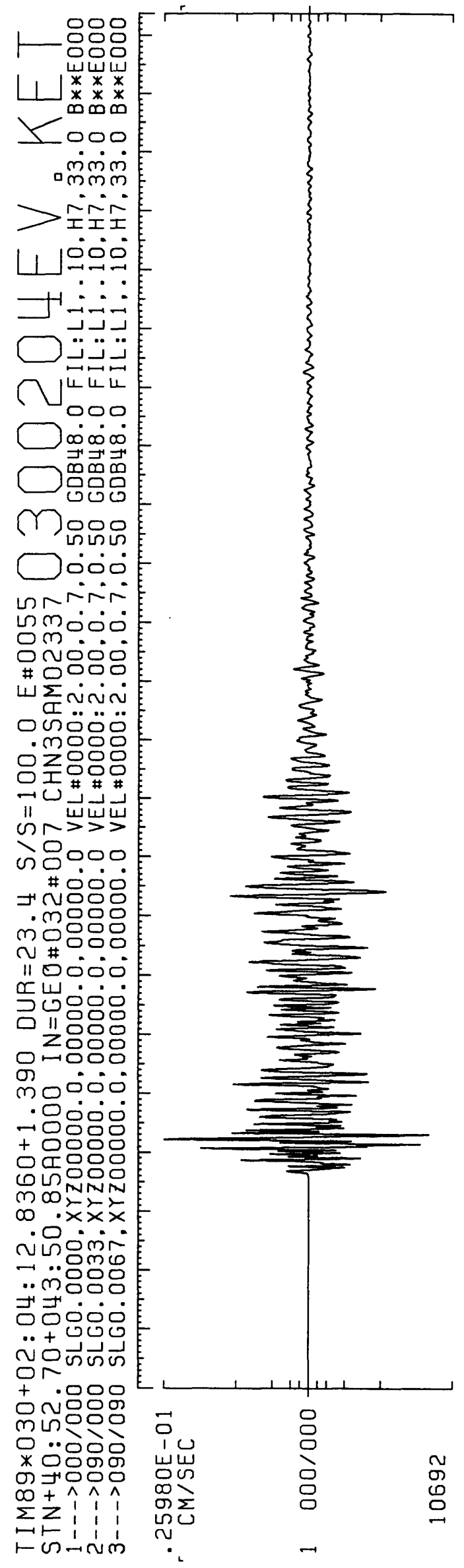

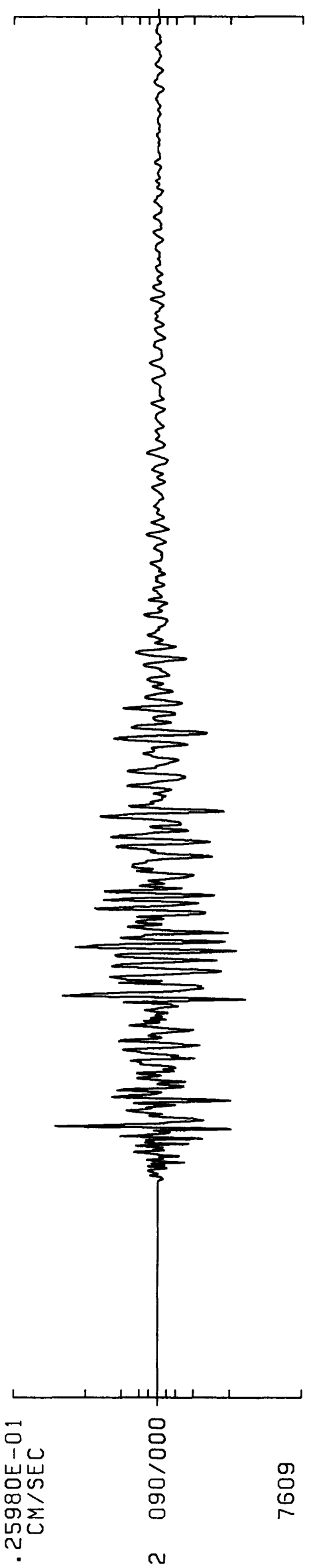

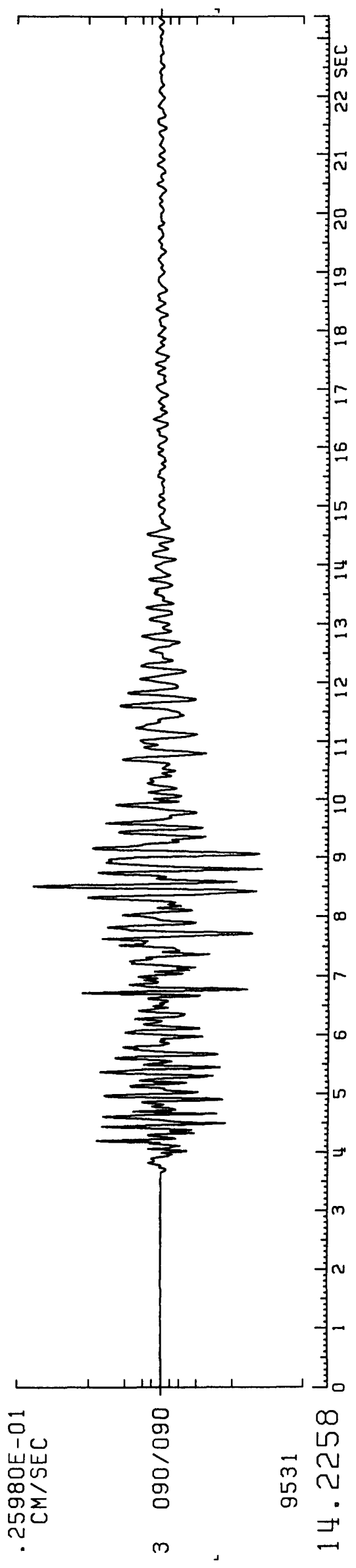




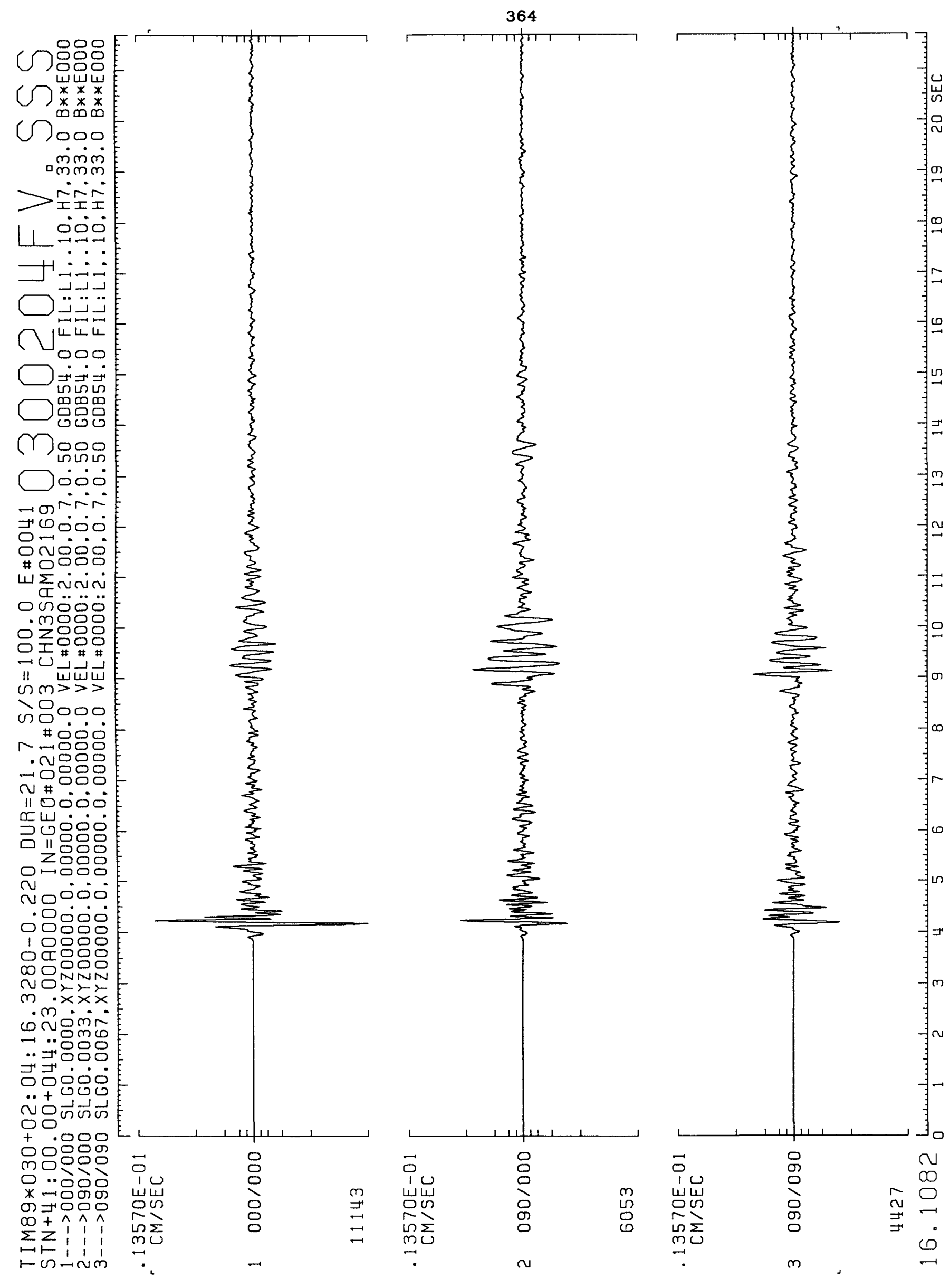




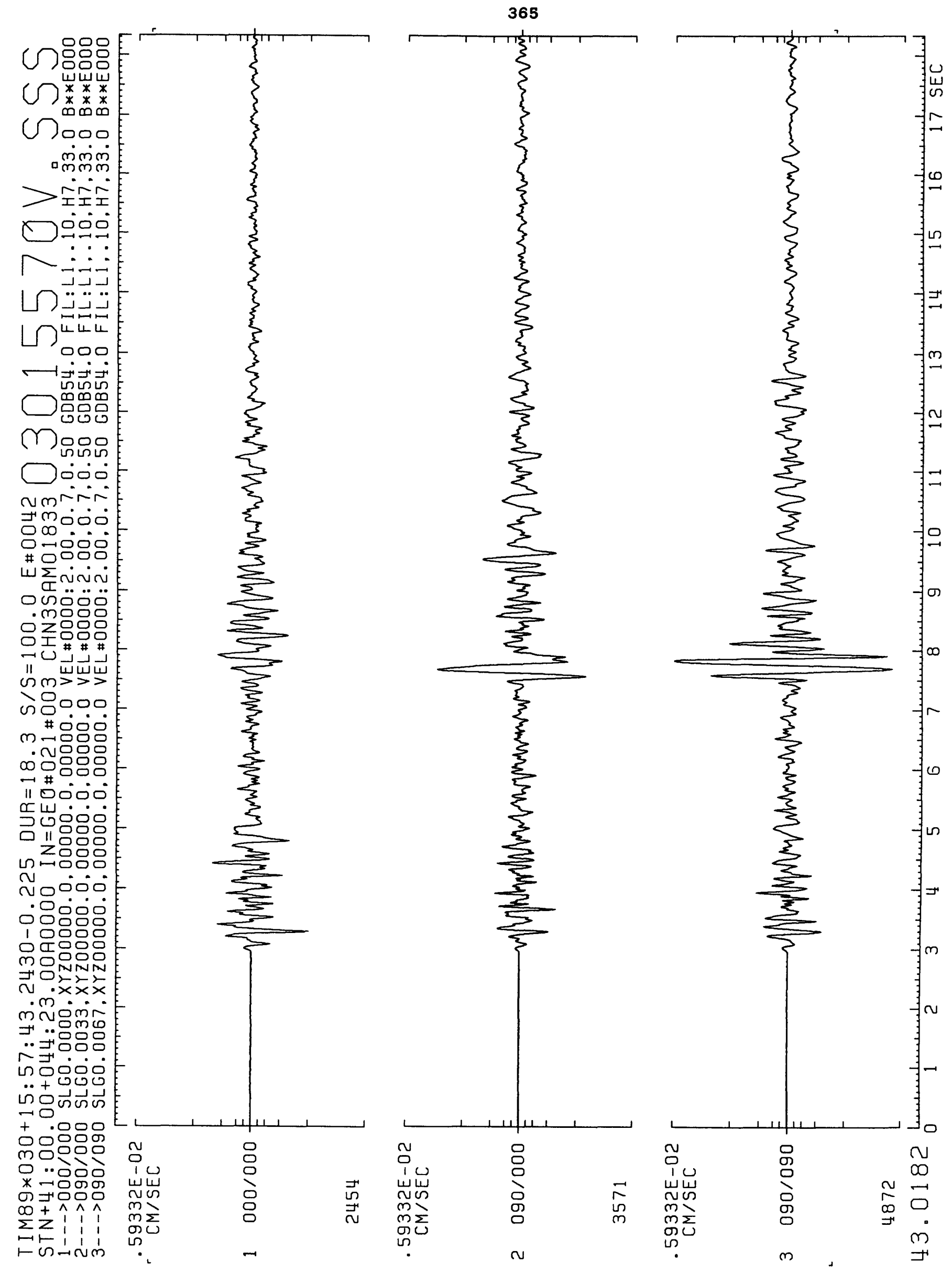




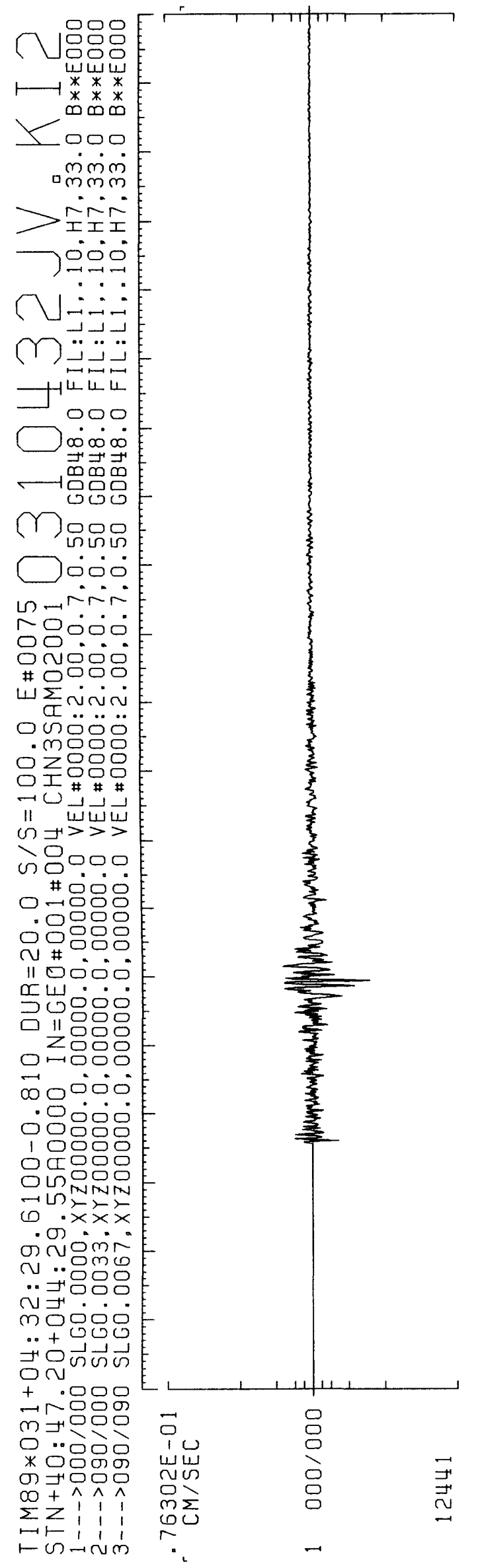

366

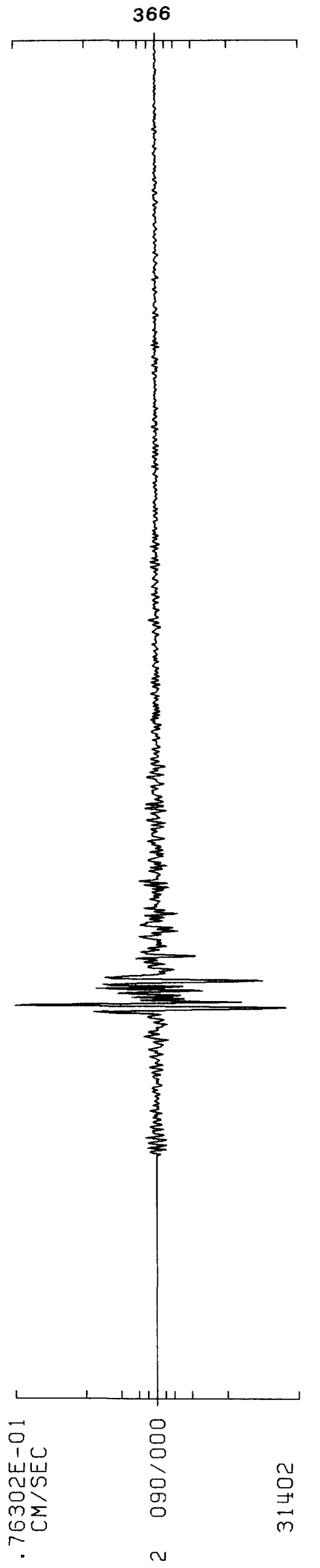

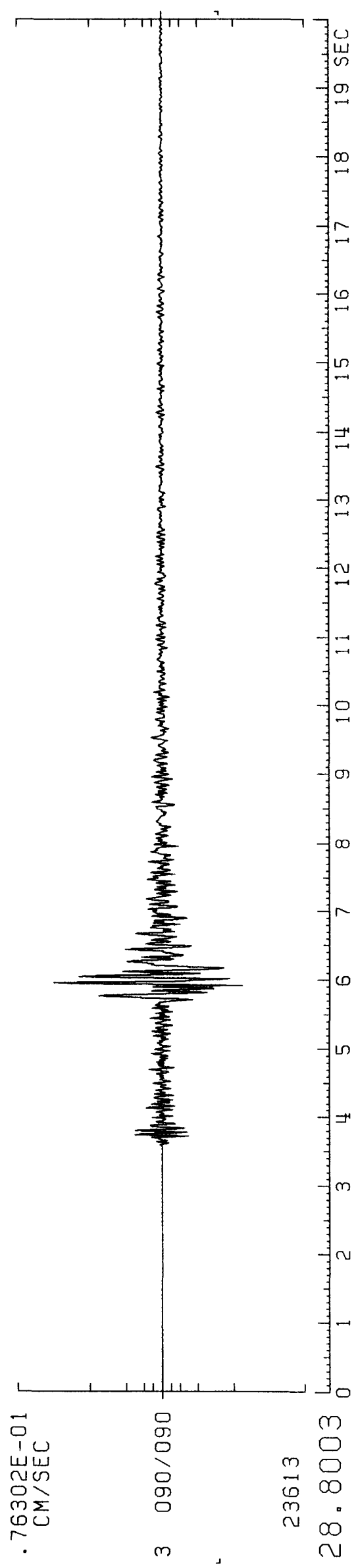



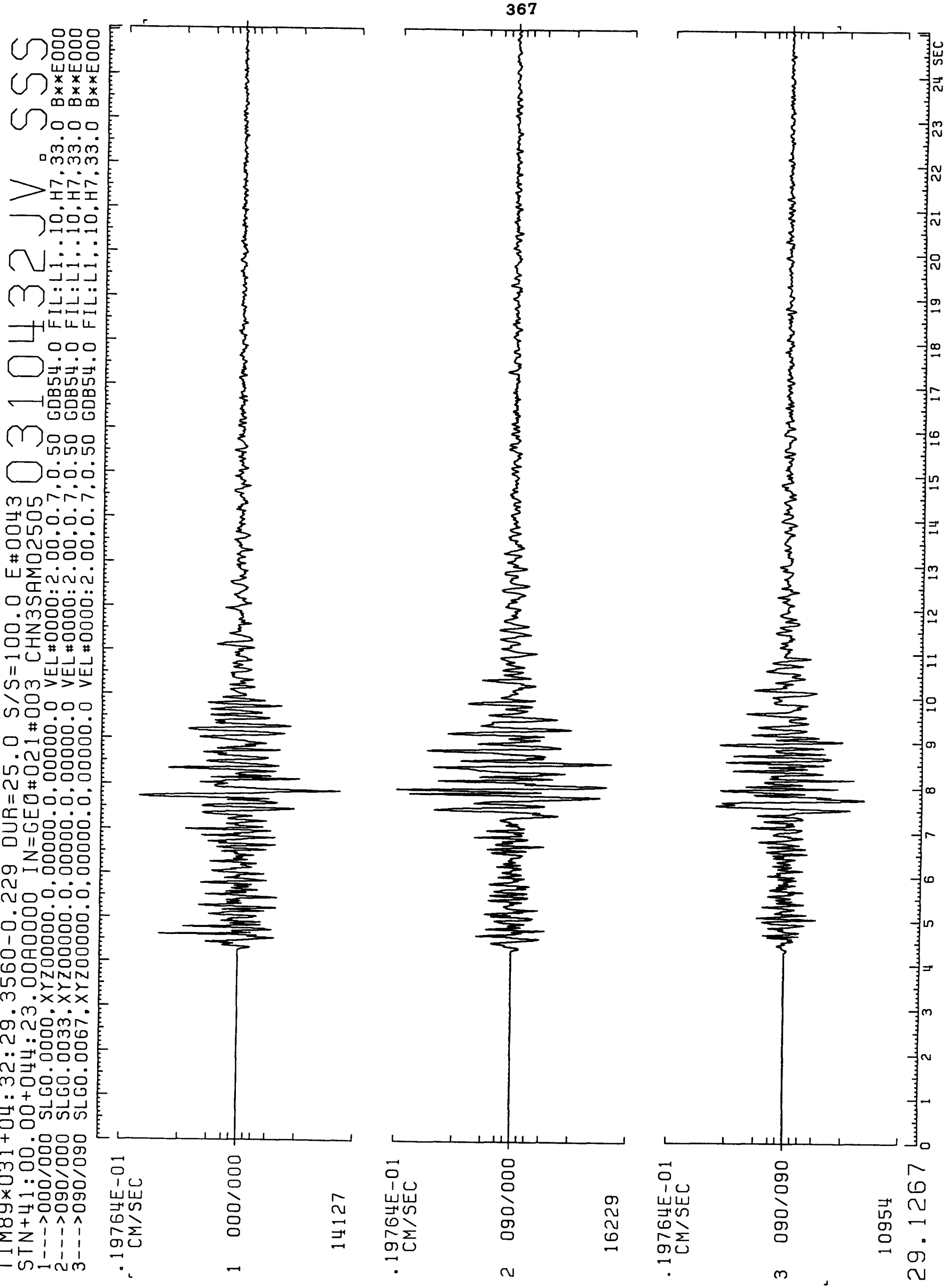


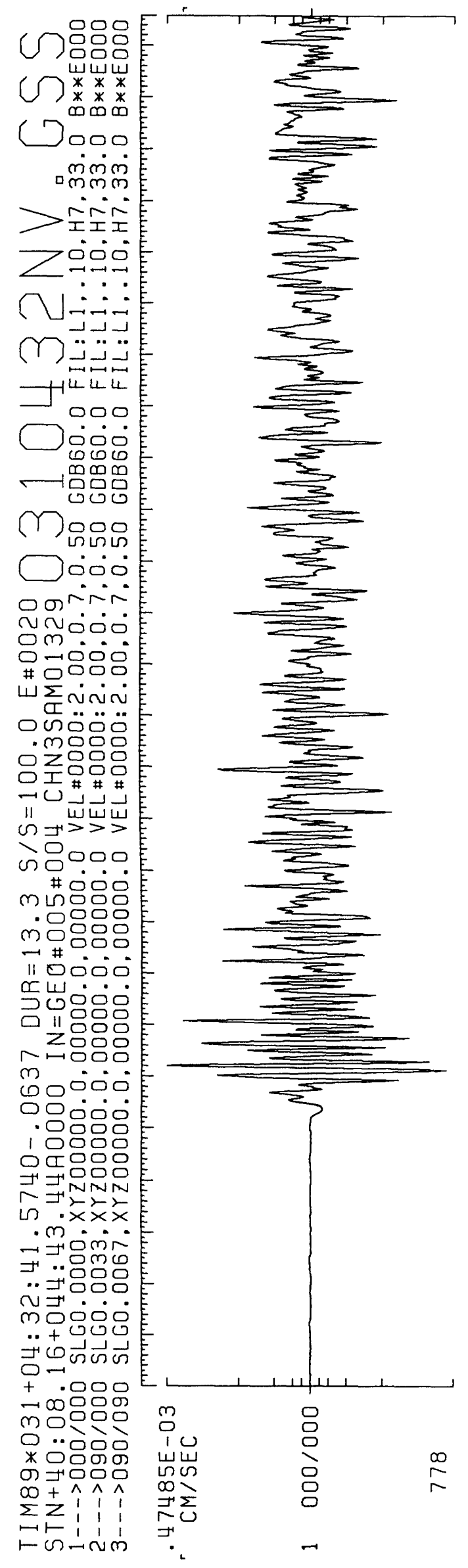

368

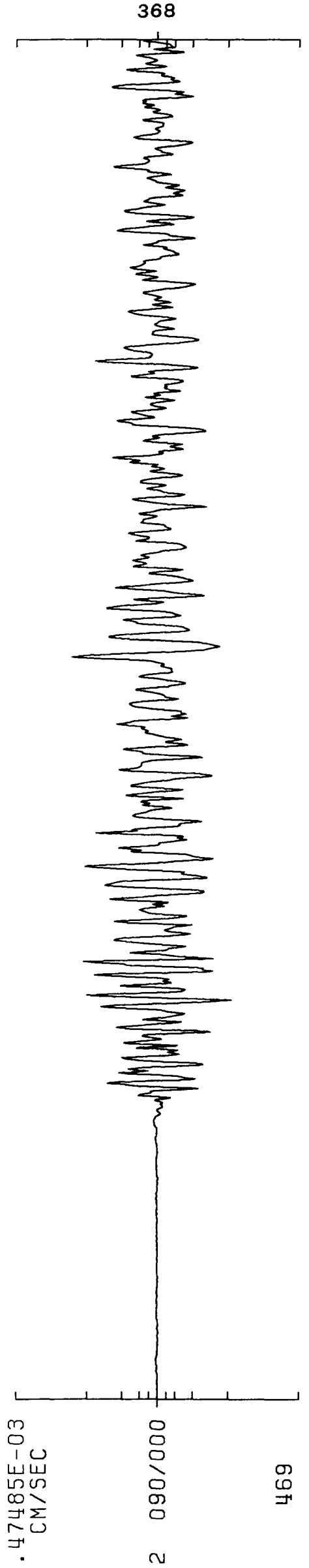

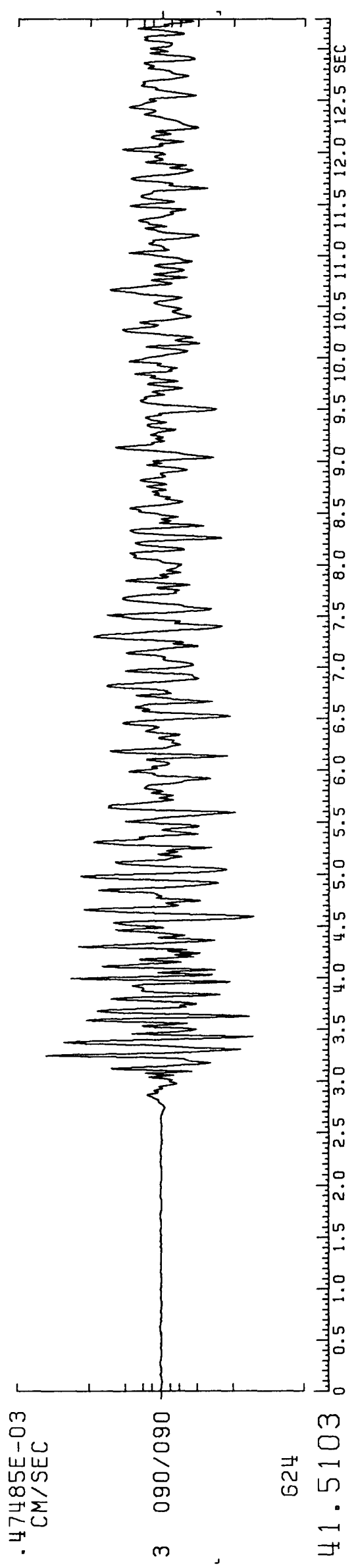



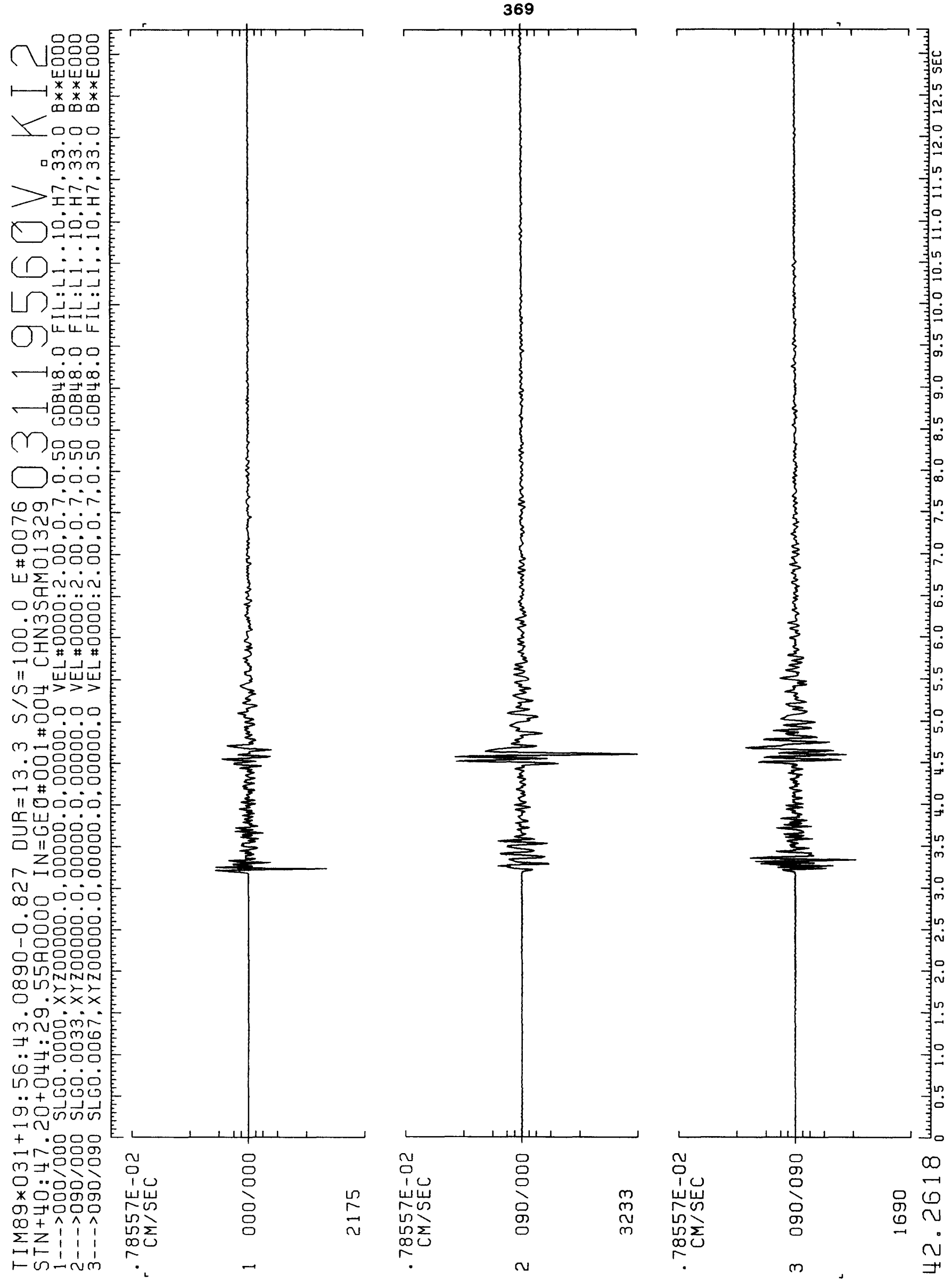
370

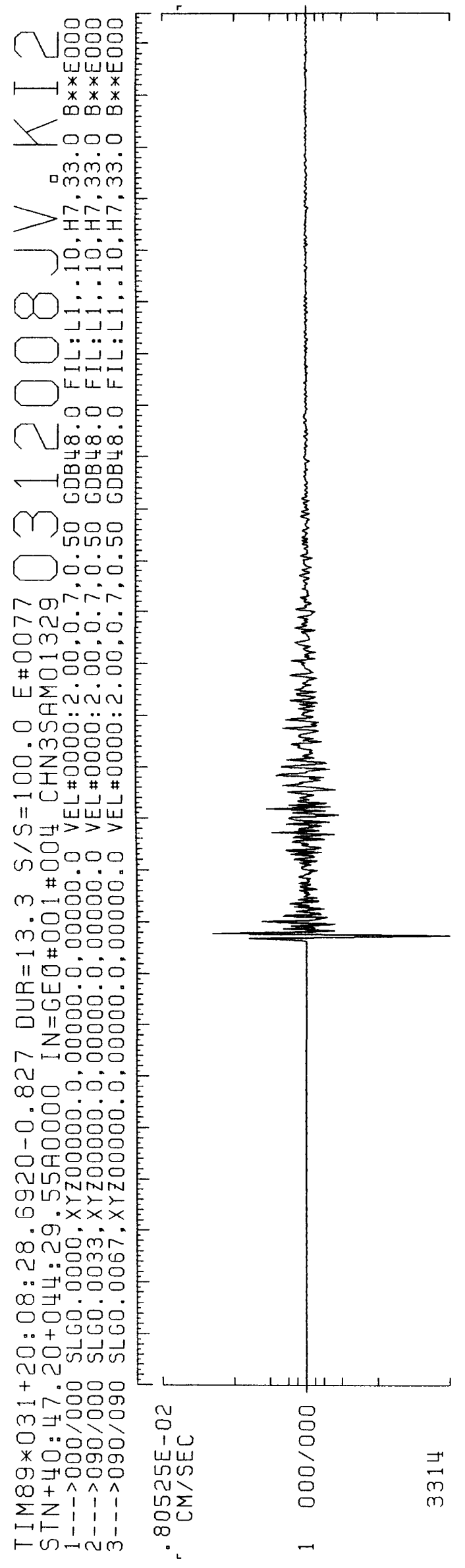

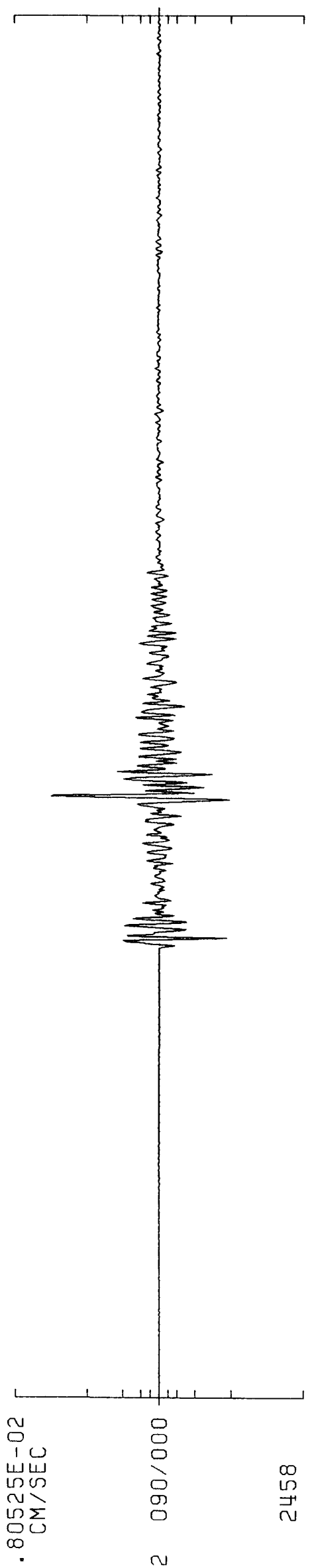

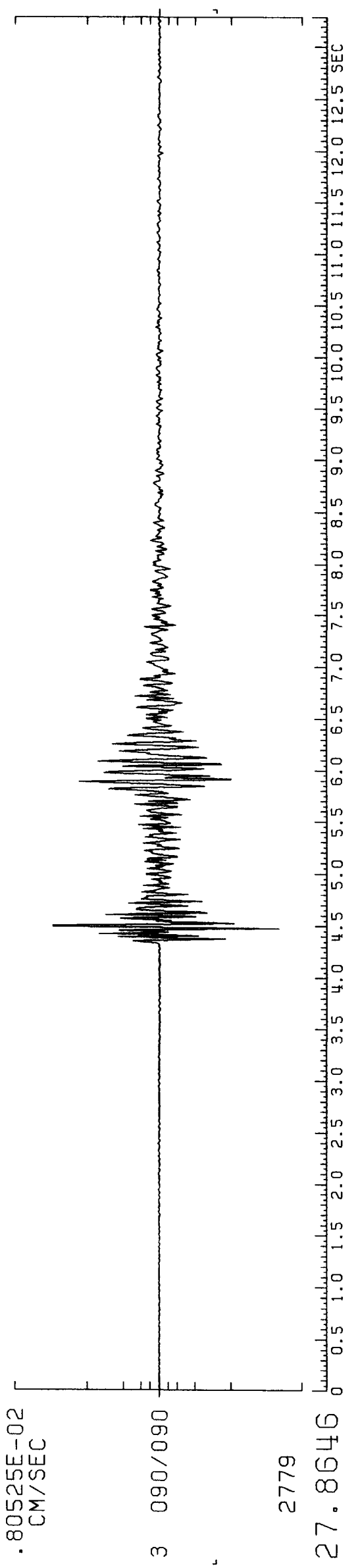


371

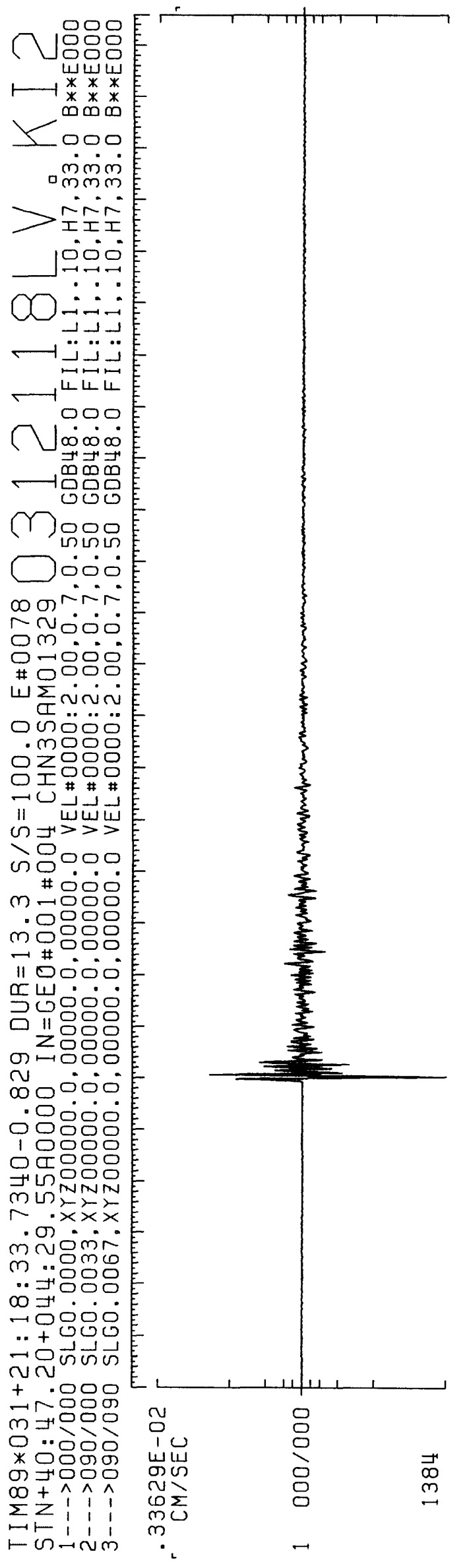

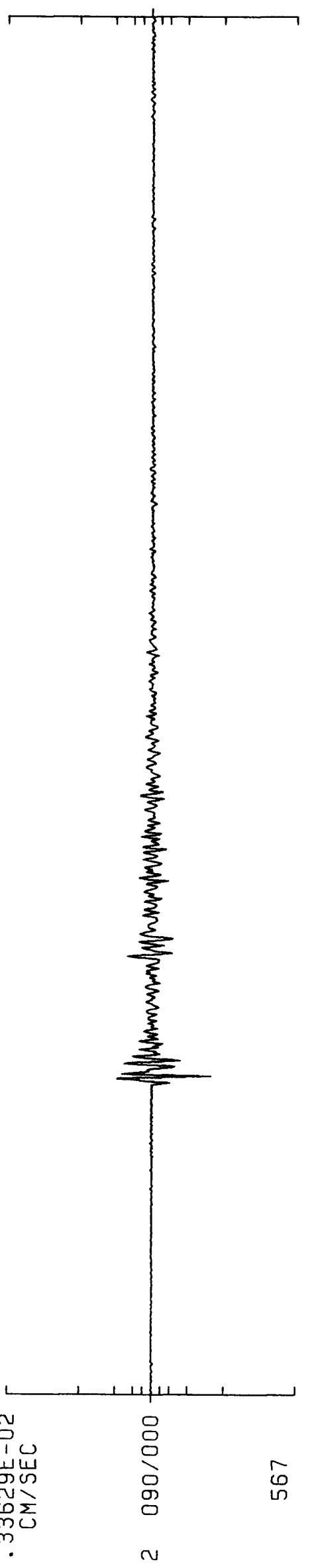

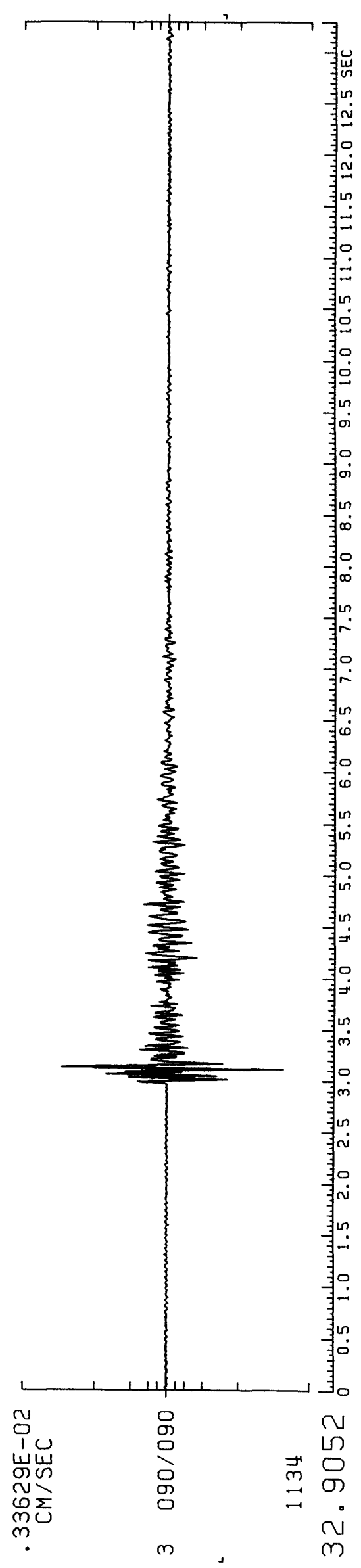


372

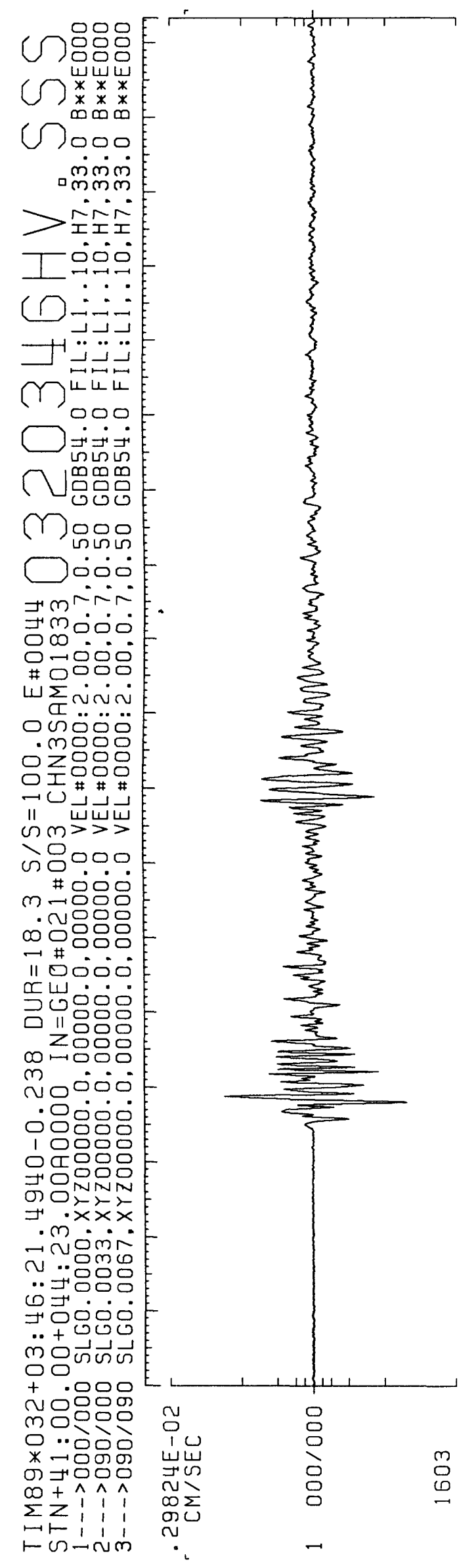

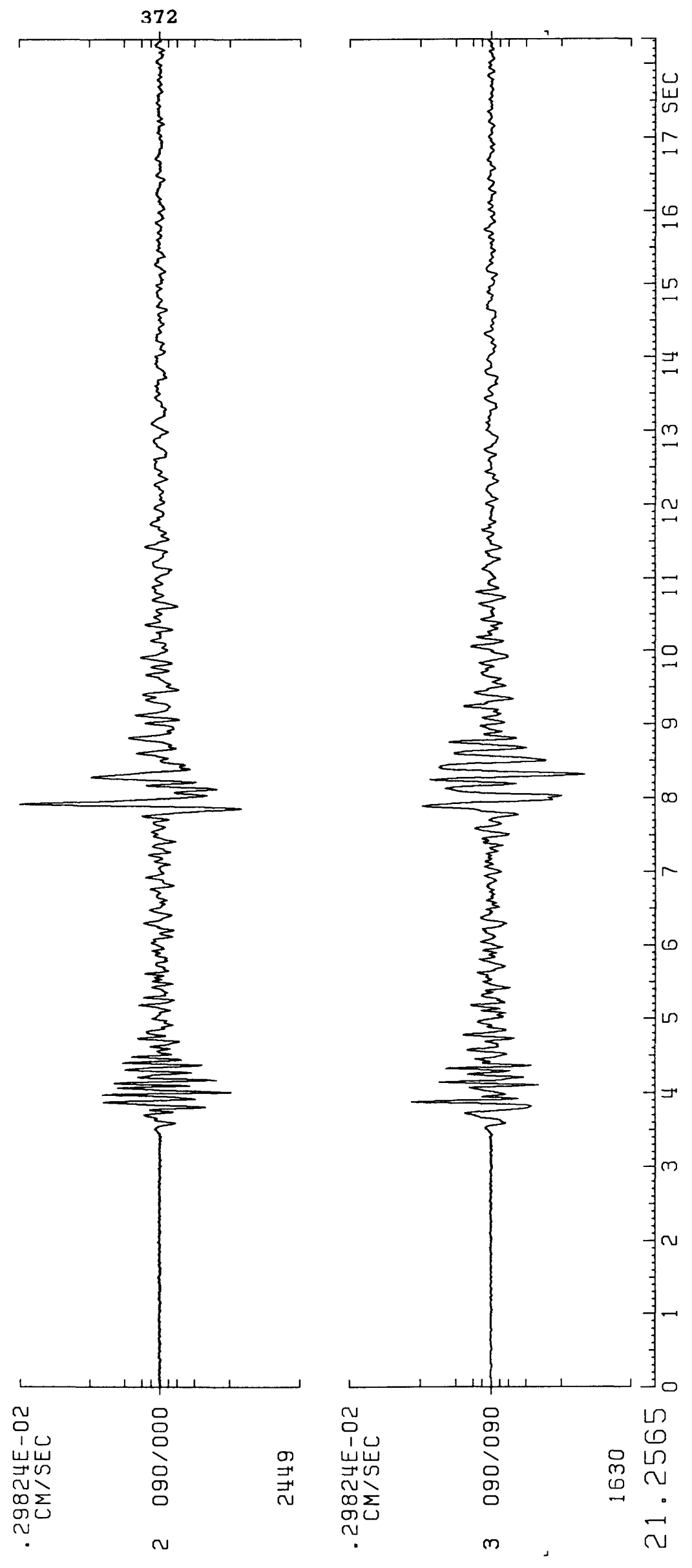



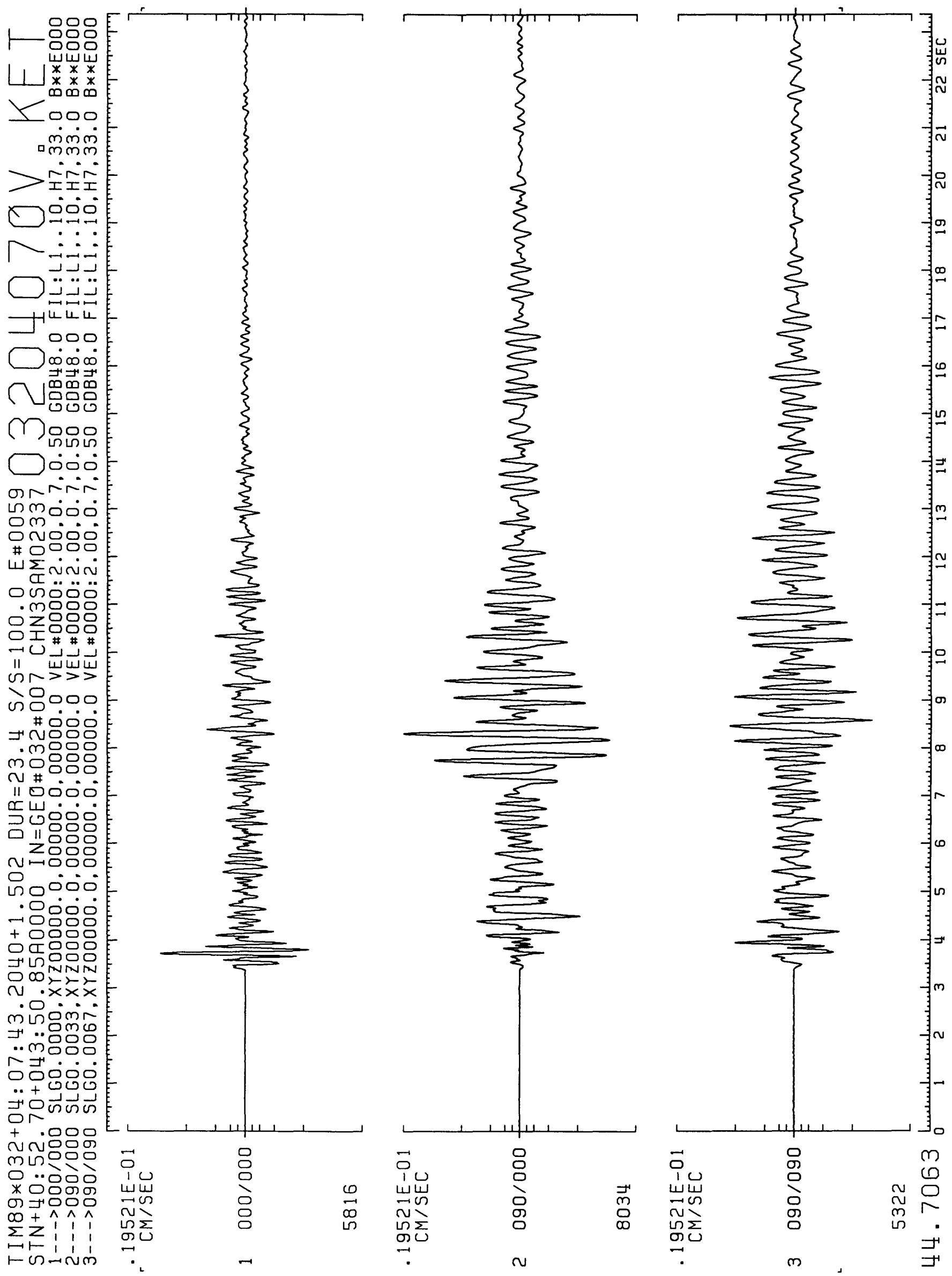
374

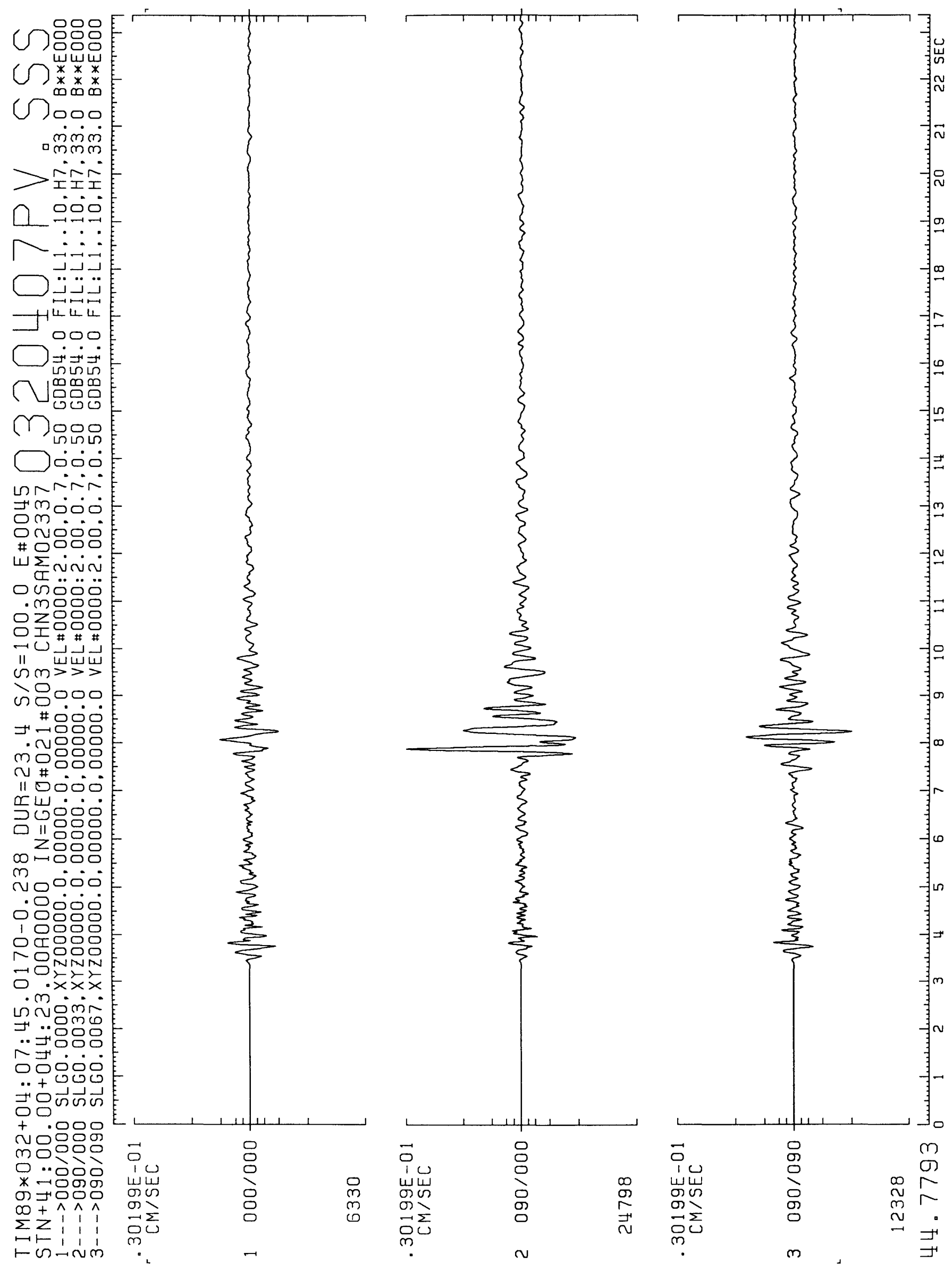



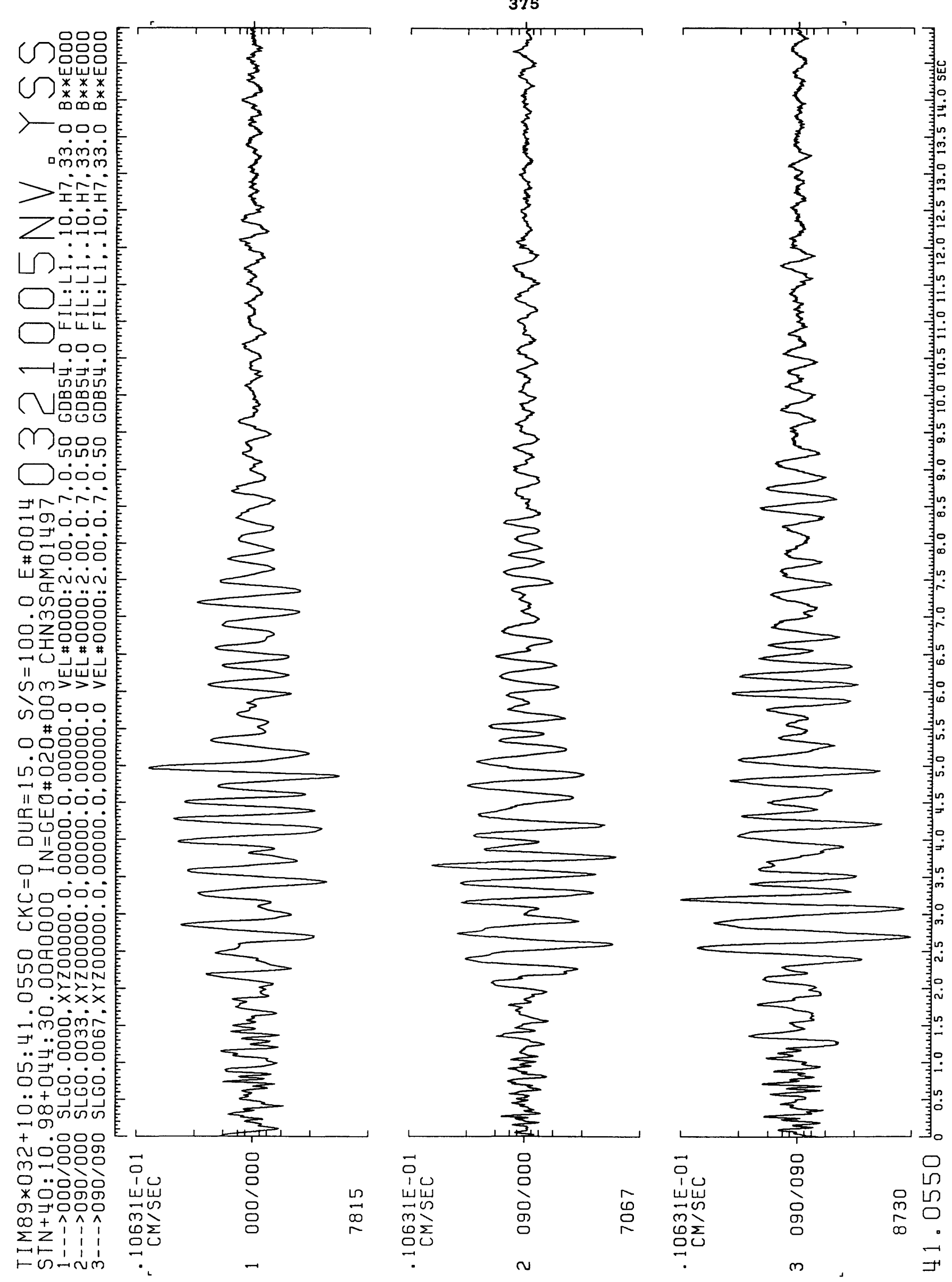
376

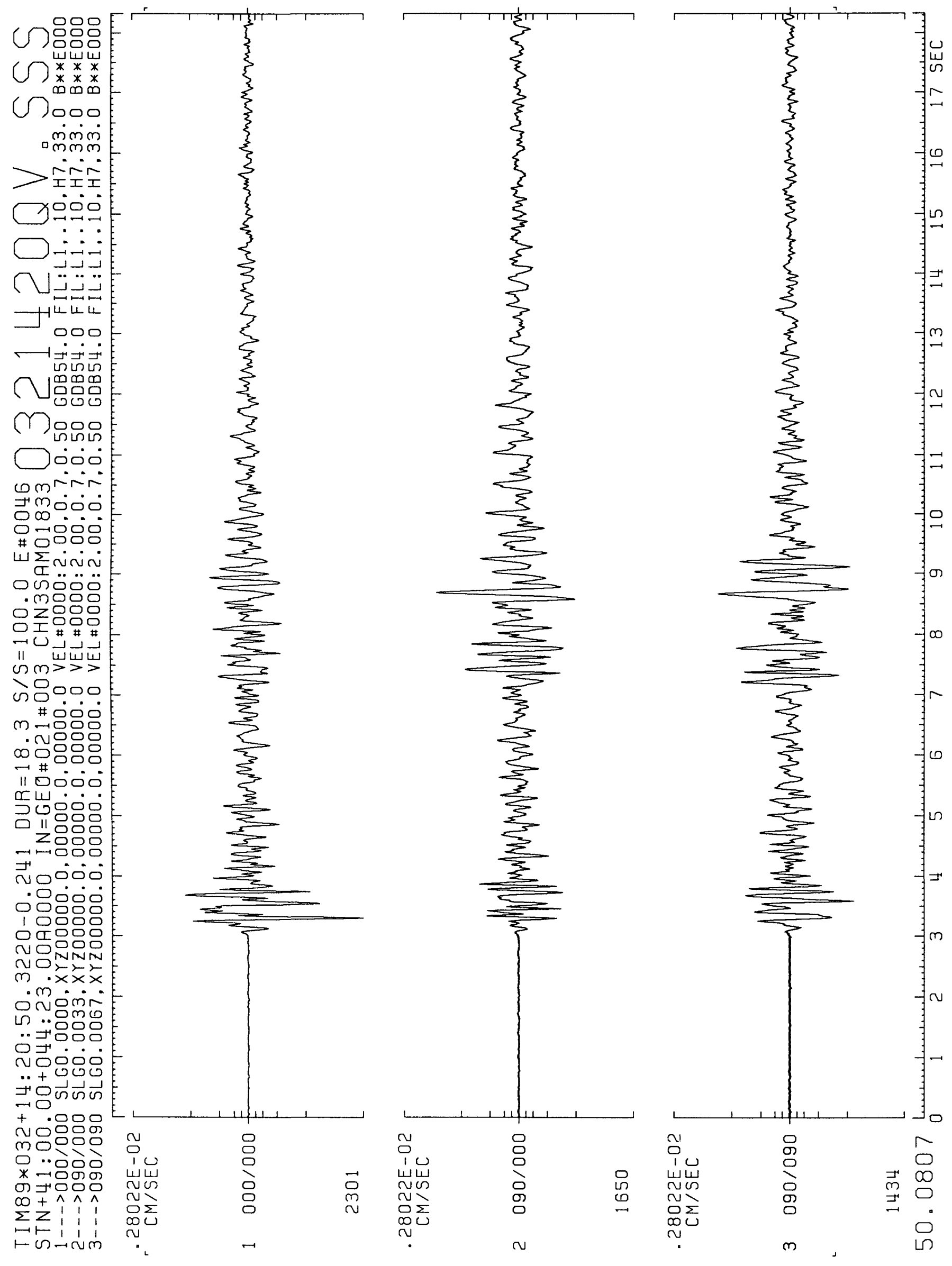




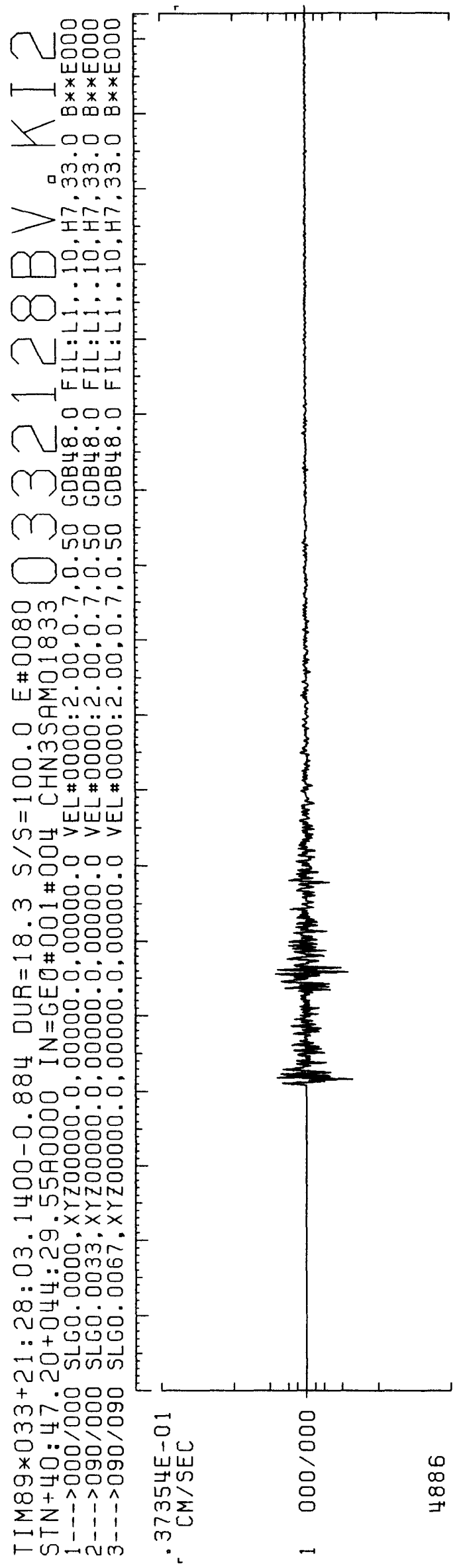

377

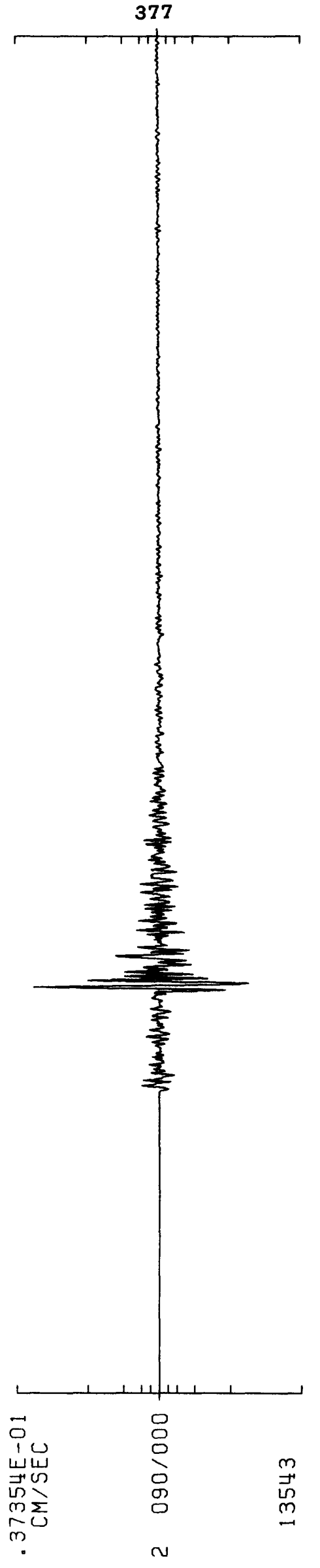

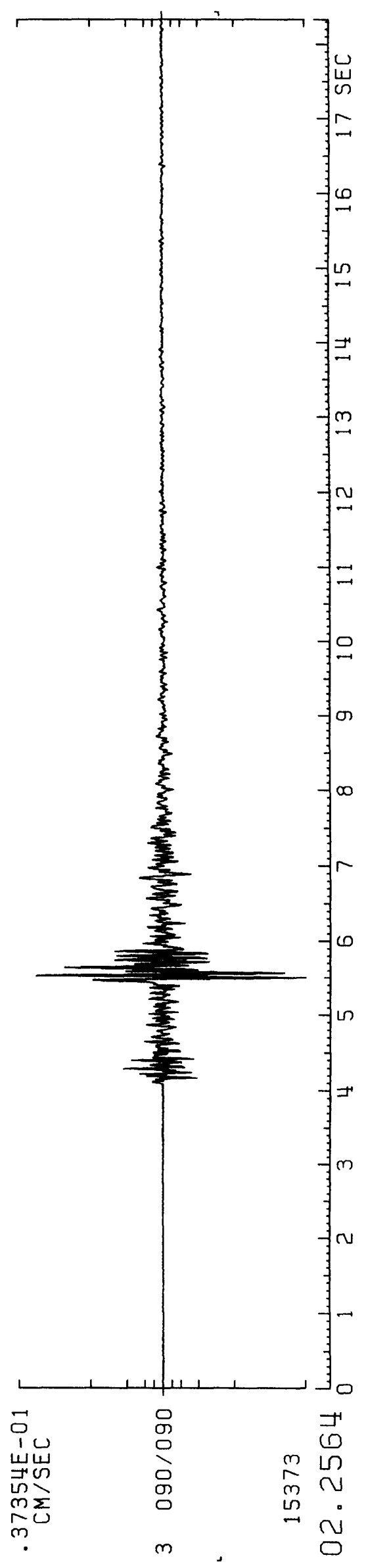



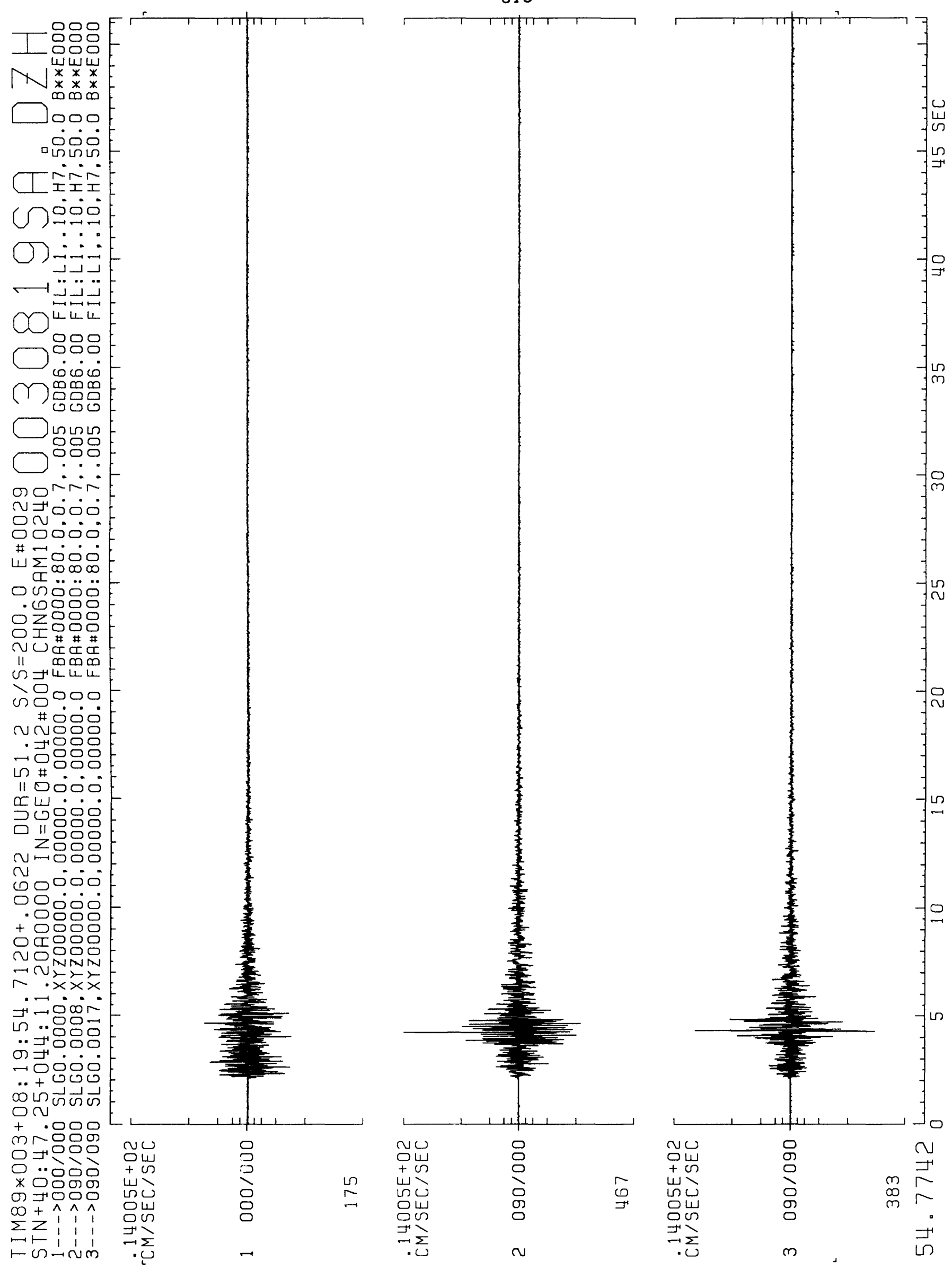

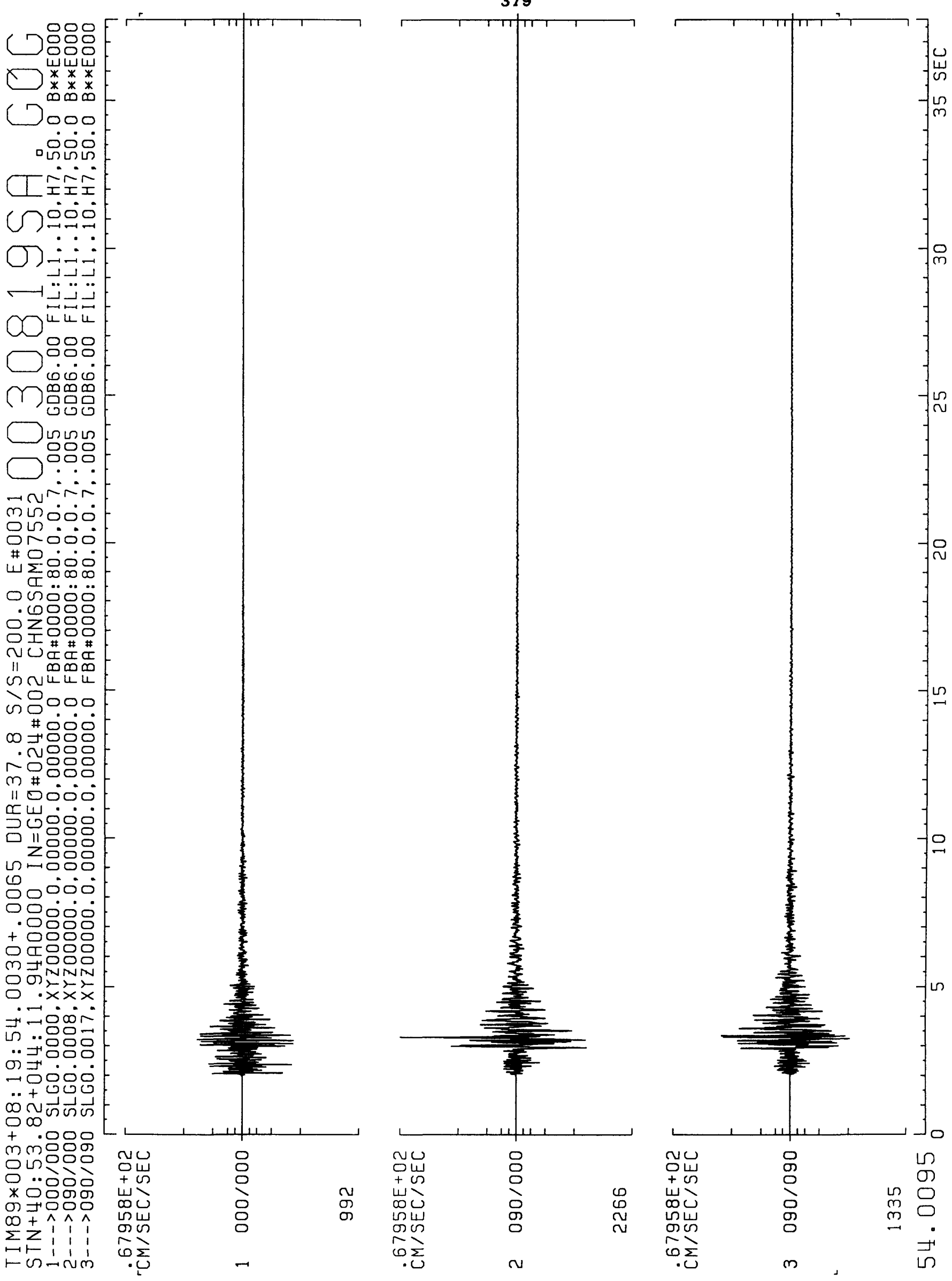


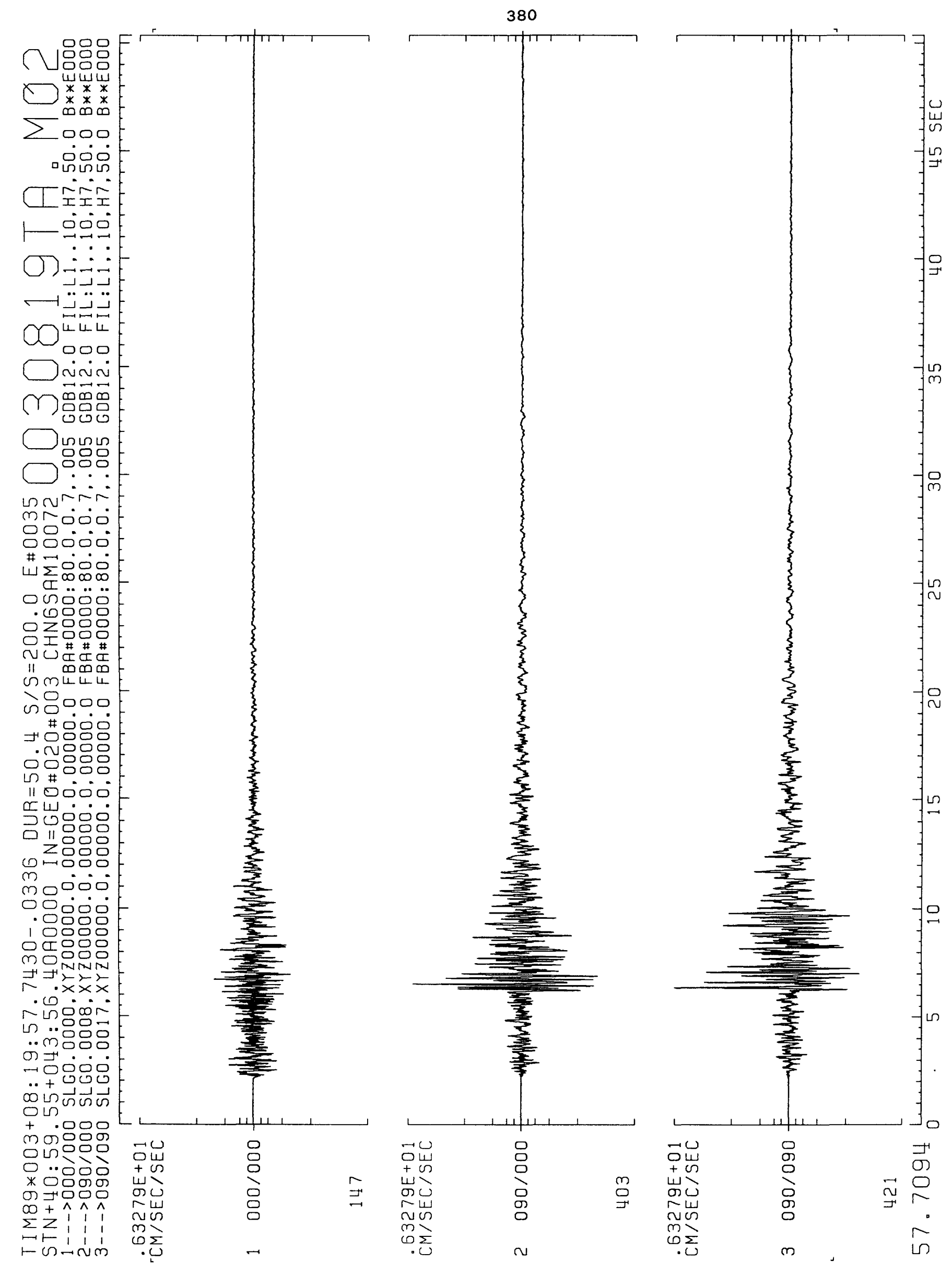


381

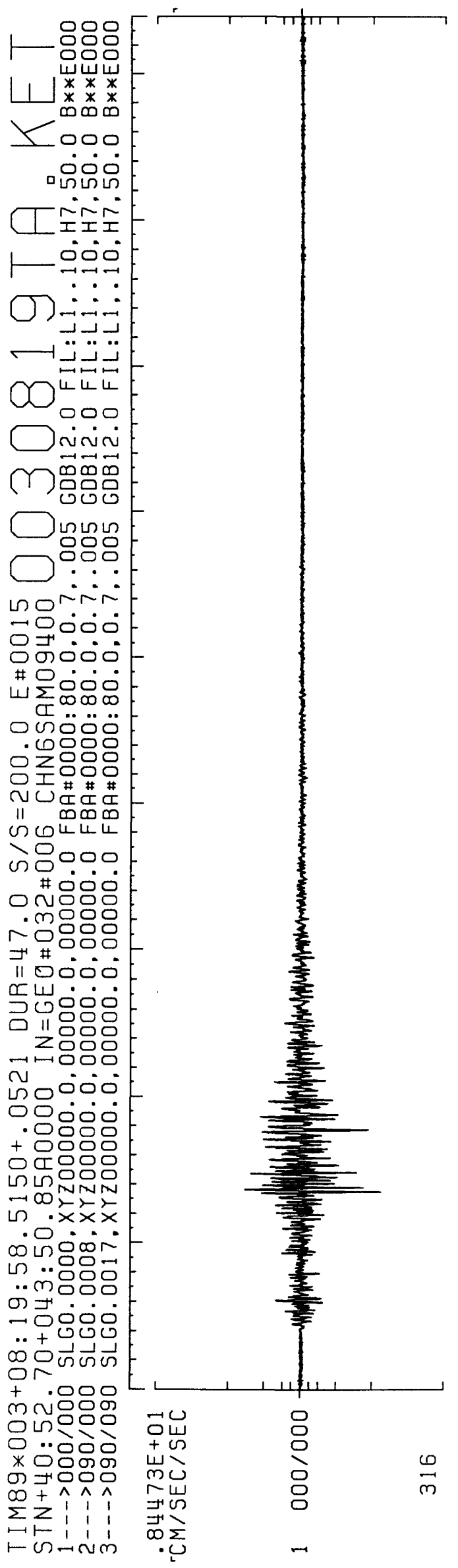

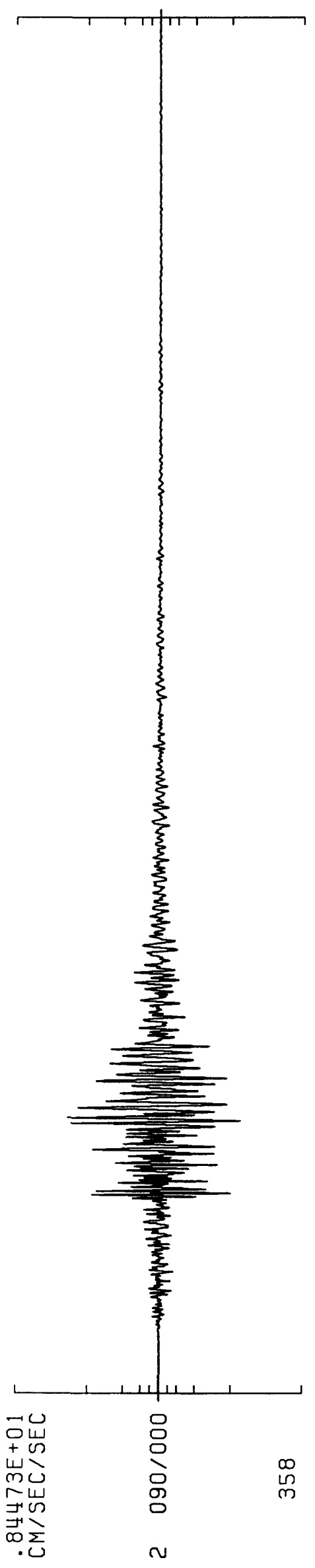

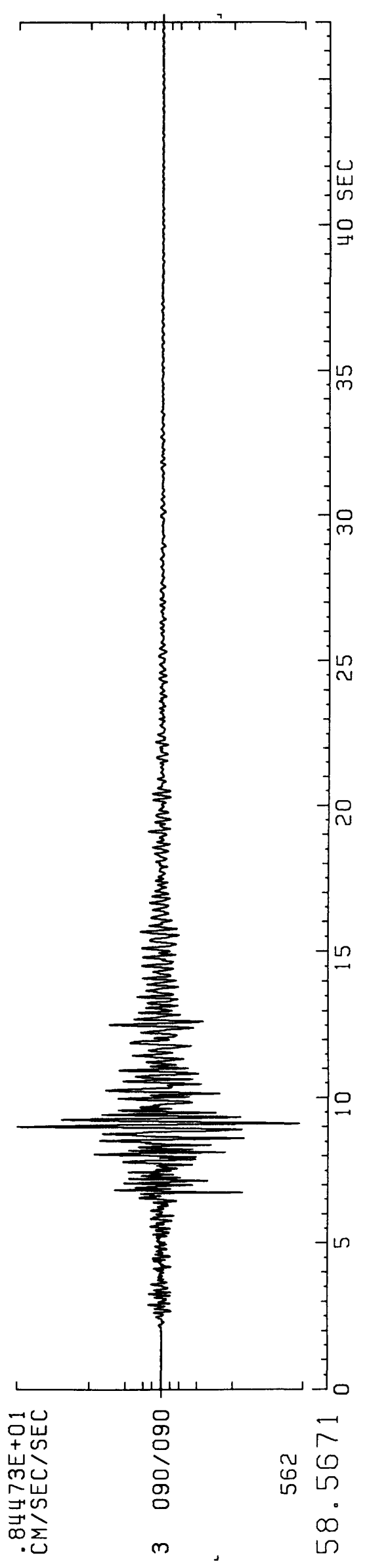




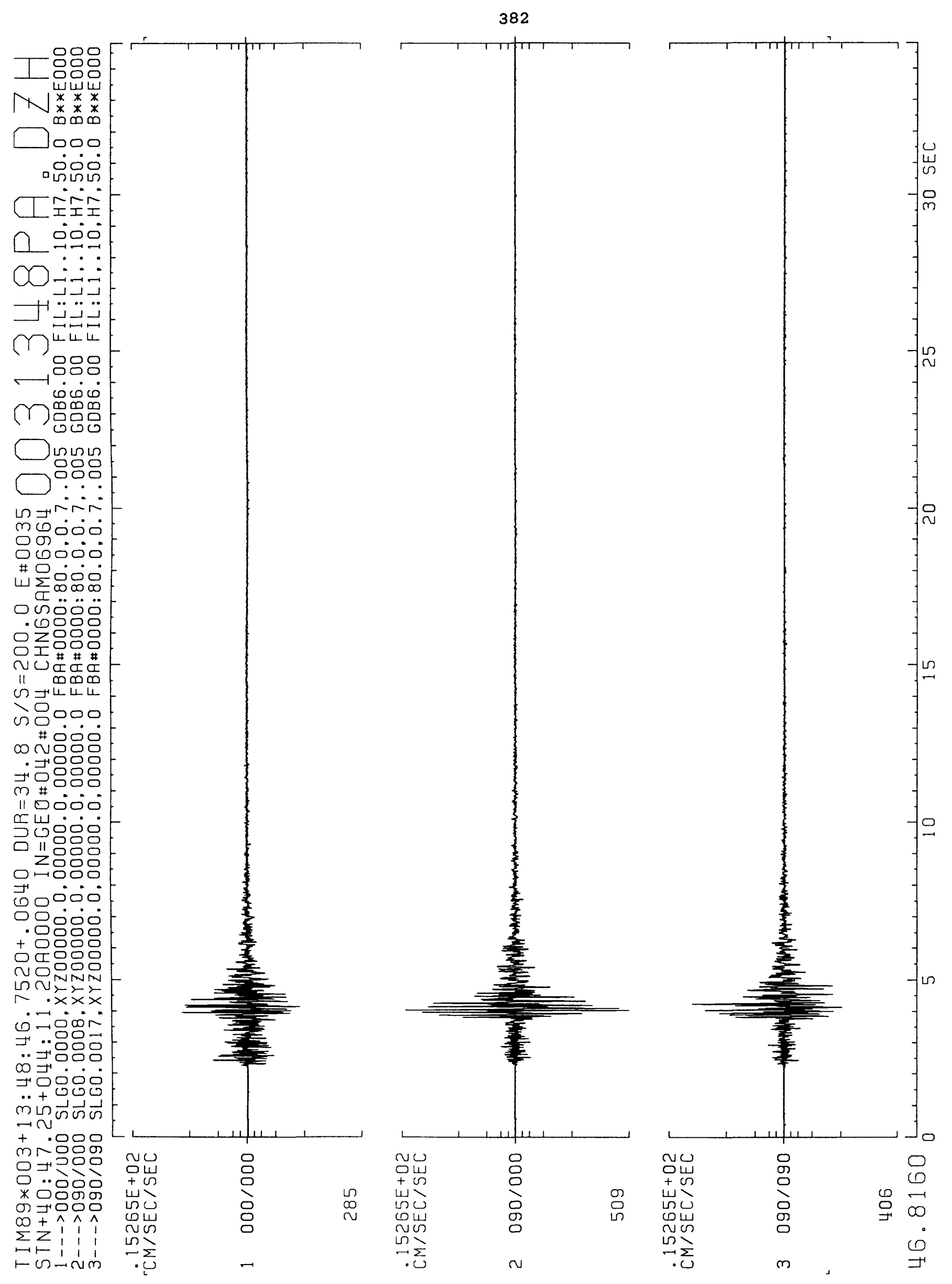




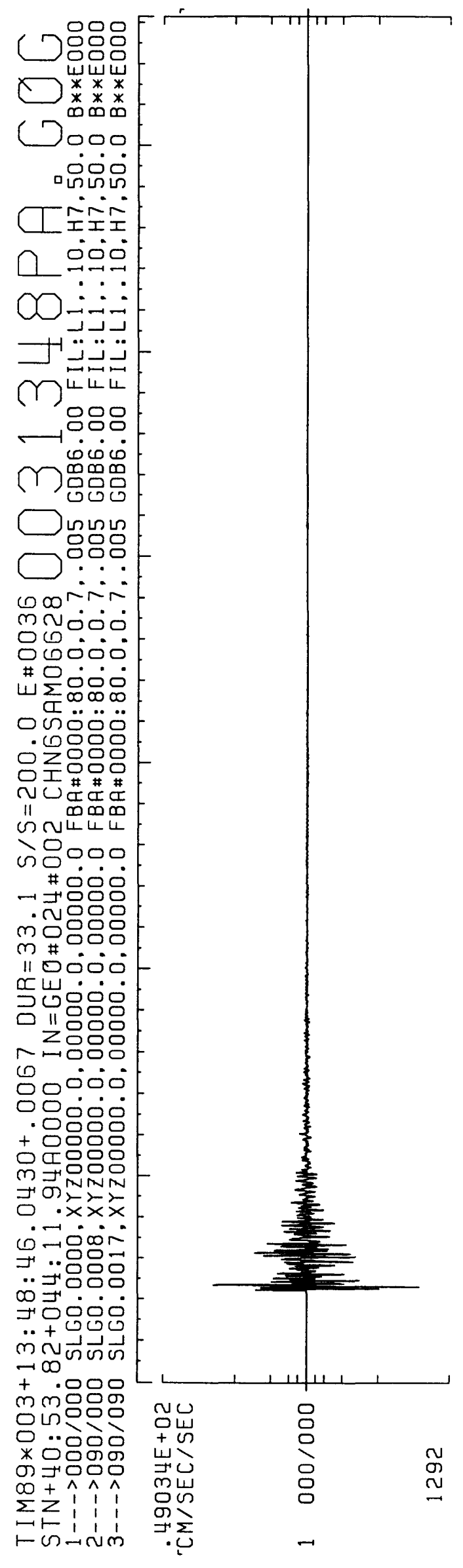

383

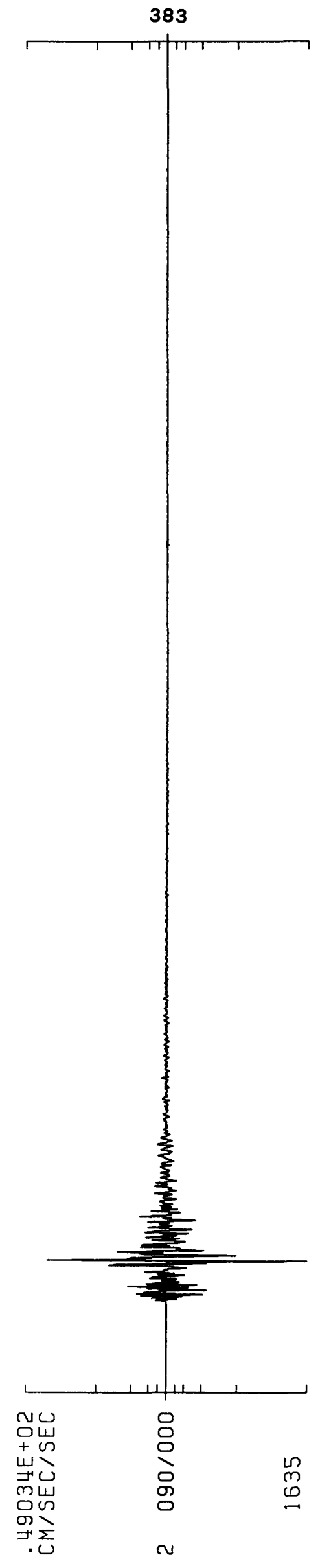

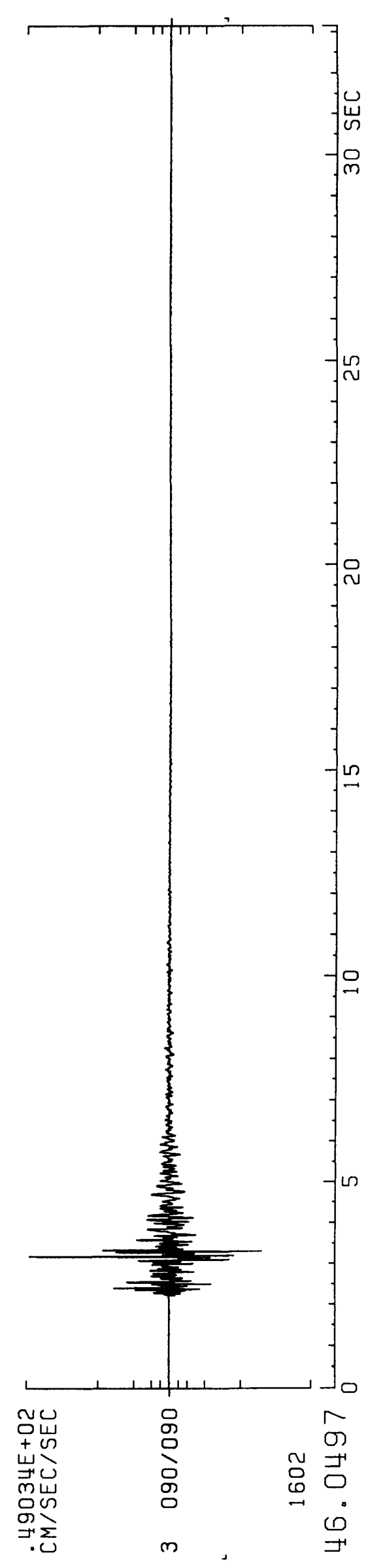




$$
+1
$$



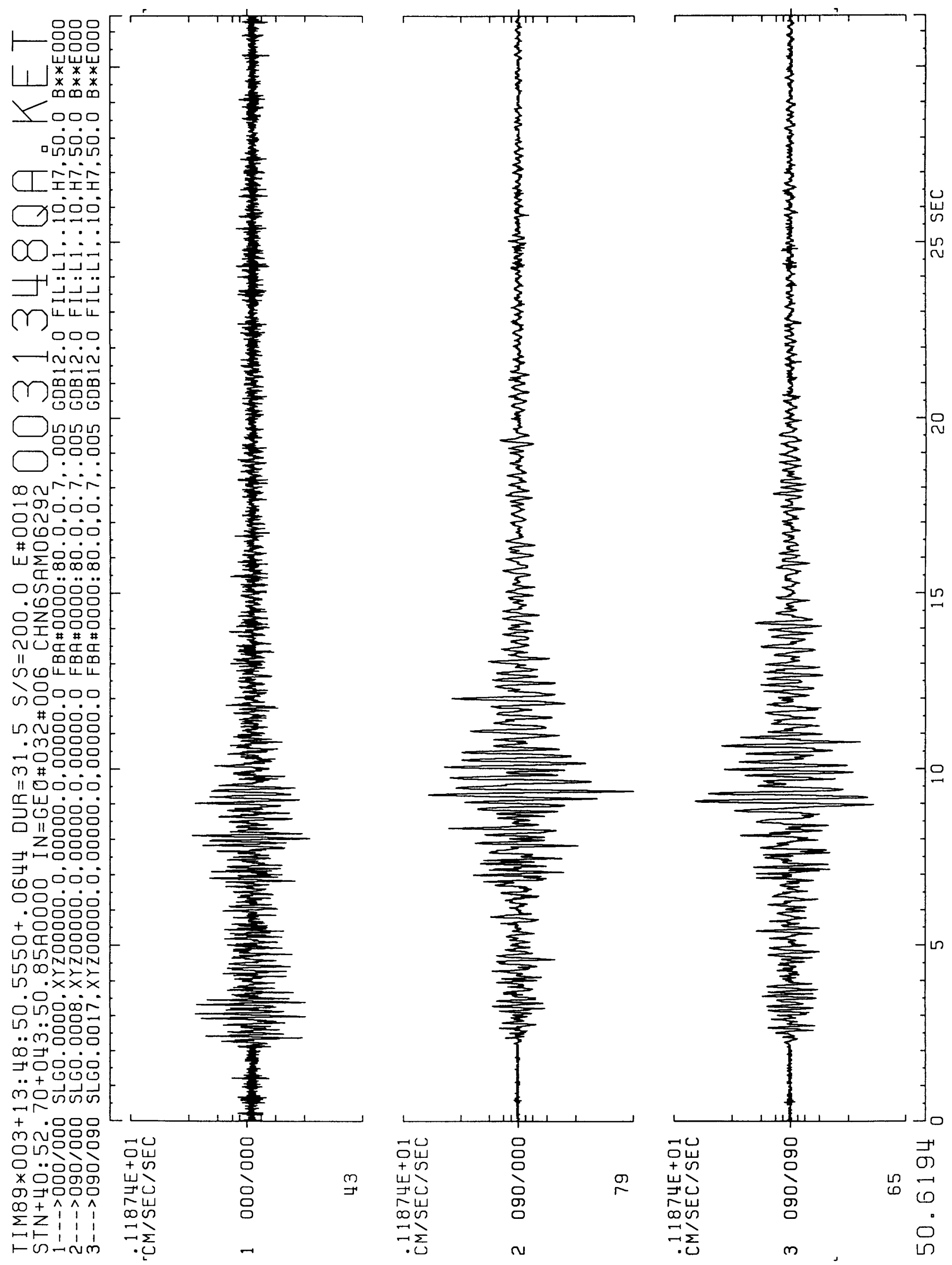


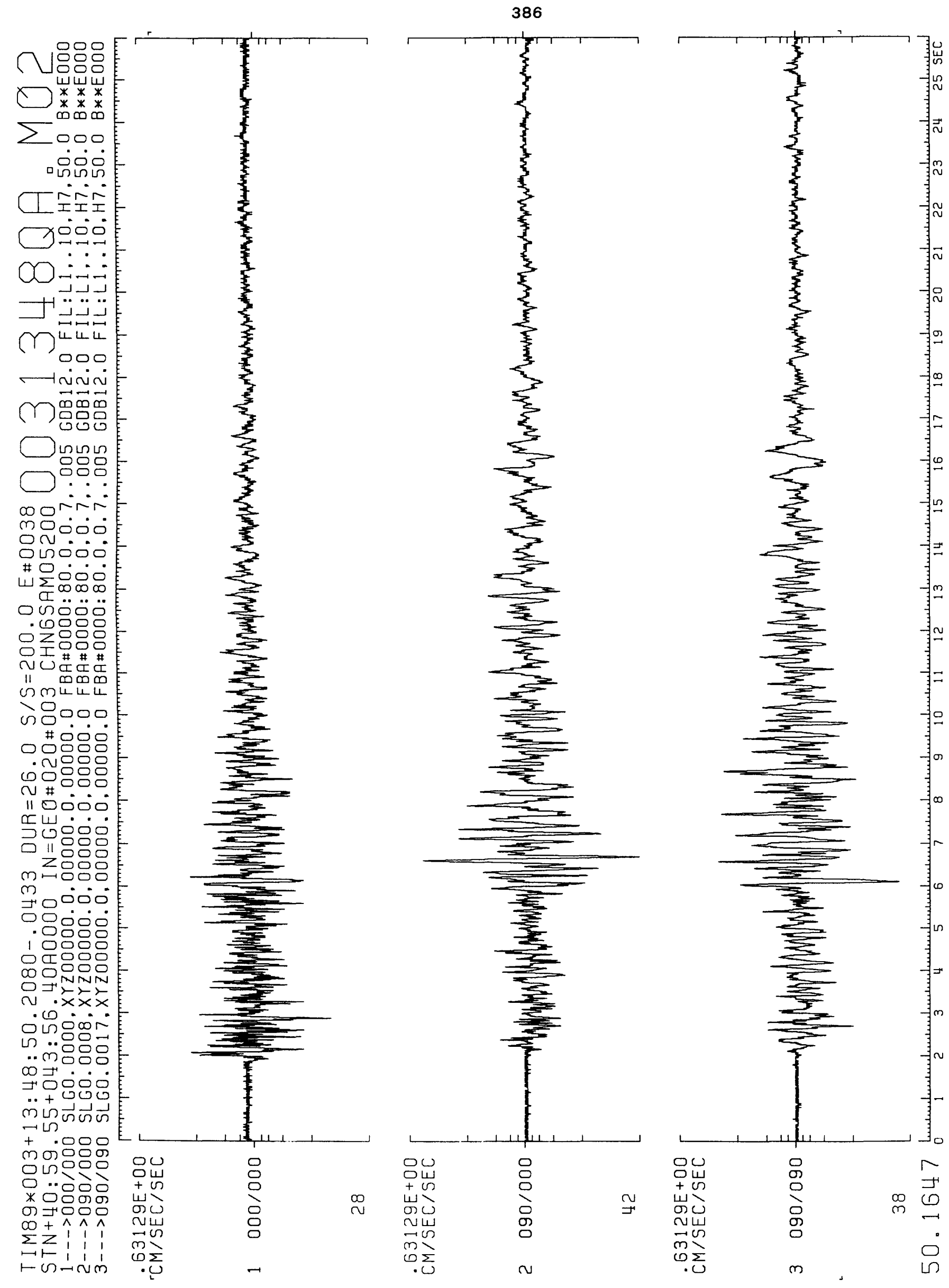


387

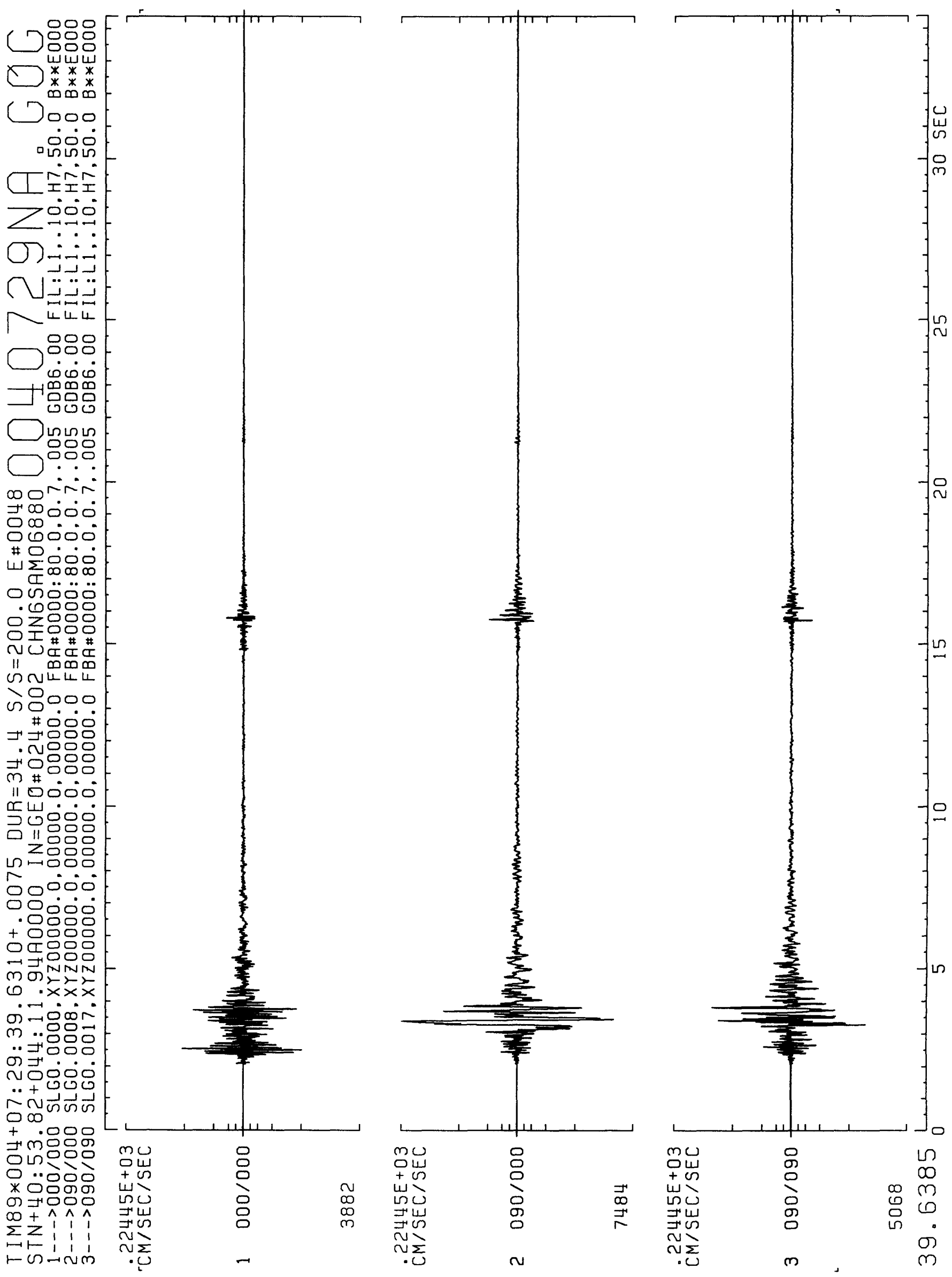



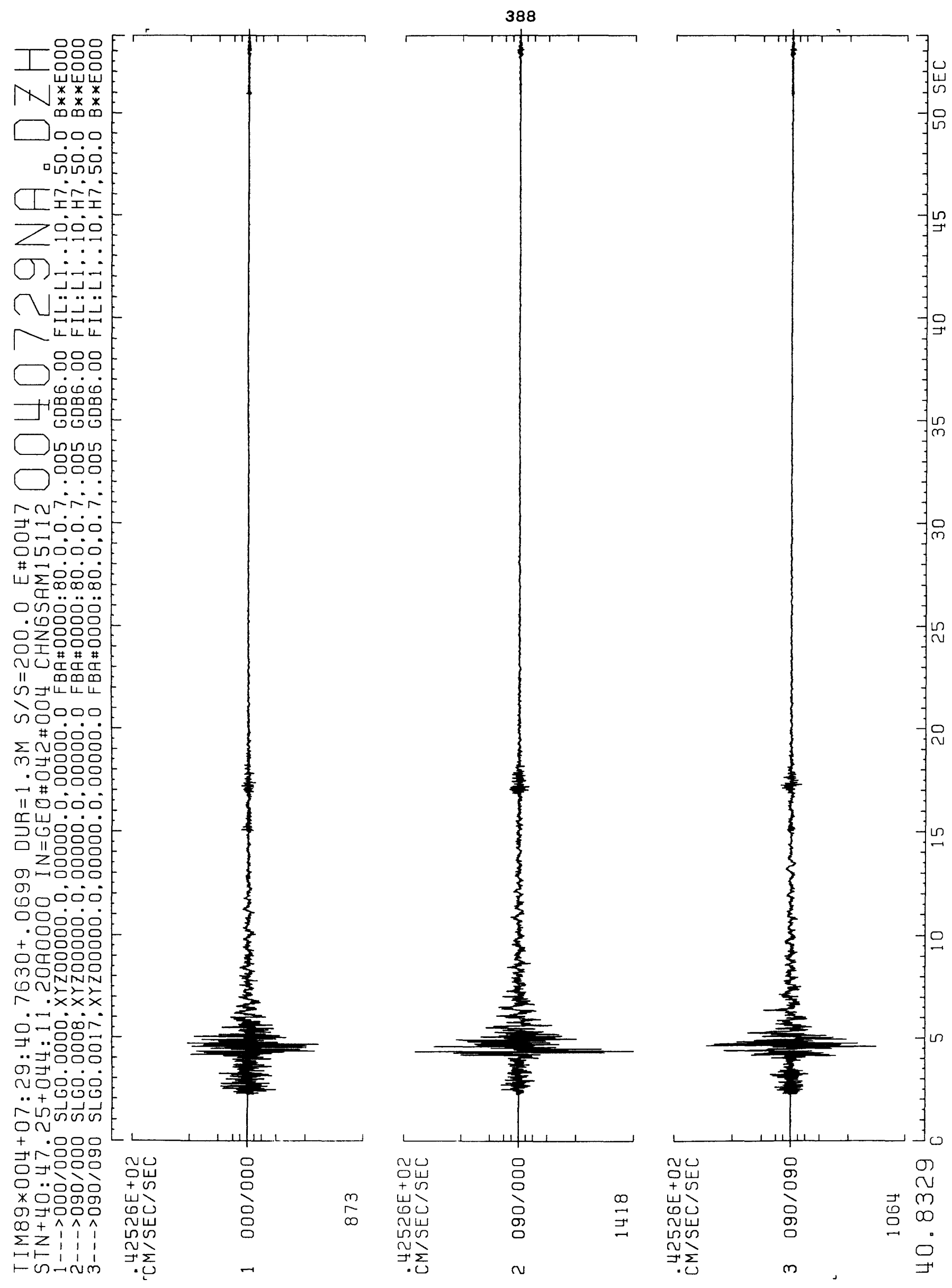

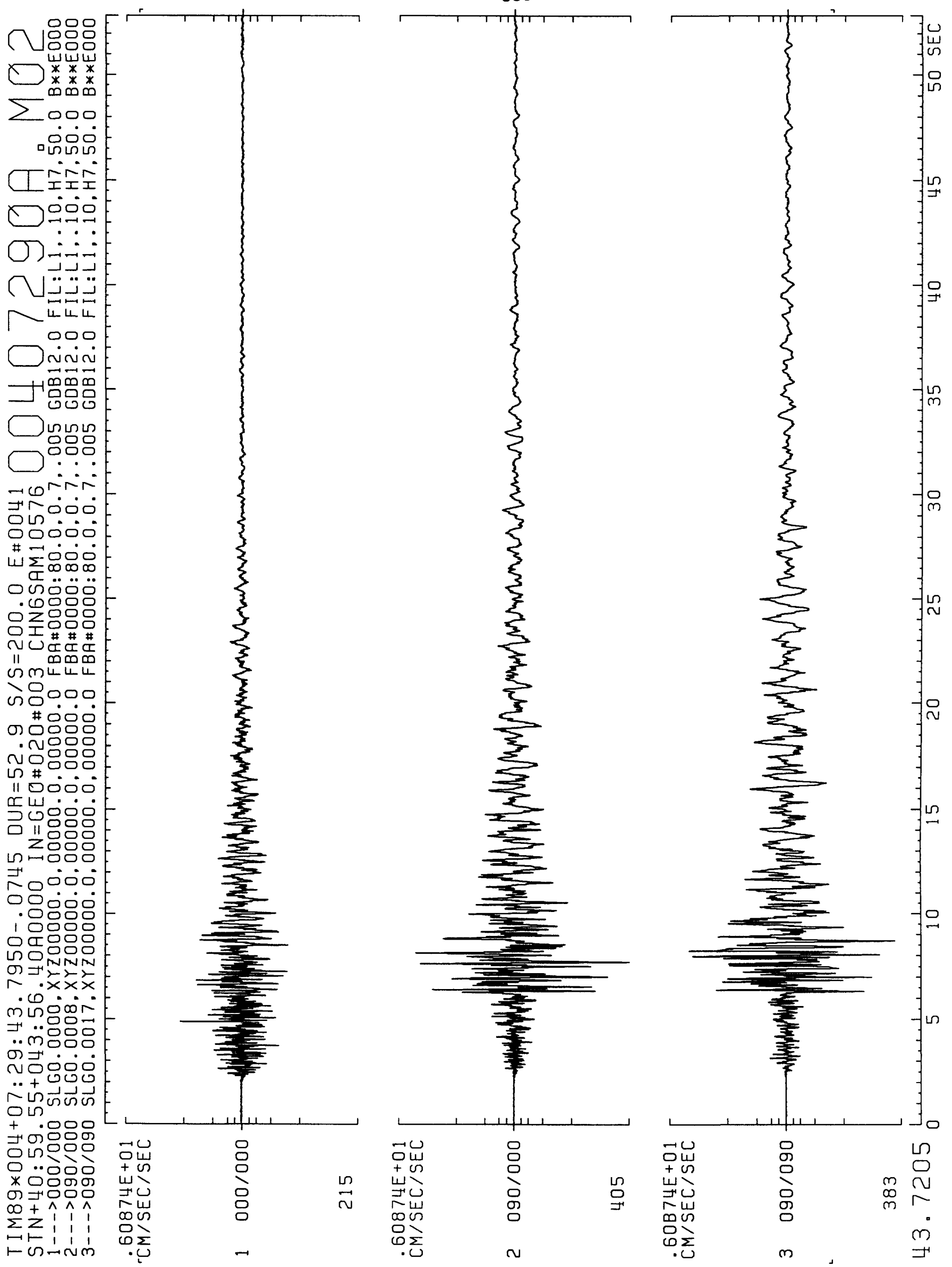


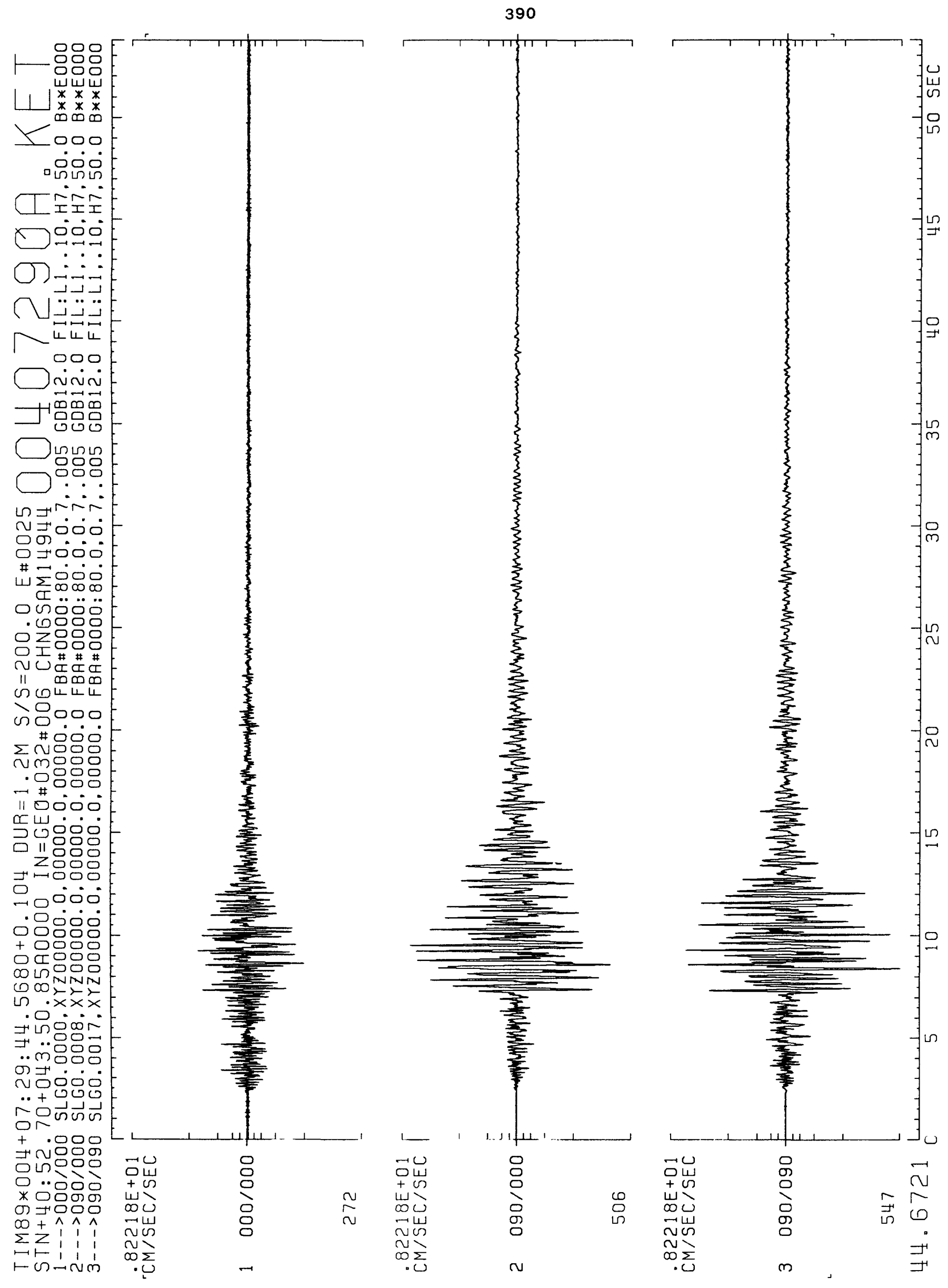


391

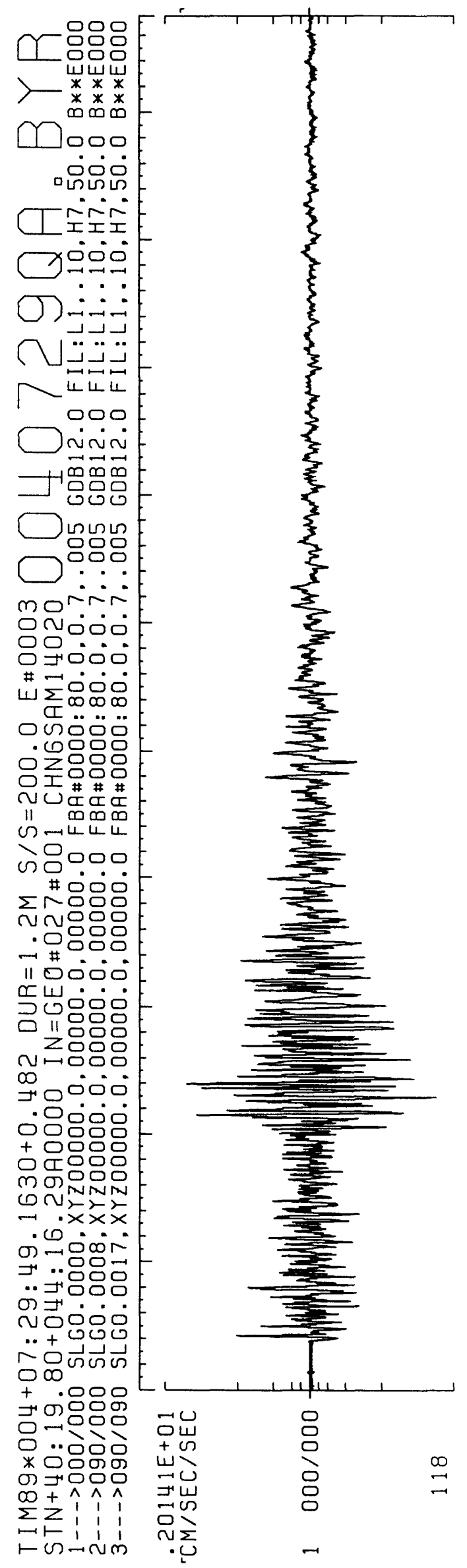

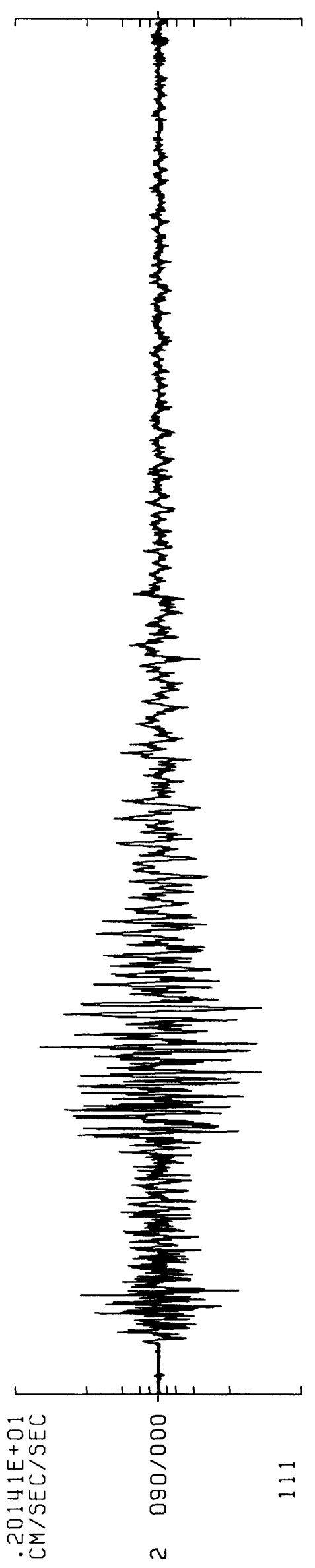

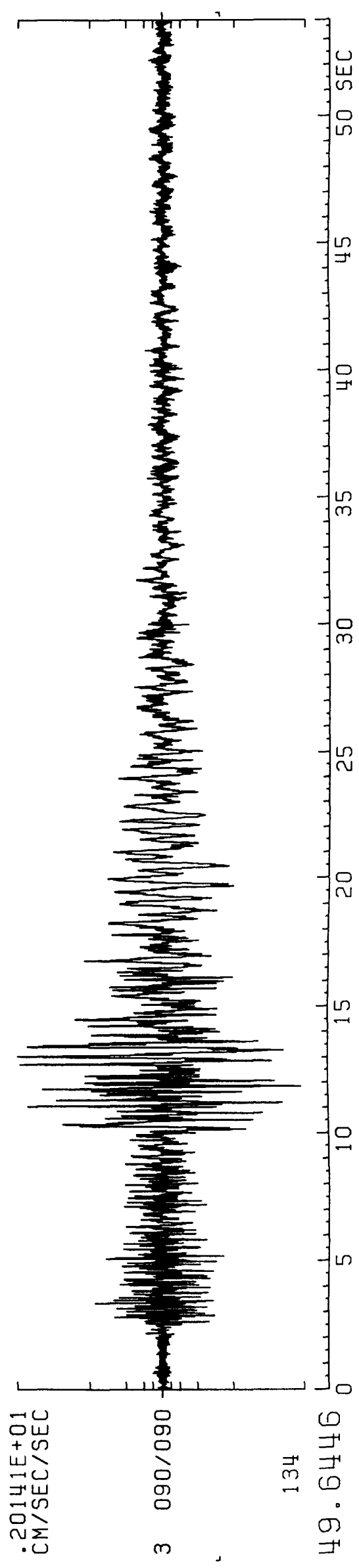


392

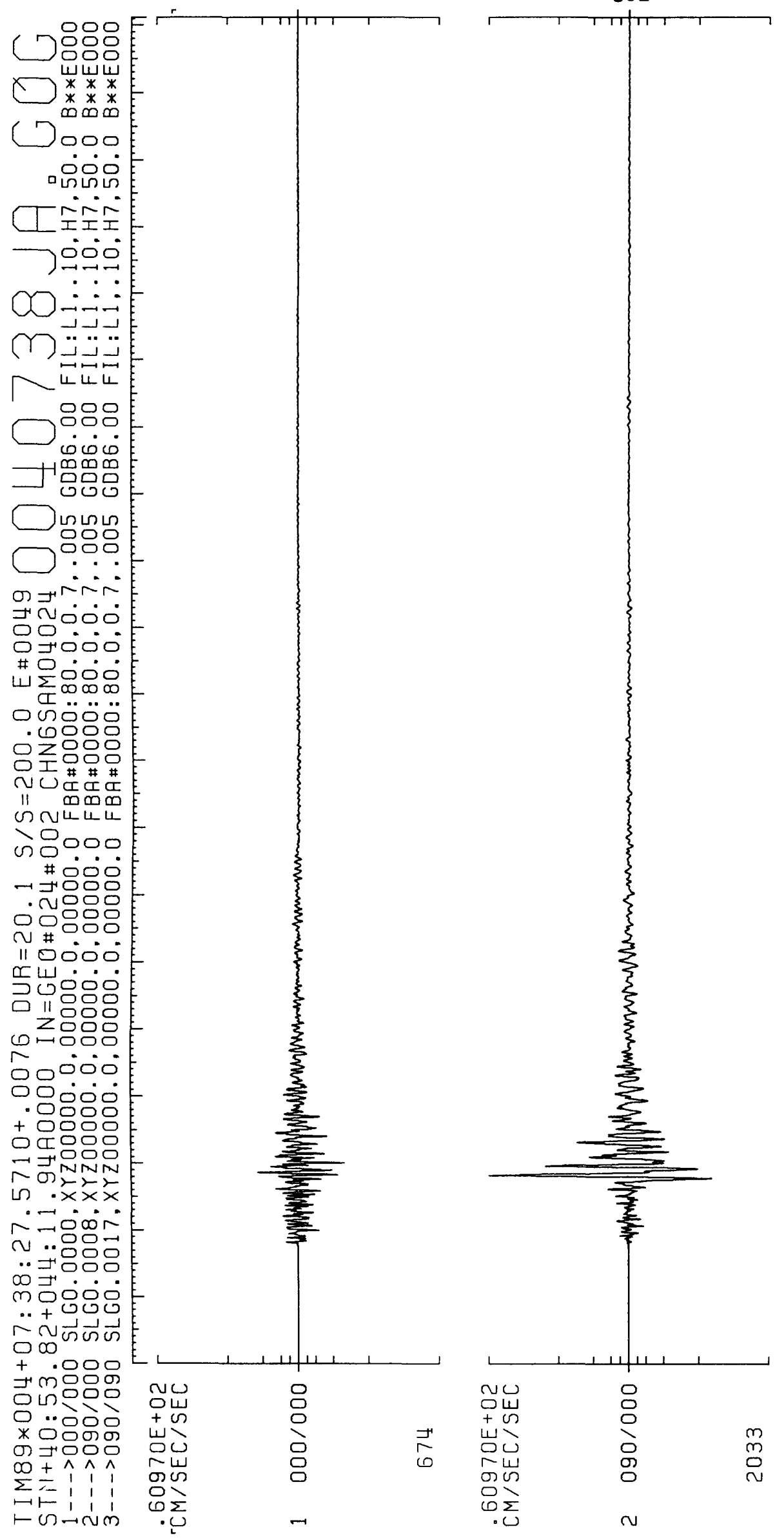

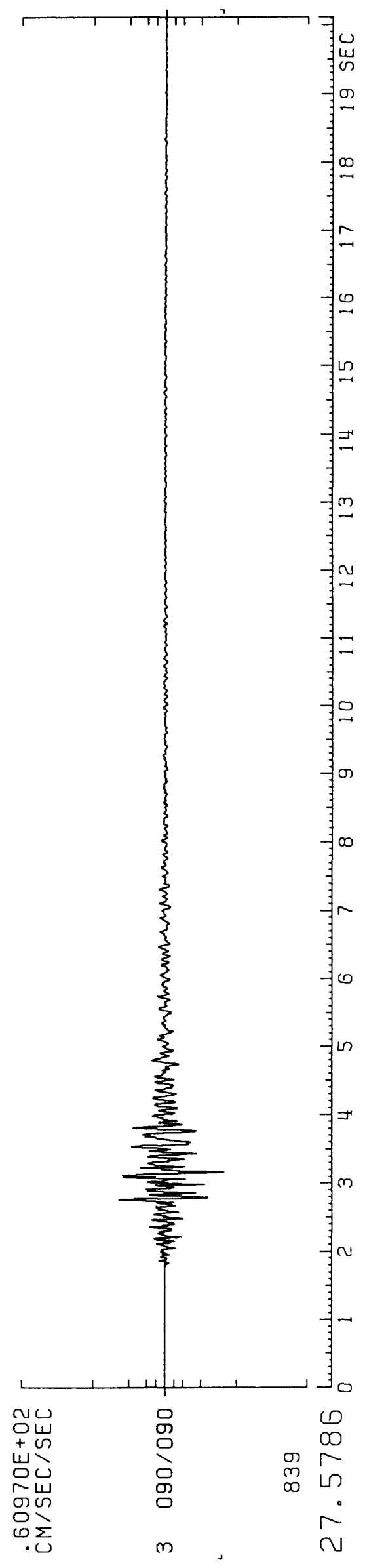


393
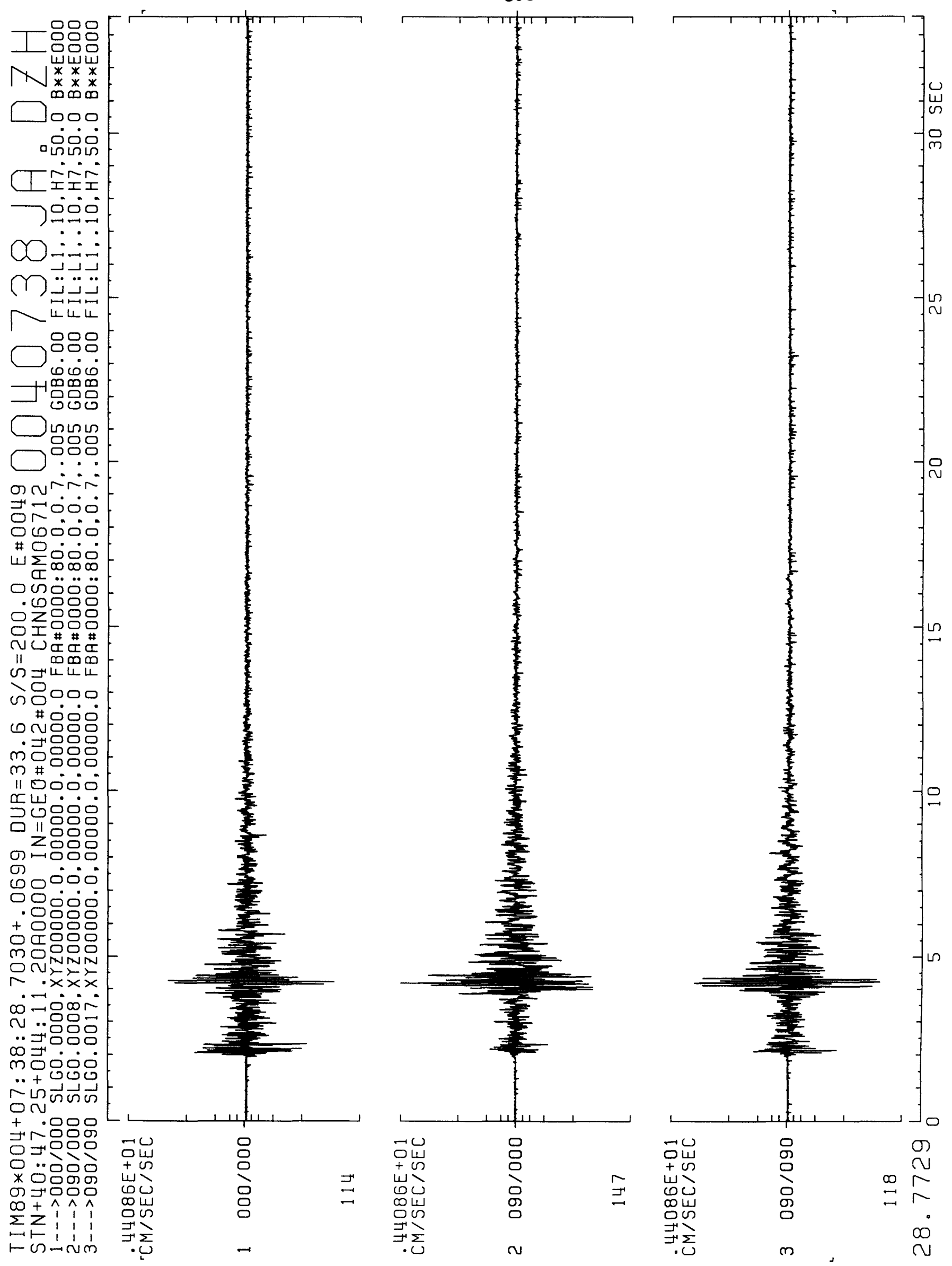

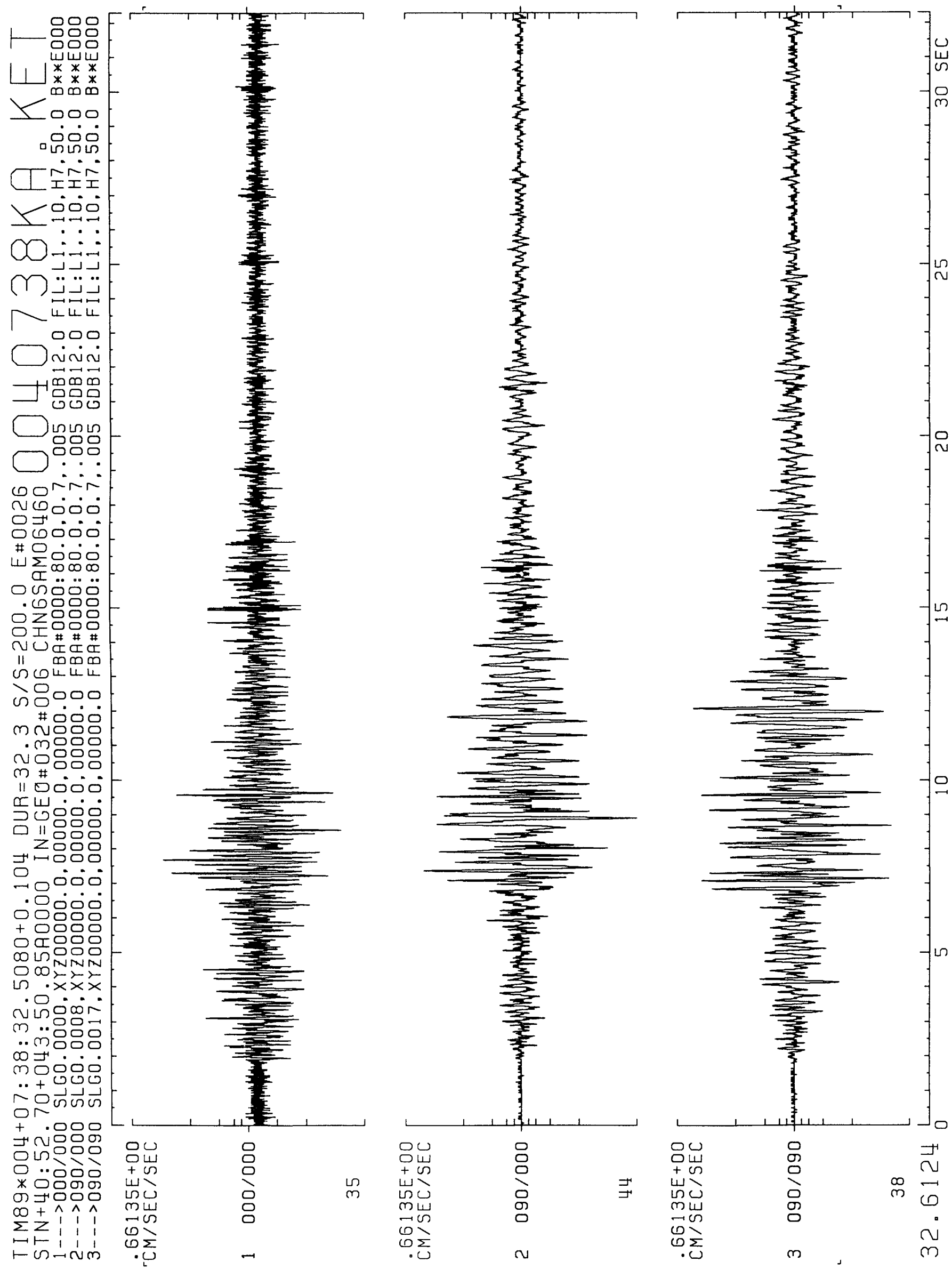

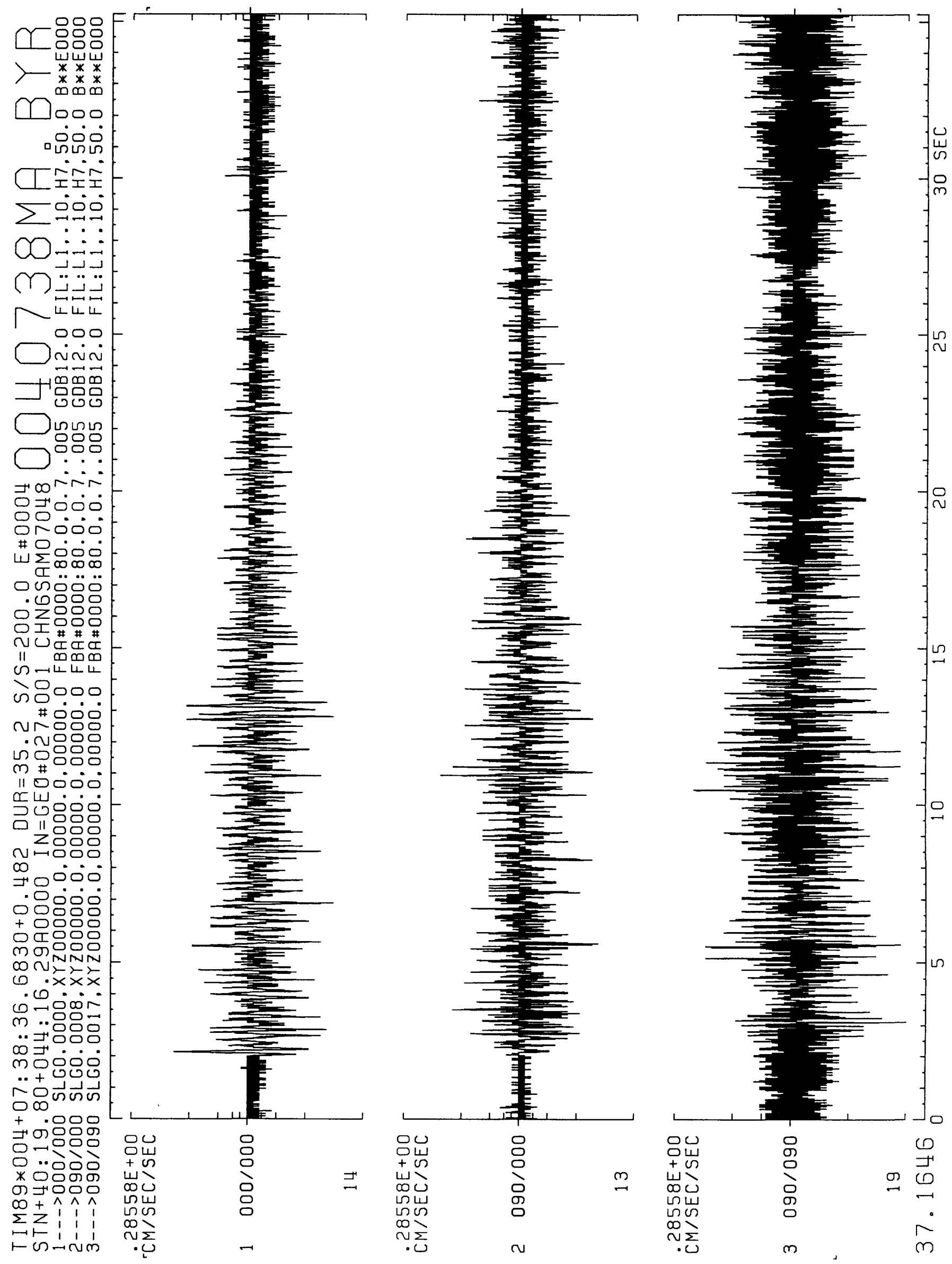


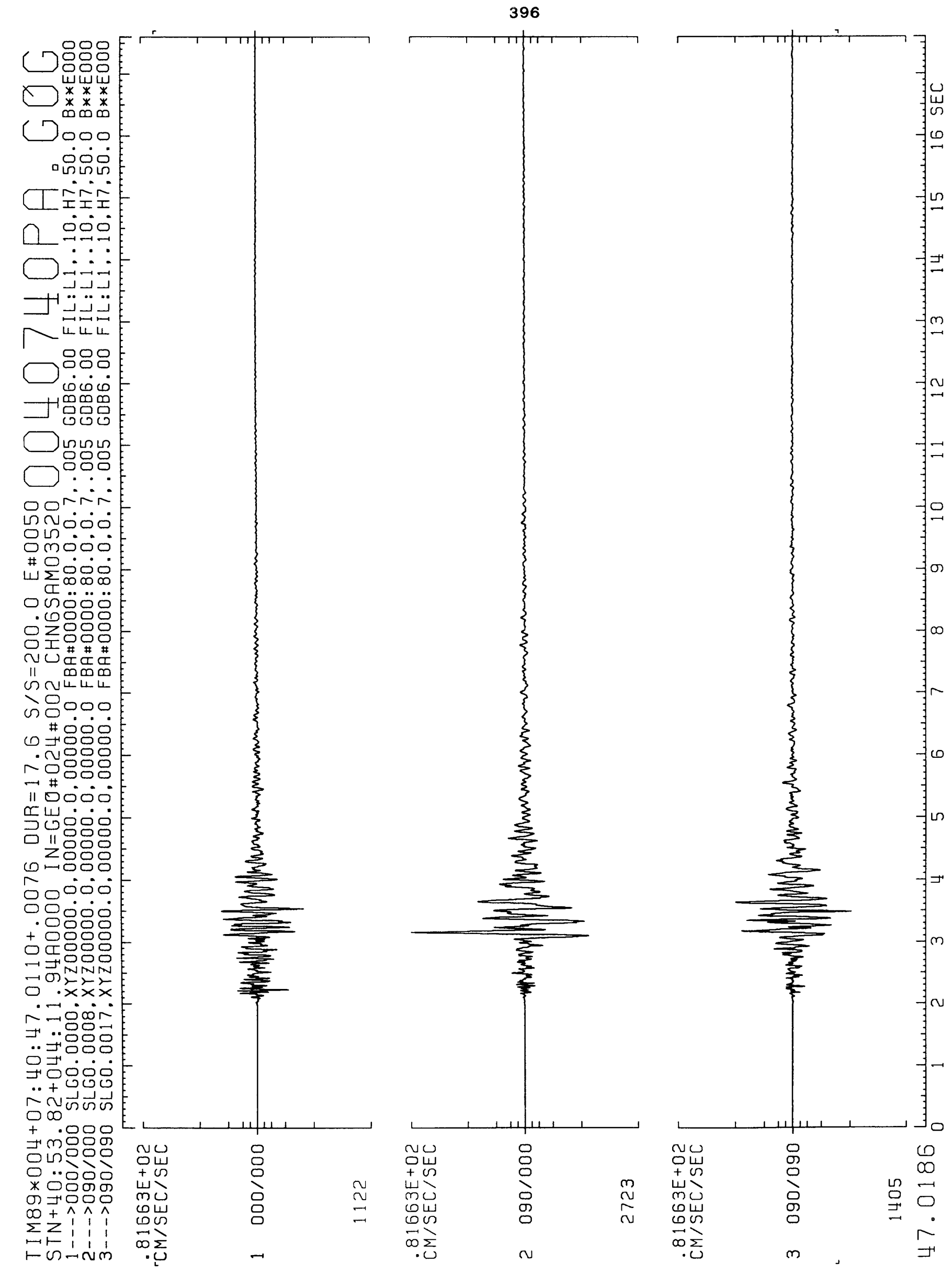



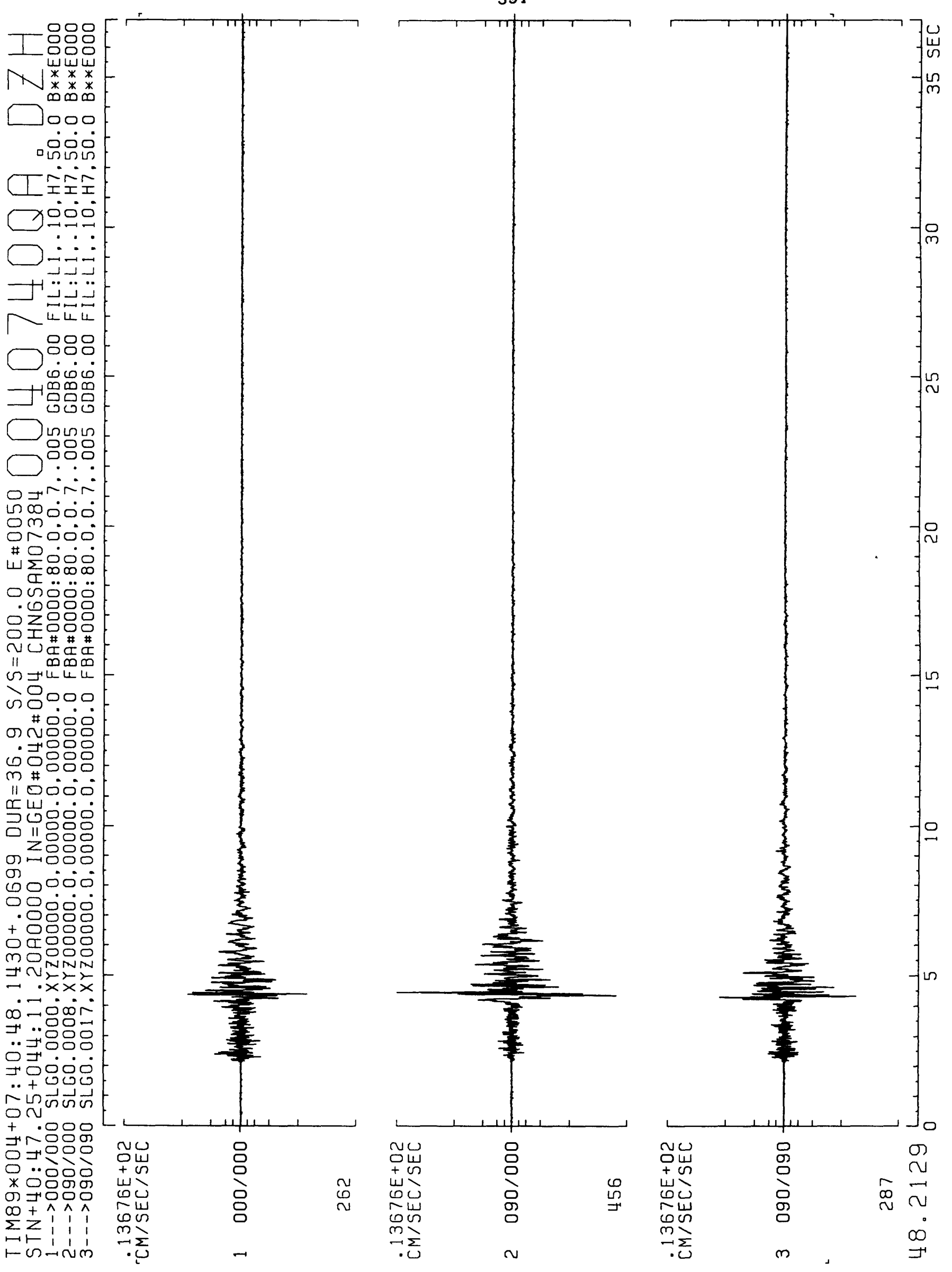

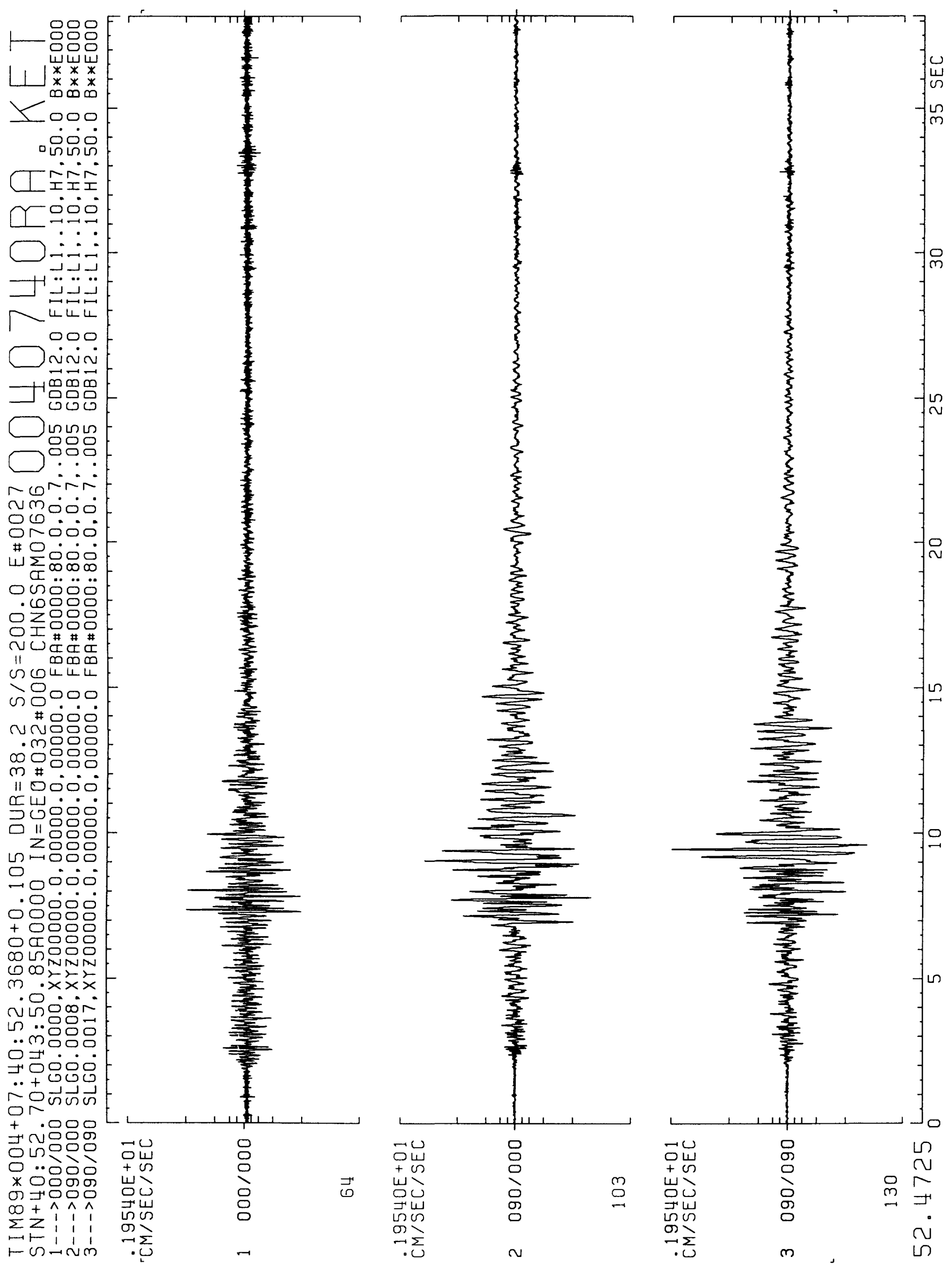
399

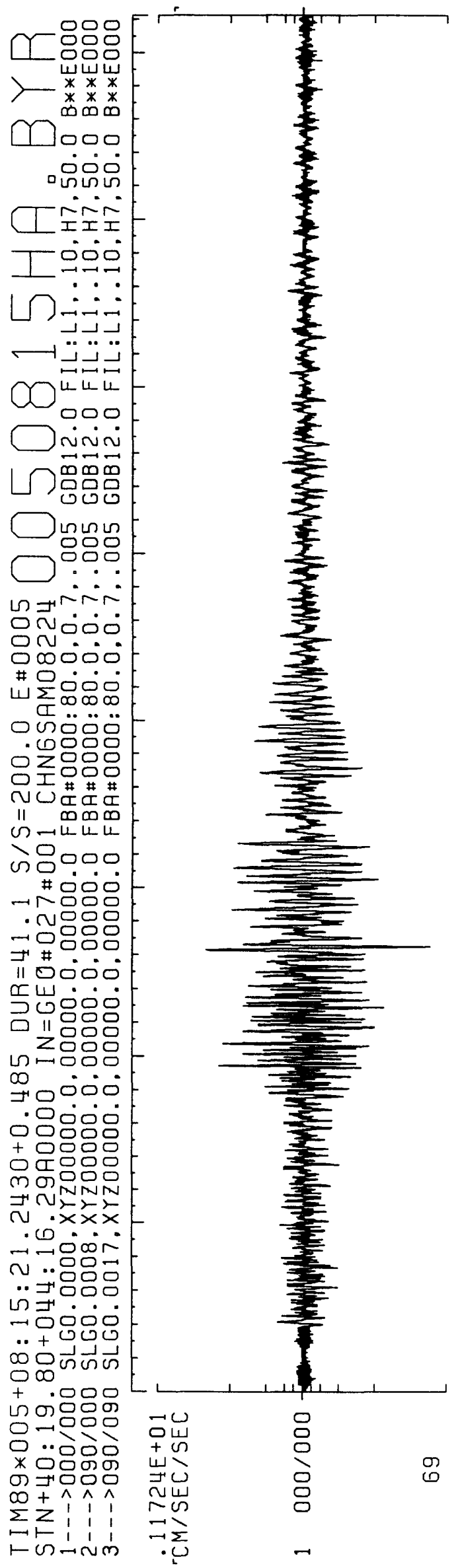

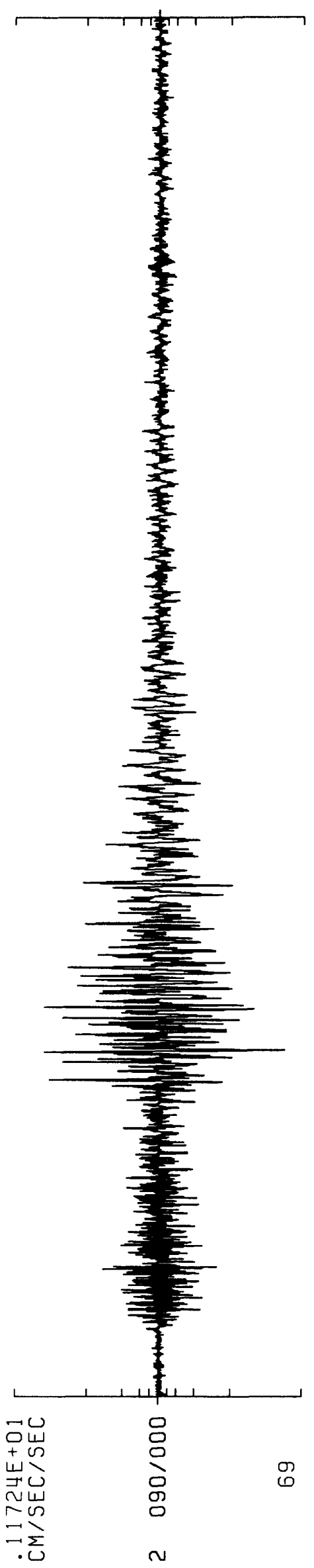

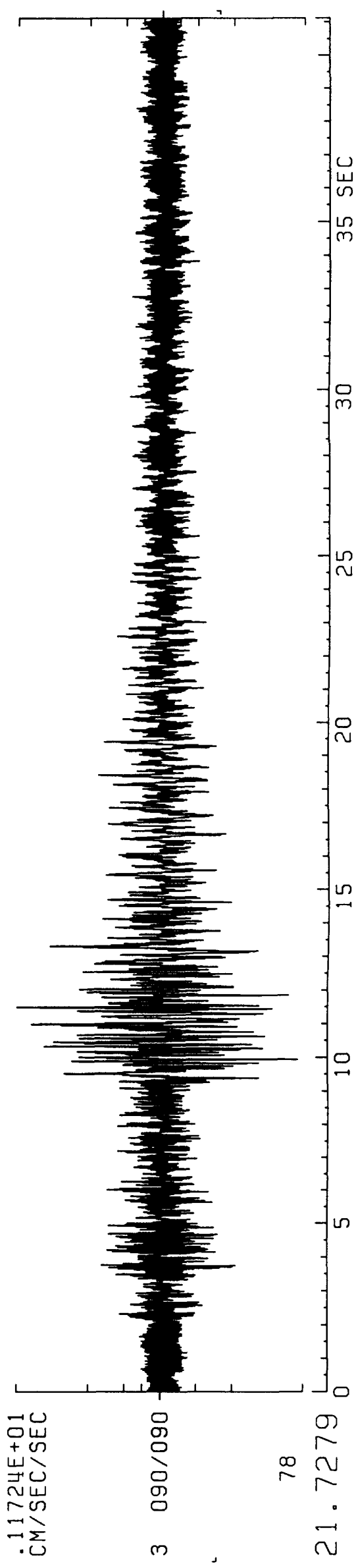




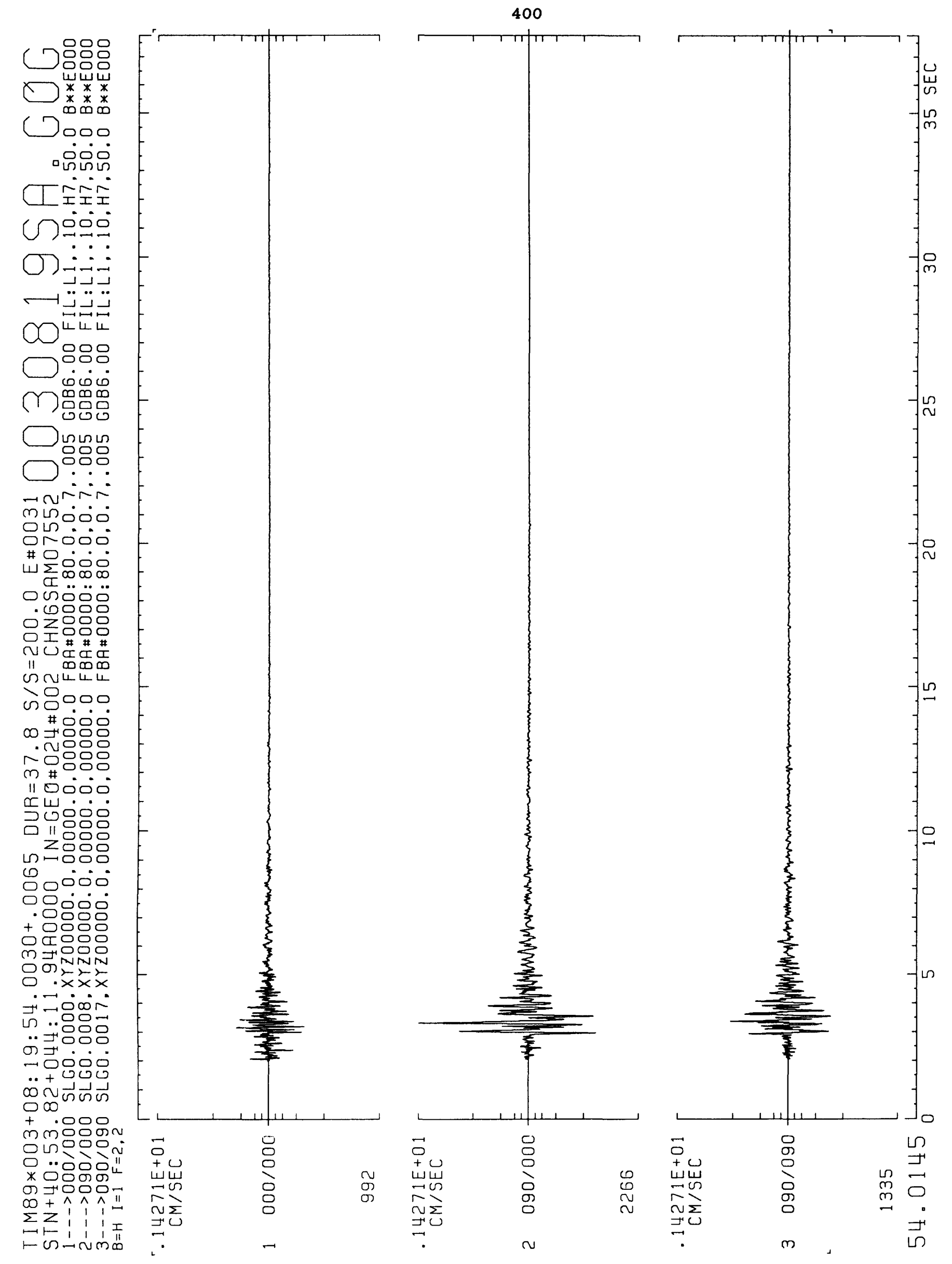


401

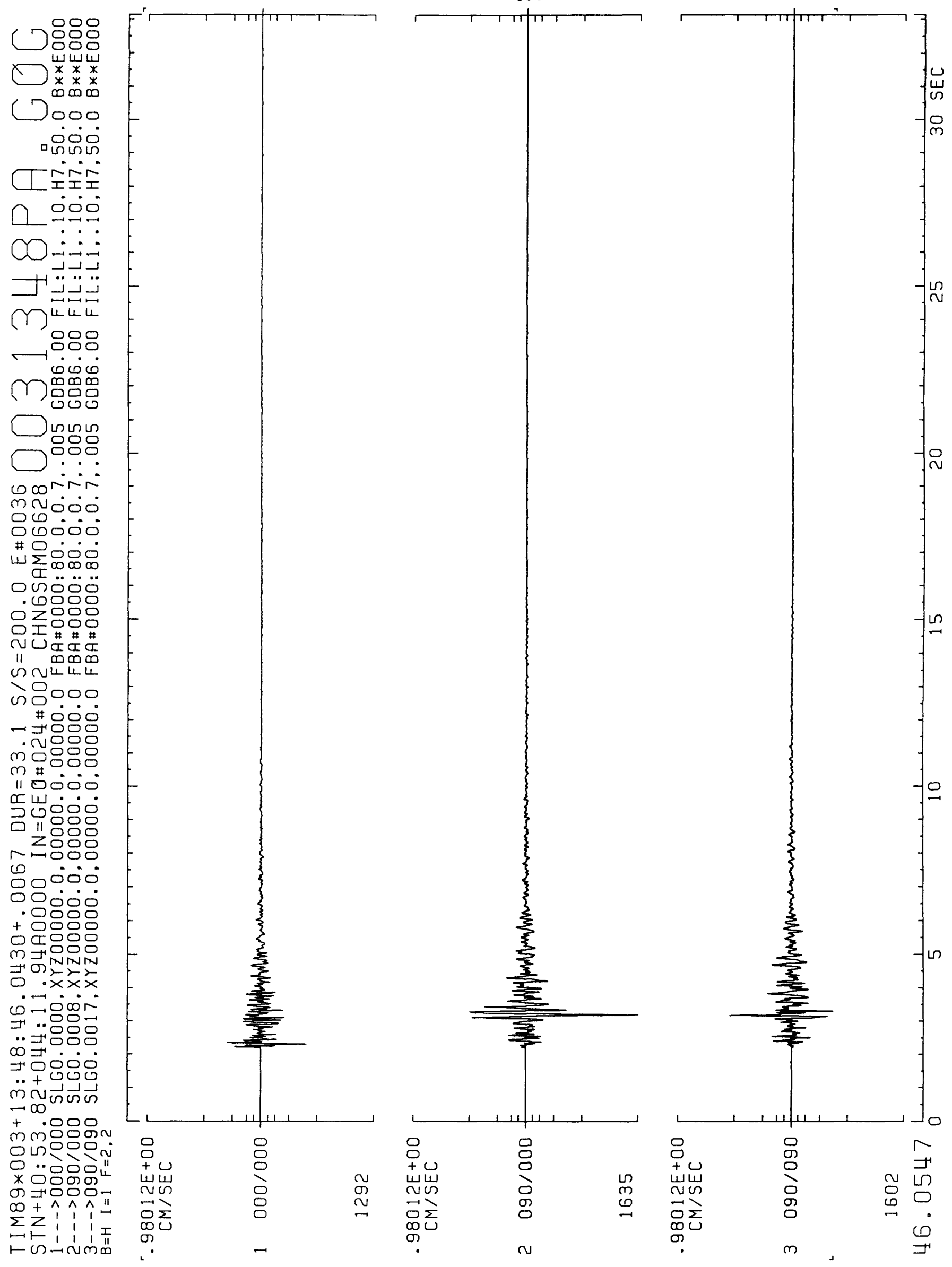




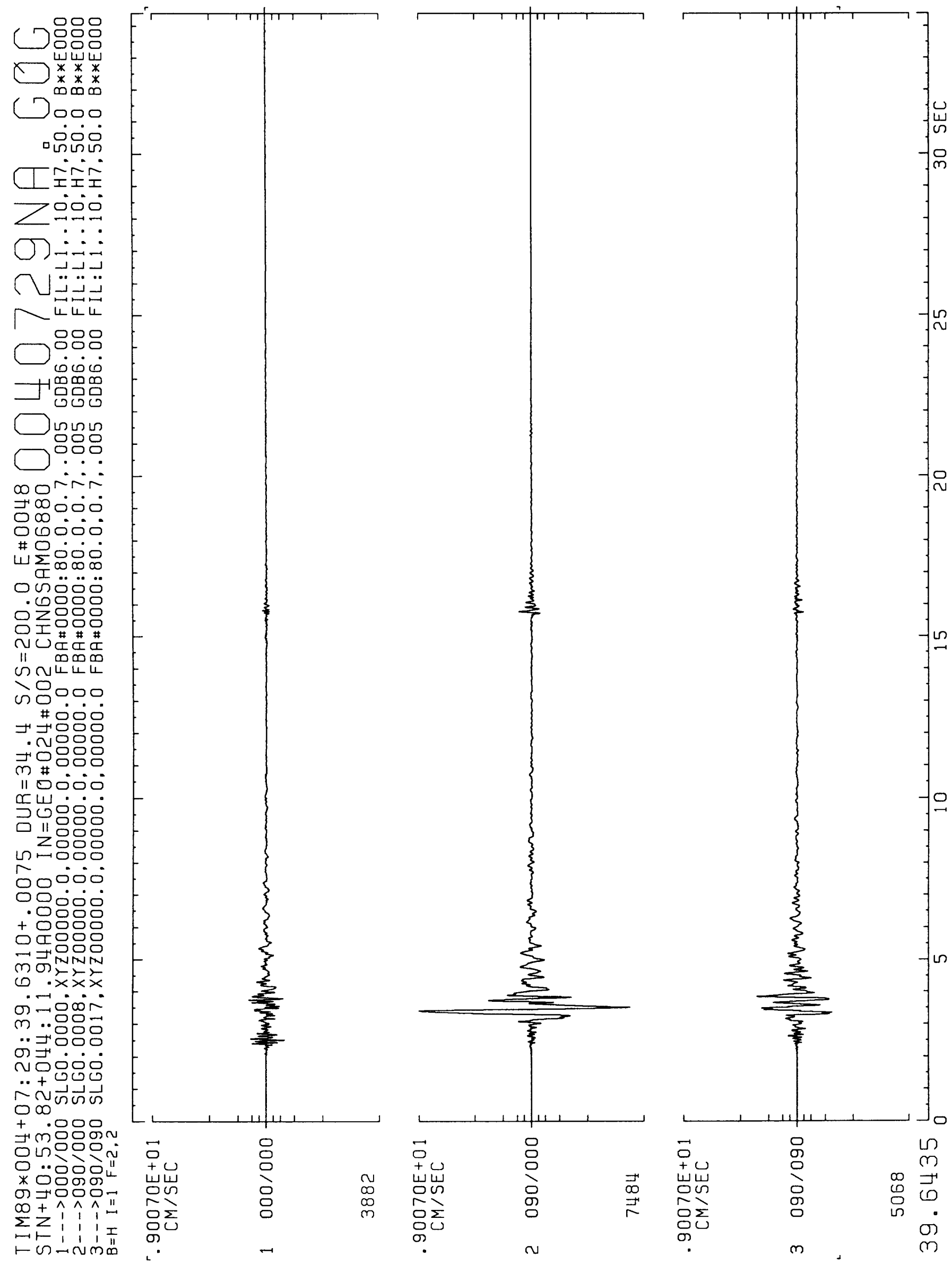


403
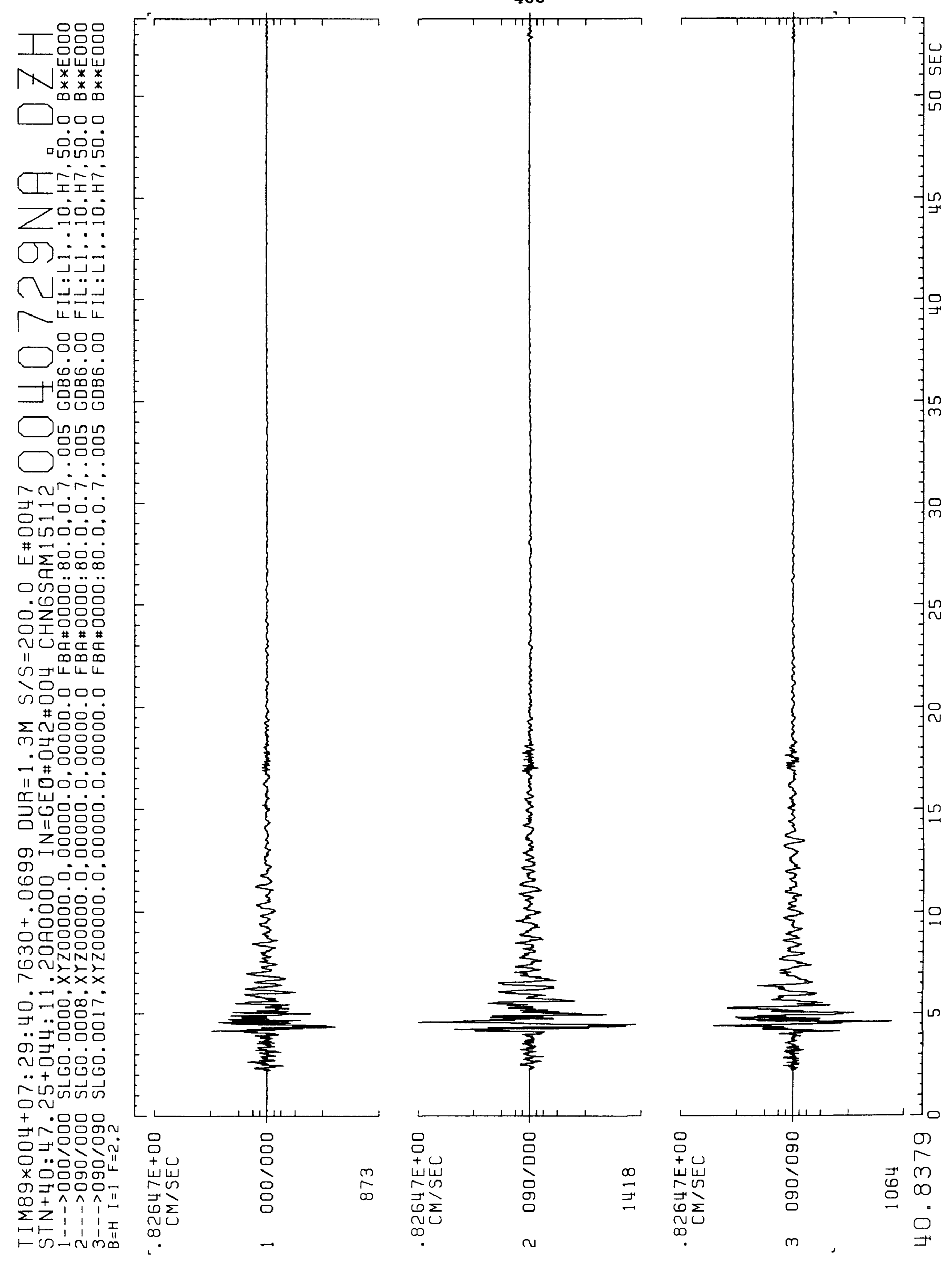


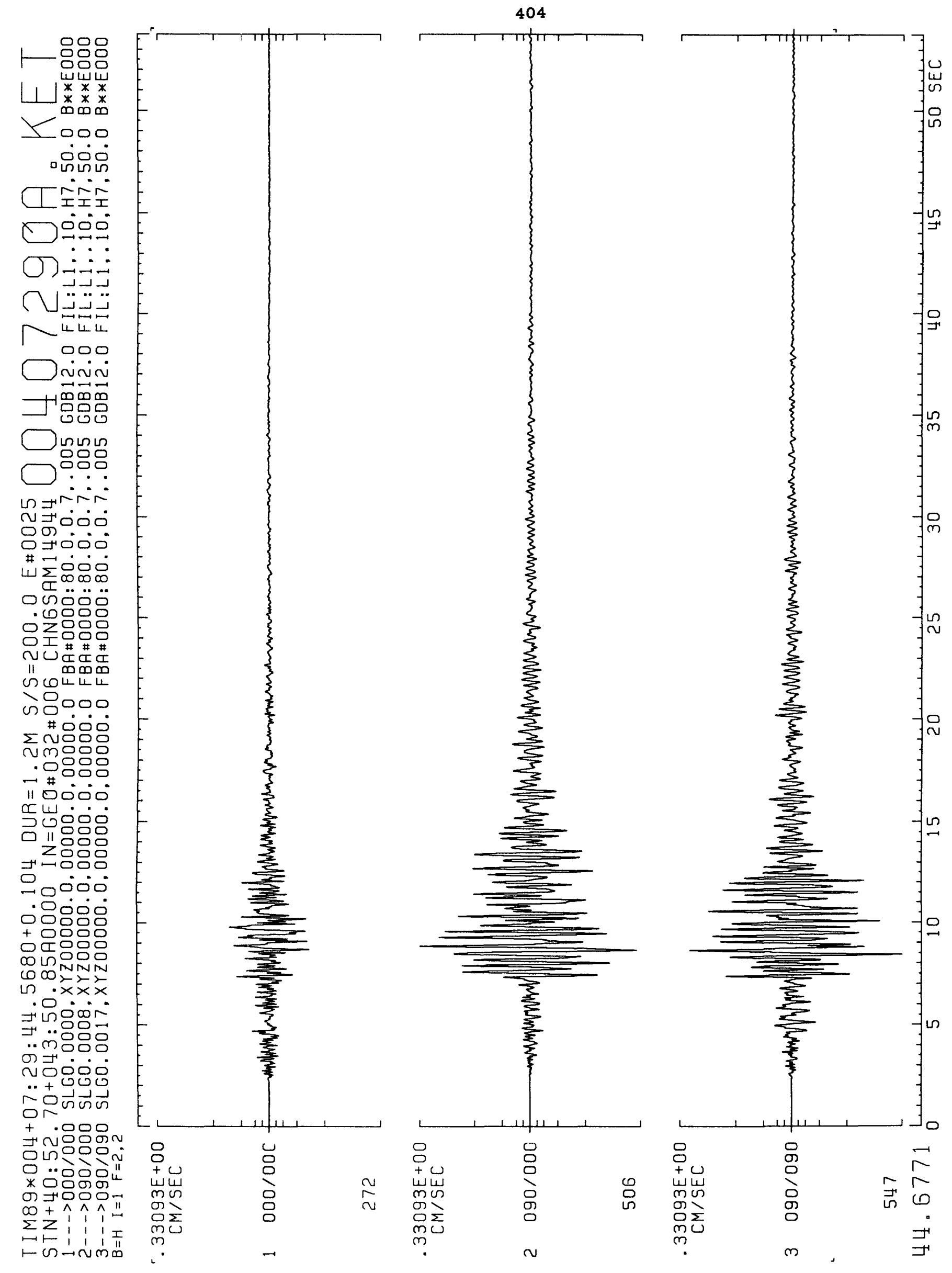




\section{5}
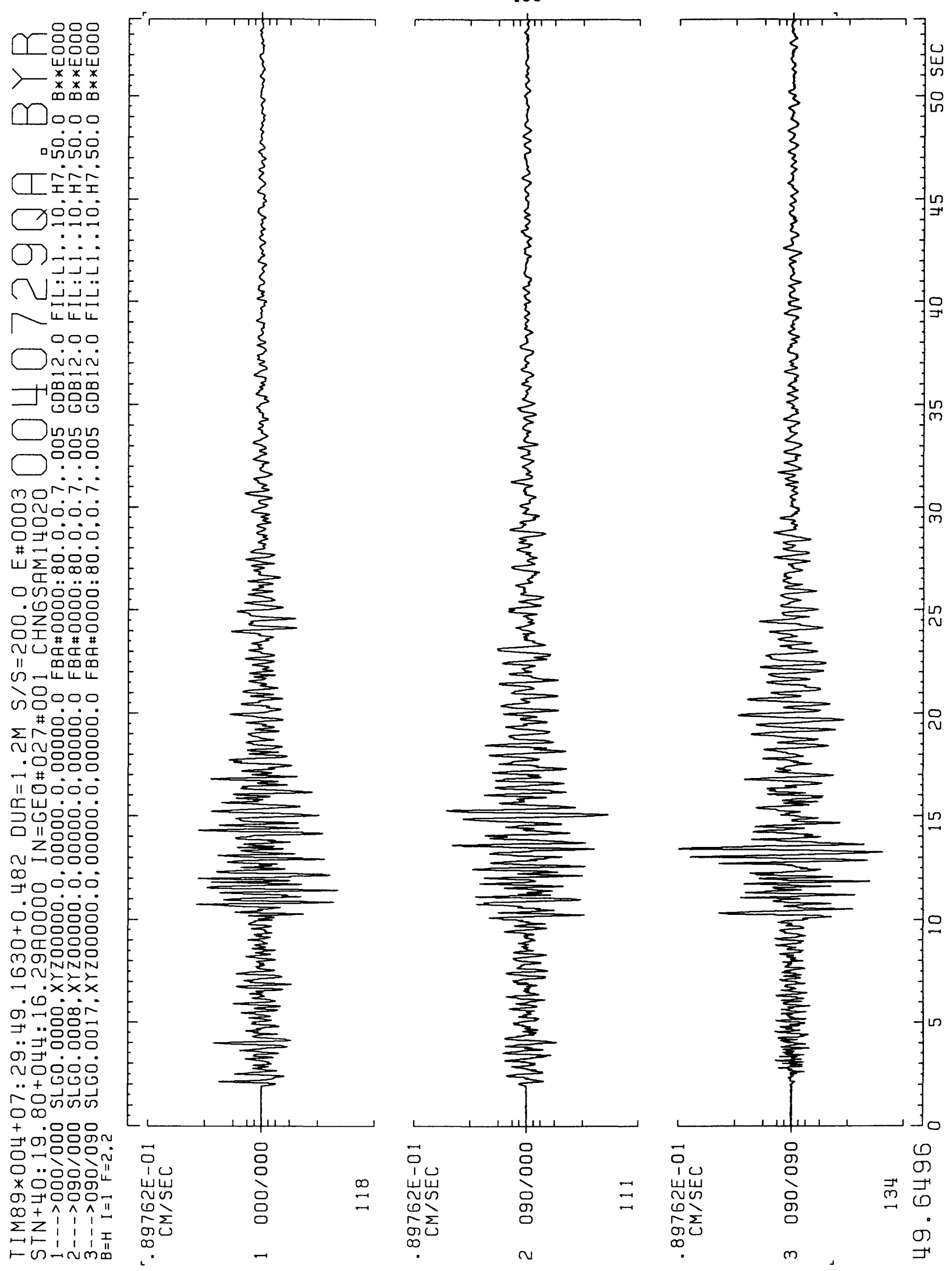


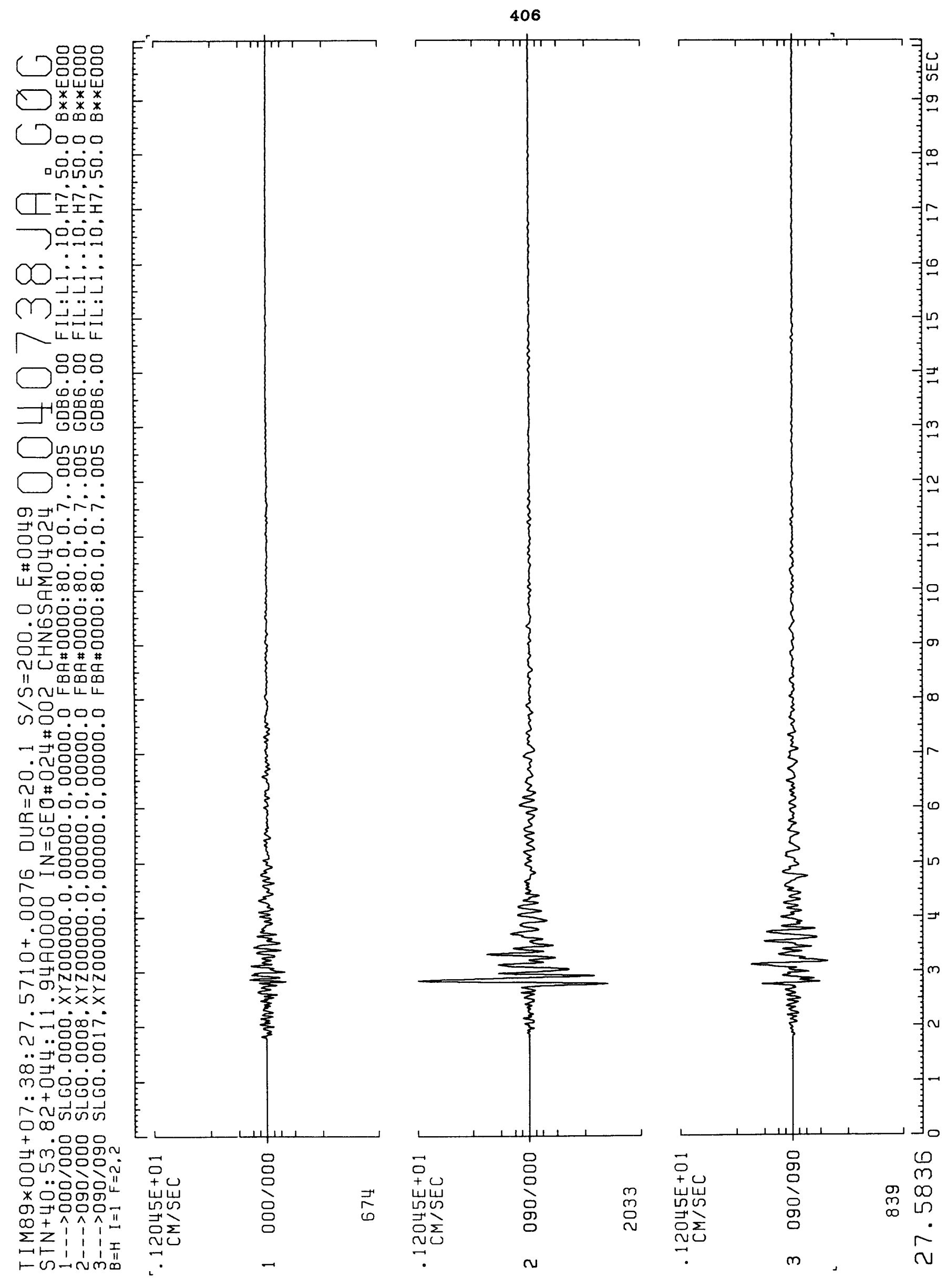




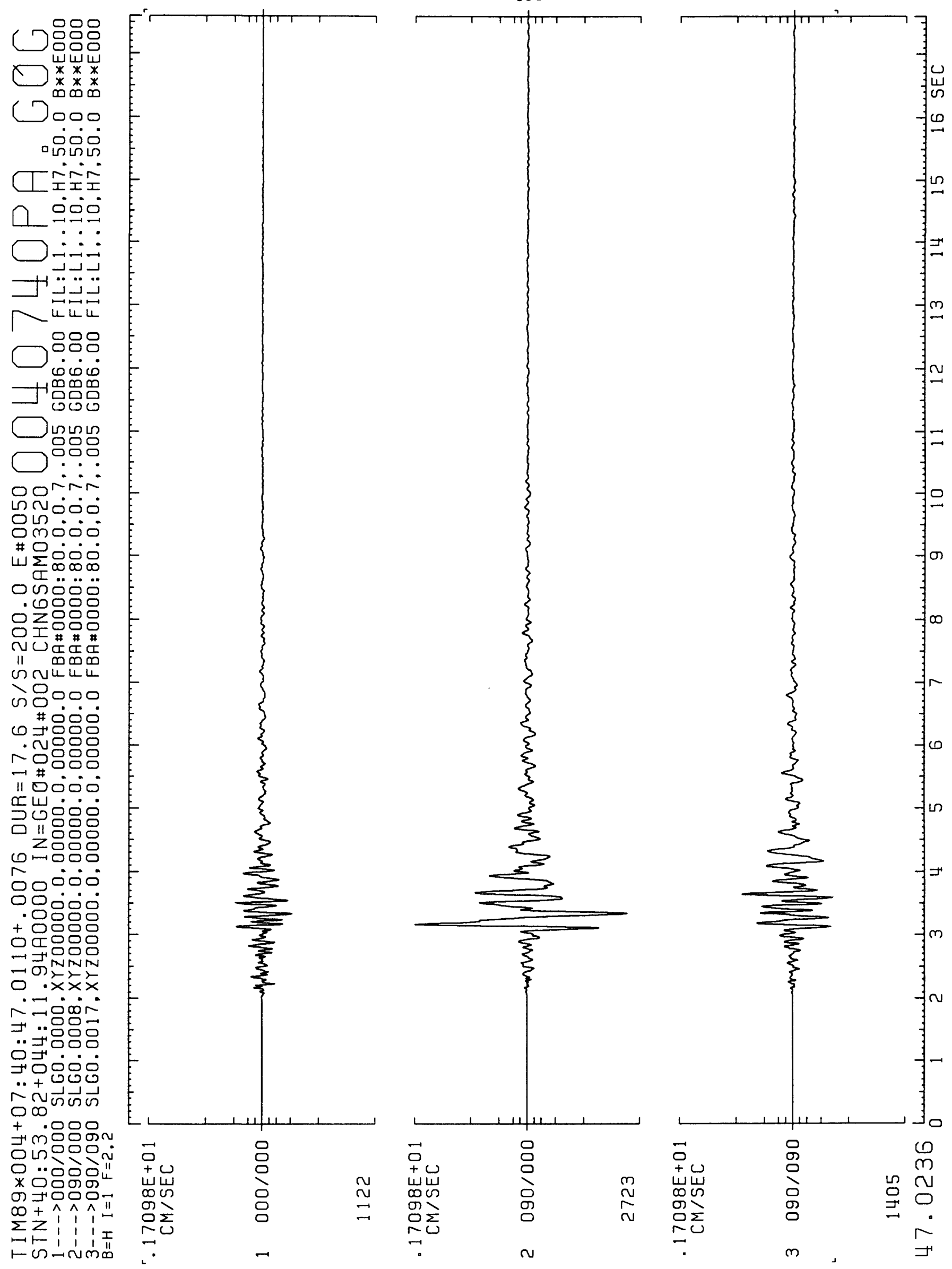

